\title{
COHOMOLOGY RINGS OF FINITE-DIMENSIONAL POINTED HOPF ALGEBRAS OVER ABELIAN GROUPS
}

\author{
N. ANDRUSKIEWITSCH, I. ANGIONO, J. PEVTSOVA, S. WITHERSPOON
}

\begin{abstract}
We show that the cohomology ring of a finite-dimensional complex pointed Hopf algebra with an abelian group of group-like elements is finitely generated. Our strategy has three major steps. We first reduce the problem to the finite generation of cohomology of finite dimensional Nichols algebras of diagonal type. For the Nichols algebras we do a detailed analysis of cohomology via the Anick resolution reducing the problem further to specific combinatorial properties. Finally, to check these properties we turn to the classification of Nichols algebras of diagonal type due to Heckenberger. In this paper we complete the verification of these combinatorial properties for major parametric families, including Nichols algebras of Cartan and super types and develop all the theoretical foundations necessary for the case-by-case analysis. The remaining discrete families are addressed in a separate publication. As an application of the main theorem we deduce finite generation of cohomology for other classes of finite-dimensional Hopf algebras, including basic Hopf algebras with abelian groups of characters and finite quotients of quantum groups at roots of one.
\end{abstract}

\section{Contents}

1. Introduction

1.1. Antecedents

1.2. The main result and applications

1.3. Scheme of the proof of Theorem 1.2.1

1.4. Finite-generation of cohomology for Nichols algebras

1.5. Future directions/applications

1.6. Organization of the paper

1.7. Conventions

Acknowledgements

\section{Part I. From cohomology of Nichols algebras to cohomology of Hopf} algebras

2. Finite-dimensional Hopf algebras

2.1. Morita equivalence of Hopf algebras

2.2. The role of Nichols algebras

2.3. Nichols algebras of diagonal type

2.4. Realizations

2.5. The Drinfeld double of a bosonization

3. Cohomology

Date: August 3, 2021.

MSC 2020: 16E40;16T20. 
3.1. Invariants

3.2. Subalgebras, extensions

16

3.3. Evens Lemma

17

3.4. The May spectral sequence

18

4. The Anick resolution

20

4.1. The setup

4.2. Application to Nichols algebras

20

4.3. Quantum linear spaces

4.4. Cohomology of graded algebras with convex PBW basis 26

5. Cohomology of the Drinfeld double

Part II. Permanent cocycles for Nichols algebras of diagonal type

6. The strategy

6.1. The setup

6.2. Degree 2 cocycles

6.3. Higher degree cocycles

6.4. Summary of the algorithm

7. Classical types

7.1. Types $A_{\theta}$ and $\mathbf{A}(j \mid \theta-j), \theta \geq 1, j \in \mathbb{I}_{\left\lfloor\frac{\theta+1}{2}\right\rfloor}$

7.2. Types $B_{\theta}$ and $\mathbf{B}(j \mid \theta-j), \theta \geq 1, j \in \mathbb{I}_{\theta-1}$

7.3. Type $B_{\theta, j}$ standard, $j \in \mathbb{I}_{\theta-1}$

7.4. Type $C_{\theta}$

7.5. Type $D_{\theta}$

7.6. Type $\mathbf{D}(j \mid \theta-j), \theta \geq 1, j \in \mathbb{I}_{\theta-1}$

8. Exceptional types

8.1. Type $E_{\theta}$

8.2. Type $F_{4}$

8.3. Type $G_{2}$ Cartan

8.4. Type $G_{2}$ standard

8.5. Type $\mathbf{D}(2,1 ; \alpha)$

8.6. Type $\mathbf{F}(4)$

8.7. Type $\mathbf{G}(3)$

9. Parametric modular types 74

9.1. Modular type wk(4) 74

9.2. Modular type br(2)

10. Proofs of the Computational Lemmas

10.1 .

References 


\section{INTRODUCTION}

1.1. Antecedents. A fundamental result in representation theory of a finite group scheme [FS, Theorem 1.1] is that its cohomology satisfies the finite generation property. Using the language of Hopf algebras it can be phrased as follows. Let $H$ be a finite-dimensional cocommutative Hopf algebra over a field $k$. Then

(fgc-a) The cohomology ring $\mathrm{H}(H, \mathbb{k})$ is finitely generated.

(fgc-b) For any finitely generated $H$-module $M, \mathrm{H}(H, M)$ is a finitely generated module over $\mathrm{H}(H, \mathbb{k})$.

Prior to the Friedlander-Suslin theorem, the result was known for group algebras of finite groups [G, $\mathrm{V}, \mathrm{Ev}$, restricted enveloping algebras [FP, AJ] and finite dimensional subalgebras of the Steenrod algebra [Wi]. At the end of the introduction of [FS], the authors observe that the cohomology ring of a finite-dimensional commutative Hopf algebra is easily seen to be finitely generated using the structure as in Wa and add:

We do not know whether it is reasonable to expect finite generation of the cohomology of an arbitrary finite-dimensional Hopf algebra.

Slowly, evidence confirming that this is indeed a reasonable question has emerged. In GK the cohomology ring of Lusztig's small quantum groups $u_{q}(\mathfrak{g}$ ) (in characteristic 0) under some restrictions on the parameters was identified as the coordinate ring of the nilpotent cone of the Lie algebra $\mathfrak{g}$. The restrictions on the parameters were weakened in BNPP]. The finite generation of cohomology was established for the duals of Lusztig's small quantum groups (in characteristic 0) [Go], for Lusztig's small quantum groups in positive characteristic [Dru1, for finite supergroup schemes [Dru2, for finite-dimensional complex pointed Hopf algebras whose group of grouplike elements is abelian and has order coprime to 210 [MPSW], for some pointed Hopf algebras of dimension $p^{3}$ [NWW, EOW] (in characteristic $p>0$ ), for the bosonizations of the Fomin-Kirillov algebra $\mathcal{F} \mathcal{K}_{3}$ with the group algebra of $\mathbb{S}_{3}$ and its dual [SV], for Drinfeld doubles of finite group schemes [FN,, $\mathbb{N}$ ]. In all the cases above, the approach is based to a greater or lesser extent on the knowledge of the structure of the Hopf algebras under consideration.

Finite tensor categories were introduced in [EO], where it was also conjectured that finite generation holds in this more general context. A systematic study of this question was started in [NP].

1.2. The main result and applications. In the present paper we work over an algebraically closed field $\mathbb{k}$ of characteristic 0 . For brevity we shall say that an augmented algebra $H$ has finitely generated cohomology (abbreviated as fgc) when both (fgc-a) and (fgc-b) hold. Our main result is the following:

Theorem 1.2.1. Let $H$ be a finite-dimensional pointed Hopf algebra whose group of grouplike elements is abelian. Then $H$ has finitely generated cohomology.

The class of finite-dimensional pointed Hopf algebras is the best understood and the subclass of those with abelian group of group-like elements is the only one whose classification is essentially complete. Theorem 1.2.1 goes beyond the situation treated in [MPSW] but uses the same approach to the classification of pointed Hopf algebras proposed in [AS1, AS2]. Let us mention the main differences between the setting of [MPSW], that invoked the classification result [AS3], and the present work. In the former, the associated 
braided vector space $V$ (described below) was of Cartan type and the deformations of the defining relations in the liftings took values in the group algebras. These restrictions, in the terminology introduced in $[\mathrm{NPe}$, guaranteed that the Nichols algebra $\mathscr{B}(V)$ had a smooth integration $Q \rightarrow \mathscr{B}(V)$ by an algebra of finite global dimension. That property, though not stated as explicitly, was crucial for the techniques in [MPSW]. When the restriction on the order of the group of group-like elements $G(H)$ is dropped, $V$ belongs to the list in the celebrated classification of $[\mathrm{H} 2$ but is not necessarily of Cartan type. The defining relations of the Nichols algebras and their deformations are more involved, see An1, An2, AAG, AnG, GaJ, $\mathrm{He}$ and conceptually different resulting in the absence of the crucial smooth integration property. In particular, as our results demonstrate, generating classes of the cohomology ring of a general Nichols algebra of diagonal type can lie in arbitrary large degrees whereas in the context of [MPSW] and whenever the algebra is smoothly integrable, generating classes lie in degree 2. We do have control over the degrees of the generators: they depend on vanishing of certain coefficients as stated in Remark 6.1.1. In the very computationally heavy Section [10 we calculate these coefficients which turn out to be quantum integers determined by the defining parameters of the Nichols algebra. By choosing different Nichols algebras from the classification list one can obtain various high values for the minimal degrees of the generators of the cohomology ring. Hence, handling this more general case of all Nichols algebras of diagonal type calls for development of new techniques which we present in this work.

We state two direct applications of Theorem 1.2.1 extending further the number of types of finite-dimensional Hopf algebras with finitely generated cohomology. We also observe that Theorem 3.1.7 provides another class of Hopf algebras satisfying fgc.

Theorem 1.2.2. Let $H$ be a finite-dimensional basic Hopf algebra whose group of characters is abelian. Then $H$ has finitely generated cohomology.

Basic Hopf algebras with abelian group of characters are just the duals of the Hopf algebras in Theorem 1.2.1, thus Theorem 1.2.2, that generalizes [Go], follows from Theorem 1.2.1, Lemma 2.1.1, Corollary 3.2.3 and Theorem 5.0.6

Theorem 1.2.3. Let $H$ be a finite-dimensional Hopf algebra that fits into an extension $\mathbb{k} \rightarrow K \rightarrow H \rightarrow L \rightarrow \mathbb{k}$, where $K$ is semisimple and $L$ is either pointed with abelian group of group-like elements or else basic with abelian group of characters. Then $H$ has fgc.

Theorem 1.2.3 follows from Lemma 3.2.5 and one of the previous two theorems. Quotients of algebras of functions on quantum groups at roots of one (of various kinds) were classified in $\mathrm{AG}, \mathrm{Ga}, \mathrm{GaG}$. In particular, these results provide families of Hopf algebras $H$ that fit into an extension $\mathbb{k} \rightarrow \mathbb{k}^{|G|} \rightarrow H \rightarrow L \rightarrow \mathbb{k}$ where $G$ is a finite group and $L$ is a finite-dimensional basic Hopf algebra with abelian group of characters; thus Theorem 1.2 .3 applies to them.

1.3. Scheme of the proof of Theorem 1.2.1. Let $H$ be a finite-dimensional pointed Hopf algebra with abelian group of group-like elements $\Gamma:=G(H)$ so that the coradical of $H$ is $H_{0} \simeq \mathbb{k} \Gamma$. Let $D(H)$ be the the Drinfeld double of $H$, let gr $H$ be the graded Hopf algebra associated to the coradical filtration and let $V$ be the infinitesimal braiding of $H$, see 2.2. We know that gr $H \simeq \mathscr{B}(V) \# \mathbb{k} \Gamma$ [An2]. Then the Nichols algebra $\mathscr{B}(V)$ 
is finite-dimensional. We shall use $V^{\#}=\operatorname{Hom}_{k}(V, k)$ to denote the $k$-linear dual. The key point in the proof of Theorem 1.2.1 is the following.

Theorem 1.3.1. Let $U$ be a braided vector space of diagonal type such that the Nichols algebra $\mathscr{B}(U)$ has finite dimension. Then $\mathscr{B}(U)$ has fgc.

This Theorem being proved, the rest of the proof proceeds in the following steps:

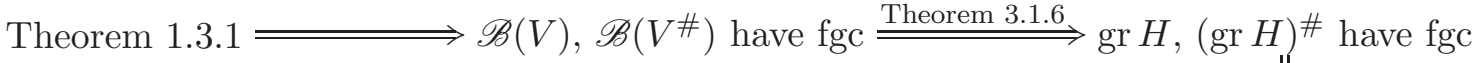

$$
\begin{aligned}
& \text { Theorem } 5.0 .6
\end{aligned}
$$

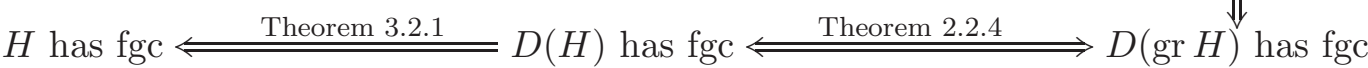

1.4. Finite-generation of cohomology for Nichols algebras. We next outline the proof of Theorem 1.3.1 referring to 2.3 for unexplained terminology.

1.4.1. Reduction to the connected case. By Theorem 5.0.1, we conclude that Theorem 1.3.1 holds for $U$ if and only if it holds for $U_{J}$ for every connected component $J \in \mathcal{X}$.

We assume for the rest of this Subsection that the Dynkin diagram of $U$ is connected.

1.4.2. The Anick resolution. The Nichols algebra $\mathscr{B}(U)$ has a convex PBW-basis, hence a suitable filtration. Its associated graded ring gr $\mathscr{B}(U)$ is a quantum linear space. The cohomology ring of gr $\mathscr{B}(U)$ is well-known, but we provide a computation using the Anick resolution Ani specifically in order to relate it to permanent cycles in a suitable spectral sequence. See 4.3

Since the Anick resolution is compatible with the mentioned filtration on $\mathscr{B}(U)$, we may use a spectral sequence argument based on Evens Lemma 3.3 .2 to reduce the finite generation of $\mathrm{H}(\mathscr{B}(U), \mathbb{k})$ to the verification of the following statement.

Condition 1.4.1. For every positive root $\gamma \in \Delta_{+}^{U}$, there exists $L_{\gamma} \in \mathbb{N}$ such that the cochain $\left(\mathrm{x}_{\gamma}^{L_{\gamma}}\right)^{*}$ is a cocycle, that is, represents an element in $\mathrm{H}(\mathscr{B}(U), \mathbb{k})$.

The elements $\left(\mathrm{x}_{\gamma}^{L_{\gamma}}\right)^{*}$ are cochains of the complex $\operatorname{Hom}\left(C_{*}(\mathscr{B}(U), \mathbb{k})\right.$ which computes $\mathrm{H}(\mathscr{B}(U), \mathbb{k})$ and are defined prior to Theorem 4.4.3. If Condition 1.4.1 holds, then Theorem 4.4 .3 implies that $\mathscr{B}(U)$ has fgc.

1.4.3. Reduction to Weyl-equivalence. In practice, given $U$ we shall prove that Condition 1.4.1 holds for any braided vector space with the same Dynkin diagram as $U$, particularly for $U^{\#}$. Let $G$ be any finite abelian group such that $U$ is realized in $\mathbb{k}_{\mathbb{k} G}^{\mathbb{R} G} \mathcal{Y} \mathcal{D}$. By Theorem 3.1 .6 and Theorem 5.0.6 we see that $D(\mathscr{B}(U) \# \mathbb{k} G)$ has fgc.

We apply this last claim as follows: let $U^{\prime}$ be a braided vector space of diagonal type which is Weyl-equivalent to $U$ (see $\S$ 2.3.2). This implies that $U^{\prime}$ is realized as YetterDrinfeld module over $G$ and there is an algebra isomorphism

$$
D(\mathscr{B}(U) \# \mathbb{k} G) \simeq D\left(\mathscr{B}\left(U^{\prime}\right) \# \mathbb{k} G\right) .
$$

By Corollary $3.2 .2 \mathscr{B}\left(U^{\prime}\right)$ has fgc. That is, we only need to deal with one representative of each Weyl-equivalence class which drastically reduced the amount of computations we need to perform to finish the proof. 
1.4.4. Verification of Condition [1.4.1. We argue case-by-case using the list of [H2]; by the preceding discussion we just need to consider one representative in each Weyl-equivalence class - and we could choose the most convenient for our purpose. We also argue recursively on $\operatorname{dim} U$. All in all, we reduce the verification to claims on Nichols algebras of diagonal type, see 6.2 and we deal with them using information on the PBW-basis from AA.

1.5. Future directions/applications. We expect that our methods can be applied to prove fgc property for finite-dimensional Hopf algebras beyond the setting of Theorem 1.2.1, for instance, pointed but with non-abelian group of group-like elements $G(H)$. We outline here the steps which will need to be taken to follow our general strategy.

Let $H$ be a finite-dimensional Hopf algebra whose coradical $H_{0}$ is a Hopf subalgebra. Let $V$ be the infinitesimal braiding of $H$. Then gr $H \simeq R \# H_{0}$ where $R$ is a connected graded Hopf algebra in the category of Yetter-Drinfeld modules over $H_{0},{ }_{H_{0}}^{H_{0}} \mathcal{Y D}$, and $\mathscr{B}(V)$ is a graded Hopf subalgebra of $R$. To prove that $H$ has fgc following the scheme presented in 1.3 one would need to address these problems:

(i) Prove that $\mathscr{B}(V)$ and $\mathscr{B}\left(V^{\#}\right)$ have fgc.

(ii) Is $R=\mathscr{B}(V)$ ? (in all known examples in characteristic 0 the answer is positive). If not, prove that $R$ and $R^{\#}$ have fgc.

(iii) Prove Lemma 3.1.4 for any semisimple Hopf algebra (and not just for group algebras and their duals). Together with (ii) this would give that gr $H$, (gr $H)^{\#}$ have fgc.

(iv) Extend Theorem 5.0.6 to prove that $D($ gr $H)$ has fgc. Even in the pointed case, we would need Lemma 3.1 .4 for $D(\mathbb{k} G(H))$ to prove this conjectural extension.

(v) Is $H$ a cocycle deformation of gr $H$ or at least Morita equivalent to gr $H$ as in 22.1? (in all known examples in characteristic 0 the answer is positive). This would imply that $D(H)$, and a fortiori $H$, have fgc since Theorem 3.2.1 holds in general.

We also notice that a large part of this approach could be used in positive characteristic under appropriate assumptions, e.g. the coradical $H_{0}$ needs to be a semisimple Hopf subalgebra.

1.6. Organization of the paper. Part $\square$ starts with a recollection of facts on Hopf and Nichols algebras in Section 2. Section 3 contains several preliminary results on cohomology including the passage from the cohomology of $\mathscr{B}(V)$ to the cohomology of $\mathscr{B}(V) \# \mathbb{k} \Gamma$ and versions of the Evens Lemma and the May spectral sequence crucial for our arguments. Section 4 presents the Anick resolution and the reduction to Condition 1.4.1. In the last Section 5 of this Part it is shown that the Drinfeld double of $\mathscr{B}(V) \# \mathbb{k} \Gamma$ has fgc provided that $\mathscr{B}(V)$ has via considerations of cohomology for twisted tensor products.

Parts Ii is devoted to the proof of Condition 1.4.1. Section 6 presents the strategy of the verification with proofs of technical Lemmas postponed to Section 10, We verify Condition 1.4.1 for finite-dimensional Nichols algebras of diagonal type belonging to families with continuous parameter. We proceed case by case in Sections 7 , 8 and 9 corresponding respectively to classical (Cartan, standard and super) types, exceptional (Cartan, standard and super) types, and Nichols algebras with the same root systems as the modular Lie 
algebras wk(4) and $b r(2)$. The remaining Nichols algebras of diagonal type are dealt with in a separate publication AAPPW of more computational nature.

1.7. Conventions. For $\ell<\theta \in \mathbb{N}_{0}$, we set $\mathbb{I}_{\ell, \theta}=\{\ell, \ell+1, \ldots, \theta\}, \mathbb{I}_{\theta}=\mathbb{I}_{1, \theta}$. Let $\mathbb{G}_{N}$ be the group of roots of unity of order $N$ in $\mathbb{k}$ and $\mathbb{G}_{N}^{\prime}$ the subset of primitive roots of order $N ; \mathbb{G}_{\infty}=\bigcup_{N \in \mathbb{N}} \mathbb{G}_{N}$ and $\mathbb{G}_{\infty}^{\prime}=\mathbb{G}_{\infty}-\{1\}$. If $L \in \mathbb{N}$ and $q \in \mathbb{K}^{\times}$, then $(L)_{q}:=\sum_{j=0}^{L-1} q^{j}$.

All vector spaces, algebras and tensor products are over $\mathbb{k}$. We use $V^{\#}$ to denote the linear dual to a vector space $V, V^{\#}=\operatorname{Hom}_{k}(V, k)$.

By abuse of notation, $\left\langle a_{i}: i \in I\right\rangle$ denotes either the group, the subgroup or the vector subspace generated by all $a_{i}$ for $i$ in an indexing set $I$, the meaning being clear from the context. Instead, the subalgebra generated by all $a_{i}$ for $i \in I$ is denoted by $\mathbb{k}\left\langle a_{i}: i \in I\right\rangle$.

If $A$ is an associative augmented algebra and $M$ is an $A$-module, then we set

$$
\mathrm{H}^{n}(A, M)=\operatorname{Ext}_{A}^{n}(\mathbb{k}, M), \quad \mathrm{H}(A, M)=\oplus_{n \in \mathbb{N}_{0}} \mathrm{H}^{n}(A, M) .
$$

Then $\mathrm{H}(A, \mathbb{k})=\oplus_{n \in \mathbb{N}_{0}} \mathrm{H}^{n}(A, \mathbb{k})$ is isomorphic to the Hochschild cohomology $\operatorname{HH}(A, \mathbb{k})=$ $\oplus_{n \in \mathbb{N}_{0}} \operatorname{Ext}_{A \otimes A^{\text {op }}}^{n}(A, \mathbb{k})$ via an equivalence of bar complexes; see for example [MPSW, (2.4.1)].

Let $P_{*}(A)$ be the normalized bar resolution of $\mathbb{k}$ in the category of left $A$-modules and let $\Omega^{*}(A)=\operatorname{Hom}_{A}\left(P_{*}(A), \mathbb{k}\right)$, in particular $\Omega^{n}(A)=\operatorname{Hom}_{\mathbb{k}}\left(A_{+}^{\otimes n}, \mathbb{k}\right)$, where $A_{+}$is the augmentation ideal.

Let $\widehat{\Gamma}=\operatorname{Hom}_{\mathrm{ab}}\left(\Gamma, \mathbb{k}^{\times}\right)$be the character group of an abelian group $\Gamma$.

Acknowledgements. J. P. thanks Eric Friedlander for introducing her to the problem of finite generation and for many invaluable discussions about it. S. W. and J. P. thank Jon Carlson for bestowing his computer prowess and wisdom on them in the early stages of this project. N. A. thanks Cris Negron and Sonia Natale for very useful conversations. I. A. thanks Leandro Vendramin for joint work on the program to compute root systems of Nichols algebras. We also thank the referee for the careful reading of our work and very valuable comments.

The authors are extremely grateful to the hospitality and support of the American Institute for Mathematics (AIM) via their SQuaREs program. N. A. was supported by the NSF under Grant No. DMS-1440140, while he was in residence at the Mathematical Sciences Research Institute in Berkeley, California, in the Spring semester of 2020. J. P. was supported by the NSF grants DMS-1501146, DMS-1901854 and Brian and Tiffinie Pang faculty fellowship. S. W. was supported by the NSF grants DMS-1401016 and DMS1665286. The work of N. A. and I. A. was partially supported by CONICET and Secyt (UNC).

\section{Part I. From cohomology of Nichols algebras to cohomology of Hopf algebras}

\section{Finite-Dimensional Hopf Algebras}

2.1. Morita equivalence of Hopf algebras. In this subsection, no restrictions on the the field $\mathbb{k}$ are needed. Let $H$ be a finite-dimensional Hopf algebra. We refer to $[\mathbb{R}]$ for 
the definitions of the Drinfeld double $D(H)$ of $H$ and of the (braided tensor) category ${ }_{H}^{H} \mathcal{Y} \mathcal{D}$ of Yetter-Drinfeld modules over $H$. It is well-known that ${ }_{H}^{H} \mathcal{Y} \mathcal{D}$ is the Drinfeld center of the category of $H$-modules and that it is braided tensor equivalent to the category of $D(H)$-modules.

Let $H^{\prime}$ be another finite-dimensional Hopf algebra. Borrowing terminology from $\mathbf{M u}$, ENO, we say that $H$ and $H^{\prime}$ are Morita equivalent, denoted $H \sim_{\text {Mor }} H^{\prime}$, if there is an isomorphism of quasitriangular Hopf algebras $D(H) \simeq D\left(H^{\prime}\right)$. This is not the same as Morita equivalent as algebras!

Lemma 2.1.1. $H$ is Morita equivalent to $H^{\prime}$ in the following cases:

(a) $H^{\prime} \simeq H^{\#}$, the dual Hopf algebra.

(b) $H^{\prime} \simeq H^{F}$ is a twist of $H[\mathrm{Dr}, \mathrm{Re}$, i.e. there exists $F \in H \otimes H$ invertible such that $H^{F}=H$ as algebra and has the comultiplication $\Delta^{F}=F \Delta F^{-1}$.

(c) $H^{\prime} \simeq H_{\sigma}$ is a cocycle deformation of $H$ [DoT], i.e. there exists an invertible 2-cocycle $\sigma: H \otimes H \rightarrow \mathbb{k}$ such that $H_{\sigma}=H$ as coalgebra and has the multiplication $x \cdot{ }_{\sigma} y=$ $\sigma\left(x_{(1)} \otimes y_{(1)}\right) x_{(2)} y_{(2)} \sigma^{-1}\left(x_{(3)} \otimes y_{(3)}\right)$.

Proof. (a) This follows from [AGr, Proposition 2.2.1] because of the identification of the category of Yetter-Drinfeld modules over $\mathrm{H}$ with the category of representations of $\mathrm{D}(\mathrm{H})$ as mentioned in Subsection 2.1. (b) follows since the categories of $H$ and $H^{F}$-modules are tensor equivalent $[\mathrm{Dr}$, p. 1422]. Finally $(\mathrm{c})$ is a consequence of the preceding, as $\left(H_{\sigma}\right)^{\#} \simeq\left(H^{\#}\right)^{F}$ where $F=\sigma$ in $H^{\#} \otimes H^{\#}$.

2.2. The role of Nichols algebras. Even though our primary interest is in cohomology of finite dimensional Hopf algebras, the Nichols algebras which originated in independent work of Nichols and Woronowicz show up very naturally in our study. Here we give a very brief account of the general approach to the classification of finite dimensional Hopf algebras over an algebraically closed field $\mathbb{k}$ of characteristic 0 , highlighting the importance of Nichols algebras in their structure. The reader can find all the precise definitions and details in the surveys AS2, A.

Let $H$ be a Hopf algebra, $H_{0}$ its coradical $\left[\underline{\mathbb{R}}\right.$ and $H_{[0]}=\mathbb{k}\left\langle H_{0}\right\rangle$ its Hopf coradical, a Hopf subalgebra of $H[\mathrm{AC}$. The classification of finite-dimensional Hopf algebras can be organized in four classes, according to the relative behavior of $H_{0}$ and $H_{[0]}$ :

(a) $H=H_{0}$, i.e. $H$ is cosemisimple.

(c) $H \neq H_{[0]}=H_{0}$.

(b) $H=H_{[0]} \neq H_{0}$.

(d) $H \neq H_{[0]} \neq H_{0}$.

Hopf algebras in class (a) are semisimple by a theorem of Larson and Radford. Albeit families of examples and some classification results in low dimension are known, no systematic approach to the classification is available. Being semisimple, they are not interesting for our cohomology explorations since cohomology simply vanishes in positive degrees.

Similarly to (a), the class (b) has no proposed method to deal with the classification, but contrary to (a), cohomology rings are far from trivial. Even though there is no classification, many examples are known; some of them have the fgc property by Theorem 1.2.2.

Hopf algebras in classes (c) and (d) have interesting cohomology rings and this is where Nichols algebras become highly relevant. The approach we develop allows us to deal with 
class (c) under some additional assumptions on $H_{0}$ though we expect that it can be applied more generally. The class (d) is not as rigidly structured as the class (c) and even though the Nichols algebras still play a central role we do not know enough about the structure to make conclusions about cohomology yet. We will mention a little bit more about case (d) towards the end of this subsection.

Now let us consider case (c) which is the case of interest for this paper. If $H$ is in class (c) then $H_{0}$ is a proper Hopf subalgebra. Let gr $H$ be the graded Hopf algebra associated to the coradical filtration of $H$; then

$$
\text { gr } H \simeq R \# H_{0}
$$

where $R=\oplus_{n \in \mathbb{N}_{0}} R^{n}$ is a connected graded Hopf algebra in the braided monoidal category ${ }_{H_{0}}^{H_{0}} \mathcal{Y D}$, called the diagram of $H$. We also say that $H$ is a lifting of $R$, or of $R \# H_{0}$. Then $R$ is coradically graded, hence its subalgebra generated by $V:=R^{1}$ is isomorphic to the Nichols algebra $\mathscr{B}(V)$; see AS2 for details. The braided vector space $V$ is an important invariant of $H$ called its infinitesimal braiding.

We make the following additional assumptions on $H$. Assume that $H$ is pointed, that is $H_{0}=k \Gamma$; also assume that $\Gamma$ is abelian. In that case the infinitesimal braiding $V$ is of diagonal type (see Subsection 2.3. $)$. Then the following two properties hold:

$$
\begin{gathered}
R \simeq \mathscr{B}(V), \\
\exists \sigma: \operatorname{gr} H \otimes \operatorname{gr} H \rightarrow \mathbb{k} \text { such that }(\operatorname{gr} H)_{\sigma} \simeq H .
\end{gathered}
$$

The first property (2.2.2) holds by [An2, Theorem 2], see also [AS3, Theorem 5.5]; notice that the proof uses the classification in $\mathrm{H} 2$ and the main result on convex orders from An1. The second property (2.2.3) holds by [AnG, Theorem 1.1], based on previous studies of the lifting question and the explicit relations from [An2, Theorem 3.1], that again uses [H2, An1]. Summarizing, we get the following structure theorem:

Theorem 2.2.4. Let $H$ be a finite-dimensional pointed Hopf algebra such that the group of group-like elements $G(H)$ is abelian. Then $H$ is a cocycle deformation of the bosonization of a Nichols algebra of diagonal type: $H \simeq(\mathscr{B}(V) \# \mathbb{k} G(H))_{\sigma}$. Hence $H \sim_{\text {Mor }}$ gr $H$.

The theorem implies that to verify fgc for $H$ it suffices to verify it for the Nichols algebra $\mathscr{B}(V)$ and for the Drinfeld double $D(\operatorname{gr} H)$ which is what we do in Theorem 3.1.7 and Corollary 3.2.3.

We point out that (2.2.2) and (2.2.3) have been verified in most known examples in class (c) beyond pointed Hopf algebras with abelian group of group-like elements and are expected to hold generally. Hence, generalizing Theorem 3.1.7 and Corollary 3.2.3 to the other cases in class (c) one should be able to reduce the question of fgc for $H$ again to the same question for $\mathscr{B}(V)$ and the Drinfeld double for gr $H$. We do not pursue this direction in this paper but we do expect our methods to be fruitful in these cases as well, potentially allowing one to finish off proving fgc property for all Hopf algebras in class (c).

Finally, let $H$ be in class (d). Then one considers the graded Hopf algebra gr $H$ associated to the standard filtration of $H[\mathrm{AC}]$; again

$$
\text { gr } H \simeq R \# H_{[0]}
$$


where $R=\oplus_{n \in \mathbb{N}_{0}} R^{n}$ is a connected graded Hopf algebra in the braided monoidal category $H_{[0]}^{H_{[0]}} \mathcal{Y D}$. But it is not known whether $R$ is coradically graded, or its subalgebra $R^{\prime}$ generated by $V:=R^{1}$ is isomorphic to the Nichols algebra $\mathscr{B}(V)$. We do know that $\mathscr{B}(V)$ is a quotient of $R^{\prime}$ but the present approach does not allow to reduce the question of finitely generated cohomology for $H$ to the analogous question for $\mathscr{B}(V)$. So fgc property for class (d) is completely open.

2.3. Nichols algebras of diagonal type. Since finite-dimensional Nichols algebras of diagonal type are central in this paper, we present here the features more relevant for our goals and refer to $\mathrm{AA}$ for an exposition. The input is a matrix of non-zero scalars $\mathfrak{q}=\left(q_{i j}\right)_{i, j \in \mathbb{I}}$ where $\mathbb{I}=\mathbb{I}_{\theta}, \theta \in \mathbb{N}$. To this datum we attach a braided vector space of diagonal type $V$ with a basis $\left(x_{i}\right)_{i \in \mathbb{I}}$ and braiding $c^{\mathfrak{q}} \in G L(V \otimes V)$ given by

$$
c^{\mathfrak{q}}\left(x_{i} \otimes x_{j}\right)=q_{i j} x_{j} \otimes x_{i}, \quad i, j \in \mathbb{I} .
$$

The corresponding Nichols algebra is a graded connected algebra with strong properties denoted here mostly as $\mathscr{B}_{\mathfrak{q}}$ instead of $\mathscr{B}(V)$. For these Nichols algebras substantial information is available.

2.3.1. Dynkin diagrams and positive roots. We codify as usual the matrix $\mathfrak{q}$ in a (generalized) Dynkin diagram $\mathcal{D}$ with vertices numbered by $\mathbb{I}$ and labelled with $q_{i i}$, while two different vertices $i$ and $j$ are joined by an edge only if $\widetilde{q}_{i j}:=q_{i j} q_{j i} \neq 1$ in which case the edge is labelled with $\widetilde{q}_{i j}$ :

$$
\underset{i}{q_{i i}} \stackrel{\widetilde{q}_{i j}}{\underset{i}{q_{j j}}} \underset{j}{\stackrel{q_{j}}{j}}
$$

Two different matrices with the same Dynkin diagram are called twist-equivalent AS2.

The Nichols algebra $\mathscr{B}_{\mathfrak{q}}$ has a very useful $\mathbb{N}_{0}^{\mathbb{I}}$-grading given by the rule $\operatorname{deg} x_{i}=\alpha_{i}, i \in \mathbb{I}$, where $\left(\alpha_{i}\right)_{i \in \mathbb{I}}$ is the canonical basis of $\mathbb{Z}^{\theta}$. By [Kh, Theorem 2.2], $\mathscr{B}_{\mathfrak{q}}$ has a PBW basis

$$
B=\left\{s_{1}^{e_{1}} \ldots s_{t}^{e_{t}}: t \in \mathbb{N}_{0}, s_{i} \in S, s_{1}>\cdots>s_{t}, 0<e_{i}<h\left(s_{i}\right)\right\} .
$$

where $S$ is an ordered set of $\mathbb{N}_{0}^{\mathbb{I}}$-homogeneous elements and $h: S \mapsto \mathbb{N} \cup\{\infty\}$ is a function called the height. The following set does not depend on the choice of $B$ :

$$
\Delta_{+}^{\mathfrak{q}}:=\{\operatorname{deg} s: s \in S\} \subset \mathbb{N}_{0}^{\mathbb{I}} .
$$

Occasionally we set $\Delta_{+}^{V}=\Delta_{+}^{\mathfrak{q}}$. The elements of $\Delta_{+}^{\mathfrak{q}}$ are called the (positive) roots of $\mathscr{B}_{\mathfrak{q}}$. We assume from now on that

$$
\operatorname{dim} \mathscr{B}_{\mathfrak{q}}<\infty .
$$

Then $\Delta_{+}^{\mathfrak{q}}$ is a finite set and the map $S \rightarrow \Delta_{+}^{\mathfrak{q}}, s \mapsto \operatorname{deg} s$, is bijective. Also $\Delta_{+}^{\mathfrak{q}}$ admits a convex (total) order in the sense

$$
\alpha, \beta, \alpha+\beta \in \Delta_{+}^{\mathfrak{q}}, \alpha<\beta \Longrightarrow \alpha<\alpha+\beta<\beta .
$$

See [An1. The convex order is not unique; in the case-by-case analysis below we use that of [AA] except when a more suitable choice is possible that we mention explicitly. 
A connected component of $\mathcal{D}$ is a subset $J$ of $\mathbb{I}$ such that the matrix $\mathfrak{q}_{J}=\left(q_{i j}\right)_{i, j \in J}$ gives rise to a connected Dynkin subdiagram $\mathcal{D}_{J}$ of $\mathcal{D}$ and is maximal with this property. Let $\mathcal{X}$ be the set of connected components of $\mathcal{D}$. For $J \subset \mathbb{I}$, we say that a positive root (an element of $\Delta_{+}^{\mathfrak{q}}$ ) is supported on $J$ if it belongs to the subset $\mathbb{N}_{0}^{J} \subset \mathbb{N}_{0}^{\mathbb{I}}$ where the containment is induced by the inclusion $J \subset \mathbb{I}$. We say that a root has full support if it's not supported on any proper subset of $\mathbb{I}$. If $J \subset \mathbb{I}$, we identify $\Delta_{+}^{\mathfrak{q}_{J}}$ with the subset $\Delta_{+}^{J}$ of $\Delta_{+}^{\mathfrak{q}}$ of roots with support in $J$. We also denote by $V_{J}$ the subspace of $V$ spanned by $\left(x_{j}\right)_{j \in J}$. By a result of Graña, we have

$$
\mathscr{B}_{\mathfrak{q}} \simeq \underline{\bigotimes}_{J \in \mathcal{X}} \mathscr{B}_{\mathfrak{q}_{J}}, \quad \Delta_{+}^{\mathfrak{q}}=\coprod_{J \in \mathcal{X}} \Delta_{+}^{J} .
$$

Here $\underline{\otimes}$ means the braided tensor product of algebras.

2.3.2. Weyl equivalence. Let $\mathfrak{q}$ be such that $\operatorname{dim} \mathscr{B}_{\mathfrak{q}}<\infty$ and let $G$ be a finite abelian group such that $\left(V, c^{\mathfrak{q}}\right)$ is realized in $\mathbb{k} G G_{\mathbb{K} G}^{\mathbb{R} G} \mathcal{D}$. Given $i \in \mathbb{I}$, one defines a matrix $\rho_{i}(\mathfrak{q})$ by a precise rule [H1] or [AA, (2.25)]. Then, although the matrices $\mathfrak{q}$ and $\rho_{i}(\mathfrak{q})$ might be quite different, there is an algebra isomorphism $T_{i}: D\left(\mathscr{B}_{\mathfrak{q}} \# \mathbb{k} G\right) \rightarrow D\left(\mathscr{B}_{\rho_{i}(\mathfrak{q})} \# \mathbb{k} G\right)$, see [H1]. The assignments $\mathfrak{q} \mapsto \rho_{i}(\mathfrak{q})$, for all $i \in \mathbb{I}$, generate the so-called Weyl groupoid of $\mathfrak{q}$ [H1]. Two matrices $\mathfrak{q}$ and $\mathfrak{q}^{\prime}$ are Weyl-equivalent if there exists a sequence $i_{1}, \ldots, i_{N} \in \mathbb{I}$ such that $\mathfrak{q}^{\prime}=\rho_{i_{1}} \ldots \rho_{i_{s}}(\mathfrak{q})$. In this case one also says that the braided vector spaces $\left(V, c^{\mathfrak{q}}\right)$ and $\left(V^{\prime}, c^{\mathfrak{q}^{\prime}}\right)$ are Weylequivalent. Hence there is an algebra isomorphism $D\left(\mathscr{B}_{\mathfrak{q}} \# \mathbb{k} G\right) \rightarrow D\left(\mathscr{B}_{\mathfrak{q}^{\prime}} \# \mathbb{k} G\right)$. See $\underline{\mathrm{AA}}$, Section 2.6] for an exposition.

2.3.3. Classification. The classification of the matrices $\mathfrak{q}$ such that $\operatorname{dim} \mathscr{B}_{\mathfrak{q}}<\infty$ was achieved in [H2 (the result is slightly more general). By the preceding discussion we may assume that $\mathcal{D}$ is connected. As in $[\mathrm{AA}]$ we organize the classification in 4 types:

(a) Cartan type.

(b) Super type. (c) Modular type.

(d) UFO type.

The type refers to the connection with different parts of Lie theory, see loc. cit. We shall check Condition 1.4.1 for each entry of the classification of [H2].

2.3.4. Root vectors. For brevity, we set

$$
x_{i j}=\operatorname{ad}_{c} x_{i}\left(x_{j}\right), \quad i \neq j \in \mathbb{I}
$$

more generally, the iterated braided commutators are

$$
x_{i_{1} i_{2} \cdots i_{k}}:=\left(\operatorname{ad}_{c} x_{i_{1}}\right) \cdots\left(\operatorname{ad}_{c} x_{i_{k-1}}\right)\left(x_{i_{k}}\right), \quad i_{1}, i_{2}, \cdots, i_{k} \in \mathbb{I} .
$$

In particular, we will use repeatedly the following further abbreviation:

$$
x_{(k l)}:=x_{k(k+1)(k+2) \ldots l}, \quad k<l .
$$

Using a fixed convex order, we define the root vector $x_{\alpha} \in \mathscr{B}_{\mathfrak{q}}$ for every $\alpha \in \Delta_{+}^{\mathfrak{q}}$ as iterated braided commutators proceeding case-by-case, see [AA]. 
For those $\mathfrak{q}$ with $\operatorname{dim} \mathscr{B}_{\mathfrak{q}}<\infty$, the defining relations of $\mathscr{B}_{\mathfrak{q}}$ were given in [An1, An2, again see $\mathrm{AA}$, but these are not needed in this paper. Instead, we use systematically that for $\alpha<\beta \in \Delta_{+}^{\mathfrak{q}}$ and a suitable defined $q_{\alpha \beta} \in \mathbb{k}^{\times}$, we have

$$
x_{\alpha} x_{\beta}-q_{\alpha \beta} x_{\beta} x_{\alpha} \in \sum_{\alpha<\gamma_{1} \leq \gamma_{2} \leq \cdots \leq \gamma_{t}<\beta \in \Delta_{+}^{\mathfrak{q}}} \mathbb{k} x_{\gamma_{1}} x_{\gamma_{2}} \ldots x_{\gamma_{t}} .
$$

See [An1, Theorem 4.9], inspired by the original work of [DCK].

2.4. Realizations. Let $\Gamma$ be a finite abelian group. A Yetter-Drinfeld module $V$ over $\mathbb{k} \Gamma$ is determined by families $\left(g_{i}\right)_{i \in \mathbb{I}_{\theta}}$ of elements of $\Gamma$ and characters $\left(\chi_{i}\right)_{i \in \mathbb{I}_{\theta}}$ in $\widehat{\Gamma}$. Then $V$ is a braided vector space of diagonal type with braiding matrix $\mathfrak{q}=\left(q_{i j}\right)_{i, j \in \mathbb{I}}$ with respect to a basis $\left(x_{i}\right)_{i \in \mathbb{I}}$, i.e. $c\left(x_{i} \otimes x_{j}\right)=q_{i j} x_{j} \otimes x_{i}, i, j \in \mathbb{I}$, where $q_{i j}=\chi_{j}\left(g_{i}\right)$. That is, the same braided vector space $V$ with braiding matrix $\mathfrak{q}=\left(q_{i j}\right)_{i, j \in \mathbb{I}}$ can be realized in many ways over many $\Gamma$. Even more, it can be realized over other Hopf algebras than group algebras over abelian groups. To discuss the possible realizations we need the notion of a YD-pair.

Let $H$ be a Hopf algebra. A pair $(g, \chi) \in G(H) \times \operatorname{Hom}_{\text {alg }}(H, \mathbb{k})$ is a $Y D$-pair for $H$ if

$$
\chi(h) g=\chi\left(h_{(2)}\right) h_{(1)} g \mathcal{S}\left(h_{(3)}\right), \quad h \in H .
$$

When this is the case, $g \in Z(G(H))$; also $\mathbb{k}_{g}^{\chi}=\mathbb{k}$ with action and coaction given by $\chi$ and $g$ respectively, is in ${ }_{H}^{H} \mathcal{Y D}$. YD-pairs classify the 1-dimensional objects in ${ }_{H}^{H} \mathcal{Y D}$. Note that, if $\operatorname{dim} H<\infty$, then $(g, \chi)$ is a YD-pair for $H$ if and only if $(\chi, g)$ is a YD-pair for $H^{\#}$.

Definition 2.4.2. Let $V$ be a braided vector space of diagonal type with braiding matrix $\mathfrak{q}=\left(q_{i j}\right)_{i, j \in \mathbb{I}}$. A principal realization of $V$ over $H$ is a family $\left(g_{i}, \chi_{i}\right)_{i \in \mathbb{I}}$ of YD-pairs such that $\chi_{j}\left(g_{i}\right)=q_{i j}, i, j \in \mathbb{I}$, so that $V \in{ }_{H}^{H} \mathcal{Y D}$ via $\mathbb{k} x_{i} \simeq \mathbb{k}_{g_{i}}^{\chi_{i}}$, and the braiding $c$ is the categorical one from ${ }_{H}^{H} \mathcal{Y D}$.

Given a principal realization of $V$ over $H$, we have $\Gamma:=\left\langle g_{1}, \ldots, g_{\theta}\right\rangle \leq Z(G(H))$; hence we can also realize $V$ as an object in $\underset{\mathbb{k} \Gamma}{\mathbb{k} \Gamma} \mathcal{Y} \mathcal{D}$.

Example 2.4.3. If $\Gamma$ is a finite group, then the YD-pairs of $H=\mathbb{k} \Gamma$ are of the form $(g, \chi) \in$ $Z(\Gamma) \times \operatorname{Hom}_{\text {grp }}\left(\Gamma, \mathbb{k}^{\times}\right)$. For example, for $\Gamma=G L_{n}\left(\mathbb{F}_{p}\right)$, the YD-pairs are $\left(\operatorname{diag}(t), \varphi \operatorname{det}^{n}\right)$, where $t \in \mathbb{F}_{p}^{\times}$and $\varphi \in \widehat{\mathbb{F}_{p}^{\times}}$.

Example 2.4.4. Not every realization is principal: if $g \in Z(\Gamma)$ and $\rho \in \operatorname{Irrep} \Gamma$ with $\operatorname{dim} \rho=d>1$ and $\rho(g)=\zeta \mathrm{id}$, then the simple Yetter-Drinfeld module $M(g, \rho)$, Example 24] is a braided vector space of diagonal type with braiding matrix $\left(q_{i j}\right)_{i, j \in \mathbb{I}_{d}}$ where $q_{i j}=\zeta$ for all $i, j$. Other examples arise from simple Yetter-Drinfeld modules $M(g, \rho)$ such that the elements in the conjugacy class of $g$ commute with each other.

2.5. The Drinfeld double of a bosonization. Recall that char $\mathbb{k}=0$. Let $\mathcal{L}$ be a Hopf algebra whose coradical $\mathcal{L}_{0}$ is a Hopf subalgebra and let $\mathcal{H}$ be another Hopf subalgebra of $\mathcal{L}$. Then $\mathcal{H}_{0}=\mathcal{L}_{0} \cap \mathcal{H}$ by $[\mathrm{R}, 4.2 .2]$ and this is a Hopf subalgebra of $\mathcal{H}$. By $[\underline{\mathrm{R}}, 4.4 .11]$ we have an injective map of graded Hopf algebras gr $\mathcal{H} \hookrightarrow \operatorname{gr} \mathcal{L}$. Let $R$ and $S$ be the diagrams of $\mathcal{H}$ and $\mathcal{L}$ respectively, see (2.2.1). Hence we have an injective map of graded braided Hopf algebras $R \hookrightarrow S$. 
Let $\mathcal{L}$ be a finite-dimensional Hopf algebra. The Drinfeld double of $\mathcal{L}$, denoted by $D(\mathcal{L})$, is a Hopf algebra whose underlying coalgebra is $\mathcal{L} \otimes \mathcal{L}^{\# \text { op }}$. Let $\langle-,-\rangle: \mathcal{L} \otimes \mathcal{L}^{\#} \rightarrow \mathbb{k}$ denote the evaluation map. The multiplication on $D(\mathcal{L})$ is given by the following formula:

$$
(h \bowtie f)\left(h^{\prime} \bowtie f^{\prime}\right)=\left\langle f_{(1)}, h_{(1)}^{\prime}\right\rangle\left\langle f_{(3)}, \mathcal{S}\left(h_{(3)}^{\prime}\right)\right\rangle\left(h h_{(2)}^{\prime} \bowtie f^{\prime} f_{(2)}\right), \quad f, f^{\prime} \in \mathcal{L}^{\#} \quad h, h^{\prime} \in \mathcal{L},
$$

where $h \bowtie f:=h \otimes f$ in $D(\mathcal{L})$ and $f r=m(f \otimes r)$ is the multiplication in $\mathcal{L}^{\#}$ rather than in $\mathcal{L}^{\# \text { op }}$.

Let $K$ be a semisimple Hopf algebra, hence cosemisimple by the Larson-Radford theorem. Let $V \in{ }_{K}^{K} \mathcal{Y D}$ with $\operatorname{dim} \mathscr{B}(V)<\infty$ and $H=\mathscr{B}(V) \# K$. Then $V^{\#} \in{ }_{K}^{\#} \mathcal{Y} \mathcal{D}$ appropriately and $H^{\#} \simeq \mathscr{B}\left(V^{\#}\right) \# K^{\#}$. Since $D(H) \simeq H^{\#^{\text {cop }}} \otimes H$ as coalgebras, the coradical of $H$, respectively $H^{\#}$, can be identified with $K$, respectively $K^{\#}$. We identify $D(K)$ with a Hopf subalgebra of $D(H)$ in a natural way.

The following result generalizes, with an analogous proof, Theorem 2.5 in [Be].

Proposition 2.5.1. The Drinfeld double $D(H)$ is a lifting of a Nichols algebra $\mathscr{B}(W)$ where $W=V \oplus V^{\#}$ and $V$ braided commutes with $V^{\#}$.

Proof. First, the coradical $D(H)_{0}$ of $D(H)$ equals $D(K)$; this follows from $[\mathbb{R}$, 4.1.8]. Hence the coradical filtration of $D(H)$ is a Hopf algebra filtration and $\operatorname{gr} D(H) \simeq R \# D(K)$, where $R=\oplus_{n \geq 0} R^{n}$ is the diagram of $D(H)$. Let $W=R^{1}$. By the preceding paragraph applied to $\mathcal{L}=D(H)$ and either $\mathcal{H}=H$ or $\mathcal{H}=H^{\#}$, we have morphisms of braided vector spaces $V \hookrightarrow W$ and $V^{\#} \hookrightarrow W$; we have $V \oplus V^{\#} \hookrightarrow W$ by comparing the $D(K)$-comodule structures. Recall that $\operatorname{dim} \mathscr{B}(V) \operatorname{dim} \mathscr{B}\left(V^{\#}\right) \leq \operatorname{dim} \mathscr{B}\left(V \oplus V^{\#}\right)$ and the equality holds iff $V$ and $V^{\#}$ braided commute [Gr, Theorem 2.2]. Then

$$
\begin{aligned}
\operatorname{dim} D(H) & =\operatorname{dim} \mathscr{B}(V) \operatorname{dim} K \operatorname{dim} \mathscr{B}\left(V^{\#}\right) \operatorname{dim} K^{\#} \\
& \leq \operatorname{dim} \mathscr{B}(W) \operatorname{dim} D(K) \leq \operatorname{dim} R \operatorname{dim} D(K)=\operatorname{dim} D(H),
\end{aligned}
$$

hence $R=\mathscr{B}(W)=\mathscr{B}\left(V \oplus V^{\#}\right)$ and $V$ and $V^{\#}$ braided commute.

Assume next that $K=\mathbb{k} \Gamma$ where $\Gamma$ is a finite abelian group; recall that $\widehat{\Gamma}$ is the group of characters of $\Gamma$. Then

$$
\begin{aligned}
\mathbb{k} \widehat{\Gamma} & \cong(\mathbb{k} \Gamma)^{\#} \\
D(\mathbb{k} \Gamma) & \cong \mathbb{k}(\Gamma \times \widehat{\Gamma}) \cong \mathbb{k} \Gamma \otimes(k \Gamma)^{\#} .
\end{aligned}
$$

Let $\left(g_{i}\right)_{i \in \mathbb{I}_{\theta}}$ and $\left(\chi_{i}\right)_{i \in \mathbb{I}_{\theta}}$ be (dual) generating families in $\Gamma$ and $\widehat{\Gamma}$ respectively. Let $V \in$ $\mathbb{k}_{\mathbb{k} \Gamma} \mathcal{Y} \mathcal{D}$ with a basis $\left(x_{i}\right)_{i \in \mathbb{I}}$ such that the action and coaction of $\Gamma$ on $x_{i}$ are given by $\chi_{i}$ and $g_{i}$ respectively, $i \in \mathbb{I}$. Assume that $\mathscr{B}(V)$ has finite dimension and let $H=\mathscr{B}(V) \# \mathbb{k} \Gamma$. Let $\left(y_{i}\right)_{i \in \mathbb{I}}$ be the basis of $V^{\#}$ dual to $\left(x_{i}\right)_{i \in \mathbb{I}}$. Then $H^{\# \text { cop }} \simeq \mathscr{B}\left(V^{\#}\right) \# \mathbb{k} \widehat{\Gamma}$ where the action and coaction of $\widehat{\Gamma}$ on $y_{i}$ are given by $g_{i}$ and $\chi_{i}^{-1}$ respectively, $i \in \mathbb{I}$. Also $W=V \oplus V^{\#}$ can be realized in $\mathbb{k}_{\mathbb{k}(\Gamma \times \widehat{\Gamma})} \mathcal{Y} \mathcal{D}$ extending these structures. See [Be, Theorem 2.5] for details. Let $\mathcal{I}(V)=\operatorname{ker}(T(V) \rightarrow \mathscr{B}(V))$ be the ideal of defining relations of the Nichols algebra $\mathscr{B}(V)$. The following statement is well-known. 
Proposition 2.5.2. $D(H)$ is isomorphic to the quotient of $T(W) \# \mathbb{k}(\Gamma \times \widehat{\Gamma})$ by the ideal generated by $\mathcal{I}(V), \mathcal{I}\left(V^{\#}\right)$ and the relations

$$
x_{i} y_{j}-\chi_{j}^{-1}\left(g_{i}\right) y_{j} x_{i}=\delta_{i, j} \chi_{i}^{-1}\left(g_{i}\right)\left(1-g_{i} \chi_{i}\right), \quad i, j \in \mathbb{I} .
$$

Outline of the proof. By the preceding discussion there is a morphism of Hopf algebras $T(W) \# \mathbb{k}(\Gamma \times \widehat{\Gamma}) \rightarrow D(H)$ whose kernel $\mathcal{J}$ contains $\mathcal{I}(V), \mathcal{I}\left(V^{\#}\right)$ and the relations (2.5.3), see the proof of [Be, Theorem 2.5]. The induced map $T(W) \# \mathbb{k}(\Gamma \times \widehat{\Gamma}) / \mathcal{J} \rightarrow D(H)$ is clearly surjective and preserves the coradical filtration. Since the associated graded map is injective, the claim follows.

\section{Cohomology}

3.1. Invariants. Let $H$ be a Hopf algebra and $A$ an $H$-module algebra. The ring of invariants is the subalgebra

$$
A^{H}=\left\{x \in A: h \cdot x=\varepsilon_{H}(h) x \quad \forall h \in H\right\} .
$$

Let now $K$ be a semisimple, hence finite-dimensional, Hopf algebra. Let $t \in K$ be a normalized integral, that is $k t=\varepsilon(k) t=t k$ for all $k \in K$ and $\varepsilon(t)=1$. In other words $t$ is a left, hence, right, integral [Mo, 2.2.4].

Let $A$ be a $K$-module algebra and let $\mathcal{R}: A \rightarrow A$ be the Reynolds operator $\mathcal{R}(x)=t \cdot x$, $x \in A$. Then

○ The Reynolds operator is a projector, $\mathcal{R}^{2}=\mathcal{R}$, and $\operatorname{im} \mathcal{R}=A^{K}$.

$\circ \mathcal{R}$ is a morphism of $K$-modules.

$\circ \mathcal{R}$ is a morphism of $R^{K}$-bimodules: $\mathcal{R}(x y z)=x \mathcal{R}(y) z$, for $x, z \in A^{K}$ and $y \in A$.

The following result, a variation of a classical argument by Hilbert, is well-known.

Lemma 3.1.1. Let $K$ be a semisimple Hopf algebra. Let $A=\oplus_{n \in \mathbb{N}_{0}} A^{n}$ be a graded $K$ module algebra that is connected and (right) Noetherian. Let $M$ be a finitely generated $A \# K$-module. Then $A^{K}$ is finitely generated and $M^{K}$ is a finitely generated $A^{K}$-module.

Proof. Let $I=A\left(A^{K}\right)_{+}$be the left ideal of $A$ generated by the augmentation ideal of $A^{K}$. Since $A$ is Noetherian, $I$ is finitely generated; we may assume that $I=\left\langle f_{1}, \ldots, f_{M}\right\rangle$, where $f_{i} \in A^{K}$ is homogeneous of degree $d_{i}$. We claim that $A^{K}=\mathbb{k}\left\langle f_{1}, \ldots, f_{M}\right\rangle$. For this we shall prove that any $f \in A^{K}$ homogeneous of degree $d$ belongs to $\mathbb{k}\left\langle f_{1}, \ldots, f_{M}\right\rangle$. If $d=0$, this follows by connectedness. If $d>0$, then we may write $f=\sum_{i} a_{i} f_{i}$ with $a_{i}$ either 0 or else homogeneous of degree $d-d_{i}$. Then $f=\mathcal{R}(f)=\sum_{i} \mathcal{R}\left(a_{i}\right) f_{i}$ and $\mathcal{R}\left(a_{i}\right) \in \mathbb{k}\left\langle f_{1}, \ldots, f_{M}\right\rangle$ by the recursive hypothesis, so $f \in \mathbb{k}\left\langle f_{1}, \ldots, f_{M}\right\rangle$.

For the module statement, note that the hypothesis of [Mo, Theorem 4.4.2] holds; namely the map denoted there $\hat{t}$ is the Reynolds operator $\mathcal{R}$. Hence $A$ is a right Noetherian $A^{K_{-}}$ module, and thus finitely generated over $A^{K}$. Thus $M$ is a Noetherian $A^{K}$-module. Since $M^{K}$ is an $A^{K}$-submodule, it is also Noetherian, therefore finitely generated over $A^{K}$.

If $R$ is an $H$-module algebra, then $H$ acts on $\Omega^{*}(R)$ via the comultiplication and the antipode, and a fortiori on $\mathrm{H}(R, \mathbb{k})$. The following proposition is well-known. 
Proposition 3.1.2. Let $K$ be a semisimple Hopf algebra and let $R$ be a finite-dimensional $K$-module algebra. Let $M$ be an $R \# K$-module. Then

$$
\mathrm{H}(R \# K, \mathbb{k}) \simeq \mathrm{H}(R, \mathbb{k})^{K}, \quad \mathrm{H}(R \# K, M) \simeq \mathrm{H}(R, M)^{K},
$$

and the action of $\mathrm{H}(R \# K, \mathbb{k})$ on $\mathrm{H}(R \# K, M)$ is precisely that induced by the isomorphisms and the action of $\mathrm{H}(R, \mathbb{k})$ on $\mathrm{H}(R, M)$.

Proof. This is well-known; the first isomorphism is for example [SV, Theorem 2.17]. In case $K$ is a group algebra, the relevant spectral sequence is the Lyndon-Hochschild-Serre spectral sequence [Ev, $\S \S 7.2,7.3]$ which collapses since $K$ is semisimple.

Now we pass to algebras in ${ }_{H}^{H} \mathcal{Y} \mathcal{D}$. An algebra $A$ in ${ }_{H}^{H} \mathcal{Y D}$ is braided commutative if the multiplication $m_{A}$ satisfies $m_{A}=m_{A} c_{A, A}$, that is

$$
x y=\left(x_{(-1)} \cdot y\right) x_{(0)}, \quad x, y \in A .
$$

If $A$ is braided commutative, then $A^{H}$ is central in $A$. We elaborate on an idea of [MPSW]; for this we do not need the commutativity of $\Gamma$.

Lemma 3.1.4. Let $\Gamma$ be a finite group and let $A$ be a braided commutative algebra either in $\underset{\mathbb{k} \Gamma}{\mathbb{k} \Gamma} \mathcal{Y} \mathcal{D}$ or in ${\mathbb{\mathbb { k } ^ { \Gamma }}}^{\mathrm{N}} \mathcal{Y} \mathcal{D}$. Assume that $A$ is finitely generated (as an algebra). Then $A$ is Noetherian.

Proof. Let $N$ be the exponent of $\Gamma$. We deal first with $\underset{\mathbb{k} \Gamma}{\mathbb{k} \Gamma} \mathcal{Y} \mathcal{D}$. As an object in ${ }_{\mathbb{k} \Gamma}^{\mathrm{k}} \Gamma \mathcal{Y} \mathcal{D}$, $A$ is $\Gamma$-graded: $A=\oplus_{g \in \Gamma} A_{g}$. Thus, if $A=\mathbb{k}\left\langle f_{1}, \ldots, f_{M}\right\rangle$, then we may assume that each $f_{i}$ belongs to $A_{g_{i}}$ for some $g_{i} \in \Gamma$. Then $f_{i}^{N} \in A_{g_{i}^{N}}=A_{e}$. Since $A$ is braided commutative, $f_{i}^{N} f_{j}=\left(g_{i}^{N} \cdot f_{j}\right) f_{i}^{N}=f_{j} f_{i}^{N}$ for all $i, j$. Then $B=\mathbb{k}\left\langle f_{1}^{N}, \ldots, f_{M}^{N}\right\rangle$ is a central subalgebra of $A$ and is Noetherian by Hilbert's Basissatz. Now $A$ is a finitely generated $B$-module, actually $A=\sum_{0 \leq a_{i} \leq N} B f_{1}^{a_{1}} \ldots, f_{M}^{a_{M}}$. Thus $A$ is a Noetherian $B$-module hence a Noetherian algebra. We deal next with ${ }_{\mathbb{k}^{\Gamma}}^{\mathbb{R}^{\Gamma}} \mathcal{Y} \mathcal{D}$. Since $H=\mathbb{k}^{\Gamma}$ has a basis of idempotents $\delta_{g}, g \in \Gamma$, again $A$ is $\Gamma$-graded: $A=\oplus_{g \in \Gamma} A_{g}$ where $A_{g}=\delta_{g} A$. Thus, if $A=\mathbb{k}\left\langle f_{1}, \ldots, f_{M}\right\rangle$, with each $f_{i} \in A_{g_{i}}$ for some $g_{i} \in \Gamma$, then $f_{i}^{N} \in A_{g_{i}^{N}}=A_{e}=\delta_{e} A$. But $\delta_{e}$ is the integral of $\mathbb{k}^{\Gamma}$, thus again $f_{i}^{N} \in A^{H}$ is central. Then we proceed as previously.

We wonder whether any finitely generated braided commutative algebra is Noetherian. We need the following result from [MPSW].

Proposition 3.1.5. [MPSW, Corollary 3.13] Let $H$ be a Hopf algebra and let $R$ be a bialgebra in ${ }_{H}^{H} \mathcal{Y} \mathcal{D}$. Assume that either $H$ or $R$ is finite-dimensional. Then the (opposite of) the Hochschild cohomology $\mathrm{HH}(R, \mathbb{k})$ is a braided commutative graded algebra in ${ }_{H}^{H} \mathcal{Y D}$.

Actually [MPSW, Theorem 3.12] gives more: the claim is true if $R$ is a bialgebra in an abelian braided monoidal category $\mathcal{C}$ where the needed hom-objects exist.

Theorem 3.1.6. Let $\Gamma$ be a finite group and let $R$ be a finite-dimensional Hopf algebra in ${ }_{\mathbb{k} \Gamma} \Gamma \mathcal{Y} \mathcal{D}$. f $R$ has fgc, then so does $R \# \mathbb{k} \Gamma$.

Proof. By Proposition 3.1.5, Lemma 3.1.4 and the hypothesis, $\mathrm{H}(R, \mathbb{k})$ is Noetherian. Then $\mathrm{H}(R, \mathbb{k})^{\Gamma}$ is finitely generated by Lemma 3.1.1. By Proposition 3.1.2, $\mathrm{H}(R \# \mathbb{k} \Gamma, \mathbb{k}) \simeq$ $\mathrm{H}(R, \mathbb{k})^{\Gamma}$ is finitely generated. We next prove: If $M$ is a finitely generated module, then 
$\mathrm{H}(R \# \mathbb{k} \Gamma, M)$ is finitely generated as an $\mathrm{H}(R \# \mathbb{k} \Gamma, \mathbb{k})$-module. For this, we may induct on the length of the composition series of $M$, and so it suffices to prove it in case $M$ is simple. Let $R_{+}$denote the augmentation ideal of $R$. Note that $R_{+} M$ is an $R \# \mathbb{k} \Gamma$-submodule of $M$ and therefore $R_{+} M=0$ (by Nakayama), that is, $\left.M\right|_{R}$ is a trivial $R$-module. We conclude that $\mathrm{H}(R, M)$ is finitely generated as an $\mathrm{H}(R, \mathbb{k})$-module. By Lemma 3.1.1, it follows that $\mathrm{H}(R, M)^{K}$ is finitely generated over $\mathrm{H}(R, \mathbb{k})^{K}$.

We are ready for one of our main results.

Theorem 3.1.7. Let $V$ be a braided vector space of diagonal type such that

(a) the Nichols algebra $\mathscr{B}(V)$ is finite-dimensional,

(b) $V$ is realizable over a finite abelian group,

(c) $\mathrm{H}(\mathscr{B}(V), \mathbb{k})$ is finitely generated.

Let $K$ be a semisimple Hopf algebra and assume that $V$ is realizable over $K$. Then $\mathscr{B}(V) \# K$ has fgc.

Proof. The proof is the same as for the previous result. By (b), (c), Proposition 3.1.5 and Lemma 3.1.4. $\mathrm{H}(\mathscr{B}(V), \mathbb{k})$ is Noetherian. Then $\mathrm{H}(\mathscr{B}(V), \mathbb{k})^{K}$ is finitely generated by Lemma 3.1.1. By Proposition 3.1.2 and (a), $\mathrm{H}(\mathscr{B}(V) \# K, \mathbb{k}) \simeq \mathrm{H}(\mathscr{B}(V), \mathbb{k})^{K}$ is finitely generated. The proof of the module statement is similar.

Observation 3.1.8. If $V$ admits a principal realization over $K$, then (b) holds. Notice that (a) does not imply (b)] take $V$ of dimension 2 with braiding matrix $\left(\begin{array}{ll}q_{11} & q_{12} \\ q_{12}^{-1} & q_{22}\end{array}\right)$ where $q_{11} \in \mathbb{G}_{M}^{\prime}, q_{12} \notin \mathbb{G}_{\infty}, q_{22} \in \mathbb{G}_{N}^{\prime}, N, M>1$. However we do not know if $V$ being realizable over $K$ semisimple implies (b).

\subsection{Subalgebras, extensions.}

Theorem 3.2.1. Let $R$ be an augmented subalgebra of a finite-dimensional augmented algebra $\mathcal{A}$, over which $\mathcal{A}$ is projective as a right $R$-module under multiplication. If $\mathcal{A}$ has fgc, then so does $R$.

Proof. By the right module version of the Eckmann-Shapiro Lemma [Ben, Corollary 2.8.4], for each $n$, and any $R$-module $M$, there is an isomorphism of vector spaces,

$$
\mathrm{H}^{n}(R, M) \simeq \operatorname{Ext}_{\mathcal{A}}^{n}\left(\mathbb{k}, \operatorname{Hom}_{R}(\mathcal{A}, M)\right)=\mathrm{H}^{n}\left(\mathcal{A}, \operatorname{Hom}_{R}(\mathcal{A}, M)\right),
$$

where $\operatorname{Hom}_{R}(\mathcal{A}, M)$ is the coinduced right $\mathcal{A}$-module. (The action is given by $(f \cdot a)(b)=$ $f(a b)$ for all $a, b \in \mathcal{A}, f \in \operatorname{Hom}_{R}(A, M)$. Then $f \cdot a$ is indeed a right $R$-module homomorphism.) These isomorphisms, one for each $n$, provide an isomorphism of $\mathrm{H}(A, \mathbb{k})$ modules $\mathrm{H}(R, M) \simeq \mathrm{H}\left(\mathcal{A}, \operatorname{Hom}_{R}(\mathcal{A}, M)\right)$. Now when $M$ is a finite-dimensional $R$-module, $\operatorname{Hom}_{R}(\mathcal{A}, M)$ is finite-dimensional as a vector space. For $M=\mathbb{k}$, a set of generators of $\mathrm{H}\left(\mathcal{A}, \operatorname{Hom}_{R}(\mathcal{A}, \mathbb{k})\right)$ as a module for $\mathrm{H}(\mathcal{A}, \mathbb{k})$, together with the restriction to $R$ of a set of generators of $\mathrm{H}(\mathcal{A}, \mathbb{k})$, generates $\mathrm{H}(R, \mathbb{k})$ as a $k$-algebra. For an arbitrary finite-dimensional module $M, \operatorname{Hom}_{R}(\mathcal{A}, M)$ is then a finite-dimensional module over $\mathrm{H}(\mathcal{A}, \mathbb{k})$ and, hence, over $\mathrm{H}(R, \mathbb{k})$. 
If $K$ is a Hopf subalgebra of a finite-dimensional Hopf algebra $H$, then $H$ is free as a left or right module over $K$ with respect to multiplication by the Nichols-Zöller Theorem. Thus Theorem 3.2.1 applies to inclusions of Hopf algebras, in particular to the inclusion of a finite-dimensional Hopf algebra into its Drinfeld double, see [NP, Theorem 3.4]. For further reference we state a useful application of Theorem 3.2.1.

Corollary 3.2.2. Let $H$ be a finite-dimensional Hopf algebra and $V \in{ }_{H}^{H} \mathcal{Y D}$ such that $\operatorname{dim} \mathscr{B}(V)<\infty$. If $D(\mathscr{B}(V) \# H)$ has fgc, then so does $\mathscr{B}(V)$.

These ideas apply in particular to Morita equivalence of Hopf algebras as in 2.1 .

Corollary 3.2.3. If $H \sim_{\text {Mor }} H^{\prime}$ and $D(H)$ has fgc, then so does $H^{\prime}$.

Question 3.2.4. Is the fgc property for Hopf algebras invariant under Morita equivalence in the sense of 2.1 ?

The reader might want to compare this question to [NP, Conjecture 1.1].

Lemma 3.2.5. Let $\mathbb{k} \rightarrow K \rightarrow H \rightarrow L \rightarrow \mathbb{k}$ be an extension of finite-dimensional Hopf algebras. If $K$ is semisimple and $L$ has finitely generated cohomology, then so does $H$.

The proof makes use of a variation of the classical Hochschild-Serre spectral sequence.

Proof. Let $M$ be an $L$-module and $N$ an $H$-module. By [CE, Chapter 16, Theorem 6.1] there exists a convergent spectral sequence

$$
H^{p}\left(L, H^{q}(K, N)\right) \Longrightarrow H^{p+q}(H, N) .
$$

Since $K$ is semisimple, we have $H^{q}(K, N)=0$ when $q>0$. Hence, the spectral sequence degenerates giving an isomorphism $H(H, N) \cong H\left(L, N^{K}\right)$. For $N=\mathbb{k}$ we immediately get that $H(H, \mathbb{k}) \cong H(L, \mathbb{k})$ is finitely generated. For an arbitrary finitely generated $H$ module $N$, we have that $N^{K}$ is a finitely generated $L$-module, and, hence, by assumption $H\left(L, N^{K}\right)$ is a finite $H(L, \mathbb{k}) \cong H(H, \mathbb{k})$-module.

3.3. Evens Lemma. Let $R=\oplus_{n \in \mathbb{N}_{0}} R^{n}$ be an $\mathbb{N}_{0}$-graded ring with a decreasing algebra filtration $F^{n} R, n \in \mathbb{N}_{0}$, compatible with the grading. We shall assume that $F^{i} R^{n}=0$ for $i$ sufficiently large. Then the associated graded ring $E_{0}(R)=\sum_{i} F^{i} R / F^{i+1} R$ is $\mathbb{N}_{0}^{2}$-graded.

Similarly, the graded $E_{0}(R)$-module associated to an $\mathbb{N}_{0}$-graded $R$-module $N$ with a decreasing module filtration is $\mathbb{N}_{0}^{2}$-graded. Again, $F^{i} N^{j}=0$ for $i$ sufficiently large. The following proposition is [Ev, Section 2, Proposition 2.1].

Proposition 3.3.1. Let $R$ be a graded filtered ring and $N$ a graded filtered $R$-module as above. If $E_{0}(N)$ is (left) Noetherian over $E_{0}(R)$, then $N$ is Noetherian over $R$.

The following result is a non-commutative version of [MPSW, Lemma 2.5], adapted in turn from [FS, Lemma 1.6] and inspired by early work of Evens.

Let $E_{1}^{p, q} \Rightarrow E_{\infty}^{p+q}$ be a multiplicative spectral sequence of bigraded $\mathbb{k}$-algebras concentrated in the half plane $p+q \geq 0$. Recall that $x \in E_{r}^{p, q}$ is called a permanent cycle if $d_{i}(x)=0$ for all $i \geq r$. More precisely, if $i>r, d_{i}$ is applied to the image of $x$ in $E_{i}$.

Lemma 3.3.2. $[\mathrm{Shr}$, Lemma 2.6]

(a) Let $C^{*, *}$ be a bigraded $\mathbb{k}$-algebra such that for each fixed $q, C^{p, q}=0$ for $p$ sufficiently large. Assume that there exists a bigraded map of algebras $\phi: C^{*, *} \rightarrow E_{1}^{*, *}$ such that 
(1) $\phi$ makes $E_{1}^{*, *}$ into a left Noetherian $C^{*, *}$-module, and

(2) the image of $C^{*, *}$ in $E_{1}^{*, *}$ consists of permanent cycles.

Then $E_{\infty}^{*}$ is a left Noetherian module over $\operatorname{Tot}\left(C^{*, *}\right)$.

(b) Let $\widetilde{E}_{1}^{p, q} \Rightarrow \widetilde{E}_{\infty}^{p+q}$ be a spectral sequence that is a bigraded module over the spectral sequence $E^{*, *}$. Assume that $\widetilde{E}_{1}^{*, *}$ is a left Noetherian module over $C^{*, *}$ where $C^{*, *}$ acts on $\widetilde{E}_{1}^{*, *}$ via the map $\phi$. Then $\widetilde{E}_{\infty}^{*}$ is a finitely generated $E_{\infty}^{*}$-module.

3.4. The May spectral sequence. Let $A$ be a Hopf algebra equipped with an increasing multiplicative filtration $A_{0} \subset A_{1} \subset A_{2} \ldots \subset A$. We fix a (non-canonical) vector space splitting $A \simeq A_{0} \oplus A_{+}$so that $A / A_{+} \simeq A_{0}$. Let $(P, d)=\left(V_{n} \otimes A, d\right)$ be a free resolution of the trivial module $k$ satisfying the following properties.

Condition 3.4.1. (1) $V_{n}$ is a finite-dimensional vector space, the action of $A$ on $P_{n}$ is on the last factor $A$.

(2) $P$. is equipped with an increasing filtration $\ldots F_{i} P_{n} \subset F_{i+1} P_{n} \ldots$

(3) For any $x \in F_{i} V_{n}=F_{i}\left(V_{n} \otimes 1\right):=F_{i} P_{n} \cap\left(V_{n} \otimes 1\right)$, we have

$$
d(x) \in F_{i} V_{n-1} \otimes A_{0}+F_{i-1} V_{n-1} \otimes A_{+} .
$$

Example 3.4.2. We will apply this setting in at least two different situations.

(i) When $P_{0}$ is the bar resolution and $F_{0}$ is the coradical filtration, see Theorem 5.0.6,

(ii) When $P$. is the Anick resolution of $k$ for $\mathscr{B}(V)$, and the filtration is given by the PBW basis induced by the convex ordering of the roots, see Theorem 4.4.3. In this case $A_{0}=k$ and we identify $A_{+}$with the augmentation ideal.

We set up a version of May spectral sequence analogous to the one in GK, 5.5]. We follow the construction in May May but without assuming that the module $M$ is filtered. Such a spectral sequence is also constructed in [BKN, §9] using a non-canonical filtration on $M$ induced by the filtration on $A$.

Theorem 3.4.3. Let $A$ be a filtered finite-dimensional Hopf algebra, $(P ., d)$ be a projective resolution of the trivial module $k$, and assume that $A$ and $P$. satisfy Condition 3.4.1. Let $M$ be an A-module. Then there exists a converging cohomological spectral sequence

$$
\widetilde{E}_{1}=H\left(\operatorname{gr} A, M_{A_{0}}\right) \Rightarrow H(A, M)
$$

equipped with a natural module structure over the multiplicative spectral sequence

$$
E_{1}=H(\operatorname{gr} A, k) \Rightarrow H(A, k) .
$$

The action of $\operatorname{gr} A$ on $M_{A_{0}}$ is via the projection gr $A \rightarrow A_{0}$ and then restricting the action of $A$ on $M$ to the action of the subalgebra $A_{0} \subset A$.

Proof. Let $C^{\bullet}(A, M):=\operatorname{Hom}_{A}(P ., M)$ be the complex computing $H(A, M)$. Let

$$
F^{i} C^{n}(A, M):=\left\{f \in \operatorname{Hom}_{A}\left(P_{n}, A\right) \mid f \downarrow_{F_{i-1} P_{n}}=0\right\} \subset C^{n}(A, M)
$$

be a decreasing filtration on $C^{*}(A, M)$ making it into a filtered complex.

As $V_{n} \subset P_{n}$, we have an induced filtration (of vector spaces) on $V_{\bullet}: F_{i} V_{n}=F_{i}\left(V_{n} \otimes 1\right)=$ $F_{i} P_{n} \cap\left(V_{n} \otimes 1\right)$. Using the isomorphism $\operatorname{Hom}_{A}\left(V_{n} \otimes A, M\right) \simeq \operatorname{Hom}_{k}\left(V_{n}, M\right)$, we make the 
identifications:

$$
\begin{aligned}
\frac{F^{i} C^{n}(A, M)}{F^{i+1} C^{n}(A, M)}= & \frac{\left\{f: V_{n} \rightarrow M \mid f \downarrow_{F_{i-1} V_{n}}=0\right\}}{\left\{f: V_{n} \rightarrow M \mid f \downarrow_{F_{i} V_{n}}=0\right\}} \\
& \simeq \frac{\operatorname{Hom}_{k}\left(V_{n} / F_{i-1} V_{n}, M\right)}{\operatorname{Hom}_{k}\left(V_{n} / F_{i} V_{n}, M\right)} \\
& \simeq \operatorname{Hom}_{k}\left(\frac{F_{i} V_{n}}{F_{i-1} V_{n}}, M\right) .
\end{aligned}
$$

Letting $n$ be the total and $i$ be the internal degree, we have

$$
\operatorname{Hom}_{k}\left(\frac{F_{i} V_{n}}{F_{i-1} V_{n}}, M\right) \simeq \operatorname{Hom}_{\mathrm{gr} A}\left(\frac{F_{i} P_{n}}{F_{i-1} P_{n}}, M_{\mathrm{tr}}\right) \simeq H^{n, i}\left(\operatorname{gr} A, M_{\mathrm{tr}}\right) .
$$

Let $\widetilde{E}_{0}^{i, n-i}(M):=\frac{F^{i} C^{n}(A, M)}{F^{i+1} C^{n}(A, M)}$. Since $A$ is finite-dimensional, the filtration is finite and, hence, this defines the 0 page of the spectral sequence of a filtered complex $C^{\bullet}(A, M)$ converging to $H(A, M)$. We have identified the terms of the double complex $\widetilde{E}_{0}(M)$ with the complex $C^{\bullet}\left(\operatorname{gr} A, M_{A_{0}}\right)$ computing cohomology $H\left(\operatorname{gr} A, M_{A_{0}}\right)$. To identify $\widetilde{E}_{1}(M)$ and $H\left(\operatorname{gr} A, M_{A_{0}}\right)$ as complexes, it suffices to show that the differentials in $\widetilde{E}(M)$ and $\widetilde{E}\left(M_{A_{0}}\right)$ are the same, that is, that the differential $d_{M}$ in the spectral sequence only depends on the $A_{0}$-module structure on $M$.

Consider the differential $d_{M}$ :

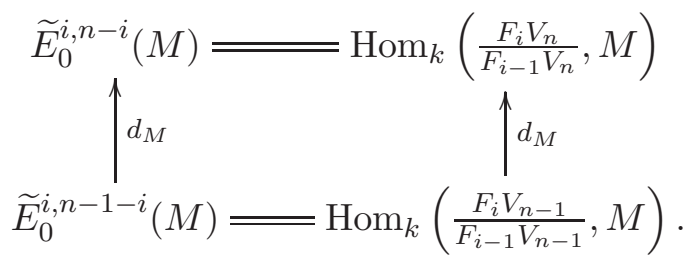

Let $f \in \operatorname{Hom}_{k}\left(\frac{F_{i} V_{n-1}}{F_{i-1} V_{n-1}}, M\right), \bar{x} \in \frac{F_{i} V_{n}}{F_{i-1} V_{n}}$, and let $x \in F_{i} V_{n}$ be a representative of $\bar{x}$. By Condition 3.4.1(3) we can write $d(x)=v_{0} \otimes a_{0}+v^{\prime} \otimes a^{\prime}$ with $a_{0} \in A_{0}$ and $v^{\prime} \in F_{i-1} V_{n-1}$. We now compute

$$
\begin{aligned}
d_{M}(f)(\bar{x}) & =f\left(\overline{d_{M}(x)}\right) \\
& =f\left(\bar{v}_{0}\right) a_{0}+f\left(\bar{v}^{\prime}\right) a^{\prime} \\
& \stackrel{*}{=} f\left(\bar{v}_{0}\right) a_{0} \\
& =f\left(\overline{d_{M_{A_{0}}}(x)}\right)=d_{M_{A_{0}}}(f)(\bar{x})
\end{aligned}
$$

The equality $(*)$ holds since $\bar{v}^{\prime}=v^{\prime} \bmod F_{i-1} V_{n-1}=0$.

The statement about the action of $E_{1}(k)$ acting on $\widetilde{E}_{1}(M)$ follows from the construction of the spectral sequence. 


\section{The ANICK RESOLUTION}

4.1. The setup. In this section we discuss the Anick graph and the construction of the Anick resolution [Ani, $\mathrm{Fa}, \mathrm{CoU}$. Let $V$ be a finite-dimensional vector space with a basis $\left(x_{i}\right)_{i \in \mathbb{I}}$, and $\mathcal{I}$ an ideal of $T(V)$ such that $\epsilon(\mathcal{I})=0$, where $\epsilon: T(V) \rightarrow \mathbb{k}$ is the standard augmentation map, $\epsilon\left(x_{i}\right)=0$ for all $i \in \mathbb{I}$. Thus the algebra $\mathcal{A}:=T(V) / \mathcal{I}$ has an augmentation map $\epsilon: \mathcal{A} \rightarrow \mathbb{k}$.

4.1.1. The tips. Let $X$ be the set of words on the letters $\left(x_{i}\right)_{i \in \mathbb{I}}$ (including the empty word $1)$. Notice that $X$ is a basis of $T(V)$. Let $x, y \in X$. We say that $x$ is a subword of $y$ if there exist $w, z \in X$ such that $y=w x z$. If $w=1$, respectively $z=1$, then we say that $x$ is a prefix, respectively a suffix, of $y$.

Let $\ell: X \rightarrow \mathbb{N}_{0}$ be the length function. The lex-length order $<$ on $X$ is defined as follows: given $v, w \in X$, we say that $v<w$ if either $\ell(v)<\ell(w)$ or else $\ell(v)=\ell(w)$ and $v$ is less than $w$ for the lexicographical order (induced by the numeration of the basis). This is a total order on $X$ compatible with left and right multiplication.

Here is a way to give a set of generators of the ideal $\mathcal{I}$. Given $f \in \mathcal{I}-0$, write $f$ as a linear combination of elements of $X$; let $x_{f}$ be the largest element of $X$ (with respect to $<)$ with non-zero coefficient. Then $x_{f}$ is called the tip of $f$. Consider the set of all tips of all elements in $\mathcal{I}-0$. A tip $t$ is minimal if each subword of $x$ is not a tip (Anick calls a minimal tip an obstruction). Let $\mathcal{T}$ be the set of minimal tips of $\mathcal{I}$. For each $t \in \mathcal{T}$ we pick $\omega_{t} \in \mathcal{I}$ such that $t$ is the tip of $\omega_{t}$ (which is not unique in general). Arguing recursively on $<$, it is possible to show that

$$
\mathcal{I}=\left\langle\omega_{t}: t \in \mathcal{T}\right\rangle .
$$

For each $w \in X$ we also denote by $w$ its image in $\mathcal{A}=T(V) / \mathcal{I}$. By Ani, Lemmas 1.1 and 1.2], the set

$$
\mathrm{B}=\{w \in X: t \text { is not a subword of } w \forall t \in \mathcal{T}\}
$$

is a basis of $\mathcal{A}$.

4.1.2. The chains. Let $n \in \mathbb{N}_{0}$. We describe the $n$-chains which are words defined from the minimal tips; they will provide a basis of the $n$-th term of the Anick resolution of $\mathcal{A}$. The unique 0 -chain is the empty word 1 . The 1 -chains are the letters, i.e. the $x_{i}$ 's. Let $n \geq 1$. An $n$-chain is a word $w$ such that:

(a) $w$ admits a factorization $w=u v$ such that $u$ is an $(n-1)$-chain and the suffix $v$ does not contain any minimal tip as a subword (i.e., does not contain any tip);

(b) for every suffix $y \neq 1$ of $u$ as in (a), the word $y v$ contains a minimal tip as a subword;

(c) any other prefix $w^{\prime}$ of $w$ does not satisfy (a) and (b) simultaneously.

Let $\mathrm{M}(n)$ be the set of $n$-chains. We urge the reader to check that $\mathrm{M}(2)$ is the set of minimal tips - all requirements are needed.

There exists an alternative way to express the definition of $n$-chains. A word $w=$ $x_{i_{1}} \cdots x_{i_{t}}, i_{j} \in \mathbb{I}$, is an $(n+1)$-chain if there exist integers $a_{j}, b_{j}, 1 \leq j \leq n$, such that 
(1) $1=a_{1}<a_{2} \leq b_{1}<a_{3} \leq b_{2}<\cdots<a_{n} \leq b_{n-1}<b_{n}=t$;

(2) $x_{i_{a_{j}}} x_{i_{a_{j}+1}} \ldots x_{i_{b_{j}-1}} x_{i_{b_{j}}} \in \mathcal{T}$ for all $1 \leq j \leq n$;

(3) for all $1 \leq m \leq n$, the words $x_{i_{1}} \cdots x_{i_{s}}, s<b_{m}$, are not $m$-chains.

By [Ani, Lemma 1.3] the integers $a_{j}, b_{j}$ satisfying (1)-(3) are uniquely determined and

- $x_{i_{1}} \cdots x_{i_{b_{n-1}}}$ is the unique prefix which is an $(n-1)$-chain, and

- $x_{i_{b_{n-1}+1}} \cdots x_{i_{t}}$ does not contain any element of $\mathcal{T}$ as a subword.

Example 4.1.2. We fix $N \geq 2, V$ of dimension $1, x \in V-0, \mathcal{I}=\left\langle x^{N}\right\rangle$. Thus $\mathcal{T}=\left\{x^{N}\right\}$. Now $\mathrm{M}(0)=\{1\}, \mathrm{M}(1)=\{x\}$, and we claim that

$$
\mathrm{M}(2 k)=\left\{x^{N k}\right\}, \quad \mathrm{M}(2 k+1)=\left\{x^{N k+1}\right\}, \quad k \geq 1 .
$$

Moreover $a_{2 i-1}=(i-1) N+1, a_{2 i}=(i-1) N+2, b_{2 i-1}=i N, b_{2 i}=i N+1$. We proceed by induction on $\mathbb{k}$. If $k=2$, then $\mathrm{M}(1)=\left\{x^{N}\right\}$ since this is the unique minimal tip, and $\left\{x^{N+1}\right\}$ is a 2-chain with $a_{1}=1, a_{2}=2, b_{1}=N, b_{2}=N+1$; thus, each word $x^{N+j}, j>1$, is not a 2-chain since $x^{N+1}$ is a prefix of $x^{N+j}$.

Assume that $k \geq 2$ and the statement holds for $\mathbb{k}$. To compute $\mathrm{M}(2 k+2)$, we start with the unique $(2 k+1)$-chain $x^{k N+1}$ and the integers $a_{j}, b_{j}$ already determined: $a_{2 k+1}$ should satisfy $b_{2 k-1}=k N<a_{2 k+1} \leq b_{2 k}=k N+1$, hence $a_{2 k+1}=k N+1$. Hence $\mathrm{M}(2 k+2)=\left\{x^{(k+1) N}\right\}$. For $\mathrm{M}(2 k+3)$, we have that $x^{(k+1) N+1}$ is a $(2 k+3)$-chain, hence this is the unique $(2 k+3)$-chain: indeed, if $w=x^{s} \in \mathrm{M}(2 k+3)$, then $s>(k+1) N$ since $w$ should contain the $(2 k+2)$-chain $x^{(k+1) N}$ as a prefix; but if $s>(k+1) N$, then $w$ contains the $(2 k+3)$-chain $x^{(k+1) N+1}$ as a prefix so it cannot be a $(2 k+3)$-chain.

Let $\mathrm{V}(n)$ be the $\mathbb{k}$-vector space with basis $\mathrm{M}(n)$. Then

$$
\widehat{\mathrm{M}}(n):=\{u \otimes w: u \in \mathbb{M}(n), w \in \mathrm{B}\}
$$

is a basis of $\mathrm{V}(n) \otimes \mathcal{A}$. Given $u \otimes w, v \otimes z \in \widehat{\mathrm{M}}(n)$, if $u w=v z$, then $u=v, w=z$; indeed, if $\ell(u) \leq \ell(v)$, then the $n$-chain $u$ is a prefix of the $n$-chain $v$ and (3) implies that $u=v$. Hence the order on $X$ induces an order on $\widehat{\mathbb{M}}(n): u \otimes w<v \otimes z$ if $u w<v z$.

4.1.3. The Anick graph. We next introduce a graph which helps to compute the chains of the Anick resolution $\mathrm{CoU}$. Let $\Gamma$ be the graph whose set of vertices is given by the union of $\{1\}, X$ and the set of all proper suffixes of the minimal tips. For the arrows, there exists one arrow from $1 \rightarrow x$ for each $x \in X$, and one arrow $u \rightarrow v$ if the word $u v$ contains a unique minimal tip such that it is a suffix of $u v$ (possibly the word $u v$ ).

A basis of the free module of $n$-chains of the Anick resolution is given by paths of length $n$ starting at 1 . Thus:

- There exists a unique 0-chain: 1 .

- The set $X$ gives a basis of the 1-chains.

- The set of minimal tips gives a basis of the 2-chains.

Notice that vertices $v$ not connected to 1 (that is, without a path from 1 to $v$ ) do not contribute new elements for the basis of chains, hence we may omit them and the related arrows. 
Example 4.1.3. Let $\zeta \in \mathbb{G}_{12}^{\prime}, q \in \mathbb{k}^{\times}$. We want to determine the Anick graph of the Nichols algebra $\mathscr{B}_{\mathfrak{q}}$ of $[\mathrm{AA}, \S 10.7 .5]$ (The scalar $q$ corresponds to $q_{12}$ in loc. cit.) In terms of the PBW generators, $\mathscr{B}_{\mathfrak{q}}$ is presented by generators $x_{1}, x_{1112}, x_{112}, x_{12}, x_{2}$, and relations

$$
\begin{aligned}
& x_{1}^{4}=0, \quad x_{1} x_{1112}=\zeta^{3} q x_{1112} x_{1}, \quad x_{1} x_{112}=-q x_{112} x_{1}+x_{1112}, \\
& x_{1112}^{2}=0, \quad x_{1} x_{12}=\zeta^{9} q x_{12} x_{1}+x_{112}, \quad x_{1} x_{2}=q x_{2} x_{1}+x_{12} \\
& x_{112}^{3}=0, \quad x_{1112} x_{112}=\zeta^{2} q x_{112} x_{1112}, \quad x_{1112} x_{12}=\zeta^{10} q^{2} x_{12} x_{1112}-q(1+\zeta) x_{112}^{2}, \\
& x_{12}^{3}=0, \quad x_{112} x_{12}=\zeta q x_{12} x_{112}, \quad x_{1112} x_{2}=-q^{3} x_{2} x_{1112}+\zeta^{5} q^{2} x_{12} x_{112}, \\
& x_{2}^{2}=0, \quad x_{12} x_{2}=-q x_{2} x_{12}, \quad x_{112} x_{2}=-q^{2} x_{2} x_{112}-q_{12}\left(1+\zeta^{9}\right) x_{12}^{2} \text {. }
\end{aligned}
$$

Thus the set of 2-chains (equivalently, minimal tips or obstructions) is

$$
\begin{aligned}
\mathrm{M}(2)= & \left\{x_{1}^{4}, x_{1112}^{2}, x_{112}^{3}, x_{12}^{3}, x_{2}^{2}, x_{1} x_{1112}, x_{1} x_{112}, x_{1} x_{12}, x_{1} x_{2},\right. \\
& \left.x_{1112} x_{112}, x_{1112} x_{12}, x_{1112} x_{2}, x_{112} x_{12}, x_{112} x_{2}, x_{12} x_{2}\right\}
\end{aligned}
$$

and the Anick graph is

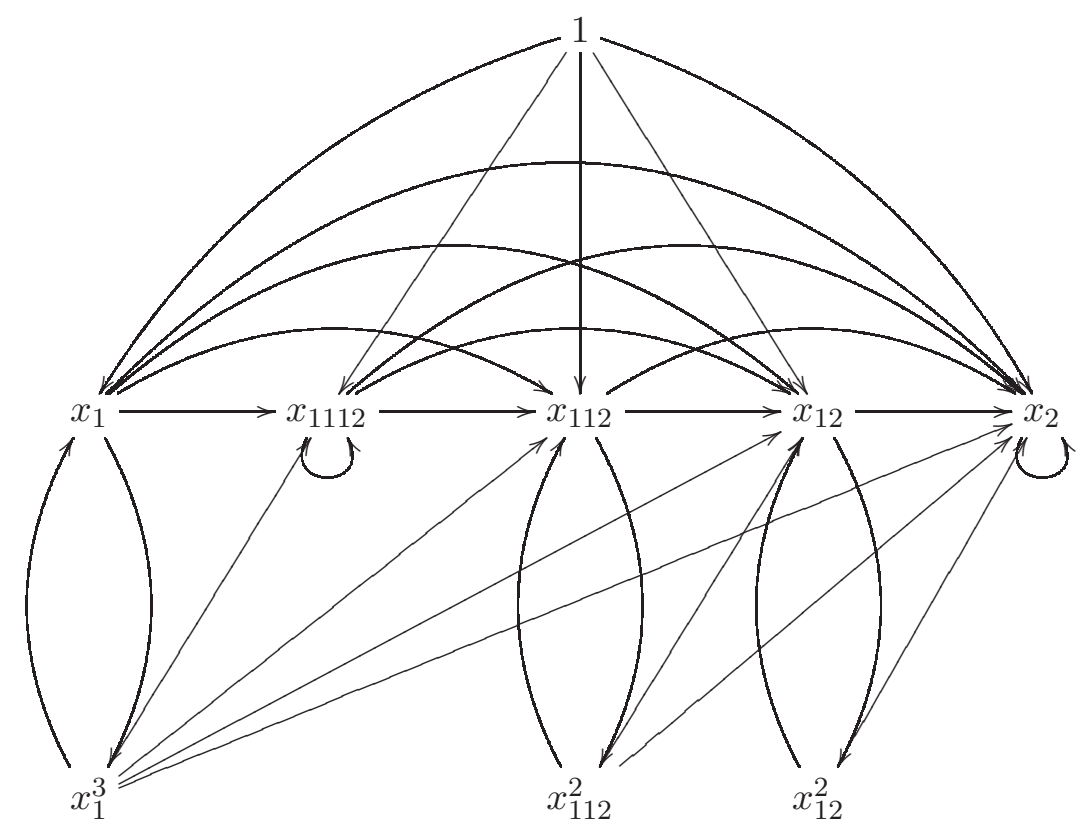

There exists a vertex $x_{1}^{2}$ with a loop on itself which we omit since this vertex is not connected to 1 . Using the graph we compute

$$
\begin{aligned}
\mathrm{M}(3)= & \left\{x_{1}^{5}, x_{1}^{4} x_{1112}, x_{1}^{4} x_{112}, x_{1}^{4} x_{12}, x_{1}^{4} x_{2}, x_{1} x_{1112}^{2}, x_{1112}^{3}, x_{1112}^{2} x_{112}, x_{1112}^{2} x_{12}, x_{1112}^{2} x_{2},\right. \\
& x_{1} x_{112}^{3}, x_{1112} x_{112}^{3}, x_{112}^{4}, x_{112}^{3} x_{12}, x_{112}^{3} x_{2}, x_{1} x_{12}^{3}, x_{1112} x_{12}^{3}, x_{112} x_{12}^{3}, x_{12}^{4}, x_{12}^{3} x_{2}, \\
& x_{1} x_{2}^{2}, x_{1112} x_{2}^{2}, x_{112} x_{2}^{2}, x_{12} x_{2}^{2}, x_{2}^{3}, x_{1} x_{1112} x_{112}, x_{1} x_{1112} x_{12}, x_{1} x_{1112} x_{2}, x_{1} x_{112} x_{12}, \\
& \left.x_{1} x_{112} x_{2}, x_{1} x_{12} x_{2}, x_{1112} x_{112} x_{12}, x_{1112} x_{112} x_{2}, x_{1112} x_{12} x_{2}, x_{112} x_{12} x_{2}\right\} .
\end{aligned}
$$


4.1.4. The resolution. We consider the Anick resolution of the $\mathcal{A}$-module $\mathbb{k}$. Recall that $\mathrm{V}(n)$ is the $\mathbb{k}$-vector space with basis $\mathrm{M}(n)$. In [Ani, Theorem 1.4] Anick introduced an $\mathcal{A}$-free complex

$$
\ldots \ldots \ldots . . . \mathrm{V}(n) \otimes \mathcal{A} \stackrel{d_{n}}{\longrightarrow} \mathrm{V}(n-1) \otimes \mathcal{A} \stackrel{d_{n-1}}{\longrightarrow} \ldots \ldots \ldots \ldots . . . \mathrm{V}(1) \otimes \mathcal{A} \stackrel{d_{1}}{\longrightarrow} \mathcal{A} \stackrel{\epsilon}{\longrightarrow} \mathbb{k} \longrightarrow 0
$$

and $\mathbb{k}$-linear maps $s_{n}: \mathrm{V}(n) \otimes \mathcal{A} \rightarrow \mathrm{V}(n+1) \otimes \mathcal{A}, n \in \mathbb{N}_{0}$, such that (4.1.4) is an $\mathcal{A}$-resolution of $\mathbb{k}$ and $s$. is a contracting homotopy:

$$
d_{n+1} s_{n}+s_{n-1} d_{n}=\mathrm{id}_{\mathrm{V}(n) \otimes \mathcal{A}} \quad \text { for all } n \in \mathbb{N} .
$$

The maps $d_{n}, s_{n-1}$ are defined recursively; see for example [NWW, §4.1] for a left module version. For $n=1$, the map $d_{1}$ is determined by

$$
d_{1}(x \otimes 1)=x \quad \text { for all } x \in \mathrm{M}(1),
$$

while for $s_{0}$ we give the values on each $w \in \mathrm{B}$ :

$$
s_{0}(w)=x \otimes z, \quad w=x z, x \in \mathrm{M}(1), z \in \mathrm{B} .
$$

Now assume that $d_{1}, \ldots, d_{n-1}, s_{0}, \ldots, s_{n-2}$ were already defined and satisfy:

$$
\text { (4.1.5) } \quad d_{i-1} d_{i}=0, \quad s_{i-2} s_{i-1}=0, \quad \text { for all } 1 \leq i \leq n-1 \text {. }
$$

The morphisms of $\mathcal{A}$-modules $d_{n}: \mathrm{V}(n) \otimes \mathcal{A} \rightarrow \mathrm{V}(n-1) \otimes \mathcal{A}$ are determined by

$$
d_{n}(u \otimes 1)=v \otimes t-s_{n-2} d_{n-1}(v \otimes t), \quad u \in \mathrm{M}(n), u=v t, v \in \mathrm{M}(n-1), t \in \mathrm{B} .
$$

Now we define $s_{n-1}$. From (4.1.5), V $(n-1) \otimes \mathcal{A}=\operatorname{ker} d_{n-1} \oplus \mathrm{im} s_{n-2}$. We start by defining $\left(s_{n-1}\right)_{\mid \text {im } s_{n-2}} \equiv 0$, so $s_{n-1} s_{n-2}=0$. Now we define $\left(s_{n-1}\right)_{\mid \operatorname{ker} d_{n-1}}$ recursively on the order of the leading term of each element of ker $d_{n-1}$, which we write in terms of the basis $\widehat{\mathrm{M}}(n-1)$. We require $d_{n}\left(s_{n-1}\right)_{\mid \operatorname{ker} d_{n-1}}=\operatorname{id}_{\operatorname{ker} d_{n-1}}$. Let

$$
K=\sum_{j \in \mathbb{I}_{m}} a_{j} u_{j} \otimes b_{j} \in \operatorname{ker} d_{n-1}, \quad \quad a_{j} \in \mathbb{k}^{\times}, u_{j} \in \mathrm{M}(n-1), b_{j} \in \mathrm{B} .
$$

We assume that $u_{1} \otimes b_{1}$ is bigger than $u_{j} \otimes b_{j}$ for all $j>1$. We write $u_{j}=v_{j} t_{j}$, where $v_{j} \in \mathrm{M}(n-2), t_{j} \in \mathrm{B}$. Hence

$$
0=d_{n-1}(K)=a_{1} v_{1} \otimes t_{1} b_{1}+\widehat{K}, \quad \widehat{K}:=\sum_{j \geq 2} a_{j} d_{n-1}\left(u_{j} \otimes b_{j}\right)-s_{n-3} d_{n-2}\left(a_{1} v_{1} \otimes t_{1} b_{1}\right)
$$

Hence $t_{1} b_{1} \notin \mathrm{B}$, otherwise $v_{1} \otimes t_{1} b_{1}$ is the biggest element of $\widehat{\mathrm{M}}(n-1)$ in the previous expression of $d_{n-1}(K)$ with non-zero coefficient. Now we write $b_{1}=w_{1} y_{1}$, where $w_{1}$ is the shortest prefix of $b_{1}$ such that $t_{1} w_{1} \notin \mathrm{B}$. Hence $u_{1} w_{1}=v_{1} t_{1} w_{1} \in \mathrm{M}(n)$. Hence we set

$$
s_{n-1}(K):=a_{1} u_{1} w_{1} \otimes y_{1}+s_{n-1}\left(\sum_{j \in \mathbb{I}_{m}} a_{j} u_{j} \otimes b_{j}-d_{n}\left(a_{1} u_{1} w_{1} \otimes y_{1}\right)\right) .
$$

Below the differentials $d_{n}$ and the maps $s_{n}$ will be denoted simply by $d$ and $s$.

We shall refer to the complex (4.1.4) as the Anick resolution of $\mathcal{A}$, and denote it by $\left(C_{*}(\mathcal{A}), d\right)$ :

$$
\ldots \ldots \ldots \ldots . . . C_{n}(\mathcal{A}) \stackrel{d_{n}}{\longrightarrow} C_{n-1}(\mathcal{A}) \stackrel{d_{n-1}}{\longrightarrow} \ldots \ldots \ldots . . . C_{1}(\mathcal{A}) \stackrel{d_{1}}{\longrightarrow} \mathcal{A} \stackrel{\epsilon}{\longrightarrow} \mathbb{k} \longrightarrow 0
$$


so that $C_{n}(\mathcal{A})=\mathrm{V}(n) \otimes \mathcal{A}$.

4.2. Application to Nichols algebras. We consider now the Anick resolution of a Nichols algebra $\mathscr{B}_{\mathfrak{q}}$ of diagonal type associated to the presentation given by PBW generators $\left(x_{\beta}\right)_{\beta \in \Delta_{+}^{\mathfrak{q}}}$. We fix a convex order on $\Delta_{+}^{\mathfrak{q}}$, which induces a total order on the letters: $x_{\beta_{1}}>x_{\beta_{2}}>\cdots>x_{\beta_{m}}$. We set $N_{\beta}=$ ord $\mathfrak{q}_{\beta \beta}$.

The defining relations of $\mathscr{B}_{\mathfrak{q}}$ are

$$
\begin{aligned}
x_{\beta}^{N_{\beta}} & =0, & & N_{\beta} \text { finite; } \\
{\left[x_{\beta_{i}}, x_{\beta_{j}}\right]_{c} } & =\sum_{n_{i+1}, \ldots, n_{j-1} \in \mathbb{N}_{0}} c_{n_{i+1}, \ldots, n_{j-1}}^{(i, j)} x_{\beta_{j-1}}^{n_{j-1}} \ldots x_{\beta_{i+1}}^{n_{i+1}}, & & i<j .
\end{aligned}
$$

where $c_{n_{i+1}, \ldots, n_{j-1}}^{(i, j)} \in \mathbb{k}$ can be computed explicitly [An1, Lemma 4.5].

We denote by $\mathrm{x}_{\beta}, \beta \in \Delta_{+}^{\mathfrak{q}}$, the set of letters for the tips and the chains, to distinguish them from the generators $x_{\beta}$ of the Nichols algebra $\mathscr{B}_{\mathfrak{q}}$. The minimal tips are the following:

$$
\mathrm{x}_{\alpha} \mathrm{x}_{\beta}, \quad \alpha>\beta \in \beta \in \Delta_{+}^{\mathfrak{q}} ; \quad \mathrm{x}_{\beta}^{N_{\beta}}, \quad \beta \in \Delta_{+}^{\mathfrak{q}} .
$$

Hence the Anick graph looks locally as

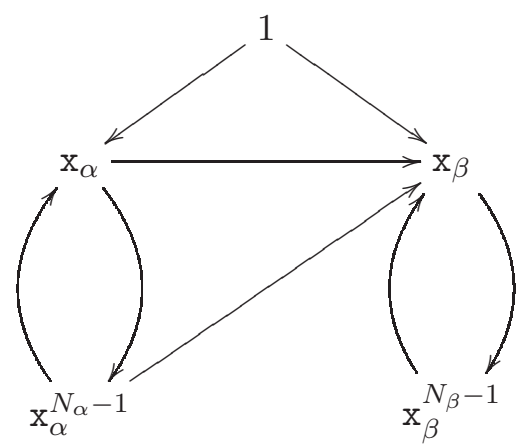

for $\alpha>\beta$, where if $N_{\alpha}=2$, then the loop between $x_{\alpha}$ and $x_{\alpha}^{N_{\alpha}-1}$ is understood to be collapsed to a loop from $x_{\alpha}$ to itself, and similarly for $x_{\beta}$. If $N_{\alpha}>3$, then there exist vertices $\mathrm{x}_{\alpha}^{t}$ and $\mathrm{x}_{\alpha}^{N_{\alpha}-t}$ for each $2 \leq t \leq N_{\alpha}-2$, and arrows between them. These vertices are not connected to 1 and are omitted.

Now we describe the set of chains. For each $\delta \in \Delta_{+}^{\mathfrak{q}}$ we set

$$
f_{\delta}: \mathbb{N}_{0} \rightarrow \mathbb{N}_{0}, \quad f_{\delta}(2 k)=N_{\beta} k, \quad f_{\delta}(2 k+1)=N_{\beta} k+1, \quad k \in \mathbb{N}_{0} .
$$

The set of all $n$-chains, $n \in \mathbb{N}$, is given by

$$
\mathrm{M}(n)=\left\{\mathrm{x}_{\beta_{1}}^{f_{\beta_{1}}\left(n_{1}\right)} \mathrm{x}_{\beta_{2}}^{f_{\beta_{2}}\left(n_{2}\right)} \ldots \mathrm{x}_{\beta_{m}}^{f_{\beta_{m}}\left(n_{m}\right)}: \sum n_{j}=n\right\} .
$$

4.3. Quantum linear spaces. These are the less complicated Nichols algebras of diagonal type. Let $\mathfrak{q}=\left(q_{i j}\right)_{i, j \in \mathbb{I}}$ be as above and assume that $q_{i j} q_{j i}=1$ for all $i \neq j \in \mathbb{I}$. Let $\mathbb{I}^{\prime}=\left\{i \in \mathbb{I}: q_{i i} \in \mathbb{G}_{\infty}^{\prime}\right\}$ and for $i \in \mathbb{I}^{\prime}$, set $N_{i}=\operatorname{ord} q_{i i}$. Then $\mathscr{B}_{\mathfrak{q}}$ is presented by generators $y_{i}, i \in \mathbb{I}$, with relations

$$
y_{i} y_{j}=q_{i j} y_{j} y_{i}, \quad i<j \in \mathbb{I},
$$




$$
y_{i}^{N_{i}}=0, \quad i \in I^{\prime} .
$$

Proposition 4.3.3. The cohomology ring of $\mathscr{B}_{\mathfrak{q}}$ is generated by $\eta_{i}$ for $i \in \mathbb{I}$ and $\xi_{j}$, for $j \in \mathbb{I}^{\prime}$ with relations

$$
\begin{aligned}
\xi_{h} \xi_{j} & =q_{h j}^{N_{h} N_{j}} \xi_{j} \xi_{h}, & & h<j \in \mathbb{I}^{\prime}, \\
\eta_{i} \xi_{j} & =q_{i j}^{N_{j}} \xi_{j} \eta_{i}, & & i \in \mathbb{I}, \quad j \in \mathbb{I}^{\prime}, \\
\eta_{i} \eta_{k} & =-q_{i k} \eta_{k} \eta_{i}, & & i<k \in \mathbb{I}, \\
\eta_{i}^{2} & =0, & & i \in \mathbb{I}, N_{i}>2, \\
\eta_{i} \xi_{i} & =\xi_{i} \eta_{i}, & & i \in \mathbb{I}^{\prime} .
\end{aligned}
$$

If $N_{i}=2$, then $\eta_{i}^{2}=\xi_{i}$. If $M$ is a finitely generated $\mathscr{B}_{\mathfrak{q}}$-module, then $\mathrm{H}\left(\mathscr{B}_{\mathfrak{q}}, M\right)$ is finitely generated as a module over $\mathrm{H}\left(\mathscr{B}_{\mathfrak{q}}, \mathbb{k}\right)$.

Proof. We will construct the Anick resolution $K$. of $\mathbb{k}$ as a $\mathscr{B}_{\mathfrak{q}}$-module. The following notation will be helpful. For each $i, 1 \leq i \leq \theta$, let $\sigma_{i}, \tau_{i}: \mathbb{N}_{0} \rightarrow \mathbb{N}_{0}$ be the functions defined by

$$
\sigma_{i}(a)=\left\{\begin{array}{cl}
1, & \text { if } a \text { is odd } \\
N_{i}-1, & \text { if } a \text { is even }
\end{array}\right.
$$

and $\tau_{i}(a)=\sum_{j=1}^{a} \sigma_{i}(j)$ for $a \geq 1, \tau(0)=0$.

We claim that the differential $d$ of the Anick resolution is given as follows:

$d\left(\mathrm{y}_{1}^{\tau_{1}\left(a_{1}\right)} \cdots \mathrm{y}_{\theta}^{\tau_{\theta}\left(a_{\theta}\right)} \otimes 1\right)=\sum_{i=1}^{\theta}\left(\prod_{\ell<i}(-1)^{a_{\ell}} q_{\ell i}^{-\sigma_{i}\left(a_{i}\right) \tau_{\ell}\left(a_{\ell}\right)}\right) \mathrm{y}_{1}^{\tau_{1}\left(a_{1}\right)} \cdots \mathrm{y}_{i}^{\tau_{i}\left(a_{i}-1\right)} \cdots y_{\theta}^{\tau_{\theta}\left(a_{\theta}\right)} \otimes y_{i}^{\sigma_{i}\left(a_{i}\right)}$,

where we set $y_{i}^{\sigma_{i}(0)}=0$. This is the right module analog of a formula from [MPSW, $\S 4$ ], for an explicitly constructed minimal resolution of $\mathbb{k}$ as a $\mathscr{B}_{\mathfrak{q}}$-module. (There is a slight difference in comparison of our formulas to those in [MPSW] since we are working with right modules. This also leads to a slight difference in relations among the generators.) The resolution from [MPSW] is in fact the Anick resolution: The modules in the Anick resolution will have the same vector space dimension in each degree by construction, implying that the Anick resolution is also minimal in this case, and so comparison maps between the Anick resolution and this one must be isomorphisms in each degree.

Next apply $\operatorname{Hom}_{S}(-, \mathbb{k})$ to $K$. in order to compute $\operatorname{Ext}_{S}^{*}(\mathbb{k}, \mathbb{k})$. Note that $d^{*}$ is the zero map since $y_{i}^{\sigma_{i}\left(a_{i}\right)}$ acts as 0 on $\mathbb{k}$. Thus in degree $n$ the cohomology is a vector space of dimension $\left(\begin{array}{c}n+\theta-1 \\ \theta-1\end{array}\right)$. Now let $\xi_{i} \in \operatorname{Hom}_{S}\left(K_{2}, \mathbb{k}\right)$ be the function dual to $\mathrm{y}_{i}^{N_{i}} \otimes 1$ and $\eta_{i} \in \operatorname{Hom}_{S}\left(K_{1}, \mathbb{k}\right)$ be the function dual to $\mathrm{y}_{i} \otimes 1$. Identify these functions with the corresponding elements in $\mathrm{H}^{2}(S, \mathbb{k})$ and $\mathrm{H}^{1}(S, \mathbb{k})$, respectively. We claim that $\xi_{i}$, $\eta_{i}$ generate $\mathrm{H}(S, \mathbb{k})$. We also denote by $\xi_{i}$ and $\eta_{i}$ the corresponding chain maps $\xi_{i}: K_{n} \rightarrow K_{n-2}$ and $\eta_{i}: K_{n} \rightarrow K_{n-1}$ given by

$$
\xi_{i}\left(\mathrm{y}_{1}^{\tau_{1}\left(a_{1}\right)} \cdots \mathrm{y}_{\theta}^{\tau_{\theta}\left(a_{\theta}\right)} \otimes 1\right)=\prod_{\ell>i} q_{i \ell}^{-N_{i} \tau_{\ell}\left(a_{\ell}\right)} \mathrm{y}_{1}^{\tau_{1}\left(a_{1}\right)} \cdots \mathrm{y}_{i}^{\tau_{i}\left(a_{i}-2\right)} \cdots \mathrm{y}_{\theta}^{\tau_{\theta}\left(a_{\theta}\right)} \otimes 1
$$




$$
\begin{gathered}
\eta_{i}\left(\mathrm{y}_{1}^{\tau_{1}\left(a_{1}\right)} \cdots \mathrm{y}_{\theta}^{\tau_{\theta}\left(a_{\theta}\right)} \otimes 1\right)= \\
\prod_{\ell<i} q_{\ell i}^{-\left(\sigma_{i}\left(a_{i}\right)-1\right) \tau_{\ell}\left(a_{\ell}\right)} \prod_{\ell>i}(-1)^{a_{\ell}} q_{i \ell}^{-\tau_{\ell}\left(a_{\ell}\right)} \mathrm{y}_{1}^{\tau_{1}\left(a_{1}\right)} \cdots \mathrm{y}_{i}^{\tau_{i}\left(a_{i}-1\right)} \cdots \mathrm{y}_{\theta}^{\tau_{\theta}\left(a_{\theta}\right)} \otimes y_{i}^{\sigma_{i}\left(a_{i}\right)-1} .
\end{gathered}
$$

Note this implies that if $a_{i}$ is even and $N_{i}>2$, then $\eta_{i}\left(\mathrm{y}_{1}^{\tau_{1}\left(a_{1}\right)} \cdots \mathrm{y}_{\theta}^{\tau_{\theta}\left(a_{\theta}\right)} \otimes 1\right)=0$, since $y_{i}^{N_{i}-2}$ acts as 0 on $\mathbb{k}$. Calculations show that these maps satisfy the following equations:

$$
\xi_{i} \xi_{j}=q_{i j}^{N_{i} N_{j}} \xi_{j} \xi_{i}, \quad \eta_{i} \xi_{j}=q_{i j}^{N_{j}} \xi_{j} \eta_{i}, \quad \text { and } \eta_{i} \eta_{j}=-q_{i j} \eta_{j} \eta_{i}
$$

for all $i<j$, and $\eta_{i} \xi_{i}=\xi_{i} \eta_{i}$ for all $i$, with one exception: If $N_{i}=2$, then $\eta_{i}^{2}=\xi_{i}$ (so that we may leave $\xi_{i}$ out of our choice of generators, or not, as is convenient), while if $N_{i}>2$, then $\eta_{i}^{2}=0$. Due to these equations, any element in the subalgebra of $\operatorname{Ext}_{S}^{*}(k, k)$ generated by the $\xi_{i}$ and $\eta_{i}$ may be written as a linear combination of elements of the form $\xi_{1}^{b_{1}} \cdots \xi_{\theta}^{b_{\theta}} \eta_{1}^{c_{1}} \cdots \eta_{\theta}^{c_{\theta}}$ with $b_{i} \geq 0$ and $c_{i} \in\{0,1\}$. Such an element takes $\mathrm{y}^{b_{1} N_{1}+c_{1}} \cdots \mathrm{y}_{\theta}^{b_{\theta} N_{\theta}+c_{\theta}} \otimes 1$ to a nonzero scalar multiple of 1 and all other $S$-basis elements of $K_{\sum\left(2 b_{i}+c_{i}\right)}$ to 0 . Recall that the dimension of $\mathrm{H}^{n}(S, \mathbb{k})$ is $\left(\begin{array}{c}n+\theta-1 \\ \theta-1\end{array}\right)$; consequently, the elements $\xi_{1}^{b_{1}} \cdots \xi_{\theta}^{b_{\theta}} \eta_{1}^{c_{1}} \cdots \eta_{\theta}^{c_{\theta}}$ form a $\mathbb{k}$-basis for $\mathrm{H}(S, \mathbb{k})$.

For the last statement, we may induct on the length of a composition series of $M$, and it suffices to prove the statement in the case that $M$ is a simple module. The generators of $\mathscr{B}_{\mathfrak{q}}$ are all nilpotent, and so the only simple module is the trivial module $\mathbb{k}$, for which the statement is clear.

4.4. Cohomology of graded algebras with convex PBW basis. Here we consider a graded connected algebra $R=\oplus_{n \in \mathbb{N}_{0}} R^{n}$ with a finite PBW-basis $B=B(\{1\}, \mathrm{X},<, h)$; that is $\mathrm{X}$ is a finite subset of $R$ with $r=|\mathrm{X}|$ elements, $<$ a total order on $\mathrm{X}$ (with a numeration $\mathrm{X}=\left\{x_{1}, x_{2}, \ldots\right\}$ such that $i<j$ if and only if $x_{i}<x_{j}$ ) and a function $h: \mathrm{X} \rightarrow \mathbb{N} \cup\{\infty\}$, $x_{i} \mapsto N_{i}$ ( $h$ is called the height), such that

$$
B=\left\{x_{r}^{e_{r}} x_{r-1}^{e_{r-1}} \ldots x_{2}^{e_{2}} x_{1}^{e_{1}}: 0 \leq e_{i}<N_{i}\right\}
$$

is a $\mathbb{k}$-basis of $R$. Let $\mathbb{I}=\{1, \ldots, r\}$ and $\mathbb{I}^{\prime}=\left\{i \in \mathbb{I}: N_{i}<\infty\right\}$. We assume that the elements of $\mathrm{X}$ are homogeneous: $x_{j} \in R^{d_{j}}, d_{j} \in \mathbb{N}$. We set

$$
\operatorname{deg} b=\left(e_{1}, \ldots, e_{r}, \sum_{j} e_{j} d_{j}\right) \in \mathbb{N}_{0}^{r+1}, \quad b=x_{r}^{e_{r}} x_{r-1}^{e_{r}-1} \ldots x_{2}^{e_{2}} x_{1}^{e_{1}} \in B .
$$

Let $\preceq$ be the lexicographical order, reading from the right, on $\mathbb{N}_{0}^{r+1}$. We consider the $\mathbb{N}_{0}^{r+1}$-filtration on $R$ given by

$$
R_{f}=\langle b \in B: \operatorname{deg} b \preceq f\rangle, \quad f=\left(f_{1}, \ldots, f_{r+1}\right) \in \mathbb{N}_{0}^{r+1} .
$$

Inspired by [DCK], we also assume that the PBW-basis $B$ is convex, i.e. $\left(R_{f}\right)_{f \in \mathbb{N}_{0}^{r+1}}$ is an algebra filtration. It can be shown that the PBW-basis $B$ is convex if and only if

(a) for every $i, j \in \mathbb{I}$ with $i<j$, there exists $q_{i j} \in \mathbb{k}$ such that

$$
x_{i} x_{j}=q_{i j} x_{j} x_{i}+\sum_{f \prec \operatorname{deg} x_{i}+\operatorname{deg} x_{j}} R_{f} ;
$$


(b) for every $i \in \mathbb{I}^{\prime}$,

$$
x_{i}^{N_{i}} \in \sum_{f \prec N_{i} \operatorname{deg} x_{i}} R_{f} .
$$

See $\underline{\mathrm{AAH}}$. We call this the PBW-filtration. Assume that in (4.4.1), $q_{i j} \neq 0$ for all $i<j$. Then the associated graded algebra $S:=\operatorname{gr} R$ is a quantum linear space, i.e. it is presented by generators $y_{i}$ (the class of $x_{i}$ ) and relations (4.3.1), (4.3.2).

The PBW basis gives rise to the Anick resolution $C_{*}(R)$ with $C_{n}(R)=\mathrm{V}(n) \otimes R$ computing $\mathrm{H}(R, k)$ and $\mathrm{H}(R, M)$ for an $R$-module $M$ as in \$4.1. By construction of the resolution, $\mathrm{x}_{i}^{\ell_{i} N_{i}}$ (or rather $\mathrm{x}_{i}^{\ell_{i} N_{i}} \otimes 1$ ) are homogeneous elements of cohomological degree $2 \ell_{i}$. Moreover, again by construction, $\mathrm{x}_{i}^{\ell_{i} N_{i}}$ is a basis element in $\mathrm{V}\left(2 \ell_{i}\right)$. We denote by $\left(\mathrm{x}_{i}^{\ell_{i} N_{i}}\right)^{*}$ the corresponding element in the dual basis of $\operatorname{Hom}_{\mathbb{k}}\left(\mathrm{V}\left(2 \ell_{i}\right), k\right) \cong \operatorname{Hom}_{R}\left(C_{2 \ell_{i}}(R), \mathbb{k}\right)$, that is the function which evaluates to 1 on $\mathrm{x}_{i}^{\ell_{i} N_{i}}$ and to 0 on all other basis elements. By construction, these are homogeneous elements (or cochains) in the complex $\operatorname{Hom}_{R}\left(C_{*}(R), \mathbb{k}\right)$ which computes $\mathrm{H}(R, \mathbb{k})$.

In the next theorem we prove that if all cochains $\left(\mathrm{x}_{i}^{\ell_{i} N_{i}}\right)^{*}$ are cocycles, then $\mathrm{H}(R, \mathbb{k})$ has fgc.

Theorem 4.4.3. Let $R$ be a graded connected algebra with a finite convex $P B W$-basis satisfying all of the assumptions above; let $C_{*}(R)$ be the Anick resolution of $R$. Suppose that there exist positive integers $\ell_{i}$ for any $i$ with $N_{i}<\infty$, such that the cochains $\left(\mathrm{x}_{i}^{\ell_{i} N_{i}}\right)^{*}$ are cocycles in $\operatorname{Hom}\left(C_{*}(R), \mathbb{k}\right)$, that is, represent elements in $\mathrm{H}(R, \mathbb{k})$. Then $\mathrm{H}(R, \mathbb{k})$ is finitely generated and $\mathrm{H}(R, M)$ is finitely generated as a module over $\mathrm{H}(R, \mathbb{k})$ for any finitely generated $R$-module $M$.

Proof. Observe that gr $R$ is a quantum linear space. By Proposition 4.3.3, the cohomology $\mathrm{H}($ gr $R, \mathbb{k})$ is finitely generated over its subalgebra generated by $\xi_{i}^{\ell_{i}}$ for all $i \in \mathbb{I}^{\prime}$, since it is generated by all $\xi_{i}, \eta_{i}$. Since the Anick resolution for $R$ is compatible with the PBWfiltration on $R$, there exists an associated spectral sequence $E$ convergent to $\mathrm{H}(R, \mathbb{k})$ whose $E_{1}$-page is $\mathrm{H}(\mathrm{gr} R, \mathbb{k})$; see Theorem 3.4.3. Moreover, the cochains $\left(\mathrm{x}_{i}^{\ell_{i} N_{i}}\right)^{*}$ are the images of $\xi_{i}^{\ell_{i}}$ in the spectral sequence (see the proof of Proposition 4.3.3) and so by assumption, the $\xi_{i}^{\ell_{i}}$ are permanent cycles. Thus the hypotheses of Lemma 3.3.2 are satisfied, and, hence, $\mathrm{H}(R, \mathbb{k})$ is left Noetherian. (That is, $\operatorname{gr} \mathrm{H}(R, \mathbb{k})$ is Noetherian, from which it follows that $\mathrm{H}(R, \mathbb{k})$ is Noetherian.) Finite generation follows from Lemma 3.1.1 taking $K$ to be a trivial algebra there.

If $M$ is a finitely generated $R$-module, then since the Condition 3.4 .1 is satisfied, Theorem 3.4.3 implies that $\mathrm{H}(R, M)$ is finitely generated as an $\mathrm{H}(R, \mathbb{k})$-module.

The following corollary is immediate since Nichols algebras of diagonal type have convex $P B W$ bases.

Corollary 4.4.4. Let $\mathscr{B}_{q}$ be a finite-dimensional Nichols algebra of diagonal type. If $\mathscr{B}_{q}$ satisfies Condition 1.4.1, then it has fgc. 


\section{Cohomology of the Drinfeld double}

In this section we prove that if the bosonization of a Nichols algebra of diagonal type has fgc then so does its Drinfeld double.

We briefly recall the general definition of a twisting map $\tau$ for two algebras $A$ and $B$ : Let $\tau: B \otimes A \rightarrow A \otimes B$ be a bijective $\mathbb{k}$-linear map for which $\tau\left(1_{B} \otimes a\right)=a \otimes 1_{B}$, $\tau\left(b \otimes 1_{A}\right)=1_{A} \otimes b$ for all $a \in A$ and $b \in B$, and the following compositions of maps from $B \otimes B \otimes A \otimes A$ to $A \otimes B$ are equal:

$$
\tau \circ\left(m_{B} \otimes m_{A}\right)=\left(m_{A} \otimes m_{B}\right)(1 \otimes \tau \otimes 1)(\tau \otimes \tau)(1 \otimes \tau \otimes 1),
$$

where $m_{A}$ (respectively, $m_{B}$ ) denotes multiplication on $A$ (respectively, on $B$ ), and 1 denotes an identity map. The twisted tensor product algebra $A \otimes_{\tau} B$ is $A \otimes B$ as a vector space, and its multiplication is the composition $\left(m_{A} \otimes m_{B}\right)(1 \otimes \tau \otimes 1)$.

If $A$ and $B$ are Hopf algebras, we say that $\tau$ is a Hopf twisting if $A \otimes_{\tau} B$ is a Hopf algebra with coalgebra structure being the usual tensor product of coalgebras (no twisting), and $A, B$ are Hopf subalgebras. The augmentation map is $\epsilon_{A} \otimes \epsilon_{B}: A \otimes_{\tau} B \rightarrow \mathbb{k}$.

We assume that the Hopf twisting $\tau$ is compatible with coradical filtrations, that is for

$$
C_{0}^{A} \subset C_{1}^{A} \subset \cdots \quad \text { and } C_{0}^{B} \subset C_{1}^{B} \subset \cdots
$$

the coradical filtrations of $A$ and $B$, we have

$$
\tau\left(C_{b}^{B} \otimes C_{a}^{A}\right) \subset \sum_{r+s \leq a+b} C_{r}^{A} \otimes C_{s}^{B} .
$$

Then the associated graded space $\operatorname{gr}\left(A \otimes_{\tau} B\right)$ is again a Hopf algebra.

We will need a special case of the twisting construction to apply it to Nichols algebras: Assume that $A$ and $B$ are graded by abelian groups $\Gamma$ and $\Gamma^{\prime}$. Let $t: \Gamma \times \Gamma^{\prime} \rightarrow \mathbb{k}^{\times}$be a bicharacter (that is, it induces a homomorphism $\Gamma \otimes_{\mathbb{Z}} \Gamma^{\prime} \rightarrow \mathbb{k}^{\times}$of abelian groups). Define $\tau: B \otimes A \rightarrow A \otimes B$ by $\tau(b \otimes a)=t(|a|,|b|) a \otimes b$ for all homogeneous $a \in A, b \in B$, where $|a| \in \Gamma,|b| \in \Gamma^{\prime}$ denote grading. In order to distinguish a twisted tensor product algebra $A \otimes_{\tau} B$ for which the twisting $\tau$ is defined by a bicharacter $t$ in this way, we will write $A \otimes{ }^{t} B$ for this twisted tensor product algebra.

Due to the following result of Bergh and Oppermann [BO, Theorem 3.7], the cohomology of $A \otimes^{t} B$ can be computed.

Theorem 5.0.1. Let $A$ and $B$ be augmented algebras graded by abelian groups $\Gamma$ and $\Gamma^{\prime}$. Let $t$ be a bicharacter on $\Gamma \times \Gamma^{\prime}$. There is a twisting map $\hat{t}$, induced by the bicharacter $t$, for which

$$
\mathrm{H}\left(A \otimes^{t} B, \mathbb{k}\right) \cong \mathrm{H}(A, \mathbb{k}) \otimes^{\hat{t}} \mathrm{H}(B, \mathbb{k})
$$

Let $A=R \# \mathbb{k} \Gamma$ and $B=(R \# \mathbb{k} \Gamma)^{\#}$ with $R=\mathscr{B}(V)$. Let $D=D(A)$ be the Drinfeld double of $A$. Since $A$ and $B$ are subalgebras of $D=D(A)$ and, as a vector space, $D$ is isomorphic to $A \otimes B$, there is an isomorphism of algebras,

$$
D \cong A \otimes_{\tau} B,
$$

where $A \otimes_{\tau} B$ is a twisted tensor product algebra whose twisting map $\tau: B \otimes A \rightarrow A \otimes B$ is defined to correspond to multiplication in $D$. The augmentation map on $D(A)$ is

$$
\epsilon_{D}=\epsilon_{A} \otimes \epsilon_{B}
$$


Recall that $A$ and $B$ are both coradically graded. With respect to the coradical filtration on $D$, there is indeed an isomorphism

$$
\operatorname{gr} D \cong A \otimes^{t} B
$$

for some bicharacter $t$ on grading groups of $A$ and $B$. The bicharacter $t$ is defined by the braiding $c$ and the group action. See, for example, Be. Hence, as a consequence of Theorem 5.0.1, $\mathrm{H}(\mathrm{gr} D, \mathbb{k})$ can be computed in terms of $\mathrm{H}(A, \mathbb{k})$ and $\mathrm{H}(B, \mathbb{k})$. Moreover, since gr $D$ is a Hopf algebra, its cohomology is graded commutative so the bicharacter $\hat{t}$ takes values \pm 1 .

To show that $D$ has finitely generated cohomology we will establish that $\mathrm{H}(D, \mathbb{k})$ has "enough" cocycles and apply Evens Lemma (3.3.2).

Let $P$., $Q$. be bar resolutions of $\mathbb{k}$ as an $A$-module and as a $B$-module. Then as in $[\mathrm{BO}$, SW] for left modules, we may form the twisted tensor product resolutions $P_{\bullet} \otimes_{\tau} Q$. and $P$. $\otimes^{t} Q$. of $\mathbb{k}$ as a right $A \otimes_{\tau} B$-module and a right $A \otimes^{t} B$-module, respectively. We recall here briefly this construction, and translate to right modules: As a complex of vector spaces, each of $P_{\bullet} \otimes_{\tau} Q_{\bullet}$ and $P_{\bullet} \otimes^{t} Q_{\bullet}$ is simply $P_{\bullet} \otimes Q_{\bullet}$, and it remains to define the $A \otimes_{\tau} B$ and $A \otimes^{t} B$-module structures on each vector space $P_{i} \otimes Q_{j}$. We will do this for $A \otimes_{\tau} B$, and $A \otimes^{t} B$ is similar. For each $j$, define $\tau_{j}: Q_{j} \otimes A \rightarrow A \otimes Q_{j}$ by iterating $\tau$. The right module structure is defined by the following composition of maps:

$$
P_{i} \otimes Q_{j} \otimes A \otimes B \stackrel{1 \otimes \tau_{j} \otimes 1}{\longrightarrow} P_{i} \otimes A \otimes Q_{j} \otimes B \stackrel{\rho_{P_{i}} \otimes \rho_{Q_{j}}}{\longrightarrow} P_{i} \otimes Q_{j},
$$

where $\rho_{P_{i}}$ and $\rho_{Q_{j}}$ denote the module structure maps.

Lemma 5.0.3. Let $f \in \operatorname{Hom}_{A}\left(P_{i}, \mathbb{k}\right)$ be a cocycle. Then $f$ extends to a cocycle representing an element in $H^{*}(D, \mathbb{k})$. A similar statement holds for $\operatorname{Hom}_{B}\left(Q_{j}, \mathbb{k}\right)$.

Proof. The first statement will follow from the construction of the resolution $P \cdot \otimes_{\tau} Q \cdot$ and the definitions. The second statement involves switching the order of $P_{\text {. and }} Q_{\text {.. }}$ This asymmetry in the proof is due to the asymmetry of choosing to work with right modules instead of left modules.

Let $f \in \operatorname{Hom}_{A}\left(P_{i}, \mathbb{k}\right)$ be a cocycle. We first claim that $f \otimes \epsilon_{B}$, as a function on $P_{i} \otimes_{\tau} Q_{0}=$ $P_{i} \otimes_{\tau} B$, is an $A \otimes_{\tau} B$-module homomorphism.

Consider

$$
\left(f \otimes \epsilon_{B}\right)((x \otimes y) \cdot(a \otimes b))
$$

where $x \in P_{i}, a \in A$, and $b, y \in B$. Expression (5.0.4) can be evaluated by first applying $1 \otimes \tau \otimes 1$ to $x \otimes y \otimes a \otimes b$, then applying $f \otimes \epsilon_{A} \otimes \epsilon_{B} \otimes \epsilon_{B}$ to the result, since $f$ is an $A$-module homomorphism and $A$ acts trivially on $\mathbb{k}$. We wish to show this is equal to

$$
\left(\left(f \otimes \epsilon_{B}\right)(x \otimes y)\right) \cdot(a \otimes b)=\left(f \otimes \epsilon_{B}\right)(x \otimes y) \epsilon_{D(A)}(a \otimes b)=f(x) \epsilon_{B}(y) \epsilon_{A}(a) \epsilon_{B}(b) .
$$

It suffices to show this for all $y$ from a set of generators of $B$, for all $a$ from a set of generators of $A$, and for all $x \in P_{i}$ and $b \in B$. If either $a$ or $y$ is an element of the field $\mathbb{k}$, the expression (5.0.4) is equal to

$$
\left(f \otimes \epsilon_{B}\right)((x \cdot a) \otimes(y \cdot b))=f(x \cdot a) \epsilon_{B}(y \cdot b)=f(x) \epsilon_{B}(y) \epsilon_{A}(a) \epsilon_{B}(b),
$$


as desired. Now assume that $a$ and $y$ are generators in the kernel of the augmentation maps for $A$ and $B$, respectively, so each is either a root vector or a difference of group elements. The case where either is a difference of group elements is straightforward since applying $\epsilon$ to either of the middle two factors will yield 0 after applying $\tau$. Now assume that $a$ and $y$ are both root vectors. Then by Proposition 2.5.2 we have that in $D(A)$,

$$
y a=\lambda a y+\kappa(1-g \chi)
$$

where $\lambda, \kappa$ are scalars, $g \in \Gamma, \chi \in \widehat{\Gamma}$. Then

$$
\begin{array}{r}
\left(f \otimes \epsilon_{B}\right)((x \otimes y) \cdot(a \otimes b))= \\
\left(f \otimes \epsilon_{A} \otimes \epsilon_{B} \otimes \epsilon_{B}\right)(\lambda x \otimes a \otimes y \otimes b+\kappa x \otimes 1 \otimes 1 \otimes b-\kappa x \otimes g \otimes \chi \otimes b)= \\
\lambda f(x) \epsilon_{A}(a) \epsilon_{B}(y) \epsilon_{B}(b)+\kappa\left(f(x) \epsilon_{B}(b)-f(x) \epsilon_{A}(g) \epsilon_{B}(\chi) \epsilon_{B}(b)=\right.
\end{array}
$$

where the first term disappears since $\epsilon_{B}(y)=0$ and the second two terms cancel out since $\epsilon_{A}(g)=\epsilon_{B}(\chi)=1$. We also have $f(x) \epsilon_{B}(y) \epsilon_{A}(a) \epsilon_{B}(b)=0$ since $\epsilon_{B}(y)=0$. Therefore the expressions (5.0.4) and (5.0.5) are equal, as desired. It follows that $f \otimes \epsilon_{B}$ is an $A \otimes_{\tau} B$-module homomorphism, that is, $f \otimes \epsilon_{B} \in \operatorname{Hom}_{A \otimes_{\tau} B}\left(P_{i} \otimes Q_{0}, \mathbb{k}\right)$.

By hypothesis, $0=d_{i+1}^{*}(f)=f d_{i+1}$ where $d_{i+1}: P_{i+1} \rightarrow P_{i}$ is the differential. Letting $d$ denote the differential on $P \cdot \otimes_{\tau} Q_{\bullet}$,

$$
d^{*}\left(f \otimes \epsilon_{B}\right)=\left(f \otimes \epsilon_{B}\right)\left(d_{i+1} \otimes 1+(-1)^{i} \otimes d_{1}\right)=f d_{i+1} \otimes \epsilon_{B}+(-1)^{i} f \otimes \epsilon_{B} d_{1}=0 .
$$

Therefore $f \otimes \epsilon_{B}$ is a cocycle representing an element of $\mathrm{H}(D, \mathbb{k})$.

Now let $g \in \operatorname{Hom}_{B}\left(Q_{j}, \mathbb{k}\right)$ be a cocycle representing an element of $\mathrm{H}(B, k)$. Note that $A \otimes_{\tau} B \cong B \otimes_{\tau^{-1}} A$. Let $Q \cdot \otimes_{\tau^{-1}} P$. be the twisted tensor product resolution corresponding to this inverse twisting $\tau^{-1}$. By the above arguments, $g \otimes \epsilon$ is a cocycle representing an element of $\mathrm{H}\left(A \otimes_{\tau} B, k\right)$. Since $P \cdot \otimes_{\tau} Q$. is quasi-isomorphic to $Q \cdot \otimes_{\tau^{-1}} P$. (in fact, a comparison map is given by iterating $\tau$ ), there is a cocycle $g^{\prime}$ defined on the resolution $P$. $\otimes_{\tau} Q$. corresponding to $g \otimes \epsilon$ on $Q_{i} \otimes_{\tau^{-1}} P_{0}$. Note that in general $g^{\prime}$ will not equal $\epsilon_{A} \otimes g$, due to the twisting.

Theorem 5.0.6. If the Nichols algebra $R=\mathscr{B}(V)$ and its dual $R^{\#}$ have fgc, then the Drinfeld double $D=D(\mathscr{B}(V) \# \mathbb{k} \Gamma)$ of the bosonization $\mathscr{B}(V) \# \mathbb{k} \Gamma$ has fgc.

Proof. Let $A=R \# \mathbb{k} \Gamma$ and $B=A^{\#}$. By hypothesis, $A$ and $B$ have finitely generated cohomology, specifically, the cohomology $\mathrm{H}(A, \mathbb{k})$ is a finite module over a finitely generated commutative subalgebra, and similarly for $\mathrm{H}(B, \mathbb{k})$. Choose generators of these commutative subalgebras and representative cocycles on $P$. and $Q_{\bullet}$; we will use these in a spectral sequence argument in combination with Theorem 5.0.1.

As a consequence of the Theorem 5.0.1, $A \otimes^{t} B$ has finitely generated cohomology since both $A$ and $B$ do. Of necessity, since $A \otimes^{t} B$ is also a Hopf algebra, $\mathrm{H}\left(\operatorname{gr}\left(A \otimes_{\tau} B\right), \mathbb{k}\right)$ is graded commutative, and so $\hat{t}$ will in the end only take values \pm 1 . We will next show that $A \otimes_{\tau} B$ also has finitely generated cohomology. This relies on existence of needed cocycles. Let $f \in \operatorname{Hom}_{A}\left(P_{i}, \mathbb{k}\right)$ be a cocycle representing an element of $\mathrm{H}(A, \mathbb{k})$. Then by Lemma 5.0.3, $f$ extends to a cocycle representing an element of $\mathrm{H}\left(A \otimes_{\tau} B, \mathbb{k}\right)$. A similar statement holds for $\operatorname{Hom}_{B}\left(Q_{j}, \mathbb{k}\right)$. 
Next note that the filtration on $A \otimes_{\tau} B$ induces a filtration on the resolution $P \cdot \otimes_{\tau} Q$. . Let $E$ be the corresponding spectral sequence. Page $E_{1}$ is $\mathrm{H}\left(A \otimes{ }^{t} B, \mathbb{k}\right)$, which by Theorem 5.0 .1 is isomorphic to $\mathrm{H}(A, \mathbb{k}) \otimes^{\hat{t}} \mathrm{H}(B, \mathbb{k})$. The cohomology $\mathrm{H}\left(A \otimes^{\tau} B, \mathbb{k}\right)$ is the homology of the total complex of the bicomplex

$$
\operatorname{Hom}_{A \otimes_{\tau} B}\left(P \cdot \otimes_{\tau} Q \cdot, \mathbb{k}\right) .
$$

By the above argument, a cocycle $f \in \operatorname{Hom}_{A}\left(P_{i}, \mathbb{k}\right)$ representing an element of $\mathrm{H}^{i}(A, \mathbb{k})$ may be extended to a cocycle $f \otimes \epsilon \in \operatorname{Hom}_{A \otimes_{\tau} B}\left(P_{i} \otimes_{\tau} Q_{0}, \mathbb{k}\right)$ representing an element of $\mathrm{H}\left(A \otimes^{\tau} B, \mathbb{k}\right)$. This is thus a permanent cocycle in the spectral sequence $E$. Moreover, it corresponds to $f \otimes \epsilon$, this time representing an element of the $E_{1}$-page $\mathrm{H}(A, \mathbb{k}) \otimes{ }^{\hat{t}} \mathrm{H}(B, \mathbb{k})$. Similarly, a cocycle $g \in \operatorname{Hom}_{B}\left(Q_{j}, \mathbb{k}\right)$ representing an element of $\mathrm{H}^{j}(B, \mathbb{k})$ may be extended to a cocycle $g^{\prime} \in \operatorname{Hom}_{A \otimes_{\tau} B}\left(P_{0} \otimes_{\tau} Q_{j}, \mathbb{k}\right)$ representing an element of $\mathrm{H}\left(A \otimes_{\tau} B, \mathbb{k}\right)$. Thus we obtain, for each chosen generator of $\mathrm{H}\left(\operatorname{gr} A \otimes_{\tau} B, \mathbb{k}\right)$, a permanent cocycle in the spectral sequence. Applying the spectral sequence Lemma 3.3.2, since $\mathrm{H}\left(A \otimes^{t} B, \mathbb{k}\right)$ is a finite module over a finitely generated (commutative) subalgebra, $\mathrm{H}\left(A \otimes_{\tau} B, \mathbb{k}\right)$ is finitely generated. (A commutative subalgebra can be found by taking high enough powers of the chosen generators since the defining parameters and thus also the values of the bicharacter $\hat{t}$ are all roots of unity.)

Now let $M$ be a finitely generated $A \otimes_{\tau} B$-module. Then $\mathrm{H}\left(A \otimes_{\tau} B, M\right)$ is a graded module over $\mathrm{H}\left(A \otimes_{\tau} B, \mathbb{k}\right)$. The coradical filtration on $A \otimes_{\tau} B$ induces a filtration on the bar resolution $K$. of $\mathbb{k}$ as $A \otimes_{\tau} B$-module and thus on $\operatorname{Hom}_{A \otimes_{\tau} B}\left(K_{\bullet}, M\right)$. Let $\widetilde{E^{*}}$ be the corresponding spectral sequence. Arguing as in [Ja, §I.9.13], we get a spectral sequence

$$
\widetilde{E}_{M}^{*}=\mathrm{H}\left(\operatorname{gr} A \otimes_{\tau} B, \mathbb{k}\right) \otimes M \Rightarrow \mathrm{H}\left(A \otimes_{\tau} B, M\right) .
$$

which is a module over the spectral sequence $\widetilde{E^{*}}$. By Lemma 3.3.2, $\mathrm{H}\left(A \otimes_{\tau} B, M\right)$ is finitely generated over $\mathrm{H}\left(A \otimes_{\tau} B, \mathbb{k}\right)$.

\section{Part II. Permanent cocycles for Nichols algebras of diagonal type}

In this Part we deal with

Condition 1.4.1. Let $U$ be a braided vector space of diagonal type whose Nichols algebra is finite-dimensional. For every positive root $\gamma \in \Delta_{+}^{U}$, there exists $L_{\gamma} \in \mathbb{N}$ such that the cochain $\left(\mathrm{x}_{\gamma}^{L_{\gamma}}\right)^{*}$ is a cocycle, that is, represents an element in $\mathrm{H}(\mathscr{B}(U), \mathbb{k})$.

We shall prove that Condition 1.4.1 holds for one representative $U$ of each Weylequivalence class in the classification of [H2]. By Theorem 4.4 .3 this shows that $\mathscr{B}(U)$ has fgc and as explained in \$1.4, this implies Theorem 1.3.1. We argue also by induction on $\operatorname{dim} U$; in other words we often assume that the root $\gamma$ has full support, i.e. $\operatorname{supp} \gamma=\mathbb{I}$. Towards this, we choose the representative $U$ in the Weyl-equivalence class in such a way that Condition 1.4.1 was already verified for any proper subdiagram. 
We discuss the strategy in Section [6, a summary been given in $\S 6.4$. Proofs of the technical statements in this Section are deferred to Section 10. We proceed case by case in Sections 7 (classical Cartan and super types), 8 (exceptional Cartan and super types) and 9 (modular types wk(4) and $\operatorname{br}(2)$ ). The remaining Nichols algebras of diagonal type in the classification are dealt with in [AAPPW].

\section{The STRATEGY}

6.1. The setup. Let $\mathfrak{q}$ be the braiding matrix of $U$ and denote by $\mathscr{B}_{\mathfrak{q}}$ the corresponding Nichols algebra as before. For $\delta \in \Delta_{+}^{\mathfrak{q}}$ recall that $N_{\delta}=$ ord $q_{\delta \delta}$. Recall that the set of $n$-chains, $n \in \mathbb{N}$, is given by

$$
\mathrm{M}(n)=\left\{\mathrm{x}_{\delta_{1}}^{f_{\delta_{1}}\left(n_{1}\right)} \mathrm{x}_{\delta_{2}}^{f_{\delta_{2}}\left(n_{2}\right)} \cdots \mathrm{x}_{\delta_{m}}^{f_{\delta_{m}}\left(n_{m}\right)}: \sum n_{j}=n\right\},
$$

where for $\delta \in \Delta_{+}^{\mathfrak{q}}$ we introduce $f_{\delta}: \mathbb{N}_{0} \rightarrow \mathbb{N}_{0}$ by

$$
f_{\delta}(2 k)=N_{\delta} k, \quad f_{\delta}(2 k+1)=N_{\delta} k+1, \quad k \in \mathbb{N}_{0} .
$$

The starting point is the following straightforward observation.

Remark 6.1.1. Let $\gamma \in \Delta_{+}^{\mathfrak{q}}$ and $L \in \mathbb{N}$.

(a) $\mathrm{x}_{\gamma}^{L}$ is a chain if and only if $L$ is of the form $\ell N_{\gamma}$ or $\ell N_{\gamma}+1$, for some $\ell \in \mathbb{N}$.

(b) A cochain $\left(\mathrm{x}_{\gamma}^{L}\right)^{*}$ is a cocycle if and only if for any chain $c \in \mathbb{M}(n+1)$ such that $d(c \otimes 1) \in \mathrm{V}(n) \otimes \mathscr{B}_{\mathfrak{q}}$, when written as a linear combination of basis elements, the term $\mathrm{x}_{\gamma}^{L} \otimes 1$ has zero coefficient.

Because of Theorem 4.4.3 and Remark 6.1.1 (a) we shall assume that $L=\ell N_{\gamma}$ for $\ell \in \mathbb{N}$.

We reduce the set of chains $c \in \mathrm{M}(n+1)$ to be considered in (b) using degree and grading constraints. First, since the relations of $\mathscr{B}_{\mathfrak{q}}$ are $\mathbb{N}_{0}^{\mathbb{I}}$-homogeneous by definition we have:

Lemma 6.1.2. The differential of the Anick resolution preserves the $\mathbb{N}^{\mathbb{I}}$-grading.

Let $c=\mathrm{x}_{\delta_{1}}^{f_{\delta_{1}}\left(n_{1}\right)} \mathrm{x}_{\delta_{2}}^{f_{\delta_{2}}\left(n_{2}\right)} \ldots \mathrm{x}_{\delta_{m}}^{f_{\delta_{m}}\left(n_{m}\right)} \in \mathrm{M}(n+1)$ such that

$$
d(c \otimes 1)=\ldots+\lambda \mathrm{x}_{\gamma}^{\ell N_{\gamma}} \otimes 1+\ldots, \quad \lambda \neq 0,
$$

as a linear combination of basis elements. By Lemma 6.1.2 and since $\mathrm{x}_{\gamma}^{\ell N_{\gamma}}$ is a $2 \ell$-chain, we have the following constraints:

$$
\begin{aligned}
f_{\delta_{1}}\left(n_{1}\right) \delta_{1}+\cdots+f_{\delta_{m}}\left(n_{m}\right) \delta_{m} & =\ell N_{\gamma} \gamma, \\
n_{1}+\cdots+n_{m} & =2 \ell+1 .
\end{aligned}
$$

Henceforth we refer to the conditions (6.1.3) and (6.1.4) on the chains as the $\mathbb{N}_{0}^{\mathbb{I}}$-grading and homological degree constraints. Writing the roots as linear combinations of simple roots, (6.1.3) and (6.1.4) boil down to a system of equations on $\left\{n_{1}, \ldots, n_{m}, \ell\right\}$.

Let $\gamma \in \Delta_{+}^{\mathfrak{q}}$ and $\ell \in \mathbb{N}$. We summarize now the approaches to verify that $\left(\mathrm{x}_{\gamma}^{\ell N_{\gamma}}\right)^{*}$ is a cocycle using Remark 6.1.1](b)] Thus we need to consider the chains $c \in \mathrm{M}(n+1)$ in (b) up to degree and grading constraints.

○ We introduce integers $P_{\gamma}, Q_{\gamma}$ in 6.2 . If $N_{\gamma}>P_{\gamma}, Q_{\gamma}$, then $\left(\mathrm{x}_{\gamma}^{N_{\gamma}}\right)^{*}$ is a cocycle of degree 2, by Lemma 6.2.5. Here $\ell=1$. 
We may assume that $\gamma$ is not simple, otherwise it is covered by the previous discussion. Then there are $\beta, \delta \in \Delta_{+}^{\mathfrak{q}}$ such that $\beta<\gamma<\delta, \gamma=\beta+\delta$. If $N_{\gamma}$ is small, typically 2 or 3 , then the condition $N_{\gamma}>P_{\gamma}, Q_{\gamma}$ does not hold.

- Assume that $N_{\gamma}=2$ and condition (6.2.9) holds. Then $\left(\mathrm{x}_{\gamma}^{N_{\gamma}}\right)^{*}$ is a 2-cocycle by Lemma 6.2.8. Again $\ell=1$.

- Condition (6.2.9) is about the relations between the root vectors $x_{\beta}, x_{\gamma}, x_{\delta}$. If it does not hold, then a finer analysis is needed. We summarize in Proposition 6.3.2 all possible cases that we need to check in this setting when $N_{\gamma}=2$.

- Similarly we summarize in Proposition 6.3.23 all possible cases to check when $N_{\gamma}>2$ and the condition $N_{\gamma}>P_{\gamma}, Q_{\gamma}$ does not hold.

Propositions 6.3.2 and 6.3.23 depend on several Lemmas whose proof is deferred to Section 10

The techniques presented in Lemma 6.2.5. Lemma 6.2.8, Proposition 6.3.2 and Proposition 6.3.23 are applied to those Dynkin diagrams in the list of $\mathrm{H}_{2}$ with a continuous parameter in Sections 7, 8 and 9. The remaining diagrams in the classification are treated in Part III.

6.2. Degree 2 cocycles. We discuss two techniques to get generators of degree 2 in cohomology from root vectors. First we introduce $P_{\gamma}$ and $Q_{\gamma}$ that under suitable conditions imply the existence of the cocycles.

Definition 6.2.1. Let $\gamma \in \Delta_{+}^{\mathfrak{q}}$. We define

$$
\begin{aligned}
& P_{\gamma}=\max \left\{p \in \mathbb{N}: \exists \text { distinct } \delta_{1}, \delta_{2}, \delta_{3} \in \Delta_{+}^{\mathfrak{q}} \text { such that } \delta_{1}+\delta_{2}+\delta_{3}=p \gamma\right\}, \\
& Q_{\gamma}=\max \left\{q \in \mathbb{N}: \exists \text { distinct } \delta_{1}, \delta_{2} \in \Delta_{+}^{\mathfrak{q}} \text { such that } N_{\delta_{1}} \delta_{1}+\delta_{2}=q \gamma\right\} .
\end{aligned}
$$

We set $P_{\gamma}=0$, respectively $Q_{\gamma}=0$ if no such relation exists.

Remark 6.2.2. For any specific $\gamma \in \Delta_{+}^{\mathfrak{q}}$, the computation of $P_{\gamma}, Q_{\gamma}$ depends only on the combinatorics of the corresponding root system, see for example Lemma 6.2.7. We will leave these calculations for an interested reader in the later sections as they are straightforward to do in any specific case.

Example 6.2.3. If $\gamma$ is simple, then $P_{\gamma}=Q_{\gamma}=0$. Also, if $\gamma$ is not simple, then

$$
P_{\gamma} \geq 2 \text {. }
$$

For, since $\gamma$ is not simple, $\gamma=\beta+\delta$ for some distinct $\beta, \delta \in \Delta_{+}^{\mathfrak{q}}$, hence $2 \gamma=\gamma+\beta+\delta$.

Lemma 6.2.5. Let $\gamma \in \Delta_{+}^{\mathfrak{q}}$. If $N_{\gamma}>P_{\gamma}, Q_{\gamma}$, then $\left(\mathrm{x}_{\gamma}^{N_{\gamma}}\right)^{*}$ is a cocycle of degree 2. In particular, if $\gamma$ is simple, then $\left(\mathrm{x}_{\gamma}^{N_{\gamma}}\right)^{*}$ is a cocycle of degree 2 .

Proof. To show that $\left(\mathrm{x}_{\gamma}^{N_{\gamma}}\right)^{*}$ is a cocycle, we use Remark 6.1.1 (b). That is, we show that there is no chain $c \in C_{3}$ such that $x_{\gamma}^{N_{\gamma}} \otimes 1$ is among the terms, with nonzero coefficient, of $d_{3}(c \otimes 1) \in C_{2} \otimes A$.

The chains in $C_{3}$ are of the form $\mathrm{x}_{\beta}^{N_{\beta}+1}, \mathrm{x}_{\beta}^{N_{\beta}} \mathrm{x}_{\delta}, \mathrm{x}_{\beta} \mathrm{x}_{\delta}^{N_{\delta}}$ and $\mathrm{x}_{\beta} \mathrm{x}_{\delta} \mathrm{x}_{\eta}$. We consider these cases separately. 
For $c=\mathrm{x}_{\beta}^{N_{\beta}+1}, d_{3}(c)=\mathrm{x}_{\beta}^{N_{\beta}} \otimes x_{\beta}$, which is not of the required form.

Let $c=\mathrm{x}_{\beta}^{N_{\beta}} \mathrm{x}_{\delta}$. If $\mathrm{x}_{\gamma}^{N_{\gamma}} \otimes 1$ is present in $d_{3}(c \otimes 1)$, then Lemma 6.1.2 implies that we have a numerical relation

$$
N_{\beta} \beta+\delta=N_{\gamma} \gamma
$$

which contradicts the assumption $N_{\gamma}>Q_{\gamma}$. The case $\mathrm{x}_{\beta} \mathrm{x}_{\delta}^{N_{\delta}}$ is similar.

Finally, if $c=\mathrm{x}_{\beta} \mathrm{x}_{\delta} \mathrm{x}_{\eta}$, and $\mathrm{x}_{\gamma}^{N_{\gamma}} \otimes 1$ is present in $d_{3}(c \otimes 1)$, then we have a relation

$$
\beta+\delta+\eta=N_{\gamma} \gamma
$$

which again contradicts the assumption $N_{\gamma}>P_{\gamma}$.

Because of the previous Lemma we need to compute $P_{\gamma}$ and $Q_{\gamma}$; this is simplified via the following result. Let $\mathcal{W}$ be the Weyl groupoid of the Nichols algebra $\mathscr{B}_{\mathfrak{q}}$, see [H1] or AA.

Lemma 6.2.6. Let $\delta_{1}, \delta_{2}, \delta_{3} \in \Delta_{+}^{\mathfrak{q}}$. Then there exist $w \in \mathcal{W}$ and $\tau \in \mathbb{S}_{\theta}$ such that

$$
w\left(\delta_{i}\right) \in \Delta_{+}^{\mathfrak{p}} \cap\left(\mathbb{Z} \gamma_{\tau(1)}+\mathbb{Z} \gamma_{\tau(2)}+\mathbb{Z} \gamma_{\tau(3)}\right), \quad i=1,2,3,
$$

for a suitable $\mathfrak{p}$.

Proof. See [CuH, Theorem 2.3].

Lemma 6.2.7. Assume that $\mathfrak{q}$ is of Cartan type and that $\gamma$ is not simple.

(a) In types $A_{\theta}, D_{\theta}$ and $E_{\theta}$, we have $P_{\gamma}=2$ and $Q_{\gamma}=1$.

(b) In types $B_{\theta}, C_{\theta}$ and $F_{4}$, we have $P_{\gamma} \leq 3$ and $Q_{\gamma}=2$.

(c) In type $G_{2}, P_{\gamma} \leq 4$ and $Q_{\gamma} \leq 3$.

Proof. (a) Type $A$ : Let $\gamma=\gamma_{i j}$ with $i<j$. Suppose that there exists $P \in \mathbb{N}$ such that $P \gamma_{i j}=\gamma_{k \ell}+\gamma_{m n}+\gamma_{s u}$ with $k \leq m \leq s$ (and the three roots in the right are different). Then the coefficient of $\gamma_{k}$ in the right hand side is at most 3 , so $P \leq 3$. If $P=3$, then $k=m=s=i$ and $\ell, n, u$ are all different. If, say, $u$ is the largest of them, then the coefficient of $\gamma_{u}$ in the right hand side is 1 , a contradiction. Thus $P_{\gamma}=2$ by (6.2.4). Next, suppose that there exists $P, t \in \mathbb{N}$ such that $P \gamma_{i j}=t \gamma_{k \ell}+\gamma_{m n}$ (and the two roots in the right are different). Arguing as before, we see that $P \leq 1$, and (6.2.4 applies. Types $D, E$ : this follows from Type $A$ and Lemma 6.2.6.

(b) Type $B$ : Let $\gamma_{1}, \ldots, \gamma_{\theta}$ be the simple roots with $\gamma_{\theta}$ the short root. Then the roots come in three flavors: $\gamma_{i}+\ldots+\gamma_{j}, i<j<\theta, \gamma_{i}+\ldots+\gamma_{\theta}$, and $\gamma_{i}+\ldots+\gamma_{j-1}+2 \gamma_{j}+\ldots+2 \gamma_{\theta}$, $i<j \leq \theta$. Hence, the maximum $P$ is 3 and $P_{\gamma} \leq 3$; similarly, $Q_{\gamma} \leq 2$. Note that $P_{\gamma}=3$ and $Q_{\gamma}=2$ can occur, e.g.

$$
\left(\gamma_{\theta-2}+\gamma_{\theta-1}\right)+\left(\gamma_{\theta-2}+\gamma_{\theta-1}+\gamma_{\theta}\right)+\left(\gamma_{\theta-2}+\gamma_{\theta-1}+2 \gamma_{\theta}\right)=3\left(\gamma_{\theta-2}+\gamma_{\theta-1}+\gamma_{\theta}\right) \text {. }
$$

Type $C$ : The coefficient of $\gamma_{\theta}$ in $\gamma$ is 0 or 1 , but in the former, $\gamma$ belongs to a sub-diagram of type $A_{\theta-1}$ that was already settled. Looking at the coefficient of $\gamma_{\theta}$ in both sides of $\delta_{1}+\delta_{2}+\delta_{3}=P \gamma$, we conclude that $P \leq 3$. Similarly, $Q_{\gamma} \leq 2$. Note that $P_{\gamma}=3$ and $Q_{\gamma}=2$ can occur, e.g.

$$
\left(2 \gamma_{\theta-2}+2 \gamma_{\theta-1}+\gamma_{\theta}\right)+\left(\gamma_{\theta-2}+\gamma_{\theta-1}+\gamma_{\theta}\right)+\gamma_{\theta}=3\left(\gamma_{\theta-2}+\gamma_{\theta-1}+\gamma_{\theta}\right) .
$$

Type $F$ : this follows from Types $B$ and $C$ and Lemma 6.2.6.

(c) By inspection. 
By Lemma 6.2.5 we may assume $\gamma$ is not simple. If $N_{\gamma}=2$, then the Lemma does not apply. The second technique provides an explicit computation of the differential of a suitable chain in this setting. Let $s=s_{n}$ be as in (4.1.6).

Lemma 6.2.8. Let $\gamma \in \Delta_{+}^{\mathfrak{q}}$ be such that $N_{\gamma}=2$ and the following conditions hold:

(a) For all $\beta, \delta \in \Delta_{+}^{\mathfrak{q}}, \beta<\delta, \gamma=\beta+\delta$,

$$
x_{\beta} x_{\gamma}=q_{\beta \gamma} x_{\gamma} x_{\beta}, \quad x_{\gamma} x_{\delta}=q_{\gamma \delta} x_{\delta} x_{\gamma}, \quad q_{\beta \beta}=q_{\delta \delta} .
$$

(b) If $\gamma_{1}, \gamma_{2}, \gamma_{3} \in \Delta_{+}^{q}$ are three different roots, $\gamma_{i} \neq \gamma$, then $\gamma_{1}+\gamma_{2}+\gamma_{3} \neq 2 \gamma$.

(c) If $\gamma_{1}, \gamma_{2} \in \Delta_{+}^{\mathfrak{q}}, \gamma_{1} \neq \gamma_{2}$, then $N_{\gamma_{1}} \gamma_{1}+\gamma_{2} \neq 2 \gamma$.

Then $\left(\mathrm{x}_{\gamma}^{2}\right)^{*}$ is a cocycle of degree two.

Proof. By Remark 6.1.1 we have to check that the coefficient of $\mathrm{x}_{\gamma}^{2} \otimes 1$ in $d(c \otimes 1)$ is zero for all 3-chains $c$ of degree $2 \gamma$. By (b) and (c) we have to deal with $c=\mathbf{x}_{\beta} \mathbf{x}_{\gamma} \mathbf{x}_{\delta}$, where $\beta, \delta \in \Delta_{+}^{\mathfrak{q}}, \beta<\delta, \gamma=\beta+\delta$ : Here we use the convexity to deduce that $\beta<\gamma<\delta$.

Fix $\beta, \delta \in \Delta_{+}^{\mathfrak{q}}$ such that $\beta<\delta$ and $\gamma=\beta+\delta$. By (4.2.2),

$$
x_{\beta} x_{\delta}=q_{\beta \delta} x_{\delta} x_{\beta}+\mathrm{b} x_{\gamma}+\sum_{\beta<\nu_{1} \leq \cdots \leq \nu_{k}<\delta: \sum \nu_{i}=\gamma} \mathrm{b}_{\nu_{1}, \ldots, \nu_{k}} x_{\nu_{k}} \ldots x_{\nu_{1}}
$$

for some $\mathrm{b} x_{\gamma}, \mathrm{b}_{\nu_{1}, \ldots, \nu_{k}} \in \mathbb{k}$. Using the convexity again we see that if $\nu_{1} \leq \cdots \leq \nu_{k}$ are such that $\sum \nu_{i}=\gamma$, then $\nu_{1}<\gamma<\nu_{k}$.

By definition of the differential on 2-chains and (a)

$$
\begin{aligned}
& d\left(\mathrm{x}_{\beta} \mathrm{x}_{\delta} \otimes 1\right)=\mathrm{x}_{\beta} \otimes x_{\delta}-q_{\beta \delta} \mathrm{x}_{\delta} \otimes x_{\beta}-\mathrm{bx}_{\gamma} \otimes 1-\sum \mathrm{b}_{\nu_{1}, \ldots, \nu_{k}} \mathrm{x}_{\nu_{k}} \otimes x_{\nu_{k-1}} \ldots x_{\nu_{1}}, \\
& d\left(\mathrm{x}_{\beta} \mathrm{x}_{\gamma} \otimes 1\right)=\mathrm{x}_{\beta} \otimes x_{\gamma}-q_{\beta \gamma} \mathrm{x}_{\gamma} \otimes x_{\beta}, \quad d\left(\mathrm{x}_{\gamma} \mathrm{x}_{\delta} \otimes 1\right)=\mathrm{x}_{\gamma} \otimes x_{\delta}-q_{\gamma \delta} \mathrm{x}_{\delta} \otimes x_{\gamma} .
\end{aligned}
$$

Using these computations and (a)

$$
\begin{aligned}
d\left(\mathrm{x}_{\beta} \mathrm{x}_{\gamma} \mathrm{x}_{\delta} \otimes 1\right)=\mathrm{x}_{\beta} \mathrm{x}_{\gamma} \otimes x_{\delta}-s d\left(\mathrm{x}_{\beta} \mathrm{x}_{\gamma} \otimes x_{\delta}\right)=\mathrm{x}_{\beta} \mathrm{x}_{\gamma} \otimes x_{\delta}-s\left(\mathrm{x}_{\beta} \otimes x_{\gamma} x_{\delta}-q_{\beta \gamma} \mathrm{x}_{\gamma} \otimes x_{\beta} x_{\delta}\right) \\
=\mathrm{x}_{\beta} \mathrm{x}_{\gamma} \otimes x_{\delta}-s\left(q_{\gamma \delta} \mathrm{x}_{\beta} \otimes x_{\delta} x_{\gamma}-q_{\beta \gamma} \mathrm{x}_{\gamma} \otimes\left(q_{\beta \delta} x_{\delta} x_{\beta}+\mathrm{b} x_{\gamma}+\sum \mathrm{b}_{\nu_{1}, \ldots, \nu_{k}} x_{\nu_{k}} \ldots x_{\nu_{1}}\right)\right) \\
=\mathrm{x}_{\beta} \mathrm{x}_{\gamma} \otimes x_{\delta}-q_{\gamma \delta} \mathrm{x}_{\beta} \mathrm{x}_{\delta} \otimes x_{\gamma}-s\left(\left(q_{\gamma \delta}-q_{\beta \gamma}\right) \mathrm{b} \mathrm{x}_{\gamma} \otimes x_{\gamma}-q_{\beta \gamma} q_{\beta \delta} \mathrm{x}_{\gamma} \otimes x_{\delta} x_{\beta}\right. \\
\left.\quad+\sum \mathrm{b}_{\nu_{1}, \ldots, \nu_{k}}\left(q_{\gamma \delta} \mathrm{x}_{\nu_{k}} \otimes x_{\nu_{k-1}} \ldots x_{\nu_{1}} x_{\gamma}-q_{\beta \gamma} \mathrm{x}_{\gamma} \otimes x_{\nu_{k}} \ldots x_{\nu_{1}}\right)+q_{\beta \gamma} q_{\beta \delta} q_{\gamma \delta} \mathrm{x}_{\delta} \otimes x_{\gamma} x_{\beta}\right) \\
\quad=\mathrm{x}_{\beta} \mathrm{x}_{\gamma} \otimes x_{\delta}-q_{\gamma \delta} \mathrm{x}_{\beta} \mathrm{x}_{\delta} \otimes x_{\gamma}+\left(q_{\beta \gamma}-q_{\gamma \delta}\right) \mathrm{b} \mathrm{x}_{\gamma}^{2} \otimes 1+q_{\beta \gamma} q_{\beta \delta} \mathrm{x}_{\gamma} \mathrm{x}_{\delta} \otimes x_{\beta} \\
\quad+\sum \mathrm{b}_{\nu_{1}, \ldots, \nu_{k}} q_{\beta \gamma} \mathrm{x}_{\gamma} \mathrm{x}_{\nu_{k}} \otimes x_{\nu_{k-1}} \ldots x_{\nu_{1}} \\
\quad-s\left(\sum \mathrm{b}_{\nu_{1}, \ldots, \nu_{k}} \mathrm{x}_{\nu_{k}} \otimes\left(q_{\gamma \delta} x_{\nu_{k-1}} \ldots x_{\nu_{1}} x_{\gamma}-q_{\beta \gamma} q_{\gamma \nu_{k}} x_{\gamma} x_{\nu_{k-1}} \ldots x_{\nu_{1}}\right)\right. \\
\left.\quad-\sum \mathrm{b}_{\nu_{1}, \ldots, \nu_{k}} q_{\beta \gamma} s\left(f_{x_{\gamma}, x_{\nu_{k}}} x_{\nu_{k-1}} \ldots x_{\nu_{1}}\right)\right)
\end{aligned}
$$

Here $f_{x_{\gamma}, x_{\nu_{k}}}=\left[x_{\gamma}, x_{\nu_{k}}\right]_{c}-x_{\gamma} x_{\nu_{k}}+q_{\gamma \nu_{k}} \mathrm{x}_{\nu_{k}} \otimes x_{\gamma}$. We claim that

$$
\begin{aligned}
& d\left(\mathrm{x}_{\beta} \mathrm{x}_{\gamma} \mathrm{x}_{\delta} \otimes 1\right)=\mathrm{x}_{\beta} \mathrm{x}_{\gamma} \otimes x_{\delta}-q_{\gamma \delta} \mathrm{x}_{\beta} \mathrm{x}_{\delta} \otimes x_{\gamma}+\left(q_{\beta \gamma}-q_{\gamma \delta}\right) \mathrm{bx}_{\gamma}^{2} \otimes 1+q_{\beta \gamma} q_{\beta \delta} \mathrm{x}_{\gamma} \mathrm{x}_{\delta} \otimes x_{\beta} \\
& \quad+\sum \mathrm{b}_{\nu_{1}, \ldots, \nu_{k}} q_{\beta \gamma} \mathrm{x}_{\gamma} \mathrm{x}_{\nu_{k}} \otimes x_{\nu_{k-1}} \ldots x_{\nu_{1}} .
\end{aligned}
$$


According with the previous computation we should prove that $s$ annihilates

$$
\mathrm{x}_{\nu_{k}} \otimes\left(q_{\gamma \delta} x_{\nu_{k-1}} \ldots x_{\nu_{1}} x_{\gamma}-q_{\beta \gamma} q_{\gamma \nu_{k}} x_{\gamma} x_{\nu_{k-1}} \ldots x_{\nu_{1}}\right), \quad s\left(f_{x_{\gamma}, x_{\nu_{k}}} x_{\nu_{k-1}} \ldots x_{\nu_{1}}\right) .
$$

For the elements on the right of (6.2.10) we use that $s^{2}=0$. For the elements on the left of (6.2.10), due to the convexity of the PBW basis, $x_{\nu_{k-1}} \ldots x_{\nu_{1}} x_{\gamma}$ and $x_{\gamma} x_{\nu_{k-1}} \ldots x_{\nu_{1}}$ are linear combinations of products $x_{\mu_{j}} \ldots x_{\mu_{1}}$, with $\nu_{1} \leq \mu_{1} \leq \cdots \leq \mu_{j} \leq \nu_{k-1}$ and $\mu_{j} \leq \gamma$. Hence $\mu_{j} \leq \nu_{k}$, so they are linear combinations of

$$
\mathrm{x}_{\nu_{k}} \otimes x_{\mu_{j}} \ldots x_{\mu_{1}}=s\left(x_{\nu_{k}} x_{\mu_{j}} \ldots x_{\mu_{1}}\right)
$$

and we use again that $s^{2}=0$. Finally, using the claim and that

$$
q_{\beta \gamma}-q_{\gamma \delta}=q_{\beta \beta} q_{\beta \delta}-q_{\beta \delta} q_{\delta \delta} \stackrel{6.2 .90}{=} 0
$$

the coefficient of $\mathrm{x}_{\gamma}^{2} \otimes 1$ is zero.

6.3. Higher degree cocycles. We now assume that we are not in the situations of 6.2 . We shall compute all chains $c \in \mathrm{M}(2 \ell+1)$ satisfying the degree and grading constraints (6.1.3) and (6.1.4) and verify that the condition in Remark 6.1.1 (b) is satisfied. As before $\gamma \in \Delta_{+}^{\mathfrak{q}}$ is fixed.

Let $f_{\delta}: \mathbb{N}_{0} \rightarrow \mathbb{N}_{0}$ be the function defined in (4.2.4) for $\delta \in \Delta_{+}$.

6.3.1. $N_{\gamma}=2$. Here the constraints (6.1.3) and (6.1.4) take the form

$$
\sum_{\delta \in \Delta_{+}} f_{\delta}\left(n_{\delta}\right) \delta=L \gamma, \quad \sum_{\delta \in \Delta_{+}} n_{\delta}=L+1
$$

In the following mega statement we collect all possible conditions that we may need to verify on $\gamma$ to conclude that $\left(\mathrm{x}_{\gamma}^{L}\right)^{*}$ is an $L$-cocycle (that is, a cocycle of degree $L$ ). We explain the scheme of the proof up to the specific computation of differentials that is postponed to Section 10 .

Proposition 6.3.2. Let $L=2 \ell \in \mathbb{N}$ even. Assume that each solution $\left(n_{\delta}\right)_{\delta \in \Delta_{+}} \in \mathbb{N}_{0}^{\Delta_{+}}$of the equations (6.3.1) is of one of the forms $(\mathrm{A}),(\mathrm{B}),(\mathrm{C}),(\mathrm{D}),(\mathrm{E}),(\mathrm{F}),(\mathrm{G}),(\mathrm{H}),(\mathrm{I})$ or $(\mathrm{J})$. Then $\left(\mathrm{x}_{\gamma}^{L}\right)^{*}$ is an L-cocycle.

(A) $n_{\gamma}=L-1, n_{\alpha}=n_{\beta}=1$ and $n_{\varphi}=0$ for the other $\varphi \in \Delta_{+}$; where $\alpha, \beta \in \Delta_{+}$satisfy

$$
\alpha<\beta, \quad \alpha+\beta=\gamma,
$$

the corresponding PBW generators satisfy (10.1.8),

$$
\text { and } L \text { satisfies }(L)_{-\frac{q_{\alpha \alpha}}{q_{\beta \beta}}}=0 \text {. }
$$

(B) $n_{\gamma}=L-2, n_{\alpha}=n_{\beta}=n_{\delta}=1$ and $n_{\varphi}=0$ for the other $\varphi \in \Delta_{+}$. where $\alpha, \beta, \delta, \eta \in \Delta_{+}$ satisfy

$$
\alpha<\eta<\gamma<\beta<\delta, \quad \gamma+\eta=\alpha+\beta, \quad \eta+\delta=\gamma,
$$
the corresponding PBW generators satisfy (10.1.11), and $L$ satisfies $\mathrm{c}_{\alpha \beta \gamma}^{(L)}:=\sum_{k=0}^{L}\left(-\widetilde{q}_{\alpha \gamma}\right)^{k}(k+1)_{\widetilde{q}_{\beta \gamma}}=0$. 
(C) $n_{\gamma}=L-3, n_{\alpha}=2, n_{\beta}=n_{\delta}=1$ and $n_{\varphi}=0$ for the other $\varphi \in \Delta_{+}$where $\alpha, \beta, \delta, \eta, \tau$ satisfy

$$
\begin{aligned}
& \alpha<\eta<\gamma<\tau<\beta<\delta, \quad \gamma+\tau=\alpha+\beta, \quad \eta+\delta=\gamma, \\
& N_{\alpha}=2 \quad \alpha+\tau=\gamma+\eta, \quad \eta+\beta=2 \tau,
\end{aligned}
$$

the corresponding PBW generators satisfy (10.1.16),

and $L$ satisfies $(L)_{\widetilde{q}_{\gamma \alpha} \widetilde{q}_{\gamma \beta}}+\sum_{j=1}^{L-1} \mathrm{c}_{\alpha \tau \gamma}^{(j)}=0$.

(D) $n_{\gamma}=L-2, n_{\alpha}=n_{\beta}=n_{\delta}=1$ and $n_{\varphi}=0$ for the other $\varphi \in \Delta_{+}$, where $\alpha, \beta, \delta, \eta, \tau \in$ $\Delta_{+}$satisfy

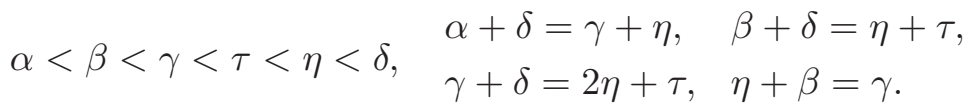

the corresponding PBW generators satisfy (10.1.24), and $L$ satisfies $\mathrm{c}_{\alpha \beta \gamma}^{(L)}=0$.

(E) $n_{\gamma}=L-3, n_{\alpha}=n_{\beta}=n_{\delta}=n_{\eta}=1$ and $n_{\varphi}=0$ for the other $\varphi \in \Delta_{+}$, where $\alpha, \beta, \delta, \tau, \mu, \eta \in \Delta_{+}$satisfy

$$
\alpha<\beta<\delta<\gamma<\tau<\mu<\eta, \quad \alpha+\mu=\gamma=\beta+\tau, \quad \eta+\delta=\gamma+\tau+\mu .
$$

the corresponding PBW generators satisfy (10.1.33),

$$
\text { and L satisfies } \mathrm{d}_{\alpha \beta \delta \gamma}^{(L)}:=\sum_{k=0}^{L-1} \widetilde{q}_{\delta \gamma}^{k}(k+1)_{\widetilde{q}_{\alpha \gamma}}(k+2)_{\widetilde{q}_{\beta \gamma}}=0 \text {. }
$$

(F) $n_{\gamma}=L-2, n_{\alpha}=n_{\beta}=n_{\delta}=1$ and $n_{\varphi}=0$ for the other $\varphi \in \Delta_{+}$, where $\alpha, \beta, \delta, \eta \in \Delta_{+}$ satisfy

$$
\alpha<\eta<\gamma<\beta<\delta, \quad \gamma+\eta=\alpha+\delta, \quad \eta+\beta=\gamma,
$$

the corresponding PBW generators satisfy (10.1.43),

(G) $n_{\gamma}=L-2, n_{\alpha}=n_{\beta}=n_{\delta}=1$ and $n_{\varphi}=0$ for the other $\varphi \in \Delta_{+}$, where $\alpha, \beta, \delta, \eta \in \Delta_{+}$ satisfy

$$
\alpha<\beta<\gamma<\eta<\delta, \quad \gamma+\eta=\beta+\delta, \quad \eta+\alpha=\gamma,
$$

the corresponding PBW generators satisfy (10.1.49),

$$
\text { and } L \text { satisfies } \mathrm{c}_{-\delta \alpha \gamma}^{(L)}=0 \text {. }
$$

(H) $n_{\gamma}=L-2, n_{\alpha}=n_{\beta}=n_{\delta}=1$ and $n_{\varphi}=0$ for the other $\varphi \in \Delta_{+}$, where $\alpha, \beta, \delta, \eta, \tau, \mu, \nu \in \Delta_{+}$satisfy

$$
\begin{aligned}
& \alpha<\tau<\beta<\gamma<\mu<\nu<\eta<\delta, \quad \alpha+\delta=\eta+\tau, \quad \beta+\delta=\nu+\gamma, \\
& \beta+\eta=\mu+\gamma, \quad \alpha+\nu=\gamma,
\end{aligned}
$$

the corresponding PBW generators satisfy (10.1.55), 


$$
\text { and } L \text { satisfies } \mathrm{c}_{-\delta \alpha \gamma}^{(L)}=0 \text {. }
$$

(I) $n_{\gamma}=L-3, n_{\alpha}=n_{\beta}=n_{\delta}=n_{\eta}=1$ and $n_{\varphi}=0$ for the other $\varphi \in \Delta_{+}$, where $\alpha, \beta, \nu, \mu, \delta, \eta \in \Delta_{+}$satisfy

$$
\alpha<\beta<\nu<\gamma<\mu<\delta<\eta, \quad \beta+\delta=\gamma+\nu, \quad \nu+\eta=\mu+\gamma, \quad \alpha+\mu=\gamma,
$$

the corresponding PBW generators satisfy (10.1.63),

(J) $n_{\gamma}=L-3, n_{\alpha}=n_{\beta}=n_{\delta}=n_{\eta}=1$ and $n_{\varphi}=0$ for the other $\varphi \in \Delta_{+}$, where $\alpha, \beta, \delta, \eta, \nu, \mu \in \Delta_{+}$satisfy

$$
\alpha<\beta<\delta<\gamma<\mu<\nu<\eta, \quad \beta+\eta=\gamma+\nu, \quad \delta+\nu=\mu+\gamma, \quad \alpha+\mu=\gamma,
$$

the corresponding PBW generators satisfy (10.1.63),

and $L$ satisfies $\mathrm{d}_{\beta-\nu \alpha \gamma}^{(L)}=0$.

Proof. As $N_{\gamma}=2, \mathrm{x}_{\gamma}^{n} \otimes 1$ is a $n$-chain for all $n \in \mathbb{N}$; hence $\mathrm{x}_{\gamma}^{L} \otimes 1$ is so. By assumption all $(L+1)$-chains of degree $L \gamma$ are one of the following forms: $\mathbf{x}_{\alpha} \mathrm{x}_{\gamma}^{L-1} \mathrm{x}_{\beta}$, for a pair $(\alpha, \beta)$ satisfying (6.3.3); $\mathrm{x}_{\alpha} \mathrm{x}_{\gamma}^{L-2} \mathrm{x}_{\beta} \mathrm{x}_{\delta}$, for a 4-tuple $(\alpha, \beta, \delta, \eta)$ satisfying (6.3.5); $\mathrm{x}_{\alpha}^{2} \mathrm{x}_{\gamma}^{L-3} \mathrm{x}_{\beta} \mathrm{x}_{\delta}$, for a 5-tuple $(\alpha, \beta, \delta, \eta, \tau)$ satisfying (6.3.7), $\mathrm{x}_{\alpha} \mathrm{x}_{\beta} \mathrm{x}_{\gamma}^{L-2} \mathrm{x}_{\delta}$, for a 5-tuple $(\alpha, \beta, \delta, \eta, \tau)$ satisfying (6.3.9), $\mathrm{x}_{\alpha} \mathrm{x}_{\beta} \mathrm{x}_{\delta} \mathrm{x}_{\gamma}^{L-2} \mathrm{x}_{\eta}$, for a 6-tuple $(\alpha, \beta, \delta, \tau, \varphi, \eta)$ satisfying (6.3.11), $\mathrm{x}_{\alpha} \mathrm{x}_{\gamma}^{L-2} \mathrm{x}_{\beta} \mathrm{x}_{\delta}$, for a 4-tuple $(\alpha, \beta, \delta, \eta)$ satisfying (6.3.13), $\mathrm{x}_{\alpha} \mathrm{x}_{\beta} \mathrm{x}_{\gamma}^{L-2} \mathrm{x}_{\delta}$, for a 4-tuple $(\alpha, \beta, \delta, \eta)$ satisfying (6.3.15), $\mathrm{x}_{\alpha} \mathrm{x}_{\beta} \mathrm{x}_{\gamma}^{L-2} \mathrm{x}_{\delta}$, for a 7-tuple $(\alpha, \beta, \delta, \eta, \tau, \mu, \nu)$ satisfying (6.3.17), $\mathrm{x}_{\alpha} \mathrm{x}_{\beta} \mathrm{x}_{\gamma}^{L-3} \mathrm{x}_{\delta} \mathrm{x}_{\eta}$, for a 6-tuple $(\alpha, \beta, \nu, \mu \delta, \eta)$ satisfying (6.3.19).

- Fix a pair $(\alpha, \beta)$ satisfying (6.3.3). To simplify the notation, call $\zeta:=-\frac{q_{\alpha \gamma}}{q_{\gamma \beta}}=-\frac{q_{\alpha \alpha}}{q_{\beta \beta}}$.

We can apply Lemma 10.1.7 since conditions (10.1.8) hold by hypothesis. Assume first that $L=2 a+1$ is odd. Hence the coefficient of $\mathrm{x}_{\gamma}^{L} \otimes 1$ in $d\left(\mathrm{x}_{\alpha} \mathrm{x}_{\gamma}^{L-1} \mathrm{x}_{\beta} \otimes 1\right)$ is

$$
\mathrm{b} q_{\gamma \beta}^{2 a}\left\{(-\zeta-1)(a)_{(-\zeta)^{2}}-(-\zeta)^{2 a}\right\}=-\mathrm{b} q_{\gamma \beta}^{L-1}\left\{(1+\zeta)(a)_{\zeta^{2}}+\zeta^{2 a}\right\}=-\mathrm{b} q_{\gamma \beta}^{L-1}(L)_{\zeta} .
$$

If $L=2 a$ is even, then the coefficient of $\mathrm{x}_{\gamma}^{L} \otimes 1$ in $d\left(\mathrm{x}_{\alpha} \mathrm{x}_{\gamma}^{L-1} \mathrm{x}_{\beta} \otimes 1\right)$ is

$$
\mathrm{b} q_{\gamma \beta}^{2 a-1}(-\zeta-1)(a)_{(-\zeta)^{2}}=-\mathrm{b} q_{\gamma \beta}^{L-1}(1+\zeta)(a)_{\zeta^{2}}=-\mathrm{b} q_{\gamma \beta}^{L-1}(L)_{\zeta} .
$$

By (A) such coefficient is zero in both cases.

- Fix a 4-tuple $(\alpha, \beta, \delta, \eta)$ satisfying (6.3.5). We can apply Lemma 10.1.10 since conditions (10.1.11) hold by hypothesis. Hence the coefficient of $\mathrm{x}_{\gamma}^{L} \otimes 1$ in $d\left(\mathrm{x}_{\alpha} \mathrm{x}_{\gamma}^{L-2} \mathrm{x}_{\beta} \mathrm{x}_{\delta} \otimes 1\right)$ is zero by (B).

- Fix a 5-tuple $(\alpha, \beta, \delta, \eta, \tau)$ satisfying (6.3.7). We can apply Lemma 10.1.15 since conditions (10.1.16) hold by hypothesis. Hence the coefficient of $\mathrm{x}_{\gamma}^{L} \otimes 1$ in $d\left(\mathrm{x}_{\alpha}^{2} \mathrm{x}_{\gamma}^{L-3} \mathrm{x}_{\beta} \mathrm{x}_{\delta} \otimes 1\right)$ is zero by $(\mathrm{C})$

- Fix a 5-tuple $(\alpha, \beta, \delta, \eta, \tau)$ satisfying (6.3.9). We can apply Lemma 10.1 .23 since conditions (10.1.24) hold by hypothesis. Hence the coefficient of $\mathrm{x}_{\gamma}^{L} \otimes 1$ in $d\left(\mathrm{x}_{\alpha} \mathrm{x}_{\beta} \mathrm{x}_{\gamma}^{L-2} \mathrm{x}_{\delta} \otimes 1\right)$ is zero by (D). 
- Fix a 6-tuple $(\alpha, \beta, \delta, \tau, \varphi, \eta)$ satisfying (6.3.11). We can apply Lemma 10.1.32 since conditions (10.1.33) hold by hypothesis. Hence the coefficient of $\mathrm{x}_{\gamma}^{L} \otimes 1$ in $d\left(\mathrm{x}_{\alpha} \mathrm{x}_{\beta} \mathrm{x}_{\delta} \mathrm{x}_{\gamma}^{L-2} \mathrm{x}_{\eta} \otimes\right.$ $1)$ is zero by (E).

- Fix a 4-tuple $(\alpha, \beta, \delta, \eta)$ satisfying (6.3.13). We can apply Lemma 10.1.42 since 10.1.43) holds by hypothesis. Hence the coefficient of $\mathrm{x}_{\gamma}^{L} \otimes 1$ in $d\left(\mathrm{x}_{\alpha} \mathrm{x}_{\gamma}^{L-2} \mathrm{x}_{\beta} \mathrm{x}_{\delta} \otimes 1\right)$ is zero by (F),

- Fix a 4-tuple $(\alpha, \beta, \delta, \eta)$ satisfying (6.3.15). We apply Lemma 10.1.48 since (10.1.49) holds by hypothesis: The coefficient of $\mathrm{x}_{\gamma}^{L} \otimes 1$ in $d\left(\mathrm{x}_{\alpha} \mathrm{x}_{\beta} \mathrm{x}_{\gamma}^{L-2} \mathrm{x}_{\delta} \otimes 1\right)$ is zero by $(\mathrm{G})$,

- Fix a 7 -tuple $(\alpha, \beta, \delta, \eta, \tau, \mu, \nu)$ satisfying (6.3.17). We can apply Lemma 10.1.54 since (10.1.55) holds by hypothesis: The coefficient of $\mathrm{x}_{\gamma}^{L} \otimes 1$ in $d\left(\mathrm{x}_{\alpha} \mathrm{x}_{\beta} \mathrm{x}_{\gamma}^{L-2} \mathrm{x}_{\delta} \otimes 1\right)$ is zero by $(\mathrm{H})$.

○ Fix a 6-tuple $(\alpha, \beta, \nu, \mu, \delta, \eta)$ satisfying (6.3.19). We apply Lemma10.1.62 since (10.1.63) holds by hypothesis: The coefficient of $\mathrm{x}_{\gamma}^{L} \otimes 1$ in $d\left(\mathrm{x}_{\alpha} \mathrm{x}_{\beta} \mathrm{x}_{\gamma}^{L-3} \mathrm{x}_{\delta} \mathrm{x}_{\eta} \otimes 1\right)$ is zero by (I).

○ Fix a 6-tuple $(\alpha, \beta, \delta, \eta, \nu, \mu)$ satisfying (6.3.21). We apply Lemma 10.1.70 since (10.1.71) holds by hypothesis: The coefficient of $\mathrm{x}_{\gamma}^{L} \otimes 1$ in $d\left(\mathrm{x}_{\alpha} \mathrm{x}_{\beta} \mathrm{x}_{\delta} \mathrm{x}_{\gamma}^{L-3} \mathrm{x}_{\eta} \otimes 1\right)$ is zero by $(\mathrm{J})$,

Thus the coefficient of $\mathbf{x}_{\gamma}^{L} \otimes 1$ in $d(c)$ is zero for all $c \in \mathrm{M}(L+1)$ and Remark 6.1.1 applies.

6.3.2. $N_{\gamma}>2$. We carry out a similar analysis when the assumption is $N_{\gamma}>2$ instead.

Proposition 6.3.23. Let $\gamma \in \Delta_{+}$be such that $N_{\gamma}>2$ and for all pairs $(\alpha, \beta) \in \Delta_{+}^{2}$ such that

$$
\alpha<\beta \quad \text { and } \quad \alpha+\beta=\left(N_{\gamma}-1\right) \gamma,
$$

the corresponding PBW generators satisfy (10.1.8). Let $f_{\delta}: \mathbb{N}_{0} \rightarrow \mathbb{N}_{0}$ be the function defined in (4.2.4) for each $\delta \in \Delta_{+}$. Assume that $\ell \in \mathbb{N}$ satisfies the following two conditions:

(a) For each pair $(\alpha, \beta) \in \Delta_{+}^{2}$ satisfying (6.3.24) the scalars $q_{\alpha \gamma}$ and $q_{\gamma \beta}$ satisfy

$$
\left(\frac{q_{\alpha \gamma}}{q_{\gamma \beta}}-1\right)(\ell)_{\left(\frac{q_{\alpha \gamma}}{q_{\gamma \beta}}\right)^{N_{\gamma}}}=0 .
$$

(b) The solutions $\left(n_{\delta}\right)_{\delta \in \Delta_{+}} \in \mathbb{N}_{0}^{\Delta_{+}}$of the equations

$$
\sum_{\delta \in \Delta_{+}} f_{\delta}\left(n_{\delta}\right) \delta=\ell N_{\gamma} \gamma, \quad \sum_{\delta \in \Delta_{+}} n_{\delta}=2 \ell+1
$$

are all of the form $n_{\gamma}=2(\ell-1)+1, n_{\alpha}=n_{\beta}=1$, for any pair $(\alpha, \beta)$ satisfying (6.3.24), and $n_{\delta}=0$ for the remaining $\delta \in \Delta_{+}$.

Then $\left(\mathrm{x}_{\gamma}^{\ell N_{\gamma}}\right)^{*}$ is a $2 \ell$-cocycle.

Proof. Fix a pair of positive roots $(\alpha, \beta)$ satisfying (6.3.24). We can apply Lemma 10.1.7 since conditions (10.1.8) hold by hypothesis and conclude that the coefficient of $\mathrm{x}_{\gamma}^{L N_{\gamma}} \otimes 1$ in $d\left(\mathrm{x}_{\alpha} \mathrm{x}_{\gamma}^{N_{\gamma}(L-1)+1} \mathrm{x}_{\beta} \otimes 1\right)$ is zero by (a).

By (b) all $(2 L+1)$-chains of degree $L N_{\gamma} \gamma$ are of the form $\mathbf{x}_{\alpha} \mathbf{x}_{\gamma}^{N_{\gamma}(L-1)+1} \mathbf{x}_{\beta}$, for a pair $(\alpha, \beta)$ satisfying (6.3.24). Thus the coefficient of $\mathrm{x}_{\gamma}^{L N_{\gamma}} \otimes 1$ in $d(c)$ is zero for all $c \in \mathrm{M}(2 L+1)$ and Remark 6.1.1 applies. 
Next we deal with the scalars $\mathrm{c}_{\alpha \beta \gamma}^{(L)}$ and $\mathrm{d}_{\alpha \beta \delta \gamma}^{(L)}$. Given $r, s, t \in \mathbb{k}$, let

$$
\mathrm{c}_{r, s}^{(L)}:=\sum_{k=0}^{L-1} r^{k}(k+1)_{s}, \quad \mathrm{~d}_{r, s, t}^{(L)}:=\sum_{k=0}^{L-1} r^{k}(k+1)_{s}(k+2)_{t} .
$$

Notice that $\mathrm{c}_{\alpha \beta \gamma}^{(L)}=\mathrm{c}_{\widetilde{q}_{\alpha \gamma}, \widetilde{q}_{\beta \gamma}}^{(L)}$ and $\mathrm{d}_{\alpha \delta \beta \gamma}^{(L)}=\mathrm{d}_{\widetilde{q}_{\alpha \gamma}, \widetilde{q}_{\beta \gamma}, \widetilde{q}_{\delta \gamma}}^{(L)}$.

Lemma 6.3.28. (a) Assume that $(L)_{r}=0$. Then $\mathrm{c}_{r, s}^{(L)}=-s \mathrm{c}_{s, r}^{(L)}$

(b) Assume that $(L)_{r}=0=(L)_{s}$ and $r s \neq 1$. Then $\mathrm{c}_{r, s}^{(L)}=0$.

(c) Assume that $(L)_{r}=0=(L)_{s}$ for $L \geq 3$ and $r s \neq 1$. Then $\mathrm{d}_{r, s, s}^{(L)}=0$.

Proof. For (a) we compute

$$
\begin{aligned}
\mathrm{c}_{r, s}^{(L)} & =\sum_{k=0}^{L-1} r^{k}\left(\sum_{j=0}^{k} s^{j}\right)=\sum_{i=0}^{L-1} s^{i}\left(\sum_{k=i}^{L-1} r^{k}\right)=\sum_{i=1}^{L-1} s^{i}\left((L)_{r}-(i)_{r}\right)=-\sum_{i=1}^{L-1} s^{i}(i)_{r} \\
& =-\sum_{k=0}^{L-2} s^{k+1}(k+1)_{r}=-s \sum_{k=0}^{L-1} s^{k}(k+1)_{r}=-s \mathrm{c}_{s, r}^{(L)} .
\end{aligned}
$$

Now (b) follows using (a). Indeed, we have that $\mathrm{c}_{r, s}^{(L)}=-s \mathrm{c}_{s, r}^{(L)}=r s \mathrm{c}_{r, s}^{(L)}$; as $r s \neq 1$ by hypothesis, we have that $\mathrm{c}_{r, s}^{(L)}=0$.

Next we deal with (c). As $r s \neq 1$ and $(L)_{r}=(L)_{s}=0$, we have that $(L)_{r s}=0$; thus $\mathrm{c}_{r s, s}^{(L)}=0$ by (b). Also, $\left(\begin{array}{c}L+1 \\ 2\end{array}\right)_{s}=0$. Then we compute

$$
\begin{aligned}
\mathrm{d}_{r, s, s}^{(L)} & =(2)_{s} \sum_{k=0}^{L-1} r^{k}\left(\begin{array}{c}
k+2 \\
2
\end{array}\right)_{s}=(2)_{s} \sum_{k=0}^{L-1} r^{k}\left(\left(\begin{array}{c}
k+1 \\
2
\end{array}\right)_{s}+s^{k}(k+1)_{s}\right) \\
& =(2)_{s} \sum_{k=1}^{L-1} r^{k}\left(\begin{array}{c}
k+1 \\
2
\end{array}\right)_{s}+(2)_{s} \mathrm{c}_{r s, s}^{(L)}=(2)_{s} \sum_{j=0}^{L-2} r^{j+1}\left(\begin{array}{c}
j+2 \\
2
\end{array}\right)_{s} \\
& =(2)_{s} r \sum_{j=0}^{L-1} r^{j}\left(\begin{array}{c}
j+2 \\
2
\end{array}\right)_{s}=r \mathrm{~d}_{r, s, s}^{(L)} .
\end{aligned}
$$

As $r \neq 1$ we have that $\mathrm{d}_{r, s, s}^{(L)}=0$.

For each $\delta \in \Delta_{+}$, let $a_{i}^{\delta} \in \mathbb{N}_{0}$ be the coordinate of $\alpha_{i}$ in $\delta$ : that is, $\delta=\sum_{i \in \mathbb{I}} a_{i}^{\delta} \alpha_{i}$.

6.4. Summary of the algorithm. As we have seen in Part \, to prove finite generation it is remained to establish Condition (1.4.1) for Nichols algebras of diagonal type. Now that we introduced all the necessary players we can describe the procedure we will follow to establish this condition. Going through these steps will occupy the remainder of the paper.

- We fix one type in the classification of Nichols algebras of diagonal type. We choose a representative of the Weyl-equivalence (as defined in $\S$ 2.3.2) with the care that the proper subdiagrams were already treated. 
- We fix $\gamma \in \Delta_{+}^{\mathfrak{q}}$. We assume that $\gamma$ has full support (see definition in $\S[2.3$ ); recall that Lemma 6.2.5 takes care of simple roots.

○ We compute $N_{\gamma}, P_{\gamma}, Q_{\gamma}$.

Then we apply one of the following criteria:

(I) If $N_{\gamma}>P_{\gamma}, Q_{\gamma}$, then $\left(\mathrm{x}_{\gamma}^{N_{\gamma}}\right)^{*}$ is a cocycle of degree 2 .

(II) If $N_{\gamma}=2$ and (6.2.9) holds, then $\left(\mathrm{x}_{\gamma}^{N_{\gamma}}\right)^{*}$ is a 2-cocycle.

(III) Assume that $N_{\gamma}=2$ but (6.2.9) does not hold. We define

$$
L_{\gamma}=\operatorname{lcm}\left(\{2\} \cup\left\{\operatorname{ord}\left(-\frac{q_{\alpha \alpha}}{q_{\beta \beta}}\right): \gamma=\alpha+\beta, \alpha, \beta \in \Delta_{+}^{\mathfrak{q}}\right\}\right) .
$$

We find all families $\left(n_{\delta}\right)_{\delta \in \Delta_{+}^{\mathfrak{q}}}$ of non-negative integers satisfying (6.3.1) with $L=L_{\gamma}$.

We check that any of these families $\left(n_{\delta}\right)_{\delta \in \Delta_{+}^{\mathfrak{q}}}$ has one of the forms $(\mathrm{A}), \ldots$, or $(\mathrm{J})$ in Proposition 6.3.2. Then $\left(\mathrm{x}_{\gamma}^{L_{\gamma}}\right)^{*}$ is a cocycle.

(IV) Assume that $N_{\gamma}>2$ but the inequality in (I) does not hold. We define

$$
L_{\gamma}=\operatorname{lcm}\left(\left\{N_{\gamma}\right\} \cup\left\{\operatorname{ord}\left(\left(\frac{q_{\alpha \gamma}}{q_{\gamma \beta}}\right)^{N_{\gamma}}\right):\left(N_{\gamma}-1\right) \gamma=\alpha+\beta, \alpha, \beta \in \Delta_{+}^{\mathfrak{q}}\right\}\right) .
$$

We find all families $\left(n_{\delta}\right)_{\delta \in \Delta_{+}^{q}}$ of non-negative integers satisfying (6.3.26) with $L=$ $L_{\gamma}$. We check that any of these families $\left(n_{\delta}\right)_{\delta \in \Delta_{+}^{\mathfrak{q}}}$ has one of the forms (a), . . in Proposition 6.3.23. Then $\left(\mathrm{x}_{\gamma}^{L_{\gamma}}\right)^{*}$ is a cocycle.

Actually we distinguish two classes of types in the classification of finite-dimensional Nichols algebras of diagonal type. In the first the braiding matrices have continuous parameters and correspondingly the values of $N_{\gamma}$ might depend on these parameters. Then arguments by hand are needed. These are the types treated in Sections 7 , 8 and 9

The second class consists of the remaining types where the braiding matrices are so to say discrete. For them we compute $N_{\gamma}, P_{\gamma}, Q_{\gamma}$ and the suitable families $\left(n_{\delta}\right)_{\delta \in \Delta_{+}^{q}}$ using a computer program developed by Héctor Peña Pollastri towards these goals. We then check whether $\left(n_{\delta}\right)_{\delta \in \Delta_{+}^{\mathfrak{q}}}$ has one of the forms (A), ..., or (J), respectively (a), ... by hand using the defining relations, or at least the convex order, of $\mathscr{B}_{\mathfrak{q}}$. The types in this class are those treated in Part III (to appear later).

The implicit numeration of any generalized Dynkin diagram is from the left to the right and from bottom to top; otherwise, the numeration appears below the vertices.

\section{Classical types}

We shall use notations and conventions introduced in $\S 1.7$. For a positive integer $\theta$ defining the Dynkin type let $\mathbb{I}=\mathbb{I}_{\theta}$. 
7.1. Types $A_{\theta}$ and $\mathbf{A}(j \mid \theta-j), \theta \geq 1, j \in \mathbb{I}_{\left\lfloor\frac{\theta+1}{2}\right\rfloor}$. Let $q$ be a root of 1 of order $N \geq 2$. In this subsection, we deal with the Nichols algebra $\mathscr{B}_{\mathfrak{q}}$ of standard diagonal type $A_{\theta}$, that is associated to the Dynkin diagram

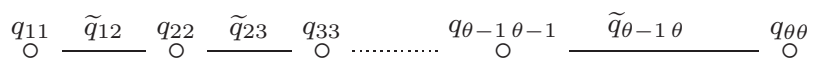

where the $q_{i i}$ 's are either $-1, q$ or $q^{-1}$ and locally the edges are of the following forms:

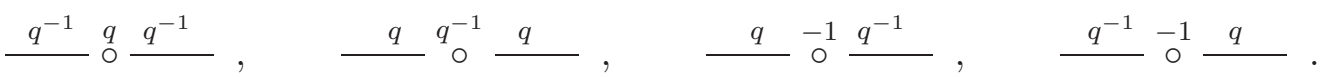

For more information, see $\mathrm{AA}, \S 4.1, \S 5.1]$. The aim of this Section is to prove that Condition 1.4.1 holds for types $A_{\theta}$ and $\mathbf{A}(j \mid \theta-j), \theta \geq 1, j \in \mathbb{I}_{\left\lfloor\frac{\theta+1}{2}\right\rfloor}$. That is,

Proposition 7.1.1. For every $\gamma \in \Delta_{+}^{\mathfrak{q}}$, there exists $L_{\gamma} \in \mathbb{N}$ such that $\left(\mathrm{x}_{\gamma}^{L_{\gamma}}\right)^{*}$ is a cocycle.

We start by setting the notation. Let

$$
\alpha_{i j}=\sum_{k \in \mathbb{I}_{i, j}} \alpha_{k}, \quad i \leq j \in \mathbb{I} .
$$

The set of positive roots is $\Delta_{+}=\left\{\alpha_{i j} \mid i, j \in \mathbb{I}, i \leq j\right\}$; this set is ordered lexicographically on the subindex $(i, j)$. Let $r=\left|\Delta_{+}\right|=\left(\begin{array}{c}\theta+1 \\ 2\end{array}\right)$; we have a numeration $\Delta_{+}=\left\{\beta_{i} \mid i \in \mathbb{I}_{r}\right\}$ so that $\beta_{k}<\beta_{\ell}$ if $k<\ell$.

We set $N_{i j}:=\operatorname{ord} q_{\alpha_{i j}}$, that is $N_{i j}=N_{\alpha}$ if $\alpha=\alpha_{i j}$. For simplicity we set $N_{i}=N_{i i}$ for all $i$. Then

$$
N_{i j}= \begin{cases}2 & \left|\left\{k \in \mathbb{I}_{i, j}: q_{k k}=-1\right\}\right| \text { is odd } \\ N & \left|\left\{k \in \mathbb{I}_{i, j}: q_{k k}=-1\right\}\right| \text { is even }\end{cases}
$$

The root vectors are

$$
\begin{array}{rlrl}
x_{\alpha_{i i}} & =x_{\alpha_{i}}=x_{i}, & i \in \mathbb{I}, \\
x_{\alpha_{i j}}=x_{(i j)}=\left[x_{i}, x_{\alpha_{i+1} j}\right]_{c}, & i<j \in \mathbb{I},
\end{array}
$$

see (2.3.5); we order them lexicographically: $x_{1}<x_{(12)}<\cdots<x_{2}<\cdots<x_{\theta}$. Thus

$$
\left\{x_{\theta}^{n_{\theta \theta}} x_{(\theta-1 \theta)}^{n_{\theta-1 \theta}} x_{\theta-1}^{n_{\theta-1 \theta-1}} \ldots x_{(1 \theta)}^{n_{1 \theta}} \ldots x_{1}^{n_{11}} \mid 0 \leq n_{i j}<N_{i j}\right\}
$$

is a PBW-basis of $\mathscr{B}_{\mathfrak{q}}$. The defining relations in terms of the PBW-generators are

$$
\begin{aligned}
x_{(i j)} x_{(i k)} & =q_{\alpha_{i j} \alpha_{i k}} x_{(i k)} x_{(i j)}, & & i \leq j<k ; \\
x_{(i k)} x_{(j k)} & =q_{\alpha_{i k} \alpha_{j k}} x_{(j k)} x_{(i k)}, & & i<j \leq k ; \\
x_{(i j)} x_{(j+1 k)} & =q_{\alpha_{i j} \alpha_{j+1}} x_{(j+1 k)} x_{(i j)}+x_{(i k)}, & & i \leq j<k ; \\
x_{(i j)} x_{(k \ell)} & =q_{\alpha_{i j} \alpha_{k \ell}} x_{(k \ell)} x_{(i j)}, & & i \leq j<k-1 \leq \ell-1 ; \\
x_{(i \ell)} x_{(j k)} & =q_{\alpha_{i \ell} \alpha_{j k}} x_{(j k)} x_{(i \ell)}, & & i<j \leq k<\ell ; \\
x_{(i j)} x_{(k \ell)} & =q_{\alpha_{i j} \alpha_{k \ell}} x_{(k \ell)} x_{(i j)}+\left(1-\widetilde{q}_{j, j+1}\right) q_{\alpha_{i j} \alpha_{k j} x_{(i \ell)} x_{(k j)},}, & & i<k \leq j<\ell ; \\
x_{(i j)}^{N_{i j}} & =0, & & i \leq j .
\end{aligned}
$$


The relations are homogeneous with respect to the $\mathbb{N}_{0}^{\theta}$-grading, an observation that will be useful later. As an example we draw the Cojocaru-Ufnarovski graph that encodes the Anick resolution for the Nichols algebra $\mathscr{B}_{\mathfrak{q}}$ of type $A_{2}$ in case $N_{1}, N_{12}, N_{2}>2$ :

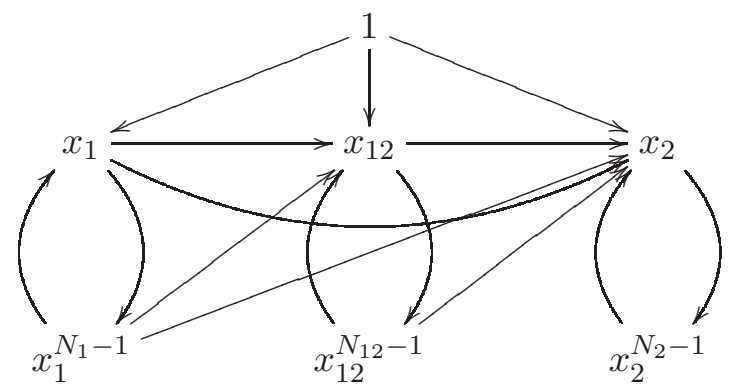

Observe that in this case $N_{1}=N_{12}=N_{2}$ and we are in Cartan type. The chains are then $\mathrm{M}(0)=\left\{\mathrm{x}_{1}, \mathrm{x}_{12}, \mathrm{x}_{2}\right\}$, $\mathrm{M}(1)=\left\{\mathrm{x}_{1}^{N_{1}}, \mathrm{x}_{12}^{N_{12}}, \mathrm{x}_{2}^{N_{2}}, \mathrm{x}_{1} \mathrm{x}_{12}, \mathrm{x}_{1} \mathrm{x}_{2}, \mathrm{x}_{12} \mathrm{x}_{2}\right\}$, $\mathrm{M}(2)=\left\{\mathrm{x}_{1}^{N_{1}+1}, \mathrm{x}_{12}^{N_{12}+1}, \mathrm{x}_{2}^{N_{2}+1}, \mathrm{x}_{1}^{N_{1}} \mathrm{x}_{12}, \mathrm{x}_{1}^{N_{1}} \mathrm{x}_{2}, \mathrm{x}_{1} \mathrm{x}_{12}^{N_{12}}, \mathrm{x}_{1} \mathrm{x}_{2}^{N_{2}}, \mathrm{x}_{12} \mathrm{x}_{2}^{N_{2}}, \mathrm{x}_{12}^{N_{12}} \mathrm{x}_{2}, \mathrm{x}_{1} \mathrm{x}_{12} \mathrm{x}_{2}\right\}$, $\mathrm{M}(3)=\left\{\mathrm{x}_{1}^{2 N_{1}}, \mathrm{x}_{12}^{2 N_{12}}, \mathrm{x}_{2}^{2 N_{2}}, \mathrm{x}_{1}^{N_{1}} \mathrm{x}_{12}^{N_{12}}, \mathrm{x}_{1}^{N_{1}} \mathrm{x}_{12} \mathrm{x}_{2}, \mathrm{x}_{1}^{N_{1}} \mathrm{x}_{2}^{N_{2}}, \mathrm{x}_{1}^{N_{1}+1} \mathrm{x}_{12}, \mathrm{x}_{1}^{N_{1}+1} \mathrm{x}_{2}\right.$, $\left.\mathrm{x}_{1} \mathrm{x}_{12}^{N_{12}+1}, \mathrm{x}_{1} \mathrm{x}_{2}^{N_{2}+1}, \mathrm{x}_{1} \mathrm{x}_{12} \mathrm{x}_{2}^{N_{2}}, \mathrm{x}_{12}^{N_{12}+1} \mathrm{x}_{2}, \mathrm{x}_{12}^{N_{12}} \mathrm{x}_{2}^{N_{2}}, \mathrm{x}_{12} \mathrm{x}_{2}^{N_{2}+1}\right\}$,

and so on. In case $N_{1}=2$, the loop between $\mathrm{x}_{1}$ and $\mathrm{x}_{1}^{N_{1}-1}$ is understood to be collapsed to a loop from $\mathrm{x}_{1}$ to itself, and similarly for the other root vectors.

In order to apply Remark 6.1.1, we start by the following Claim.

Claim 1. Let $\alpha$ be a non-simple root with $N_{\alpha}=2$. Let $C_{*}\left(\mathscr{B}_{\mathfrak{q}}\right)$ be the Anick resolution of the Nichols algebra $\mathscr{B}_{\mathfrak{q}}$. Let $c \in \mathrm{M}(N+1)$ and assume that $d(c \otimes 1) \in C_{N}\left(\mathscr{B}_{\mathfrak{q}}\right)$ has a term $\mathrm{x}_{\alpha}^{N} \otimes 1$ with nonzero coefficient. Write $c=\mathrm{x}_{\beta_{1}}^{a_{1}} \ldots \mathrm{x}_{\beta_{r}}^{a_{r}}$, where $a_{j} \in \mathbb{N}_{0}$ for all $j$. If $N_{\beta_{i}}>2$, then $a_{i}=0,1$ and

$$
\sum_{i \in \mathbb{I}} a_{i}=N+1
$$

Indeed, we may safely assume that $\alpha=\alpha_{1 \theta}=\alpha_{1}+\cdots+\alpha_{\theta}$ for simplicity. Since $d$ is homogeneous, $N \alpha_{1 \theta} \stackrel{*}{=} \sum_{i \in \mathbb{I}_{r}} a_{i} \beta_{i}$. Assume that

$$
\mathfrak{S}:=\left\{i \in \mathbb{I}_{r}: N_{\beta_{i}}>2, a_{i}>1\right\}
$$

is non-empty. If $i \in \mathfrak{S}$, then $a_{i} \geq N$ by looking at the Anick graph, hence $a_{i}=N$ by $*$. Also, the supports of the $\beta_{i}$ with $i \in \mathfrak{S}$ are disjoint. Let $\mathfrak{K}=\left\{k \in \mathbb{I}: k \notin \operatorname{supp} \beta_{i}, i \in \mathfrak{S}\right\}$; observe that $\mathfrak{K}$ is non-empty because $N_{\alpha}=2$, cf. (7.1.3). Now

$$
\sum_{i \notin \mathfrak{S}} a_{i} \beta_{i} \stackrel{\text { by }}{=}{ }^{*} N \alpha-N \sum_{i \in \mathfrak{S}} \beta_{i}=N \sum_{k \in \mathfrak{K}} \alpha_{k} .
$$

Pick $k \in \mathfrak{K}$ and compute the coefficient of $\alpha_{k}$ in the last expression; then

$$
\sum_{i \notin \mathfrak{S}} a_{i} \geq \sum_{i \notin \mathfrak{S}, k \in \operatorname{supp} \beta_{i}} a_{i}=N .
$$


Now the cohomological degree of $x_{\beta_{1}}^{a_{1}} \ldots x_{\beta_{r}}^{a_{r}}$ sums up $a_{i}$ for each $i \notin \mathfrak{S}$ and 2 for each $i \in \mathfrak{S}$ (one arrow in, one out). Thus

$$
N+1=\sum_{i \notin \mathfrak{S}} a_{i}+2|\mathfrak{S}| \geq N+2 .
$$

This contradiction shows that $\mathfrak{S}=\emptyset$ and the claim is proved.

The following result on root systems of type A should be well-known; we provide a proof for completeness of the argument.

Lemma 7.1.5. Let $\gamma, \gamma_{1}, \ldots, \gamma_{n+1} \in \Delta_{+}$with $\gamma_{1} \leq \gamma_{2} \leq \ldots \leq \gamma_{n+1}$. Assume that

$$
\gamma_{1}+\ldots+\gamma_{n+1}=n \gamma \text {. }
$$

Then

$$
\gamma_{2}=\gamma_{3}=\ldots=\gamma_{n}=\gamma, \quad \gamma=\gamma_{1}+\gamma_{n+1} .
$$

Proof. We may assume that $\gamma=\alpha_{1}+\ldots+\alpha_{\theta}$ for otherwise we reduce to a smaller $\theta$. Since we have $n+1$ roots in the sum which contains each simple root with coefficient exactly $n$, we must have that $\gamma_{1}, \ldots, \gamma_{n}$ have $\alpha_{1}$ in the support but $\gamma_{n+1}$ does not. Similarly, $\gamma_{2}, \ldots, \gamma_{n+1}$ must have $\alpha_{\theta}$ in their support but $\gamma_{1}$ does not. Hence, the supports of $\gamma_{2}, \ldots, \gamma_{n}$ contain all simple roots. We conclude that $\gamma_{2}=\ldots=\gamma_{n}=\gamma$, thus $\gamma_{1}+\gamma_{n+1}=\gamma$ by (7.1.6).

Claim 2. Let $\gamma \in \Delta_{+}$and let $c \in \mathrm{M}(n)$. Assume that

(a) $d(c \otimes 1)=\ldots+C \mathbf{x}_{\gamma}^{n} \otimes 1+\ldots$ for some $C \neq 0$,

(b) The polynomial degree of $c$ is $n+1$.

Then there exist $\alpha, \beta \in \Delta_{+}$such that

$$
c=\mathrm{x}_{\alpha} \mathrm{x}_{\gamma}^{n-1} \mathrm{x}_{\beta}, \quad \gamma=\alpha+\beta .
$$

Proof. By (b), we may write $c=\mathrm{x}_{\gamma_{1}} \ldots \mathrm{x}_{\gamma_{n+1}} \otimes 1$, where $\gamma_{1} \leq \gamma_{2} \leq \ldots \leq \gamma_{n+1} \in \Delta_{+}$. Since $d$ is $\mathbb{N}_{0}^{\theta}$-homogeneous, (7.1.6) holds by (a). Thus Lemma [7.1.5 applies.

Claim 3. Let $\alpha, \beta, \gamma \in \Delta_{+}$with $\gamma=\alpha+\beta$. Let $\wp=-\frac{q_{\alpha \alpha}}{q_{\beta \beta}}$. Assume that $N_{\gamma}=2$. Then

$$
\begin{aligned}
d\left(\mathrm{x}_{\alpha} \mathrm{x}_{\beta}^{j-1} \mathrm{x}_{\gamma} \otimes 1\right)=\mathrm{x}_{\alpha} \mathrm{x}_{\gamma}^{j-1} \otimes x_{\beta}-q_{\gamma, \beta} \mathrm{x}_{\alpha} \mathrm{x}_{\gamma}^{j-2} \mathbf{x}_{\beta} \otimes x_{\gamma} \\
+(-1)^{j} q_{\alpha, \beta} q_{\alpha, \gamma}^{j-1} \mathbf{x}_{\gamma}^{j-1} \mathbf{x}_{\beta} \otimes x_{\alpha}-q_{\gamma, \beta}^{j-1}(j)_{\wp} \mathbf{x}_{\gamma}^{j} \otimes 1 .
\end{aligned}
$$

Proof. The defining relations say that

$$
x_{\alpha} x_{\gamma}=q_{\alpha, \beta} x_{\gamma} x_{\alpha}+x_{\gamma}, \quad x_{\alpha} x_{\beta}=q_{\alpha, \gamma} x_{\beta} x_{\alpha}, \quad x_{\beta} x_{\gamma}=q_{\gamma, \beta} x_{\gamma} x_{\beta} .
$$

Hence we are in the setting of Lemma 10.1.7. We consider two cases.

First we assume that $j=2 a+1$ is odd. The only thing we need to prove is that the coefficient of $\mathrm{x}_{\gamma}^{j} \otimes 1$ is of the given form. We compute

$$
\begin{aligned}
& q_{\gamma, \beta}^{j-1}\left\{\left(\frac{q_{\alpha, \gamma}}{q_{\gamma, \beta}}-1\right)(a)_{\left(\frac{q_{\alpha, \gamma}}{q_{\gamma, \beta}}\right)^{2}}-\left(\frac{q_{\alpha, \gamma}}{q_{\gamma, \beta}}\right)^{2 a}\right\}=q_{\gamma, \beta}^{j-1}\left((-\wp-1)(a)_{(-\wp)^{2}}-(-\wp)^{2 a}\right) \\
& =-q_{\gamma, \beta}^{j-1}(1+\wp)\left(1+\wp^{2}+\ldots+\wp^{2 a-2}+\wp^{2 a}\right)=-q_{\gamma, \beta}^{j-1}(2 a+1)_{\wp}=-q_{\gamma, \beta}^{j-1}(j)_{\wp} .
\end{aligned}
$$


Next we assume that $j=2 a+2$ is even. We do a similar calculation for the coefficient of $\mathrm{x}_{\gamma}^{j} \otimes 1$.

$$
\begin{aligned}
& q_{\gamma, \beta}^{j-1}\left(\frac{q_{\alpha, \gamma}}{q_{\gamma, \beta}}-1\right)(a+1)\left(\frac{q_{\alpha, \gamma}}{q_{\gamma, \beta}}\right)^{2}=q_{\gamma, \beta}^{j-1}(-\wp-1)(a+1)_{(-\wp)^{2}} \\
& =-q_{\gamma, \beta}^{j-1}(1+\wp)\left(1+\wp^{2}+\ldots+\wp^{2 a}\right)=-q_{\gamma, \beta}^{j-1}(2 a+2)_{\wp}=-q_{\gamma, \beta}^{j-1}(j)_{\wp} .
\end{aligned}
$$

Proof of Proposition 7.1.1. First, if $N_{\gamma}>2$, then $\left(\mathrm{x}_{\gamma}^{N_{\gamma}}\right)^{*}$ is a cocycle in degree 2 by Lemma 6.2.5, cf. Lemma 6.2.7 (a), Let $\gamma$ be a non-simple root with $N_{\gamma}=2$.

By Remark 6.1.1, it suffices to show that there is no $c \in C_{N}(R)$ such that $d(c)$ contains $\mathrm{x}_{\gamma}^{N} \otimes 1$ with a non-zero coefficient. Assume that this happens. By Claim 1, $c$ satisfies the hypothesis (b) of Claim 2. Hence $c=\mathrm{x}_{\alpha} \mathrm{x}_{\gamma}^{N-1} \mathrm{x}_{\beta} \otimes 1$ for some $\alpha$ and $\beta$. But $\mathrm{x}_{\gamma}^{N} \otimes 1$ does not enter in $d\left(\mathrm{x}_{\alpha} \mathrm{x}_{\gamma}^{N-1} \mathrm{x}_{\beta} \otimes 1\right)$ with a non-zero coefficient for any $\alpha, \beta, \gamma \in \Delta^{+}$with $\gamma=\alpha+\beta$. Indeed, $\wp=-\frac{q_{\alpha \alpha}}{q_{\beta \beta}}=q^{ \pm 1}$, see (7.1.3); taking $j=N$, the coefficient in Lemma 3 is $q_{\gamma, \beta}^{j-1}(j)_{\wp}=0$.

7.2. Types $B_{\theta}$ and $\mathbf{B}(j \mid \theta-j), \theta \geq 1, j \in \mathbb{I}_{\theta-1}$. Let $q$ be a root of 1 of order $N>2$. In this subsection, we deal with the Nichols algebras $\mathscr{B}_{\mathfrak{q}}$ of diagonal types $B_{\theta}$ or $\mathbf{B}(j \mid \theta-j)$. In the first case, the Dynkin diagram is

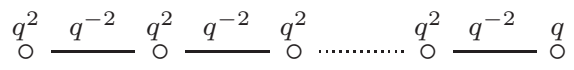

while in the second we may assume that the corresponding diagram is

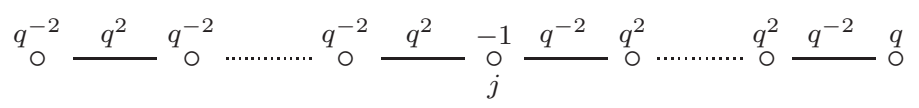

The set of positive roots in both cases is

$$
\Delta_{+}=\left\{\alpha_{i k} \mid i \leq k \in \mathbb{I}\right\} \cup\left\{\alpha_{i \theta}+\alpha_{k \theta} \mid i<k \in \mathbb{I}\right\} .
$$

We fix the following convex order:

$$
\begin{aligned}
& \alpha_{1}<\alpha_{12}<\cdots<\alpha_{1 \theta}<\alpha_{1 \theta}+\alpha_{\theta}<\cdots<\alpha_{1 \theta}+\alpha_{2 \theta} \\
& <\alpha_{2}<\alpha_{23}<\cdots<\alpha_{\theta-1}<\alpha_{\theta-1 \theta}<\alpha_{\theta-1 \theta}+\alpha_{\theta}<\alpha_{\theta} .
\end{aligned}
$$

We set $N_{i k}:=\operatorname{ord} q_{\alpha_{i k}}, M_{i k}:=\operatorname{ord} q_{\alpha_{i \theta}+\alpha_{k \theta}}$, that is $N_{i k}=N_{\alpha}$ if $\alpha=\alpha_{i k}, M_{i k}=N_{\alpha}$ if $\alpha=\alpha_{i \theta}+\alpha_{k \theta}$. Let $M=\operatorname{ord} q^{2}, P=\operatorname{ord}(-q)$. Then

$$
N_{i k}=\left\{\begin{array}{ll}
2 & i \leq j \leq k<\theta, \\
M & i \leq k<j \text { or } j<i \leq k<\theta ; \\
P & i \leq j<k=\theta, \\
N & j<i<k=\theta ;
\end{array} \quad M_{i k}= \begin{cases}2 & i \leq j<k \\
M & i<k \leq j \text { or } j<i<k .\end{cases}\right.
$$

Here we set $j=0$ if $\mathfrak{q}$ is of Cartan type. For more information, see [AA, §4.2, §5.2]. 
In this Section we prove that Condition 1.4 .1 holds for types $B_{\theta}$ and $\mathbf{B}(j \mid \theta-j), \theta \geq 1$, $j \in \mathbb{I}_{\theta-1}$. We need the following technical result.

Lemma 7.2.3. Let $\gamma=\alpha_{1 \theta}$, assume $N_{\gamma}=3$, and let $(\alpha, \beta)$ be a pair of positive roots such that

(1) $\alpha+\beta=2 \gamma$,

(2) $\alpha=\alpha_{1 k}, \beta=\alpha_{1 \theta}+\alpha_{k+1 \theta}$ for some $k \in \mathbb{I}_{\theta-1}$,

(in particular, $(\alpha, \beta)$ satisfy $\alpha<\beta)$.

Then the relations between the root vectors $x_{\alpha}, x_{\beta}$ and $x_{\gamma}$ are of the form

$$
x_{\alpha} x_{\gamma}=q_{\alpha \gamma} x_{\gamma} x_{\alpha}, \quad x_{\gamma} x_{\beta}=q_{\gamma \beta} x_{\beta} x_{\gamma}, \quad x_{\alpha} x_{\beta}=q_{\alpha \beta} x_{\beta} x_{\alpha}+\mathrm{b} x_{\gamma}^{2}, \quad \mathrm{~b} \in \mathbb{k}
$$

Proof. The statement follows by the repeated application of the convexity and homogeneity of the relations. We prove $x_{\alpha} x_{\gamma}=q_{\alpha \gamma} x_{\gamma} x_{\alpha}$ first. For any $\eta \in \Delta_{+}$such that $\alpha<\eta<\gamma$, we must have $\eta=\alpha_{1 r}$ for some $k<r<\theta$ by (7.2.2). From here, we see that is impossible to find $\eta_{1}, \ldots, \eta_{t}$ such that $\alpha+\gamma \neq \eta_{1}+\cdots+\eta_{t}$ and $\alpha<\eta_{i}<\gamma$. Hence, $x_{\alpha} x_{\gamma}=q_{\alpha \gamma} x_{\gamma} x_{\alpha}$ by (2.3.6).

For the second relation, if $\eta \in \Delta_{+}$is such that $\gamma<\eta<\beta$, then $\eta=\alpha_{1 \theta}+\alpha_{r \theta}$ for some $k<r<\theta$. Hence $\beta+\gamma \neq \eta_{1}+\cdots+\eta_{t}$ if $\alpha<\eta_{i}<\gamma$, so $x_{\gamma} x_{\beta}=q_{\gamma \beta} x_{\beta} x_{\gamma}$.

The last relation follows similarly: $\alpha<\eta_{1} \leq \cdots \leq \eta_{t}<\beta$ are such that $\sum_{i} \eta_{i}=\alpha+\beta$ if and only if $k=2$ and $\eta_{1}=\eta_{2}=\gamma$.

Proposition 7.2.4. For every $\gamma \in \Delta_{+}^{\mathfrak{q}}$, there exists $L_{\gamma} \in \mathbb{N}$ such that $\left(\mathrm{x}_{\gamma}^{L_{\gamma}}\right)^{*}$ is a cocycle.

Proof. Let $\gamma$ be a positive non-simple root. Arguing recursively we may assume that $\gamma$ has full support. Hence, either $\gamma=\alpha_{1 \theta}$ or else there exists $k \in \mathbb{I}_{2, \theta}$ such that $\gamma=\alpha_{1 \theta}+\alpha_{k \theta}$.

First we consider $\gamma=\alpha_{1 \theta}$. It is easy to check that $P_{\gamma}=3$ and $Q_{\gamma}=1$. Hence, if $N_{\gamma}>3$, then Lemma 6.2.5 applies and $\left(\mathrm{x}_{\gamma}^{N_{\gamma}}\right)^{*}$ is a 2-cocycle. Next we consider the case $N_{\gamma}=3$ : that is, either $N=3$ if the braiding is of Cartan type, or else $N=6$ if not.

All the pairs $(\alpha, \beta)$ as in (6.3.24) are of the form $\alpha=\alpha_{1 k}, \beta=\alpha_{1 \theta}+\alpha_{k+1 \theta}$ for some $k \in \mathbb{I}_{\theta-1}$. Fix a pair $(\alpha, \beta)$. By Lemma 7.2.3, $x_{\alpha}, x_{\beta}, x_{\gamma}$ satisfy (10.1.8). Also, $\frac{q_{\alpha \gamma}}{q_{\gamma \beta}}=1$ for all of them, so we take $L=1$. Now we look for solutions of (6.3.26). That is, $\sum_{\delta \in \Delta_{+}} f_{\delta}\left(n_{\delta}\right) \delta=3 \gamma, \sum_{\delta \in \Delta_{+}} n_{\delta}=3$. We check that there is no solution with $n_{\delta}=3$ neither with $n_{\delta}=2, \delta \in \Delta_{+}$. Hence we are forced to look for solutions with $n_{\gamma_{t}}=1$ for three different roots $\gamma_{t} \in \Delta_{+}$(and 0 for the other roots). We write $\gamma_{t}=\sum_{i \in \mathbb{I}} a_{i}^{(t)} \alpha_{i}$. As $\sum_{t} a_{i}^{(t)}=3$ for all $i \in \mathbb{I}$, we have $a_{1}^{(t)}=1$ for all $t \in \mathbb{I}_{3}$, and either $a_{\theta}^{(t)}=1$ for all $t \in \mathbb{I}_{3}$, or $a_{\theta}^{(t)}=2$ for some $t \in \mathbb{I}_{3}$. If $a_{\theta}^{(t)}=1$ for all $t \in \mathbb{I}_{3}$, then $\gamma_{t}=\gamma$ for all $t$, a contradiction. Hence we may assume $a_{\theta}^{(1)}=0, a_{\theta}^{(2)}=2, a_{\theta}^{(3)}=1$. Then $\gamma_{1}=\alpha_{1 k}, \gamma_{2}=\alpha_{1 \theta}+\alpha_{k+1 \theta}$, $\gamma_{3}=\gamma$, for some $k \in \mathbb{I}_{\theta-1}$. Thus Proposition 6.3.23 applies and $\left(\mathrm{x}_{\gamma}^{3}\right)^{*}$ is a 2-cocycle.

Now we consider $\gamma=\alpha_{1 \theta}+\alpha_{k \theta}, k \in \mathbb{I}_{2, \theta}$. Here, $P_{\gamma}=2$ and $Q_{\gamma}=1$. If $N_{\gamma}>2$, then Lemma 6.2.5 applies and $\left(\mathrm{x}_{\gamma}^{N_{\gamma}}\right)^{*}$ is a 2-cocycle. Next we consider the case $N_{\gamma}=2$ : that is, either $N=4$ if the braiding is of Cartan type, or else $k>j$ if the braiding is of type $\mathbf{B}(j \mid \theta-j)$. Let $\alpha<\beta$ be a pair of positive roots as in (6.3.3). We have several possibilities: 
$\circ \alpha=\alpha_{1 i-1}, \beta=\alpha_{i \theta}+\alpha_{k \theta}, i<k$. Arguing as in Lemma 7.2.3, the relations between the root vectors are of the form:

$$
x_{\alpha} x_{\gamma}=q_{\alpha \gamma} x_{\gamma} x_{\alpha}, \quad x_{\gamma} x_{\beta}=q_{\gamma \beta} x_{\beta} x_{\gamma}, \quad x_{\alpha} x_{\beta}=q_{\alpha \beta} x_{\beta} x_{\alpha}+\mathrm{b} x_{\gamma}, \quad \mathrm{b} \in \mathbb{k} .
$$

$\circ \alpha=\alpha_{1 i-1}, \beta=\alpha_{k \theta}+\alpha_{i \theta}, k<i \leq \theta$. The relations between root vectors are of the form $x_{\alpha} x_{\gamma}=q_{\alpha \gamma} x_{\gamma} x_{\alpha}, \quad x_{\gamma} x_{\beta}=q_{\gamma \beta} x_{\beta} x_{\gamma}, \quad x_{\alpha} x_{\beta}=q_{\alpha \beta} x_{\beta} x_{\alpha}+\mathrm{b} x_{\gamma}+\sum_{t=k+1}^{i-1} \mathrm{~b}_{t} x_{\alpha_{k \theta}+\alpha_{t \theta}} x_{\alpha_{1 t-1}}$,

for some $\mathrm{b}, \mathrm{b}_{t} \in \mathbb{k}$.

$\circ \alpha=\alpha_{1 \theta}, \beta=\alpha_{k \theta}$. Arguing as in Lemma 7.2.3.

$$
x_{\alpha} x_{\gamma}=q_{\alpha \gamma} x_{\gamma} x_{\alpha}, \quad x_{\gamma} x_{\beta}=q_{\gamma \beta} x_{\beta} x_{\gamma}, \quad x_{\alpha} x_{\beta}=q_{\alpha \beta} x_{\beta} x_{\alpha}+\mathrm{b} x_{\gamma}+\sum_{t=k+1}^{\theta-1} \mathrm{~b}_{t} x_{\alpha_{1 \theta}+\alpha_{t \theta}} x_{\alpha_{k t-1}} \text {, }
$$

for some $\mathrm{b}, \mathrm{b}_{t} \in \mathbb{k}$.

In all cases the root vectors satisfy (10.1.8), and $-\frac{q_{\alpha \alpha}}{q_{\beta \beta}}=q^{ \pm 2}$, so we take $L=M$.

Next we look for solutions of (6.3.1). In the Cartan case with $N=4$, we have $\sum_{\delta \in \Delta_{+}} n_{\delta}=3$ : we easily discard the possibility that $n_{\delta} \geq 2$ for some $\delta \in \Delta_{+}$, so exactly three of them are one, and $n_{\varphi}=0$ for the remaining $\varphi \in \Delta_{+}$. Arguing as in the case $\gamma=\alpha_{1 \theta}$ we check that all solutions are of form $n_{\gamma}=n_{\alpha}=n_{\beta}=1$ for a pair $(\alpha, \beta)$ satisfying (6.3.3). Next we consider the case $\mathbf{B}(j \mid \theta-j), k>j$. We have that $\sum_{\delta \in \Delta_{+}: 1 \in \operatorname{supp} \delta} f_{\delta}\left(n_{\delta}\right)=M$. Let $\eta \in \Delta_{+}$such that $1 \in \operatorname{supp} \eta$ and $n_{\eta}>0$.

- If $\eta=\alpha_{1 \theta}$, then $n_{\eta}=1$ : otherwise, $f_{\eta}\left(n_{\eta}\right) \geq P=2 M>M=\sum_{\delta \in \Delta_{+}: 1 \in \operatorname{supp} \delta} f_{\delta}\left(n_{\delta}\right)$, a contradiction.

- If $\eta=\alpha_{1 i}, i<j$, then $n_{\eta}=1$. Suppose on the contrary that $n_{\eta}>1$. Then $n_{\eta}=2$, since $f_{\eta}\left(n_{\eta}\right) \leq M=N_{\eta}$. This implies that $n_{\delta}=0$ for all $\delta \neq \alpha_{1 i}$ such that $i \in \operatorname{supp} \delta$.

Let $\delta \in \Delta_{+}$such that $a_{i+1}^{\delta}=2$. Then $n_{\delta}=0$ since $i \in \operatorname{supp} \delta$. Thus

$$
M=\sum_{\delta \in \Delta_{+}: a_{i+1}^{\delta}=1} f_{\delta}\left(n_{\delta}\right), \quad \sum_{\delta \in \Delta_{+}: a_{i+1}^{\delta}=1} n_{\delta}<M .
$$

Then $n_{i+1 \ell}=2$ for some $\ell>i$, which implies that $n_{\delta}=0$ for all $\delta \neq \alpha_{i+1 \ell}$ such that $\ell \in \operatorname{supp} \delta$. Recursively, there exist $i_{0}=1<i_{1}=i<i_{2}<\cdots<i_{s}=j-1$ such that

$$
n_{\delta}= \begin{cases}2 & \delta=\alpha_{i_{r-1} i_{r}}, r \in \mathbb{I}_{s}, \\ 0 & \delta \neq \alpha_{i_{r-1} i_{r}}, \operatorname{supp} \delta \cap \mathbb{I}_{j-1} \neq \emptyset .\end{cases}
$$

On the other hand, if $a_{j}^{\delta}=1$, then either $N_{\delta}=1$, or $\delta=\alpha_{i \theta}, i<j$, in which case $n_{\delta} \leq 1$ since $N_{\delta}=P=2 M$. Hence $n_{\delta}=f_{\delta}\left(n_{\delta}\right)$ for all $\delta$ such that $a_{j}^{\delta}=1$. Using this fact,

$$
\begin{aligned}
M & =\sum_{\delta \in \Delta_{+}} f_{\delta}\left(n_{\delta}\right) a_{j}^{\delta}=\sum_{\delta \in \Delta_{+}: a_{j}^{\delta}=1} n_{\delta}+2 \sum_{\delta \in \Delta_{+}: a_{j}^{\delta}=2} f_{\delta}\left(n_{\delta}\right) \\
& \leq \sum_{\delta \in \Delta_{+}-\{\eta\}} n_{\delta}+2 \sum_{\delta \in \Delta_{+}: a_{j}^{\delta}=2} f_{\delta}\left(n_{\delta}\right) \leq M-1+2 \sum_{\delta \in \Delta_{+}: a_{j}^{\delta}=2} f_{\delta}\left(n_{\delta}\right) .
\end{aligned}
$$

Thus $n_{\mu} \neq 0$ for some $\mu \in \Delta_{+}$such that $a_{j}^{\mu}=2$; but $j-1 \in \operatorname{supp} \mu$, a contradiction. 
- If either $\eta=\alpha_{1 i}$ or else $\eta=\alpha_{1 \theta}+\alpha_{i \theta}, j \leq i<\theta$, then $N_{\eta}=2$, so $f_{\eta}\left(n_{\eta}\right)=n_{\eta}$.

- If $\eta=\alpha_{1 \theta}+\alpha_{i \theta}, i<j$, then $n_{\eta}=1$. Otherwise,

$$
M=\sum_{\delta \in \Delta_{+}: j-1 \in \operatorname{supp} \delta} f_{\delta}\left(n_{\delta}\right) a_{j-1}^{\delta} \geq 2 f_{\eta}\left(n_{\eta}\right) \geq 2 M,
$$

and we get a contradiction.

Hence $f_{\delta}\left(n_{\delta}\right)=n_{\delta}$ for all $\delta$ such that $1 \in \operatorname{supp} \delta$. Therefore,

$$
\sum_{\delta \in \Delta_{+}: 1 \notin \operatorname{supp} \delta} n_{\delta}=M+1-\sum_{\delta \in \Delta_{+}: 1 \in \operatorname{supp} \delta} n_{\delta}=M+1-\sum_{\delta \in \Delta_{+}: 1 \in \operatorname{supp} \delta} f_{\delta}\left(n_{\delta}\right)=1 .
$$

That is, there exists a unique root $\beta \in \Delta_{+}$such that $1 \notin \operatorname{supp} \beta$ and $n_{\beta} \neq 0$; moreover $n_{\beta}=1$ for this root $\beta$. Thus $f_{\delta}\left(n_{\delta}\right)=n_{\delta}$ for all $\delta$ and we may translate to solve (6.3.1) as follows: find $\gamma_{t} \in \Delta_{+}, t \in \mathbb{I}_{M+1}$, not necessarily different such that $\sum_{t} \gamma_{t}=M \gamma$. Write $\gamma_{t}=\sum_{i \in \mathbb{I}} a_{i}^{(t)} \alpha_{i}$. Hence we may assume that $a_{1}^{(t)}=1$ for $t \in \mathbb{I}_{M}, a_{1}^{(M+1)}=0$. As $\sum_{t \in \mathbb{I}_{M+1}} a_{k}^{(t)}=2 M$ and each $a_{k}^{(t)} \leq 2$, at least $M-1$ of them are 2 , and for the other two $a_{k}^{(t)}$ 's, either both are 1 , or else one of them is 2 and the other is 0 . Hence we may assume that $a_{k}^{(t)}=1$ for $t \in \mathbb{I}_{M-1}$. This forces to have $\gamma_{t}=\gamma$ for $t \in \mathbb{I}_{M-1}, \gamma_{M}+\gamma_{M+1}=\gamma$.

Hence all the hypotheses of Proposition 6.3.2 hold, and $\left(\mathrm{x}_{\gamma}^{L_{\gamma}}\right)^{*}$ is a cocycle.

7.3. Type $B_{\theta, j}$ standard, $j \in \mathbb{I}_{\theta-1}$. Here $\zeta \in \mathbb{G}_{3}^{\prime}$. In this subsection, we deal with Nichols algebras $\mathscr{B}_{\mathfrak{q}}$ of standard type $B_{\theta, j}$. We assume that the corresponding diagram is

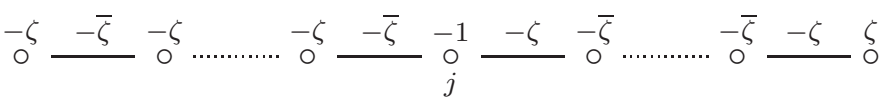

The set of positive roots is (7.2.1), and we fix the same convex order, see (17.2.2). For more information, see $\left[\mathrm{AA}, \S 6.1\right.$. We prove that Condition 1.4.1 holds for type $B_{\theta, j}$ standard:

Proposition 7.3.1. For every $\gamma \in \Delta_{+}^{\mathfrak{q}}$, there exists $L_{\gamma} \in \mathbb{N}$ such that $\left(\mathrm{x}_{\gamma}^{L_{\gamma}}\right)^{*}$ is a cocycle.

Proof. Let $\gamma$ be a positive non-simple root. Arguing recursively we may assume that $\gamma$ has full support. Hence, either $\gamma=\alpha_{1 \theta}$ or else there exists $k \in \mathbb{I}_{2, \theta}$ such that $\gamma=\alpha_{1 \theta}+\alpha_{k \theta}$.

First we consider $\gamma=\alpha_{1 \theta}$. Here, $P_{\gamma}=3$ and $Q_{\gamma}=2, N_{\gamma}=3$. All the pairs $(\alpha, \beta)$ as in (6.3.24) are of the form $\alpha=\alpha_{1 k}, \beta=\alpha_{1 \theta}+\alpha_{k+1 \theta}$ for some $k \in \mathbb{I}_{\theta-1}$. By Lemma 7.2.3,

$$
x_{\alpha} x_{\gamma}=q_{\alpha \gamma} x_{\gamma} x_{\alpha}, \quad x_{\gamma} x_{\beta}=q_{\gamma \beta} x_{\beta} x_{\gamma}, \quad x_{\alpha} x_{\beta}=q_{\alpha \beta} x_{\beta} x_{\alpha}+\mathrm{b}_{k} x_{\gamma}^{2}, \quad \text { for some } \mathrm{b}_{k} \in \mathbb{k},
$$

so the root vectors satisfy (10.1.8). Also, $\frac{q_{\alpha \gamma}}{q_{\gamma \beta}}=1$ for all of them, so we take $L=1$. Now we look for solutions of (6.3.26). As in the proof of Proposition 7.2.4, there exists a pair $(\alpha, \beta)$ as in (6.3.24) such that $n_{\gamma}=n_{\alpha}=n_{\beta}=1$, and $n_{\delta}=0$ for the remaining roots $\delta \in \Delta_{+}^{\mathfrak{q}}$. Thus Proposition 6.3 .23 applies and $\left(\mathrm{x}_{\gamma}^{3}\right)^{*}$ is a 2-cocycle.

Now we consider $\gamma=\alpha_{1 \theta}+\alpha_{k \theta}, k \in \mathbb{I}_{2, \theta}$. Here we have $P_{\gamma}=2$ and $Q_{\gamma}=1$. Hence, if $k \leq j$, then Lemma 6.2.5 applies since $N_{\gamma}=6$, so $\left(\mathrm{x}_{\gamma}^{6}\right)^{*}$ is a 2-cocycle. Next we consider the case $k>j$, so $N_{\gamma}=2$. The pairs $\alpha<\beta$ as in (6.3.3) are the same as in Proposition 7.2.4, the root vectors satisfy (10.1.8) and $-\frac{q_{\alpha \alpha}}{q_{\beta \beta}}=-\zeta^{ \pm 1}$, so we take $L=6$. Also, the same argument as in the case $\mathbf{B}(j \mid \theta-j)$ in Proposition 7.2.4 shows that there exists a pair 
$(\alpha, \beta)$ as in (6.3.24) such that $n_{\gamma}=4, n_{\alpha}=n_{\beta}=1$, and $n_{\delta}=0$ for the remaining roots $\delta \in \Delta_{+}^{\mathfrak{q}}$. Hence all the hypotheses of Proposition 6.3 .2 hold, and $\left(\mathrm{x}_{\gamma}^{6}\right)^{*}$ is a cocycle.

7.4. Type $C_{\theta}$. Let $q$ be a root of 1 of order $N>2$. In this subsection, we deal with the Nichols algebras $\mathscr{B}_{\mathfrak{q}}$ of diagonal type $C_{\theta}$ : the Dynkin diagram is

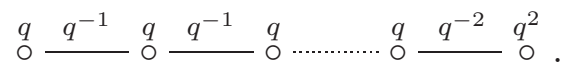

The set of positive roots is

$$
\Delta_{+}=\left\{\alpha_{i j} \mid i \leq j \in \mathbb{I}\right\} \cup\left\{\alpha_{i \theta}+\alpha_{j \theta-1} \mid i \leq j \in \mathbb{I}_{\theta-1}\right\} .
$$

We fix the following convex order:

$$
\begin{aligned}
& \alpha_{1}<\alpha_{12}<\cdots<\alpha_{1 \theta-1}<\alpha_{1 \theta}+\alpha_{1 \theta-1}<\alpha_{1 \theta}+\alpha_{\theta-1}<\cdots<\alpha_{1 \theta}+\alpha_{2 \theta-1} \\
& <\alpha_{1 \theta}<\alpha_{2}<\alpha_{23}<\cdots<\alpha_{\theta-1}<\alpha_{\theta-1 \theta}<\alpha_{\theta-1 \theta}+\alpha_{\theta-1}<\alpha_{\theta} .
\end{aligned}
$$

Let $M=\operatorname{ord} q^{2}$. It follows from the definition that

$$
N_{\gamma}= \begin{cases}M, & \gamma=\alpha_{i \theta}+\alpha_{i \theta-1} \text { or } \gamma=\alpha_{\theta} ; \\ N, & \gamma=\alpha_{i \theta}+\alpha_{j \theta-1}, i<j, \text { or } \gamma=\alpha_{i j},(i, j) \neq(\theta, \theta) .\end{cases}
$$

For more information, see [AA, §4.3]. The aim of this Section is to prove that Condition 1.4.1 holds for type $C_{\theta}$. More precisely,

Proposition 7.4.3. For every $\gamma \in \Delta_{+}^{\mathfrak{q}},\left(\mathrm{x}_{\gamma}^{N_{\gamma}}\right)^{*}$ is a 2-cocycle.

Proof. Let $\gamma$ be a positive non-simple root. Arguing recursively we may assume that $\gamma$ has full support. Hence, either $\gamma=\alpha_{1 \theta}$ or else there exists $j \in \mathbb{I}_{\theta-1}$ such that $\gamma=\alpha_{1 \theta}+\alpha_{j \theta-1}$.

First we consider $\gamma=\alpha_{1 \theta}$. In this case we have $P_{\gamma}=3$ and $Q_{\gamma}=1$. Hence, if $N_{\gamma}>3$, then Lemma 6.2.5 applies and $\left(\mathrm{x}_{\gamma}^{N_{\gamma}}\right)^{*}$ is a 2-cocycle.

Now assume that $N_{\gamma}=3$ : that is, $N=3$. The unique pair $(\alpha, \beta)$ as in (6.3.24) is $\alpha=\alpha_{1 \theta}+\alpha_{1 \theta-1}, \beta=\alpha_{\theta}$. Arguing as in Lemma 7.2.3, the relations are of the form 1

$$
x_{\alpha} x_{\gamma}=q_{\alpha \gamma} x_{\gamma} x_{\alpha}, \quad x_{\gamma} x_{\beta}=q_{\gamma \beta} x_{\beta} x_{\gamma}, \quad x_{\alpha} x_{\beta}=q_{\alpha \beta} x_{\beta} x_{\alpha}+\mathrm{b} x_{\gamma}^{2} \quad \text { for some } \mathrm{b} \in \mathbb{k},
$$

and then the root vectors satisfy (10.1.8). Also, $\frac{q_{\alpha \gamma}}{q_{\gamma \beta}}=1$ for all of them, so we take $L=1$. Now we look for solutions of (6.3.26). That is, $\sum_{\delta \in \Delta_{+}} f_{\delta}\left(n_{\delta}\right) \delta=3 \gamma, \sum_{\delta \in \Delta_{+}} n_{\delta}=3$. We check that there is no solution with $n_{\delta}=3$ neither with $n_{\delta}=2, \delta \in \Delta_{+}$. Hence we look for solutions with $n_{\gamma_{t}}=1$, for three different roots $\gamma_{t} \in \Delta_{+}$(and $n_{\delta}=0$ for the other roots). We write $\gamma_{t}=\sum_{i \in \mathbb{I}} a_{i}^{(t)} \alpha_{i}$. As $\sum_{t} a_{i}^{(t)}=3$ for all $i \in \mathbb{I}$, we have $a_{\theta}^{(t)}=1$ for all $t \in \mathbb{I}_{3}$, and either $a_{1}^{(t)}=1$ for all $t \in \mathbb{I}_{3}$, or $a_{1}^{(t)}=2$ for some $t \in \mathbb{I}_{3}$. The case $a_{\theta}^{(t)}=1$ for all $t \in \mathbb{I}_{3}$ gives a contradiction: either $\gamma_{t}=\gamma$ for all $t$, or else $\sum_{t} a_{\theta-1}^{(t)}>3$. Hence we may assume $a_{1}^{(1)}=2, a_{1}^{(2)}=0, a_{1}^{(3)}=1$. Then $\gamma_{1}=\alpha_{1 \theta}+\alpha_{1 \theta-1}, \gamma_{2}=\alpha_{\theta}, \gamma_{3}=\gamma$. Thus Proposition 6.3 .23 applies and $\left(\mathrm{x}_{\gamma}^{3}\right)^{*}$ is a 2-cocycle.

\footnotetext{
${ }^{1}$ Indeed, $x_{\alpha}=\left[x_{1 \theta-1}, x_{1 \theta}\right]_{c}, x_{\gamma}=x_{1 \theta}$, and using $q$-Jacobi identity we have that $\mathrm{b}=q(q-1) \prod_{i \in \mathbb{I}_{\theta-1}} q_{i \theta}$.
} 
Now we consider $\gamma=\alpha_{1 \theta}+\alpha_{j \theta-1}, j \in \mathbb{I}_{\theta-1}$. In this case, $P_{\gamma}=2$ and $Q_{\gamma}=1$. If $N_{\gamma}>2$, then Lemma 6.2.5 applies and $\left(\mathrm{x}_{\gamma}^{N_{\gamma}}\right)^{*}$ is a 2-cocycle. Hence we need to study the case $N_{\gamma}=2$ : that is, $N=4$ and $\gamma=\alpha_{1 \theta}+\alpha_{1 \theta-1}$. Let $\alpha<\beta$ be a pair of positive roots as in (6.3.3). We have the following possibilities:

$\circ \alpha=\alpha_{1 i-1}, \beta=\alpha_{1 \theta}+\alpha_{i \theta}, i \in \mathbb{I}_{2, \theta-1}$. There exist $\mathrm{b}, \mathrm{b}_{t} \in \mathbb{k}$ such that

$$
x_{\alpha} x_{\gamma}=q_{\alpha \gamma} x_{\gamma} x_{\alpha}, \quad x_{\gamma} x_{\beta}=q_{\gamma \beta} x_{\beta} x_{\gamma}, \quad x_{\alpha} x_{\beta}=q_{\alpha \beta} x_{\beta} x_{\alpha}+\mathrm{b} x_{\gamma}+\sum_{t=i+1}^{\theta-1} \mathrm{~b}_{t} x_{\alpha_{1 \theta}+\alpha_{t \theta-1}} x_{\alpha_{1-1}} \text {. }
$$

$\circ \alpha=\alpha_{1 \theta}, \beta=\alpha_{1 \theta-1}$. In this case,

$$
x_{\alpha} x_{\gamma}=q_{\alpha \gamma} x_{\gamma} x_{\alpha}, \quad x_{\gamma} x_{\beta}=q_{\gamma \beta} x_{\beta} x_{\gamma}, \quad x_{\alpha} x_{\beta}=q_{\alpha \beta} x_{\beta} x_{\alpha}+x_{\gamma} .
$$

In all cases the root vectors satisfy (10.1.8), and $-\frac{q_{\alpha \alpha}}{q_{\beta \beta}}=-1$, so we take $L=2$.

Next we look for solutions of (6.3.1). That is, $\sum_{\delta \in \Delta_{+}} f_{\delta}\left(n_{\delta}\right) \delta=2 \gamma, \sum_{\delta \in \Delta_{+}} n_{\delta}=3$. Let $\mu \in \Delta_{+}$be such that $n_{\mu} \neq 0, \theta \in \operatorname{supp} \mu$. Notice that

$$
2=\sum_{\delta \in \Delta_{+}: \theta \in \operatorname{supp} \delta} f_{\delta}\left(n_{\delta}\right) a_{\theta}^{\delta}=\sum_{\delta \in \Delta_{+}: \theta \in \operatorname{supp} \delta} f_{\delta}\left(n_{\delta}\right),
$$

so $n_{\mu} \leq f_{\mu}\left(n_{\mu}\right) \leq 2$. Suppose that $n_{\mu}=2$. Then $\mu=\alpha_{i \theta}+\alpha_{i \theta-1}$ for some $i \in \mathbb{I}_{\theta-1}$ since $N_{\mu}=f_{\mu}(2) \leq 2$, and there exists $\eta \neq \mu$ such that $n_{\eta}=1, n_{\delta}=0$ if $\delta \neq \mu, \eta$. But then

$$
4 \alpha_{1 i-1}=2 \gamma-2 \mu=\sum_{\delta \neq \mu} f_{\delta}\left(n_{\delta}\right) \delta=f_{\eta}(1) \eta=\eta
$$

a contradiction. Hence $n_{\mu}=1$ for all $\mu \in \Delta_{+}$such that $n_{\mu} \neq 0, \theta \in \operatorname{supp} \mu$. Then there exist three different roots $\gamma_{i} \in \Delta_{+}$such that $n_{\gamma_{i}}=1$, and we may assume that $\theta \in \operatorname{supp} \gamma_{2} \cap \operatorname{supp} \gamma_{3}, \theta \notin \operatorname{supp} \gamma_{1}$. As $\gamma_{2} \neq \gamma_{3}$, we may assume $\gamma_{2} \neq \gamma$, so $a_{1}^{\gamma_{2}}=1$. This implies that $a_{1}^{\gamma_{1}}=1$, so $\gamma_{1}=\alpha_{1 i}$ for some $i \in \mathbb{I}_{\theta-1}$, and $a_{1}^{\gamma_{3}}=2$, so $\gamma_{3}=\gamma$.

Hence all the hypotheses of Proposition 6.3.2 hold, and $\left(\mathrm{x}_{\gamma}^{N_{\gamma}}\right)^{*}$ is a 2-cocycle.

7.5. Type $D_{\theta}$. Let $q \in \mathbb{G}_{N}^{\prime}, n \geq 2$. Let $\mathscr{B}_{\mathfrak{q}}$ be a Nichols algebra of type $D_{\theta}$. That is, the generalized Dynkin diagram of $\mathscr{B}_{\mathfrak{q}}$ has the form

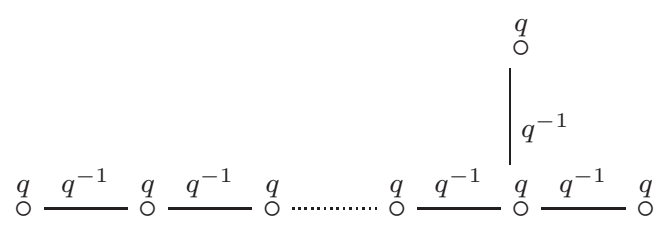

The set of positive roots is

$$
\begin{aligned}
\Delta_{+}^{\mathfrak{q}}= & \left\{\alpha_{i j} \mid i \leq j \in \mathbb{I},(i, j) \neq(\theta-1, \theta)\right\} \\
& \cup\left\{\alpha_{i \theta-2}+\alpha_{\theta} \mid i \in \mathbb{I}_{\theta-2}\right\} \cup\left\{\alpha_{i \theta}+\alpha_{j \theta-2} \mid i<j \in \mathbb{I}_{\theta-2}\right\} .
\end{aligned}
$$

We fix the following convex order:

$$
\begin{aligned}
& \alpha_{11}<\alpha_{12}<\cdots<\alpha_{1 \theta-1}<\alpha_{1 \theta-2}+\alpha_{\theta}<\alpha_{1 \theta}<\alpha_{1 \theta}+\alpha_{\theta-2}<\ldots \\
& <\alpha_{1 \theta}+\alpha_{2 \theta-2}<\alpha_{22}<\alpha_{23}<\cdots<\alpha_{\theta-2}<\alpha_{\theta-2 \theta-1}<\alpha_{\theta-2}+\alpha_{\theta}
\end{aligned}
$$




$$
<\alpha_{\theta-2 \theta}<\alpha_{\theta-1}<\alpha_{\theta}
$$

For more information, see [AA, §4.4]. The aim of this Section is to prove that Condition 1.4.1 holds for type $D_{\theta}$. More precisely,

Proposition 7.5.2. For every $\gamma \in \Delta_{+}^{\mathfrak{q}},\left(\mathrm{x}_{\gamma}^{N_{\gamma}}\right)^{*}$ is a 2-cocycle.

Proof. By Lemma 6.2.7 $P_{\alpha}=2$ and $Q_{\alpha}=1$ for all non-simple roots $\alpha$. Hence, if $N>2$, then Lemma 6.2.5 applies and $\left(\mathrm{x}_{\gamma}^{N_{\gamma}}\right)^{*}$ is a 2-cocycle for all roots $\alpha$.

Next we assume $N=2$, that is, $q=-1$. We will apply Proposition 6.3.2. Let $\gamma$ be a positive non-simple root. Arguing recursively we may assume that $\gamma$ has full support. Hence, either $\gamma=\alpha_{1 \theta}$ or else there exists $k \in \mathbb{I}_{2, \theta-2}$ such that $\gamma=\alpha_{1 \theta}+\alpha_{k \theta-2}$. We look for pairs $\alpha<\beta \in \Delta_{+}$such that $\gamma=\alpha+\beta$. Notice that $q_{\alpha \alpha}=q_{\beta \beta}=-1=-\frac{q_{\alpha \alpha}}{q_{\beta \beta}}$ in any case so we may guess that $L=2$.

First we consider $\gamma=\alpha_{1 \theta}$. All the pairs $(\alpha, \beta)$ as in (6.3.3) are of the form $\alpha=\alpha_{1 j}$, $\beta=\alpha_{j+1 \theta}$ for some $j \in \mathbb{I}_{\theta-1}$. Similar to Lemma 7.2.3.

$$
x_{\alpha} x_{\gamma}=q_{\alpha \gamma} x_{\gamma} x_{\alpha}, \quad x_{\gamma} x_{\beta}=q_{\gamma \beta} x_{\beta} x_{\gamma}, \quad x_{\alpha} x_{\beta}=q_{\alpha \beta} x_{\beta} x_{\alpha}+\mathrm{b} x_{\gamma} \quad \text { for some } \mathrm{b} \in \mathbb{k},
$$

so the root vectors satisfy (10.1.8). Next we look for solutions of (6.3.1). That is, $2 \gamma=$ $\delta_{1}+\delta_{2}+\delta_{3}, \delta_{i} \in \Delta_{+}$. We write $\delta_{j}=\sum_{i \in \mathbb{I}} a_{i}^{(j)} \alpha_{i}$. As $a_{i}^{(j)}$ is 0 or 1 for $i=1, \theta-1, \theta$, we may fix $a_{1}^{(1)}=a_{1}^{(2)}=1, a_{1}^{(3)}=0$ and see the possible pairs of roots such that $a_{\theta-1}^{(m)}=a_{\theta-1}^{(n)}=1$, respectively $a_{\theta}^{(p)}=a_{\theta}^{(r)}=1$. Suppose that no one of the $\delta_{i}$ 's has coefficient 1 for the three simple roots simultaneously. Then we may assume $a_{\theta-1}^{(1)}=a_{\theta-1}^{(3)}=1, a_{\theta}^{(2)}=a_{\theta}^{(3)}=1$, so $a_{\theta-2}^{(j)}>0$ for all $j \in \mathbb{I}_{3}$, a contradiction. Hence we assume $a_{\theta-1}^{(1)}=a_{\theta}^{(1)}=1$, so $a_{i}^{(1)} \geq 1$ for all $i \in \mathbb{I}$. If either $a_{\theta-1}^{(2)}=1$ or $a_{\theta}^{(2)}=1$, then $a_{\theta-2}^{(2)} \geq 1$, which implies $a_{\theta-2}^{(1)}=1$ and so $\delta_{1}=\gamma$. Otherwise $a_{\theta-1}^{(3)}=1=a_{\theta}^{(3)}$, then $a_{\theta-2}^{(3)} \geq 1$, which implies again $\delta_{1}=\gamma$.

Finally, let $\gamma=\alpha_{1 \theta}+\alpha_{k \theta-2}$. Let $\alpha<\beta$ be a pair of positive roots as in (6.3.3). Then the coefficient of $\alpha_{1}$ is one for just one of them (and zero for the other): it should be $\alpha$, since $\alpha<\beta$. We have several possibilities:

$\circ \alpha=\alpha_{1 j-1}, \beta=\alpha_{j \theta}+\alpha_{k \theta-2}, j<k$. Then

$$
x_{\alpha} x_{\gamma}=q_{\alpha \gamma} x_{\gamma} x_{\alpha}, \quad x_{\gamma} x_{\beta}=q_{\gamma \beta} x_{\beta} x_{\gamma}, \quad x_{\alpha} x_{\beta}=q_{\alpha \beta} x_{\beta} x_{\alpha}+\mathrm{b} x_{\gamma} \quad \text { for some } \mathrm{b} \in \mathbb{k} \text {. }
$$

○ $\alpha=\alpha_{1 j-1}, \beta=\alpha_{k \theta}+\alpha_{j \theta-2}, k<j \leq \theta-2$. Then

$$
x_{\alpha} x_{\gamma}=q_{\alpha \gamma} x_{\gamma} x_{\alpha}, \quad x_{\gamma} x_{\beta}=q_{\gamma \beta} x_{\beta} x_{\gamma}, \quad x_{\alpha} x_{\beta}=q_{\alpha \beta} x_{\beta} x_{\alpha}+\mathrm{b} x_{\gamma}+\sum_{t=k+2}^{j-1} \mathrm{~b}_{t} x_{\alpha_{k \theta}+\alpha_{t \theta-2}} x_{\alpha_{1 t-1}} \text {, }
$$

for some $\mathrm{b}, \mathrm{b}_{t} \in \mathbb{k}$.

○ $\alpha=\alpha_{1 \theta-1}, \beta=\alpha_{k \theta-2}+\alpha_{\theta}$. Then

$$
x_{\alpha} x_{\gamma}=q_{\alpha \gamma} x_{\gamma} x_{\alpha}, \quad x_{\gamma} x_{\beta}=q_{\gamma \beta} x_{\beta} x_{\gamma}, \quad x_{\alpha} x_{\beta}=q_{\alpha \beta} x_{\beta} x_{\alpha}+\mathrm{b} x_{\gamma},
$$

for some $\mathrm{b}, \mathrm{b}_{t} \in \mathbb{k}$. 
$\circ \alpha=\alpha_{1 \theta}, \beta=\alpha_{k \theta-2}$. Then

$x_{\alpha} x_{\gamma}=q_{\alpha \gamma} x_{\gamma} x_{\alpha}, \quad x_{\gamma} x_{\beta}=q_{\gamma \beta} x_{\beta} x_{\gamma}, \quad x_{\alpha} x_{\beta}=q_{\alpha \beta} x_{\beta} x_{\alpha}+\mathrm{b} x_{\gamma}+\sum_{t=k+1}^{j-1} \mathrm{~b}_{t} x_{\alpha_{1 \theta}+\alpha_{t \theta-2}} x_{\alpha_{k t-1}}$,

for some $\mathrm{b}, \mathrm{b}_{t} \in \mathbb{k}$.

○ $\alpha=\alpha_{1 \theta}+\alpha_{j \theta-2}, \beta=\alpha_{k j-1}, k<j \leq \theta-2$. Then

$x_{\alpha} x_{\gamma}=q_{\alpha \gamma} x_{\gamma} x_{\alpha}, \quad x_{\gamma} x_{\beta}=q_{\gamma \beta} x_{\beta} x_{\gamma}, \quad x_{\alpha} x_{\beta}=q_{\alpha \beta} x_{\beta} x_{\alpha}+\mathrm{b} x_{\gamma}+\sum_{t=k+1}^{j-1} \mathrm{~b}_{t} x_{\alpha_{1 \theta}+\alpha_{t \theta-2}} x_{\alpha_{k t-1}}$,

for some $\mathrm{b}, \mathrm{b}_{t} \in \mathbb{k}$.

In each case the justification relies on the homogeneity of the relations and is similar to Lemma [7.2.3, we leave the details to an interested reader. Therefore the root vectors satisfy (10.1.8). Next we look for solutions of (6.3.1). That is, $2 \gamma=\delta_{1}+\delta_{2}+\delta_{3}, \delta_{i} \in \Delta_{+}$. We write $\delta_{j}=\sum_{i \in \mathbb{I}} a_{i}^{(j)} \alpha_{i}$.

When $k=\theta-2$, first consider the case $\delta_{1}=\alpha_{\theta-2}$. Then $a_{j}^{(i)}=1$ for $i=1, \theta-1, \theta$ and $j=2,3$, so $\delta_{2}, \delta_{3}$ have full support. This implies that $a_{j}^{(i)}=1$ for $2 \leq i<\theta-2$ and $j=2,3$, and we need that $a_{j}^{(\theta-2)}=1$ for one of them; that is, either $\delta_{2}=\gamma$ or else $\delta_{3}=\gamma$. If $\delta_{j} \neq \alpha_{\theta-2}$ for all $j \in \mathbb{I}_{3}$, then

$$
2 \alpha_{1 \theta}=2 s_{\theta-2}(\gamma)=s_{\theta-2}\left(\delta_{1}\right)+s_{\theta-2}\left(\delta_{2}\right)+s_{\theta-2}\left(\delta_{3}\right), \quad s_{\theta-2}\left(\delta_{j}\right) \in \Delta_{+} .
$$

Applying the previous case, $s_{\theta-2}\left(\delta_{j}\right)=\alpha_{1 \theta}$ for some $j \in \mathbb{I}_{3}$, so $\delta_{j}=\gamma$.

If $k<\theta-2$, then we argue recursively. Indeed, we first consider the case $\delta_{1}=\alpha_{k}$ and argue as in the case $k=\theta-2$ to show that either $\delta_{2}=\gamma$ or else $\delta_{3}=\gamma$. If $\delta_{j} \neq \alpha_{\theta-2}$ for all $j \in \mathbb{I}_{3}$, then

$$
2 \alpha_{1 \theta}+\alpha_{k+1 \theta-2}=2 s_{k}(\gamma)=s_{k}\left(\delta_{1}\right)+s_{k}\left(\delta_{2}\right)+s_{k}\left(\delta_{3}\right), \quad s_{k}\left(\delta_{j}\right) \in \Delta_{+} .
$$

Hence $s_{k}\left(\delta_{j}\right)=\alpha_{1 \theta}+\alpha_{k+1 \theta-2}$ for some $j \in \mathbb{I}_{3}$, which means that $\delta_{j}=\gamma$ for some $j \in \mathbb{I}_{3}$.

Hence all the hypotheses of Proposition 6.3.2 hold, and $\left(\mathrm{x}_{\gamma}^{2}\right)^{*}$ is a 2-cocycle.

7.6. Type $\mathbf{D}(j \mid \theta-j), \theta \geq 1, j \in \mathbb{I}_{\theta-1}$. Let $q$ be a root of 1 of order $N>2$. In this subsection, we deal with the Nichols algebras $\mathscr{B}_{\mathfrak{q}}$ of type $\mathbf{D}(j \mid \theta-j)$. We may assume that the corresponding diagram is

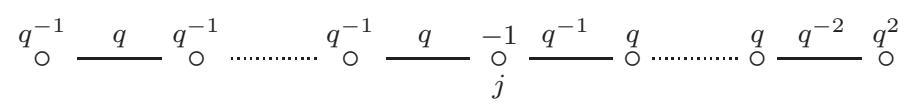

The set of positive roots is

$$
\begin{aligned}
\Delta_{+}^{\mathfrak{q}}= & \left\{\alpha_{i k} \mid i \leq k \in \mathbb{I}\right\} \cup\left\{\alpha_{i \theta}+\alpha_{k \theta-1} \mid i<k \in \mathbb{I}_{\theta-1}\right\} \\
& \cup\left\{\alpha_{i \theta}+\alpha_{i \theta-1} \mid i \in \mathbb{I}_{j+1, \theta-1}\right\} .
\end{aligned}
$$

Thus (7.6.1) is a subset of the set (7.4.1) of positive roots of type $C_{\theta}$ : We fix the convex order in $\Delta_{+}^{\mathfrak{q}}$ obtained from (7.4.2). For more information, see [AA, §5.3]. We prove Condition 1.4 .1 for type $\mathbf{D}(j \mid \theta-j)$ :

Proposition 7.6.2. For every $\gamma \in \Delta_{+}^{\mathfrak{q}}$, there exists $L_{\gamma} \in \mathbb{N}$ such that $\left(\mathrm{x}_{\gamma}^{L_{\gamma}}\right)^{*}$ is a cocycle. 
Proof. Let $\gamma$ be a positive non-simple root. Arguing recursively we may assume that $\gamma$ has full support. Hence, either $\gamma=\alpha_{1 \theta}$ or else there exists $k \in \mathbb{I}_{\theta-1}$ such that $\gamma=\alpha_{1 \theta}+\alpha_{k \theta-1}$.

First we consider $\gamma=\alpha_{1 \theta}$. Again, one can easily check that $N_{\gamma}=2, P_{\gamma}=3$ and $Q_{\gamma}=1$. Let $\alpha<\beta$ be a pair of positive roots as in 6.3.3). Then there exists $i \in \mathbb{I}_{\theta-1}$ such that $\alpha=\alpha_{1 i}, \beta=\alpha_{i+1 \theta}$. Now,

$$
x_{\alpha} x_{\gamma}=q_{\alpha \gamma} x_{\gamma} x_{\alpha}, \quad x_{\gamma} x_{\beta}=q_{\gamma \beta} x_{\beta} x_{\gamma}, \quad x_{\alpha} x_{\beta}=q_{\alpha \beta} x_{\beta} x_{\alpha}+\mathrm{b} x_{\gamma} \quad \text { for some } \mathrm{b} \in \mathbb{k},
$$

hence the root vectors satisfy (10.1.8), and $-\frac{q_{\alpha \alpha}}{q_{\beta \beta}} \in\left\{q^{-1}, q^{-2}\right\}$, so we take $L=N$.

There exist 4-tuples $(\alpha, \beta, \delta, \eta) \in \Delta_{+}^{4}$ as in (6.3.5):

$$
\alpha=\alpha_{1 i}<\eta=\alpha_{1 \theta-1}<\gamma<\beta=\alpha_{1 \theta}+\alpha_{i+1 \theta-1}<\delta=\alpha_{\theta} .
$$

The corresponding PBW generators satisfy (10.1.11); indeed, there exists $\mathrm{b} \in \mathbb{k}$ such that $\left[x_{\alpha}, x_{\beta}\right]_{c}=q_{\alpha \beta} x_{\beta} x_{\alpha}+\mathrm{b} x_{\gamma} x_{\eta}, x_{\eta} x_{\delta}=q_{\eta \delta} x_{\delta} x_{\eta}+x_{\gamma}$, and the other pairs of root vectors $q$-commute. Now $\mathrm{c}_{\alpha \beta \gamma}^{(N)}=0$ by Lemma 6.3.28 (b) since $\widetilde{q}_{\alpha \gamma}=\widetilde{q}_{\beta \gamma}=q^{-1}$.

Next we look for solutions of (6.3.1). We claim that $f_{\eta}\left(n_{\eta}\right)=n_{\eta}$ for all $\eta \in \Delta_{+}$.

○ If $N_{\eta}=2$, then this holds by definition of $f_{\eta}$.

o If $\eta=\alpha_{i \theta}+\alpha_{k \theta-1}, i<k \leq \theta-1$, then $N_{\eta}=N$, so $f_{\eta}(k) \geq N$ if $k \geq 2$. As

$$
2 f_{\eta}\left(n_{\eta}\right) \leq \sum_{\delta \in \Delta_{+}^{\mathfrak{q}}} f_{\eta}\left(n_{\eta}\right) a_{\theta-1}^{\delta}=N,
$$

we have that $n_{\eta} \leq 1$, so $f_{\eta}\left(n_{\eta}\right)=n_{\eta}$.

o Let $\eta=\alpha_{i k}$, with $i \leq k<j$. Then $N_{\eta}=N$. Suppose that $n_{\eta} \geq 2$ : as $f_{\eta}(s)>N$ if $s>2$, we may have $n_{\eta}=2$ : moreover, $n_{\delta}=0$ for all $\delta \neq \alpha_{i k}$ such that $\delta \cap \mathbb{I}_{i, k} \neq \emptyset$ since

$$
N=\sum_{\delta \in \Delta_{+}^{\mathfrak{q}}} f_{\delta}\left(n_{\delta}\right) a_{t}^{\delta}=N+\sum_{\delta \neq \alpha_{i k}: t \in \operatorname{supp} \delta} f_{\delta}\left(n_{\delta}\right) a_{t}^{\delta} \quad \text { for all } t \in \mathbb{I}_{i, k} .
$$

Now if $k+1<j$, then $n_{\delta}=0$ for all $\delta \neq \alpha_{k+1 t}, t \geq k+1$ : as $\sum_{\delta \in \Delta_{+}^{\mathfrak{q}}} f_{\delta}\left(n_{\delta}\right) a_{k+1}^{\delta}=N$, we have $n_{\alpha_{k+1 t}}=2$ for some $k+1 \leq t<j$. Thus we may assume $k=j-1$. Let $\delta \in \Delta_{+}^{\mathfrak{q}}$ be such that $j \in \operatorname{supp} \delta$. Then $n_{\delta}=0$ if $j-1 \in \operatorname{supp} \delta$, and $N_{\delta}=2$ if $j-1 \notin \operatorname{supp} \delta$, so

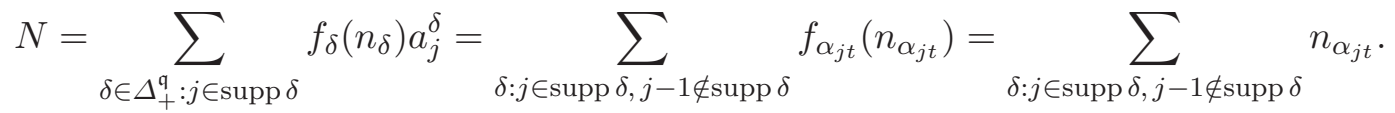

This implies that $\sum_{\delta \in \Delta_{+}^{\mathfrak{q}}} n_{\delta} \geq N+2$, a contradiction. Then $n_{\eta} \leq 1$, so $f_{\eta}\left(n_{\eta}\right)=n_{\eta}$.

- Let $\eta=\alpha_{i k}, j<i \leq k$. Then $N_{\eta}=N$ and an argument as in the previous case shows that we have that $n_{\eta} \leq 1$, so $f_{\eta}\left(n_{\eta}\right)=n_{\eta}$.

- Similar situation holds for $\eta=\alpha_{i \theta}+\alpha_{i \theta-1}: N_{\eta}=M$ but again $n_{\eta} \leq 1$, so $f_{\eta}\left(n_{\eta}\right)=n_{\eta}$.

As the claim holds, we may rewrite the problem as follows: find $\gamma_{i} \in \Delta_{+}^{\mathfrak{q}}, i \in \mathbb{I}_{N+1}$, such that $\sum \gamma_{i}=N \gamma$. As $a_{1}^{\delta}=1$ if $1 \in \operatorname{supp} \delta, a_{\theta}^{\delta}=1$ if $\theta \in \operatorname{supp} \delta$, there exist $\theta-1$ roots such that $1, \theta \in \operatorname{supp} \gamma_{i}$ : we may fix that $1, \theta \in \operatorname{supp} \gamma_{i}$ for $i \geq 3$. As $N=\sum_{i=3}^{N+1} a_{\theta-1}^{\gamma_{i}}$ and $a_{\theta-1}^{\gamma_{i}} \geq 1$, there exists at most one $i \geq 3$ such that $a_{\theta-1}^{\gamma_{i}}=2$ :

- if $a_{\theta-1}^{\gamma_{i}}=1$ for all $i \geq 3$, then $\gamma_{i}=\gamma$ for all $i \geq 3$ and $\gamma_{1}+\gamma_{2}=\gamma$.

- if $a_{\theta-1}^{\gamma_{3}}=2$, then $\gamma_{i}=\gamma$ for all $i \geq 4$ and $\gamma_{3}=\alpha_{1 \theta}+\alpha_{k \theta-1}$ for some $k \in \mathbb{I}_{2, \theta-1}$. Hence $\gamma_{1}+\gamma_{2}=\alpha_{1 k-1}+\alpha_{\theta}$, so $\gamma_{1}, \gamma_{2}$ are $\alpha_{1 k-1}, \alpha_{\theta}$. 
Hence all the hypotheses of Proposition 6.3.2 hold, and $\left(\mathrm{x}_{\gamma}^{L_{\gamma}}\right)^{*}$ is a cocycle.

Now we consider $\gamma=\alpha_{1 \theta}+\alpha_{i \theta-1}, i \in \mathbb{I}_{2, \theta-1}$. In this case, $P_{\gamma}=2$ and $Q_{\gamma}=1$. Let $i \leq j$. Then $N_{\gamma}=N>2$, so Lemma 6.2.5 applies and $\left(\mathrm{x}_{\gamma}^{N_{\gamma}}\right)^{*}$ is a 2-cocycle.

Next we assume that $i>j$, so $N_{\gamma}=2$. The pairs $(\alpha, \beta)$ as in (6.3.3) are the following: $\circ \alpha=\alpha_{1 \theta}, \beta=\alpha_{i \theta-1}$. As in Lemma 7.2.3,

$$
x_{\alpha} x_{\gamma}=q_{\alpha \gamma} x_{\gamma} x_{\alpha}, \quad x_{\gamma} x_{\beta}=q_{\gamma \beta} x_{\beta} x_{\gamma}, \quad x_{\alpha} x_{\beta}=q_{\alpha \beta} x_{\beta} x_{\alpha}+\mathrm{b} x_{\gamma} \quad \text { for some } \mathrm{b} \in \mathbb{k} .
$$

○ $\alpha=\alpha_{1 \theta}+\alpha_{k \theta}, \beta=\alpha_{i k-1}, k \in \mathbb{I}_{i+1, \theta-1}$. Then

$$
x_{\alpha} x_{\gamma}=q_{\alpha \gamma} x_{\gamma} x_{\alpha}, \quad x_{\gamma} x_{\beta}=q_{\gamma \beta} x_{\beta} x_{\gamma}, \quad x_{\alpha} x_{\beta}=q_{\alpha \beta} x_{\beta} x_{\alpha}+\mathrm{b} x_{\gamma}+\sum_{t=k+1}^{\theta-1} \mathrm{~b}_{t} x_{\alpha_{i t-1}} x_{\alpha_{1 \theta}+\alpha_{t \theta}} \text {, }
$$

for some $\mathrm{b}, \mathrm{b}_{t} \in \mathbb{k}$.

$\circ \alpha=\alpha_{1 k-1}, \beta=\alpha_{k \theta}+\alpha_{i \theta-1}, k \in \mathbb{I}_{j+1, i-1}$. Then

$$
x_{\alpha} x_{\gamma}=q_{\alpha \gamma} x_{\gamma} x_{\alpha}, \quad x_{\gamma} x_{\beta}=q_{\gamma \beta} x_{\beta} x_{\gamma}, \quad x_{\alpha} x_{\beta}=q_{\alpha \beta} x_{\beta} x_{\alpha}+\mathrm{b} x_{\gamma}+\sum_{t=k+1}^{i-1} \mathrm{~b}_{t} x_{\alpha_{t \theta}+\alpha_{i \theta}} x_{\alpha_{1-1}}
$$

for some $\mathrm{b}, \mathrm{b}_{t} \in \mathbb{k}$.

$\circ \alpha=\alpha_{1 i-1}, \beta=\alpha_{i \theta}+\alpha_{i \theta-1}$. In this case,

$$
x_{\alpha} x_{\gamma}=q_{\alpha \gamma} x_{\gamma} x_{\alpha}, \quad x_{\gamma} x_{\beta}=q_{\gamma \beta} x_{\beta} x_{\gamma}, \quad x_{\alpha} x_{\beta}=q_{\alpha \beta} x_{\beta} x_{\alpha}+\mathrm{b} x_{\gamma} \quad \text { for some } \mathrm{b} \in \mathbb{k} .
$$

Hence all the pairs of root vectors satisfy (10.1.8) and $-\frac{q_{\alpha \alpha}}{q_{\beta \beta}} \in\left\{q^{-1}, q^{-2}\right\}$, so we take $L=N$. Now we look for solutions of (6.3.1); i.e. $\sum_{\delta \in \Delta_{+}} f_{\delta}\left(n_{\delta}\right) \delta=N \gamma, \sum_{\delta \in \Delta_{+}} n_{\delta}=N+1$. Let $\eta \in \Delta_{+}^{\mathfrak{q}}$ such that $1 \in \operatorname{supp} \eta, N_{\eta}>2$ and $n_{\eta} \neq 0$. Suppose that $n_{\eta} \geq 2$. Arguing as for the case $\gamma=\alpha_{1 \theta}$, there exists $t \in \mathbb{I}_{j-1}$ such that $\eta=\alpha_{1 t}$ and $n_{\eta}=2$. This implies that $n_{\delta}=0$ for all $\delta \in \Delta_{+}^{\mathfrak{q}}$ such that $\operatorname{supp} \delta \cap \mathbb{I}_{t} \neq \emptyset$. Recursively, there exist $t_{0}=0<t_{1}=t<t_{2}<\cdots<t_{s}=j-1$ such that $n_{\eta}=2$ if $\eta=\alpha_{i_{r-1}+1} i_{r}, r \in \mathbb{I}_{s}$, and $n_{\delta}=0$ for all $\delta \in \Delta_{+}^{\mathfrak{q}}$ such that $\operatorname{supp} \delta \cap \mathbb{I}_{j-1} \neq \emptyset$. Now, if $j \in \operatorname{supp} \delta$, then either $j-1 \operatorname{supp} \delta$ (so $n_{\delta}=0$ by the previous argument) or $a_{j}^{\delta}=1, N_{\delta}=2$, so $f_{\delta}\left(n_{\delta}\right)=n_{\delta}$. Thus,

$$
N=\sum_{\delta \in \Delta_{+}^{\mathfrak{q}}: j \in \operatorname{supp} \delta} f_{\delta}\left(n_{\delta}\right) a_{j}^{\delta}=\sum_{\delta \in \Delta_{+}^{\mathfrak{q}}: j \in \operatorname{supp} \delta} n_{\delta} .
$$

But then

$$
N+1=\sum_{\delta \in \Delta_{+}^{\mathfrak{q}}} n_{\delta} \geq n_{\eta}+\sum_{\delta \in \Delta_{+}^{\mathfrak{q}}: j \in \operatorname{supp} \delta} n_{\delta}=N+2,
$$

a contradiction. Thus we have that $f_{\eta}\left(n_{\eta}\right)=n_{\eta}$ for all $\eta \in \Delta_{+}^{\mathfrak{q}}$ such that $1 \in \operatorname{supp} \eta$ since either $N_{\eta}=2$ or else $n_{\eta} \geq 1$. From here,

$$
N=\sum_{\delta \in \Delta_{+}^{\mathfrak{q}}: 1 \in \operatorname{supp} \delta} f_{\delta}\left(n_{\delta}\right) a_{j}^{\delta}=\sum_{\delta \in \Delta_{+}^{\mathfrak{q}}: 1 \in \operatorname{supp} \delta} n_{\delta},
$$

As $\sum_{\delta \in \Delta_{+}^{q}} n_{\delta}=N+1$, there exists a unique $\eta \in \Delta_{+}$such that $n_{\eta} \neq 0$ and $1 \notin \operatorname{supp} \eta$; moreover, $n_{\eta}=1$. Again we may rewrite the problem as follows: find $\gamma_{k} \in \Delta_{+}^{\mathfrak{q}}, k \in \mathbb{I}_{N+1}$, such that $\sum_{k} \gamma_{k}=N \gamma$. As $a_{1}^{\delta}=1$ if $1 \in \operatorname{supp} \delta, a_{\theta}^{\delta}=1$ if $\theta \in \operatorname{supp} \delta$, there exist $\theta-1$ 
roots such that $1, \theta \in \operatorname{supp} \gamma_{k}$ : we may fix that $1, \theta \in \operatorname{supp} \gamma_{k}$ for $k \geq 3$. Also, $a_{i}^{\gamma_{k}} \leq 2$ and $\sum_{t} a_{i}^{\gamma_{k}}=2 N$, so either $a_{i}^{\gamma_{k}}=2$ for exactly $N$ of them and 0 for the remaining one $a_{i}^{\gamma_{k}}$, or else $a_{i}^{\gamma_{k}}=2$ for exactly $N-1$ of them and 1 for the remaining two $a_{i}^{\gamma_{k}}$ 's. A detailed study case-by-case shows that $\gamma_{i}=\gamma$ for $i \geq 3$, and $\gamma_{1}+\gamma_{2}=\gamma$. Hence all the hypotheses of Proposition 6.3.2 hold, and $\left(\mathrm{x}_{\gamma}^{N}\right)^{*}$ is a cocycle.

\section{EXCEPTIONAL TYPES}

8.1. Type $E_{\theta}$. Let $q \in \mathbb{G}_{N}^{\prime}, n \geq 2$. Let $\mathscr{B}_{\mathfrak{q}}$ be a Nichols algebra of type $E_{\theta}, 6 \leq \theta \leq 8$. That is, the generalized Dynkin diagram of $\mathscr{B}_{\mathfrak{q}}$ has the form

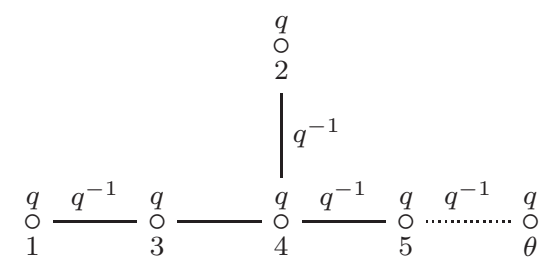

Here $\Delta^{\mathfrak{q}}=\Delta$ is a root system of type $E_{\theta}$. We fix the following convex orders on the sets of positive roots:

$\mathbf{E}_{\mathbf{6}}: 1,2,13,3,1234,134,234,24,34,4,123^{2} 4^{2} 5,1234^{2} 5,234^{2} 5,12345,1345,2345,345,245$, $45,5,12^{2} 3^{2} 4^{3} 5^{2} 6,123^{2} 4^{3} 5^{2} 6,123^{2} 4^{2} 5^{2} 6,123^{2} 4^{2} 56,1234^{2} 5^{2} 6,23^{2} 4^{2} 5^{2} 6,1234^{2} 56,234^{2} 56$, $123456,23456,2456,13456,3456,456,56,6$;

$\mathbf{E}_{\mathbf{7}}$ : roots of support contained in $\mathbb{I}_{6}$ ordered as for $E_{6}$ followed by $1^{2} 2^{2} 3^{3} 4^{4} 5^{3} 6^{2} 7,12^{2} 3^{3} 4^{4} 5^{3} 6^{2} 7,12^{2} 3^{2} 4^{4} 5^{3} 6^{2} 7,12^{2} 3^{2} 4^{3} 5^{3} 6^{2} 7,123^{2} 4^{3} 5^{3} 6^{2} 7,12^{2} 3^{2} 4^{3} 5^{2} 6^{2} 7$, $12^{2} 3^{2} 4^{3} 5^{2} 67,123^{2} 4^{3} 5^{2} 6^{2} 7,123^{2} 4^{3} 5^{2} 67,123^{2} 4^{2} 5^{2} 6^{2} 7,123^{2} 4^{2} 5^{2} 67,123^{2} 4^{2} 567$, $1234^{2} 5^{2} 6^{2} 7,1234^{2} 5^{2} 67,1234^{2} 567,1234567,134567,234^{2} 5^{2} 6^{2} 7,234^{2} 5^{2} 67,234^{2} 567$, $234567,24567,34567,4567,567,67,7$;

$\mathbf{E}_{\mathbf{8}}$ : roots of support contained in $\mathbb{I}_{7}$ ordered as for $E_{7}$ followed by $1^{2} 2^{3} 3^{4} 4^{6} 5^{5} 6^{4} 7^{3} 8,1^{2} 2^{3} 3^{4} 4^{6} 5^{5} 6^{4} 7^{2} 8,1^{2} 2^{3} 3^{4} 4^{6} 5^{5} 6^{3} 7^{2} 8,1^{2} 2^{3} 3^{4} 4^{6} 5^{4} 6^{3} 7^{2} 8,1^{2} 2^{3} 3^{4} 4^{5} 5^{4} 6^{3} 7^{2} 8$, $1^{2} 2^{2} 3^{4} 4^{5} 5^{4} 6^{3} 7^{2} 8,1^{2} 2^{3} 3^{3} 4^{5} 5^{4} 6^{3} 7^{2} 8,12^{3} 3^{3} 4^{5} 5^{4} 6^{3} 7^{2} 8,1^{2} 2^{2} 3^{3} 4^{5} 5^{4} 6^{3} 7^{2} 8,12^{2} 3^{3} 4^{5} 5^{4} 6^{3} 7^{2} 8$, $1^{2} 2^{2} 3^{3} 4^{4} 5^{4} 6^{3} 7^{2} 8,12^{2} 3^{3} 4^{4} 5^{4} 6^{3} 7^{2} 8,12^{2} 3^{2} 4^{4} 5^{4} 6^{3} 7^{2} 8,1^{2} 2^{2} 3^{3} 4^{4} 5^{3} 6^{3} 7^{2} 8,12^{2} 3^{3} 4^{4} 5^{3} 6^{3} 7^{2} 8$, $12^{2} 3^{2} 4^{4} 5^{3} 6^{3} 7^{2} 8,12^{2} 3^{2} 4^{3} 5^{3} 6^{3} 7^{2} 8,123^{2} 4^{3} 5^{3} 6^{3} 7^{2} 8,1^{2} 2^{2} 3^{3} 4^{4} 5^{3} 6^{2} 7^{2} 8,12^{2} 3^{3} 4^{4} 5^{3} 6^{2} 7^{2} 8$, $12^{2} 3^{2} 4^{4} 5^{3} 6^{2} 7^{2} 8,12^{2} 3^{2} 4^{3} 5^{3} 6^{2} 7^{2} 8,12^{2} 3^{2} 4^{3} 5^{2} 6^{2} 7^{2} 8,123^{2} 4^{3} 5^{3} 6^{2} 7^{2} 8,123^{2} 4^{3} 5^{2} 6^{2} 7^{2} 8$, $123^{2} 4^{2} 5^{2} 6^{2} 7^{2} 8,1234^{2} 5^{2} 6^{2} 7^{2} 8,234^{2} 5^{2} 6^{2} 7^{2} 8,1^{2} 2^{3} 3^{4} 4^{6} 5^{5} 6^{4} 7^{3} 8^{2}, 1^{2} 2^{2} 3^{3} 4^{4} 5^{3} 6^{2} 78$, $12^{2} 3^{3} 4^{4} 5^{3} 6^{2} 78,12^{2} 3^{2} 4^{4} 5^{3} 6^{2} 78,12^{2} 3^{2} 4^{3} 5^{3} 6^{2} 78,123^{2} 4^{3} 5^{3} 6^{2} 78,12^{2} 3^{2} 4^{3} 5^{2} 6^{2} 78$, $12^{2} 3^{2} 4^{3} 5^{2} 678,123^{2} 4^{3} 5^{2} 6^{2} 78,123^{2} 4^{3} 5^{2} 678,123^{2} 4^{2} 5^{2} 6^{2} 78,123^{2} 4^{2} 5^{2} 678,123^{2} 4^{2} 5678$, $1234^{2} 5^{2} 6^{2} 78,1234^{2} 5^{2} 678,1234^{2} 5678,12345678,1345678,234^{2} 5^{2} 6^{2} 78,234^{2} 5^{2} 678$, $234^{2} 5678,2345678,245678,345678,45678,5678,678,78,8$. 
For more information, see [AA, §4.5]. The aim of this Section is to prove that Condition 1.4.1 holds for type $E_{\theta}$. We need first the following result.

Lemma 8.1.1. Let $\beta<\delta \in \Delta_{+}^{\mathfrak{q}}$ be such that $\gamma=\beta+\delta \in \Delta_{+}^{\mathfrak{q}}$.

(a) If $\mu_{1} \leq \cdots \leq \mu_{k} \in \Delta_{+}$satisfy $\sum_{i} \mu_{i}=\beta+\gamma$, then either $\mu_{1} \leq \beta$ or else $\mu_{k} \geq \gamma$.

(b) If $\mu_{1} \leq \cdots \leq \mu_{k} \in \Delta_{+}$satisfy $\sum_{i} \mu_{i}=\delta+\gamma$, then either $\mu_{1} \leq \gamma$ or else $\mu_{k} \geq \delta$.

Proof. Let $(\cdot, \cdot)$ be the symmetric positive definite form on $\mathbb{R}^{\theta}$ such that $(\nu, \nu)=2$ for all $\nu \in \Delta$. Then $-1 \leq\left(\mu, \mu^{\prime}\right) \leq 1$ if $\nu \neq \nu^{\prime} \in \Delta$. As $\beta+\delta \in \Delta,(\beta, \delta)=-1$; thus $(\beta, \gamma)=1$.

Next we prove (a) the proof of (b) is analogous. Let $\mu_{1} \leq \cdots \leq \mu_{k}$ be such that $\sum_{i} \mu_{i}=\beta+\gamma$. Note that $k \geq 2$, since $\beta+\gamma=2 \beta+\delta \notin \Delta$. Suppose on the contrary that $\beta<\mu_{1} \leq \cdots \leq \mu_{k}<\gamma$. Then $k \geq 3$, since $\left(\mu_{i}, \beta\right) \leq 1$ and

$$
3=(\beta+\gamma, \beta)=\sum_{i}\left(\mu_{i}, \beta\right) .
$$

Assume that $k \geq 4$. Then there exist $j \neq \ell$ such that $\left(\mu_{j}, \mu_{\ell}\right)=-1$ since

$$
6=(\beta+\gamma, \beta+\gamma)=\sum_{i}\left(\mu_{i}, \mu_{i}\right)+\sum_{i \neq j}\left(\mu_{i}, \mu_{j}\right) \leq 8+\sum_{i \neq j}\left(\mu_{i}, \mu_{j}\right) .
$$

Thus $\mu_{j}+\mu_{\ell} \in \Delta_{+}, \mu_{j}<\mu_{j}+\mu_{\ell}<\mu_{\ell}$ and we can replace the set $\left\{\mu_{i}\right\}_{i \in \mathbb{I}_{k}}$ by

$$
\left(\left\{\mu_{i}\right\}_{i \in \mathbb{I}_{k}}-\left\{\mu_{j}, \mu_{\ell}\right\}\right) \cup\left\{\mu_{j}+\mu_{\ell}\right\} .
$$

Hence, recursively, we may assume that $k=3$. But using the computer we check that $\nu_{1}+\nu_{2}+\nu_{3} \neq \beta+\gamma$ for all the 3 -uples $\beta<\nu_{1} \leq \nu_{2} \leq \nu_{3}<\gamma$ so we get a contradiction.

Proposition 8.1.2. For every $\gamma \in \Delta_{+}^{\mathfrak{q}},\left(\mathrm{x}_{\gamma}^{N_{\gamma}}\right)^{*}$ is a 2-cocycle.

Proof. By Lemma 6.2.7, $P_{\gamma}=2$ and $Q_{\gamma}=1$ for all non-simple roots $\gamma$. Hence, if $N>2$, then Lemma 6.2.5 applies and $\left(\mathrm{x}_{\gamma}^{N_{\gamma}}\right)^{*}$ is a 2-cocycle for all roots $\gamma$.

Next we assume $N=2$, that is, $q=-1$. We will apply Lemma 6.2.8, Let $\gamma$ be a positive non-simple root. For each pair $\beta<\delta \in \Delta_{+}^{\mathfrak{q}}$ such that $\gamma=\beta+\delta$, we have that

$$
x_{\beta} x_{\gamma}=q_{\beta \gamma} x_{\gamma} x_{\beta}, \quad x_{\gamma} x_{\delta}=q_{\gamma \delta} x_{\delta} x_{\gamma}
$$

by (4.2.2) and Lemma 8.1.1, and $q_{\beta \beta}=q_{\delta \delta}=-1$. Hence (6.2.9) holds.

Let $\gamma_{1}, \gamma_{2}, \gamma_{3} \in \Delta_{+}^{\mathfrak{q}}$ be three different roots such that $\gamma_{1}+\gamma_{2}+\gamma_{3}=2 \gamma$. By Lemma 6.2.6 there exists $w \in \mathcal{W}$ such that the support of $w\left(\gamma_{i}\right)$ is of size $\leq 3$, and a fortiori $\gamma$ too. As $w\left(\gamma_{1}\right)+w\left(\gamma_{2}\right)+w\left(\gamma_{3}\right)=2 w(\gamma)$ and these roots are contained in a subdiagram of type $A_{3}$ or $A_{2} \times A_{1}$, we conclude that $\gamma_{i}=\gamma$ for some $i \in \mathbb{I}_{3}$. Using a similar argument we also check that $2 \gamma_{1}+\gamma_{2} \neq 2 \gamma$ for all $\gamma_{1} \neq \gamma_{2} \in \Delta_{+}^{\mathfrak{q}}$. Hence all the hypothesis of Lemma 6.2.8 hold, and $\left(\mathrm{x}_{\gamma}^{2}\right)^{*}$ is a 2-cocycle.

8.2. Type $F_{4}$. Let $q$ be a root of 1 of order $N>2, M=\operatorname{ord} q^{2}$. In this section, we deal with a Nichols algebra $\mathscr{B}_{\mathfrak{q}}$ of Cartan type $F_{4}$, that is associated to the Dynkin diagram

$$
\stackrel{q}{\circ} \stackrel{q^{-1}}{\sim} \stackrel{q}{\circ} \stackrel{q^{-2}}{\longrightarrow} \stackrel{q^{2}}{\circ} \stackrel{q^{-2}}{\longrightarrow} \stackrel{q^{2}}{\circ}
$$


For more information, see [AA, §4.6]. The set of positive roots with full support is

$$
\left\{1^{2} 2^{4} 3^{3} 4,1^{2} 2^{4} 3^{2} 4,1^{2} 2^{3} 3^{2} 4,1^{2} 2^{2} 3^{2} 4,1^{2} 2^{2} 34,12^{3} 3^{2} 4,12^{2} 3^{2} 4,1^{2} 2^{4} 3^{3} 4^{2}, 12^{2} 34,1234\right\} .
$$

The aim of this Section is to prove that Condition 1.4.1 holds for type $F_{4}$. More precisely,

Proposition 8.2.1. For every $\gamma \in \Delta_{+}^{\mathfrak{q}},\left(\mathrm{x}_{\gamma}^{N_{\gamma}}\right)^{*}$ is a 2-cocycle.

Proof. By induction on the rank it is enough to consider $\gamma$ with full support. We have:

$$
\begin{aligned}
& \text { if } \gamma \in\left\{1^{2} 2^{3} 3^{2} 4,12^{3} 3^{2} 4,12^{2} 3^{2} 4,12^{2} 34,1234\right\}, \text { then } \quad N_{\gamma}=N, P_{\gamma}=3, Q_{\gamma}=2 ; \\
& \text { if } \gamma \in\left\{1^{2} 2^{4} 3^{3} 4,1^{2} 2^{4} 3^{2} 4,1^{2} 2^{2} 3^{2} 4,1^{2} 2^{2} 34,1^{2} 2^{4} 3^{3} 4^{2}\right\}, \text { then } \quad N_{\gamma}=M, P_{\gamma}=2, Q_{\gamma}=2 .
\end{aligned}
$$

Hence, if $N>4$, then $N_{\gamma}>P_{\gamma}, Q_{\gamma}$ for all $\gamma$ with full support. Thus $\left(\mathrm{x}_{\gamma}^{N_{\gamma}}\right)^{*}$ is a 2-cocycle for all $\gamma$ with full support by Lemma 6.2.5.

Next we assume $N=4$. If $\gamma \in\left\{1^{2} 2^{3} 3^{2} 4,12^{3} 3^{2} 4,12^{2} 3^{2} 4,12^{2} 34,1234\right\}$, then $N_{\gamma}=4>$ $P_{\gamma}, Q_{\gamma}$, so $\left(\mathrm{x}_{\gamma}^{N_{\gamma}}\right)^{*}$ is a 2 -cocycle by Lemma 6.2.5.

Now we consider $\gamma \in\left\{1^{2} 2^{4} 3^{3} 4,1^{2} 2^{4} 3^{2} 4,1^{2} 2^{2} 3^{2} 4,1^{2} 2^{2} 34,1^{2} 2^{4} 3^{3} 4^{2}\right\}$. Let $\alpha<\beta$ be a pair of positive roots as in (6.3.3). We have the following possibilities:

○ $\gamma=1^{2} 2^{4} 3^{3} 4, \alpha=3, \beta=1^{2} 2^{4} 3^{2} 4$. There exists $\mathrm{b} \in \mathbb{k}$ such that $\left[x_{\alpha}, x_{\beta}\right]_{c}=\mathrm{b} x_{\gamma}$.

$\circ \gamma=1^{2} 2^{4} 3^{3} 4, \alpha=23, \beta=1^{2} 2^{3} 3^{2} 4$. There exist $\mathrm{b}, \mathrm{b}_{1} \in \mathbb{k}$ such that

$$
\left[x_{\alpha}, x_{\beta}\right]_{c}=\mathrm{b} x_{\gamma}+\mathrm{b}_{1} x_{12} 2^{4} 3^{2} x_{3} .
$$

$\circ \gamma=1^{2} 2^{4} 3^{3} 4, \alpha=2^{2} 3, \beta=1^{2} 2^{2} 3^{2} 4$. There exist $\mathrm{b}, \mathrm{b}_{t} \in \mathbb{k}$ such that

$$
\left[x_{\alpha}, x_{\beta}\right]_{c}=\mathrm{b} x_{\gamma}+\mathrm{b}_{1} x_{1^{2} 2^{4} 3^{2} 4} x_{3}+\mathrm{b}_{2} x_{1^{2} 2^{3} 3^{2} 4} x_{23} .
$$

$\circ \gamma=1^{2} 2^{4} 3^{3} 4, \alpha=123, \beta=12^{3} 3^{2} 4$. There exist $\mathrm{b}, \mathrm{b}_{t} \in \mathbb{k}$ such that

$$
\left[x_{\alpha}, x_{\beta}\right]_{c}=\mathrm{b} x_{\gamma}+\mathrm{b}_{1} x_{1^{2} 2^{4} 3^{2}{ }^{2}} x_{3}+\mathrm{b}_{2} x_{1^{2} 2^{3} 3^{2}{ }^{4}} x_{23}+\mathrm{b}_{3} x_{1^{2} 2^{2} 3^{2}{ }^{2}} x_{2^{2} 3} .
$$

$\circ \gamma=122^{4} 3^{3} 4, \alpha=12^{2} 3, \beta=12^{2} 3^{2} 4$. There exist $\mathrm{b}, \mathrm{b}_{t} \in \mathbb{k}$ such that

$$
\left[x_{\alpha}, x_{\beta}\right]_{c}=\mathrm{b} x_{\gamma}+\mathrm{b}_{1} x_{12^{2} 2^{2}{ }^{2}} x_{3}+\mathrm{b}_{2} x_{12^{2} 2^{3} 3^{2} 4} x_{23}+\mathrm{b}_{3} x_{1^{2} 2^{2} 3^{2} 4^{4}} x_{2^{2} 3}+\mathrm{b}_{4} x_{12^{3} 3^{2} x_{123}} .
$$

$\circ \gamma=1^{2} 2^{4} 3^{3} 4, \alpha=1^{2} 2^{2} 3, \beta=2^{2} 3^{2} 4$. There exist $\mathrm{b}, \mathrm{b}_{t} \in \mathbb{k}$ such that

$$
\begin{aligned}
{\left[x_{\alpha}, x_{\beta}\right]_{c}=} & \mathrm{b} x_{\gamma}+\mathrm{b}_{1} x_{12} 2^{4} 3^{2} x_{3}+\mathrm{b}_{2} x_{1^{2} 2^{3} 3^{2} 4_{23}}+\mathrm{b}_{3} x_{122^{2} 3^{2} 4 x_{2^{2} 3}} \\
& +\mathrm{b}_{4} x_{12^{3} 3^{2}{ }^{2}} x_{123}+\mathrm{b}_{5} x_{12^{2} 3^{2}{ }^{2}} x_{123} .
\end{aligned}
$$

$\circ \gamma=1^{2} 2^{4} 3^{2} 4, \alpha=2^{2} 3, \beta=1^{2} 2^{2} 34$. There exist $\mathrm{b} \in \mathbb{k}$ such that $\left[x_{\alpha}, x_{\beta}\right]_{c}=\mathrm{b} x_{\gamma}$.

$\circ \gamma=1^{2} 2^{4} 3^{2} 4, \alpha=12^{2} 3, \beta=12^{2} 34$. There exist $\mathrm{b}, \mathrm{b}_{1} \in \mathbb{k}$ such that

$$
\left[x_{\alpha}, x_{\beta}\right]_{c}=\mathrm{b} x_{\gamma}+\mathrm{b}_{1} x_{1^{2} 2^{2} 34} x_{2^{2} 3} .
$$

$\circ \gamma=1^{2} 2^{4} 3^{2} 4, \alpha=1^{2} 2^{2} 3, \beta=2^{2} 34$. There exist $\mathrm{b}, \mathrm{b}_{t} \in \mathbb{k}$ such that

$$
\left[x_{\alpha}, x_{\beta}\right]_{c}=\mathrm{b} x_{\gamma}+\mathrm{b}_{1} x_{1^{2} 2^{2} 34} x_{2^{2} 3}+\mathrm{b}_{2} x_{12^{2} 34} x_{12^{2} 3} \text {. }
$$

$\circ \gamma=1^{2} 2^{4} 3^{2} 4, \alpha=2, \beta=1^{2} 2^{3} 3^{2} 4$. There exists $\mathrm{b} \in \mathbb{k}$ such that $\left[x_{\alpha}, x_{\beta}\right]_{c}=\mathrm{b} x_{\gamma}$. 
$\circ \gamma=1^{2} 2^{4} 3^{2} 4, \alpha=12, \beta=12^{3} 3^{2} 4$. There exist $\mathrm{b}, \mathrm{b}_{t} \in \mathbb{k}$ such that

$$
\left[x_{\alpha}, x_{\beta}\right]_{c}=\mathrm{b} x_{\gamma}+\mathrm{b}_{1} x_{1^{2} 2^{2} 34} x_{2^{2} 3}+\mathrm{b}_{2} x_{1^{2} 2^{3} 3^{2} 4} x_{2} .
$$

$\circ \gamma=1^{2} 2^{2} 3^{2} 4, \alpha=3, \beta=1^{2} 2^{2} 34$. There exists $\mathrm{b} \in \mathbb{k}$ such that $\left[x_{\alpha}, x_{\beta}\right]_{c}=\mathrm{b} x_{\gamma}$.

$\circ \gamma=1^{2} 2^{2} 3^{2} 4, \alpha=123, \beta=1234$. There exist $\mathrm{b}, \mathrm{b}_{1} \in \mathbb{k}$ such that

$$
\left[x_{\alpha}, x_{\beta}\right]_{c}=\mathrm{b} x_{\gamma}+\mathrm{b}_{1} x_{1^{2} 2^{2} 34} x_{3} .
$$

○ $\gamma=1^{2} 2^{2} 3^{2} 4, \alpha=1^{2} 2^{2} 3, \beta=34$. There exist $\mathrm{b}, \mathrm{b}_{t} \in \mathbb{k}$ such that

$$
\left[x_{\alpha}, x_{\beta}\right]_{c}=\mathrm{b} x_{\gamma}+\mathrm{b}_{1} x_{1^{2} 2^{2} 34} x_{3}+\mathrm{b}_{2} x_{1234} x_{123} .
$$

○ $\gamma=1^{2} 2^{2} 3^{2} 4, \alpha=1, \beta=12^{2} 3^{2} 4$. There exist $\mathrm{b}, \mathrm{b}_{1} \in \mathbb{k}$ such that

$$
\left[x_{\alpha}, x_{\beta}\right]_{c}=\mathrm{b} x_{\gamma}+\mathrm{b}_{1} x_{1^{2} 2^{2} 34} x_{3} .
$$

$\circ \gamma=1^{2} 2^{2} 34,(\alpha, \beta)$ one of the pairs $(12,1234),\left(1^{2} 2^{2} 3,4\right),\left(1,12^{2} 34\right)$. There exists $\mathrm{b} \in \mathbb{k}$ such that $\left[x_{\alpha}, x_{\beta}\right]_{c}=\mathrm{b} x_{\gamma}$.

$\circ \gamma=1^{2} 2^{4} 3^{3} 4^{2}, \alpha=12^{2} 3^{2} 4, \beta=12^{2} 34$. There exists $\mathrm{b} \in \mathbb{k}$ such that $\left[x_{\alpha}, x_{\beta}\right]_{c}=\mathrm{b} x_{\gamma}$.

○ $\gamma=1^{2} 2^{4} 3^{3} 4^{2}, \alpha=12^{3} 3^{2} 4, \beta=1234$. There exist $\mathrm{b}, \mathrm{b}_{1} \in \mathbb{k}$ such that

$$
\left[x_{\alpha}, x_{\beta}\right]_{c}=\mathrm{b} x_{\gamma}+\mathrm{b}_{1} x_{12^{2} 34} x_{12^{2} 3^{2} 4} .
$$

○ $\gamma=1^{2} 2^{4} 3^{3} 4^{2}, \alpha=1^{2} 2^{2} 34, \beta=2^{2} 3^{2} 4$. There exist $\mathrm{b}, \mathrm{b}_{t} \in \mathbb{k}$ such that

$$
\left[x_{\alpha}, x_{\beta}\right]_{c}=\mathrm{b} x_{\gamma}+\mathrm{b}_{1} x_{12^{2} 34} x_{12^{2} 3^{2} 4}+\mathrm{b}_{2} x_{1234} x_{12^{3} 3^{2} 4} .
$$

$\circ \gamma=1^{2} 2^{4} 3^{3} 4^{2}, \alpha=1^{2} 2^{2} 3^{2} 4, \beta=2^{2} 34$. There exist $\mathrm{b}, \mathrm{b}_{t} \in \mathbb{k}$ such that

$$
\left[x_{\alpha}, x_{\beta}\right]_{c}=\mathrm{b} x_{\gamma}+\mathrm{b}_{1} x_{12^{2} 34} x_{12^{2} 3^{2} 4}+\mathrm{b}_{2} x_{1234} x_{12^{3} 3^{2} 4}+\mathrm{b}_{3} x_{2^{2} 3^{2} x_{1} x_{12} 2^{2} 34} .
$$

○ $\gamma=1^{2} 2^{4} 3^{3} 4^{2}, \alpha=1^{2} 2^{3} 3^{2} 4, \beta=234$. There exist $\mathrm{b}, \mathrm{b}_{t} \in \mathbb{k}$ such that

$$
\left[x_{\alpha}, x_{\beta}\right]_{c}=\mathrm{b} x_{\gamma}+\mathrm{b}_{1} x_{12^{2} 34} x_{12^{2} 3^{2} 4}+\mathrm{b}_{2} x_{1234} x_{12^{3} 3^{2} 4}+\mathrm{b}_{3} x_{2^{2} 3^{2} 4} x_{1^{2} 2^{2} 34}+\mathrm{b}_{4} x_{234} x_{1^{2} 2^{3} 3^{2} 4} \text {. }
$$

○ $\gamma=1^{2} 2^{4} 3^{3} 4^{2}, \alpha=1^{2} 2^{4} 3^{2} 4, \beta=34$. There exist $\mathrm{b}, \mathrm{b}_{t} \in \mathbb{k}$ such that

$$
\begin{aligned}
{\left[x_{\alpha}, x_{\beta}\right]_{c}=} & \mathrm{b} x_{\gamma}+\mathrm{b}_{1} x_{12^{2} 34} x_{12^{2} 3^{2} 4}+\mathrm{b}_{2} x_{1234} x_{12^{3} 3^{2} 4}+\mathrm{b}_{3} x_{2^{2} 3^{2} 4_{4}} x_{1^{2} 2^{2} 34} \\
& +\mathrm{b}_{4} x_{234} x_{1^{2} 2^{3} 3^{2} 4}+\mathrm{b}_{5} x_{234} x_{122^{3} 3^{2} 4} .
\end{aligned}
$$

$\circ \gamma=1^{2} 2^{4} 3^{3} 4^{2}, \alpha=1^{2} 2^{4} 3^{3} 4, \beta=4$. There exist $\mathrm{b}, \mathrm{b}_{t} \in \mathbb{k}$ such that

$$
\begin{aligned}
{\left[x_{\alpha}, x_{\beta}\right]_{c}=} & \mathrm{b} x_{\gamma}+\mathrm{b}_{1} x_{12^{2} 34} x_{12^{2} 3^{2} 4}+\mathrm{b}_{2} x_{1234} x_{12^{3} 3^{2} 4}+\mathrm{b}_{3} x_{2^{2} 3^{2} 4_{4}} x_{1^{2} 2^{2} 34} \\
& +\mathrm{b}_{4} x_{234} x_{1^{2} 2^{3} 3^{2} 4}+\mathrm{b}_{5} x_{234} x_{12^{2} 2^{3} 3^{2}}+\mathrm{b}_{6} x_{34} x_{1^{2} 2^{4} 3^{2} 4} .
\end{aligned}
$$

In all cases $\left[x_{\alpha}, x_{\gamma}\right]_{c}=0=\left[x_{\gamma}, x_{\beta}\right]_{c}$ : the proof of all these relations follow as in Lemma 7.2.3. Hence the root vectors satisfy (10.1.8), and $-\frac{q_{\alpha \alpha}}{q_{\beta \beta}}=-1$; then we take $L=2$.

Next we look for solutions of (6.3.1). That is, $\sum_{\delta \in \Delta_{+}^{\mathfrak{q}}} f_{\delta}\left(n_{\delta}\right) \delta=2 \gamma, \sum_{\delta \in \Delta_{+}^{\mathfrak{q}}} n_{\delta}=3$. Notice that $n_{\delta} \neq 3$ for all $\delta \in \Delta_{+}^{q}$ : otherwise $n_{\eta}=0$ for all $\eta \neq \delta$, so $2 \gamma=f_{\delta}(3) \delta$, a contradiction. Hence, either $2 \gamma=f_{\gamma_{1}}(2) \gamma_{1}+\gamma_{2}$ or else $2 \gamma=\gamma_{1}+\gamma_{2}+\gamma_{3}$ for some $\gamma_{i} \neq \gamma_{j} \in \Delta_{+}^{q}$. By Lemma 6.2.6 there exists $w \in W$ such that $\gamma_{i}^{\prime}=w\left(\gamma_{i}\right)$ have support in a rank 3 subdiagram, so $\gamma^{\prime}=w(\gamma)$ has the same support: this subdiagram is either of Cartan type $B_{3}$ or $C_{3}$. Looking at the corresponding cases (see the proofs of Propositions 7.2.4 and 7.4.3) the solutions for $\gamma^{\prime}$ are $\gamma_{3}^{\prime}=\gamma^{\prime}, \gamma_{1}^{\prime}+\gamma_{2}^{\prime}=\gamma^{\prime}$. Hence, all solutions for $\gamma$ are 
of form $n_{\gamma}=n_{\alpha}=n_{\beta}=1$ for a pair $(\alpha, \beta)$ satisfying (6.3.3), and $n_{\varphi}=0$ for the remaining $\varphi \in \Delta_{+}$. Hence all the hypotheses of Proposition 6.3.2 hold, and $\left(\mathrm{x}_{\gamma}^{L_{\gamma}}\right)^{*}$ is a 2-cocycle.

Finally we consider $N=3$. If $\gamma \in\left\{1^{2} 2^{4} 3^{3} 4,1^{2} 2^{4} 3^{2} 4,1^{2} 2^{2} 3^{2} 4,1^{2} 2^{2} 34,1^{2} 2^{4} 3^{3} 4^{2}\right\}$, then $N_{\gamma}=3>P_{\gamma}, Q_{\gamma}$, so $\left(\mathrm{x}_{\gamma}^{N_{\gamma}}\right)^{*}$ is a 2 -cocycle by Lemma 6.2.5.

Now we consider $\gamma \in\left\{1^{2} 2^{3} 3^{2} 4,12^{3} 3^{2} 4,12^{2} 3^{2} 4,12^{2} 34,1234\right\}$. Let $\alpha<\beta$ be a pair of positive roots as in (6.3.24). We have the following possibilities:

$\circ \gamma=1^{2} 2^{3} 3^{2} 4, \alpha=1^{2} 2^{4} 3^{2} 4, \beta=1^{2} 2^{2} 3^{2} 4$. There exist $\mathrm{b} \in \mathbb{k}$ such that $\left[x_{\alpha}, x_{\beta}\right]_{c}=\mathrm{b} x_{\gamma}^{2}$.

$\circ \gamma=1^{2} 2^{3} 3^{2} 4, \alpha=1^{2} 2^{4} 3^{3} 4, \beta=1^{2} 2^{2} 34$. There exist $\mathrm{b}, \mathrm{b}^{\prime} \in \mathbb{k}$ such that

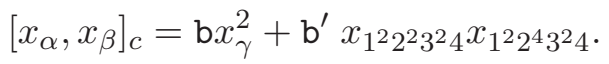

○ $\gamma=12^{3} 3^{2} 4, \alpha=1^{2} 2^{4} 3^{2} 4, \beta=2^{2} 3^{2} 4$. There exist $\mathrm{b} \in \mathbb{k}$ such that $\left[x_{\alpha}, x_{\beta}\right]_{c}=\mathrm{b} x_{\gamma}^{2}$.

○ $\gamma=12^{3} 3^{2} 4, \alpha=1^{2} 2^{4} 3^{3} 4, \beta=2^{2} 34$. There exist $\mathrm{b}, \mathrm{b}^{\prime} \in \mathbb{k}$ such that

$$
\left[x_{\alpha}, x_{\beta}\right]_{c}=\mathrm{b} x_{\gamma}^{2}+\mathrm{b}^{\prime} x_{2^{2} 3^{2} 4^{2}} x_{1^{2} 2^{4} 3^{2} 4} .
$$

○ $\gamma=12^{2} 3^{2} 4, \alpha=1^{2} 2^{2} 3^{2} 4, \beta=2^{2} 3^{2} 4$. There exist $\mathrm{b} \in \mathbb{k}$ such that $\left[x_{\alpha}, x_{\beta}\right]_{c}=\mathrm{b} x_{\gamma}^{2}$.

$\circ \gamma=12^{2} 3^{2} 4, \alpha=1^{2} 2^{4} 3^{3} 4, \beta=34$. There exist $\mathrm{b}, \mathrm{b}^{\prime} \in \mathbb{k}$ such that

$$
\left[x_{\alpha}, x_{\beta}\right]_{c}=\mathrm{b} x_{\gamma}^{2}+\mathrm{b}^{\prime} x_{2^{2} 3^{2} 4} x_{1^{2} 2^{2} 3^{2} 4} .
$$

$\circ \gamma=12^{2} 34, \alpha=1^{2} 2^{2} 34, \beta=2^{2} 34$. There exist $\mathrm{b} \in \mathbb{k}$ such that $\left[x_{\alpha}, x_{\beta}\right]_{c}=\mathrm{b} x_{\gamma}^{2}$.

$\circ \gamma=12^{2} 34, \alpha=1^{2} 2^{4} 3^{2} 4, \beta=4$. There exist $\mathrm{b}, \mathrm{b}^{\prime} \in \mathbb{k}$ such that

$$
\left[x_{\alpha}, x_{\beta}\right]_{c}=\mathrm{b} x_{\gamma}^{2}+\mathrm{b}^{\prime} x_{2^{2} 34} x_{1^{2} 2^{2} 34} .
$$

$\circ \gamma=1234, \alpha=1^{2} 2^{2} 34, \beta=34$. There exist $\mathrm{b} \in \mathbb{k}$ such that $\left[x_{\alpha}, x_{\beta}\right]_{c}=\mathrm{b} x_{\gamma}^{2}$.

$\circ \gamma=1234, \alpha=1^{2} 2^{2} 3^{2} 4, \beta=4$. There exist $\mathrm{b}, \mathrm{b}^{\prime} \in \mathbb{k}$ such that

$$
\left[x_{\alpha}, x_{\beta}\right]_{c}=\mathrm{b} x_{\gamma}^{2}+\mathrm{b}^{\prime} x_{34} x_{1^{2} 2^{2} 34} .
$$

In all cases $\left[x_{\alpha}, x_{\gamma}\right]_{c}=0=\left[x_{\gamma}, x_{\beta}\right]_{c}$, and $\frac{q_{\alpha \gamma}}{q_{\gamma \beta}}=1$ : the proof of all these relations follow as in Lemma 7.2.3. Therefore the root vectors satisfy (10.1.8), so we take $L=1$.

Next we look for solutions of (6.3.1). That is, $\sum_{\delta \in \Delta_{+}^{q}} f_{\delta}\left(n_{\delta}\right) \delta=3 \gamma, \sum_{\delta \in \Delta_{+}^{\mathfrak{q}}} n_{\delta}=3$. Notice that $n_{\delta} \neq 3$ for all $\delta \in \Delta_{+}^{\mathfrak{q}}$ : otherwise $n_{\eta}=0$ for all $\eta \neq \delta$, so $3 \gamma=f_{\delta}(3) \delta$, a contradiction. Hence, either $3 \gamma=f_{\gamma_{1}}(2) \gamma_{1}+\gamma_{2}$ or else $3 \gamma=\gamma_{1}+\gamma_{2}+\gamma_{3}$ for some $\gamma_{i} \neq \gamma_{j} \in \Delta_{+}^{\mathfrak{q}}$. Again we use Lemma 6.2.6 to reduce to rank 3 subdiagrams and looking at the proofs of Propositions 7.2.4 and 7.4.3 we conclude that all the solutions for $\gamma$ are of form $n_{\gamma}=n_{\alpha}=n_{\beta}=1$ for a pair $(\alpha, \beta)$ satisfying (6.3.24), and $n_{\varphi}=0$ for the remaining $\varphi \in \Delta_{+}$. Hence all the hypotheses of Proposition 6.3.23 hold, and $\left(\mathrm{x}_{\gamma}^{N_{\gamma}}\right)^{*}$ is a 2-cocycle. 
8.3. Type $G_{2}$ Cartan. Let $q$ be a root of 1 of order $N>3$. Set

$$
M= \begin{cases}N, & 3 \text { does not divide } N \\ N / 3, & 3 \text { divides } N .\end{cases}
$$

In this section, we deal with a Nichols algebra $\mathscr{B}_{\mathfrak{q}}$ of Cartan type $G_{2}$, with Dynkin diagram

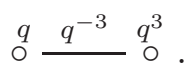

The set of positive roots is

$$
\Delta_{+}=\left\{1,1^{3} 2,1^{2} 2,1^{3} 2^{2}, 12,2\right\} .
$$

We take as generators $x_{1}, x_{2}$, as well as

$$
\begin{aligned}
x_{1112} & :=\left(\operatorname{ad}_{c} x_{1}\right)^{3} x_{2}, \quad x_{112}:=\left(\operatorname{ad}_{c} x_{1}\right)^{2} x_{2}, \\
x_{11212} & :=\left[x_{112}, x_{12}\right]_{c}, \quad x_{12}:=\left(\operatorname{ad}_{c} x_{1}\right) x_{2} .
\end{aligned}
$$

We order these root vectors: $x_{1}<x_{1112}<x_{112}<x_{11212}<x_{12}<x_{2}$.

The aim of this Section is to prove that Condition 1.4.1 holds for type $G_{2}$. More precisely,

Proposition 8.3.3. For every $\gamma \in \Delta_{+}^{\mathfrak{q}},\left(\mathrm{x}_{\gamma}^{N_{\gamma}}\right)^{*}$ is a 2-cocycle.

Proof. $\circ$ For $\gamma=1^{3} 2$, the case $N_{1112}>2$ follows by Lemma 6.2.5 again. Assume now that $N_{1112}=2$ (that is, $N=6$ ). We will apply Proposition 6.3.2. The unique pair as in (6.3.3) is $\alpha=\alpha_{1}, \beta=2 \alpha_{1}+\alpha_{2}$, since the following relations hold:

$$
x_{1} x_{112}=x_{1112}+q^{2} q_{12} x_{112} x_{1}, \quad x_{1} x_{1112}=q^{3} q_{12} x_{1112} x_{1}, \quad x_{1112} x_{112}=q^{3} q_{12} x_{112} x_{1112} .
$$

In this case, $-\frac{q_{\alpha \alpha}}{q_{\beta \beta}}=-1$ so we take $L=2$. The unique solution of (6.3.1) is $n_{1}=n_{1^{3} 2}=$ $n_{1^{2} 2}=1$, and $n_{\delta}=0$ for the remaining roots. Hence Proposition 6.3.2 applies and $\left(\mathrm{x}_{1112}^{2}\right)^{*}$ is a 2-cocycle.

○ For $\gamma=1^{2} 2$, the case $N_{112}>4$ follows by Lemma 6.2.5. Assume now that $N_{112}=4$. We will apply Proposition 6.3.23. The unique pair as in (6.3.24) is $\alpha=3 \alpha_{1}+\alpha_{2}, \beta=3 \alpha_{1}+2 \alpha_{2}$, since the following relations hold:

$$
\begin{aligned}
x_{1112} x_{11212}=-(q+1) q_{12}^{2} x_{112}^{3}-q_{12}^{3} x_{11212} x_{1112}, & x_{1112} x_{112} \\
= & q^{3} q_{12} x_{112} x_{1112}, \\
x_{112} x_{11212} & =q^{3} q_{12} x_{11212} x_{112} .
\end{aligned}
$$

Here $\frac{q_{\alpha \gamma}}{q_{\gamma \beta}}=1$, so we take $L=1$. The unique solution of (6.3.26) is $n_{1^{3} 2}=n_{1^{2} 2}=n_{1^{3} 2^{2}}=1$, and $n_{\delta}=0$ for the remaining roots. Hence Proposition 6.3.23 applies: $\left(\mathrm{x}_{112}^{4}\right)^{*}$ is a 2-cocycle. - For $\gamma=1^{3} 2^{2}$, the case $N_{11212}>2$ follows by Lemma 6.2.5. Assume now that $N_{11212}=2$ (that is, $N=6$ ). We will apply Proposition 6.3.2. The pairs as in (6.3.3) are $\alpha=2 \alpha_{1}+\alpha_{2}$, $\beta=\alpha_{1}+\alpha_{2}$, and $\alpha=3 \alpha_{1}+\alpha_{2}, \beta=\alpha_{2}$, since the following relations hold:

$$
\begin{aligned}
& x_{112} x_{12}=x_{11212}+q^{2} q_{12} x_{12} x_{112}, \quad x_{112} x_{11212}=q_{12}^{2} x_{11212} x_{112} \text {, } \\
& x_{11212} x_{12}=q_{12}^{2} x_{12} x_{11212} ; \\
& x_{1112} x_{2}=-(3)_{q} q_{12} x_{11212}-q_{12}^{3} x_{2} x_{1112}-2 q^{2} q_{12}^{2} x_{12} x_{112}, \quad x_{1112} x_{11212}=q_{12}^{3} x_{11212} x_{1112} \text {, }
\end{aligned}
$$




$$
x_{11212} x_{2}=q_{12}^{3} x_{2} x_{11212} .
$$

In both cases, $-\frac{q_{\alpha \alpha}}{q_{\beta \beta}}=-1$ so we take $L=2$. The solutions of (6.3.1) are

- $n_{112}=n_{1^{3} 2^{2}}=n_{12}=1$, and $n_{\delta}=0$ for the remaining roots, or

- $n_{1^{3} 2}=n_{1^{3} 2^{2}}=n_{2}=1$, and $n_{\delta}=0$ for the remaining roots.

Hence Proposition 6.3.2 applies and $\left(\mathrm{x}_{11212}^{2}\right)^{*}$ is a 2-cocycle.

- For $\gamma=12$, the case $N_{12}>4$ follows by Lemma 6.2.5. Assume now that $N_{12}=4$. We will apply Proposition 6.3.23. The unique pair as in 6.3.24) is $\alpha=3 \alpha_{1}+2 \alpha_{2}, \beta=\alpha_{2}$, since the following relations hold:

$$
\begin{aligned}
& x_{11212} x_{2}=-2(1+q) q_{12}^{2} x_{12}^{3}+q q_{12}^{4} x_{2} x_{11212}, \quad x_{11212} x_{12} \\
&=q^{3} q_{12} x_{12} x_{11212}, \\
& x_{12} x_{2}=q^{3} q_{12} x_{2} x_{12} .
\end{aligned}
$$

Here $\frac{q_{\alpha \gamma}}{q_{\gamma \beta}}=1$, so we take $L=1$. The unique solution of (6.3.26) is $n_{1^{3} 2^{2}}=n_{12}=n_{2}=1$,

\begin{tabular}{|c|c|c|c|c|c|c|c|c|}
\hline$\gamma$ & $N_{\gamma}$, Cartan & $N_{\gamma}, 8.4 .1 \mathrm{~b}$ & $P_{\gamma}$ & $Q_{\gamma}$ & $\gamma_{1}$ & $\gamma_{2}$ & $L_{\gamma}$, Cartan & $L_{\gamma}$,8.4.1) b \\
\hline $1^{3} 2$ & $M$ & 8 & 2 & 2 & 1 & $1^{2} 2$ & $M$ & 8 \\
\hline $1^{2} 2$ & $N$ & 4 & 4 & 3 & 1 & 12 & $N$ & 8 \\
\hline $1^{3} 2^{2}$ & $M$ & 2 & 2 & 1 & $\begin{array}{l}1^{2} 2 \\
1^{3} 2\end{array}$ & & $M$ & 8 \\
\hline 12 & $N$ & 8 & 4 & 3 & 1 & 2 & $N$ & 8 \\
\hline
\end{tabular}
$n_{\delta}=0$ for the remaining roots. Hence Proposition 6.3.23 applies: $\left(\mathrm{x}_{12}^{4}\right)^{*}$ is a 2-cocycle.

TABLE 1. The roots with full support of $G_{2} ; \gamma_{1}<\gamma=\gamma_{1}+\gamma_{2}<\gamma_{2}$

8.4. Type $G_{2}$ standard. Let $\zeta \in \mathbb{G}_{8}^{\prime}$. In this section, we deal with a Nichols algebra $\mathscr{B}_{\mathfrak{q}}$ of standard type $G_{2}$ associated to any of the Dynkin diagrams
a: $\quad \zeta^{2}$
$\mathrm{b}: \quad \zeta^{2} \stackrel{\zeta^{3}}{-1} \stackrel{-1}{\circ}$
c: $\zeta^{\zeta} \stackrel{\zeta^{5}}{-1}{ }^{-1}$.

The set of positive roots is again (8.3.1). Thus we take as generators $x_{1}, x_{2}$, as well as (8.3.2) with the same order for these root vectors: $x_{1}<x_{1112}<x_{112}<x_{11212}<x_{12}<x_{2}$. For more information, see [AA, §6.2]. We prove Condition 1.4.1 for type $G_{2}$ standard:

Proposition 8.4.2. For every $\gamma \in \Delta_{+}^{\mathfrak{q}}$, there exists $L_{\gamma} \in \mathbb{N}$ such that $\left(\mathrm{x}_{\gamma}^{L_{\gamma}}\right)^{*}$ is a cocycle.

Proof. We just consider the diagram (8.4.1) b.

○ For $\gamma=1^{3} 2,\left(\mathrm{x}_{1112}^{8}\right)^{*}$ is a 2-cocycle by Lemma 6.2.5. 
○ For $\gamma=1^{2} 2$, we will use Proposition 6.3.23. The unique pair as in (6.3.24) is $\alpha=3 \alpha_{1}+\alpha_{2}$, $\beta=3 \alpha_{1}+2 \alpha_{2}$, since the following relations hold:

$$
\begin{aligned}
x_{1112} x_{11212}=q_{12}^{2} x_{112}^{3}-q_{12}^{3} x_{11212} x_{1112}, \quad & x_{1112} x_{112} \\
x_{112} x_{11212} & =-\zeta_{12} x_{112} x_{12} x_{11212} x_{112}
\end{aligned}
$$

In this case, $\left(\frac{q_{\alpha \gamma}}{q_{\gamma \beta}}\right)^{N_{\gamma}}=-1$ so we take $L=2$. The unique solution of (6.3.26) is $n_{1^{2} 2}=3$, $n_{1^{3} 2}=n_{1^{3} 2^{2}}=1, n_{\delta}=0$ for the remaining roots. Hence Proposition 6.3.23 applies and $\left(\mathrm{x}_{112}^{8}\right)^{*}$ is a 4-cocycle.

○ For $\gamma=1^{3} 2^{2}$, we will use Proposition 6.3.2. The pairs as in 6.3.3) are $\alpha=2 \alpha_{1}+\alpha_{2}$, $\beta=\alpha_{1}+\alpha_{2}$, and $\alpha=3 \alpha_{1}+\alpha_{2}, \beta=\alpha_{2}$, since the following relations hold:

$$
\begin{aligned}
x_{112} x_{12} & =x_{11212}+\zeta^{3} q_{12} x_{12} x_{112}, \\
x_{112} x_{11212} & =\zeta^{7} q_{12} x_{11212} x_{112}, \\
x_{11212} x_{12} & =q_{12} x_{12} x_{11212} \\
x_{1112} x_{2} & =\zeta^{3}(4)_{\zeta^{5}} q_{12} x_{11212}-q_{12}^{3} x_{2} x_{1112}-\zeta^{2}(2)_{\zeta^{3}} q_{12}^{2} x_{12} x_{112}, \\
x_{1112} x_{11212} & =\zeta^{3} q_{12}^{3} x_{11212} x_{1112}, \\
x_{11212} x_{2} & =q_{12}^{3} x_{2} x_{11212} .
\end{aligned}
$$

In both cases, $-\frac{q_{\alpha \alpha}}{q_{\beta \beta}}=\zeta^{7}$ so we take $L=8$. The solutions of (6.3.1) are

- $n_{1^{3} 2^{2}}=7, n_{112}=n_{12}=1$, and $n_{\delta}=0$ for the remaining roots, or

- $n_{1^{3} 2^{2}}=7, n_{1^{3} 2}=n_{2}=1$, and $n_{\delta}=0$ for the remaining roots.

Hence Proposition 6.3.2 applies and $\left(\mathrm{x}_{11212}^{8}\right)^{*}$ is an 8-cocycle.

$\circ$ For $\gamma=12,\left(\mathrm{x}_{12}^{8}\right)^{*}$ is a 2-cocycle by Lemma 6.2.5.

8.5. Type $\mathbf{D}(2,1 ; \alpha)$. Here $q, r, s \neq 1$, qr $s=1 ; N=\operatorname{ord} q, M=\operatorname{ord} r, P=\operatorname{ord} s$. In this section, we deal with a Nichols algebra $\mathscr{B}_{\mathfrak{q}}$ of super type $\mathbf{D}(2,1 ; \alpha)$ with Dynkin diagram

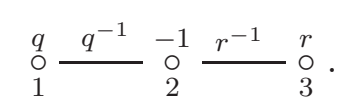

We fix the following convex order on the set of positive roots:

$$
1<12<123<12^{2} 3<2<23<3
$$

For more information, see [AA, §5.4]. We prove Condition 1.4.1 for type $\mathbf{D}(2,1 ; \alpha)$ :

Proposition 8.5.1. For every $\gamma \in \Delta_{+}^{\mathfrak{q}}$, there exists $L_{\gamma} \in \mathbb{N}$ such that $\left(\mathrm{x}_{\gamma}^{L_{\gamma}}\right)^{*}$ is a cocycle.

Proof. By induction on the rank it is enough to consider $\gamma$ with full support. We start with $\gamma=123$. The pairs $(\alpha, \beta)$ of positive roots as in (6.3.3) are $(1,23)$ and $(12,3)$. As

$$
\begin{aligned}
& x_{1} x_{23}=q_{12} q_{13} x_{23} x_{1}+x_{123}, \quad x_{1} x_{123}=q q_{12} q_{13} x_{123} x_{1}, \quad x_{123} x_{23}=-q_{12} q_{13} x_{23} x_{123} ; \\
& x_{12} x_{3}=q_{13} q_{23} x_{3} x_{12}+x_{123}, \quad x_{12} x_{123}=-q_{13} q_{23} x_{123} x_{12}, \quad x_{123} x_{3}=r q_{13} q_{23} x_{3} x_{123} .
\end{aligned}
$$

the root vectors satisfy (10.1.8). As $-\frac{q_{\alpha \alpha}}{q_{\beta \beta}}=q$, respectively $=r$, we may take $L=$ $\operatorname{lcm}\{N, M, P\}$. There exists a 4 -tuples $(\alpha, \beta, \delta, \eta) \in \Delta_{+}^{4}$ as in (6.3.5):

$$
\alpha=1<\eta=12<\gamma=123<\beta=12^{2} 3<\delta=3 .
$$


The corresponding PBW generators satisfy (10.1.11); indeed,

$$
x_{\alpha} x_{\beta}=q_{\alpha \beta} x_{\beta} x_{\alpha}+q q_{12} q_{13}(1-s) x_{\gamma} x_{\eta}, \quad x_{\eta} x_{\delta}=q_{\eta \delta} x_{\delta} x_{\eta}+x_{\gamma},
$$

and the other pairs of root vectors $q$-commute. Now $c_{\alpha \beta \gamma}^{(N)}=0$ by Lemma 6.3.28 (b) since $\widetilde{q}_{\alpha \gamma}=q, \widetilde{q}_{\beta \gamma}=s$. Next we look for solutions of (6.3.1). There exist three solutions:

○ $n_{123}=L-1, n_{1}=n_{23}=1, n_{\delta}=0$ if $\delta \neq 123,1,23$;

○ $n_{123}=L-1, n_{12}=n_{3}=1, n_{\delta}=0$ if $\delta \neq 123,12,3$;

○ $n_{123}=L-2, n_{1}=n_{12^{3}}=n_{3}=1, n_{\delta}=0$ if $\delta \neq 123,1,12^{3}, 3$;

Hence all the hypotheses of Proposition 6.3.2 hold, and $\left(\mathrm{x}_{\gamma}^{L_{\gamma}}\right)^{*}$ is a cocycle.

Now we consider $\gamma=12^{2} 3$. If $N_{\gamma}>2=P_{\gamma}$, then Lemma 6.2.5 applies and $\left(\mathrm{x}_{\gamma}^{N_{\gamma}}\right)^{*}$ is a 2-cocycle. Now assume that $N_{\gamma}=2$. The following relations between root vectors hold:

$$
\begin{aligned}
& x_{123} x_{2}=-q_{12} q_{32} x_{2} x_{123}+x_{12^{2} 3}, \\
& x_{123} x_{12^{2} 3}=q_{12} q_{32} x_{12{ }_{3} 3} x_{123}, \quad x_{12{ }^{2} 3} x_{2}=q_{12} q_{32} x_{2} x_{122} 3 \\
& x_{12} x_{23}=-q_{12} q_{13} q_{23} x_{23} x_{12}-q_{23} x_{12^{2} 3}+q_{12}(r-1) x_{2} x_{123}, \\
& x_{12} x_{12^{2} 3}=-q_{12} q_{13} q_{23} x_{12^{3}} x_{12}, \quad x_{12^{2} 3} x_{23}=-q_{12} q_{13} q_{23} x_{23} x_{123} .
\end{aligned}
$$

Hence for each pair $(\alpha, \beta)$ as in (6.3.3), the PBW generators satisfy (10.1.8). Now $L=2$ satifies the hypothesis of Proposition 6.3.2, so $\left(\mathrm{x}_{\gamma}^{2}\right)^{*}$ is a 2-cocycle.

8.6. Type $\mathbf{F}(4)$. Let $q$ be a root of 1 of order $N>3$. Set $M=\operatorname{ord} q^{2}, P=\operatorname{ord} q^{3}$.

In this subsection, we deal with the Nichols algebras $\mathscr{B}_{\mathfrak{q}}$ of diagonal type $\mathbf{F}(4)$. We may assume that the corresponding diagram is

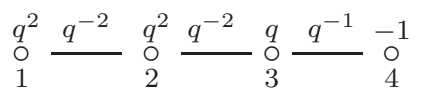

We fix the following convex order on $\Delta_{+}^{\mathfrak{q}}$ :

$$
1,12,123,123^{2}, 12^{2} 3^{2}, 2,23,23^{2}, 3,12^{2} 3^{3} 4,12^{2} 3^{2} 4,123^{2} 4,23^{2} 4,12^{2} 3^{3} 4^{2}, 1234,234,34,4 .
$$

For more information, see [AA, §5.5]. The aim of this Section is to prove:

Proposition 8.6.1. For every positive root $\gamma$, there exists a positive integer $L_{\gamma}$ such that $\left(\mathrm{x}_{\gamma}^{L_{\gamma}}\right)^{*}$ is a cocycle.

Proof. Let $\gamma$ be a positive non simple root. Arguing recursively we may assume that $\gamma$ has full support. That is, $\gamma \in\left\{12^{2} 3^{3} 4,12^{2} 3^{2} 4,123^{2} 4,12^{2} 3^{3} 4^{2}, 1234\right\}$.

If $\gamma=12^{2} 3^{2} 4$, then $N_{\gamma}=2=P_{\gamma}$. First we look for pairs $\alpha<\beta$ as in (6.3.3). We have the following posibilities:

$\circ(\alpha, \beta)=\left(2,123^{2} 4\right)$ or $(\alpha, \beta)=(23,1234)$. There exists $\mathrm{b} \in \mathbb{k}$ such that $\left[x_{\alpha}, x_{\beta}\right]_{c}=\mathrm{b} x_{\gamma}$.

$\circ(\alpha, \beta)=\left(12,23^{2} 4\right)$. There exist $\mathrm{b}, \mathrm{b}_{1} \in \mathbb{k}$ such that

$$
\left[x_{\alpha}, x_{\beta}\right]_{c}=\mathrm{b} x_{\gamma}+\mathrm{b}_{1} x_{123^{2} 4} x_{2} .
$$

○ $(\alpha, \beta)=(123,234)$ or $(\alpha, \beta)=\left(12^{2} 3^{2}, 4\right)$. There exist $\mathrm{b}, \mathrm{b}_{1} \in \mathbb{k}$ such that

$$
\left[x_{\alpha}, x_{\beta}\right]_{c}=\mathrm{b} x_{\gamma}+\mathrm{b}_{1} x_{1234} x_{23} \text {. }
$$


In all cases $\left[x_{\alpha}, x_{\gamma}\right]_{c}=0=\left[x_{\gamma}, x_{\beta}\right]_{c}$, so the root vectors satisfy (10.1.8), and $\left(-\frac{q_{\alpha \alpha}}{q_{\beta \beta}}\right)^{N}=1$ : the proof of all these relations follow as in Lemma 7.2.3. Hence we take $L=N$.

Next we check that $\left(12,2,12^{2} 3^{3} 4,123^{2} 4,23^{2} 4,12^{2} 3^{3} 4^{2}\right)$ is a 6 -uple $(\alpha, \beta, \delta, \tau, \varphi, \eta)$ satisfying (6.3.11). As $\widetilde{q}_{\alpha \gamma}=q^{2}=\widetilde{q}_{\beta \gamma}, \widetilde{q}_{\delta \gamma}=q^{-1}$, we have that $\mathrm{d}_{\alpha \beta \delta \gamma}^{(L)}=\mathrm{d}_{q^{-1}, q^{2}, q^{2}}^{(L)}=0$ by Lemma 6.3.28 (c), Hence (E) holds in this case.

Now we look for 4 -uples $(\alpha, \beta, \delta, \eta)$ satisfying (6.3.13). There are three possibilities: $\left(2,123,123^{2} 4,12^{2} 3^{3} 4^{2}\right),\left(2,12^{2} 3^{3} 4,123^{2} 4,1234\right)$ and $\left(12^{2} 3^{2}, 1234,234,23\right)$. In these cases, $\widetilde{q}_{\alpha \gamma}=q^{2}, \widetilde{q}_{\beta \gamma}=q^{-1}$. Hence $\mathrm{c}_{\alpha \beta \gamma}^{(L)}=\mathrm{c}_{q^{2}, q^{-1}}^{(L)}=0$ by Lemma 6.3.28 (b), and (F) holds.

We look for 4-uples $(\alpha, \beta, \delta, \eta)$ satisfying (6.3.15). A possibility is $\left(12,23,12^{2} 3^{3} 4^{2}, 23^{2} 4\right)$. As $\widetilde{q}_{\alpha \gamma}=q^{2}$ and $\widetilde{q}_{\delta \gamma}=q^{-1}$; thus $c_{-\delta \alpha \gamma}^{(L)}=\mathrm{c}_{q, q^{2}}^{(L)}=0$ by Lemma 6.3.28 (b) and (G) holds.

Also, $\left(12,12^{2} 3^{3} 4,234,12^{2} 3^{3} 4^{2}, 1234,2,123^{2} 4,23^{2} 4\right)$ is a 7 -uple $(\alpha, \beta, \delta, \eta, \tau, \mu, \nu)$ satisfying (6.3.17). As $\widetilde{q}_{\alpha \gamma}=q^{2}, \widetilde{q}_{\delta \gamma}=q^{-1}$, we have that $\mathrm{c}_{-\delta, \alpha, \gamma}^{(L)}=\mathrm{c}_{q, q^{2}}^{(L)}=0$ by Lemma 6.3 .28 (a). Hence $(\mathrm{H})$ holds in this case. Now $\left(2,12^{2} 3^{2}, 12^{2} 3^{3} 4,123^{2} 4,12^{2} 3^{3} 4^{2}, 1234\right)$ and $\left(12,12^{2} 3^{2}, 12^{2} 3^{3} 4,23^{2} 4,12^{2} 3^{3} 4^{2}, 234\right)$ are 6 -uples $(\alpha, \beta, \nu, \mu, \delta, \eta)$ satisfying (6.3.19). As $\widetilde{q}_{\alpha \gamma}=\widetilde{q}_{\beta \gamma}=q^{2}$, and $\widetilde{q}_{\delta \gamma}=q^{-1}$ in both cases, we have that $\mathrm{d}_{\alpha+\beta, \delta, \alpha, \gamma}^{(L)}=\mathrm{d}_{q^{4}, q^{-1}, q^{2}}^{(L)}=0$ by Lemma 6.3.28 (b), Thus (I) holds in this case.

Notice that $\gamma_{1}=2, \gamma_{2}=12, \gamma_{3}=12^{2} 3^{2}, \gamma_{4}=\gamma_{5}=12^{2} 3^{3} 4^{2}$ satisfy $\sum_{i \in \mathbb{I}_{5}} \gamma_{i}=4 \gamma$. Hence, if $N_{12^{2} 3^{3} 4^{2}}=2$, then $n_{2}=n_{12}=n_{12^{2} 3^{2}}=1, n_{\gamma}=n_{12^{2} 3^{3} 4^{2}}=2$ is a solution of (6.3.1). The coefficient of $\mathrm{x}_{\gamma}^{6} \otimes 1$ is zero in $d\left(\mathrm{x}_{2} \mathrm{x}_{12} \mathrm{x}_{12^{2} 32} \mathrm{x}_{\gamma}^{2} \mathrm{x}_{12^{2} 3^{3} 4^{2}}^{2} \otimes 1\right)$ by Lemma 10.1.79,

Finally we look for solutions of (6.3.1), i.e. $\sum_{\delta \in \Delta_{+}^{\mathfrak{q}}} f_{\delta}\left(n_{\delta}\right) \delta=N \gamma, \sum_{\delta \in \Delta_{+}^{\mathfrak{q}}} n_{\delta}=N+1$. Set $\eta=12^{2} 3^{3} 4^{2}$. Looking at the coefficient of $\alpha_{4}$ :

$$
N=\sum_{\delta \in \Delta_{+}^{\mathfrak{q}}} f_{\delta}\left(n_{\delta}\right) a_{4}^{\delta}=2 f_{\eta}\left(n_{\eta}\right)+\sum_{\delta \neq \eta, 4 \in \operatorname{supp} \delta} n_{\delta} \geq 2 f_{\eta}\left(n_{\eta}\right) .
$$

As $N_{\eta}=M$, we have that $n_{\eta} \leq 3$. Suppose that $n_{\eta}=3$ : necessarily $N=3 M$ and

$$
\sum_{\delta \neq \eta, 3,4 \in \operatorname{supp} \delta} n_{\delta}=3 M-n_{4}-2 f_{\eta}(3)=M-n_{4}-2 .
$$

Looking at the coefficient of $\alpha_{3}$ :

$$
\begin{aligned}
\sum_{\delta: 3 \in \operatorname{supp} \delta, 4 \notin \operatorname{supp} \delta} f_{\delta}\left(n_{\delta}\right) a_{3}^{\delta}=6 M-\sum_{\delta \neq \eta, 3,4 \in \operatorname{supp} \delta} n_{\delta} a_{3}^{\delta}-3 f_{\eta}(3) \\
\quad \leq 6 M-\sum_{\delta \neq \eta, 3,4 \in \operatorname{supp} \delta} n_{\delta}-3 f_{\eta}(3)=2 M+n_{4}-1<3 M=N .
\end{aligned}
$$

By inspection, $f_{\delta}(2) a_{3}^{\delta}=N_{\delta} a_{3}^{\delta}=N$ for all $\delta$ such that $3 \in \operatorname{supp} \delta, 4 \notin \operatorname{supp} \delta$, so $n_{\delta} \leq 1$ for those $\delta$. This implies that $f_{\delta}\left(n_{\delta}\right)=n_{\delta}$ for all $\delta \neq \eta$ such that $3 \in \operatorname{supp} \delta$. Using this fact, the coefficients of $\alpha_{2}$ and $\alpha_{3}$ give the following equalities:

$$
\begin{aligned}
& 6 M=\sum_{\delta: 2 \in \operatorname{supp} \delta} f_{\delta}\left(n_{\delta}\right) a_{2}^{\delta}=f_{12}\left(n_{12}\right)+f_{2}\left(n_{2}\right)+2(M+1)+\sum_{\delta \neq \eta: 2,3 \in \operatorname{supp} \delta} n_{\delta} a_{2}^{\delta} \\
& 6 M=\sum_{\delta: 3 \in \operatorname{supp} \delta} f_{\delta}\left(n_{\delta}\right) a_{3}^{\delta}=3(M+1)+\sum_{\delta \neq \eta: 3 \in \operatorname{supp} \delta} n_{\delta} a_{3}^{\delta}
\end{aligned}
$$


From these two equalities:

$$
\begin{aligned}
f_{12}\left(n_{12}\right)+f_{2}\left(n_{2}\right) & =M+1+\sum_{\delta \neq \eta: 3 \in \operatorname{supp} \delta} n_{\delta} a_{3}^{\delta}-\sum_{\delta \neq \eta: 2,3 \in \operatorname{supp} \delta} n_{\delta} a_{2}^{\delta} \\
& =M+1+n_{123^{2}}+n_{23^{2}}+n_{3}+n_{12^{2} 3^{3} 4}+n_{123^{2} 4}+n_{23^{2} 4}+n_{34} \geq M+1 .
\end{aligned}
$$

As $n_{12^{2} 3^{3} 4}+n_{123^{2} 4}+n_{23^{2} 4}+n_{34} \leq M-n_{4}-2$ and $n_{123^{2}}, n_{23^{2}}, n_{3} \leq 1$, we have that

$$
f_{12}\left(n_{12}\right)+f_{2}\left(n_{2}\right) \leq M+1+3+M-n_{4}-2 \geq 2 M+2-n_{4} \leq 2 M-2 .
$$

But this is a contradiction since $N_{2}=N_{12}=P$. A similar argument holds if we suppose that $n_{\eta}=2$, so $n_{\eta} \leq 1$.

Hence $n_{\delta}=f_{\delta}\left(n_{\delta}\right)$ for all $\delta$ such that $4 \in \operatorname{supp} \delta$, so we may translate the equations to the following problem: Find $\gamma_{i} \in \Delta_{+}^{\mathfrak{q}}, i \in \mathbb{I}_{N+1}$, such that $\sum_{i \in \mathbb{I}_{N+1}} \gamma_{i}=N \gamma$. As $\sum a_{1}^{\gamma_{i}}=N$ and $a_{1}^{\delta} \leq 1$ for all $\delta \in \Delta_{+}^{\mathfrak{q}}$, we may assume that $a_{1}^{\gamma_{i}}=1$ for $i \in \mathbb{I}_{N}, a_{1}^{\gamma_{N+1}}=0$. As $\sum a_{2}^{\gamma_{i}}=2 N$ and $a_{2}^{\delta} \leq 2$ for all $\delta \in \Delta_{+}^{\mathfrak{q}}$, there are two possible cases: either $a_{2}^{\gamma_{i}}=2$ for $N$ of them, $a_{2}^{\gamma_{i}}=0$ for the remaining root, or else $a_{2}^{\gamma_{i}}=2$ for $N-1$ of them, $a_{2}^{\gamma_{i}}=1$ for the remaining two roots. In any case $N-1$ roots have $a_{2}^{\gamma_{i}}=2$, and as $a_{2}^{\delta}=2$ implies that $a_{1}^{\delta}=1$, we may assume that $a_{2}^{\gamma_{i}}=2$ for all $i \in \mathbb{I}_{N-1}$, so $a_{3}^{\gamma_{i}} \geq 2$ for all $i \in \mathbb{I}_{N-1}$. As $\sum a_{3}^{\gamma_{i}}=2 N$, at most two of $a_{3}^{\gamma_{i}}$ s s are equal to 3 . Therefore we have three cases:

(a) $a_{3}^{\gamma_{1}}=a_{3}^{\gamma_{2}}=3, a_{3}^{\gamma_{i}}=2$ for $i \in \mathbb{I}_{3, N-1}$. Hence $a_{3}^{\gamma_{N}}=a_{3}^{\gamma_{N+1}}=0$, which implies that $a_{4}^{\gamma_{N}}=a_{4}^{\gamma_{N+1}}=0$. As $\sum a_{4}^{\gamma_{i}}=2 N$, at least one of them is equal to 2 . With all these conditions we find exactly two solutions:

$$
\begin{aligned}
& \gamma_{1}=12^{2} 3^{3} 4^{2}, \quad \gamma_{2}=12^{2} 3^{3} 4, \quad \gamma_{i}=\gamma, i \in \mathbb{I}_{3, N-1}, \quad \gamma_{N}=12, \quad \gamma_{N+1}=2 ; \\
& \gamma_{1}=\gamma_{2}=12^{2} 3^{3} 4^{2}, \quad \gamma_{3}=12^{2} 3^{2}, \quad \gamma_{i}=\gamma, i \in \mathbb{I}_{4, N-1}, \quad \gamma_{N}=12, \quad \gamma_{N+1}=2 .
\end{aligned}
$$

The last solution requires $2=f_{12^{2} 3^{3} 4^{2}}(n)$ for some $n \in \mathbb{N}$ : the unique possibility is $N_{12^{2} 3^{3} 4^{2}}=2, n=2$.

(b) $a_{3}^{\gamma_{1}}=3, a_{3}^{\gamma_{i}}=2$ for $i \in \mathbb{I}_{2, N-1}$. Hence either $a_{3}^{\gamma_{N}}=1, a_{3}^{\gamma_{N+1}}=0$ or else $a_{3}^{\gamma_{N}}=0$, $a_{3}^{\gamma_{N+1}}=1$. In the first case, $\gamma_{N+1}=2$, so $a_{2}^{N}=1$. The solutions are:

$$
\begin{aligned}
& \gamma_{1}=12^{2} 3^{3} 4^{2}, \quad \gamma_{i}=\gamma, i \in \mathbb{I}_{2, N-2}, \quad \gamma_{N-1}=12^{2} 3^{2}, \quad \gamma_{N}=1234, \quad \gamma_{N+1}=2 ; \\
& \gamma_{1}=12^{2} 3^{3} 4^{2}, \quad \gamma_{i}=\gamma, i \in \mathbb{I}_{2, N-1}, \quad \gamma_{N}=123, \quad \gamma_{N+1}=2 ; \\
& \gamma_{1}=12^{2} 3^{3} 4, \quad \gamma_{i}=\gamma, i \in \mathbb{I}_{2, N-1}, \quad \gamma_{N}=1234, \quad \gamma_{N+1}=2 .
\end{aligned}
$$

Now we consider $a_{3}^{\gamma_{N}}=0, a_{3}^{\gamma_{N+1}}=1$. Notice that $a_{2}^{\gamma_{N}}, a_{2}^{\gamma_{N+1}} \leq 1$, so $a_{2}^{\gamma_{N}}=a_{2}^{\gamma_{N+1}}=1$. This implies that $\gamma_{N}=12$. We have three solutions:

$$
\begin{aligned}
& \gamma_{1}=12^{2} 3^{3} 4^{2}, \quad \gamma_{i}=\gamma, i \in \mathbb{I}_{2, N-2}, \quad \gamma_{N-1}=12^{2} 3^{2}, \quad \gamma_{N}=12, \quad \gamma_{N+1}=234 ; \\
& \gamma_{1}=12^{2} 3^{3} 4^{2}, \quad \gamma_{i}=\gamma, i \in \mathbb{I}_{2, N-1}, \quad \gamma_{N}=12, \quad \gamma_{N+1}=23 \text {; } \\
& \gamma_{1}=12^{2} 3^{3} 4, \quad \gamma_{i}=\gamma, i \in \mathbb{I}_{2, N-1}, \quad \gamma_{N}=12, \quad \gamma_{N+1}=234 .
\end{aligned}
$$

(c) $a_{3}^{\gamma_{i}}=2$ for all $i \in \mathbb{I}_{N-1}$. In this case, exactly $N$ of the $a_{4}^{\gamma_{i}}$ 's are 1 , and the remaining one is 0 . Hence either

$$
\gamma_{1}=12^{2} 3^{2}, \quad \gamma_{i}=\gamma, i \in \mathbb{I}_{2, N-1}, \quad \gamma_{N}=1234, \quad \gamma_{N+1}=234,
$$


or $\gamma_{i}=\gamma$, for all $i \in \mathbb{I}_{N-1}$, so $\gamma_{N}+\gamma_{N+1}=\gamma$ : the possible pairs $\left(\gamma_{N}, \gamma_{N+1}\right)$ are $(123,234),(1234,23),\left(2,123^{4}\right),\left(12,23^{2} 4\right),\left(12^{2} 3^{2}, 4\right)$.

Hence all the hypothesis of Proposition 6.3.2 hold, and $\left(\mathrm{x}_{\gamma}^{L_{\gamma}}\right)^{*}$ is a cocycle.

If $\gamma=12^{2} 3^{3} 4$, then $N_{\gamma}=2=P_{\gamma}$. First we look for pairs $\alpha<\beta$ as in (6.3.3). We have the following posibilities:
$\left(12^{2} 3^{2}, 34\right)$
$\left(123^{2}, 234\right)$,
$\left(23^{2}, 1234\right)$
$\left(3,12^{2} 3^{2} 4\right)$
$\left(23,123^{2} 4\right), \quad\left(123,23^{2} 4\right)$.

In all cases $\left[x_{\alpha}, x_{\gamma}\right]_{c}=0=\left[x_{\gamma}, x_{\beta}\right]_{c}$, so the root vectors satisfy (10.1.8), and $\left(-\frac{q_{\alpha \alpha}}{q_{\beta \beta}}\right)^{N}=1$. Hence we take $L=N$.

Next we check that $\left(123^{2}, 23^{2}, 12^{2} 3^{2} 4,1234,234,12^{2} 3^{3} 4^{2}\right)$ is a 6 -uple $(\alpha, \beta, \delta, \tau, \varphi, \eta)$ satisfying (6.3.11). As $\widetilde{q}_{\alpha \gamma}=q^{2}=\widetilde{q}_{\beta \gamma}, \widetilde{q}_{\delta \gamma}=q^{-1}$, we have that $\mathrm{d}_{\alpha \beta \delta \gamma}^{(L)}=\mathrm{d}_{q^{-1}, q^{2}, q^{2}}^{(L)}=0$ by Lemma 6.3.28 (c) Hence (E) holds in this case.

Now we look for 4 -uples $(\alpha, \beta, \delta, \eta)$ satisfying (6.3.13). There are five possibilities:

$$
\begin{array}{lll}
\left(123^{2}, 23,12^{2} 3^{3} 4^{2}, 123^{2} 4\right), & \left(12^{2} 3^{2}, 3,12^{2} 3^{3} 4^{2}, 12^{2} 3^{2} 4\right), & \left(123^{2}, 12^{2} 3^{2} 4,23^{2} 4,3\right), \\
\left(23^{2}, 12^{2} 3^{2} 4,123^{2} 4,3\right), & \left(12^{2} 3^{2}, 123^{2} 4,23^{2} 4,23\right) .
\end{array}
$$

Here $\widetilde{q}_{\alpha \gamma}=q^{2}, \widetilde{q}_{\beta \gamma}=q^{-1}$. Hence $\mathrm{c}_{\alpha \beta \gamma}^{(L)}=\mathrm{c}_{q^{2}, q^{-1}}^{(L)}=0$ by Lemma 6.3.28 (b), and (F) holds.

We look for 4-uples $(\alpha, \beta, \delta, \eta)$ satisfying (6.3.15): the unique is $\left(123,23^{2}, 12^{2} 3^{3} 4^{2}, 23^{2} 4\right)$. As $\widetilde{q}_{\alpha \gamma}=q^{2}$ and $\widetilde{q}_{\delta \gamma}=q^{-1}$; thus $\mathrm{c}_{-\delta \alpha \gamma}^{(L)}=\mathrm{c}_{q, q^{2}}^{(L)}=0$ by Lemma 6.3.28 (b), and (G) holds.

There are two 6-uples $(\alpha, \beta, \nu, \mu, \delta, \eta)$ satisfying (6.3.19):

$$
\left(23^{2}, 12^{2} 3^{2}, 12^{2} 3^{2} 4,1234,12^{2} 3^{3} 4^{2}, 123^{2} 4\right) \text { and }\left(123^{2}, 12^{2} 3^{2}, 12^{2} 3^{2} 4,234,12^{2} 3^{3} 4^{2}, 23^{2} 4\right) \text {. }
$$

As $\widetilde{q}_{\alpha \gamma}=\widetilde{q}_{\beta \gamma}=q^{2}$, and $\widetilde{q}_{\delta \gamma}=q^{-1}$ in both cases, we have that $\mathrm{d}_{\alpha+\beta, \delta, \alpha, \gamma}^{(L)}=\mathrm{d}_{q^{4}, q^{-1}, q^{2}}^{(L)}=0$ by Lemma 6.3.28 (b), Thus (I) holds.

Also $\gamma_{1}=12^{2} 3^{2}, \gamma_{2}=123^{2}, \gamma_{3}=23^{2}, \gamma_{4}=\gamma_{5}=12^{2} 3^{3} 4^{2}$ satisfy $\sum_{i \in \mathbb{I}_{5}} \gamma_{i}=4 \gamma$. Hence, if $N_{12^{2} 3^{3} 4^{2}}=2$, then $n_{12^{2} 3^{2}}=n_{123^{2}}=n_{23^{2}}=1, n_{\gamma}=n_{12^{2} 3^{3} 4^{2}}=2$ is a solution of (6.3.1).

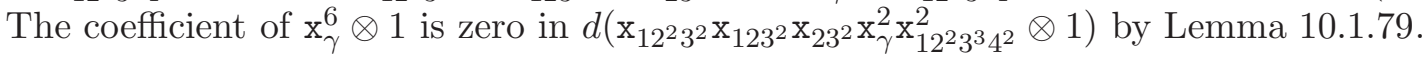

Finally we look for solutions of (6.3.1), i.e. $\sum_{\delta \in \Delta_{+}^{\mathfrak{q}}} f_{\delta}\left(n_{\delta}\right) \delta=N \gamma, \sum_{\delta \in \Delta_{+}^{\mathfrak{q}}} n_{\delta}=N+1$. Looking at the coefficient of $\alpha_{4}$ we get as in the previous case that $n_{\delta}=f_{\delta}^{+}\left(n_{\delta}\right)$ for all $\delta$ such that $4 \in \operatorname{supp} \delta$, so we may translate the equations to the following problem: Find $\gamma_{i} \in \Delta_{+}^{\mathfrak{q}}, i \in \mathbb{I}_{N+1}$, such that $\sum_{i \in \mathbb{I}_{N+1}} \gamma_{i}=N \gamma$. As $\sum a_{1}^{\gamma_{i}}=N$ and $a_{1}^{\delta} \leq 1$ for all $\delta \in \Delta_{+}^{\mathfrak{q}}$, we may assume that $a_{1}^{\gamma_{i}}=1$ for $i \in \mathbb{I}_{N}, a_{1}^{\gamma_{N+1}}=0$. As $\sum a_{3}^{\gamma_{i}}=3 N$ and $a_{3}^{\delta} \leq 3$ for all $\delta \in \Delta_{+}^{\mathfrak{q}}$, at least $N-2$ of these roots satisfy $a_{3}^{\gamma_{i}}=3$ and we have three cases:

(a) $a_{3}^{\gamma_{1}}=0, a_{3}^{\gamma_{i}}=3$ for $i \in \mathbb{I}_{2, N+1}$. Then $a_{1}^{\gamma_{i}}=1, a_{2}^{\gamma_{i}}=2, a_{4}^{\gamma_{i}} \geq 1$ for $i \in \mathbb{I}_{2, N+1}$ but there is no solution in this case.

(b) $a_{3}^{\gamma_{1}}=1, a_{3}^{\gamma_{2}}=2, a_{3}^{\gamma_{i}}=3$ for $i \in \mathbb{I}_{3, N+1}$. Then $a_{1}^{\gamma_{i}}=1, a_{2}^{\gamma_{i}}=2, a_{4}^{\gamma_{i}} \geq 1$ if $i \geq 3$, so

$$
a_{1}^{\gamma_{1}}+a_{1}^{\gamma_{2}}=1, \quad a_{2}^{\gamma_{1}}+a_{2}^{\gamma_{2}}=2, \quad a_{4}^{\gamma_{1}}+a_{4}^{\gamma_{2}} \leq 1 .
$$

If $a_{4}^{\gamma_{1}}=a_{4}^{\gamma_{2}}=0$, then we obtain the following solutions

$$
\gamma_{1}=123, \quad \gamma_{2}=23^{2}, \quad \gamma_{3}=12^{2} 3^{3} 4^{2}, \quad \gamma_{i}=\gamma \text { if } i \in \mathbb{I}_{4, N+1} ;
$$




$$
\begin{array}{llll}
\gamma_{1}=123^{2}, & \gamma_{2}=23, & \gamma_{3}=12^{2} 3^{3} 4^{2}, & \gamma_{i}=\gamma \text { if } i \in \mathbb{I}_{4, N+1} ; \\
\gamma_{1}=12^{2} 3^{2}, & \gamma_{2}=3, & \gamma_{3}=12^{2} 3^{3} 4^{2}, & \gamma_{i}=\gamma \text { if } i \in \mathbb{I}_{4, N+1} .
\end{array}
$$

Otherwise $\gamma_{i}=\gamma$ for all $i \geq 3$; that is, $\gamma_{1}+\gamma_{2}=\gamma$, and the possible pairs $\left(\gamma_{1}, \gamma_{2}\right)$ are

$$
\left(12^{2} 3^{2}, 34\right), \quad\left(123^{2}, 234\right), \quad\left(23^{2}, 1234\right), \quad\left(3,12^{2} 3^{2} 4\right), \quad\left(23,123^{2} 4\right), \quad\left(123,23^{2} 4\right) .
$$

(c) $a_{3}^{\gamma_{i}}=2$ for $i \in \mathbb{I}_{3}, a_{3}^{\gamma_{i}}=3$ for $i \in \mathbb{I}_{4, N+1}$. Then $a_{1}^{\gamma_{i}}=1, a_{2}^{\gamma_{i}}=2, a_{4}^{\gamma_{i}} \geq 1$ if $i \geq 4$, so

$$
a_{1}^{\gamma_{1}}+a_{1}^{\gamma_{2}}+a_{1}^{\gamma_{3}}=2, \quad a_{2}^{\gamma_{1}}+a_{2}^{\gamma_{2}}+a_{2}^{\gamma_{3}}=4, \quad a_{4}^{\gamma_{1}}+a_{4}^{\gamma_{2}}+a_{4}^{\gamma_{3}} \leq 2 .
$$

If $a_{4}^{\gamma_{1}}=a_{4}^{\gamma_{2}}=a_{4}^{\gamma_{3}}=0$, then the unique solution is

$$
\gamma_{1}=12^{2} 3^{2}, \quad \gamma_{2}=123^{2}, \quad \gamma_{3}=23^{2}, \quad \gamma_{4}=\gamma_{5}=12^{2} 3^{3} 4^{2}, \quad \gamma_{i}=\gamma \text { if } i \in \mathbb{I}_{6, N+1} .
$$

For this solution we need $N_{12^{2} 3^{3} 4^{2}}=2$, which implies that $N=6$.

If $a_{4}^{\gamma_{1}}=1, a_{4}^{\gamma_{2}}=a_{4}^{\gamma_{3}}=0$, then the solutions are

$$
\begin{array}{lllll}
\gamma_{1}=12^{2} 3^{2} 4, & \gamma_{2}=123^{2}, & \gamma_{3}=23^{2}, & \gamma_{4}=12^{2} 3^{3} 4^{2}, & \gamma_{i}=\gamma \text { if } i \in \mathbb{I}_{5, N+1} ; \\
\gamma_{1}=123^{2} 4, & \gamma_{2}=12^{2} 3^{2}, & \gamma_{3}=23^{2}, & \gamma_{4}=12^{2} 3^{3} 4^{2}, & \gamma_{i}=\gamma \text { if } i \in \mathbb{I}_{5, N+1} ; \\
\gamma_{1}=23^{2} 4, & \gamma_{2}=123^{2}, & \gamma_{3}=12^{2} 3^{2}, & \gamma_{4}=12^{2} 3^{3} 4^{2}, & \gamma_{i}=\gamma \text { if } i \in \mathbb{I}_{5, N+1} .
\end{array}
$$

If $a_{4}^{\gamma_{1}}=a_{4}^{\gamma_{2}}=1, a_{4}^{\gamma_{3}}=0$, then the unique solution is

$$
\begin{array}{llll}
\gamma_{1}=12^{2} 3^{2} 4, & \gamma_{2}=23^{2} 4, & \gamma_{3}=123^{2}, & \gamma_{i}=\gamma \text { if } i \in \mathbb{I}_{4, N+1} ; \\
\gamma_{1}=123^{2} 4, & \gamma_{2}=12^{2} 3^{2} 4, & \gamma_{3}=23^{2}, & \gamma_{i}=\gamma \text { if } i \in \mathbb{I}_{4, N+1} ; \\
\gamma_{1}=23^{2} 4, & \gamma_{2}=123^{2} 4, & \gamma_{3}=12^{2} 3^{2}, & \gamma_{i}=\gamma \text { if } i \in \mathbb{I}_{4, N+1} .
\end{array}
$$

Hence all the hypothesis of Proposition 6.3.2 hold, and $\left(\mathrm{x}_{\gamma}^{L_{\gamma}}\right)^{*}$ is a cocycle.

If $\gamma=123^{2} 4$, then $N_{\gamma}=2=P_{\gamma}$. The pairs $\alpha<\beta$ as in (6.3.3) are $\left(1,23^{2} 4\right),(123,34)$, $(\alpha, \beta)=\left(123^{2}, 4\right)$. In all cases $\left[x_{\alpha}, x_{\gamma}\right]_{c}=0=\left[x_{\gamma}, x_{\beta}\right]_{c}$, so the root vectors satisfy (10.1.8), and $\left(-\frac{q_{\alpha \alpha}}{q_{\beta \beta}}\right)^{N}=1$. Hence we take $L=N$.

Now $\left(123^{2}, 1234,34,3\right)$ is a 4 -uples $(\alpha, \beta, \delta, \eta)$ satisfying (6.3.13). Here, $\widetilde{q}_{\alpha \gamma}=q^{2}, \widetilde{q}_{\beta \gamma}=$ $q^{-1}$, so $\mathrm{c}_{\alpha \beta \gamma}^{(L)}=\mathrm{c}_{q^{2}, q^{-1}}^{(L)}=0$ by Lemma 6.3.28 (b) and (F) holds.

Next we check that $\left(1,3,12^{2} 3^{3} 4^{2}, 23^{2} 4\right)$ and $\left(1,12^{2} 3^{3} 4,34,23^{2} 4\right)$ are 4 -uples $(\alpha, \beta, \delta, \eta)$ satisfying (6.3.15). As $\widetilde{q}_{\alpha \gamma}=q^{2}$ and $\widetilde{q}_{\delta \gamma}=q^{-1}$ in both cases, $\mathrm{c}_{-\delta \alpha \gamma}^{(L)}=\mathrm{c}_{q, q^{2}}^{(L)}=0$ by Lemma 6.3.28 (b) and (G) holds.

Also, $\left(1,123^{2}, 12^{2} 3^{3} 4,23^{2} 4,12^{2} 3^{3} 4^{2}, 34\right)$ is a 6-uple satisfying (6.3.19). As $\widetilde{q}_{\alpha \gamma}=\widetilde{q}_{\beta \gamma}=q^{2}$ and $\widetilde{q}_{\delta \gamma}=q^{-1}$, we have that $\mathrm{d}_{\alpha+\beta, \delta, \alpha, \gamma}^{(L)}=\mathrm{d}_{q^{4}, q^{-1}, q^{2}}^{(L)}=0$ by Lemma 6.3.28 (b). Thus (I) holds in this case.

Finally we look for solutions of (6.3.1), i.e. $\sum_{\delta \in \Delta_{+}^{\mathfrak{q}}} f_{\delta}\left(n_{\delta}\right) \delta=N \gamma, \sum_{\delta \in \Delta_{+}^{\mathfrak{q}}} n_{\delta}=N+1$. Looking at the coefficient of $\alpha_{4}$ and arguing as in the case $\gamma=12^{2} 3^{2}$, we find that $n_{\delta}=f_{\delta}\left(n_{\delta}\right)$ for all $\delta$, so we translate the equations to the following problem: Find $\gamma_{i} \in \Delta_{+}^{\mathfrak{q}}$, $i \in \mathbb{I}_{N+1}$, such that $\sum_{i \in \mathbb{I}_{N+1}} \gamma_{i}=N \gamma$. As $\sum a_{1}^{\gamma_{i}}=N$ and $a_{1}^{\delta} \leq 1$ for all $\delta \in \Delta_{+}^{\mathfrak{q}}$, we may 
assume that $a_{1}^{\gamma_{i}}=1$ for $i \in \mathbb{I}_{N}, a_{1}^{\gamma_{N+1}}=0$. Using a detailed study as the previous case we check that the solutions are

$$
\begin{aligned}
& \gamma_{1}=12^{2} 3^{3} 4^{2}, \quad \gamma_{i}=\gamma, i \in \mathbb{I}_{2, N-2}, \quad \gamma_{N-1}=123^{2}, \quad \gamma_{N}=1, \quad \gamma_{N+1}=34 \\
& \gamma_{1}=12^{2} 3^{3} 4^{2}, \quad \gamma_{i}=\gamma, i \in \mathbb{I}_{2, N-1}, \quad \gamma_{N}=1, \quad \gamma_{N+1}=3 ; \\
& \gamma_{1}=12^{2} 3^{3} 4, \quad \gamma_{i}=\gamma, i \in \mathbb{I}_{2, N-1}, \quad \gamma_{N}=1, \quad \gamma_{N+1}=34 . \\
& \gamma_{1}=123^{2}, \quad \gamma_{i}=\gamma, i \in \mathbb{I}_{2, N-1}, \quad \gamma_{N}=1234, \quad \gamma_{N+1}=34,
\end{aligned}
$$

or $\gamma_{i}=\gamma$, for all $i \in \mathbb{I}_{N-1}$, so $\gamma_{N}+\gamma_{N+1}=\gamma$ : the possible pairs $\left(\gamma_{N}, \gamma_{N+1}\right)$ are $(123,34)$, $(1234,3),\left(1,23^{2} 4\right),\left(123^{2}, 4\right)$. Hence all the hypothesis of Proposition 6.3.2 hold, and $\left(\mathrm{x}_{\gamma}^{L_{\gamma}}\right)^{*}$ is a cocycle.

If $\gamma=1234$, then $N_{\gamma}=2=P_{\gamma}$. The pairs $\alpha<\beta$ as in (6.3.3) are $(1,234),(123,4)$, $(12,34)$. In all cases $\left[x_{\alpha}, x_{\gamma}\right]_{c}=0=\left[x_{\gamma}, x_{\beta}\right]_{c}$, so the root vectors satisfy (10.1.8), and $\left(-\frac{q_{\alpha \alpha}}{q_{\beta \beta}}\right)^{N}=1$. Hence we take $L=N$.

Next we check that $\left(1,12^{2} 3^{2} 4^{2}, 234,4\right)$ and $\left(12,123^{2} 4,4,34\right)$ are 4 -uples $(\alpha, \beta, \delta, \eta)$ satisfying (6.3.15). As $\widetilde{q}_{\alpha \gamma}=q^{2}$ and $\widetilde{q}_{\delta \gamma}=q^{-1}$ in both cases, $\mathrm{c}_{-\delta \alpha \gamma}^{(L)}=\mathrm{c}_{q, q^{2}}^{(L)}=0$ by Lemma 6.3 .28 (b) so (G) holds.

Notice that $\gamma_{1}=1, \gamma_{2}=12, \gamma_{3}=12^{2} 3^{3} 4^{2}, \gamma_{4}=4$ satisfy $\sum_{i \in \mathbb{I}_{4}} \gamma_{i}=3 \gamma$. The corresponding root vectors $q$-commute so the coefficient of $\mathrm{x}_{\gamma}^{6} \otimes 1$ in $d\left(\mathrm{x}_{1} \mathrm{x}_{12} \mathrm{x}_{12} 3^{3} 4^{2} \mathrm{x}_{\gamma}^{N-3} \mathrm{x}_{4} \otimes 1\right)$ is zero by Remark 10.1.2.

Finally we look for solutions of (6.3.1), i.e. $\sum_{\delta \in \Delta_{+}^{\mathfrak{q}}} f_{\delta}\left(n_{\delta}\right) \delta=N \gamma, \sum_{\delta \in \Delta_{+}^{\mathfrak{q}}} n_{\delta}=N+1$. Looking at the coefficient of $\alpha_{4}$ and arguing as in the case $\gamma=12^{2} 3^{2} 4$, we find that $n_{\delta}=f_{\delta}\left(n_{\delta}\right)$ for all $\delta$, so we translate the equations to the following problem: Find $\gamma_{i} \in \Delta_{+}^{\mathfrak{q}}$, $i \in \mathbb{I}_{N+1}$, such that $\sum_{i \in \mathbb{I}_{N+1}} \gamma_{i}=N \gamma$. As $\sum a_{1}^{\gamma_{i}}=N$ and $a_{1}^{\delta} \leq 1$ for all $\delta \in \Delta_{+}^{\mathfrak{q}}$, we may assume that $a_{1}^{\gamma_{i}}=1$ for $i \in \mathbb{I}_{N}, a_{1}^{\gamma_{N+1}}=0$. Using a detailed study as the previous case we check that the solutions are

$$
\begin{aligned}
& \gamma_{1}=12^{2} 3^{3} 4^{2}, \quad \gamma_{i}=\gamma, i \in \mathbb{I}_{2, N-2}, \quad \gamma_{N-1}=12, \quad \gamma_{N}=1, \quad \gamma_{N+1}=4 ; \\
& \gamma_{1}=12^{2} 3^{2} 4, \quad \gamma_{i}=\gamma, i \in \mathbb{I}_{2, N-1}, \quad \gamma_{N}=1, \quad \gamma_{N+1}=4 . \\
& \gamma_{1}=12, \quad \gamma_{i}=\gamma, i \in \mathbb{I}_{2, N-1}, \quad \gamma_{N}=123^{2} 4, \quad \gamma_{N+1}=4 \text {, }
\end{aligned}
$$

or $\gamma_{i}=\gamma$, for all $i \in \mathbb{I}_{N-1}$, so $\gamma_{N}+\gamma_{N+1}=\gamma$ : the possible pairs $\left(\gamma_{N}, \gamma_{N+1}\right)$ are $(123,4)$, $(1,234),(12,34)$. Hence Proposition 6.3.2 applies and $\left(\mathrm{x}_{\gamma}^{L_{\gamma}}\right)^{*}$ is a cocycle.

Finally, if $\gamma=12^{2} 3^{3} 4^{2}$, then $N_{\gamma}=M, P_{\gamma}=2, Q_{\gamma}=1$. If $N \neq 6$, then $N_{\gamma}>P_{\gamma}=2, Q_{\gamma}$, so $\left(\mathrm{x}_{\gamma}^{N_{\gamma}}\right)^{*}$ is a 2-cocycle by Lemma 6.2.5. Next we assume $N=6$; that is, $N_{\gamma}=2$. Let $\alpha<\beta$ be a pair of positive roots as in (6.3.3). We have the following posibilities:

$\circ \alpha=23^{2} 4, \beta=1234$. There exists $\mathrm{b} \in \mathbb{k}$ such that $\left[x_{\alpha}, x_{\beta}\right]_{c}=\mathrm{b} x_{\gamma}$.

$\circ \alpha=123^{2} 4, \beta=234$. There exist $\mathrm{b}, \mathrm{b}_{1} \in \mathbb{k}$ such that

$$
\left[x_{\alpha}, x_{\beta}\right]_{c}=\mathrm{b} x_{\gamma}+\mathrm{b}_{1} x_{1234} x_{23^{2} 4} .
$$


$\circ \alpha=12^{2} 3^{2} 4, \beta=34$. There exist $\mathrm{b}, \mathrm{b}_{t} \in \mathbb{k}$ such that

$$
\left[x_{\alpha}, x_{\beta}\right]_{c}=\mathrm{b} x_{\gamma}+\mathrm{b}_{1} x_{1234} x_{23^{2} 4}+\mathrm{b}_{2} x_{234} x_{123^{2} 4} .
$$

○ $\alpha=12^{2} 3^{3} 4, \beta=4$. There exist $\mathrm{b}, \mathrm{b}_{t} \in \mathbb{k}$ such that

$$
\left[x_{\alpha}, x_{\beta}\right]_{c}=\mathrm{b} x_{\gamma}+\mathrm{b}_{1} x_{1234} x_{23^{2} 4}+\mathrm{b}_{2} x_{234} x_{123^{2} 4}+\mathrm{b}_{3} x_{34} x_{12^{2} 3^{2} 4} .
$$

In all cases $\left[x_{\alpha}, x_{\gamma}\right]_{c}=0=\left[x_{\gamma}, x_{\beta}\right]_{c}$, so the root vectors satisfy (10.1.8), and $-\frac{q_{\alpha \alpha}}{q_{\beta \beta}}=-1$ : the proof of all these relations follow as in Lemma 7.2.3. Hence we take $L=2$.

Next we look for solutions of (6.3.1). That is, $\sum_{\delta \in \Delta_{+}^{\mathfrak{q}}} f_{\delta}\left(n_{\delta}\right) \delta=2 \gamma, \sum_{\delta \in \Delta_{+}^{\mathfrak{q}}} n_{\delta}=3$. Suppose that $n_{\eta}=3$ for some $\eta \in \Delta_{+}^{\mathfrak{q}}$, then $n_{\delta}=0$ for $\delta \neq \eta$ and $2 \gamma=\left(N_{\eta}+1\right) \eta$, a contradiction. Now suppose that $n_{\eta}=2, n_{\tau}=1$ for $\eta \neq \tau: 2 \gamma=N_{\eta} \eta+\tau$. As $a_{1}^{\eta}, a_{1}^{\tau} \leq 1$ and $2=N_{\eta} a_{1}^{\eta}+a_{1}^{\tau}$, we have that $a_{1}^{\eta}=1, N_{\eta}=2, a_{1}^{\tau}=0$. As $4=2 a_{2}^{\eta}+a_{2}^{\tau}$ and $a_{2}^{\eta} \leq 2$, $a_{2}^{\tau} \leq 1$, we have that $a_{2}^{\eta}=2, a_{2}^{\tau}=0$. Thus $\tau \in\{3,34,4\}$, but there is no solution for these cases, a contradiction.

Therefore, $n_{\eta}=n_{\tau}=n_{\mu}=1$ for three different roots $\eta, \tau, \mu$. As $a_{1}^{\eta}, a_{1}^{\tau}, a_{1}^{\mu} \leq 1$ and $2=a_{1}^{\eta}+a_{1}^{\tau}+a_{1}^{\mu}$, we may assume $a_{1}^{\eta}=a_{1}^{\tau}=1, a_{1}^{\mu}=0$. As $a_{2}^{\eta}, a_{2}^{\tau} \leq 2, a_{2}^{\mu} \leq 1$ and $4=a_{2}^{\eta}+a_{2}^{\tau}+a_{2}^{\mu}$, either $a_{2}^{\eta}=a_{2}^{\tau}=2, a_{2}^{\mu}=0$ or else $a_{2}^{\eta}=2, a_{2}^{\tau}=a_{2}^{\mu}=1$. In the first case, $a_{3}^{\eta}, a_{3}^{\tau} \leq 3, a_{3}^{\mu} \leq 1$ and $6=a_{3}^{\eta}+a_{3}^{\tau}+a_{3}^{\mu}$, so either $a_{3}^{\eta}=a_{3}^{\tau}=3, a_{3}^{\mu}=0$ or else $a_{3}^{\eta}=3, a_{3}^{\tau}=2, a_{3}^{\mu}=1$; in both cases we are forced to get $\eta=\gamma=\tau+\mu$, and moreover we obtain only two possibilities, either $\tau=12^{2} 3^{2} 4, \mu=34$ or else $\tau=12^{2} 3^{3} 4, \mu=4$. In the second case, $a_{4}^{\eta} \leq 2, a_{4}^{\tau}, a_{4}^{\mu} \leq 1$ and $4=a_{4}^{\eta}+a_{4}^{\tau}+a_{4}^{\mu}$, so $a_{4}^{\eta}=2, a_{4}^{\tau}=a_{4}^{\mu}=1$, and again we are forced to get $\eta=\gamma=\tau+\mu$, and moreover we obtain only two possibilities, either $\tau=123^{2} 4, \mu=234$ or else $\tau=1234, \mu=23^{2} 4$.

Hence all the hypothesis of Proposition 6.3.23 hold, and $\left(\mathrm{x}_{\gamma}^{N_{\gamma}}\right)^{*}$ is a 2-cocycle.

8.7. Type $\mathbf{G}(3)$. Let $q$ be a root of 1 of order $N>3$. In this section, we deal with a Nichols algebra $\mathscr{B}_{\mathfrak{q}}$ of super type $\mathbf{G}(3)$, associated to the Dynkin diagram

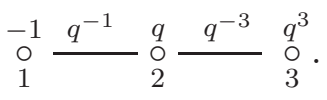

For more information, see $[\mathrm{AA}, \S 5.6]$. The set of positive roots with full support is

$$
\left\{123,12^{2} 3,12^{3} 3,12^{3} 3^{2}, 12^{4} 3^{2}\right\} \text {. }
$$

We fix the following convex order of $\Delta_{+}^{\mathfrak{q}}$ :

$$
1<12<123<12^{2} 3<12^{3} 3<12^{3} 3^{2}<12^{4} 3^{2}<2<2^{3} 3<2^{2} 3<2^{3} 3^{2}<23<3 .
$$

It comes from Lyndon words once we fix the order of the letters $1<2<3$ and differs from the one in $\mathrm{AA}, \S 5.6]$. We prove Condition 1.4 .1 for type $\mathbf{G}(3)$ :

Proposition 8.7.1. For every $\gamma \in \Delta_{+}^{\mathfrak{q}}$, there exists $L_{\gamma} \in \mathbb{N}$ such that $\left(\mathrm{x}_{\gamma}^{L_{\gamma}}\right)^{*}$ is a cocycle.

Proof. It is enough to prove the statement for $\gamma$ with full support.

- For $\gamma=123$, we apply Proposition 6.3.2. The pairs as in (6.3.3) are $\alpha=\alpha_{1}, \beta=\alpha_{2}+\alpha_{3}$, and $\alpha=\alpha_{1}+\alpha_{2}, \beta=\alpha_{3}$ since the following relations hold:

$$
x_{\alpha} x_{\beta}=x_{\gamma}+q_{\alpha \beta} x_{\beta} x_{\alpha}, \quad x_{\alpha} x_{\gamma}=q_{\alpha} x_{\gamma} x_{\alpha}, \quad x_{\gamma} x_{\beta}=q_{\beta} x_{\beta} x_{\gamma} .
$$


As $-\frac{q_{\alpha \alpha}}{q_{\beta \beta}}=q$, respectively $q^{3}, L$ should be a multiple of $N$.

Let $L=\operatorname{ord}(-q)$. Now 6.3.5 holds for $\alpha=1, \beta=12^{2} 3, \delta=3, \eta=12$, and the root vectors satisfy (10.1.11); the scalars $\widetilde{q}_{\alpha \gamma}=q^{-1}$ and $\widetilde{q}_{\beta \gamma}=q^{-2}$ satisfy $c_{\alpha \beta \gamma}^{(L)}=0$.

Also, (6.3.7) holds for $\alpha=1, \beta=12^{3} 3^{2}, \delta=3, \eta=12, \tau=12^{2} 3$, and the root vectors satisfy (10.1.16); the scalars $\widetilde{q}_{\alpha \gamma}=q^{-1}, \widetilde{q}_{\beta \gamma}=q^{-1}, \widetilde{q}_{\tau \gamma}=q^{-2}$ satisfy (6.3.8).

Let $\left(n_{\delta}\right)_{\delta \in \Delta_{+}^{\mathfrak{q}}}$ be a solution of (6.3.1). If $n_{3}=0$, then

$$
L\left(\alpha_{1}+\alpha_{2}\right)=s_{3}(L \gamma)=\sum_{\delta \in \Delta_{+}^{\mathfrak{q}}} f_{\delta}\left(n_{\delta}\right) s_{3}(\delta),
$$

and $s_{3}(\delta) \in \Delta_{+}^{\mathfrak{q}}$ if $\delta \neq \alpha_{3}$. As $N_{\delta}=N_{s_{3}(\delta)}$, we have that $f_{\delta}=f_{s_{3}(\delta)}$, so we have a system as in (6.3.1) for $\alpha_{1}+\alpha_{2}$ in place of $\gamma$ and we may restrict the support to $\alpha_{1}, \alpha_{2}$. The new system has a unique solution, which gives place to the solution of the original system:

- $n_{1}=n_{23}=1, n_{123}=L-1, n_{\delta}=0$ for all the other $\delta \in \Delta_{+}^{\mathfrak{q}}$.

Next we assume $n_{3} \neq 0$. Suppose that $n_{12^{2} 3}>1$. Then $f_{12^{2} 3}\left(n_{12^{2} 3}\right) \geq N$, so the coefficient of $\alpha_{2}$ in $\sum_{\delta \in \Delta_{+}^{\mathfrak{q}}} f_{\delta}\left(n_{\delta}\right) \delta$ is $\geq 2 N$, a contradiction. Hence $n_{12^{2} 3} \leq 1$, and then $f_{12^{2} 3}\left(n_{12^{2} 3}\right)=$ $n_{12^{2} 3} \leq 1$. The coefficient of $\alpha_{1}$ in this sum is

$$
n_{1}+n_{12}+n_{123}+n_{12^{2} 3}+n_{12^{3} 3}+n_{12^{3} 3^{2}}+n_{12^{4} 3^{2}}=L .
$$

As the sum of all $n_{\delta}$ 's is $L+1$ and $n_{3} \neq 0$, we have $n_{3}=1, n_{2}=n_{2^{3} 3}=n_{2^{2} 3}=n_{2^{3} 3^{2}}=$ $n_{23}=0$. Now we look at the coefficients of $\alpha_{2}, \alpha_{3}$ in the equality $\sum_{\delta \in \Delta_{+}^{\mathfrak{q}}} f_{\delta}\left(n_{\delta}\right) \delta=L \gamma$ :

$$
\begin{aligned}
& L=n_{12}+n_{123}+2 n_{12^{2} 3}+3 n_{12^{3} 3}+3 n_{12^{3} 3^{2}}+4 n_{12^{4} 3^{2}}, \\
& L=1+n_{123}+n_{12^{2} 3}+n_{12^{3} 3}+2 n_{12^{3} 3^{2}}+2 n_{12^{4} 3^{2}} .
\end{aligned}
$$

Thus $n_{12}+n_{12^{2} 3}+2 n_{12^{3} 3}+n_{12^{3} 3^{2}}+2 n_{12^{4} 3^{2}}=1$, which implies that $n_{12^{3} 3}=n_{12^{4} 3^{2}}=0$ and two of the three numbers $n_{12}, n_{12^{2} 3}, n_{12^{3} 3^{2}}$ are zero (the remaining one being 1 ). Looking at the three possibilities, we have three solutions:

- $n_{12}=n_{3}=1, n_{123}=L-1, n_{\delta}=0$ for all the other $\delta \in \Delta_{+}^{\mathfrak{q}}$;

- $n_{1}=n_{3}=n_{12^{2} 3}=1, n_{123}=L-2, n_{\delta}=0$ for all the other $\delta \in \Delta_{+}^{\mathfrak{q}}$;

- $n_{1}=2, n_{12^{3} 3^{2}}=n_{3}=1, n_{123}=L-3, n_{\delta}=0$ for all the other $\delta \in \Delta_{+}^{\mathfrak{q}}$.

Hence Proposition 6.3.2 applies and $\left(\mathrm{x}_{123}^{L}\right)^{*}$ is a cocycle.

○ For $\gamma=12^{2} 3$, the case $N_{\gamma}>3$ follows by Lemma 6.2.5. Assume now that $N_{\gamma}=3$. We will work as in Proposition 6.3.23. The pairs as in (6.3.24) are $\left(1,12^{4} 3^{2}\right),\left(12,12^{3} 3^{2}\right)$, $\left(123,12^{3} 3\right)$, since for each one of these pairs the following relations hold:

$$
x_{\alpha} x_{\beta}=\mathrm{b}_{\alpha \beta} x_{\gamma}^{2}+q_{\alpha \beta} x_{\beta} x_{\alpha}, \quad x_{\alpha} x_{\gamma}=q_{\alpha \gamma} x_{\gamma} x_{\alpha}, \quad x_{\gamma} x_{\beta}=q_{\gamma \beta} x_{\beta} x_{112}, \quad \mathrm{~b}_{\alpha \beta} \in \mathbb{k} .
$$

As $\frac{q_{\alpha \gamma}}{q_{\gamma \beta}}=1$ for the three cases, we take $L=1$. We look for solutions of (6.3.26):

$$
\sum_{\delta \in \Delta_{+}^{\mathfrak{q}}} n_{\delta}=3, \quad \sum_{\delta \in \Delta_{+}^{\mathfrak{q}}} f_{\delta}\left(n_{\delta}\right) \delta=3 \gamma .
$$

If $n_{\gamma} \geq 2$, then $f_{\gamma}\left(n_{\gamma}\right) \geq 3$, a contradiction. Then $n_{\gamma} \leq 1$, so $f_{\gamma}\left(n_{\gamma}\right)=n_{\gamma}$. Looking at the coefficient of $\alpha_{1}$ we get the equation:

$$
3=n_{1}+n_{12}+n_{123}+n_{12^{2} 3}+n_{12^{3} 3}+n_{12^{3} 3^{2}}+n_{12^{4} 3^{2}} .
$$


Hence $n_{\delta}=0$ for $\delta=2,2^{3} 3,2^{2} 3,2^{3} 3^{2}, 23,3$. Looking at the coefficients of $\alpha_{2}$ and $\alpha_{3}$,

$$
\begin{aligned}
& 6=n_{12}+n_{123}+2 n_{12^{2} 3}+3 n_{12^{3} 3}+3 n_{12^{3} 3^{2}}+4 n_{12^{4} 3^{2}}, \\
& 3=n_{123}+n_{12^{2} 3}+n_{12^{3} 3}+2 n_{12^{3} 3^{2}}+2 n_{12^{4} 3^{2} .} .
\end{aligned}
$$

From (8.7.2) and (8.7.4), $n_{1}+n_{12}=n_{12^{3} 3^{2}}+n_{12^{4} 3^{2}}$. From (8.7.4), $n_{12^{3} 3^{2}}+n_{12^{4} 3^{2}} \leq 1$. If $n_{1}=n_{12}=0$, then $n_{12^{3} 3^{2}}=n_{12^{4} 3^{2}}=0$ : the solution is $n_{123}=n_{12^{2} 3}=n_{12^{3} 3}=1$. Next we assume $n_{1}+n_{12}=1=n_{12^{3} 3^{2}}+n_{12^{4} 3^{2}}$. If $n_{12^{2} 3}=1$, then the solutions give pairs as in (6.3.24). Otherwise we have a unique solution: $n_{12}=n_{123}=n_{12^{4} 3^{2}}=1, n_{\delta}=0$ otherwise. Hence we have to compute $d\left(\mathrm{x}_{12} \mathrm{x}_{123} \mathrm{x}_{12^{4} 3^{2}} \otimes 1\right)$. Notice that

$$
\begin{aligned}
x_{12} x_{12^{4} 3^{2}} & =-q^{3} q_{12}^{3} q_{13}^{2} q_{23}^{2} x_{12^{4} 3^{2}} x_{12}+\mathrm{b}_{1} x_{12^{3} 3^{3}} x_{12^{2} 3}, \\
x_{123} x_{12^{4} 3^{2}} & =-q^{3} q_{12}^{3} q_{13} q_{32}^{2} x_{12^{4} 3^{2}} x_{123}+\mathrm{b}_{2} x_{12^{3} 3^{2}} x_{12^{2} 3}, \\
x_{12} x_{12^{3} 3^{2}} & =-q^{2} q_{12}^{2} q_{13}^{2} q_{23}^{2} x_{12^{3} 3^{2}} x_{12}+\mathrm{b}_{3} x_{12^{2} 3}^{2}+\mathrm{b}_{4} x_{12^{3} 3} x_{123}, \\
x_{123} x_{12^{3} 3} & =-q^{2} q_{12}^{2} q_{32}^{2} x_{12^{3} 3} x_{123}+\mathrm{b}_{5} x_{12^{2} 3}^{2},
\end{aligned}
$$

for some $\mathrm{b}_{j} \in \mathbb{k}$. Using these relations, we get

$$
\begin{aligned}
& d\left(\mathrm{x}_{12} \mathrm{x}_{123} \mathrm{x}_{12^{4} 3^{2}} \otimes 1\right)=\mathrm{x}_{12} \mathrm{x}_{123} \otimes x_{12^{4} 3^{2}}-s\left(\mathrm{x}_{12} \otimes x_{123} x_{12^{4} 3^{2}}+q_{13} q_{23} \mathrm{x}_{123} \otimes x_{12} x_{12^{4} 3^{2}}\right) \\
& =\mathrm{x}_{12} \mathrm{x}_{123} \otimes x_{12^{4} 3^{2}}-s\left(-q^{3} q_{12}^{3} q_{13} q_{32}^{2} \mathrm{x}_{12} \otimes x_{12^{4} 3^{2}} x_{123}+\mathrm{b}_{2} \mathrm{x}_{12} \otimes x_{12^{3} 3^{2}} x_{12^{2} 3}\right. \\
& \left.-q^{3} q_{12}^{3} q_{13}^{3} q_{23}^{3} \mathrm{x}_{123} \otimes x_{12^{4} 3^{2}} x_{12}+q_{13} q_{23} \mathrm{~b}_{1} \mathrm{x}_{123} \otimes x_{12^{3} 3} x_{12^{2} 3}\right) \\
& =\mathrm{x}_{12} \mathrm{x}_{123} \otimes x_{12^{4} 3^{2}}-\mathrm{b}_{2} \mathrm{x}_{12} \mathrm{x}_{12^{3} 3^{2}} \otimes x_{12^{2} 3}-s\left(-q^{3} q_{12}^{3} q_{13} q_{32}^{2} \mathrm{x}_{12} \otimes x_{12^{4} 3^{2}} x_{123}\right. \\
& -\mathrm{b}_{2} q^{2} q_{12}^{2} q_{13}^{2} q_{23}^{2} \mathrm{x}_{12^{3} 3^{2}} \otimes x_{12} x_{12^{2} 3}+\mathrm{b}_{2} \mathrm{~b}_{3} \mathrm{x}_{12^{2} 3} \otimes x_{12^{2} 3}^{2}+\mathrm{b}_{2} \mathrm{~b}_{4} \mathrm{x}_{12^{3} 3} \otimes x_{123} x_{12^{2} 3} \\
& \left.-q^{3} q_{12}^{3} q_{13}^{3} q_{23}^{3} \mathrm{x}_{123} \otimes x_{12^{4} 3^{2}} x_{12}+q_{13} q_{23} \mathrm{~b}_{1} \mathrm{x}_{123} \otimes x_{12^{3} 3} x_{12^{2} 3}\right) \\
& =\mathrm{x}_{12} \mathrm{x}_{123} \otimes x_{12^{4} 3^{2}}-\mathrm{b}_{2} \mathrm{x}_{12} \mathrm{x}_{12^{3} 3^{2}} \otimes x_{12^{2} 3}+q^{3} q_{12}^{3} q_{13} q_{32}^{2} \mathrm{x}_{12} \mathrm{x}_{12^{4} 3^{2}} \otimes x_{123} \\
& -s\left(q_{12}^{7} q_{23} q_{13}^{3} \mathrm{x}_{12^{4} 3^{2}} \otimes x_{123} x_{12}-\mathrm{b}_{1} q^{3} q_{12}^{3} q_{13} q_{32}^{2} \mathrm{x}_{123}{ }^{3} \otimes x_{12^{2} 3} x_{123}\right. \\
& +\mathrm{b}_{2} q^{3} q_{12}^{3} q_{13}^{3} q_{23}^{3} \mathrm{x}_{12^{3} 3^{2}} \otimes x_{12^{2} 3} x_{12}+\mathrm{b}_{2} \mathrm{~b}_{3} \mathrm{x}_{12^{2} 3} \otimes x_{12^{2} 3}^{2}-q q_{12} q_{32} \mathrm{~b}_{2} \mathrm{~b}_{4} \mathrm{x}_{12^{3} 3} \otimes x_{12^{2} 3^{2}} x_{123} \\
& \left.-q^{3} q_{12}^{3} q_{13}^{3} q_{23}^{3} \mathrm{x}_{123} \otimes x_{12^{4} 3^{2}} x_{12}+q_{13} q_{23} \mathrm{~b}_{1} \mathrm{x}_{123} \otimes x_{12^{3} 3} x_{12^{2} 3}\right) \\
& =\mathrm{x}_{12} \mathrm{x}_{123} \otimes x_{12^{4} 3^{2}}-\mathrm{b}_{2} \mathrm{x}_{12} \mathrm{x}_{12^{3} 3^{2}} \otimes x_{12^{2} 3}+q^{3} q_{12}^{3} q_{13} q_{32}^{2} \mathrm{x}_{12} \mathrm{x}_{12^{4} 3^{2}} \otimes x_{123} \\
& -q_{13} q_{23} \mathrm{~b}_{1} \mathrm{x}_{123} \mathrm{x}_{12^{3} 3} \otimes x_{12^{2} 3}+q^{3} q_{12}^{3} q_{13}^{3} q_{23}^{3} \mathrm{x}_{123} \mathrm{x}_{12^{4} 3^{2}} \otimes x_{12} \\
& +\left(q_{13} q_{23} \mathrm{~b}_{1} \mathrm{~b}_{5}-\mathrm{b}_{2} \mathrm{~b}_{3}\right) \mathrm{x}_{12^{2} 3}^{3} \otimes 1 \text {. }
\end{aligned}
$$

We compute the scalars $\mathrm{b}_{j}$ using the form of the Lyndon words and the $q$-Jacobi identity: $\mathrm{b}_{1}=q_{12}^{2} q_{13} q_{23} q(1-q), \mathrm{b}_{2}=q_{12}^{2} q_{13} q(1-q), \mathrm{b}_{3}=q_{12} q_{13} q_{23}^{3} q(1+q), \mathrm{b}_{5}=q_{12} q_{23} q(1+q)$.

Hence $q_{13} q_{23} \mathrm{~b}_{1} \mathrm{~b}_{5}-\mathrm{b}_{2} \mathrm{~b}_{3}=0$. Thus the coefficient of $\mathrm{x}_{12{ }_{2} 3}^{3} \otimes 1$ in $d(c)$ is zero for all 2-chains $c$, so $\left(\mathrm{x}_{12^{2} 3}^{3}\right)^{*}$ is a 2-cocycle.

○ For $\gamma=12^{3} 3$, we will apply Proposition 6.3.2. The pairs $(\alpha, \beta)$ as in (6.3.3) are $\left(1,2^{3} 3\right)$, $\left(12,2^{2} 3\right),\left(12^{2} 3,2\right)$, since the following relations hold:

$$
x_{\alpha} x_{\beta}=x_{\gamma}+q_{\alpha \beta} x_{\beta} x_{\alpha}, \quad x_{\alpha} x_{\gamma}=q_{\alpha} x_{\gamma} x_{\alpha}, \quad x_{\gamma} x_{\beta}=q_{\beta} x_{\beta} x_{\gamma} .
$$

As $-\frac{q_{\alpha \alpha}}{q_{\beta \beta}}=q$, respectively $q^{2}, q^{3}, L$ should be a multiple of $N$. 
Let $L=\operatorname{ord}(-q)$. Now (6.3.9) holds for $\alpha=12, \beta=12^{2} 3, \delta=2^{3} 3$, and the root vectors satisfy (10.1.11); the scalars $\widetilde{q}_{\alpha \gamma}=q^{-1}, \widetilde{q}_{\beta \gamma}=q^{-2}$ satisfy (6.3.10).

Also, (6.3.7) holds for $\alpha=12, \beta=12^{4} 3^{2}, \delta=2^{3} 3, \eta=123, \tau=12^{2} 3$, and the root vectors satisfy (10.1.16); the scalars $\widetilde{q}_{\alpha \gamma}=q^{-1}, \widetilde{q}_{\beta \gamma}=q^{-1}, \widetilde{q}_{\tau \gamma}=q^{-2}$ satisfy (6.3.8).

Let $\left(n_{\delta}\right)_{\delta \in \Delta_{+}^{\mathfrak{q}}}$ be a solution of (6.3.1). If $n_{2}=0$, then

$$
N\left(\alpha_{1}+\alpha_{2}+\alpha_{3}\right)=s_{2}(N \gamma)=\sum_{\delta \in \Delta_{+}^{\mathfrak{q}}} f_{\delta}\left(n_{\delta}\right) s_{3}(\delta),
$$

and $s_{2}(\delta) \in \Delta_{+}^{\mathfrak{q}}$ if $\delta \neq \alpha_{2}$. As $N_{\delta}=N_{s_{2}(\delta)}$, we have that $f_{\delta}=f_{s_{2}(\delta)}$, so we have a system as in (6.3.1) for $\alpha_{1}+\alpha_{2}+\alpha_{3}$ in place of $\gamma$. The new system has four solutions, which gives place to the following solutions of the original system:

- $n_{12}=n_{2^{2} 3}=1, n_{12^{3} 3}=N-1, n_{\delta}=0$ for all the other $\delta \in \Delta_{+}^{\mathfrak{q}}$;

- $n_{1}=n_{2^{3} 3}=1, n_{12^{3} 3}=N-1, n_{\delta}=0$ for all the other $\delta \in \Delta_{+}^{\mathfrak{q}}$;

- $n_{12}=n_{2^{3} 3}=n_{12^{2} 3}=1, n_{12^{3} 3}=N-2, n_{\delta}=0$ for all the other $\delta \in \Delta_{+}^{\mathfrak{q}}$;

- $n_{12}=2, n_{12^{4} 3^{2}}=n_{2^{3} 3}=1, n_{12^{3} 3}=N-3, n_{\delta}=0$ for all the other $\delta \in \Delta_{+}^{\mathfrak{q}}$.

Next we assume $n_{2} \neq 0$. Suppose that $n_{12^{2} 3}>1$. Then $f_{12^{2} 3}\left(n_{12^{2} 3}\right) \geq N$, so the coefficient of $\alpha_{1}$ in $\sum_{\delta \in \Delta_{+}^{\mathrm{q}}} f_{\delta}\left(n_{\delta}\right) \delta$ is $\geq N$; this forces to $n_{\delta}=0$ for any $\delta \neq \alpha_{2}$ since $f_{\delta}\left(n_{\delta}\right) \delta$ must have $\alpha_{1}, \alpha_{3}$ with coefficient zero, and this gives a contradiction. Hence $n_{12^{2} 3}=f_{12^{2} 3}\left(n_{12^{2} 3}\right) \leq 1$. The coefficcient of $\alpha_{1}$ in this sum is

$$
n_{1}+n_{12}+n_{123}+n_{12^{2} 3}+n_{12^{3} 3}+n_{12^{3} 3^{2}}+n_{12^{4} 3^{2}}=N \text {. }
$$

As the sum of all $n_{\delta}$ 's is $N+1$ and $n_{2} \neq 0$, we have $n_{2}=1, n_{3}=n_{2^{3} 3}=n_{2^{2} 3}=n_{2^{3} 3^{2}}=$ $n_{23}=0$. Now we look at the coefficients of $\alpha_{2}, \alpha_{3}$ in the equality $\sum_{\delta \in \Delta_{+}^{\mathfrak{q}}} f_{\delta}\left(n_{\delta}\right) \delta=N \gamma$ :

$$
\begin{aligned}
3 N & =1+n_{12}+n_{123}+2 n_{12^{2} 3}+3 n_{12^{3} 3}+3 n_{12^{3} 3^{2}}+4 n_{12^{4} 3^{2}}, \\
N & =n_{123}+n_{12^{2} 3}+n_{12^{3} 3}+2 n_{12^{3} 3^{2}}+2 n_{12^{4} 3^{2}} .
\end{aligned}
$$

Thus $n_{1}+2 n_{123}+n_{12^{3} 3}+2 n_{12^{3} 3^{2}}+4 n_{12^{4} 3^{2}}=1$, which implies that $n_{123}=n_{12^{3} 3^{2}}=0$ and two of the three numbers $n_{1}, n_{12^{3} 3}, n_{12^{4} 3^{2}}$ are zero (the remaining one being 1 ). Reducing the three previous equations, we get $n_{12}+n_{12^{3} 3}=N-1, n_{12}+3 n_{12^{3} 3} \geq 3 N-3$, so we have a unique solution:

- $n_{12^{2} 3}=n_{2}=1, n_{12^{3} 3}=N-1, n_{\delta}=0$ for all the other $\delta \in \Delta_{+}^{\mathfrak{q}}$.

Hence Proposition 6.3.2 applies and $\left(\mathrm{x}_{12^{3} 3}^{L}\right)^{*}$ is a cocycle.

○ For $\gamma=12^{3} 3^{2}$, we will apply Proposition 6.3.2. The pairs $(\alpha, \beta)$ as in (6.3.3) are $\left(1,2^{3} 3^{2}\right)$, $\left(123,2^{2} 3\right),\left(12^{2} 3,23\right),\left(12^{3} 3,3\right)$, since the following relations hold:

$$
x_{\alpha} x_{\beta}=x_{\gamma}+q_{\alpha \beta} x_{\beta} x_{\alpha}, \quad x_{\alpha} x_{\gamma}=q_{\alpha} x_{\gamma} x_{\alpha}, \quad x_{\gamma} x_{\beta}=q_{\beta} x_{\beta} x_{\gamma} .
$$

As $-\frac{q_{\alpha \alpha}}{q_{\beta \beta}} \in\left\{q, q^{2}, q^{3}\right\}, L$ should be a multiple of $N$.

Let $L=\operatorname{ord}(-q)$. Now 6.3 .9 holds for $\alpha=123, \beta=12^{2} 3, \delta=2^{3} 3^{2}$, and the root vectors satisfy (10.1.11); the scalars $\widetilde{q}_{\alpha \gamma}=q^{-1}, \widetilde{q}_{\beta \gamma}=q^{-2}$ satisfy (6.3.10).

Also, (6.3.7) holds for $\alpha=123, \beta=12^{4} 3^{2}, \delta=2^{3} 3^{2}, \eta=12, \tau=12^{2} 3$, and the root vectors satisfy (10.1.16); the scalars $\widetilde{q}_{\alpha \gamma}=q^{-1}, \widetilde{q}_{\beta \gamma}=q^{-1}, \widetilde{q}_{\tau \gamma}=q^{-2}$ satisfy (6.3.8). 
Let $\left(n_{\delta}\right)_{\delta \in \Delta_{+}^{\mathfrak{q}}}$ be a solution of (6.3.1). If $n_{3}=0$, then

$$
N\left(\alpha_{1}+3 \alpha_{2}+\alpha_{3}\right)=s_{3}(N \gamma)=\sum_{\delta \in \Delta_{+}^{\mathfrak{q}}} f_{\delta}\left(n_{\delta}\right) s_{3}(\delta),
$$

and $s_{3}(\delta) \in \Delta_{+}^{\mathfrak{q}}$ if $\delta \neq \alpha_{3}$. As $N_{\delta}=N_{s_{3}(\delta)}$, we have that $f_{\delta}=f_{s_{3}(\delta)}$, so we have a system as in (6.3.1) for $\alpha_{1}+3 \alpha_{2}+\alpha_{3}$ in place of $\gamma$. The new system has five solutions, which gives place to the following solutions of the original system:

- $n_{12^{2} 3}=n_{23}=1, n_{12^{3} 3^{2}}=N-1, n_{\delta}=0$ for all the other $\delta \in \Delta_{+}^{\mathfrak{q}}$;

- $n_{123}=n_{2^{2} 3}=1, n_{12^{3} 3^{2}}=N-1, n_{\delta}=0$ for all the other $\delta \in \Delta_{+}^{\mathfrak{q}}$;

- $n_{1}=n_{2^{3} 3^{2}}=1, n_{12^{3} 3^{2}}=N-1, n_{\delta}=0$ for all the other $\delta \in \Delta_{+}^{\mathfrak{q}}$;

- $n_{123}=n_{2^{3} 3^{2}}=n_{12^{2} 3}=1, n_{12^{3} 3^{2}}=N-2, n_{\delta}=0$ for all the other $\delta \in \Delta_{+}^{\mathfrak{q}}$;

- $n_{123}=2, n_{12^{4} 3^{2}}=n_{2^{3} 3^{2}}=1, n_{12^{3} 3^{2}}=N-3, n_{\delta}=0$ for all the other $\delta \in \Delta_{+}^{\mathfrak{q}}$.

Next we assume $n_{3} \neq 0$. An analogous analysis as for the root $12^{3} 3$ shows that the unique solution is:

- $n_{12^{3} 3}=n_{3}=1, n_{12^{3} 3^{2}}=N-1, n_{\delta}=0$ for all the other $\delta \in \Delta_{+}^{\mathfrak{q}}$.

Hence Proposition 6.3 .2 applies and $\left(\mathrm{x}_{12^{3} 3^{2}}^{L}\right)^{*}$ is a cocycle.

- For $\gamma=12^{4} 3^{2}$, we apply Proposition 6.3.2 again. The pairs $(\alpha, \beta)$ as in (6.3.3) are $\left(12,2^{3} 3^{2}\right),\left(12^{3} 3,23\right),\left(12^{2} 3,2^{2} 3\right),\left(123,2^{3} 3\right),\left(12^{3} 3^{2}, 2\right)$, since the following relations hold:

$$
x_{\alpha} x_{\beta}=x_{\gamma}+q_{\alpha \beta} x_{\beta} x_{\alpha}, \quad x_{\alpha} x_{\gamma}=q_{\alpha} x_{\gamma} x_{\alpha}, \quad x_{\gamma} x_{\beta}=q_{\beta} x_{\beta} x_{\gamma} .
$$

As $-\frac{q_{\alpha \alpha}}{q_{\beta \beta}} \in\left\{q, q^{2}, q^{3}\right\}, L$ should be a multiple of $N$.

Let $L=\operatorname{ord}(-q)$. Now (6.3.9) holds for $\alpha=12^{3} 3, \beta=12^{2} 3, \delta=2^{3} 3^{2}$, and the root vectors satisfy (10.1.11); the scalars $\widetilde{q}_{\alpha \gamma}=q^{-1}, \widetilde{q}_{\beta \gamma}=q^{-2}$ satisfy (6.3.10).

Also, 6.3.7 holds for $\alpha=12^{3} 3, \beta=12^{3} 3^{2}, \delta=2^{3} 3^{2}, \eta=1, \tau=12^{2} 3$, and the root vectors satisfy (10.1.16); the scalars $\widetilde{q}_{\alpha \gamma}=q^{-1}, \widetilde{q}_{\beta \gamma}=q^{-1}, \widetilde{q}_{\tau \gamma}=q^{-2}$ satisfy (6.3.8).

Let $\left(n_{\delta}\right)_{\delta \in \Delta_{+}^{\mathfrak{q}}}$ be a solution of (6.3.1). If $n_{2}=0$, then

$$
N\left(\alpha_{1}+3 \alpha_{2}+2 \alpha_{3}\right)=s_{2}(N \gamma)=\sum_{\delta \in \Delta_{+}^{\mathfrak{q}}} f_{\delta}\left(n_{\delta}\right) s_{2}(\delta),
$$

and $s_{2}(\delta) \in \Delta_{+}^{\mathfrak{q}}$ if $\delta \neq \alpha_{2}$. As $N_{\delta}=N_{s_{2}(\delta)}$, we have that $f_{\delta}=f_{s_{2}(\delta)}$, so we have a system as in (6.3.1) for $\alpha_{1}+3 \alpha_{2}+2 \alpha_{3}$ in place of $\gamma$. The new system has six solutions, which gives place to the following solutions of the original system:

- $n_{12^{2} 3}=n_{2^{2} 3}=1, n_{12^{4} 3^{2}}=N-1, n_{\delta}=0$ for all the other $\delta \in \Delta_{+}^{\mathfrak{q}}$;

- $n_{12^{3} 3^{2}}=n_{2^{2} 3}=1, n_{12^{4} 3^{2}}=N-1, n_{\delta}=0$ for all the other $\delta \in \Delta_{+}^{\mathfrak{q}}$;

- $n_{12}=n_{2^{3} 3^{2}}=1, n_{12^{4} 3^{2}}=N-1, n_{\delta}=0$ for all the other $\delta \in \Delta_{+}^{\mathfrak{q}}$;

- $n_{123}=n_{2^{3} 3}=1, n_{12^{4} 3^{2}}=N-1, n_{\delta}=0$ for all the other $\delta \in \Delta_{+}^{\mathfrak{q}}$;

- $n_{12^{3} 3}=n_{2^{3} 3^{2}}=n_{12^{2} 3}=1, n_{12^{4} 3^{2}}=N-2, n_{\delta}=0$ for all the other $\delta \in \Delta_{+}^{\mathfrak{q}}$;

- $n_{12^{3} 3}=2, n_{12^{3} 3^{2}}=n_{2^{3} 3^{2}}=1, n_{12^{4} 3^{2}}=N-3, n_{\delta}=0$ for all the other $\delta \in \Delta_{+}^{\mathfrak{q}}$.

Next we assume $n_{2} \neq 0$. An analogous analysis as for the root $12^{3} 3$ shows that the unique solution is:

- $n_{12^{3} 3^{2}}=n_{2}=1, n_{12^{4} 3^{2}}=N-1, n_{\delta}=0$ for all the other $\delta \in \Delta_{+}^{\mathfrak{q}}$. 
Hence Proposition 6.3 .2 applies and $\left(\mathrm{x}_{12^{4} 3^{2}}^{L}\right)^{*}$ is a cocycle.

\section{Parametric modular types}

9.1. Modular type wk(4). Here $\theta=4, q \neq \pm 1$. In this subsection, we deal with a Nichols algebra $\mathscr{B}_{\mathfrak{q}}$ of diagonal type wk(4). We may assume that the corresponding diagram is

$$
\stackrel{q}{\circ} \stackrel{q^{-1}}{-1} \stackrel{-1}{\circ} \stackrel{-1}{\circ} \stackrel{-q}{\circ} \stackrel{-q^{-1}}{\circ} .
$$

We fix the following convex order on $\Delta_{+}^{\mathfrak{q}}$ :

$$
1,12,2,12^{2} 3,123,23,3,12^{2} 3^{2} 4,123^{2} 4,23^{2} 4,12^{2} 34,1234,234,34,4 \text {. }
$$

For more information, see $\underline{\underline{\mathrm{AA}}}, \S 7.1]$. Let $M=\operatorname{ord}(-q)$ : We may assume that $N \leq M$. Note that

$$
N_{\delta}= \begin{cases}N & \text { if } \delta \in\left\{1,123^{2} 4,23^{2} 4\right\}, \\ M & \text { if } \delta \in\left\{4,12^{2} 34,12^{2} 3\right\}, \\ 2 & \text { otherwise. }\end{cases}
$$

We prove Condition 1.4.1 for type wk(4):

Proposition 9.1.2. For every $\gamma \in \Delta_{+}^{\mathfrak{q}}$, there exists $L_{\gamma} \in \mathbb{N}$ such that $\left(\mathrm{x}_{\gamma}^{L_{\gamma}}\right)^{*}$ is a cocycle.

Proof. We may assume that $\gamma$ has full support i.e. $\gamma \in\left\{12^{2} 3^{2} 4,1234,123^{2} 4,12^{2} 34\right\}$.

First we consider $\gamma=12^{2} 3^{2} 4$. Here $N_{\gamma}=2$. The pairs $\alpha<\beta$ such that $\alpha+\beta=\gamma$ are:

$$
\left(3,12^{2} 34\right), \quad(23,1234), \quad(123,234), \quad\left(12^{2} 3,34\right), \quad\left(2,123^{2} 4\right), \quad\left(12,23^{2} 4\right) .
$$

For all pairs, $x_{\alpha} x_{\gamma}=q_{\alpha \gamma} x_{\gamma} x_{\alpha}, x_{\gamma} x_{\beta}=q_{\gamma \beta} x_{\beta} x_{\gamma}$. Also, there exist $\mathrm{b}_{i} \in \mathbb{k}$ such that

$$
\begin{array}{ll}
{\left[x_{3}, x_{122^{2} 34}\right]_{c}=\mathrm{b}_{1} x_{\gamma},} & {\left[x_{123}, x_{234}\right]_{c}=\mathrm{b}_{2} x_{\gamma}+\mathrm{b}_{3} x_{1234} x_{23},} \\
{\left[x_{23}, x_{1234}\right]_{c}=\mathrm{b}_{4} x_{\gamma},} & {\left[x_{122_{3} 3}, x_{34}\right]_{c}=\mathrm{b}_{5} x_{\gamma}+\mathrm{b}_{6} x_{3} x_{12^{2} 34}+\mathrm{b}_{7} x_{23} x_{1234}+\mathrm{b}_{8} x_{234} x_{123},} \\
{\left[x_{2}, x_{123^{2} 4}\right]_{c}=\mathrm{b}_{9} x_{\gamma},} & {\left[x_{12}, x_{23^{2} 4}\right]_{c}=\mathrm{b}_{10} x_{\gamma}+\mathrm{b}_{11} x_{123^{2} 4} x_{2} .}
\end{array}
$$

Thus the root vectors satisfy (10.1.8), and $-\frac{q_{\alpha \alpha}}{q_{\beta} \beta} \in\left\{-1, \pm q^{ \pm 1}\right\}$; hence we take $L=M$.

Next we check that $\left(23,123^{2} 4,12^{2} 34,3\right),\left(123,23^{2} 4,12^{2} 34,3\right)$ and $\left(12^{2} 3,123^{2} 4,234,123\right)$ are 4-tuples $(\alpha, \beta, \delta, \eta)$ satisfying (6.3.5). As $\widetilde{q}_{\alpha \gamma}=-1, \widetilde{q}_{\beta \gamma}=q$, we have that $c_{\alpha \beta \gamma}^{(L)}=$ $\mathrm{c}_{-1, q}^{(L)}=0$ by Lemma 6.3.28 (b). Hence (B) holds.

Finally we compute the solutions of (6.3.1). That is,

$$
\begin{aligned}
\sum_{\delta: 1 \in \operatorname{supp} \delta} f_{\delta}\left(n_{\delta}\right) & =M, \quad \sum_{\delta: 4 \in \operatorname{supp} \delta} f_{\delta}\left(n_{\delta}\right)=M \\
\sum_{\delta: 2 \in \operatorname{supp} \delta} f_{\delta}\left(n_{\delta}\right) a_{2}^{\delta} & =2 M, \quad \sum_{\delta: 3 \in \operatorname{supp} \delta} f_{\delta}\left(n_{\delta}\right) a_{3}^{\delta}=2 M . \\
\sum_{\delta \in \Delta_{+}^{\mathfrak{q}}} n_{\delta} & =M+1 .
\end{aligned}
$$


Let $\left(n_{\delta}\right)$ be a solution of (6.3.1). We claim that $n_{\delta} \leq 1$ if $N_{\delta} \neq 2$. To prove it, first we note that $n_{4}, n_{12^{2} 3}, n_{12^{2} 34} \leq 2$ by (9.1.3). If $n_{4}=2$, then $n_{\delta}=0$ if $4 \in \operatorname{supp} \delta, \delta \neq 4$, so

$$
\begin{aligned}
2 M & =\sum_{\delta \in \Delta_{+}^{\mathfrak{q}}} f_{\delta}\left(n_{\delta}\right) a_{3}^{\delta}=f_{12^{2} 3}\left(n_{12^{2} 3}\right)+n_{123}+n_{23}+n_{3} \leq f_{12^{2} 3}(2)+\sum_{\delta \neq 4,12^{2} 3} n_{\delta} \\
& \leq M+(M-1)=2 M-1,
\end{aligned}
$$

a contradiction. Hence $n_{4} \leq 1$. Next we suppose that $n_{12^{2} 3}=2$. Then $n_{\delta}=0$ if either $1 \in \operatorname{supp} \delta$ or else $2 \in \operatorname{supp} \delta, \delta \neq 12^{2} 3$. From (9.1.5), $n_{3}+n_{34}+n_{4}=M+1-n_{12^{2} 3}=M-1$, but we check directly that there are no solutions of (9.1.3) and (9.1.4) with these conditions. Hence $n_{12^{2} 3} \leq 1$. Analogously, $n_{12^{2} 34} \leq 1$.

Next we check that $n_{1}, n_{23^{2} 4}, n_{123^{2} 4} \leq 1$. The proof is analogous to the cases $n_{4}, n_{12^{2} 3}$, $n_{12^{2} 34}$ if $N=M$. Thus we assume that $N<M$ : that is, $M=2 N, N$ odd. By (9.1.3), $n_{1}, n_{23^{2} 4}, n_{123^{2} 4} \leq 4$. We deal first with $n_{123^{2} 4}$.

- Suppose that $n_{123^{2} 4}=4$. Then $n_{\delta}=0$ if $\operatorname{supp} \delta \cap\{1,3,4\} \neq \emptyset$ by (9.1.3) and (9.1.4), and also $n_{2}=2 N$. But from (9.1.5), $n_{2}=2 N-3$, a contradiction.

- Suppose that $n_{123^{2} 4}=3$. Then $\sum_{\delta \neq 123^{2} 4} n_{\delta}=2 N-2$. By (9.1.4),

$$
n_{12^{2} 3^{2} 4}=f_{12^{2} 3^{2} 4}\left(n_{12^{2} 3^{2} 4}\right) a_{4}^{12^{2} 3^{2} 4} \leq N-1 .
$$

By the first equality of (9.1.4) and the previous computations

$$
\begin{aligned}
3 N-1 & =\sum_{\delta \neq 123^{2} 4} f_{\delta}\left(n_{\delta}\right) a_{2}^{\delta}=2 f_{12^{2} 3^{2} 4}\left(n_{12^{2} 3^{2} 4}\right)+2 f_{12^{2} 3}\left(n_{12^{2} 3}\right)+2 f_{12^{2} 34}\left(n_{12^{2} 34}\right) \\
& +\sum_{\delta: a_{2}^{\delta}=1} n_{\delta} \leq 2 n_{12^{2} 3^{2} 4}+2+2+\left(2 N-2-n_{12^{2} 3^{2} 4}\right) \leq 3 N-1 .
\end{aligned}
$$

The equality holds if and only if $n_{12^{2} 3^{2} 4}=N-1, n_{12^{2} 3}=n_{12^{2} 34}=1$, but in this case the second equation of (9.1.4) does not hold.

- Suppose that $n_{123^{2} 4}=2$. Then $\sum_{\delta \neq 123^{2} 4} n_{\delta}=2 N-1$, and by (9.1.4), $n_{12^{2} 3^{2} 4} \leq$ $N$. A similar computation as for the previous case shows that the equality $3 N-1=$ $\sum_{\delta \neq 123^{2} 4} f_{\delta}\left(n_{\delta}\right) a_{2}^{\delta}$ holds if and only if $n_{12^{2} 3^{2} 4}=N, n_{12^{2} 3}=n_{12^{2} 34}=1$, but again the second equation of (9.1.4) does not hold.

The same argument applies for $n_{23^{2} 4}$. Finally we check that $n_{1} \leq 1$. If $n_{1}=4$, then $n_{\delta}=0$ for all $\delta \neq 4$ such that $4 \in \operatorname{supp} \delta$, so

$$
2 M=\sum_{\delta: 3 \in \operatorname{supp} \delta} f_{\delta}\left(n_{\delta}\right) a_{3}^{\delta}=n_{3}+n_{23}+n_{123}+n_{12^{2} 3} \leq \sum_{\delta \neq 4} n_{\delta} \stackrel{9.1 .5)}{=} M-3,
$$

a contradiction. Now suppose that $2 \leq n_{1} \leq 3$ : by (9.1.3), $n_{12^{2} 3^{2} 4} \leq N$ and

$$
4 N=\sum_{\delta \neq 123^{2} 4} f_{\delta}\left(n_{\delta}\right) a_{3}^{\delta} \leq 2 n_{12^{2} 3^{2} 4}+2+2+\left(2 N-2-n_{12^{2} 3^{2} 4}\right) \leq 3 N+2,
$$

a contradiction. Hence $n_{\delta} \leq 1$ if $N_{\delta} \neq 2$, so $f_{\delta}\left(n_{\delta}\right)=n_{\delta}$ for all $\delta \in \Delta_{+}^{\mathfrak{q}}$. Then we look for $\gamma_{i} \in \Delta_{+}^{\mathfrak{q}}, i \in \mathbb{I}_{M+1}$, such that $\sum_{i \in \mathbb{I}_{M+1}} \gamma_{i}=M \gamma$. As $a_{3}^{\delta} \leq 2$ for all $\delta \in \Delta_{+}^{\mathfrak{q}}$, there exist two possibilities up to permutations of these roots: 
$\circ a_{3}^{\gamma_{i}}=2$ for $\mathbb{I}_{M}, a_{3}^{\gamma_{M+1}}=0$. As $a_{2}^{\delta} \leq 2$ for all $\delta \in \Delta_{+}^{\mathfrak{q}}$, at least $M-1$ roots satisfy that $a_{2}^{\gamma_{i}}=2$, and we know that $a_{2}^{\gamma_{M+1}} \leq 1$, so $\gamma_{i}=\gamma$ for $i \in \mathbb{I}_{M-1}$ up to permutation, and $\gamma_{M}+\gamma_{M+1}=\gamma$.

$\circ a_{3}^{\gamma_{i}}=2$ for $\mathbb{I}_{M-1}, a_{3}^{\gamma_{M}}=a_{3}^{\gamma_{M+1}}=1$. Again at least $M-1$ roots satisfy that $a_{2}^{\gamma_{i}}=2$. If these roots are $\gamma_{i}, i \in \mathbb{I}_{M-1}$, then $\gamma_{i}=\gamma$ for $i \in \mathbb{I}_{M-1}$ up to permutation, and $\gamma_{M}+\gamma_{M+1}=\gamma$. Otherwise we may assume that $\gamma_{i}=\gamma$ for $i \in \mathbb{I}_{M-2}, a_{2}^{\gamma_{M-1}}=a_{2}^{\gamma_{M}}=1$, $a_{2}^{\gamma_{M+1}}=2$. We have three possibilities for $\left(\gamma_{M-1}, \gamma_{M}, \gamma_{M+1}\right)$ :

$$
\left(123^{2} 4,23,12^{2} 34\right), \quad\left(23^{2} 4,123,12^{2} 34\right), \quad\left(123^{2} 4,234,12^{2} 3\right) .
$$

Hence all the hypotheses of Proposition 6.3.2 hold, and $\left(\mathrm{x}_{\gamma}^{M}\right)^{*}$ is a cocycle.

Next we consider $\gamma=1234$. Here $N_{\gamma}=2$. The pairs $\alpha<\beta$ such that $\alpha+\beta=\gamma$ are $(1,234),(12,34),(123,4)$. For all pairs, $x_{\alpha} x_{\gamma}=q_{\alpha \gamma} x_{\gamma} x_{\alpha}, x_{\gamma} x_{\beta}=q_{\gamma \beta} x_{\beta} x_{\gamma}$ and there exist $\mathrm{b} \in \mathbb{k}$ such that $\left[x_{\alpha}, x_{\beta}\right]_{c}=\mathrm{b} x_{\gamma}$. Thus the root vectors satisfy (10.1.8) and $-\frac{q_{\alpha \alpha}}{q_{\beta \beta}} \in$ $\{-1, \pm q\}$; hence we take $L=M$.

Next we check that $\left(12,123^{2} 4,4,34\right),\left(1,123^{2} 4,34,234\right)$ and $\left(1,12^{2} 3^{2} 4,4,234\right)$ are 4 tuples $(\alpha, \beta, \delta, \eta)$ satisfying (6.3.15). As $\left(\widetilde{q}_{\delta \gamma}, \widetilde{q}_{\beta \gamma}\right)$ are respectively $\left(-q^{-1},-1\right),(-1, q)$, $\left(-q^{-1}, q\right)$, we have that $\mathrm{c}_{-\delta \alpha \gamma}^{(L)}=0$ by Lemma 6.3.28 (b). Hence $(\mathrm{G})$ holds.

Finally we check that $\left(1,123^{2} 4,12^{2} 34,4,234,34\right)$ is a 6 -tuple $(\alpha, \beta, \delta, \eta, \mu, \nu)$ satisfying (6.3.21). As $\widetilde{q}_{\alpha \gamma}=q=\widetilde{q}_{\beta \gamma}, \widetilde{q}_{\nu \gamma}=-1$, we have that $\mathrm{d}_{\beta-\nu \alpha \gamma}^{(L)}=0$ by Lemma 6.3.28 (c). Hence $(\mathrm{J})$ holds.

Next we look for solutions of (6.3.1). That is, (9.1.5) and

$$
\begin{array}{cc}
\sum_{\delta: 1 \in \operatorname{supp} \delta} f_{\delta}\left(n_{\delta}\right) & =M, \quad \sum_{\delta: 4 \in \operatorname{supp} \delta} f_{\delta}\left(n_{\delta}\right)=M, \\
\sum_{\delta: 2 \in \operatorname{supp} \delta} f_{\delta}\left(n_{\delta}\right) a_{2}^{\delta} & =M, \quad \sum_{\delta: 3 \in \operatorname{supp} \delta} f_{\delta}\left(n_{\delta}\right) a_{3}^{\delta}=M .
\end{array}
$$

Let $\left(n_{\delta}\right)$ be a solution of (6.3.1). We claim that $n_{\delta} \leq 1$ if $N_{\delta} \neq 2$. By (9.1.7),

$$
M \geq 2 f_{12^{2} 3}\left(n_{12^{2} 3}\right), 2 f_{12^{2} 34}\left(n_{12^{2} 34}\right) .
$$

As $N_{12^{2} 3}=N_{12^{2} 34}=M$ we have that $n_{12^{2} 3}, n_{12^{2} 34} \leq 1$. Now suppose that $n_{4} \geq 2$. By (9.1.6) we have that $n_{4}=2$. Then $n_{\delta}=0$ for all $\delta \neq 4$ such that $4 \in \operatorname{supp} \delta$. By (9.1.7),

$$
M=\sum_{\delta: 3 \in \operatorname{supp} \delta} f_{\delta}\left(n_{\delta}\right) a_{3}^{\delta}=n_{123}+n_{23}+n_{3} \leq \sum_{\delta \neq 4} n_{\delta}=M-1,
$$

a contradiction.

We also have that $n_{23^{2} 4}, n_{123^{2} 4} \leq 1$ if $N=M$, and $n_{23^{2} 4}, n_{123^{2} 4} \leq 2$ if $M=2 N$. Suppose that $M=2 N$ and $n_{1^{a} 23^{2} 4}=2, a \in\{0,1\}$. We have that $n_{\delta}=0$ for all $\delta$ such that $3 \in \operatorname{supp} \delta, \delta \neq 1^{a} 23^{2} 4$. By (9.1.6),$f_{4}\left(n_{4}\right)=M$ : that is, $n_{4}=2$, a contradiction.

Finally suppose that $n_{1} \geq 2$. By (9.1.6),

$$
M=\sum_{\delta: 4 \in \operatorname{supp} \delta} f_{\delta}\left(n_{\delta}\right)=\sum_{\delta: 4 \in \operatorname{supp} \delta} n_{\delta} \leq \sum_{\delta \neq 1} n_{\delta} \leq M-1,
$$


a contradiction. Hence $n_{\delta} \leq 1$ if $N_{\delta} \neq 2$, so $f_{\delta}\left(n_{\delta}\right)=n_{\delta}$ for all $\delta \in \Delta_{+}^{\mathfrak{q}}$. Then we look for $\gamma_{i} \in \Delta_{+}^{\mathfrak{q}}, i \in \mathbb{I}_{M+1}$, such that $\sum_{i \in \mathbb{I}_{M+1}} \gamma_{i}=M \gamma$. As $a_{1}^{\delta}, a_{4}^{\delta} \leq 1$ for all $\delta \in \Delta_{+}^{\mathfrak{q}}$, there exist exactly $M$ roots such that $a_{1}^{\gamma_{i}}=1$, respectively $a_{4}^{\gamma_{i}}=1$. Thus there exist $M-1$ roots $\gamma_{i}$ such that $a_{1}^{\delta}=a_{4}^{\delta}=1$, which implies that $\operatorname{supp} \gamma_{i}=\{1,2,3,4\}$ for these roots. We may assume that $a_{1}^{\gamma_{i}}=a_{4}^{\gamma_{i}}=1$ for all $i \in \mathbb{I}_{M-1}$ and we have three possibilities up to permutations of the roots: either $a_{1}^{\gamma_{M}}=a_{4}^{\gamma_{M}}=1, a_{1}^{\gamma_{M+1}}=a_{4}^{\gamma_{M+1}}=0$, or else $a_{1}^{\gamma_{M}}=a_{4}^{\gamma_{M+1}}=1, a_{1}^{\gamma_{M+1}}=a_{4}^{\gamma_{M}}=0$. For the first case, $a_{2}^{\gamma_{i}}, a_{3}^{\gamma_{i}} \geq 1$ for all $i \in \mathbb{I}_{M}$ and we have a contradiction. Hence $a_{1}^{\gamma_{M}}=a_{4}^{\gamma_{M+1}}=1, a_{1}^{\gamma_{M+1}}=a_{4}^{\gamma_{M}}=0$. For $i \in \mathbb{I}_{M-1}$ we write $\gamma_{i}=12^{a_{i}} 3^{b_{i}} 4, a_{i}, b_{i} \in \mathbb{I}_{2}$. We also write $\gamma_{M}=12^{a_{M}} 3^{b_{M}}, \gamma_{M+1}=2^{a_{M+1}} 3^{b_{M+1}} 4$. At most one of the $a_{i}$, respectively $b_{i}$, is 2 for $i \in \mathbb{I}_{M-1}$. We analyze each case.

$\circ a_{i}=1=b_{i}=1$ for all $i \in \mathbb{I}_{M-1}$. Hence $\gamma_{i}=\gamma$ for all $i \in \mathbb{I}_{M-1}$ and $\gamma_{M}+\gamma_{M+1}=\gamma$.

$\circ a_{i}=1$ for all $i \in \mathbb{I}_{M-1}, b_{i}=1$ for all $i \in \mathbb{I}_{M-2}, b_{M-1}=2$. Here $b_{M}=b_{M+1}=0$, so

$$
\gamma_{i}=\gamma \text { for all } i \in \mathbb{I}_{M-2}, \quad \gamma_{M-1}=123^{2} 4, \quad \gamma_{M}=12, \quad \gamma_{M+1}=4 .
$$

○ $a_{i}=1$ for all $i \in \mathbb{I}_{M-2}, b_{i}=1$ for all $i \in \mathbb{I}_{M-1}, a_{M-1}=2$. Here $a_{M}=a_{M+1}=0$, so

$$
\gamma_{i}=\gamma \text { for all } i \in \mathbb{I}_{M-2}, \quad \gamma_{M-1}=12^{2} 34, \quad \gamma_{M}=1, \quad \gamma_{M+1}=34 .
$$

$\circ a_{i}=b_{i}=1$ for all $i \in \mathbb{I}_{M-2}, a_{M-1}=b_{M-1}=2$. Here $a_{M}=b_{M}=a_{M+1}=b_{M+1}=0$, so

$$
\gamma_{i}=\gamma \text { for all } i \in \mathbb{I}_{M-2}, \quad \gamma_{M-1}=12^{2} 3^{2} 4, \quad \gamma_{M}=1, \quad \gamma_{M+1}=4 .
$$

$\circ a_{i}=1$ for all $i \in \mathbb{I}_{M-2}, b_{i}=1$ for all $i \in \mathbb{I}_{M-1}-\{M-2\}, a_{M-1}=b_{M-2}=2$. Here

$$
\gamma_{i}=\gamma \text { for all } i \in \mathbb{I}_{M-3}, \quad \gamma_{M-2}=123^{2} 4, \quad \gamma_{M-1}=12^{2} 34, \quad \gamma_{M}=1, \quad \gamma_{M+1}=4 .
$$

Hence all the hypotheses of Proposition 6.3.2 hold, and $\left(\mathrm{x}_{\gamma}^{M}\right)^{*}$ is a cocycle.

Finally we consider $\gamma=123^{2} 4,12^{2} 34$. We have $P_{\gamma}=2, Q_{\gamma}=1$, so $P_{\gamma}, Q_{\gamma}<N_{\gamma}$. By Lemma 6.2.5 $\left(\mathrm{x}_{\gamma}^{N_{\gamma}}\right)^{*}$ is a 2-cocycle.

9.2. Modular type $\operatorname{br}(2)$. Here $\theta=2, \zeta \in \mathbb{G}_{3}, q \notin \mathbb{G}_{3}$. In this subsection, we deal with a Nichols algebra $\mathscr{B}_{\mathfrak{q}}$ of modular type $\operatorname{br}(2)$, that is associated to any of the Dynkin diagrams

$$
a_{1} \longmapsto \stackrel{\zeta}{\circ} \stackrel{q^{-1}}{q} \stackrel{q}{\circ}, \quad a_{2} \longmapsto \stackrel{\zeta}{\zeta^{2} q} \stackrel{\zeta q^{-1}}{\circ} .
$$

For more information, see [AA, §7.2]. Since (9.2.1 b) has the same shape as (9.2.1 a) but with $\zeta q^{-1}$ instead of $q$, we just discuss the latter. Essentially this is very similar to standard $B_{2}$. The corresponding set of positive roots with full support is

$$
\left\{2 \alpha_{1}+\alpha_{2}, \alpha_{1}+\alpha_{2}\right\} \text {. }
$$

Let $M=\operatorname{ord}\left(\zeta q^{-1}\right)$. We order the root vectors: $x_{1}<x_{112}<x_{12}<x_{2}$.

We prove Condition 1.4 .1 for type $\operatorname{br}(2)$.

Proposition 9.2.2. For every $\gamma \in \Delta_{+}^{\mathfrak{q}},\left(\mathrm{x}_{\gamma}^{N_{\gamma}}\right)^{*}$ is a 2-cocycle.

Proof. As before we just consider non-simple roots, i.e. with full support. 
TABLE 2. The roots with full support of $\operatorname{br}(2) ; \gamma_{1}<\gamma_{2},\left(N_{\gamma}-1\right) \gamma=\gamma_{1}+\gamma_{2}$

\begin{tabular}{|c|c|c|c|cc|c|}
\hline$\gamma$ & $N_{\gamma}$ & $P_{\gamma}$ & $Q_{\gamma}$ & $\gamma_{1}$ & $\gamma_{2}$ & $L_{\gamma}$ \\
\hline $1^{2} 2$ & $M$ & 2 & 1 & 1 & 12 & 2 \\
\hline 12 & 3 & 3 & 2 & $1^{2} 2$ & 2 & $\operatorname{ord} q^{3}$ \\
\hline
\end{tabular}

- For $\gamma=1^{2} 2$, the case $N_{112}>2$ follows by Lemma 6.2.5. Assume now that $N_{112}=2$. We will apply Proposition 6.3.2. The unique pair as in (6.3.3) is $\alpha=\alpha_{1}, \beta=\alpha_{1}+\alpha_{2}$, since the following relations hold:

$$
x_{1} x_{12}=x_{112}+\zeta q_{12} x_{12} x_{1}, \quad x_{1} x_{112}=\zeta^{2} q_{12} x_{112} x_{1}, \quad x_{112} x_{12}=\zeta^{2} q_{12} x_{12} x_{112} .
$$

As $-\frac{q_{\alpha \alpha}}{q_{\beta \beta}}=-1$, we take $L=2$. The unique solution of (6.3.1) is $n_{112}=n_{1}=n_{12}=1$, and $n_{2}=0$. Hence Proposition 6.3 .2 applies and $\left(\mathrm{x}_{112}^{2}\right)^{*}$ is a 2-cocycle.

- For $\gamma=12$, we will apply Proposition 6.3.23. The unique pair as in (6.3.24) is $\alpha=$ $2 \alpha_{1}+\alpha_{2}, \beta=\alpha_{2}$, since the following relations hold:

$$
\begin{aligned}
& x_{112} x_{2}=(q-\zeta) q_{12} x_{12}^{2}+q q_{12}^{2} x_{2} x_{112}, \quad x_{112} x_{12}=\zeta^{2} q_{12} x_{12} x_{112}, \\
& x_{12} x_{2}=q q_{12} x_{2} x_{12} \text {. }
\end{aligned}
$$

In this case, $\frac{q_{\alpha \gamma}}{q_{\gamma \beta}}=\zeta^{2} q^{-1}$ : as $q \neq \zeta^{2}$, we take $L=$ ord $q^{3}$. The unique solution of (6.3.26) is $n_{12}=L-1, n_{12}=n_{2}=1$, and $n_{2}=0$, so Proposition 6.3.23 applies and $\left(\mathrm{x}_{12}^{L}\right)^{*}$ is a $2 L$-cocycle.

\section{Proofs of the Computational Lemmas}

10.1. Given $\gamma \in \Delta_{+}$, let $g_{\gamma}: \mathbb{N}_{0} \rightarrow \mathbb{N}_{0}$ be the function

$$
g_{\gamma}(n):=f_{\gamma}(n)-f_{\gamma}(n-1)= \begin{cases}1, & n \text { odd, } \\ N_{\gamma}-1 & n \text { even. }\end{cases}
$$

Remark 10.1.2. Let $\beta_{1}<\beta_{2}<\beta_{3}$ be positive roots such that the corresponding root vectors $q$-commute:

$$
x_{\beta_{i}} x_{\beta_{j}}=q_{\beta_{i} \beta_{j}} x_{\beta_{j}} x_{\beta_{i}}, \quad \text { for all } i<j .
$$

For each $n \in \mathbb{N}$,

$$
\begin{aligned}
d\left(\mathrm{x}_{\beta_{1}} \mathrm{x}_{\beta_{2}}^{f_{\beta_{2}}(n)} \otimes 1\right)= & \mathrm{x}_{\beta_{1}} \mathrm{x}_{\beta_{2}}^{f_{\beta_{2}}(n-1)} \otimes x_{\beta_{2}}^{g_{\beta_{2}}(n)}+(-1)^{n} q_{\beta_{1} \beta_{2}}^{f_{\beta_{2}}(n)} \mathrm{x}_{\beta_{2}}^{f_{\beta_{2}}(n)} \otimes x_{\beta_{1}}, \\
d\left(\mathrm{x}_{\beta_{1}}^{f_{\beta_{1}}(n)} \mathrm{x}_{\beta_{2}} \otimes 1\right)= & \mathrm{x}_{\beta_{1}}^{f_{\beta_{1}}(n)} \otimes x_{\beta_{2}}-q_{\beta_{1} \beta_{2}}^{g_{\beta_{1}}(n)} \mathrm{x}_{\beta_{1}}^{f_{\beta_{1}}(n-1)} \mathrm{x}_{\beta_{2}} \otimes x_{\beta_{1}}^{g_{\beta_{1}}(n)} \\
d\left(\mathrm{x}_{\beta_{1}} \mathrm{x}_{\beta_{2}}^{f_{\beta_{2}}(n)} \mathrm{x}_{\beta_{3}} \otimes 1\right)= & \mathrm{x}_{\beta_{1}} \mathrm{x}_{\beta_{2}}^{f_{\beta_{2}}(n)} \otimes x_{\beta_{3}}-q_{\beta_{2} \beta_{3}}^{g_{\beta_{2}}(n)} \mathrm{x}_{\beta_{1}} \mathrm{x}_{\beta_{2}}^{f_{\beta_{2}}(n-1)} \mathrm{x}_{\beta_{3}} \otimes x_{\beta_{2}}^{g_{\beta_{2}}(n)} \\
& -(-1)^{n} q_{\beta_{1} \beta_{3}} q_{\beta_{1} \beta_{2}}^{f_{\beta_{2}}(n)} \mathrm{x}_{\beta_{2}}^{f_{\beta_{2}}(n)} \mathrm{x}_{\beta_{3}} \otimes x_{\beta_{1}}, \\
d\left(\mathrm{x}_{\beta_{1}}^{f_{\beta_{1}}(n)} \mathrm{x}_{\beta_{2}} \mathrm{x}_{\beta_{3}} \otimes 1\right)= & \mathrm{x}_{\beta_{1}}^{f_{\beta_{1}}(n)} \mathrm{x}_{\beta_{2}} \otimes x_{\beta_{3}}-q_{\beta_{2} \beta_{3}} \mathrm{x}_{\beta_{1}}^{f_{\beta_{1}}(n)} \mathrm{x}_{\beta_{3}} \otimes x_{\beta_{2}} \\
& +q_{\beta_{1} \beta_{2}}^{g_{\beta_{1}}(n)} q_{\beta_{1} \beta_{3}}^{g_{\beta_{1}}(n)} \mathrm{x}_{\beta_{1}}^{f_{\beta_{1}}(n-1)} \mathrm{x}_{\beta_{2}} \mathrm{x}_{\beta_{3}} \otimes x_{\beta_{1}}^{g_{\beta_{1}}(n)}
\end{aligned}
$$


In fact, the root vectors $q$-commute by hypothesis so we can compute the differentials as in the proof of Proposition 4.3.3.

The next results will allow us to identify some cocycles of degree higher than 2 .

Lemma 10.1.7. Let $n \in \mathbb{N}_{0}$. Let $\alpha<\delta_{1}, \ldots, \delta_{n}<\gamma<\eta_{1}, \ldots, \eta_{n}<\beta$ be positive roots. Assume that the relations among the corresponding root vectors take the form

$$
\begin{array}{ll}
x_{\alpha} x_{\beta}=q_{\alpha \beta} x_{\beta} x_{\alpha}+\mathrm{b} x_{\gamma}^{N_{\gamma}-1}+\sum_{j=1}^{n} \mathrm{~b}_{j} x_{\eta_{j}} x_{\delta_{j}}, & \\
x_{\alpha} x_{\gamma}=q_{\alpha \gamma} x_{\gamma} x_{\alpha}, & x_{\delta_{j}} x_{\gamma}=q_{\delta_{j} \gamma} x_{\gamma} x_{\delta_{j}}, \\
x_{\gamma} x_{\beta}=q_{\gamma \beta} x_{\beta} x_{\gamma}, & x_{\gamma} x_{\eta_{j}}=q_{\gamma \eta_{j}} x_{\eta_{j}} x_{\gamma},
\end{array}
$$

for some scalars $\mathrm{b}, \mathrm{b}_{1}, \ldots, \mathrm{b}_{n}$. Then, for all $a \geq 1, d\left(\mathrm{x}_{\alpha} \mathrm{x}_{\gamma}^{a N_{\gamma}} \mathrm{x}_{\beta} \otimes 1\right)=$

$$
\begin{aligned}
& \mathrm{x}_{\alpha} \mathrm{x}_{\gamma}^{a N_{\gamma}} \otimes x_{\beta}-q_{\gamma \beta}^{N_{\gamma}-1} \mathrm{x}_{\alpha} \mathrm{x}_{\gamma}^{N_{\gamma}(a-1)+1} \mathrm{x}_{\beta} \otimes x_{\gamma}^{N_{\gamma}-1} \\
& -q_{\alpha \beta} q_{\alpha \gamma}^{a N_{\gamma}} \mathrm{x}_{\gamma}^{a N_{\gamma}} \mathrm{x}_{\beta} \otimes x_{\alpha}-\sum_{j=1}^{n} \mathrm{~b}_{j} q_{\alpha \gamma}^{a N_{\gamma}} \mathrm{x}_{\gamma}^{a N_{\gamma}} \mathrm{x}_{\eta_{j}} \otimes x_{\delta_{j}} \\
& +\mathrm{b} q_{\gamma \beta}^{a N_{\gamma}}\left\{\left(\frac{q_{\alpha \gamma}}{q_{\gamma \beta}}-1\right)(a)_{\left(\frac{q_{\alpha \gamma}}{q_{\gamma \beta}}\right)^{N_{\gamma}}}-\left(\frac{q_{\alpha \gamma}}{q_{\gamma \beta}}\right)^{a N_{\gamma}}\right\} \mathrm{x}_{\gamma}^{a N_{\gamma}+1} \otimes x_{\gamma}^{N_{\gamma}-2} ;
\end{aligned}
$$

and for all $a \geq 0, d\left(\mathrm{x}_{\alpha} \mathrm{x}_{\gamma}^{a N_{\gamma}+1} \mathrm{x}_{\beta} \otimes 1\right)=$

$$
\begin{aligned}
& \mathrm{x}_{\alpha} \mathrm{x}_{\gamma}^{a N_{\gamma}+1} \otimes x_{\beta}-q_{\gamma \beta} \mathrm{x}_{\alpha} \mathrm{x}_{\gamma}^{a N_{\gamma}} \mathrm{x}_{\beta} \otimes x_{\gamma}+q_{\alpha \beta} q_{\alpha \gamma}^{a N_{\gamma}+1} \mathrm{x}_{\gamma}^{a N_{\gamma}+1} \mathrm{x}_{\beta} \otimes x_{\alpha} \\
& +\sum_{j=1}^{n} \mathrm{~b}_{j} q_{\alpha \gamma}^{a N_{\gamma}+1} \mathrm{x}_{\gamma}^{a N_{\gamma}+1} \mathrm{x}_{\eta_{j}} \otimes x_{\delta_{j}}+\mathrm{b} q_{\gamma \beta}^{a N_{\gamma}+1}\left(\frac{q_{\alpha \gamma}}{q_{\gamma \beta}}-1\right)(a+1)\left(\frac{q_{\alpha \gamma}}{q_{\gamma \beta}}\right)^{N_{\gamma} \mathbf{x}_{\gamma}^{N_{\gamma}(a+1)} \otimes 1 .}
\end{aligned}
$$

Notice that the first equality in (10.1.8) forces

$$
\left(N_{\gamma}-1\right) \gamma=\alpha+\beta=\delta_{j}+\eta_{j} \quad \text { for all } j \in \mathbb{I}_{n} .
$$

Proof. We need the following computation:

$$
d\left(\mathrm{x}_{\alpha} \mathrm{x}_{\beta} \otimes 1\right)=\mathrm{x}_{\alpha} \otimes x_{\beta}-q_{\alpha \beta} \mathrm{x}_{\beta} \otimes x_{\alpha}-\mathrm{bx}_{\gamma} \otimes x_{\gamma}^{N_{\gamma}-2}-\sum_{j=1}^{n} \mathrm{~b}_{j} \mathrm{x}_{\eta_{j}} \otimes x_{\delta_{j}}
$$

The proof of the lemma is by induction on $a$. First we compute:

$$
\begin{aligned}
& d\left(\mathrm{x}_{\alpha} \mathrm{x}_{\gamma} \mathrm{x}_{\beta} \otimes 1\right)=\mathrm{x}_{\alpha} \mathrm{x}_{\gamma} \otimes x_{\beta}-s\left(d\left(\mathrm{x}_{\alpha} \mathrm{x}_{\gamma} \otimes 1\right) x_{\beta}\right) \\
& \quad=\mathrm{x}_{\alpha} \mathrm{x}_{\gamma} \otimes x_{\beta}-s\left(\mathrm{x}_{\alpha} \otimes x_{\gamma} x_{\beta}-q_{\alpha \gamma} \mathrm{x}_{\gamma} \otimes x_{\alpha} x_{\beta}\right) \\
& \quad=\mathrm{x}_{\alpha} \mathrm{x}_{\gamma} \otimes x_{\beta}-s\left(q_{\gamma \beta} \mathrm{x}_{\alpha} \otimes x_{\beta} x_{\gamma}-q_{\alpha \gamma} \mathrm{x}_{\gamma} \otimes\left(q_{\alpha \beta} x_{\beta} x_{\alpha}+\mathrm{b} x_{\gamma}^{N_{\gamma}-1}+\sum_{j=1}^{n} \mathrm{~b}_{j} x_{\eta_{j}} x_{\delta_{j}}\right)\right) \\
& =\mathrm{x}_{\alpha} \mathrm{x}_{\gamma} \otimes x_{\beta}-q_{\gamma \beta} \mathrm{x}_{\alpha} \mathrm{x}_{\beta} \otimes x_{\gamma}+s\left(q_{\alpha \gamma} q_{\alpha \beta}\left(\mathrm{x}_{\gamma} \otimes x_{\beta}-q_{\gamma \beta} \mathrm{x}_{\beta} \otimes x_{\gamma}\right) x_{\alpha}\right.
\end{aligned}
$$




$$
\begin{aligned}
& \left.+\mathrm{b}\left(q_{\alpha \gamma}-q_{\gamma \beta}\right) \mathrm{x}_{\gamma} \otimes x_{\gamma}^{N_{\gamma}-1}+\sum_{j=1}^{n} \mathrm{~b}_{j}\left(q_{\alpha \gamma} \mathrm{x}_{\gamma} \otimes x_{\eta_{j}}-q_{\gamma \beta} q_{\delta_{j} \gamma} \mathrm{x}_{\eta_{j}} \otimes x_{\gamma}\right) x_{\delta_{j}}\right) \\
& =\mathrm{x}_{\alpha} \mathrm{x}_{\gamma} \otimes x_{\beta}-q_{\gamma \beta} \mathrm{x}_{\alpha} \mathrm{x}_{\beta} \otimes x_{\gamma}+\sum_{j=1}^{n} \mathrm{~b}_{j} q_{\alpha \gamma} \mathrm{x}_{\gamma} \mathrm{x}_{\eta_{j}} \otimes x_{\delta_{j}}+q_{\alpha \gamma} q_{\alpha \beta} \mathrm{x}_{\gamma} \mathrm{x}_{\beta} \otimes x_{\alpha} \\
& +\mathrm{b}\left(q_{\alpha \gamma}-q_{\gamma \beta}\right) \mathrm{x}_{\gamma}^{N_{\gamma}} \otimes 1+s\left(\sum_{j=1}^{n} \mathrm{~b}_{j}\left(q_{\alpha \gamma} q_{\gamma \eta_{j}}-q_{\gamma \beta} q_{\delta_{j} \gamma}\right) s\left(1 \otimes x_{\eta_{j}} x_{\gamma} x_{\delta_{j}}\right)\right)
\end{aligned}
$$

which agrees with the second formula for $a=0$ since $s \circ s=0$. Next we compute

$$
\begin{aligned}
& d\left(\mathrm{x}_{\alpha} \mathrm{x}_{\gamma}^{N_{\gamma}} \mathrm{x}_{\beta} \otimes 1\right)=\mathrm{x}_{\alpha} \mathrm{x}_{\gamma}^{N_{\gamma}} \otimes x_{\beta}-s\left(d\left(\mathrm{x}_{\alpha} \mathrm{x}_{\gamma}^{N_{\gamma}} \otimes 1\right) x_{\beta}\right) \\
& =\mathrm{x}_{\alpha} \mathrm{x}_{\gamma}^{N_{\gamma}} \otimes x_{\beta}-s\left(q_{\gamma \beta}^{N_{\gamma}-1} \mathrm{x}_{\alpha} \mathrm{x}_{\gamma} \otimes x_{\beta} x_{\gamma}^{N_{\gamma}-1}+q_{\alpha \beta} q_{\alpha \gamma}^{N_{\gamma}} \mathbf{x}_{\gamma}^{N_{\gamma}} \otimes x_{\beta} x_{\alpha}\right. \\
& \left.\quad+q_{\alpha \gamma}^{N_{\gamma}} \mathrm{b} \mathrm{x}_{\gamma}^{N_{\gamma}} \otimes x_{\gamma}^{N_{\gamma}-1}+\sum_{j=1}^{n} \mathrm{~b}_{j} q_{\alpha \gamma}^{N_{\gamma}} \mathrm{x}_{\gamma}^{N_{\gamma}} \otimes x_{\eta_{j}} x_{\delta_{j}}\right) \\
& =\mathrm{x}_{\alpha} \mathrm{x}_{\gamma}^{N_{\gamma}} \otimes x_{\beta}-q_{\gamma \beta}^{N_{\gamma}-1} \mathrm{x}_{\alpha} \mathrm{x}_{\gamma} \mathrm{x}_{\beta} \otimes x_{\gamma}^{N_{\gamma}-1} \\
& \quad-\mathrm{b}\left(q_{\alpha \gamma}^{N_{\gamma}}-q_{\gamma \beta}^{N_{\gamma}-1} q_{\alpha \gamma}+q_{\gamma \beta}^{N_{\gamma}}\right) \mathrm{x}_{\gamma}^{N_{\gamma}+1} \otimes x_{\gamma}^{N_{\gamma}-2}-\sum_{j=1}^{n} \mathrm{~b}_{j} q_{\alpha \gamma}^{N_{\gamma}} \mathrm{x}_{\gamma}^{N_{\gamma}} \mathrm{x}_{\eta_{j}} \otimes x_{\delta_{j}} \\
& \quad-q_{\alpha \beta} q_{\alpha \gamma}^{N_{\gamma}} \mathrm{x}_{\gamma}^{N_{\gamma}} \mathrm{x}_{\beta} \otimes x_{\alpha},
\end{aligned}
$$

and by (10.1.9), this agrees with the first formula in the lemma when $a=1$. Now assume the second formula given in the lemma holds when $a$ is replaced by $a-1$. Then

$$
\begin{aligned}
d\left(\mathrm{x}_{\alpha} \mathrm{x}_{\gamma}^{a N_{\gamma}} \mathrm{x}_{\beta} \otimes 1\right)=\mathrm{x}_{\alpha} \mathrm{x}_{\gamma}^{a N_{\gamma}} \otimes x_{\beta}-s\left(d\left(\mathrm{x}_{\alpha} \mathrm{x}_{\gamma}^{a N_{\gamma}} \otimes 1\right) x_{\beta}\right) \\
=\mathrm{x}_{\alpha} \mathrm{x}_{\gamma}^{a N_{\gamma}} \otimes x_{\beta}-s\left(\mathrm{x}_{\alpha} \mathrm{x}_{\gamma}^{N_{\gamma}(a-1)+1} \otimes x_{\gamma}^{N_{\gamma}-1} x_{\beta}+q_{\alpha \gamma}^{a N_{\gamma}} \mathbf{x}_{\gamma}^{a N_{\gamma}} \otimes x_{\alpha} x_{\beta}\right) \\
=\mathrm{x}_{\alpha} \mathrm{x}_{\gamma}^{a N_{\gamma}} \otimes x_{\beta}-s\left(q_{\gamma \beta}^{N_{\gamma}-1} \mathbf{x}_{\alpha} \mathrm{x}_{\gamma}^{N_{\gamma}(a-1)+1} \otimes x_{\beta} x_{\gamma}^{N_{\gamma}-1}+q_{\alpha \beta} q_{\alpha \gamma}^{a N_{\gamma}} \mathbf{x}_{\gamma}^{a N_{\gamma}} \otimes x_{\beta} x_{\alpha}\right. \\
\left.\quad+\mathrm{b} q_{\alpha \gamma}^{a N_{\gamma}} \mathrm{x}_{\gamma}^{a N_{\gamma}} \otimes x_{\gamma}^{N_{\gamma}-1}+\sum_{j=1}^{n} \mathrm{~b}_{j} q_{\alpha \gamma}^{a N_{\gamma}} \mathbf{x}_{\gamma}^{a N_{\gamma}} \otimes x_{\eta_{j}} x_{\delta_{j}}\right) .
\end{aligned}
$$

Use the induction hypothesis to rewrite the term $q_{\gamma \beta}^{N_{\gamma}-1} \mathrm{x}_{\alpha} \mathrm{x}_{\gamma}^{N_{\gamma}(a-1)+1} \otimes x_{\beta} x_{\gamma}^{N_{\gamma}-1}$ to obtain

$$
\begin{aligned}
& d\left(\mathrm{x}_{\alpha} \mathrm{x}_{\gamma}^{a N_{\gamma}} \mathrm{x}_{\beta} \otimes 1\right)=\mathrm{x}_{\alpha} \mathrm{x}_{\gamma}^{a N_{\gamma}} \otimes x_{\beta}-s\left(q_{\gamma \beta}^{N_{\gamma}-1} d\left(\mathrm{x}_{\alpha} \mathrm{x}_{\gamma}^{(a-1) N_{\gamma}+1} \mathrm{x}_{\beta} \otimes x_{\gamma}^{N_{\gamma}-1}\right)\right. \\
& -q_{\alpha \beta} q_{\alpha \gamma}^{(a-1) N_{\gamma}+1} q_{\gamma \beta}^{N_{\gamma}-1} \mathrm{x}_{\gamma}^{(a-1) N_{\gamma}+1} \mathrm{x}_{\beta} \otimes x_{\alpha} x_{\gamma}^{N_{\gamma}-1} \\
& -\mathrm{b} q_{\gamma \beta}^{a N_{\gamma}}\left(\frac{q_{\alpha \gamma}}{q_{\gamma \beta}}-1\right)(a)\left(\frac{q_{\alpha \gamma}}{q_{\gamma \beta}}\right)^{N_{\gamma} \mathrm{x}_{\gamma}^{a N_{\gamma}} \otimes x_{\gamma}^{N_{\gamma}-1}} \\
& \quad-\sum_{j=1}^{n} \mathrm{~b}_{j} q_{\gamma \beta}^{N_{\gamma}-1} q_{\alpha \gamma}^{(a-1) N_{\gamma}+1} \mathrm{x}_{\gamma}^{(a-1) N_{\gamma}+1} \mathrm{x}_{\eta_{j}} \otimes x_{\delta_{j}} x_{\gamma}^{N_{\gamma}-1}+q_{\alpha \beta} q_{\alpha \gamma}^{a N_{\gamma}} \mathrm{x}_{\gamma}^{a N_{\gamma}} \otimes x_{\beta} x_{\alpha}
\end{aligned}
$$




$$
\begin{aligned}
& \left.+\mathrm{b} q_{\alpha \gamma}^{a N_{\gamma}} \mathbf{x}_{\gamma}^{a N_{\gamma}} \otimes x_{\gamma}^{N_{\gamma}-1}+\sum_{j=1}^{n} \mathrm{~b}_{j} q_{\alpha \gamma}^{a N_{\gamma}} \mathrm{x}_{\gamma}^{a N_{\gamma}} \otimes x_{\eta_{j}} x_{\delta_{j}}\right) \\
= & \mathrm{x}_{\alpha} \mathrm{x}_{\gamma}^{a N_{\gamma}} \otimes x_{\beta}-q_{\gamma \beta}^{N_{\gamma}-1} \mathrm{x}_{\alpha} \mathrm{x}_{\gamma}^{(a-1) N_{\gamma}+1} \mathrm{x}_{\beta} \otimes x_{\gamma}^{N_{\gamma}-1} \\
& +s\left(q_{\alpha \beta} q_{\alpha \gamma}^{a N_{\gamma}} q_{\gamma \beta}^{N_{\gamma}-1} \mathrm{x}_{\gamma}^{(a-1) N_{\gamma}+1} \mathrm{x}_{\beta} \otimes x_{\gamma}^{N_{\gamma}-1} x_{\alpha}\right. \\
& +\mathrm{b} q_{\gamma \beta}^{a N_{\gamma}}\left(\left(\frac{q_{\alpha \gamma}}{q_{\gamma \beta}}-1\right)(a)\left(\frac{q \alpha \gamma}{q_{\gamma \beta}}\right)^{N_{\gamma}}-\frac{q_{\alpha \gamma}^{a N_{\gamma}}}{q_{\gamma \beta}^{a N_{\gamma}}}\right) \mathrm{x}_{\gamma}^{a N_{\gamma}} \otimes x_{\gamma}^{N_{\gamma}-1}-q_{\alpha \beta} q_{\alpha \gamma}^{a N_{\gamma}} \mathrm{x}_{\gamma}^{a N_{\gamma}} \otimes x_{\beta} x_{\alpha} \\
& \left.+\sum_{j=1}^{n} \mathrm{~b}_{j} q_{\gamma \beta}^{N_{\gamma}-1} q_{\alpha \gamma}^{(a-1) N_{\gamma}+1} q_{\delta_{j} \gamma}^{N_{\gamma}-1} \mathrm{x}_{\gamma}^{(a-1) N_{\gamma}+1} \mathrm{x}_{\eta_{j}} \otimes x_{\gamma}^{N_{\gamma}-1} x_{\delta_{j}}-\sum_{j=1}^{n} \mathrm{~b}_{j} q_{\alpha \gamma}^{a N_{\gamma}} \mathrm{x}_{\gamma}^{a N_{\gamma}} \otimes x_{\eta_{j}} x_{\delta_{j}}\right) .
\end{aligned}
$$

Now use the formula $d\left(\mathrm{x}_{\gamma}^{a N_{\gamma}+1} \otimes 1\right)=x_{\gamma}^{a N_{\gamma}} \otimes x_{\gamma}$, 10.1.4) and (10.1.9) to rewrite the above expression as

$$
\begin{aligned}
& \mathrm{x}_{\alpha} \mathrm{x}_{\gamma}^{a N_{\gamma}} \otimes x_{\beta}-q_{\gamma \beta}^{N_{\gamma}-1} \mathbf{x}_{\alpha} \mathbf{x}_{\gamma}^{N_{\gamma}(a-1)+1} \mathbf{x}_{\beta} \otimes x_{\gamma}^{N_{\gamma}-1}-q_{\alpha \beta} q_{\alpha \gamma}^{a N_{\gamma}} \mathbf{x}_{\gamma}^{a N_{\gamma}} \mathbf{x}_{\beta} \otimes x_{\alpha} \\
& \quad+\mathrm{b} q_{\gamma \beta}^{a N_{\gamma}}\left\{\left(\frac{q_{\alpha \gamma}}{q_{\gamma \beta}}-1\right)(a)_{\left(\frac{q \alpha \gamma}{q_{\gamma \beta}}\right)^{N_{\gamma}}}-\left(\frac{q_{\alpha \gamma}}{q_{\gamma \beta}}\right)^{a N_{\gamma}}\right\} \mathrm{x}_{\gamma}^{a N_{\gamma}+1} \otimes x_{\gamma}^{N_{\gamma}-2}-\sum_{j=1}^{n} \mathrm{~b}_{j} q_{\alpha \gamma}^{a N_{\gamma}} \mathbf{x}_{\gamma}^{a N_{\gamma}} \mathbf{x}_{\eta_{j}} \otimes x_{\delta_{j}} .
\end{aligned}
$$

This agrees with the first claimed formula in the lemma.

Now we use the first formula to obtain the second formula:

$$
\begin{aligned}
d\left(\mathrm{x}_{\alpha} \mathrm{x}_{\gamma}^{a N_{\gamma}+1} \mathrm{x}_{\beta} \otimes 1\right)=\mathrm{x}_{\alpha} \mathrm{x}_{\gamma}^{a N_{\gamma}+1} \otimes x_{\beta}-s\left(d\left(\mathrm{x}_{\alpha} \mathrm{x}_{\gamma}^{a N_{\gamma}+1} \otimes 1\right) x_{\beta}\right) \\
=\mathrm{x}_{\alpha} \mathrm{x}_{\gamma}^{a N_{\gamma}+1} \otimes x_{\beta}-s\left(\mathrm{x}_{\alpha} \mathrm{x}_{\gamma}^{a N_{\gamma}} \otimes x_{\gamma} x_{\beta}-q_{\alpha \gamma}^{a N_{\gamma}+1} \mathrm{x}_{\gamma}^{a N_{\gamma}+1} \otimes x_{\alpha} x_{\beta}\right) \\
=\mathrm{x}_{\alpha} \mathrm{x}_{\gamma}^{a N_{\gamma}+1} \otimes x_{\beta}-s\left(q_{\gamma \beta} \mathrm{x}_{\alpha} \mathrm{x}_{\gamma}^{a N_{\gamma}} \otimes x_{\beta} x_{\gamma}-q_{\alpha \beta} q_{\alpha \gamma}^{a N_{\gamma}+1} \mathrm{x}_{\gamma}^{a N_{\gamma}+1} \otimes x_{\beta} x_{\alpha}\right. \\
\left.\quad-\mathrm{b} q_{\alpha \gamma}^{a N_{\gamma}+1} \mathrm{x}_{\gamma}^{a N_{\gamma}+1} \otimes x_{\gamma}^{N_{\gamma}-1}-\sum_{j=1}^{n} \mathrm{~b}_{j} q_{\alpha \gamma}^{a N_{\gamma}+1} \mathrm{x}_{\gamma}^{a N_{\gamma}+1} \otimes x_{\eta_{j}} x_{\delta_{j}}\right)
\end{aligned}
$$

By our induction hypothesis, we may use the first formula in the statement of the lemma to rewrite the term $q_{\gamma \beta} \mathbf{x}_{\alpha} \mathbf{x}_{\gamma}^{a N_{\gamma}} \otimes x_{\beta} x_{\gamma}$, obtaining

$$
\begin{aligned}
& \mathrm{x}_{\alpha} \mathrm{x}_{\gamma}^{a N_{\gamma}+1} \otimes x_{\beta}-s\left(q_{\gamma \beta} d\left(\mathrm{x}_{\alpha} \mathrm{x}_{\gamma}^{a N_{\gamma}} \mathbf{x}_{\beta} \otimes x_{\gamma}\right)+q_{\alpha \beta} q_{\alpha \gamma}^{a N_{\gamma}} q_{\gamma \beta} \mathbf{x}_{\gamma}^{a N_{\gamma}} \mathbf{x}_{\beta} \otimes x_{\alpha} x_{\gamma}\right. \\
& -\mathrm{b} q_{\gamma \beta}^{a N_{\gamma}+1}\left\{\left(\frac{q_{\alpha \gamma}}{q_{\gamma \beta}}-1\right)(a)_{\left(\frac{q_{\alpha \gamma}}{q_{\gamma \beta}}\right)^{N_{\gamma}}}-\left(\frac{q_{\alpha \gamma}}{q_{\gamma \beta}}\right)^{a N_{\gamma}}\right\} \mathrm{x}_{\gamma}^{a N_{\gamma}+1} \otimes x_{\gamma}^{N_{\gamma}-1} \\
& +\sum_{j=1}^{n} \mathrm{~b}_{j} q_{\alpha \gamma}^{a N_{\gamma}} q_{\gamma \beta} \mathrm{x}_{\gamma}^{a N_{\gamma}} \mathrm{x}_{\eta_{j}} \otimes x_{\delta_{j}} x_{\gamma}-q_{\alpha \beta} q_{\alpha \gamma}^{a N_{\gamma}+1} \mathrm{x}_{\gamma}^{a N_{\gamma}+1} \otimes x_{\beta} x_{\alpha} \\
& \left.-\mathrm{b} q_{\alpha \gamma}^{a N_{\gamma}+1} \mathrm{x}_{\gamma}^{a N_{\gamma}+1} \otimes x_{\gamma}^{N_{\gamma}-1}-\sum_{j=1}^{n} \mathrm{~b}_{j} q_{\alpha \gamma}^{a N_{\gamma}+1}{ }_{\mathrm{x}_{\gamma}}^{a N_{\gamma}+1} \otimes x_{\eta_{j}} x_{\delta_{j}}\right) \\
& =\mathbf{x}_{\alpha} \mathbf{x}_{\gamma}^{a N_{\gamma}+1} \otimes x_{\beta}-q_{\gamma \beta} \mathbf{x}_{\alpha} \mathbf{x}_{\gamma}^{a N_{\gamma}} \mathbf{x}_{\beta} \otimes x_{\gamma} \\
& -s\left(q_{\alpha \beta} q_{\alpha \gamma}^{a N_{\gamma}+1}\left(q_{\gamma \beta} \mathbf{x}_{\gamma}^{a N_{\gamma}} \mathbf{x}_{\beta} \otimes x_{\gamma} x_{\alpha}-\mathrm{x}_{\gamma}^{a N_{\gamma}+1} \otimes x_{\beta} x_{\alpha}\right)\right.
\end{aligned}
$$




$$
\begin{aligned}
& -\mathrm{b} q_{\gamma \beta}^{a N_{\gamma}+1}\left\{\left(\frac{q_{\alpha \gamma}}{q_{\gamma \beta}}-1\right)(a)_{\left(\frac{q_{\alpha \gamma}}{q_{\gamma \beta}}\right)^{N_{\gamma}}}-\left(\frac{q_{\alpha \gamma}}{q_{\gamma \beta}}\right)^{a N_{\gamma}}+\left(\frac{q_{\alpha \gamma}}{q_{\gamma \beta}}\right)^{a N_{\gamma}+1}\right\} \mathrm{x}_{\gamma}^{a N_{\gamma}+1} \otimes x_{\gamma}^{N_{\gamma}-1} \\
& \left.-\sum_{j=1}^{n} \mathrm{~b}_{j} q_{\alpha \gamma}^{a N_{\gamma}}\left(q_{\alpha \gamma} \mathrm{x}_{\gamma}^{a N_{\gamma}+1} \otimes x_{\eta_{j}}-q_{\gamma \beta} q_{\delta_{j} \gamma} \mathrm{x}_{\gamma}^{a N_{\gamma}} \mathrm{x}_{\eta_{j}} \otimes x_{\gamma}\right) x_{\delta_{j}}\right) .
\end{aligned}
$$

Now we use the formula $d\left(\mathrm{x}_{\gamma}^{N_{\gamma}(a+1)} \otimes 1\right)=\mathrm{x}_{\gamma}^{a N_{\gamma}+1} \otimes x_{\gamma}^{N_{\gamma}-1}$, (10.1.4) and (10.1.9) to rewrite the above expression as

$$
\begin{aligned}
& \mathrm{x}_{\alpha} \mathrm{x}_{\gamma}^{a N_{\gamma}+1} \otimes x_{\beta}-q_{\gamma \beta} \mathrm{x}_{\alpha} \mathrm{x}_{\gamma}^{a N_{\gamma}} \mathrm{x}_{\beta} \otimes x_{\gamma}+q_{\alpha \beta} q_{\alpha \gamma}^{a N_{\gamma}+1} \mathrm{x}_{\gamma}^{a N_{\gamma}+1} \mathrm{x}_{\beta} \otimes x_{\alpha} \\
& \quad+\mathrm{b} q_{\gamma \beta}^{a N_{\gamma}+1}\left\{\left(\frac{q_{\alpha \gamma}}{q_{\gamma \beta}}-1\right)(a+1)_{\left(\frac{q \alpha \gamma}{q_{\gamma \beta}}\right)^{N_{\gamma}}}\right\} \mathrm{x}_{\gamma}^{N_{\gamma}(a+1)} \otimes 1+\sum_{j=1}^{n} \mathrm{~b}_{j} q_{\alpha \gamma}^{a N_{\gamma}+1} \mathrm{x}_{\gamma}^{a N_{\gamma}+1} \mathrm{x}_{\eta_{j}} \otimes x_{\delta_{j}},
\end{aligned}
$$

which agrees with the second claimed formula in the lemma.

Lemma 10.1.10. Let $\alpha<\eta<\gamma<\beta<\delta$ be positive roots such that $N_{\gamma}=2$ and the relations among the corresponding root vectors take the form

$$
x_{\alpha} x_{\beta}=q_{\alpha \beta} x_{\beta} x_{\alpha}+\mathrm{b}_{1} x_{\gamma} x_{\eta}, \quad x_{\eta} x_{\delta}=q_{\eta \delta} x_{\delta} x_{\eta}+\mathrm{b}_{2} x_{\gamma},
$$

for some scalars $\mathrm{b}_{1}, \mathrm{~b}_{2}$ and the other pairs of root vectors $q$-commute. Then, for all $n \geq 0$,

$$
\begin{aligned}
d\left(\mathrm{x}_{\alpha} \mathrm{x}_{\gamma}^{n} \mathrm{x}_{\beta} \mathrm{x}_{\delta} \otimes 1\right)=\mathrm{x}_{\alpha} \mathrm{x}_{\gamma}^{n} \mathrm{x}_{\beta} \otimes x_{\delta}-q_{\beta \delta} \mathrm{x}_{\alpha} \mathrm{x}_{\gamma}^{n} \mathrm{x}_{\delta} \otimes x_{\beta}+q_{\gamma \beta} q_{\gamma \delta} \mathrm{x}_{\alpha} \mathrm{x}_{\gamma}^{n-1} \mathrm{x}_{\beta} \mathrm{x}_{\delta} \otimes x_{\gamma} \\
\quad+\left(-q_{\alpha \gamma}\right)^{n} q_{\alpha \beta} q_{\alpha \delta} \mathrm{x}_{\gamma}^{n} \mathrm{x}_{\beta} \mathrm{x}_{\delta} \otimes x_{\alpha}-q_{\eta \delta} \mathrm{b}_{1}(-1)^{n-1} q_{\alpha \gamma}^{n}(n+1)_{\widetilde{q}_{\beta \gamma}} \mathrm{x}_{\gamma}^{n+1} \mathrm{x}_{\delta} \otimes x_{\eta} \\
\quad+\mathrm{b}_{1} \mathrm{~b}_{2} \mathrm{c}_{\alpha \beta \gamma}^{(n)}\left(-q_{\gamma \alpha}\right)^{-n} \mathrm{x}_{\gamma}^{n+2} \otimes 1 .
\end{aligned}
$$

where $\mathrm{c}_{\alpha \beta \gamma}^{(n)}:=\sum_{k=0}^{n}\left(-\widetilde{q}_{\alpha \gamma}\right)^{k}(k+1)_{\widetilde{q}_{\beta \gamma}}, n \in \mathbb{N}$.

Notice that the equalities in (10.1.11) force

$$
\gamma+\eta=\alpha+\beta, \quad \eta+\delta=\gamma .
$$

Hence the following equality also holds: $2 \gamma=\alpha+\beta+\delta$.

Proof. First we claim that

$$
\begin{aligned}
d\left(\mathrm{x}_{\alpha} \mathrm{x}_{\gamma}^{n} \mathrm{x}_{\beta} \otimes 1\right) & =\mathrm{x}_{\alpha} \mathrm{x}_{\gamma}^{n} \otimes x_{\beta}-q_{\gamma \beta} \mathrm{x}_{\alpha} \mathrm{x}_{\gamma}^{n-1} \mathrm{x}_{\beta} \otimes x_{\gamma}-q_{\alpha \beta}\left(-q_{\alpha \gamma}\right)^{n} \mathrm{x}_{\gamma}^{n} \mathrm{x}_{\beta} \otimes x_{\alpha} \\
& -\mathrm{b}_{1}\left(-q_{\alpha \gamma}\right)^{n}(n+1)_{\widetilde{q}_{\beta \gamma}} \mathrm{x}_{\gamma}^{n+1} \otimes x_{\eta}
\end{aligned}
$$

The proof is by induction on $n$. When $n=0$,

$$
d\left(\mathrm{x}_{\alpha} \mathrm{x}_{\beta} \otimes 1\right)=\mathrm{x}_{\alpha} \otimes x_{\beta}-s_{0} d_{1}\left(\mathrm{x}_{\alpha} \otimes x_{\beta}\right)=\mathrm{x}_{\alpha} \otimes x_{\beta}-q_{\alpha \beta} \mathrm{x}_{\beta} \otimes x_{\alpha}-\mathrm{b}_{1} \mathrm{x}_{\gamma} \otimes x_{\eta} .
$$

Now assume that (10.1.14) holds for $n$. Let $c_{n}=\left(-q_{\alpha \gamma}\right)^{n}(n+1)_{\widetilde{q}_{\beta \gamma}}$. We compute:

$$
\begin{aligned}
d\left(\mathrm{x}_{\alpha} \mathrm{x}_{\gamma}^{n+1} \mathrm{x}_{\beta} \otimes 1\right)=\mathrm{x}_{\alpha} \mathrm{x}_{\gamma}^{n+1} \otimes x_{\beta}-s d\left(\mathrm{x}_{\alpha} \mathrm{x}_{\gamma}^{n+1} \otimes x_{\beta}\right) \\
=\mathrm{x}_{\alpha} \mathrm{x}_{\gamma}^{n+1} \otimes x_{\beta}-s\left(q_{\gamma \beta} \mathrm{x}_{\alpha} \mathrm{x}_{\gamma}^{n} \otimes x_{\beta} x_{\gamma}+\left(-q_{\alpha \gamma}\right)^{n+1} \mathrm{x}_{\gamma}^{n+1} \otimes\left(q_{\alpha \beta} x_{\beta} x_{\alpha}+\mathrm{b}_{1} x_{\gamma} x_{\eta}\right)\right) \\
=\mathrm{x}_{\alpha} \mathrm{x}_{\gamma}^{n+1} \otimes x_{\beta}-q_{\gamma \beta} \mathrm{x}_{\alpha} \mathrm{x}_{\gamma}^{n} \mathrm{x}_{\beta} \otimes x_{\gamma}-s\left(\left(-q_{\alpha \gamma}\right)^{n+1} q_{\alpha \beta} \mathrm{x}_{\gamma}^{n+1} \otimes x_{\beta} x_{\alpha}\right. \\
\left.\quad+\left(-q_{\alpha \gamma}\right)^{n+1} \mathrm{~b}_{1} \mathrm{x}_{\gamma}^{n+1} \otimes x_{\gamma} x_{\eta}+q_{\gamma \beta} q_{\alpha \beta}\left(-q_{\alpha} \gamma\right)^{n} \mathrm{x}_{\gamma}^{n} \mathrm{x}_{\beta} \otimes x_{\alpha} x_{\gamma}+q_{\gamma \beta} \mathrm{b}_{1} c_{n} \mathrm{x}_{\gamma}^{n+1} \otimes x_{\eta} x_{\gamma}\right) \\
\quad=\mathrm{x}_{\alpha} \mathrm{x}_{\gamma}^{n+1} \otimes x_{\beta}-q_{\gamma \beta} \mathrm{x}_{\alpha} \mathrm{x}_{\gamma}^{n} \mathrm{x}_{\beta} \otimes x_{\gamma}-\mathrm{b}_{1}\left(q_{\gamma \beta} q_{\eta \gamma} c_{n}+\left(-q_{\alpha \gamma}\right)^{n+1}\right) \mathrm{x}_{\gamma}^{n+2} \otimes x_{\eta}
\end{aligned}
$$


$-\left(-q_{\alpha \gamma}\right)^{n+1} q_{\alpha \beta} s d\left(\mathrm{x}_{\gamma}^{n+1} \mathrm{x}_{\beta} \otimes x_{\alpha}\right)$.

Now the inductive step follows using Remark 10.1.2 and

$$
q_{\eta \gamma}=q_{\alpha \gamma} q_{\beta \gamma} q_{\gamma \gamma}^{-1}=-q_{\alpha \gamma} q_{\beta \gamma} .
$$

Next we prove by induction on $n$ that there exist $e_{n} \in \mathbb{k}$ such that:

$$
\begin{aligned}
& d\left(\mathrm{x}_{\alpha} \mathrm{x}_{\gamma}^{n} \mathrm{x}_{\beta} \mathrm{x}_{\delta} \otimes 1\right)=\mathrm{x}_{\alpha} \mathrm{x}_{\gamma}^{n} \mathrm{x}_{\beta} \otimes x_{\delta}-q_{\beta \delta} \mathrm{x}_{\alpha} \mathrm{x}_{\gamma}^{n} \mathrm{x}_{\delta} \otimes x_{\beta}+q_{\gamma \beta} q_{\gamma \delta} \mathrm{x}_{\alpha} \mathrm{x}_{\gamma}^{n-1} \mathrm{x}_{\beta} \mathrm{x}_{\delta} \otimes x_{\gamma} \\
& \quad+\left(-q_{\alpha \gamma}\right)^{n} q_{\alpha \beta} q_{\alpha \delta} \mathrm{x}_{\gamma}^{n} \mathrm{x}_{\beta} \mathrm{x}_{\delta} \otimes x_{\alpha}-q_{\eta \delta} \mathrm{b}_{1} c_{n-1} \mathrm{x}_{\gamma}^{n+1} \mathrm{x}_{\delta} \otimes x_{\eta}+\mathrm{b}_{1} \mathrm{~b}_{2} e_{n} \mathrm{x}_{\gamma}^{n+2} \otimes 1 .
\end{aligned}
$$

The proof is again by induction. When $n=0$,

$$
\begin{aligned}
& d\left(\mathrm{x}_{\alpha} \mathrm{x}_{\beta} \mathrm{x}_{\delta} \otimes 1\right)=\mathrm{x}_{\alpha} \mathrm{x}_{\beta} \otimes x_{\delta}-s_{1} d_{2}\left(\mathrm{x}_{\alpha} \mathrm{x}_{\beta} \otimes x_{\delta}\right) \\
&=\mathrm{x}_{\alpha} \mathrm{x}_{\beta} \otimes x_{\delta}-s_{1}\left(q_{\beta \delta} \mathrm{x}_{\alpha} \otimes x_{\delta} x_{\beta}-q_{\alpha \beta} q_{\alpha \delta} \mathrm{x}_{\beta} \otimes x_{\delta} x_{\alpha}-\mathrm{b}_{1} \mathrm{x}_{\gamma} \otimes\left(q_{\eta \delta} x_{\delta} x_{\eta}+\mathrm{b}_{2} x_{\gamma}\right)\right) \\
&=\mathrm{x}_{\alpha} \mathrm{x}_{\beta} \otimes x_{\delta}-q_{\beta \delta} \mathrm{x}_{\alpha} \mathrm{x}_{\delta} \otimes x_{\beta}-s_{1}\left(-q_{\alpha \beta} q_{\alpha \delta} \mathrm{x}_{\beta} \otimes x_{\delta} x_{\alpha}-q_{\eta \delta} \mathrm{b}_{1} \mathrm{x}_{\gamma} \otimes x_{\delta} x_{\eta}\right. \\
&\left.\quad-\mathrm{b}_{1} \mathrm{~b}_{2} \mathrm{x}_{\gamma} \otimes x_{\gamma}+q_{\beta \delta} q_{\alpha \delta} \mathrm{x}_{\delta} \otimes\left(q_{\alpha \beta} x_{\beta} x_{\alpha}+\mathrm{b}_{1} x_{\gamma} x_{\eta}\right)\right) \\
&=\mathrm{x}_{\alpha} \mathrm{x}_{\beta} \otimes x_{\delta}-q_{\beta \delta} \mathrm{x}_{\alpha} \mathrm{x}_{\delta} \otimes x_{\beta}+\mathrm{b}_{1} \mathrm{~b}_{2} \mathrm{x}_{\gamma}^{2} \otimes 1+q_{\eta \delta} \mathrm{b}_{1} \mathrm{x}_{\gamma} \mathrm{x}_{\delta} \otimes x_{\eta} \\
&-s_{1}\left(-q_{\alpha \beta} q_{\alpha \delta} \mathrm{x}_{\beta} \otimes x_{\delta} x_{\alpha}+q_{\beta \delta} q_{\alpha \delta} \mathrm{x}_{\delta} \otimes\left(q_{\alpha \beta} x_{\beta} x_{\alpha}+\mathrm{b}_{1} x_{\gamma} x_{\eta}\right)-q_{\gamma \delta} q_{\eta \delta} \mathrm{b}_{1} \mathrm{x}_{\delta} \otimes x_{\gamma} x_{\eta}\right) \\
&= \mathrm{x}_{\alpha} \mathrm{x}_{\beta} \otimes x_{\delta}-q_{\beta \delta} \mathrm{x}_{\alpha} \mathrm{x}_{\delta} \otimes x_{\beta}+\mathrm{b}_{1} \mathrm{~b}_{2} \mathrm{x}_{\gamma}^{2} \otimes 1+q_{\eta \delta} \mathrm{b}_{1} \mathrm{x}_{\gamma} \mathrm{x}_{\delta} \otimes x_{\eta}+q_{\alpha \beta} q_{\alpha \delta} \mathrm{x}_{\beta} x_{\delta} \otimes x_{\alpha} \\
&-\mathrm{b}_{1}\left(q_{\beta \delta} q_{\alpha \delta}-q_{\gamma \delta} q_{\eta \delta}\right) s\left(\mathrm{x}_{\delta} \otimes x_{\gamma} x_{\eta}\right) .
\end{aligned}
$$

Now assume that the formula holds for $n$. Using (10.1.14):

$$
\begin{aligned}
d\left(\mathrm{x}_{\alpha} \mathrm{x}_{\gamma}^{n+1} \mathrm{x}_{\beta} \mathrm{x}_{\delta} \otimes 1\right)=\mathrm{x}_{\alpha} \mathrm{x}_{\gamma}^{n+1} \mathrm{x}_{\beta} \otimes x_{\delta}-s d\left(\mathrm{x}_{\alpha} \mathrm{x}_{\gamma}^{n+1} \mathrm{x}_{\beta} \otimes x_{\delta}\right) \\
=\mathrm{x}_{\alpha} \mathrm{x}_{\gamma}^{n+1} \mathrm{x}_{\beta} \otimes x_{\delta}-s\left(q_{\beta \delta} \mathrm{x}_{\alpha} \mathrm{x}_{\gamma}^{n+1} \otimes x_{\delta} x_{\beta}-q_{\gamma \beta} q_{\gamma \delta} \mathrm{x}_{\alpha} \mathrm{x}_{\gamma}^{n} \mathrm{x}_{\beta} \otimes x_{\delta} x_{\gamma}\right. \\
\left.\quad-q_{\alpha \beta} q_{\alpha \delta}\left(-q_{\alpha} \gamma\right)^{n+1} \mathrm{x}_{\gamma}^{n+1} \mathrm{x}_{\beta} \otimes x_{\delta} x_{\alpha}+\mathrm{b}_{1} c_{n+1} \mathrm{x}_{\gamma}^{n+2} \otimes\left(q_{\eta \delta} x_{\delta} x_{\eta}+\mathrm{b}_{2} x_{\gamma}\right)\right) \\
=\mathrm{x}_{\alpha} \mathrm{x}_{\gamma}^{n+1} \mathrm{x}_{\beta} \otimes x_{\delta}-q_{\beta \delta} \mathrm{x}_{\alpha} \mathrm{x}_{\gamma}^{n+1} \mathrm{x}_{\delta} \otimes x_{\beta}-s\left(q_{\beta \delta} q_{\gamma \delta} q_{\gamma \beta} \mathrm{x}_{\alpha} \mathrm{x}_{\gamma}^{n} \mathrm{x}_{\delta} \otimes x_{\beta} x_{\gamma}\right. \\
\quad+q_{\beta \delta} q_{\alpha \delta}\left(-q_{\alpha \gamma}\right)^{n+1} \mathrm{x}_{\gamma}^{n+1} \mathrm{x}_{\delta} \otimes\left(q_{\alpha \beta} x_{\beta} x_{\alpha}+\mathrm{b}_{1} x_{\gamma} x_{\eta}\right)-q_{\gamma \beta} q_{\gamma \delta} \mathrm{x}_{\alpha} \mathrm{x}_{\gamma}^{n} \mathrm{x}_{\beta} \otimes x_{\delta} x_{\gamma} \\
\left.\quad-q_{\alpha \beta} q_{\alpha \delta}\left(-q_{\alpha} \gamma\right)^{n+1} \mathrm{x}_{\gamma}^{n+1} \mathrm{x}_{\beta} \otimes x_{\delta} x_{\alpha}-\mathrm{b}_{1} c_{n+1} \mathrm{x}_{\gamma}^{n+2} \otimes\left(q_{\eta \delta} x_{\delta} x_{\eta}+\mathrm{b}_{2} x_{\gamma}\right)\right)
\end{aligned}
$$

Next we use the inductive hypothesis, the relation $x_{\gamma}^{2}=0$, (10.1.5) and (10.1.6):

$$
\begin{aligned}
d\left(\mathrm{x}_{\alpha} \mathrm{x}_{\gamma}^{n+1} \mathrm{x}_{\beta} \mathrm{x}_{\delta} \otimes 1\right)=\mathrm{x}_{\alpha} \mathrm{x}_{\gamma}^{n+1} \mathrm{x}_{\beta} \otimes x_{\delta}-q_{\beta \delta} \mathrm{x}_{\alpha} \mathrm{x}_{\gamma}^{n+1} \mathrm{x}_{\delta} \otimes x_{\beta}+q_{\gamma \beta} q_{\gamma \delta} \mathrm{x}_{\alpha} \mathrm{x}_{\gamma}^{n} \mathrm{x}_{\beta} \mathrm{x}_{\delta} \otimes x_{\gamma} \\
\quad-s\left(-q_{\gamma \beta} q_{\gamma \delta}\left(-q_{\alpha \gamma}\right)^{n+1} q_{\alpha \beta} q_{\alpha \delta} \mathrm{x}_{\gamma}^{n} \mathrm{x}_{\beta} \mathrm{x}_{\delta} \otimes x_{\gamma} x_{\alpha}-q_{\gamma \beta} q_{\gamma \delta} q_{\eta \gamma} q_{\eta \delta} \mathrm{b}_{1} c_{n+1} \mathrm{x}_{\gamma}^{n+1} \mathrm{x}_{\delta} \otimes x_{\gamma} x_{\eta}\right. \\
\quad+\mathrm{b}_{1} \mathrm{~b}_{2} e_{n} q_{\gamma \beta} q_{\gamma \delta} \mathrm{x}_{\gamma}^{n+2} \otimes x_{\gamma}+q_{\beta \delta} q_{\alpha \delta}\left(-q_{\alpha \gamma}\right)^{n+1} \mathrm{x}_{\gamma}^{n+1} \mathrm{x}_{\delta} \otimes\left(q_{\alpha \beta} x_{\beta} x_{\alpha}+\mathrm{b}_{1} x_{\gamma} x_{\eta}\right) \\
\left.\quad-q_{\alpha \beta} q_{\alpha \delta}\left(-q_{\alpha \gamma}\right)^{n+1} \mathrm{x}_{\gamma}^{n+1} \mathrm{x}_{\beta} \otimes x_{\delta} x_{\alpha}-\mathrm{b}_{1} c_{n+1} \mathrm{x}_{\gamma}^{n+2} \otimes\left(q_{\eta \delta} x_{\delta} x_{\eta}+\mathrm{b}_{2} x_{\gamma}\right)\right) \\
=\mathrm{x}_{\alpha} \mathrm{x}_{\gamma}^{n+1} \mathrm{x}_{\beta} \otimes x_{\delta}-q_{\beta \delta} \mathrm{x}_{\alpha} \mathrm{x}_{\gamma}^{n+1} \mathrm{x}_{\delta} \otimes x_{\beta}+q_{\gamma \beta} q_{\gamma \delta} \mathrm{x}_{\alpha} \mathrm{x}_{\gamma}^{n} \mathrm{x}_{\beta} \mathrm{x}_{\delta} \otimes x_{\gamma} \\
\quad+q_{\alpha \beta} q_{\alpha \delta}\left(-q_{\alpha \gamma}\right)^{n+1} \mathrm{x}_{\gamma}^{n+1} \mathrm{x}_{\beta} \mathrm{x}_{\delta} \otimes x_{\alpha}-\mathrm{b}_{1} \mathrm{~b}_{2}\left(e_{n} q_{\gamma \beta} q_{\gamma \delta}-c_{n+1}\right) \mathrm{x}_{\gamma}^{n+3} \otimes 1 \\
\quad+q_{\eta \delta} \mathrm{b}_{1} c_{n+1} \mathrm{x}_{\gamma}^{n+2} \mathrm{x}_{\delta} \otimes x_{\eta},
\end{aligned}
$$

and the inductive step follows. To finish the proof we have to compute $e_{n}$. Note that

$$
e_{0}=1, \quad e_{n+1}=-e_{n} q_{\gamma \beta} q_{\gamma \delta}+c_{n+1}, \quad \text { for all } n \geq 0 .
$$


By (10.1.13) and using that $q_{\gamma \gamma}=-1$,

$$
q_{\gamma \delta}=q_{\gamma \gamma}^{2} q_{\gamma \alpha}^{-1} q_{\gamma \beta}^{-1}=q_{\gamma \alpha}^{-1} q_{\gamma \beta}^{-1} .
$$

Hence $e_{n}=\mathrm{c}_{\alpha \beta \gamma}^{(n)}\left(-q_{\gamma \alpha}\right)^{-n}$ for all $n \geq 0$.

Lemma 10.1.15. Let $\alpha<\eta<\gamma<\tau<\beta<\delta$ be positive roots such that $N_{\gamma}=N_{\alpha}=2$ and the relations among the corresponding root vectors take the form

$$
\begin{array}{ll}
x_{\alpha} x_{\beta}=q_{\alpha \beta} x_{\beta} x_{\alpha}+\mathrm{b}_{1} x_{\tau} x_{\gamma}, & x_{\eta} x_{\delta}=q_{\eta \delta} x_{\delta} x_{\eta}+\mathrm{b}_{2} x_{\gamma}, \\
x_{\alpha} x_{\tau}=q_{\alpha \tau} x_{\tau} x_{\alpha}+\mathrm{b}_{3} x_{\gamma} x_{\eta}, & x_{\eta} x_{\beta}=q_{\eta \beta} x_{\beta} x_{\eta}+\mathrm{b}_{4} x_{\tau}^{2},
\end{array}
$$

for some scalars $\mathrm{b}_{j} \in \mathbb{k}$ and the other pairs of root vectors $q$-commute. Then, for all $n \geq 0$,

$$
\begin{gathered}
d\left(\mathrm{x}_{\alpha}^{2} \mathrm{x}_{\gamma}^{n} \mathrm{x}_{\beta} \mathrm{x}_{\delta} \otimes 1\right)=\mathrm{x}_{\alpha}^{2} \mathrm{x}_{\gamma}^{n} \mathrm{x}_{\beta} \otimes x_{\delta}-q_{\beta \delta} \mathrm{x}_{\alpha}^{2} \mathrm{x}_{\gamma}^{n} \mathrm{x}_{\delta} \otimes x_{\beta}+q_{\gamma \beta} q_{\gamma \delta} \mathrm{x}_{\alpha}^{2} \mathrm{x}_{\gamma}^{n-1} \mathrm{x}_{\beta} \mathrm{x}_{\delta} \otimes x_{\gamma} \\
+\left(-q_{\alpha \gamma}\right)^{n} q_{\alpha \beta} q_{\alpha \delta} \mathrm{x}_{\alpha} \mathrm{x}_{\gamma}^{n} \mathrm{x}_{\beta} \mathrm{x}_{\delta} \otimes x_{\alpha}+q_{\gamma \beta}^{n} q_{\eta \gamma}^{n+1} q_{\eta \delta} \mathrm{b}_{1} \mathrm{~b}_{3} \mathrm{x}_{\gamma}^{n+2} \mathrm{x}_{\delta} \otimes x_{\eta} \\
+\frac{q_{\alpha \gamma}^{n+1}}{q_{\gamma \alpha}^{n+2} q_{\gamma \beta}}\left((n+1)_{\widetilde{q}_{\gamma \alpha} \widetilde{q}_{\gamma \beta}}+\sum_{j=1}^{n} \mathrm{c}_{\alpha \tau \gamma}^{(j)}\right) \mathrm{b}_{1} \mathrm{~b}_{2} \mathrm{~b}_{3} \mathrm{x}_{\gamma}^{n+3} \otimes 1 .
\end{gathered}
$$

Notice that the equalities in (10.1.16) force

$$
\gamma+\tau=\alpha+\beta, \quad \eta+\delta=\gamma, \quad \alpha+\tau=\gamma+\eta, \quad \eta+\beta=2 \tau .
$$

Thus the following equality also holds: $3 \gamma=2 \alpha+\beta+\delta$.

Proof. Let $n \in \mathbb{N}_{0}$. A computation similar to (10.1.14) proves that

$$
\begin{aligned}
d\left(\mathrm{x}_{\alpha} \mathrm{x}_{\gamma}^{n} \mathrm{x}_{\tau} \otimes 1\right)= & \mathrm{x}_{\alpha} \mathrm{x}_{\gamma}^{n} \otimes x_{\tau}-q_{\gamma \tau} \mathrm{x}_{\alpha} \mathrm{x}_{\gamma}^{n-1} \mathrm{x}_{\tau} \otimes x_{\gamma}-q_{\alpha \tau}\left(-q_{\alpha \gamma}\right)^{n} \mathrm{x}_{\gamma}^{n} \mathrm{x}_{\tau} \otimes x_{\alpha} \\
& -\mathrm{b}_{3}\left(-q_{\alpha \gamma}\right)^{n}(n+1)_{\widetilde{q}_{\tau \gamma}} \mathrm{x}_{\gamma}^{n+1} \otimes x_{\eta}, \\
d\left(\mathrm{x}_{\alpha} \mathrm{x}_{\gamma}^{n} \mathrm{x}_{\beta} \otimes 1\right)= & \mathrm{x}_{\alpha} \mathrm{x}_{\gamma}^{n} \otimes x_{\beta}-q_{\gamma \beta} \mathrm{x}_{\alpha} \mathrm{x}_{\gamma}^{n-1} \mathrm{x}_{\beta} \otimes x_{\gamma}-q_{\alpha \beta}\left(-q_{\alpha \gamma}\right)^{n} \mathrm{x}_{\gamma}^{n} \mathrm{x}_{\beta} \otimes x_{\alpha} \\
& -\left(-q_{\alpha \gamma}\right)^{n} \mathrm{~b}_{1} \mathrm{x}_{\gamma}^{n} \mathrm{x}_{\tau} \otimes x_{\gamma} .
\end{aligned}
$$

Now we compute more differentials:

$$
\begin{gathered}
d\left(\mathrm{x}_{\alpha}^{2} \mathrm{x}_{\gamma}^{n} \mathrm{x}_{\beta} \otimes 1\right)=\mathrm{x}_{\alpha}^{2} \mathrm{x}_{\gamma}^{n} \otimes x_{\beta}-q_{\gamma \beta} \mathrm{x}_{\alpha}^{2} \mathrm{x}_{\gamma}^{n-1} \mathrm{x}_{\beta} \otimes x_{\gamma}-q_{\alpha \beta}\left(-q_{\alpha \gamma}\right)^{n} \mathrm{x}_{\alpha} \mathrm{x}_{\gamma}^{n} \mathrm{x}_{\beta} \otimes x_{\alpha} \\
+q_{\gamma \beta}^{n} q_{\eta \gamma}^{n+1} \mathrm{~b}_{1} \mathrm{~b}_{3} \mathrm{x}_{\gamma}^{n+2} \otimes x_{\eta}-\left(-q_{\alpha \gamma}\right)^{n+1} \mathrm{~b}_{1} \mathrm{x}_{\alpha} \mathrm{x}_{\gamma}^{n} \mathrm{x}_{\tau} \otimes x_{\gamma}, \\
d\left(\mathrm{x}_{\alpha} \mathrm{x}_{\gamma}^{n} \mathrm{x}_{\beta} \mathrm{x}_{\delta} \otimes 1\right)=\mathrm{x}_{\alpha} \mathrm{x}_{\gamma}^{n} \mathrm{x}_{\beta} \otimes x_{\delta}-q_{\beta \delta} \mathrm{x}_{\alpha} \mathrm{x}_{\gamma}^{n} \mathrm{x}_{\delta} \otimes x_{\beta}+q_{\gamma \beta} q_{\gamma \delta} \mathrm{x}_{\alpha} \mathrm{x}_{\gamma}^{n-1} \mathbf{x}_{\beta} \mathrm{x}_{\delta} \otimes x_{\gamma} \\
+\left(-q_{\alpha \gamma}\right)^{n} q_{\alpha \beta} q_{\alpha \delta} \mathrm{x}_{\gamma}^{n} \mathrm{x}_{\beta} \mathrm{x}_{\delta} \otimes x_{\alpha}+\left(-q_{\alpha \gamma}\right)^{n} q_{\gamma \delta} \mathrm{b}_{1} \mathrm{x}_{\gamma}^{n} \mathrm{x}_{\tau} \mathrm{x}_{\delta} \otimes x_{\gamma},
\end{gathered}
$$

First we prove (10.1.21) by induction on $n$. For $n=0$,

$$
\begin{aligned}
d\left(\mathrm{x}_{\alpha}^{2} \mathrm{x}_{\beta} \otimes 1\right)=\mathrm{x}_{\alpha}^{2} \otimes x_{\beta}-s\left(q_{\alpha \beta} \mathrm{x}_{\alpha} \otimes x_{\beta} x_{\alpha}+\mathrm{b}_{1} \mathrm{x}_{\alpha} \otimes x_{\tau} x_{\gamma}\right) & \\
= & \mathrm{x}_{\alpha}^{2} \otimes x_{\beta}-\mathrm{b}_{1} \mathrm{x}_{\alpha} \mathrm{x}_{\tau} \otimes x_{\gamma}-q_{\alpha \beta} \mathrm{x}_{\alpha} \mathrm{x}_{\beta} \otimes x_{\alpha}+s\left(q_{\eta \gamma} \mathrm{b}_{1} \mathrm{~b}_{3} \mathrm{x}_{\gamma} \otimes x_{\gamma} x_{\eta}\right. \\
& \left.+\left(q_{\alpha \tau} q_{\alpha \gamma}+q_{\alpha \beta}\right) \mathrm{b}_{1} \mathrm{x}_{\tau} \otimes x_{\gamma} x_{\alpha}\right) \\
= & \mathrm{x}_{\alpha}^{2} \otimes x_{\beta}-\mathrm{b}_{1} \mathrm{x}_{\alpha} \mathrm{x}_{\tau} \otimes x_{\gamma}-q_{\alpha \beta} \mathrm{x}_{\alpha} \mathrm{x}_{\beta} \otimes x_{\alpha}+q_{\eta \gamma} \mathrm{b}_{1} \mathrm{~b}_{3} \mathrm{x}_{\gamma}^{2} \otimes x_{\eta} .
\end{aligned}
$$

Assume that (10.1.21) holds for $n$. By inductive hypothesis, $x_{\gamma}^{2}=0$, (10.1.19) and (10.1.20):

$$
d\left(\mathrm{x}_{\alpha}^{2} \mathrm{x}_{\gamma}^{n+1} \mathrm{x}_{\beta} \otimes 1\right)=\mathrm{x}_{\alpha}^{2} \mathrm{x}_{\gamma}^{n+1} \otimes x_{\beta}-s\left(q_{\gamma \beta} \mathrm{x}_{\alpha}^{2} \mathrm{x}_{\gamma}^{n} \otimes x_{\beta} x_{\gamma}+\left(-q_{\alpha \gamma}\right)^{n+1} \mathrm{x}_{\alpha} \mathrm{x}_{\gamma}^{n+1} \otimes x_{\alpha} x_{\beta}\right)
$$




$$
\begin{aligned}
= & \mathrm{x}_{\alpha}^{2} \mathrm{x}_{\gamma}^{n+1} \otimes x_{\beta}-q_{\gamma \beta} \mathrm{x}_{\alpha}^{2} \mathrm{x}_{\gamma}^{n} \mathrm{x}_{\beta} \otimes x_{\gamma}-s\left(q_{\gamma \beta} q_{\alpha \beta}\left(-q_{\alpha \gamma}\right)^{n} \mathrm{x}_{\alpha} \mathrm{x}_{\gamma}^{n} \mathrm{x}_{\beta} \otimes x_{\alpha} x_{\gamma}\right. \\
& -q_{\gamma \beta}^{n+1} q_{\eta \gamma}^{n+1} \mathrm{~b}_{1} \mathrm{~b}_{3} \mathrm{x}_{\gamma}^{n+2} \otimes x_{\eta} x_{\gamma}+\left(-q_{\alpha \gamma}\right)^{n+1} q_{\alpha \beta} \mathrm{x}_{\alpha} \mathrm{x}_{\gamma}^{n+1} \otimes x_{\beta} x_{\alpha} \\
& \left.+\left(-q_{\alpha \gamma}\right)^{n+1} \mathrm{~b}_{1} \mathrm{x}_{\alpha} \mathrm{x}_{\gamma}^{n+1} \otimes x_{\tau} x_{\gamma}\right) \\
= & \mathrm{x}_{\alpha}^{2} \mathrm{x}_{\gamma}^{n+1} \otimes x_{\beta}-q_{\gamma \beta} \mathrm{x}_{\alpha}^{2} \mathrm{x}_{\gamma}^{n} \mathrm{x}_{\beta} \otimes x_{\gamma}-\left(-q_{\alpha \gamma}\right)^{n+1} q_{\alpha \beta} \mathrm{x}_{\alpha} \mathrm{x}_{\gamma}^{n+1} \mathrm{x}_{\beta} \otimes x_{\alpha} \\
& -s\left(\left(-q_{\alpha \gamma}\right)^{n+1} \mathrm{~b}_{1} \mathrm{x}_{\alpha} \mathrm{x}_{\gamma}^{n+1} \otimes x_{\tau} x_{\gamma}-q_{\gamma \beta}^{n+1} q_{\eta \gamma}^{n+1} \mathrm{~b}_{1} \mathrm{~b}_{3} \mathrm{x}_{\gamma}^{n+2} \otimes x_{\eta} x_{\gamma}\right. \\
+ & \left.\left(-q_{\alpha \gamma}\right)^{2 n+2} q_{\alpha \beta} \mathrm{b}_{1} \mathrm{x}_{\gamma}^{n+1} \mathrm{x}_{\tau} \otimes x_{\gamma} x_{\alpha}\right) \\
= & \mathrm{x}_{\alpha}^{2} \mathrm{x}_{\gamma}^{n+1} \otimes x_{\beta}-q_{\gamma \beta} \mathrm{x}_{\alpha}^{2} \mathrm{x}_{\gamma}^{n} \mathrm{x}_{\beta} \otimes x_{\gamma}-\left(-q_{\alpha \gamma}\right)^{n+1} q_{\alpha \beta} \mathrm{x}_{\alpha} \mathrm{x}_{\gamma}^{n+1} \mathrm{x}_{\beta} \otimes x_{\alpha} \\
& \left.-\left(-q_{\alpha \gamma}\right)^{n+1} \mathrm{~b}_{1} \mathrm{x}_{\alpha} \mathrm{x}_{\gamma}^{n+1} \mathrm{x}_{\tau} \otimes x_{\gamma}+q_{\gamma \beta}^{n+1} q_{\eta \gamma}^{n+2} \mathrm{~b}_{1} \mathrm{~b}_{3} s\left(\mathrm{x}_{\gamma}^{n+2} \otimes x_{\gamma} x_{\eta}\right)\right),
\end{aligned}
$$

so (10.1.21) follows since $s\left(\mathrm{x}_{\gamma}^{n+2} \otimes x_{\gamma} x_{\eta}\right)=\mathrm{x}_{\gamma}^{n+3} \otimes x_{\eta}$.

Now we prove (10.1.22) by induction on $n$. For $n=0$,

$$
\begin{aligned}
d\left(\mathrm{x}_{\alpha} \mathrm{x}_{\beta} \mathrm{x}_{\delta} \otimes 1\right)=\mathrm{x}_{\alpha} \mathrm{x}_{\beta} \otimes x_{\delta}-s\left(q_{\beta \delta} \mathrm{x}_{\alpha} \otimes x_{\delta} x_{\beta}-q_{\alpha \beta} q_{\alpha \delta} \mathrm{x}_{\beta} \otimes x_{\delta} x_{\alpha}-q_{\gamma \delta} \mathrm{b}_{1} \mathrm{x}_{\tau} \otimes x_{\delta} x_{\gamma}\right) \\
=\mathrm{x}_{\alpha} \mathrm{x}_{\beta} \otimes x_{\delta}-q_{\beta \delta} \mathrm{x}_{\alpha} \mathrm{x}_{\delta} \otimes x_{\beta}-s\left(q_{\beta \delta} q_{\alpha \delta} \mathrm{x}_{\delta} \otimes\left(q_{\alpha \beta} x_{\beta} x_{\alpha}+\mathrm{b}_{1} x_{\tau} x_{\gamma}\right)\right. \\
\left.\quad-q_{\alpha \beta} q_{\alpha \delta} \mathrm{x}_{\beta} \otimes x_{\delta} x_{\alpha}-q_{\gamma \delta} \mathrm{b}_{1} \mathrm{x}_{\tau} \otimes x_{\delta} x_{\gamma}\right) \\
=\mathrm{x}_{\alpha} \mathrm{x}_{\beta} \otimes x_{\delta}-q_{\beta \delta} \mathrm{x}_{\alpha} \mathrm{x}_{\delta} \otimes x_{\beta}+q_{\gamma \delta} \mathrm{b}_{1} \mathrm{x}_{\tau} \mathrm{x}_{\delta} \otimes x_{\gamma}+q_{\alpha \beta} q_{\alpha \delta} \mathrm{x}_{\beta} \mathrm{x}_{\delta} \otimes x_{\alpha}
\end{aligned}
$$

Now assume that (10.1.22) holds for $n$. Using (10.1.20), Remark 10.1.2 three times, inductive hypothesis, $x_{\gamma}^{2}=0=x_{\alpha}^{2}$,

$$
\begin{aligned}
& d\left(\mathrm{x}_{\alpha} \mathrm{x}_{\gamma}^{n+1} \mathrm{x}_{\beta} \mathrm{x}_{\delta} \otimes 1\right)=\mathrm{x}_{\alpha} \mathrm{x}_{\gamma}^{n+1} \mathrm{x}_{\beta} \otimes x_{\delta}-s\left(q_{\beta \delta} \mathrm{x}_{\alpha} \mathrm{x}_{\gamma}^{n+1} \otimes x_{\delta} x_{\beta}-q_{\gamma \beta} q_{\gamma \delta} \mathrm{x}_{\alpha} \mathrm{x}_{\gamma}^{n} \mathrm{x}_{\beta} \otimes x_{\delta} x_{\gamma}\right. \\
& \left.-q_{\alpha \beta}\left(-q_{\alpha \gamma}\right)^{n+1} q_{\alpha \delta} \mathrm{x}_{\gamma}^{n+1} \mathrm{x}_{\beta} \otimes x_{\delta} x_{\alpha}-\left(-q_{\alpha \gamma}\right)^{n+1} q_{\gamma \delta} \mathrm{b}_{1} \mathrm{x}_{\gamma}^{n+1} \mathrm{x}_{\tau} \otimes x_{\delta} x_{\gamma}\right) \\
& =\mathrm{x}_{\alpha} \mathrm{x}_{\gamma}^{n+1} \mathrm{x}_{\beta} \otimes x_{\delta}-q_{\beta \delta} \mathrm{x}_{\alpha} \mathrm{x}_{\gamma}^{n+1} \mathrm{x}_{\delta} \otimes x_{\beta}-s\left(-q_{\gamma \beta} q_{\gamma \delta} \mathrm{x}_{\alpha} \mathrm{x}_{\gamma}^{n} \mathrm{x}_{\beta} \otimes x_{\delta} x_{\gamma}\right. \\
& +q_{\beta \delta} q_{\gamma \delta} q_{\gamma \beta} \mathrm{x}_{\alpha} \mathrm{x}_{\gamma}^{n} \mathrm{x}_{\delta} \otimes x_{\beta} x_{\gamma}+q_{\alpha \delta}\left(-q_{\alpha \gamma}\right)^{n+1} q_{\beta \delta} \mathrm{x}_{\gamma}^{n+1} \mathrm{x}_{\delta} \otimes\left(q_{\alpha \beta} x_{\beta} x_{\alpha}+\mathrm{b}_{1} x_{\tau} x_{\gamma}\right) \\
& \left.-q_{\alpha \beta}\left(-q_{\alpha \gamma}\right)^{n+1} q_{\alpha \delta} \mathrm{x}_{\gamma}^{n+1} \mathrm{x}_{\beta} \otimes x_{\delta} x_{\alpha}-\left(-q_{\alpha \gamma}\right)^{n+1} q_{\gamma \delta} \mathrm{b}_{1} \mathrm{x}_{\gamma}^{n+1} \mathrm{x}_{\tau} \otimes x_{\delta} x_{\gamma}\right) \\
& =\mathrm{x}_{\alpha} \mathrm{x}_{\gamma}^{n+1} \mathrm{x}_{\beta} \otimes x_{\delta}-q_{\beta \delta} \mathrm{x}_{\alpha} \mathrm{x}_{\gamma}^{n+1} \mathrm{x}_{\delta} \otimes x_{\beta}+q_{\gamma \beta} q_{\gamma \delta} \mathrm{x}_{\alpha} \mathrm{x}_{\gamma}^{n} \mathrm{x}_{\beta} \mathrm{x}_{\delta} \otimes x_{\gamma} \\
& -s\left(-q_{\gamma \beta} q_{\gamma \delta}\left(-q_{\alpha \gamma}\right)^{n+1} q_{\alpha \beta} q_{\alpha \delta} \mathrm{x}_{\gamma}^{n} \mathrm{x}_{\beta} \mathrm{x}_{\delta} \otimes x_{\gamma} x_{\alpha}\right. \\
& +q_{\alpha \beta} q_{\alpha \delta}\left(-q_{\alpha \gamma}\right)^{n+1} q_{\beta \delta} \mathrm{x}_{\gamma}^{n+1} \mathrm{x}_{\delta} \otimes x_{\beta} x_{\alpha}+q_{\alpha \delta}\left(-q_{\alpha \gamma}\right)^{n+1} q_{\beta \delta} \mathrm{b}_{1} \mathrm{x}_{\gamma}^{n+1} \mathrm{x}_{\delta} \otimes x_{\tau} x_{\gamma} \\
& \left.-q_{\alpha \beta}\left(-q_{\alpha \gamma}\right)^{n+1} q_{\alpha \delta} \mathrm{x}_{\gamma}^{n+1} \mathrm{x}_{\beta} \otimes x_{\delta} x_{\alpha}-\left(-q_{\alpha \gamma}\right)^{n+1} q_{\gamma \delta} \mathrm{b}_{1} \mathrm{x}_{\gamma}^{n+1} \mathrm{x}_{\tau} \otimes x_{\delta} x_{\gamma}\right) \\
& =\mathrm{x}_{\alpha} \mathrm{x}_{\gamma}^{n+1} \mathrm{x}_{\beta} \otimes x_{\delta}-q_{\beta \delta} \mathrm{x}_{\alpha} \mathrm{x}_{\gamma}^{n+1} \mathrm{x}_{\delta} \otimes x_{\beta}+q_{\gamma \beta} q_{\gamma \delta} \mathrm{x}_{\alpha} \mathrm{x}_{\gamma}^{n} \mathrm{x}_{\beta} \mathrm{x}_{\delta} \otimes x_{\gamma} \\
& +\left(-q_{\alpha \gamma}\right)^{n+1} q_{\gamma \delta} \mathrm{b}_{1} \mathrm{x}_{\gamma}^{n+1} \mathrm{x}_{\tau} \mathrm{x}_{\delta} \otimes x_{\gamma}-s\left(-q_{\gamma \beta} q_{\gamma \delta}\left(-q_{\alpha \gamma}\right)^{n+1} q_{\alpha \beta} q_{\alpha \delta} \mathrm{x}_{\gamma}^{n} \mathrm{x}_{\beta} \mathrm{x}_{\delta} \otimes x_{\gamma} x_{\alpha}\right. \\
& \left.+q_{\alpha \beta} q_{\alpha \delta}\left(-q_{\alpha \gamma}\right)^{n+1} q_{\beta \delta} \mathrm{x}_{\gamma}^{n+1} \mathrm{x}_{\delta} \otimes x_{\beta} x_{\alpha}-q_{\alpha \beta}\left(-q_{\alpha \gamma}\right)^{n+1} q_{\alpha \delta} \mathrm{x}_{\gamma}^{n+1} \mathrm{x}_{\beta} \otimes x_{\delta} x_{\alpha}\right) \\
& =\mathrm{x}_{\alpha} \mathrm{x}_{\gamma}^{n+1} \mathrm{x}_{\beta} \otimes x_{\delta}-q_{\beta \delta} \mathrm{x}_{\alpha} \mathrm{x}_{\gamma}^{n+1} \mathrm{x}_{\delta} \otimes x_{\beta}+q_{\gamma \beta} q_{\gamma \delta} \mathrm{x}_{\alpha} \mathrm{x}_{\gamma}^{n} \mathrm{x}_{\beta} \mathrm{x}_{\delta} \otimes x_{\gamma} \\
& +\left(-q_{\alpha \gamma}\right)^{n+1} q_{\gamma \delta} \mathrm{b}_{1} \mathrm{x}_{\gamma}^{n+1} \mathrm{x}_{\tau} \mathrm{x}_{\delta} \otimes x_{\gamma}+q_{\alpha \beta}\left(-q_{\alpha \gamma}\right)^{n+1} q_{\alpha \delta} \mathrm{x}_{\gamma}^{n+1} \mathrm{x}_{\beta} \mathrm{x}_{\delta} \otimes x_{\alpha} .
\end{aligned}
$$

Finally we prove (10.1.17) by induction on $n$. Notice that root vectors corresponding to $\alpha<\eta<\gamma<\tau<\delta$ satisfy (10.1.11), so $d\left(\mathrm{x}_{\alpha} \mathrm{x}_{\gamma}^{n} \mathrm{x}_{\tau} \mathrm{x}_{\delta} \otimes 1\right)$ is given by (10.1.12). We claim 
that

$$
\begin{aligned}
& d\left(\mathrm{x}_{\alpha}^{2} \mathrm{x}_{\gamma}^{n} \mathrm{x}_{\beta} \mathrm{x}_{\delta} \otimes 1\right)=\mathrm{x}_{\alpha}^{2} \mathrm{x}_{\gamma}^{n} \mathrm{x}_{\beta} \otimes x_{\delta}-q_{\beta \delta} \mathrm{x}_{\alpha}^{2} \mathrm{x}_{\gamma}^{n} \mathrm{x}_{\delta} \otimes x_{\beta}+q_{\gamma \beta} q_{\gamma \delta} \mathrm{x}_{\alpha}^{2} \mathrm{x}_{\gamma}^{n-1} \mathrm{x}_{\beta} \mathrm{x}_{\delta} \otimes x_{\gamma} \\
& +\left(-q_{\alpha \gamma}\right)^{n} q_{\alpha \beta} q_{\alpha \delta} \mathrm{x}_{\alpha} \mathrm{x}_{\gamma}^{n} \mathrm{x}_{\beta} \mathrm{x}_{\delta} \otimes x_{\alpha}+q_{\gamma \beta}^{n} q_{\eta \gamma}^{n+1} q_{\eta \delta} \mathrm{b}_{1} \mathrm{~b}_{3} \mathrm{x}_{\gamma}^{n+2} \mathrm{x}_{\delta} \otimes x_{\eta}+c_{n} \mathrm{~b}_{1} \mathrm{~b}_{2} \mathrm{~b}_{3} \mathrm{x}_{\gamma}^{n+3} \otimes 1
\end{aligned}
$$

for some scalar $c_{n}$. For $n=0$,

$$
\begin{aligned}
& d\left(\mathrm{x}_{\alpha}^{2} \mathrm{x}_{\beta} \mathrm{x}_{\delta} \otimes 1\right)=\mathrm{x}_{\alpha}^{2} \mathrm{x}_{\beta} \otimes x_{\delta}-s\left(q_{\beta \delta} \mathrm{x}_{\alpha}^{2} \otimes x_{\delta} x_{\beta}-q_{\gamma \delta} \mathrm{b}_{1} \mathrm{x}_{\alpha} \mathrm{x}_{\tau} \otimes x_{\delta} x_{\gamma}-q_{\alpha \beta} q_{\alpha \delta} \mathrm{x}_{\alpha} \mathrm{x}_{\beta} \otimes x_{\delta} x_{\alpha}\right. \\
&\left.\quad+q_{\eta \gamma} \mathrm{b}_{1} \mathrm{~b}_{3} \mathrm{x}_{\gamma}^{2} \otimes\left(q_{\eta \delta} x_{\delta} x_{\eta}+\mathrm{b}_{2} x_{\gamma}\right)\right) \\
&=\mathrm{x}_{\alpha}^{2} \mathrm{x}_{\beta} \otimes x_{\delta}-q_{\beta \delta} \mathrm{x}_{\alpha}^{2} \mathrm{x}_{\delta} \otimes x_{\beta}-s\left(-q_{\gamma \delta} \mathrm{b}_{1} \mathrm{x}_{\alpha} \mathrm{x}_{\tau} \otimes x_{\delta} x_{\gamma}-q_{\alpha \beta} q_{\alpha \delta} \mathrm{x}_{\alpha} \mathrm{x}_{\beta} \otimes x_{\delta} x_{\alpha}\right. \\
&\left.\quad+q_{\eta \gamma} q_{\eta \delta} \mathrm{b}_{1} \mathrm{~b}_{3} \mathrm{x}_{\gamma}^{2} \otimes x_{\delta} x_{\eta}+q_{\eta \gamma} \mathrm{b}_{1} \mathrm{~b}_{2} \mathrm{~b}_{3} \mathrm{x}_{\gamma}^{2} \otimes x_{\gamma}+q_{\beta \delta} q_{\alpha \delta} \mathrm{x}_{\alpha} \mathrm{x}_{\delta} \otimes\left(q_{\alpha \beta} x_{\beta} x_{\alpha}+\mathrm{b}_{1} x_{\tau} x_{\gamma}\right)\right) \\
&=\mathrm{x}_{\alpha}^{2} \mathrm{x}_{\beta} \otimes x_{\delta}-q_{\beta \delta} \mathrm{x}_{\alpha}^{2} \mathrm{x}_{\delta} \otimes x_{\beta}+q_{\gamma \delta} \mathrm{b}_{1} \mathrm{x}_{\alpha} \mathrm{x}_{\tau} \mathrm{x}_{\delta} \otimes x_{\gamma}-s\left(-q_{\alpha \beta} q_{\alpha \delta} \mathrm{x}_{\alpha} \mathrm{x}_{\beta} \otimes x_{\delta} x_{\alpha}\right. \\
& \quad+q_{\alpha \tau} q_{\alpha \delta} q_{\gamma \delta} \mathrm{b}_{1} \mathrm{x}_{\tau} \mathrm{x}_{\delta} \otimes x_{\alpha} x_{\gamma}+q_{\eta \delta} q_{\gamma \delta} \mathrm{b}_{1} \mathrm{~b}_{3} \mathrm{x}_{\gamma} \mathrm{x}_{\delta} \otimes x_{\eta} x_{\gamma}+q_{\eta \gamma} q_{\eta \delta} \mathrm{b}_{1} \mathrm{~b}_{3} \mathrm{x}_{\gamma}^{2} \otimes x_{\delta} x_{\eta} \\
&\left.\quad+\left(q_{\eta \gamma}+q_{\gamma \delta}\right) \mathrm{b}_{1} \mathrm{~b}_{2} \mathrm{~b}_{3} \mathrm{x}_{\gamma}^{2} \otimes x_{\gamma}+q_{\beta \delta} q_{\alpha \delta} q_{\alpha \beta} \mathrm{x}_{\alpha} \mathrm{x}_{\delta} \otimes x_{\beta} x_{\alpha}\right) \\
&=\mathrm{x}_{\alpha}^{2} \mathrm{x}_{\beta} \otimes x_{\delta}-q_{\beta \delta} \mathrm{x}_{\alpha}^{2} \mathrm{x}_{\delta} \otimes x_{\beta}+q_{\gamma \delta} \mathrm{b}_{1} \mathrm{x}_{\alpha} \mathrm{x}_{\tau} \mathrm{x}_{\delta} \otimes x_{\gamma}+q_{\alpha \beta} q_{\alpha \delta} \mathrm{x}_{\alpha} \mathrm{x}_{\beta} \mathrm{x}_{\delta} \otimes x_{\alpha} \\
& \quad+\left(q_{\eta \gamma}+q_{\gamma \delta}\right) \mathrm{b}_{1} \mathrm{~b}_{2} \mathrm{~b}_{3} \mathrm{x}_{\gamma}^{3} \otimes 1-s\left(\left(q_{\alpha \beta}+q_{\alpha \tau} q_{\alpha \gamma}\right) q_{\alpha \delta} q_{\gamma \delta} \mathrm{b}_{1} \mathrm{x}_{\tau} \mathrm{x}_{\delta} \otimes x_{\gamma} x_{\alpha}\right. \\
&\left.\quad+q_{\eta \delta} q_{\gamma \delta} \mathrm{b}_{1} \mathrm{~b}_{3} \mathrm{x}_{\gamma} \mathrm{x}_{\delta} \otimes x_{\eta} x_{\gamma}+q_{\eta \gamma} q_{\eta \delta} \mathrm{b}_{1} \mathrm{~b}_{3} \mathrm{x}_{\gamma}^{2} \otimes x_{\delta} x_{\eta}\right) \\
&= \mathrm{x}_{\alpha}^{2} \mathrm{x}_{\beta} \otimes x_{\delta}-q_{\beta \delta} \mathrm{x}_{\alpha}^{2} \mathrm{x}_{\delta} \otimes x_{\beta}+q_{\gamma \delta} \mathrm{b}_{1} \mathrm{x}_{\alpha} \mathrm{x}_{\tau} \mathrm{x}_{\delta} \otimes x_{\gamma}+q_{\alpha \beta} q_{\alpha \delta} \mathrm{x}_{\alpha} \mathrm{x}_{\beta} \mathrm{x}_{\delta} \otimes x_{\alpha} \\
&+q_{\gamma \delta}\left(1-\widetilde{q}_{\gamma \delta}^{-1}\right) \mathrm{b}_{1} \mathrm{~b}_{2} \mathrm{~b}_{3} \mathrm{x}_{\gamma}^{3} \otimes 1+q_{\eta \gamma} q_{\eta \delta} \mathrm{b}_{1} \mathrm{~b}_{3} \mathrm{x}_{\gamma}^{2} \mathrm{x}_{\delta} \otimes x_{\eta} .
\end{aligned}
$$

Now assume that (10.1.17) holds for $n$. Using (10.1.21), Remark 10.1.2, inductive hypothesis, $x_{\gamma}^{2}=0=x_{\alpha}^{2}$, (10.1.12), (10.1.22),

$$
\begin{aligned}
& d\left(\mathrm{x}_{\alpha}^{2} \mathrm{x}_{\gamma}^{n+1} \mathrm{x}_{\beta} \mathrm{x}_{\delta} \otimes 1\right)=\mathrm{x}_{\alpha}^{2} \mathrm{x}_{\gamma}^{n+1} \mathrm{x}_{\beta} \otimes x_{\delta}-s\left(q_{\beta \delta} \mathrm{x}_{\alpha}^{2} \mathrm{x}_{\gamma}^{n+1} \otimes x_{\delta} x_{\beta}-q_{\gamma \beta} q_{\gamma \delta} \mathrm{x}_{\alpha}^{2} \mathrm{x}_{\gamma}^{n} \mathrm{x}_{\beta} \otimes x_{\delta} x_{\gamma}\right. \\
& -q_{\alpha \beta}\left(-q_{\alpha \gamma}\right)^{n+1} q_{\alpha \delta} \mathrm{x}_{\alpha} \mathrm{x}_{\gamma}^{n+1} \mathrm{x}_{\beta} \otimes x_{\delta} x_{\alpha}-\left(-q_{\alpha \gamma}\right)^{n+2} q_{\gamma \delta} \mathrm{b}_{1} \mathrm{x}_{\alpha} \mathrm{x}_{\gamma}^{n+1} \mathrm{x}_{\tau} \otimes x_{\delta} x_{\gamma} \\
& \left.+q_{\gamma \beta}^{n+1} q_{\eta \gamma}^{n+2} \mathrm{~b}_{1} \mathrm{~b}_{2} \mathrm{~b}_{3} \mathrm{x}_{\gamma}^{n+3} \otimes x_{\gamma}+q_{\gamma \beta}^{n+1} q_{\eta \gamma}^{n+2} q_{\eta \delta} \mathrm{b}_{1} \mathrm{~b}_{3} \mathrm{x}_{\gamma}^{n+3} \otimes x_{\delta} x_{\eta}\right) \\
& =\mathrm{x}_{\alpha}^{2} \mathrm{x}_{\gamma}^{n+1} \mathrm{x}_{\beta} \otimes x_{\delta}-q_{\beta \delta} \mathrm{x}_{\alpha}^{2} \mathrm{x}_{\gamma}^{n+1} \mathrm{x}_{\delta} \otimes x_{\beta}-s\left(q_{\gamma \beta} q_{\gamma \delta} q_{\beta \delta} \mathrm{x}_{\alpha}^{2} \mathrm{x}_{\gamma}^{n} \mathrm{x}_{\delta} \otimes x_{\beta} x_{\gamma}\right. \\
& +q_{\alpha \delta}\left(-q_{\alpha \gamma}\right)^{n+1} q_{\beta \delta} \mathrm{x}_{\alpha} \mathrm{x}_{\gamma}^{n+1} \mathrm{x}_{\delta} \otimes\left(q_{\alpha \beta} x_{\beta} x_{\alpha}+\mathrm{b}_{1} x_{\tau} x_{\gamma}\right)-q_{\gamma \beta} q_{\gamma \delta} \mathrm{x}_{\alpha}^{2} \mathrm{x}_{\gamma}^{n} \mathrm{x}_{\beta} \otimes x_{\delta} x_{\gamma} \\
& -q_{\alpha \beta}\left(-q_{\alpha \gamma}\right)^{n+1} q_{\alpha \delta} \mathrm{x}_{\alpha} \mathrm{x}_{\gamma}^{n+1} \mathbf{x}_{\beta} \otimes x_{\delta} x_{\alpha}-\left(-q_{\alpha \gamma}\right)^{n+2} q_{\gamma \delta} \mathrm{b}_{1} \mathrm{x}_{\alpha} \mathrm{x}_{\gamma}^{n+1} \mathbf{x}_{\tau} \otimes x_{\delta} x_{\gamma} \\
& \left.+q_{\gamma \beta}^{n+1} q_{\eta \gamma}^{n+2} \mathrm{~b}_{1} \mathrm{~b}_{2} \mathrm{~b}_{3} \mathrm{x}_{\gamma}^{n+3} \otimes x_{\gamma}+q_{\gamma \beta}^{n+1} q_{\eta \gamma}^{n+2} q_{\eta \delta} \mathrm{b}_{1} \mathrm{~b}_{3} \mathrm{x}_{\gamma}^{n+3} \otimes x_{\delta} x_{\eta}\right) \\
& =\mathrm{x}_{\alpha}^{2} \mathrm{x}_{\gamma}^{n+1} \mathrm{x}_{\beta} \otimes x_{\delta}-q_{\beta \delta} \mathrm{x}_{\alpha}^{2} \mathrm{x}_{\gamma}^{n+1} \mathrm{x}_{\delta} \otimes x_{\beta}+q_{\gamma \beta} q_{\gamma \delta} \mathrm{x}_{\alpha}^{2} \mathrm{x}_{\gamma}^{n} \mathrm{x}_{\beta} \mathrm{x}_{\delta} \otimes x_{\gamma} \\
& -s\left(-q_{\gamma \beta} q_{\gamma \delta}\left(-q_{\alpha \gamma}\right)^{n+1} q_{\alpha \beta} q_{\alpha \delta} \mathrm{x}_{\alpha} \mathrm{x}_{\gamma}^{n} \mathrm{x}_{\beta} \mathrm{x}_{\delta} \otimes x_{\gamma} x_{\alpha}\right. \\
& +q_{\gamma \beta} q_{\gamma \delta} c_{n} \mathrm{~b}_{1} \mathrm{~b}_{2} \mathrm{~b}_{3} \mathrm{x}_{\gamma}^{n+3} \otimes x_{\gamma}+q_{\gamma \beta} q_{\gamma \delta} q_{\eta \gamma} q_{\gamma \beta}^{n} q_{\eta \gamma}^{n+1} q_{\eta \delta} \mathrm{b}_{1} \mathrm{~b}_{3} \mathrm{x}_{\gamma}^{n+2} \mathrm{x}_{\delta} \otimes x_{\gamma} x_{\eta} \\
& +q_{\alpha \beta} q_{\alpha \delta}\left(-q_{\alpha \gamma}\right)^{n+1} q_{\beta \delta} \mathrm{x}_{\alpha} \mathrm{x}_{\gamma}^{n+1} \mathrm{x}_{\delta} \otimes x_{\beta} x_{\alpha}+q_{\alpha \delta}\left(-q_{\alpha \gamma}\right)^{n+1} q_{\beta \delta} \mathrm{b}_{1} \mathrm{x}_{\alpha} \mathrm{x}_{\gamma}^{n+1} \mathrm{x}_{\delta} \otimes x_{\tau} x_{\gamma} \\
& -q_{\alpha \beta}\left(-q_{\alpha \gamma}\right)^{n+1} q_{\alpha \delta} \mathrm{x}_{\alpha} \mathrm{x}_{\gamma}^{n+1} \mathrm{x}_{\beta} \otimes x_{\delta} x_{\alpha}-\left(-q_{\alpha \gamma}\right)^{n+2} q_{\gamma \delta} \mathrm{b}_{1} \mathrm{x}_{\alpha} \mathrm{x}_{\gamma}^{n+1} \mathrm{x}_{\tau} \otimes x_{\delta} x_{\gamma} \\
& \left.+q_{\gamma \beta}^{n+1} q_{\eta \gamma}^{n+2} \mathrm{~b}_{1} \mathrm{~b}_{2} \mathrm{~b}_{3} \mathrm{x}_{\gamma}^{n+3} \otimes x_{\gamma}+q_{\gamma \beta}^{n+1} q_{\eta \gamma}^{n+2} q_{\eta \delta} \mathrm{b}_{1} \mathrm{~b}_{3} \mathrm{x}_{\gamma}^{n+3} \otimes x_{\delta} x_{\eta}\right) \\
& =\mathrm{x}_{\alpha}^{2} \mathrm{x}_{\gamma}^{n+1} \mathrm{x}_{\beta} \otimes x_{\delta}-q_{\beta \delta} \mathrm{x}_{\alpha}^{2} \mathrm{x}_{\gamma}^{n+1} \mathrm{x}_{\delta} \otimes x_{\beta}+q_{\gamma \beta} q_{\gamma \delta} \mathrm{x}_{\alpha}^{2} \mathrm{x}_{\gamma}^{n} \mathrm{x}_{\beta} \mathrm{x}_{\delta} \otimes x_{\gamma}
\end{aligned}
$$




$$
\begin{aligned}
& +\left(-q_{\alpha \gamma}\right)^{n+2} q_{\gamma \delta} \mathrm{b}_{1} \mathrm{x}_{\alpha} \mathrm{x}_{\gamma}^{n+1} \mathrm{x}_{\tau} \mathrm{x}_{\delta} \otimes x_{\gamma}-s\left(-q_{\gamma \beta} q_{\gamma \delta}\left(-q_{\alpha \gamma}\right)^{n+1} q_{\alpha \beta} q_{\alpha \delta} \mathrm{x}_{\alpha} \mathrm{x}_{\gamma}^{n} \mathrm{x}_{\beta} \mathrm{x}_{\delta} \otimes x_{\gamma} x_{\alpha}\right. \\
& +q_{\gamma \beta} q_{\gamma \delta} c_{n} \mathrm{~b}_{1} \mathrm{~b}_{2} \mathrm{~b}_{3} \mathrm{x}_{\gamma}^{n+3} \otimes x_{\gamma}+q_{\gamma \beta} q_{\gamma \delta} q_{\eta \gamma} q_{\gamma \beta}^{n} q_{\eta \gamma}^{n+1} q_{\eta \delta} \mathrm{b}_{1} \mathrm{~b}_{3} \mathrm{x}_{\gamma}^{n+2} \mathrm{x}_{\delta} \otimes x_{\gamma} x_{\eta} \\
& +q_{\alpha \beta} q_{\alpha \delta}\left(-q_{\alpha \gamma}\right)^{n+1} q_{\beta \delta} \mathrm{x}_{\alpha} \mathrm{x}_{\gamma}^{n+1} \mathrm{x}_{\delta} \otimes x_{\beta} x_{\alpha}-q_{\alpha \beta}\left(-q_{\alpha \gamma}\right)^{n+1} q_{\alpha \delta} \mathrm{x}_{\alpha} \mathrm{x}_{\gamma}^{n+1} \mathrm{x}_{\beta} \otimes x_{\delta} x_{\alpha} \\
& +q_{\gamma \beta}^{n+1} q_{\eta \gamma}^{n+2} \mathrm{~b}_{1} \mathrm{~b}_{2} \mathrm{~b}_{3} \mathrm{x}_{\gamma}^{n+3} \otimes x_{\gamma}+q_{\gamma \beta}^{n+1} q_{\eta \gamma}^{n+2} q_{\eta \delta} \mathrm{b}_{1} \mathrm{~b}_{3} \mathrm{x}_{\gamma}^{n+3} \otimes x_{\delta} x_{\eta} \\
& -q_{\gamma \delta} q_{\eta \gamma} q_{\eta \delta} q_{\alpha \gamma}^{2 n+3}(n+2)_{\widetilde{q}_{\tau \gamma}} \mathrm{b}_{1} \mathrm{~b}_{3} \mathrm{x}_{\gamma}^{n+2} \mathrm{x}_{\delta} \otimes x_{\gamma} x_{\eta}-q_{\gamma \delta} q_{\alpha \gamma}^{2 n+4} q_{\alpha \tau} q_{\alpha \delta} \mathrm{b}_{1} \mathrm{x}_{\gamma}^{n+1} \mathrm{x}_{\tau} \mathrm{x}_{\delta} \otimes x_{\gamma} x_{\alpha} \\
& \left.+\left(-q_{\alpha \gamma}\right)^{n+2} q_{\gamma \delta} \mathrm{b}_{1} \mathrm{~b}_{2} \mathrm{~b}_{3}\left(-q_{\gamma \alpha}\right)^{-n-1} \mathrm{c}_{\alpha \tau \gamma}^{(n+1)} \mathrm{x}_{\gamma}^{n+3} \otimes x_{\gamma}\right) \\
& =\mathrm{x}_{\alpha}^{2} \mathrm{x}_{\gamma}^{n+1} \mathrm{x}_{\beta} \otimes x_{\delta}-q_{\beta \delta} \mathrm{x}_{\alpha}^{2} \mathrm{x}_{\gamma}^{n+1} \mathrm{x}_{\delta} \otimes x_{\beta}+q_{\gamma \beta} q_{\gamma \delta} \mathrm{x}_{\alpha}^{2} \mathrm{x}_{\gamma}^{n} \mathrm{x}_{\beta} \mathrm{x}_{\delta} \otimes x_{\gamma} \\
& +\left(-q_{\alpha \gamma}\right)^{n+2} q_{\gamma \delta} \mathrm{b}_{1} \mathrm{x}_{\alpha} \mathrm{x}_{\gamma}^{n+1} \mathrm{x}_{\tau} \mathrm{x}_{\delta} \otimes x_{\gamma}+q_{\alpha \beta}\left(-q_{\alpha \gamma}\right)^{n+1} q_{\alpha \delta} \mathrm{x}_{\alpha} \mathrm{x}_{\gamma}^{n+1} \mathrm{x}_{\beta} \mathrm{x}_{\delta} \otimes x_{\alpha} \\
& -s\left(q_{\gamma \beta}^{n+1} q_{\eta \gamma}^{n+2} q_{\eta \delta} \mathrm{b}_{1} \mathrm{~b}_{3} \mathrm{x}_{\gamma}^{n+3} \otimes x_{\delta} x_{\eta}\right. \\
& +\left(q_{\gamma \beta} q_{\gamma \beta}^{n} q_{\eta \gamma}^{n+1} q_{\eta \delta}-q_{\eta \delta} q_{\alpha \gamma}^{2 n+3}(n+2)_{\widetilde{q}_{\tau \gamma}}\right) q_{\gamma \delta} q_{\eta \gamma} \mathrm{b}_{1} \mathrm{~b}_{3} \mathrm{x}_{\gamma}^{n+2} \mathrm{x}_{\delta} \otimes x_{\gamma} x_{\eta} \\
& \left.+\left(q_{\gamma \beta}^{n+1} q_{\eta \gamma}^{n+2}+q_{\gamma \beta} q_{\gamma \delta} c_{n}-q_{\alpha \gamma}^{n+2} q_{\gamma \delta} q_{\gamma \alpha}^{-n-1} c_{\alpha \tau \gamma}^{(n+1)}\right) \mathrm{b}_{1} \mathrm{~b}_{2} \mathrm{~b}_{3} \mathrm{x}_{\gamma}^{n+3} \otimes x_{\gamma}\right) .
\end{aligned}
$$

Hence the claim follows using Remark 10.1.2 and that $d\left(\mathrm{x}_{\gamma}^{n+4} \otimes 1\right)=\mathrm{x}_{\gamma}^{n+3} \otimes x_{\gamma}$. The scalars $c_{n}$ are defined recursively by the equation:

$$
c_{n+1}=q_{\gamma \beta}^{n+1} q_{\eta \gamma}^{n+2}+q_{\gamma \beta} q_{\gamma \delta} c_{n}-q_{\alpha \gamma}^{n+2} q_{\gamma \delta} q_{\gamma \alpha}^{-n-1} \mathrm{c}_{\alpha \tau \gamma}^{(n+1)} .
$$

Thus (10.1.17) follows using (10.1.18) to express all the roots in terms of $\alpha, \beta, \gamma$.

Lemma 10.1.23. Let $\alpha<\beta<\gamma<\tau<\eta<\delta$ be positive roots such that $N_{\gamma}=2$ and the relations among the corresponding root vectors take the form

$$
\begin{aligned}
& x_{\alpha} x_{\delta}=q_{\alpha \delta} x_{\delta} x_{\alpha}+\mathrm{b}_{1} x_{\eta} x_{\gamma}, \quad x_{\beta} x_{\delta}=q_{\beta \delta} x_{\delta} x_{\beta}+\mathrm{b}_{2} x_{\eta} x_{\tau}, \\
& x_{\gamma} x_{\delta}=q_{\gamma \delta} x_{\delta} x_{\gamma}+\mathrm{b}_{3} x_{\eta}^{2} x_{\tau}, \quad x_{\alpha} x_{\tau}=q_{\alpha \tau} x_{\tau} x_{\alpha}+\mathrm{b}_{4} x_{\gamma} x_{\beta}, \\
& x_{\beta} x_{\eta}=q_{\beta \eta} x_{\eta} x_{\beta}+\mathrm{b}_{5} x_{\gamma},
\end{aligned}
$$

for some scalars $\mathrm{b}_{j} \in \mathbb{k}$ and the other pairs of root vectors $q$-commute. Then, for all $n \geq 0$,

$$
\begin{aligned}
& d\left(\mathrm{x}_{\alpha} \mathrm{x}_{\beta} \mathrm{x}_{\gamma}^{n} \mathrm{x}_{\delta} \otimes 1\right)=\mathrm{x}_{\alpha} \mathrm{x}_{\beta} \mathrm{x}_{\gamma}^{n} \otimes x_{\delta}-q_{\gamma \delta} \mathrm{x}_{\alpha} \mathrm{x}_{\beta} \mathrm{x}_{\gamma}^{n-1} \mathrm{x}_{\delta} \otimes x_{\gamma} \\
& -\left(-q_{\beta \gamma}\right)^{n} q_{\beta \delta} \mathrm{x}_{\alpha} \mathrm{x}_{\gamma}^{n} \mathrm{x}_{\delta} \otimes x_{\beta}+q_{\alpha \beta}\left(-q_{\alpha \gamma}\right)^{n} q_{\alpha \delta} \mathrm{x}_{\beta} \mathrm{x}_{\gamma}^{n} \mathrm{x}_{\delta} \otimes x_{\alpha} \\
& -\left(q_{\gamma \eta}^{n-1}(n)_{-\frac{q_{\beta \gamma}}{q \gamma \eta}} \mathrm{b}_{3} \mathrm{~b}_{5}+\left(-q_{\beta \gamma}\right)^{n} \mathrm{~b}_{2}\right) \mathrm{x}_{\alpha} \mathrm{x}_{\gamma}^{n} \mathrm{x}_{\eta} \otimes x_{\tau} \\
& +q_{\alpha \beta}\left(-q_{\alpha \gamma}\right)^{n} \mathrm{~b}_{1} \mathrm{~b}_{1} \mathrm{x}_{\beta} \mathrm{x}_{\gamma}^{n} \mathrm{x}_{\eta} \otimes x_{\gamma}-\mathrm{b}_{3} \mathrm{x}_{\alpha} \mathrm{x}_{\beta} \mathrm{x}_{\gamma}^{n-1} \mathrm{x}_{\eta} \otimes x_{\eta} x_{\tau} \\
& -\frac{q_{\alpha \beta}}{q_{\alpha \gamma}^{n} q_{\gamma \beta}^{n}}\left(\sum_{k=0}^{n}\left(-\widetilde{q}_{\alpha \gamma}\right)^{k}(k+1)_{\widetilde{q}_{\beta \gamma}}\right) \mathrm{b}_{1} \mathrm{~b}_{5} \mathrm{x}_{\gamma}^{n+2} \otimes 1 .
\end{aligned}
$$

Notice that the equalities in (10.1.24) force

$$
\alpha+\delta=\gamma+\eta, \quad \beta+\delta=\eta+\tau, \quad \gamma+\delta=2 \eta+\tau, \quad \eta+\beta=\gamma .
$$

Thus the following equality also holds: $2 \gamma=\alpha+\beta+\delta$.

Proof. A recursive computation on $n \in \mathbb{N}$ shows that

$$
d\left(\mathrm{x}_{\gamma}^{n} \mathrm{x}_{\delta} \otimes 1\right)=\mathrm{x}_{\gamma}^{n} \otimes x_{\delta}-q_{\gamma \delta} \mathrm{x}_{\gamma}^{n-1} \mathrm{x}_{\delta} \otimes x_{\gamma}-\mathrm{b}_{3} \mathrm{x}_{\gamma}^{n-1} \mathrm{x}_{\eta} \otimes x_{\eta} x_{\tau} .
$$


The root vectors corresponding to $\beta<\gamma<\eta$ satisfy (10.1.8), so by Lemma 10.1.7.

$$
\begin{aligned}
& d\left(\mathrm{x}_{\beta} \mathrm{x}_{\gamma}^{n} \mathrm{x}_{\eta} \otimes 1\right)=\mathrm{x}_{\beta} \mathrm{x}_{\gamma}^{n} \otimes x_{\eta}-q_{\gamma \eta} \mathrm{x}_{\beta} \mathrm{x}_{\gamma}^{n-1} \mathrm{x}_{\eta} \otimes x_{\gamma}-q_{\beta \eta}\left(-q_{\beta \gamma}\right)^{n} \mathbf{x}_{\gamma}^{n} \mathrm{x}_{\eta} \otimes x_{\beta} \\
& \quad+\mathrm{b}_{5} q_{\gamma \eta}^{n}(n+1)_{-\frac{q_{\beta} \gamma}{q_{\gamma \eta}} \mathrm{x}_{\gamma}^{n+1} \otimes 1}
\end{aligned}
$$

We need more auxiliary results:

$$
\begin{aligned}
& d\left(\mathrm{x}_{\alpha} \mathrm{x}_{\beta} \mathrm{x}_{\gamma}^{n} \mathrm{x}_{\eta} \otimes 1\right)=\mathrm{x}_{\alpha} \mathrm{x}_{\beta} \mathrm{x}_{\gamma}^{n} \otimes x_{\eta}-q_{\gamma \eta} \mathrm{x}_{\alpha} \mathrm{x}_{\beta} \mathrm{x}_{\gamma}^{n-1} \mathrm{x}_{\eta} \otimes x_{\gamma} \\
& \quad-\left(-q_{\beta \gamma}\right)^{n} q_{\beta \eta} \mathrm{x}_{\alpha} \mathrm{x}_{\gamma}^{n} \mathrm{x}_{\eta} \otimes x_{\beta}+q_{\alpha \beta}\left(-q_{\alpha \gamma}\right)^{n} q_{\alpha \eta} \mathrm{x}_{\beta} \mathrm{x}_{\gamma}^{n} \mathrm{x}_{\eta} \otimes x_{\alpha} \\
& \quad-q_{\gamma \eta}^{n}(n+1)_{-\frac{q_{\beta \gamma}}{q \gamma \eta}} \mathrm{b}_{5} \mathrm{x}_{\alpha} \mathrm{x}_{\gamma}^{n+1} \otimes 1, \\
& d\left(\mathrm{x}_{\alpha} \mathrm{x}_{\gamma}^{n} \mathrm{x}_{\delta} \otimes 1\right)=\mathrm{x}_{\alpha} \mathrm{x}_{\gamma}^{n} \otimes x_{\delta}-q_{\gamma \delta} \mathrm{x}_{\alpha} \mathrm{x}_{\gamma}^{n-1} \mathrm{x}_{\delta} \otimes x_{\gamma}-\left(-q_{\alpha \gamma}\right)^{n} q_{\alpha \delta} \mathrm{x}_{\gamma}^{n} \mathrm{x}_{\delta} \otimes x_{\alpha} \\
& \quad-\mathrm{b}_{3} \mathrm{x}_{\alpha} \mathrm{x}_{\gamma}^{n-1} \mathrm{x}_{\eta} \otimes x_{\eta} x_{\tau}-\left(-q_{\alpha \gamma}\right)^{n} \mathrm{~b}_{1} \mathrm{x}_{\gamma}^{n} \mathrm{x}_{\eta} \otimes x_{\gamma}, \\
& d\left(\mathrm{x}_{\beta} \mathrm{x}_{\gamma}^{n} \mathrm{x}_{\delta} \otimes 1\right)=\mathrm{x}_{\beta} \mathrm{x}_{\gamma}^{n} \otimes x_{\delta}-q_{\gamma \delta} \mathrm{x}_{\beta} \mathrm{x}_{\gamma}^{n-1} \mathrm{x}_{\delta} \otimes x_{\gamma}-\left(-q_{\beta \gamma}\right)^{n} q_{\beta \delta} \mathrm{x}_{\gamma}^{n} \mathrm{x}_{\delta} \otimes x_{\beta} \\
& \quad-\mathrm{b}_{3} \mathrm{x}_{\beta} \mathrm{x}_{\gamma}^{n-1} \mathrm{x}_{\eta} \otimes x_{\eta} x_{\tau}-\left(\left(-q_{\beta \gamma}\right)^{n} \mathrm{~b}_{2}-\mathrm{b}_{3} \mathrm{~b}_{5} q_{\gamma \eta}^{n}(n)_{-\frac{q_{\beta \gamma}}{q \gamma \eta}}\right) \mathrm{x}_{\gamma}^{n} \mathrm{x}_{\eta} \otimes x_{\tau} .
\end{aligned}
$$

We start with the proof of (10.1.29) by induction on $n$. For $n=0$,

$$
\begin{gathered}
d\left(\mathrm{x}_{\alpha} \mathrm{x}_{\beta} \mathrm{x}_{\eta} \otimes 1\right)=\mathrm{x}_{\alpha} \mathrm{x}_{\beta} \otimes x_{\eta}-s\left(q_{\beta \eta} \mathrm{x}_{\alpha} \otimes x_{\eta} x_{\beta}+\mathrm{b}_{5} \mathrm{x}_{\alpha} \otimes x_{\gamma}-q_{\alpha \beta} q_{\alpha \eta} \mathrm{x}_{\beta} \otimes x_{\eta} x_{\alpha}\right) \\
=\mathrm{x}_{\alpha} \mathrm{x}_{\beta} \otimes x_{\eta}-q_{\beta \eta} \mathrm{x}_{\alpha} \mathrm{x}_{\eta} \otimes x_{\beta}+q_{\alpha \beta} q_{\alpha \eta} \mathrm{x}_{\beta} \mathrm{x}_{\eta} \otimes x_{\alpha}-\mathrm{b}_{5} \mathrm{x}_{\alpha} \mathrm{x}_{\gamma} \otimes 1 .
\end{gathered}
$$

Now assume that (10.1.29) holds for $n$. By Remark 10.1.2, inductive hypothesis and (10.1.28),

$$
\begin{aligned}
d\left(\mathrm{x}_{\alpha} \mathrm{x}_{\beta} \mathrm{x}_{\gamma}^{n+1} \mathrm{x}_{\eta} \otimes 1\right)=\mathrm{x}_{\alpha} \mathrm{x}_{\beta} \mathrm{x}_{\gamma}^{n+1} \otimes x_{\eta}-s\left(q_{\gamma \eta} \mathrm{x}_{\alpha} \mathrm{x}_{\beta} \mathrm{x}_{\gamma}^{n} \otimes x_{\eta} x_{\gamma}\right. \\
\left.\quad+\left(-q_{\beta \gamma}\right)^{n+1} \mathrm{x}_{\alpha} \mathrm{x}_{\gamma}^{n+1} \otimes\left(q_{\beta \eta} x_{\eta} x_{\beta}+\mathrm{b}_{5} x_{\gamma}\right)-q_{\alpha \beta}\left(-q_{\alpha \gamma}\right)^{n+1} q_{\alpha \eta} \mathrm{x}_{\beta} \mathrm{x}_{\gamma}^{n+1} \otimes x_{\eta} x_{\alpha}\right) \\
=\mathrm{x}_{\alpha} \mathrm{x}_{\beta} \mathrm{x}_{\gamma}^{n+1} \otimes x_{\eta}-q_{\gamma \eta} \mathrm{x}_{\alpha} \mathrm{x}_{\beta} \mathrm{x}_{\gamma}^{n} \mathrm{x}_{\eta} \otimes x_{\gamma}-s\left(\left(-q_{\beta \gamma}\right)^{n} q_{\beta \eta} q_{\gamma \eta} q_{\beta \gamma} \mathrm{x}_{\alpha} \mathrm{x}_{\gamma}^{n} \mathrm{x}_{\eta} \otimes x_{\gamma} x_{\beta}\right. \\
\quad-q_{\alpha \beta}\left(-q_{\alpha \gamma}\right)^{n} q_{\alpha \eta} q_{\gamma \eta} q_{\alpha \gamma} \mathrm{x}_{\beta} \mathrm{x}_{\gamma}^{n} \mathrm{x}_{\eta} \otimes x_{\gamma} x_{\alpha}-q_{\alpha \beta}\left(-q_{\alpha \gamma}\right)^{n+1} q_{\alpha \eta} \mathrm{x}_{\beta} \mathrm{x}_{\gamma}^{n+1} \otimes x_{\eta} x_{\alpha} \\
\left.\quad+\left(-q_{\beta \gamma}\right)^{n+1} q_{\beta \eta} \mathrm{x}_{\alpha} \mathrm{x}_{\gamma}^{n+1} \otimes x_{\eta} x_{\beta}+\left(q_{\gamma \eta}^{n+1}(n+1)_{-\frac{q_{\beta \gamma}}{q_{\gamma \eta}}}+\left(-q_{\beta \gamma}\right)^{n+1}\right) \mathrm{b}_{5} \mathrm{x}_{\alpha} \mathrm{x}_{\gamma}^{n+1} \otimes x_{\gamma}\right) \\
=\mathrm{x}_{\alpha} \mathrm{x}_{\beta} \mathrm{x}_{\gamma}^{n+1} \otimes x_{\eta}-q_{\gamma \eta} \mathrm{x}_{\alpha} \mathrm{x}_{\beta} \mathrm{x}_{\gamma}^{n} \mathrm{x}_{\eta} \otimes x_{\gamma}-q_{\gamma \eta}^{n+1}(n+2)_{-\frac{q_{\beta \gamma}}{q_{\gamma \eta}}} \mathrm{b}_{5} \mathrm{x}_{\alpha} \mathrm{x}_{\gamma}^{n+2} \otimes 1 \\
\quad-\left(-q_{\beta \gamma}\right)^{n+1} q_{\beta \eta} \mathrm{x}_{\alpha} \mathrm{x}_{\gamma}^{n+1} \mathrm{x}_{\eta} \otimes x_{\beta}+q_{\alpha \beta}\left(-q_{\alpha \gamma}\right)^{n+1} q_{\alpha \eta} \mathrm{x}_{\beta} \mathrm{x}_{\gamma}^{n+1} \mathrm{x}_{\eta} \otimes x_{\alpha} .
\end{aligned}
$$

Next we prove (10.1.30) by induction on $n$. For $n=0$,

$$
d\left(\mathrm{x}_{\alpha} \mathrm{x}_{\delta} \otimes 1\right)=\mathrm{x}_{\alpha} \otimes x_{\delta}-q_{\alpha \delta} \mathrm{x}_{\delta} \otimes x_{\alpha}-\mathrm{b}_{1} \mathrm{x}_{\eta} \otimes x_{\gamma} .
$$

Now assume that (10.1.30) holds for $n$. Using Remark 10.1.2 three times, inductive hypothesis and (10.1.27):

$$
\begin{gathered}
d\left(\mathrm{x}_{\alpha} \mathrm{x}_{\gamma}^{n+1} \mathrm{x}_{\delta} \otimes 1\right)=\mathrm{x}_{\alpha} \mathrm{x}_{\gamma}^{n+1} \otimes x_{\delta}-s\left(\mathrm{x}_{\alpha} \mathrm{x}_{\gamma}^{n} \otimes\left(q_{\gamma \delta} x_{\delta} x_{\gamma}+\mathrm{b}_{3} x_{\eta}^{2} x_{\tau}\right)\right. \\
\left.+\left(-q_{\alpha \gamma}\right)^{n+1} \mathrm{x}_{\gamma}^{n+1} \otimes\left(q_{\alpha \delta} x_{\delta} x_{\alpha}+\mathrm{b}_{1} x_{\eta} x_{\gamma}\right)\right)
\end{gathered}
$$




$$
\begin{aligned}
= & \mathrm{x}_{\alpha} \mathrm{x}_{\gamma}^{n+1} \otimes x_{\delta}-\mathrm{b}_{3} \mathrm{x}_{\alpha} \mathrm{x}_{\gamma}^{n} \mathrm{x}_{\eta} \otimes x_{\eta} x_{\tau}-q_{\gamma \delta} \mathrm{x}_{\alpha} \mathrm{x}_{\gamma}^{n} \mathrm{x}_{\delta} \otimes x_{\gamma}-s\left(\left(-q_{\alpha \gamma}\right)^{n+1} \mathrm{~b}_{1} \mathrm{x}_{\gamma}^{n+1} \otimes x_{\eta} x_{\gamma}\right. \\
& +q_{\alpha \delta}\left(-q_{\alpha \gamma}\right)^{n+1} \mathrm{x}_{\gamma}^{n+1} \otimes x_{\delta} x_{\alpha}-\left(-q_{\alpha \gamma}\right)^{n+1} q_{\alpha \delta} q_{\gamma \delta} \mathrm{x}_{\gamma}^{n} \mathrm{x}_{\delta} \otimes x_{\gamma} x_{\alpha} \\
& \left.+q_{\alpha \eta}^{2}\left(-q_{\alpha \eta}\right)^{n} q_{\alpha \tau} \mathrm{b}_{3} \mathrm{x}_{\gamma}^{n} \mathrm{x}_{\eta} \otimes x_{\eta} x_{\tau} x_{\alpha}+q_{\alpha \eta}^{2}\left(-q_{\alpha \gamma}\right)^{n} \mathrm{~b}_{3} \mathrm{~b}_{4} \mathrm{x}_{\gamma}^{n} \mathrm{x}_{\eta} \otimes x_{\eta} x_{\gamma} x_{\beta}\right) \\
= & \mathrm{x}_{\alpha} \mathrm{x}_{\gamma}^{n+1} \otimes x_{\delta}-\mathrm{b}_{3} \mathrm{x}_{\alpha} \mathrm{x}_{\gamma}^{n} \mathrm{x}_{\eta} \otimes x_{\eta} x_{\tau}-q_{\gamma \delta} \mathrm{x}_{\alpha} \mathrm{x}_{\gamma}^{n} \mathrm{x}_{\delta} \otimes x_{\gamma}-\left(-q_{\alpha \gamma}\right)^{n+1} \mathrm{~b}_{1} \mathrm{x}_{\gamma}^{n+1} \mathrm{x}_{\eta} \otimes x_{\gamma} \\
& -q_{\alpha \delta}\left(-q_{\alpha \gamma}\right)^{n+1} \mathrm{x}_{\gamma}^{n+1} \mathrm{x}_{\delta} \otimes x_{\alpha} .
\end{aligned}
$$

Now we prove (10.1.31) by induction on $n$. For $n=0$,

$$
d\left(\mathrm{x}_{\beta} \mathrm{x}_{\delta} \otimes 1\right)=\mathrm{x}_{\beta} \otimes x_{\delta}-q_{\beta \delta} \mathrm{x}_{\delta} \otimes x_{\beta}-\mathrm{b}_{2} \mathrm{x}_{\eta} \otimes x_{\tau} .
$$

We assume that (10.1.31) holds for $n$. Using Remark 10.1.2 three times, (10.1.28), inductive hypothesis and (10.1.27):

$$
\begin{aligned}
& d\left(\mathrm{x}_{\beta} \mathrm{x}_{\gamma}^{n+1} \mathrm{x}_{\delta} \otimes 1\right)=\mathrm{x}_{\beta} \mathrm{x}_{\gamma}^{n+1} \otimes x_{\delta}-s\left(q_{\gamma \delta} \mathrm{x}_{\beta} \mathrm{x}_{\gamma}^{n} \otimes x_{\delta} x_{\gamma}+\mathrm{b}_{3} \mathrm{x}_{\beta} \mathrm{x}_{\gamma}^{n} \otimes x_{\eta}^{2} x_{\tau}\right. \\
& \left.+q_{\beta \delta}\left(-q_{\beta \gamma}\right)^{n+1} \mathrm{x}_{\gamma}^{n+1} \otimes x_{\delta} x_{\beta}+\left(-q_{\beta \gamma}\right)^{n+1} \mathrm{~b}_{2} \mathrm{x}_{\gamma}^{n+1} \otimes x_{\eta} x_{\tau}\right) \\
& =\mathrm{x}_{\beta} \mathrm{x}_{\gamma}^{n+1} \otimes x_{\delta}-q_{\gamma \delta} \mathrm{x}_{\beta} \mathrm{x}_{\gamma}^{n} \mathrm{x}_{\delta} \otimes x_{\gamma}-\mathrm{b}_{3} \mathrm{x}_{\beta} \mathrm{x}_{\gamma}^{n} \mathrm{x}_{\eta} \otimes x_{\eta} x_{\tau}-s\left(d_{n} q_{\gamma \delta} \mathrm{x}_{\gamma}^{n} \mathrm{x}_{\eta} \otimes x_{\tau} x_{\gamma}\right. \\
& -q_{\gamma \delta}\left(-q_{\beta \gamma}\right)^{n+1} q_{\beta \delta} \mathrm{x}_{\gamma}^{n} \mathrm{x}_{\delta} \otimes x_{\gamma} x_{\beta}+q_{\beta \delta}\left(-q_{\beta \gamma}\right)^{n+1} \mathrm{x}_{\gamma}^{n+1} \otimes x_{\delta} x_{\beta} \\
& +q_{\beta \eta}^{2} q_{\beta \tau}\left(-q_{\beta \gamma}\right)^{n} \mathrm{~b}_{3} \mathrm{x}_{\gamma}^{n} \mathrm{x}_{\eta} \otimes x_{\eta} x_{\tau} x_{\beta}+q_{\beta \eta}\left(-q_{\beta \gamma}\right)^{n} q_{\gamma \tau} \mathrm{b}_{3} \mathrm{~b}_{5} \mathrm{x}_{\gamma}^{n} \mathrm{x}_{\eta} \otimes x_{\tau} x_{\gamma} \\
& \left.+\left(\left(-q_{\beta \gamma}\right)^{n+1} \mathrm{~b}_{2}-\mathrm{b}_{3} \mathrm{~b}_{5} q_{\gamma \eta}^{n}(n+1)_{-\frac{q_{\beta \gamma}}{q \gamma \eta}}\right) \mathrm{x}_{\gamma}^{n+1} \otimes x_{\eta} x_{\tau}\right) \\
& =\mathrm{x}_{\beta} \mathrm{x}_{\gamma}^{n+1} \otimes x_{\delta}-q_{\gamma \delta} \mathrm{x}_{\beta} \mathrm{x}_{\gamma}^{n} \mathrm{x}_{\delta} \otimes x_{\gamma}-\mathrm{b}_{3} \mathrm{x}_{\beta} \mathrm{x}_{\gamma}^{n} \mathrm{x}_{\eta} \otimes x_{\eta} x_{\tau}-q_{\beta \delta}\left(-q_{\beta \gamma}\right)^{n+1} \mathbf{x}_{\gamma}^{n+1} \mathrm{x}_{\delta} \otimes x_{\beta} \\
& -\left(\left(-q_{\beta \gamma}\right)^{n+1} \mathrm{~b}_{2}-\mathrm{b}_{3} \mathrm{~b}_{5} q_{\gamma \eta}^{n+1}(n+1)_{-\frac{q_{\beta \gamma}}{q \gamma \eta}}\right) \mathrm{x}_{\gamma}^{n+1} \mathrm{x}_{\eta} \otimes x_{\tau} \text {. }
\end{aligned}
$$

Finally we prove (10.1.25). To do so, we prove that there exist $c_{n}, d_{n}, e_{n} \in \mathbb{k}$ such that

$$
\begin{aligned}
& d\left(\mathrm{x}_{\alpha} \mathrm{x}_{\beta} \mathrm{x}_{\gamma}^{n} \mathrm{x}_{\delta} \otimes 1\right)=\mathrm{x}_{\alpha} \mathrm{x}_{\beta} \mathrm{x}_{\gamma}^{n} \otimes x_{\delta}-q_{\gamma \delta} \mathrm{x}_{\alpha} \mathrm{x}_{\beta} \mathrm{x}_{\gamma}^{n-1} \mathrm{x}_{\delta} \otimes x_{\gamma}-\left(-q_{\beta \gamma}\right)^{n} q_{\beta \delta} \mathrm{x}_{\alpha} \mathrm{x}_{\gamma}^{n} \mathrm{x}_{\delta} \otimes x_{\beta} \\
& \quad+q_{\alpha \beta}\left(-q_{\alpha \gamma}\right)^{n} q_{\alpha \delta} \mathrm{x}_{\beta} \mathrm{x}_{\gamma}^{n} \mathrm{x}_{\delta} \otimes x_{\alpha}-c_{n} \mathrm{x}_{\alpha} \mathrm{x}_{\gamma}^{n} \mathrm{x}_{\eta} \otimes x_{\tau}-\mathrm{b}_{3} \mathrm{x}_{\alpha} \mathrm{x}_{\beta} \mathrm{x}_{\gamma}^{n-1} \mathrm{x}_{\eta} \otimes x_{\eta} x_{\tau} \\
& \quad+d_{n} \mathrm{x}_{\gamma}^{n} \mathrm{x}_{\eta} \otimes x_{\gamma}-e_{n} \mathrm{~b}_{1} \mathrm{~b}_{5} \mathrm{x}_{\gamma}^{n+2} \otimes 1 .
\end{aligned}
$$

For $n=0$,

$$
\begin{aligned}
d\left(\mathrm{x}_{\alpha} \mathrm{x}_{\beta} \mathrm{x}_{\delta} \otimes 1\right)=\mathrm{x}_{\alpha} \mathrm{x}_{\beta} \otimes x_{\delta}-s\left(\mathrm{x}_{\alpha} \otimes\left(q_{\beta \delta} x_{\delta} x_{\beta}+\mathrm{b}_{2} x_{\eta} x_{\tau}\right)-q_{\alpha \beta} \mathrm{x}_{\beta} \otimes x_{\alpha} x_{\delta}\right) \\
=\mathrm{x}_{\alpha} \mathrm{x}_{\beta} \otimes x_{\delta}-q_{\beta \delta} \mathrm{x}_{\alpha} \mathrm{x}_{\delta} \otimes x_{\beta}-\mathrm{b}_{2} \mathrm{x}_{\alpha} \mathrm{x}_{\eta} \otimes x_{\tau}+s\left(q_{\alpha \beta} q_{\alpha \delta} \mathrm{x}_{\beta} \otimes x_{\delta} x_{\alpha}+q_{\alpha \beta} \mathrm{b}_{1} \mathrm{x}_{\beta} \otimes x_{\eta} x_{\gamma}\right. \\
\left.\quad-\left(q_{\beta \delta} \mathrm{b}_{1}+q_{\alpha \eta} \mathrm{b}_{2} \mathrm{~b}_{4}\right) \mathrm{x}_{\eta} \otimes x_{\gamma} x_{\beta}-q_{\alpha \tau} q_{\alpha \eta} \mathrm{b}_{2} \mathrm{x}_{\eta} \otimes x_{\tau} x_{\alpha}-q_{\alpha \beta} q_{\alpha \delta} q_{\beta \delta} \mathrm{x}_{\delta} \otimes x_{\beta} x_{\alpha}\right) \\
=\mathrm{x}_{\alpha} \mathrm{x}_{\beta} \otimes x_{\delta}-q_{\beta \delta} \mathrm{x}_{\alpha} \mathrm{x}_{\delta} \otimes x_{\beta}-\mathrm{b}_{2} \mathrm{x}_{\alpha} \mathrm{x}_{\eta} \otimes x_{\tau}+q_{\alpha \beta} q_{\alpha \delta} \mathrm{x}_{\beta} \mathrm{x}_{\delta} \otimes x_{\alpha}+q_{\alpha \beta} \mathrm{b}_{1} \mathrm{x}_{\beta} \mathrm{x}_{\eta} \otimes x_{\gamma} \\
\quad+q_{\alpha \beta} \mathrm{b}_{1} \mathrm{~b}_{5} \mathrm{x}_{\gamma}^{2} \otimes 1-s\left(\left(q_{\beta \delta} \mathrm{b}_{1}+q_{\alpha \eta} \mathrm{b}_{2} \mathrm{~b}_{4}-q_{\beta \eta} q_{\beta \gamma} q_{\alpha \beta} \mathrm{b}_{1}\right) s\left(x_{\eta} x_{\gamma} x_{\beta}\right)\right) .
\end{aligned}
$$


We assume that (10.1.25) holds for $n$. Using Remark 10.1.2 twice, (10.1.29), inductive hypothesis, (10.1.30), (10.1.28), (10.1.31)

$$
\begin{aligned}
& d\left(\mathrm{x}_{\alpha} \mathrm{x}_{\beta} \mathrm{x}_{\gamma}^{n+1} \mathbf{x}_{\delta} \otimes 1\right)=\mathrm{x}_{\alpha} \mathrm{x}_{\beta} \mathrm{x}_{\gamma}^{n+1} \otimes x_{\delta}-s\left(q_{\gamma \delta} \mathrm{x}_{\alpha} \mathrm{x}_{\beta} \mathrm{x}_{\gamma}^{n} \otimes x_{\delta} x_{\gamma}+\mathrm{b}_{3} \mathrm{x}_{\alpha} \mathrm{x}_{\beta} \mathrm{x}_{\gamma}^{n} \otimes x_{\eta}^{2} x_{\tau}\right. \\
& \left.+\left(-q_{\beta \gamma}\right)^{n+1} \mathrm{x}_{\alpha} \mathrm{x}_{\gamma}^{n+1} \otimes x_{\beta} x_{\delta}-q_{\alpha \beta}\left(-q_{\alpha \gamma}\right)^{n+1} \mathrm{x}_{\beta} \mathrm{x}_{\gamma}^{n+1} \otimes x_{\alpha} x_{\delta}\right) \\
& =\mathrm{x}_{\alpha} \mathrm{x}_{\beta} \mathrm{x}_{\gamma}^{n+1} \otimes x_{\delta}-q_{\gamma \delta} \mathrm{x}_{\alpha} \mathrm{x}_{\beta} \mathrm{x}_{\gamma}^{n} \mathrm{x}_{\delta} \otimes x_{\gamma}-\mathrm{b}_{3} \mathrm{x}_{\alpha} \mathrm{x}_{\beta} \mathrm{x}_{\gamma}^{n} \mathrm{x}_{\eta} \otimes x_{\eta} x_{\tau}-s\left(q_{\gamma \delta} e_{n} \mathrm{~b}_{1} \mathrm{~b}_{5} \mathrm{x}_{\gamma}^{n+2} \otimes x_{\gamma}\right. \\
& +q_{\gamma \delta}\left(-q_{\beta \gamma}\right)^{n} q_{\beta \gamma} q_{\beta \delta} \mathbf{x}_{\alpha} \mathbf{x}_{\gamma}^{n} \mathrm{x}_{\delta} \otimes x_{\gamma} x_{\beta}+q_{\gamma \delta} q_{\alpha \beta}\left(-q_{\alpha \gamma}\right)^{n+1} q_{\alpha \delta} \mathbf{x}_{\beta} \mathbf{x}_{\gamma}^{n} \mathbf{x}_{\delta} \otimes x_{\gamma} x_{\alpha} \\
& +\left(-q_{\beta \gamma}\right)^{n+1} q_{\beta \delta} \mathrm{x}_{\alpha} \mathrm{x}_{\gamma}^{n+1} \otimes x_{\delta} x_{\beta}+q_{\gamma \delta} c_{n} \mathrm{~b}_{2} \mathrm{x}_{\alpha} \mathrm{x}_{\gamma}^{n} \mathrm{x}_{\eta} \otimes x_{\tau} x_{\gamma} \\
& +\left(-q_{\beta \gamma}\right)^{n} q_{\beta \eta}^{2} q_{\beta \tau} \mathrm{b}_{3} \mathrm{x}_{\alpha} \mathrm{x}_{\gamma}^{n} \mathrm{x}_{\eta} \otimes x_{\eta} x_{\tau} x_{\beta}+\left(-q_{\beta \gamma}\right)^{n} q_{\beta \eta} q_{\gamma \tau} \mathrm{b}_{3} \mathrm{~b}_{5} \mathrm{x}_{\alpha} \mathrm{x}_{\gamma}^{n} \mathrm{x}_{\eta} \otimes x_{\tau} x_{\gamma} \\
& -q_{\alpha \beta}\left(-q_{\alpha \gamma}\right)^{n} q_{\alpha \eta}^{2} q_{\alpha \tau} \mathrm{b}_{3} \mathrm{x}_{\beta} \mathrm{x}_{\gamma}^{n} \mathrm{x}_{\eta} \otimes x_{\eta} x_{\tau} x_{\alpha}-q_{\alpha \beta}\left(-q_{\alpha \gamma}\right)^{n} q_{\alpha \eta}^{2} \mathrm{~b}_{3} \mathrm{~b}_{4} \mathrm{x}_{\beta} \mathrm{x}_{\gamma}^{n} \mathrm{x}_{\eta} \otimes x_{\eta} x_{\gamma} x_{\beta} \\
& +\left(q_{\gamma \eta}^{n}(n+1)_{-\frac{q_{\beta \gamma}}{q_{\gamma \eta}}} \mathrm{b}_{3} \mathrm{~b}_{5}+\left(-q_{\beta \gamma}\right)^{n+1} \mathrm{~b}_{2}\right) \mathrm{x}_{\alpha} \mathrm{x}_{\gamma}^{n+1} \otimes x_{\eta} x_{\tau} \\
& \left.-q_{\alpha \beta}\left(-q_{\alpha \gamma}\right)^{n+1} \mathrm{~b}_{1} \mathrm{x}_{\beta} \mathrm{x}_{\gamma}^{n+1} \otimes x_{\eta} x_{\gamma}-q_{\alpha \beta}\left(-q_{\alpha \gamma}\right)^{n+1} q_{\alpha \delta} \mathrm{x}_{\beta} \mathrm{x}_{\gamma}^{n+1} \otimes x_{\delta} x_{\alpha}\right) \\
& =\mathrm{x}_{\alpha} \mathrm{x}_{\beta} \mathrm{x}_{\gamma}^{n+1} \otimes x_{\delta}-q_{\gamma \delta} \mathrm{x}_{\alpha} \mathrm{x}_{\beta} \mathrm{x}_{\gamma}^{n} \mathrm{x}_{\delta} \otimes x_{\gamma}-\left(-q_{\beta \gamma}\right)^{n+1} q_{\beta \delta} \mathrm{x}_{\alpha} \mathrm{x}_{\gamma}^{n+1} \mathrm{x}_{\delta} \otimes x_{\beta} \\
& -\mathrm{b}_{3} \mathrm{x}_{\alpha} \mathrm{x}_{\beta} \mathrm{x}_{\gamma}^{n} \mathrm{x}_{\eta} \otimes x_{\eta} x_{\tau}-\left(q_{\gamma \eta}^{n}(n+1)_{-\frac{q_{\beta} \gamma}{q_{\gamma \eta}}} \mathrm{b}_{3} \mathrm{~b}_{5}+\left(-q_{\beta \gamma}\right)^{n+1} \mathrm{~b}_{2}\right) \mathrm{x}_{\alpha} \mathbf{x}_{\gamma}^{n+1} \mathbf{x}_{\eta} \otimes x_{\tau} \\
& -s\left(-q_{\alpha \beta}\left(-q_{\alpha \gamma}\right)^{n+1} \mathrm{~b}_{1} \mathrm{x}_{\beta} \mathrm{x}_{\gamma}^{n+1} \otimes x_{\eta} x_{\gamma}-q_{\alpha \beta}\left(-q_{\alpha \gamma}\right)^{n+1} q_{\alpha \delta} \mathrm{x}_{\beta} \mathrm{x}_{\gamma}^{n+1} \otimes x_{\delta} x_{\alpha}\right. \\
& +\left(q_{\gamma \eta}^{n}(n+1)_{-\frac{q_{\beta \gamma}}{q_{\gamma \eta}}} \mathrm{b}_{3} \mathrm{~b}_{5}+\left(-q_{\beta \gamma}\right)^{n+1} \mathrm{~b}_{2}\right) q_{\alpha \eta}\left(-q_{\alpha \gamma}\right)^{n+1} \mathbf{x}_{\gamma}^{n+1} \mathbf{x}_{\eta} \otimes x_{\alpha} x_{\tau} \\
& +q_{\gamma \delta} e_{n} \mathrm{~b}_{1} \mathrm{~b}_{5} \mathrm{x}_{\gamma}^{n+2} \otimes x_{\gamma}+q_{\alpha \beta} q_{\alpha \gamma}^{n+1} q_{\alpha \delta} q_{\beta \gamma}^{n+1} q_{\beta \delta} \mathrm{x}_{\gamma}^{n+1} \mathrm{x}_{\delta} \otimes x_{\beta} x_{\alpha} \\
& +q_{\gamma \delta} q_{\alpha \beta}\left(-q_{\alpha \gamma}\right)^{n+1} q_{\alpha \delta} \mathrm{x}_{\beta} \mathrm{x}_{\gamma}^{n} \mathrm{x}_{\delta} \otimes x_{\gamma} x_{\alpha}+\left(-q_{\beta \gamma}\right)^{n+1} q_{\beta \delta}\left(-q_{\alpha \gamma}\right)^{n+1} \mathrm{~b}_{1} \mathrm{x}_{\gamma}^{n+1} \mathrm{x}_{\eta} \otimes x_{\gamma} x_{\beta} \\
& \left.-q_{\alpha \beta}\left(-q_{\alpha \gamma}\right)^{n} q_{\alpha \eta}^{2} q_{\alpha \tau} \mathrm{b}_{3} \mathrm{x}_{\beta} \mathrm{x}_{\gamma}^{n} \mathrm{x}_{\eta} \otimes x_{\eta} x_{\tau} x_{\alpha}-q_{\alpha \beta}\left(-q_{\alpha \gamma}\right)^{n} q_{\alpha \eta}^{2} \mathrm{~b}_{3} \mathrm{~b}_{4} \mathrm{x}_{\beta} \mathrm{x}_{\gamma}^{n} \mathrm{x}_{\eta} \otimes x_{\eta} x_{\gamma} x_{\beta}\right) \\
& =\mathrm{x}_{\alpha} \mathrm{x}_{\beta} \mathrm{x}_{\gamma}^{n+1} \otimes x_{\delta}-q_{\gamma \delta} \mathrm{x}_{\alpha} \mathrm{x}_{\beta} \mathrm{x}_{\gamma}^{n} \mathrm{x}_{\delta} \otimes x_{\gamma}-\left(-q_{\beta \gamma}\right)^{n+1} q_{\beta \delta} \mathrm{x}_{\alpha} \mathrm{x}_{\gamma}^{n+1} \mathrm{x}_{\delta} \otimes x_{\beta} \\
& -\mathrm{b}_{3} \mathrm{x}_{\alpha} \mathrm{x}_{\beta} \mathrm{x}_{\gamma}^{n} \mathrm{x}_{\eta} \otimes x_{\eta} x_{\tau}-\left(q_{\gamma \eta}^{n}(n+1){ }_{-\frac{q_{\beta \gamma}}{q \gamma \eta}} \mathrm{b}_{3} \mathrm{~b}_{5}+\left(-q_{\beta \gamma}\right)^{n+1} \mathrm{~b}_{2}\right) \mathrm{x}_{\alpha} \mathrm{x}_{\gamma}^{n+1} \mathrm{x}_{\eta} \otimes x_{\tau} \\
& +q_{\alpha \beta}\left(-q_{\alpha \gamma}\right)^{n+1} \mathrm{~b}_{1} \mathbf{x}_{\beta} \mathrm{x}_{\gamma}^{n+1} \mathbf{x}_{\eta} \otimes x_{\gamma}+q_{\alpha \beta}\left(-q_{\alpha \gamma}\right)^{n+1} q_{\alpha \delta} \mathbf{x}_{\beta} \mathbf{x}_{\gamma}^{n+1} \mathbf{x}_{\delta} \otimes x_{\alpha} \\
& -s\left(\left(q_{\alpha \beta}\left(-q_{\alpha \gamma}\right)^{n+1} q_{\gamma \eta}^{n+1}(n+2)_{-\frac{q_{\beta \gamma}}{q_{\gamma \eta}}}+q_{\gamma \delta} e_{n}\right) \mathrm{b}_{1} \mathrm{~b}_{5} \mathrm{x}_{\gamma}^{n+2} \otimes x_{\gamma}\right. \\
& +\left(q_{\gamma \eta}^{n}(n+1)_{-\frac{q_{\beta \gamma}}{q_{\gamma \eta}}} \mathrm{b}_{3} \mathrm{~b}_{5}+\left(-q_{\beta \gamma}\right)^{n+1} \mathrm{~b}_{2}\right) q_{\alpha \eta}\left(-q_{\alpha \gamma}\right)^{n+1} \mathrm{~b}_{4} s\left(\mathrm{x}_{\gamma}^{n+1} \otimes x_{\eta} x_{\gamma} x_{\beta}\right) \\
& \left.-q_{\alpha \beta}\left(-q_{\alpha \gamma}\right)^{n} q_{\alpha \eta}^{2} \mathrm{~b}_{3} \mathrm{~b}_{4} s\left(\mathrm{x}_{\beta} \mathrm{x}_{\gamma}^{n} \otimes x_{\eta}^{2} x_{\gamma} x_{\beta}\right)\right) .
\end{aligned}
$$

Hence the inductive step follows since $s^{2}=0$ and $s\left(\mathrm{x}_{\gamma}^{n+2} \otimes x_{\gamma}\right)=\mathrm{x}_{\gamma}^{n+3} \otimes 1$; for the last step we use the equalities

$$
s\left(\mathrm{x}_{\gamma}^{n+1} \otimes x_{\eta} x_{\gamma} x_{\beta}\right)=\mathrm{x}_{\gamma}^{n+1} \mathbf{x}_{\eta} \otimes x_{\gamma} x_{\beta}, \quad s\left(\mathrm{x}_{\beta} \mathrm{x}_{\gamma}^{n} \otimes x_{\eta}^{2} x_{\gamma} x_{\beta}\right)=\mathrm{x}_{\beta} \mathrm{x}_{\gamma}^{n} \mathrm{x}_{\eta} \otimes x_{\eta} x_{\gamma} x_{\beta},
$$


which follow since $x_{\gamma}^{2}=0$ and $x_{\gamma} x_{\eta}=q_{\gamma \eta} x_{\eta} x_{\gamma}$. The formula for $c_{n}, d_{n}$ is explicit, while for the $e_{n}$ 's we have the recursive expression: $e_{0}=q_{\alpha \beta}$,

$$
e_{n+1}=q_{\alpha \beta} q_{\alpha \gamma}^{n+1} q_{\gamma \beta}^{-n-1}(n+2)_{\widetilde{q}_{\beta \gamma}}+q_{\gamma \alpha}^{-1} q_{\gamma \beta}^{-1} e_{n}, \quad n \geq 0,
$$

where we use (10.1.26) to express $\delta$ and $\eta$ in terms of $\alpha, \beta$ and $\gamma$.

Lemma 10.1.32. Let $\alpha<\beta<\delta<\gamma<\tau<\varphi<\eta$ be positive roots such that $N_{\gamma}=2$ and the relations among the corresponding root vectors take the form

$$
\begin{aligned}
& x_{\alpha} x_{\varphi}=q_{\alpha \varphi} x_{\varphi} x_{\alpha}+\mathrm{b}_{1} x_{\gamma}, \\
& x_{\beta} x_{\tau}=q_{\beta \tau} x_{\tau} x_{\beta}+\mathrm{b}_{2} x_{\gamma}, \quad x_{\delta} x_{\eta}=q_{\delta \eta} x_{\eta} x_{\delta}+\mathrm{b}_{3} x_{\varphi} x_{\tau} x_{\gamma},
\end{aligned}
$$

for some scalars $\mathrm{b}_{i}$, and the other pairs of root vectors $q$-commute. Then, for all $n \geq 0$,

$$
\begin{aligned}
& d\left(\mathrm{x}_{\alpha} \mathrm{x}_{\beta} \mathrm{x}_{\delta} \mathrm{x}_{\gamma}^{n} \mathrm{x}_{\eta} \otimes 1\right)=\mathrm{x}_{\alpha} \mathrm{x}_{\beta} \mathrm{x}_{\delta} \mathrm{x}_{\gamma}^{n} \otimes x_{\eta}-q_{\gamma \eta} \mathrm{x}_{\alpha} \mathrm{x}_{\beta} \mathrm{x}_{\delta} \mathrm{x}_{\gamma}^{n-1} \mathrm{x}_{\eta} \otimes x_{\gamma} \\
& \quad-\left(-q_{\delta \gamma}\right)^{n} q_{\delta \eta} \mathrm{x}_{\alpha} \mathrm{x}_{\beta} \mathrm{x}_{\gamma}^{n} \mathrm{x}_{\eta} \otimes x_{\delta}+q_{\beta \delta}\left(-q_{\beta \gamma}\right)^{n} q_{\beta \eta} \mathrm{x}_{\alpha} \mathrm{x}_{\delta} \mathrm{x}_{\gamma}^{n} \mathrm{x}_{\eta} \otimes x_{\beta} \\
& \quad-q_{\alpha \beta} q_{\alpha \delta}\left(-q_{\alpha \gamma}\right)^{n} q_{\alpha \eta} \mathrm{x}_{\beta} \mathrm{x}_{\delta} \mathrm{x}_{\gamma}^{n} \mathrm{x}_{\eta} \otimes x_{\alpha}-\left(-q_{\delta \gamma}\right)^{n} \mathrm{~b}_{3} \mathrm{x}_{\alpha} \mathrm{x}_{\beta} \mathrm{x}_{\gamma}^{n} \mathrm{x}_{\varphi} \otimes x_{\tau} x_{\gamma} \\
& \quad-q_{\alpha \beta} q_{\gamma \varphi}(n+1)_{\widetilde{q}_{\alpha \gamma}}\left(-q_{\delta \gamma}\right)^{n} \mathrm{~b}_{1} \mathrm{~b}_{3} \mathrm{x}_{\beta} \mathrm{x}_{\gamma}^{n+1} \mathrm{x}_{\tau} \otimes x_{\gamma}-\frac{q_{\alpha \beta} q_{\gamma \eta}}{\left(-q_{\delta \gamma}\right)^{n}} \mathrm{~d}_{\alpha \beta \delta \gamma}^{(n)} \mathrm{x}_{\gamma}^{n+3} \otimes 1,
\end{aligned}
$$

where $\mathrm{d}_{\alpha \beta \delta \gamma}^{(n)}:=\sum_{k=0}^{n} \widetilde{q}_{\delta \gamma}^{k}(k+1)_{\widetilde{q}_{\alpha \gamma}}(k+2)_{\widetilde{q}_{\beta \gamma}}, n \in \mathbb{N}$.

Notice that the equalities in (10.1.33) force

$$
\alpha+\varphi=\gamma=\beta+\tau, \quad \eta+\delta=\gamma+\tau+\varphi .
$$

Hence the following equality also holds: $3 \gamma=\alpha+\beta+\delta+\eta$.

Proof. We may apply Lemma 10.1.7 to the 3-tuples $\alpha<\gamma<\varphi$ and $\beta<\gamma<\tau$ to obtain

$$
\begin{aligned}
d\left(\mathrm{x}_{\alpha} \mathrm{x}_{\gamma}^{n} \mathrm{x}_{\varphi} \otimes 1\right)= & \mathrm{x}_{\alpha} \mathrm{x}_{\gamma}^{n} \otimes x_{\varphi}-q_{\gamma \varphi} \mathrm{x}_{\alpha} \mathrm{x}_{\gamma}^{n-1} \mathrm{x}_{\varphi} \otimes x_{\gamma}-\left(-q_{\alpha \gamma}\right)^{n} q_{\alpha \varphi} \mathrm{x}_{\gamma}^{n} \mathrm{x}_{\varphi} \otimes x_{\alpha} \\
& +\mathrm{b}_{1} q_{\gamma \varphi}(n+1)_{\widetilde{q}_{\alpha \gamma}} \mathrm{x}_{\gamma}^{n+1} \otimes 1, \\
d\left(\mathrm{x}_{\beta} \mathrm{x}_{\gamma}^{n} \mathrm{x}_{\tau} \otimes 1\right)= & \mathrm{x}_{\beta} \mathrm{x}_{\gamma}^{n} \otimes x_{\tau}-q_{\gamma \tau} \mathrm{x}_{\beta} \mathrm{x}_{\gamma}^{n-1} \mathrm{x}_{\tau} \otimes x_{\gamma}-\left(-q_{\beta \gamma}\right)^{n} q_{\beta \tau} \mathrm{x}_{\gamma}^{n} \mathrm{x}_{\tau} \otimes x_{\beta} \\
& +\mathrm{b}_{2} q_{\gamma \tau}(n+1)_{\widetilde{q}_{\beta}} \mathrm{x}_{\gamma}^{n+1} \otimes 1,
\end{aligned}
$$

for all $n \geq 0$. The next step is to prove by induction on $n$ the following equalities:

$$
\begin{aligned}
& d\left(\mathrm{x}_{\alpha} \mathrm{x}_{\beta} \mathrm{x}_{\gamma}^{n} \mathrm{x}_{\varphi} \otimes 1\right)=\mathrm{x}_{\alpha} \mathrm{x}_{\beta} \mathrm{x}_{\gamma}^{n} \otimes x_{\varphi}-q_{\gamma \varphi} \mathrm{x}_{\alpha} \mathrm{x}_{\beta} \mathrm{x}_{\gamma}^{n-1} \mathrm{x}_{\varphi} \otimes x_{\gamma} \\
& \quad-\left(-q_{\beta \gamma}\right)^{n} q_{\beta \varphi} \mathrm{x}_{\alpha} \mathrm{x}_{\gamma}^{n} \mathrm{x}_{\varphi} \otimes x_{\beta}+q_{\alpha \beta}\left(-q_{\alpha \gamma}\right)^{n} q_{\alpha \varphi} \mathrm{x}_{\beta} \mathrm{x}_{\gamma}^{n} \mathrm{x}_{\varphi} \otimes x_{\alpha} \\
& \quad+q_{\alpha \beta} q_{\gamma \varphi}^{n}(n+1)_{\widetilde{q}_{\alpha \gamma}} \mathrm{b}_{1} \mathrm{x}_{\beta} \mathrm{x}_{\gamma}^{n+1} \otimes 1 \\
& d\left(\mathrm{x}_{\delta} \mathrm{x}_{\gamma}^{n} \mathrm{x}_{\eta} \otimes 1\right)=\mathrm{x}_{\delta} \mathrm{x}_{\gamma}^{n} \otimes x_{\eta}-q_{\gamma \eta} \mathrm{x}_{\delta} \mathrm{x}_{\gamma}^{n-1} \mathrm{x}_{\eta} \otimes x_{\gamma}-\left(-q_{\delta \gamma}\right)^{n} q_{\delta \eta} \mathrm{x}_{\gamma}^{n} \mathrm{x}_{\eta} \otimes x_{\delta} \\
& \quad-\left(-q_{\delta \gamma}\right)^{n} \mathrm{~b}_{3} \mathrm{x}_{\gamma}^{n} \mathrm{x}_{\varphi} \otimes x_{\tau} x_{\gamma} \\
& d\left(\mathrm{x}_{\alpha} \mathrm{x}_{\delta} \mathrm{x}_{\gamma}^{n} \mathrm{x}_{\eta} \otimes 1\right)=\mathrm{x}_{\alpha} \mathrm{x}_{\delta} \mathrm{x}_{\gamma}^{n} \otimes x_{\eta}-q_{\gamma \eta} \mathrm{x}_{\alpha} \mathrm{x}_{\delta} \mathrm{x}_{\gamma}^{n-1} \mathrm{x}_{\eta} \otimes x_{\gamma} \\
& \quad-\left(-q_{\delta \gamma}\right)^{n} q_{\delta \eta} \mathrm{x}_{\alpha} \mathrm{x}_{\gamma}^{n} \mathrm{x}_{\eta} \otimes x_{\delta}+q_{\alpha \delta}\left(-q_{\alpha \gamma}\right)^{n} q_{\alpha \eta} \mathrm{x}_{\delta} \mathrm{x}_{\gamma}^{n} \mathrm{x}_{\eta} \otimes x_{\alpha} \\
& \quad-\left(-q_{\delta \gamma}\right)^{n} \mathrm{~b}_{3} \mathrm{x}_{\alpha} \mathrm{x}_{\gamma}^{n} \mathrm{x}_{\varphi} \otimes x_{\tau} x_{\gamma}-q_{\gamma \varphi}(n+1)_{\widetilde{q}_{\alpha \gamma}}\left(-q_{\delta \gamma}\right)^{n} \mathrm{~b}_{1} \mathrm{~b}_{3} \mathrm{x}_{\gamma}^{n+1} \mathrm{x}_{\tau} \otimes x_{\gamma},
\end{aligned}
$$




$$
\begin{aligned}
& d\left(\mathrm{x}_{\beta} \mathrm{x}_{\delta} \mathrm{x}_{\gamma}^{n} \mathrm{x}_{\eta} \otimes 1\right)=\mathbf{x}_{\beta} \mathrm{x}_{\delta} \mathbf{x}_{\gamma}^{n} \otimes x_{\eta}-q_{\gamma \eta} \mathrm{x}_{\beta} \mathrm{x}_{\delta} \mathbf{x}_{\gamma}^{n-1} \mathbf{x}_{\eta} \otimes x_{\gamma} \\
& \quad-\left(-q_{\delta \gamma}\right)^{n} q_{\delta \eta} \mathrm{x}_{\beta} \mathrm{x}_{\gamma}^{n} \mathrm{x}_{\eta} \otimes x_{\delta}+q_{\beta \delta}\left(-q_{\beta \gamma}\right)^{n} q_{\beta \eta} \mathrm{x}_{\delta} \mathrm{x}_{\gamma}^{n} \mathrm{x}_{\eta} \otimes x_{\beta} \\
& \quad-\left(-q_{\delta \gamma}\right)^{n} \mathrm{~b}_{3} \mathrm{x}_{\beta} \mathrm{x}_{\gamma}^{n} \mathbf{x}_{\varphi} \otimes x_{\tau} x_{\gamma} .
\end{aligned}
$$

We start with (10.1.38). For $n=0$ we have

$$
\begin{gathered}
d\left(\mathrm{x}_{\alpha} \mathrm{x}_{\beta} \mathrm{x}_{\varphi} \otimes 1\right)=\mathrm{x}_{\alpha} \mathrm{x}_{\beta} \otimes x_{\varphi}-s\left(q_{\beta \varphi} \mathrm{x}_{\alpha} \otimes x_{\varphi} x_{\beta}-q_{\alpha \beta} \mathrm{x}_{\beta} \otimes\left(q_{\alpha \varphi} x_{\varphi} x_{\alpha}+\mathrm{b}_{1} x_{\gamma}\right)\right) \\
=\mathrm{x}_{\alpha} \mathrm{x}_{\beta} \otimes x_{\varphi}-q_{\beta \varphi} \mathrm{x}_{\alpha} \mathrm{x}_{\varphi} \otimes x_{\beta}-s\left(q_{\beta \varphi} q_{\alpha \varphi} q_{\alpha \beta} \mathrm{x}_{\varphi} \otimes x_{\beta} x_{\alpha}+q_{\beta \varphi} \mathrm{b}_{1} \mathrm{x}_{\gamma} \otimes x_{\beta}\right. \\
\left.\quad-q_{\alpha \beta} q_{\alpha \varphi} \mathrm{x}_{\beta} \otimes x_{\varphi} x_{\alpha}-q_{\alpha \beta} \mathrm{b}_{1} \mathrm{x}_{\beta} \otimes x_{\gamma}\right) \\
=\mathrm{x}_{\alpha} \mathrm{x}_{\beta} \otimes x_{\varphi}-q_{\beta \varphi} \mathrm{x}_{\alpha} \mathrm{x}_{\varphi} \otimes x_{\beta}+q_{\alpha \beta} q_{\alpha \varphi} \mathrm{x}_{\beta} \mathrm{x}_{\varphi} \otimes x_{\alpha}+q_{\alpha \beta} \mathrm{b}_{1} \mathrm{x}_{\beta} \mathrm{x}_{\gamma} \otimes 1 .
\end{gathered}
$$

We assume that (10.1.38) holds for $n$. Using Remark 10.1.2 three times, inductive hypothesis, (10.1.36), (10.1.33) and $x_{\gamma}^{2}=0$, we compute

$$
\begin{aligned}
d\left(\mathrm{x}_{\alpha} \mathrm{x}_{\beta} \mathrm{x}_{\gamma}^{n+1} \mathrm{x}_{\varphi} \otimes 1\right)=\mathrm{x}_{\alpha} \mathrm{x}_{\beta} \mathrm{x}_{\gamma}^{n+1} \otimes x_{\varphi}-s\left(q_{\gamma \varphi} \mathrm{x}_{\alpha} \mathrm{x}_{\beta} \mathrm{x}_{\gamma}^{n} \otimes x_{\varphi} x_{\gamma}\right. \\
\left.\quad+\left(-q_{\beta \gamma}\right)^{n+1} q_{\beta \varphi} \mathrm{x}_{\alpha} \mathrm{x}_{\gamma}^{n+1} \otimes x_{\varphi} x_{\beta}-q_{\alpha \beta}\left(-q_{\alpha \gamma}\right)^{n+1} \mathrm{x}_{\beta} \mathrm{x}_{\gamma}^{n+1} \otimes\left(q_{\alpha \varphi} x_{\varphi} x_{\alpha}+\mathrm{b}_{1} x_{\gamma}\right)\right) \\
=\mathrm{x}_{\alpha} \mathrm{x}_{\beta} \mathrm{x}_{\gamma}^{n+1} \otimes x_{\varphi}-q_{\gamma \varphi} \mathrm{x}_{\alpha} \mathrm{x}_{\beta} \mathrm{x}_{\gamma}^{n} \mathrm{x}_{\varphi} \otimes x_{\gamma}-\left(-q_{\beta \gamma}\right)^{n+1} q_{\beta \varphi} \mathrm{x}_{\alpha} \mathrm{x}_{\gamma}^{n+1} \mathrm{x}_{\varphi} \otimes x_{\beta} \\
\quad-s\left(q_{\alpha \beta}\left(-q_{\alpha \gamma}\right)^{n+1} q_{\alpha \varphi} q_{\gamma \varphi} \mathrm{x}_{\beta} \mathrm{x}_{\gamma}^{n} \mathrm{x}_{\varphi} \otimes x_{\gamma} x_{\alpha}+q_{\beta \varphi} q_{\alpha \beta} q_{\alpha \gamma}^{n+1} q_{\beta \gamma}^{n+1} q_{\alpha \varphi} \mathrm{x}_{\gamma}^{n+1} \mathrm{x}_{\varphi} \otimes x_{\beta} x_{\alpha}\right. \\
\quad-q_{\alpha \beta}\left(-q_{\alpha \gamma}\right)^{n+1} q_{\alpha \varphi} \mathrm{x}_{\beta} \mathrm{x}_{\gamma}^{n+1} \otimes x_{\varphi} x_{\alpha}-\left(-q_{\beta \gamma}\right)^{n+1} q_{\beta \varphi} \mathrm{b}_{1} q_{\gamma \varphi}(n+2)_{\widetilde{q}_{\alpha \gamma}} \mathrm{x}_{\gamma}^{n+2} \otimes x_{\beta} \\
\left.\quad-\left(q_{\gamma \varphi}(n+1)_{\widetilde{q}_{\alpha \gamma}}+\left(-q_{\alpha \gamma}\right)^{n+1}\right) q_{\alpha \beta} \mathrm{b}_{1} \mathrm{x}_{\beta} \mathrm{x}_{\gamma}^{n+1} \otimes x_{\gamma}\right) \\
=\mathrm{x}_{\alpha} \mathrm{x}_{\beta} \mathrm{x}_{\gamma}^{n+1} \otimes x_{\varphi}-q_{\gamma \varphi} \mathrm{x}_{\alpha} \mathrm{x}_{\beta} \mathrm{x}_{\gamma}^{n} \mathrm{x}_{\varphi} \otimes x_{\gamma}-\left(-q_{\beta \gamma}\right)^{n+1} q_{\beta \varphi} \mathrm{x}_{\alpha} \mathrm{x}_{\gamma}^{n+1} \mathrm{x}_{\varphi} \otimes x_{\beta} \\
\quad+q_{\alpha \beta} q_{\gamma \varphi}(n+2)_{\widetilde{q}_{\alpha \gamma}} \mathrm{b}_{1} \mathrm{x}_{\beta} \mathrm{x}_{\gamma}^{n+2} \otimes 1+q_{\alpha \beta}\left(-q_{\alpha \gamma}\right)^{n+1} q_{\alpha \varphi} \mathrm{x}_{\beta} \mathrm{x}_{\gamma}^{n+1} \mathrm{x}_{\varphi} \otimes x_{\alpha} .
\end{aligned}
$$

Now we prove (10.1.39). For $n=0$,

$$
d\left(\mathrm{x}_{\delta} \mathrm{x}_{\eta} \otimes 1\right)=\mathrm{x}_{\delta} \otimes x_{\eta}-q_{\delta \eta} \mathrm{x}_{\eta} \otimes x_{\delta}-\mathrm{b}_{3} \mathrm{x}_{\varphi} \otimes x_{\tau} x_{\gamma} .
$$

We assume that (10.1.39) holds for $n$. By Remark 10.1.2 and inductive hypothesis,

$$
\begin{aligned}
& d\left(\mathrm{x}_{\delta} \mathrm{x}_{\gamma}^{n+1} \mathrm{x}_{\eta} \otimes 1\right)=\mathrm{x}_{\delta} \mathrm{x}_{\gamma}^{n+1} \otimes x_{\eta}-s\left(q_{\gamma \eta} \mathrm{x}_{\delta} \mathrm{x}_{\gamma}^{n} \otimes x_{\eta} x_{\gamma}+\left(-q_{\delta \gamma}\right)^{n+1} \mathrm{x}_{\gamma}^{n+1} \otimes x_{\delta} x_{\eta}\right) \\
& =\mathrm{x}_{\delta} \mathrm{x}_{\gamma}^{n+1} \otimes x_{\eta}-q_{\gamma \eta} \mathrm{x}_{\delta} \mathrm{x}_{\gamma}^{n} \mathrm{x}_{\eta} \otimes x_{\gamma}-\left(-q_{\delta \gamma}\right)^{n+1} \mathrm{~b}_{3} \mathrm{x}_{\gamma}^{n+1} \mathrm{x}_{\varphi} \otimes x_{\tau} x_{\gamma} \\
& \quad-\left(-q_{\delta \gamma}\right)^{n+1} q_{\delta \eta} \mathrm{x}_{\gamma}^{n+1} \mathrm{x}_{\eta} \otimes x_{\delta} .
\end{aligned}
$$

Next we prove (10.1.40). For $n=0$,

$$
\begin{aligned}
& d\left(\mathrm{x}_{\alpha} \mathrm{x}_{\delta} \mathrm{x}_{\eta} \otimes 1\right)=\mathrm{x}_{\alpha} \mathrm{x}_{\delta} \otimes x_{\eta}-s\left(q_{\delta \eta} \mathrm{x}_{\alpha} \otimes x_{\eta} x_{\delta}+\mathrm{b}_{3} \mathrm{x}_{\alpha} \otimes x_{\varphi} x_{\tau} x_{\gamma}-q_{\alpha \delta} q_{\alpha \eta} \mathrm{x}_{\delta} \otimes x_{\eta} x_{\alpha}\right) \\
& =\mathrm{x}_{\alpha} \mathrm{x}_{\delta} \otimes x_{\eta}-\mathrm{b}_{3} \mathrm{x}_{\alpha} \mathrm{x}_{\varphi} \otimes x_{\tau} x_{\gamma}-q_{\delta \eta} \mathrm{x}_{\alpha} \mathrm{x}_{\eta} \otimes x_{\delta}-s\left(q_{\delta \eta} q_{\alpha \eta} q_{\alpha \delta} \mathrm{x}_{\eta} \otimes x_{\delta} x_{\alpha}\right. \\
& \left.\quad+q_{\alpha \varphi} q_{\alpha \tau} q_{\alpha \gamma} \mathrm{b}_{3} \mathrm{x}_{\varphi} \otimes x_{\tau} x_{\gamma} x_{\alpha}+\mathrm{b}_{1} \mathrm{~b}_{3} \mathrm{x}_{\gamma} \otimes x_{\tau} x_{\gamma}-q_{\alpha \delta} q_{\alpha \eta} \mathrm{x}_{\delta} \otimes x_{\eta} x_{\alpha}\right) \\
& =\mathrm{x}_{\alpha} \mathrm{x}_{\delta} \otimes x_{\eta}-\mathrm{b}_{3} \mathrm{x}_{\alpha} \mathrm{x}_{\varphi} \otimes x_{\tau} x_{\gamma}-q_{\delta \eta} \mathrm{x}_{\alpha} \mathrm{x}_{\eta} \otimes x_{\delta}+q_{\alpha \delta} q_{\alpha \eta} \mathrm{x}_{\delta} \mathrm{x}_{\eta} \otimes x_{\alpha}-\mathrm{b}_{1} \mathrm{~b}_{3} \mathrm{x}_{\gamma} \mathrm{x}_{\tau} \otimes x_{\gamma} .
\end{aligned}
$$

We assume that (10.1.40) holds for $n$. Using Remark 10.1.2 three times, inductive hypothesis, (10.1.36), (10.1.39), we have

$$
\begin{gathered}
d\left(\mathrm{x}_{\alpha} \mathrm{x}_{\delta} \mathrm{x}_{\gamma}^{n+1} \mathrm{x}_{\eta} \otimes 1\right)=\mathrm{x}_{\alpha} \mathrm{x}_{\delta} \mathrm{x}_{\gamma}^{n+1} \otimes x_{\eta}-s\left(q_{\gamma \eta} \mathrm{x}_{\alpha} \mathrm{x}_{\delta} \mathrm{x}_{\gamma}^{n} \otimes x_{\eta} x_{\gamma}+\left(-q_{\delta \gamma}\right)^{n+1} q_{\delta \eta} \mathrm{x}_{\alpha} \mathrm{x}_{\gamma}^{n+1} \otimes x_{\eta} x_{\delta}\right. \\
\left.+\left(-q_{\delta \gamma}\right)^{n+1} \mathrm{~b}_{3} \mathrm{x}_{\alpha} \mathrm{x}_{\gamma}^{n+1} \otimes x_{\varphi} x_{\tau} x_{\gamma}+q_{\alpha \delta}\left(-q_{\alpha \gamma}\right)^{n+1} q_{\alpha \eta} \mathrm{x}_{\delta} \mathrm{x}_{\gamma}^{n+1} \otimes x_{\eta} x_{\alpha}\right)
\end{gathered}
$$




$$
\begin{aligned}
= & \mathrm{x}_{\alpha} \mathrm{x}_{\delta} \mathrm{x}_{\gamma}^{n+1} \otimes x_{\eta}-q_{\gamma \eta} \mathrm{x}_{\alpha} \mathrm{x}_{\delta} \mathrm{x}_{\gamma}^{n} \mathrm{x}_{\eta} \otimes x_{\gamma}-\left(-q_{\delta \gamma}\right)^{n+1} \mathrm{~b}_{3} \mathrm{x}_{\alpha} \mathrm{x}_{\gamma}^{n+1} \mathrm{x}_{\varphi} \otimes x_{\tau} x_{\gamma} \\
& -s\left(-\left(-q_{\delta \gamma}\right)^{n+1} q_{\delta \eta} q_{\gamma \eta} \mathrm{x}_{\alpha} \mathrm{x}_{\gamma}^{n} \mathrm{x}_{\eta} \otimes x_{\gamma} x_{\delta}+q_{\alpha \delta}\left(-q_{\alpha \gamma}\right)^{n+1} q_{\alpha \eta} q_{\gamma \eta} \mathrm{x}_{\delta} \mathrm{x}_{\gamma}^{n} \mathrm{x}_{\eta} \otimes x_{\gamma} x_{\alpha}\right. \\
& +\left(-q_{\delta \gamma}\right)^{n+1} q_{\delta \eta} \mathrm{x}_{\alpha} \mathrm{x}_{\gamma}^{n+1} \otimes x_{\eta} x_{\delta}+q_{\alpha \delta}\left(-q_{\alpha \gamma}\right)^{n+1} q_{\alpha \eta} \mathrm{x}_{\delta} \mathrm{x}_{\gamma}^{n+1} \otimes x_{\eta} x_{\alpha} \\
& \left.+q_{\alpha \gamma}^{n+2} q_{\alpha \varphi} q_{\alpha \tau} q_{\delta \gamma}^{n+1} \mathrm{~b}_{3} \mathrm{x}_{\gamma}^{n+1} \mathrm{x}_{\varphi} \otimes x_{\tau} x_{\gamma} x_{\alpha}+q_{\gamma \varphi}(n+2)_{\widetilde{q}_{\alpha \gamma}}\left(-q_{\delta \gamma}\right)^{n+1} \mathrm{~b}_{1} \mathrm{~b}_{3} \mathrm{x}_{\gamma}^{n+2} \otimes x_{\tau} x_{\gamma}\right) \\
= & \mathrm{x}_{\alpha} \mathrm{x}_{\delta} \mathrm{x}_{\gamma}^{n+1} \otimes x_{\eta}-q_{\gamma \eta} \mathrm{x}_{\alpha} \mathrm{x}_{\delta} \mathrm{x}_{\gamma}^{n} \mathrm{x}_{\eta} \otimes x_{\gamma}-\left(-q_{\delta \gamma}\right)^{n+1} \mathrm{~b}_{3} \mathrm{x}_{\alpha} \mathrm{x}_{\gamma}^{n+1} \mathrm{x}_{\varphi} \otimes x_{\tau} x_{\gamma} \\
& -\left(-q_{\delta \gamma}\right)^{n+1} q_{\delta \eta} \mathrm{x}_{\alpha} \mathrm{x}_{\gamma}^{n+1} \mathrm{x}_{\eta} \otimes x_{\delta}+q_{\alpha \delta}\left(-q_{\alpha \gamma}\right)^{n+1} q_{\alpha \eta} \mathrm{x}_{\delta} \mathrm{x}_{\gamma}^{n+1} \mathrm{x}_{\eta} \otimes x_{\alpha} \\
& -q_{\gamma \varphi}(n+2)_{\widetilde{q}_{\alpha \gamma}}\left(-q_{\delta \gamma}\right)^{n+1} \mathrm{~b}_{1} \mathrm{~b}_{3} s\left(\mathrm{x}_{\gamma}^{n+2} \otimes x_{\tau} x_{\gamma}\right) .
\end{aligned}
$$

As $x_{\gamma}^{2}=0$ and $x_{\gamma} x_{\tau}=q_{\gamma \tau} x_{\tau} x_{\gamma}$, we have that $s\left(\mathrm{x}_{\gamma}^{n+2} \otimes x_{\tau} x_{\gamma}\right)=\mathrm{x}_{\gamma}^{n+2} \mathrm{x}_{\tau} \otimes x_{\gamma}$; hence the inductive step follows.

Now we prove (10.1.41). For $n=0$,

$$
\begin{gathered}
d\left(\mathrm{x}_{\beta} \mathrm{x}_{\delta} \mathrm{x}_{\eta} \otimes 1\right)=\mathrm{x}_{\beta} \mathrm{x}_{\delta} \otimes x_{\eta}-s\left(q_{\delta \eta} \mathrm{x}_{\beta} \otimes x_{\eta} x_{\delta}+\mathrm{b}_{3} \mathrm{x}_{\beta} \otimes x_{\varphi} x_{\tau} x_{\gamma}-q_{\beta \delta} q_{\beta \eta} \mathrm{x}_{\delta} \otimes x_{\eta} x_{\beta}\right) \\
=\mathrm{x}_{\beta} \mathrm{x}_{\delta} \otimes x_{\eta}-q_{\delta \eta} \mathrm{x}_{\beta} \mathrm{x}_{\eta} \otimes x_{\delta}-\mathrm{b}_{3} \mathrm{x}_{\beta} \mathrm{x}_{\varphi} \otimes x_{\tau} x_{\gamma}-s\left(q_{\delta \eta} q_{\beta \eta} q_{\beta \delta} \mathrm{x}_{\eta} \otimes x_{\delta} x_{\beta}\right. \\
\left.\quad+q_{\beta \varphi} \mathrm{b}_{3} \mathrm{x}_{\varphi} \otimes\left(q_{\beta \tau} x_{\tau} x_{\beta}+\mathrm{b}_{2} x_{\gamma}\right) x_{\gamma}-q_{\beta \delta} q_{\beta \eta} \mathrm{x}_{\delta} \otimes x_{\eta} x_{\beta}\right) \\
=\mathrm{x}_{\beta} \mathrm{x}_{\delta} \otimes x_{\eta}-q_{\delta \eta} \mathrm{x}_{\beta} \mathrm{x}_{\eta} \otimes x_{\delta}-\mathrm{b}_{3} \mathrm{x}_{\beta} \mathrm{x}_{\varphi} \otimes x_{\tau} x_{\gamma}+q_{\beta \delta} q_{\beta \eta} \mathrm{x}_{\delta} \mathrm{x}_{\eta} \otimes x_{\beta} .
\end{gathered}
$$

We assume that (10.1.41) holds for $n$. Using Remark 10.1.2 three times, inductive hypothesis and (10.1.39), we have

$$
\begin{aligned}
d\left(\mathrm{x}_{\beta} \mathrm{x}_{\delta} \mathrm{x}_{\gamma}^{n+1} \mathrm{x}_{\eta} \otimes 1\right)=\mathrm{x}_{\beta} \mathrm{x}_{\delta} \mathrm{x}_{\gamma}^{n+1} \otimes x_{\eta}-s\left(q_{\gamma \eta} \mathrm{x}_{\beta} \mathrm{x}_{\delta} \mathrm{x}_{\gamma}^{n} \otimes x_{\eta} x_{\gamma}+\left(-q_{\delta \gamma}\right)^{n+1} q_{\delta \eta} \mathrm{x}_{\beta} \mathrm{x}_{\gamma}^{n+1} \otimes x_{\eta} x_{\delta}\right. \\
\left.\quad+\left(-q_{\delta \gamma}\right)^{n+1} \mathrm{~b}_{3} \mathrm{x}_{\beta} \mathrm{x}_{\gamma}^{n+1} \otimes x_{\varphi} x_{\tau} x_{\gamma}+q_{\beta \delta}\left(-q_{\beta \gamma}\right)^{n+1} q_{\beta \eta} \mathrm{x}_{\delta} \mathrm{x}_{\gamma}^{n+1} \otimes x_{\eta} x_{\beta}\right) \\
=\mathrm{x}_{\beta} \mathrm{x}_{\delta} \mathrm{x}_{\gamma}^{n+1} \otimes x_{\eta}-q_{\gamma \eta} \mathrm{x}_{\beta} \mathrm{x}_{\delta} \mathrm{x}_{\gamma}^{n} \mathrm{x}_{\eta} \otimes x_{\gamma}-\left(-q_{\delta \gamma}\right)^{n+1} \mathrm{~b}_{3} \mathrm{x}_{\beta} \mathrm{x}_{\gamma}^{n+1} \mathrm{x}_{\varphi} \otimes x_{\tau} x_{\gamma} \\
\quad-\left(-q_{\delta \gamma}\right)^{n+1} q_{\delta \eta} \mathrm{x}_{\beta} \mathrm{x}_{\gamma}^{n+1} \mathrm{x}_{\eta} \otimes x_{\delta}-s\left(q_{\beta \delta}\left(-q_{\beta \gamma}\right)^{n+1} q_{\beta \eta} q_{\gamma \eta} \mathrm{x}_{\delta} \mathrm{x}_{\gamma}^{n} \mathrm{x}_{\eta} \otimes x_{\gamma} x_{\beta}\right. \\
\quad+q_{\beta \delta}\left(-q_{\beta \gamma}\right)^{n+1} q_{\beta \eta} \mathrm{x}_{\delta} \mathrm{x}_{\gamma}^{n+1} \otimes x_{\eta} x_{\beta}+q_{\delta \gamma}^{n+1} q_{\beta \gamma}^{n+1} q_{\beta \varphi} \mathrm{b}_{3} \mathrm{x}_{\gamma}^{n+1} \mathrm{x}_{\varphi} \otimes\left(q_{\beta \tau} x_{\tau} x_{\beta}+\mathrm{b}_{2} x_{\gamma}\right) x_{\gamma} \\
\left.\left.\quad+q_{\delta \gamma}^{n+1} q_{\delta \eta} q_{\beta \gamma}^{n+1} q_{\beta \eta} q_{\beta \delta} \mathrm{x}_{\gamma}^{n+1} \mathrm{x}_{\eta} \otimes x_{\delta} x_{\beta}\right)\right) \\
=\mathrm{x}_{\beta} \mathrm{x}_{\delta} \mathrm{x}_{\gamma}^{n+1} \otimes x_{\eta}-q_{\gamma \eta} \mathrm{x}_{\beta} \mathrm{x}_{\delta} \mathrm{x}_{\gamma}^{n} \mathrm{x}_{\eta} \otimes x_{\gamma}-\left(-q_{\delta \gamma}\right)^{n+1} \mathrm{~b}_{3} \mathrm{x}_{\beta} \mathrm{x}_{\gamma}^{n+1} \mathrm{x}_{\varphi} \otimes x_{\tau} x_{\gamma} \\
\quad-\left(-q_{\delta \gamma}\right)^{n+1} q_{\delta \eta} \mathrm{x}_{\beta} \mathrm{x}_{\gamma}^{n+1} \mathrm{x}_{\eta} \otimes x_{\delta}+q_{\beta \delta}\left(-q_{\beta \gamma}\right)^{n+1} q_{\beta \eta} \mathrm{x}_{\delta} \mathrm{x}_{\gamma}^{n+1} \mathrm{x}_{\eta} \otimes x_{\beta} .
\end{aligned}
$$

Finally, we prove (10.1.34) by induction on $n$. For $n=0$,

$$
\begin{aligned}
& d\left(\mathrm{x}_{\alpha} \mathrm{x}_{\beta} \mathrm{x}_{\delta} \mathrm{x}_{\eta} \otimes 1\right)=\mathrm{x}_{\alpha} \mathrm{x}_{\beta} \mathrm{x}_{\delta} \otimes x_{\eta}-s\left(q_{\delta \eta} \mathrm{x}_{\alpha} \mathrm{x}_{\beta} \otimes x_{\eta} x_{\delta}+\mathrm{b}_{3} \mathrm{x}_{\alpha} \mathrm{x}_{\beta} \otimes x_{\varphi} x_{\tau} x_{\gamma}\right. \\
&\left.\quad-q_{\beta \delta} q_{\beta \eta} \mathrm{x}_{\alpha} \mathrm{x}_{\delta} \otimes x_{\eta} x_{\beta}+q_{\alpha \beta} q_{\alpha \delta} q_{\alpha \eta} \mathrm{x}_{\beta} \mathrm{x}_{\delta} \otimes x_{\eta} x_{\alpha}\right) \\
&=\mathrm{x}_{\alpha} \mathrm{x}_{\beta} \mathrm{x}_{\delta} \otimes x_{\eta}-\mathrm{b}_{3} \mathrm{x}_{\alpha} \mathrm{x}_{\beta} \mathrm{x}_{\varphi} \otimes x_{\tau} x_{\gamma}-q_{\delta \eta} \mathrm{x}_{\alpha} \mathrm{x}_{\beta} \mathrm{x}_{\eta} \otimes x_{\delta} \\
& \quad-s\left(q_{\beta \eta} q_{\beta \delta} q_{\delta \eta} \mathrm{x}_{\alpha} \mathrm{x}_{\eta} \otimes x_{\delta} x_{\beta}-q_{\alpha \beta} q_{\alpha \eta} q_{\alpha \delta} q_{\delta \eta} \mathrm{x}_{\beta} \mathrm{x}_{\eta} \otimes x_{\delta} x_{\alpha}\right. \\
& \quad+q_{\beta \varphi} q_{\beta \tau} q_{\beta \gamma} \mathrm{b}_{3} \mathrm{x}_{\alpha} \mathrm{x}_{\varphi} \otimes x_{\tau} x_{\gamma} x_{\beta}-q_{\alpha \beta} q_{\alpha \varphi} q_{\alpha \tau} q_{\alpha \gamma} \mathrm{b}_{3} \mathrm{x}_{\beta} \mathrm{x}_{\varphi} \otimes x_{\tau} x_{\gamma} x_{\alpha} \\
&\left.-q_{\alpha \beta} \mathrm{b}_{1} \mathrm{~b}_{3} \mathrm{x}_{\beta} \mathrm{x}_{\gamma} \otimes x_{\tau} x_{\gamma}-q_{\beta \delta} q_{\beta \eta} \mathrm{x}_{\alpha} \mathrm{x}_{\delta} \otimes x_{\eta} x_{\beta}+q_{\alpha \beta} q_{\alpha \delta} q_{\alpha \eta} \mathrm{x}_{\beta} \mathrm{x}_{\delta} \otimes x_{\eta} x_{\alpha}\right) \\
&= \mathrm{x}_{\alpha} \mathrm{x}_{\beta} \mathrm{x}_{\delta} \otimes x_{\eta}-\mathrm{b}_{3} \mathrm{x}_{\alpha} \mathrm{x}_{\beta} \mathrm{x}_{\varphi} \otimes x_{\tau} x_{\gamma}-q_{\delta \eta} \mathrm{x}_{\alpha} \mathrm{x}_{\beta} \mathrm{x}_{\eta} \otimes x_{\delta}+q_{\beta \delta} q_{\beta \eta} \mathrm{x}_{\alpha} \mathrm{x}_{\delta} \mathrm{x}_{\eta} \otimes x_{\beta} \\
&-q_{\alpha \beta} q_{\alpha \delta} q_{\alpha \eta} \mathrm{x}_{\beta} \mathrm{x}_{\delta} \mathrm{x}_{\eta} \otimes x_{\alpha}+q_{\alpha \beta} \mathrm{b}_{1} \mathrm{~b}_{3} \mathrm{x}_{\beta} \mathrm{x}_{\gamma} \mathrm{x}_{\tau} \otimes x_{\gamma}-q_{\alpha \beta} q_{\gamma \tau}[2]_{q_{\beta \gamma}} \mathrm{b}_{1} \mathrm{~b}_{2} \mathrm{~b}_{3} \mathrm{x}_{\gamma}^{3} \otimes 1
\end{aligned}
$$


We assume that (10.1.34) holds for $n$. Using Remark 10.1.2 twice, inductive hypothesis, (10.1.38), (10.1.40), (10.1.41), (10.1.37), we have

$$
\begin{aligned}
& d\left(\mathrm{x}_{\alpha} \mathrm{x}_{\beta} \mathrm{x}_{\delta} \mathrm{x}_{\gamma}^{n+1} \mathrm{x}_{\eta} \otimes 1\right)=\mathrm{x}_{\alpha} \mathrm{x}_{\beta} \mathrm{x}_{\delta} \mathrm{x}_{\gamma}^{n+1} \otimes x_{\eta}-s\left(q_{\gamma \eta} \mathrm{x}_{\alpha} \mathrm{x}_{\beta} \mathrm{x}_{\delta} \mathrm{x}_{\gamma}^{n} \otimes x_{\eta} x_{\gamma}\right. \\
& +\left(-q_{\delta \gamma}\right)^{n+1} q_{\delta \eta} \mathrm{x}_{\alpha} \mathrm{x}_{\beta} \mathrm{x}_{\gamma}^{n+1} \otimes x_{\eta} x_{\delta}+\left(-q_{\delta \gamma}\right)^{n+1} \mathrm{~b}_{3} \mathrm{x}_{\alpha} \mathrm{x}_{\beta} \mathrm{x}_{\gamma}^{n+1} \otimes x_{\varphi} x_{\tau} x_{\gamma} \\
& \left.-q_{\beta \delta}\left(-q_{\beta \gamma}\right)^{n+1} q_{\beta \eta} \mathrm{x}_{\alpha} \mathrm{x}_{\delta} \mathrm{x}_{\gamma}^{n+1} \otimes x_{\eta} x_{\beta}+q_{\alpha \beta} q_{\alpha \delta}\left(-q_{\alpha \gamma}\right)^{n+1} q_{\alpha \eta} \mathrm{x}_{\beta} \mathrm{x}_{\delta} \mathrm{x}_{\gamma}^{n+1} \otimes x_{\eta} x_{\alpha}\right) \\
& =\mathrm{x}_{\alpha} \mathrm{x}_{\beta} \mathrm{x}_{\delta} \mathrm{x}_{\gamma}^{n+1} \otimes x_{\eta}-q_{\gamma \eta} \mathrm{x}_{\alpha} \mathrm{x}_{\beta} \mathrm{x}_{\delta} \mathrm{x}_{\gamma}^{n} \mathrm{x}_{\eta} \otimes x_{\gamma}-\left(-q_{\delta \gamma}\right)^{n+1} \mathrm{~b}_{3} \mathrm{x}_{\alpha} \mathrm{x}_{\beta} \mathrm{x}_{\gamma}^{n+1} \mathrm{x}_{\varphi} \otimes x_{\tau} x_{\gamma} \\
& -s\left(q_{\gamma \eta}\left(-q_{\delta \gamma}\right)^{n} q_{\delta \gamma} q_{\delta \eta} \mathrm{x}_{\alpha} \mathrm{x}_{\beta} \mathrm{x}_{\gamma}^{n} \mathrm{x}_{\eta} \otimes x_{\gamma} x_{\delta}+q_{\beta \gamma}^{n+2} q_{\beta \varphi} q_{\delta \gamma}^{n+1} \mathrm{~b}_{3} \mathrm{x}_{\alpha} \mathrm{x}_{\gamma}^{n+1} \mathrm{x}_{\varphi} \otimes q_{\beta \tau} x_{\tau} x_{\gamma} x_{\beta}\right. \\
& -q_{\gamma \eta} q_{\beta \delta}\left(-q_{\beta \gamma}\right)^{n} q_{\beta \gamma} q_{\beta \eta} \mathrm{x}_{\alpha} \mathrm{x}_{\delta} \mathrm{x}_{\gamma}^{n} \mathrm{x}_{\eta} \otimes x_{\gamma} x_{\beta}-q_{\gamma \eta} q_{\alpha \beta} q_{\alpha \delta}\left(-q_{\alpha \gamma}\right)^{n+1} q_{\alpha \eta} \mathrm{x}_{\beta} \mathrm{x}_{\delta} \mathrm{x}_{\gamma}^{n} \mathrm{x}_{\eta} \otimes x_{\gamma} x_{\alpha} \\
& +\left(-q_{\delta \gamma}\right)^{n+1} q_{\delta \eta} \mathrm{x}_{\alpha} \mathrm{x}_{\beta} \mathrm{x}_{\gamma}^{n+1} \otimes x_{\eta} x_{\delta}-q_{\alpha \beta} q_{\alpha \gamma}^{n+2} q_{\alpha \varphi} q_{\alpha \tau} q_{\delta \gamma}^{n+1} \mathrm{~b}_{3} \mathrm{x}_{\beta} \mathrm{x}_{\gamma}^{n+1} \mathrm{x}_{\varphi} \otimes x_{\tau} x_{\gamma} x_{\alpha} \\
& +q_{\gamma \eta} q_{\alpha \beta} q_{\delta \gamma}^{-n-1} \mathrm{~d}_{\alpha \beta \delta \gamma}^{(n)} \mathrm{b}_{1} \mathrm{~b}_{2} \mathrm{~b}_{3} \mathrm{x}_{\gamma}^{n+3} \otimes x_{\gamma}-q_{\alpha \beta} q_{\gamma \varphi}(n+2) \widetilde{q}_{\alpha \gamma}\left(-q_{\delta \gamma}\right)^{n+1} \mathrm{~b}_{3} \mathrm{~b}_{1} \mathrm{x}_{\beta} \mathrm{x}_{\gamma}^{n+2} \otimes x_{\tau} x_{\gamma} \\
& \left.-q_{\beta \delta}\left(-q_{\beta \gamma}\right)^{n+1} q_{\beta \eta} \mathrm{x}_{\alpha} \mathrm{x}_{\delta} \mathrm{x}_{\gamma}^{n+1} \otimes x_{\eta} x_{\beta}+q_{\alpha \beta} q_{\alpha \delta}\left(-q_{\alpha \gamma}\right)^{n+1} q_{\alpha \eta} \mathrm{x}_{\beta} \mathrm{x}_{\delta} \mathrm{x}_{\gamma}^{n+1} \otimes x_{\eta} x_{\alpha}\right) \\
& =\mathrm{x}_{\alpha} \mathrm{x}_{\beta} \mathrm{x}_{\delta} \mathrm{x}_{\gamma}^{n+1} \otimes x_{\eta}-q_{\gamma \eta} \mathrm{x}_{\alpha} \mathrm{x}_{\beta} \mathrm{x}_{\delta} \mathrm{x}_{\gamma}^{n} \mathrm{x}_{\eta} \otimes x_{\gamma}-\left(-q_{\delta \gamma}\right)^{n+1} \mathrm{~b}_{3} \mathrm{x}_{\alpha} \mathrm{x}_{\beta} \mathrm{x}_{\gamma}^{n+1} \mathrm{x}_{\varphi} \otimes x_{\tau} x_{\gamma} \\
& -\left(-q_{\delta \gamma}\right)^{n+1} q_{\delta \eta} \mathrm{x}_{\alpha} \mathrm{x}_{\beta} \mathrm{x}_{\gamma}^{n+1} \mathrm{x}_{\eta} \otimes x_{\delta}+q_{\beta \delta}\left(-q_{\beta \gamma}\right)^{n+1} q_{\beta \eta} \mathrm{x}_{\alpha} \mathrm{x}_{\delta} \mathrm{x}_{\gamma}^{n+1} \mathbf{x}_{\eta} \otimes x_{\beta} \\
& -s\left(-q_{\gamma \eta} q_{\alpha \beta} q_{\alpha \delta}\left(-q_{\alpha \gamma}\right)^{n+1} q_{\alpha \eta} \mathrm{x}_{\beta} \mathrm{x}_{\delta} \mathrm{x}_{\gamma}^{n} \mathrm{x}_{\eta} \otimes x_{\gamma} x_{\alpha}\right. \\
& -q_{\alpha \beta} q_{\alpha \gamma}^{n+2} q_{\alpha \varphi} q_{\alpha \tau} q_{\delta \gamma}^{n+1} \mathrm{~b}_{3} \mathrm{x}_{\beta} \mathrm{x}_{\gamma}^{n+1} \mathrm{x}_{\varphi} \otimes x_{\tau} x_{\gamma} x_{\alpha} \\
& -q_{\gamma \eta} q_{\alpha \beta} q_{\delta \gamma}^{-n-1} \mathrm{~d}_{\alpha \beta \delta \gamma}^{(n)} \mathrm{b}_{1} \mathrm{~b}_{2} \mathrm{~b}_{3} \mathrm{x}_{\gamma}^{n+3} \otimes x_{\gamma}-q_{\alpha \beta} q_{\gamma \varphi}(n+2) \widetilde{q}_{\alpha \gamma}\left(-q_{\delta \gamma}\right)^{n+1} \mathrm{~b}_{3} \mathrm{~b}_{1} \mathrm{x}_{\beta} \mathrm{x}_{\gamma}^{n+2} \otimes x_{\tau} x_{\gamma} \\
& +q_{\alpha \beta} q_{\alpha \delta}\left(-q_{\alpha \gamma}\right)^{n+1} q_{\alpha \eta} \mathrm{x}_{\beta} \mathrm{x}_{\delta} \mathrm{x}_{\gamma}^{n+1} \otimes x_{\eta} x_{\alpha}-q_{\delta \gamma}^{n+1} q_{\delta \eta} q_{\alpha \beta} q_{\alpha \gamma}^{n+1} q_{\alpha \eta} q_{\alpha \delta} \mathrm{x}_{\beta} \mathrm{x}_{\gamma}^{n+1} \mathbf{x}_{\eta} \otimes x_{\delta} x_{\alpha} \\
& +q_{\beta \delta} q_{\beta \gamma}^{n+1} q_{\beta \eta} q_{\alpha \beta} q_{\alpha \delta} q_{\alpha \gamma}^{n+1} q_{\alpha \eta} \mathrm{x}_{\delta} \mathrm{x}_{\gamma}^{n+1} \mathrm{x}_{\eta} \otimes x_{\beta} x_{\alpha} \\
& \left.-q_{\gamma \varphi} q_{\delta \gamma}^{n+1} q_{\beta \delta} q_{\beta \gamma}^{n+1} q_{\beta \eta}(n+2)_{\widetilde{q}_{\alpha \gamma}} \mathrm{b}_{1} \mathrm{~b}_{3} \mathrm{x}_{\gamma}^{n+2} \mathrm{x}_{\tau} \otimes x_{\gamma} x_{\beta}\right) \\
& =\mathrm{x}_{\alpha} \mathrm{x}_{\beta} \mathrm{x}_{\delta} \mathrm{x}_{\gamma}^{n+1} \otimes x_{\eta}-q_{\gamma \eta} \mathrm{x}_{\alpha} \mathrm{x}_{\beta} \mathrm{x}_{\delta} \mathrm{x}_{\gamma}^{n} \mathrm{x}_{\eta} \otimes x_{\gamma}-\left(-q_{\delta \gamma}\right)^{n+1} \mathrm{~b}_{3} \mathrm{x}_{\alpha} \mathrm{x}_{\beta} \mathrm{x}_{\gamma}^{n+1} \mathrm{x}_{\varphi} \otimes x_{\tau} x_{\gamma} \\
& -\left(-q_{\delta \gamma}\right)^{n+1} q_{\delta \eta} \mathrm{x}_{\alpha} \mathrm{x}_{\beta} \mathrm{x}_{\gamma}^{n+1} \mathrm{x}_{\eta} \otimes x_{\delta}+q_{\beta \delta}\left(-q_{\beta \gamma}\right)^{n+1} q_{\beta \eta} \mathrm{x}_{\alpha} \mathrm{x}_{\delta} \mathrm{x}_{\gamma}^{n+1} \mathrm{x}_{\eta} \otimes x_{\beta} \\
& -q_{\alpha \beta} q_{\alpha \delta}\left(-q_{\alpha \gamma}\right)^{n+1} q_{\alpha \eta} \mathrm{x}_{\beta} \mathrm{x}_{\delta} \mathrm{x}_{\gamma}^{n+1} \mathrm{x}_{\eta} \otimes x_{\alpha}-s\left(q_{\alpha \beta} q_{\gamma \eta} q_{\delta \gamma}^{-n-1} \mathrm{~d}_{\alpha \beta \delta \gamma}^{(n)} \mathrm{b}_{1} \mathrm{~b}_{2} \mathrm{~b}_{3} \mathrm{x}_{\gamma}^{n+3} \otimes x_{\gamma}\right. \\
& -q_{\alpha \beta} q_{\gamma \varphi}(n+2) \tilde{q}_{\alpha \gamma}\left(-q_{\delta \gamma}\right)^{n+1} \mathrm{~b}_{3} \mathrm{~b}_{1} \mathrm{x}_{\beta} \mathrm{x}_{\gamma}^{n+2} \otimes x_{\tau} x_{\gamma} \\
& \left.-q_{\gamma \varphi} q_{\delta \gamma}^{n+1} q_{\beta \delta} q_{\beta \gamma}^{n+1} q_{\beta \eta}(n+2)_{\widetilde{q}_{\alpha \gamma}} \mathrm{b}_{1} \mathrm{~b}_{3} \mathrm{x}_{\gamma}^{n+2} \mathrm{x}_{\tau} \otimes x_{\gamma} x_{\beta}\right) \\
& =\mathrm{x}_{\alpha} \mathrm{x}_{\beta} \mathrm{x}_{\delta} \mathrm{x}_{\gamma}^{n+1} \otimes x_{\eta}-q_{\gamma \eta} \mathrm{x}_{\alpha} \mathrm{x}_{\beta} \mathrm{x}_{\delta} \mathrm{x}_{\gamma}^{n} \mathrm{x}_{\eta} \otimes x_{\gamma}-\left(-q_{\delta \gamma}\right)^{n+1} q_{\delta \eta} \mathrm{x}_{\alpha} \mathrm{x}_{\beta} \mathrm{x}_{\gamma}^{n+1} \mathrm{x}_{\eta} \otimes x_{\delta} \\
& +q_{\beta \delta}\left(-q_{\beta \gamma}\right)^{n+1} q_{\beta \eta} \mathrm{x}_{\alpha} \mathrm{x}_{\delta} \mathrm{x}_{\gamma}^{n+1} \mathrm{x}_{\eta} \otimes x_{\beta}-q_{\alpha \beta} q_{\alpha \delta}\left(-q_{\alpha \gamma}\right)^{n+1} q_{\alpha \eta} \mathrm{x}_{\beta} \mathrm{x}_{\delta} \mathrm{x}_{\gamma}^{n+1} \mathrm{x}_{\eta} \otimes x_{\alpha} \\
& -\left(-q_{\delta \gamma}\right)^{n+1} \mathrm{~b}_{3} \mathrm{x}_{\alpha} \mathrm{x}_{\beta} \mathrm{x}_{\gamma}^{n+1} \mathrm{x}_{\varphi} \otimes x_{\tau} x_{\gamma}-q_{\alpha \beta} q_{\gamma \varphi}(n+2)_{\widetilde{q}_{\alpha \gamma}}\left(-q_{\delta \gamma}\right)^{n+1} \mathrm{~b}_{1} \mathrm{~b}_{3} \mathrm{x}_{\beta} \mathrm{x}_{\gamma}^{n+2} \mathrm{x}_{\tau} \otimes x_{\gamma} \\
& -q_{\alpha \beta}\left(q_{\gamma \eta}\left(-q_{\delta \gamma}\right)^{-n} \mathrm{~d}_{\alpha \beta \delta \gamma}^{(n)}-q_{\gamma \varphi}(n+2)_{\widetilde{q}_{\alpha \gamma}}\left(-q_{\delta \gamma}\right)^{n+1} q_{\gamma \tau}(n+3)_{\widetilde{q}_{\beta \gamma}}\right) \mathrm{b}_{1} \mathrm{~b}_{2} \mathrm{~b}_{3} s\left(\mathrm{x}_{\gamma}^{n+3} \otimes x_{\gamma}\right) \text {. }
\end{aligned}
$$

Thus the proof of the inductive step follows since $q_{\gamma \delta} q_{\gamma \delta}=q_{\gamma \gamma} q_{\gamma \tau} q_{\gamma \varphi}=-q_{\gamma \tau} q_{\gamma \varphi}$ and $s\left(\mathrm{x}_{\gamma}^{n+3} \otimes x_{\gamma}\right)=\mathrm{x}_{\gamma}^{n+4} \otimes 1$. 
Lemma 10.1.42. Let $\alpha<\eta<\gamma<\beta<\delta$ be positive roots such that $N_{\gamma}=2$ and the relations among the corresponding root vectors take the form

$$
x_{\alpha} x_{\delta}=q_{\alpha \delta} x_{\delta} x_{\alpha}+\mathrm{b}_{1} x_{\gamma} x_{\eta}+\mathrm{b}_{2} x_{\beta} x_{\eta}^{2}, \quad x_{\eta} x_{\beta}=q_{\eta \beta} x_{\beta} x_{\eta}+\mathrm{b}_{3} x_{\gamma},
$$

for some scalars $\mathrm{b}_{1}, \mathrm{~b}_{2}, \mathrm{~b}_{3}$ and the other pairs of root vectors $q$-commute. Then, for all $n \geq 0$,

$$
\begin{gathered}
d\left(\mathrm{x}_{\alpha} \mathrm{x}_{\gamma}^{n} \mathrm{x}_{\beta} \mathrm{x}_{\delta} \otimes 1\right)=\mathrm{x}_{\alpha} \mathrm{x}_{\gamma}^{n} \mathrm{x}_{\beta} \otimes x_{\delta}-q_{\beta \delta} \mathrm{x}_{\alpha} \mathrm{x}_{\gamma}^{n} \mathrm{x}_{\delta} \otimes x_{\beta}+q_{\gamma \beta} q_{\gamma \delta} \mathrm{x}_{\alpha} \mathrm{x}_{\gamma}^{n-1} \mathrm{x}_{\beta} \mathrm{x}_{\delta} \otimes x_{\gamma} \\
+\left(-q_{\alpha \gamma}\right)^{n} q_{\alpha \beta} q_{\alpha \delta} \mathrm{x}_{\gamma}^{n} \mathrm{x}_{\beta} \mathrm{x}_{\delta} \otimes x_{\alpha}+\mathrm{b}_{1}\left(-q_{\alpha \gamma}\right)^{n}(n+1)_{\widetilde{q}_{\delta \gamma}} \mathrm{x}_{\gamma}^{n+1} \mathrm{x}_{\beta} \otimes x_{\eta} \\
+q_{\alpha \beta}(n+2)_{\widetilde{q}_{\beta \delta}} \delta_{N_{\beta}, 2} \mathrm{~b}_{2} \mathrm{x}_{\gamma}^{n} \mathrm{x}_{\beta}^{2} \otimes x_{\eta}^{2}-q_{\beta \delta}\left(-q_{\gamma \alpha}\right)^{-n} \mathrm{~b}_{1} \mathrm{~b}_{3} \mathrm{c}_{\alpha \delta \gamma}^{(n)} \mathrm{x}_{\gamma}^{n+2} \otimes 1 .
\end{gathered}
$$

Notice that the equalities in (10.1.43) force

$$
\gamma+\eta=\alpha+\delta, \quad \eta+\beta=\gamma .
$$

Hence the following equality also holds: $2 \gamma=\alpha+\beta+\delta$.

Proof. Lemma 10.1.7 applied to $\eta<\gamma<\beta$ says that the following formula holds for $n \geq 0$ :

$$
\begin{aligned}
d\left(\mathrm{x}_{\eta} \mathrm{x}_{\gamma}^{n} \mathrm{x}_{\beta} \otimes 1\right)= & \mathrm{x}_{\eta} \mathrm{x}_{\gamma}^{n} \otimes x_{\beta}-q_{\gamma \beta} \mathrm{x}_{\eta} \mathrm{x}_{\gamma}^{n-1} \mathrm{x}_{\beta} \otimes x_{\gamma}-\left(-q_{\eta \gamma}\right)^{n} q_{\eta \beta} \mathrm{x}_{\gamma}^{n} \mathrm{x}_{\beta} \otimes x_{\eta} \\
& +\mathrm{b}_{3} q_{\gamma \beta}(n+1)_{\widetilde{q}_{\eta \gamma}} \mathrm{x}_{\gamma}^{n+1} \otimes 1,
\end{aligned}
$$

Next we prove that the following formula holds for all $n \geq 0$ :

$$
\begin{gathered}
d\left(\mathrm{x}_{\alpha} \mathrm{x}_{\gamma}^{n} \mathrm{x}_{\delta} \otimes 1\right)=\mathrm{x}_{\alpha} \mathrm{x}_{\gamma}^{n} \otimes x_{\delta}-q_{\gamma \delta} \mathrm{x}_{\alpha} \mathrm{x}_{\gamma}^{n-1} \mathrm{x}_{\delta} \otimes x_{\gamma}-\left(-q_{\alpha \gamma}\right)^{n} \mathrm{~b}_{2} \mathrm{x}_{\gamma}^{n} \mathrm{x}_{\beta} \otimes x_{\eta}^{2} \\
-\left(-q_{\alpha \gamma}\right)^{n}(n+1)_{\widetilde{q}_{\delta \gamma}} \mathrm{b}_{1} \mathrm{x}_{\gamma}^{n+1} \otimes x_{\eta}-\left(-q_{\alpha \gamma}\right)^{n} q_{\alpha \delta} \mathrm{x}_{\gamma}^{n} \mathrm{x}_{\delta} \otimes x_{\alpha}
\end{gathered}
$$

For $n=0$ we have that

$$
\begin{gathered}
d\left(\mathrm{x}_{\alpha} \mathrm{x}_{\delta} \otimes 1\right)=\mathrm{x}_{\alpha} \otimes x_{\delta}-s\left(q_{\alpha \delta} x_{\delta} x_{\alpha}+\mathrm{b}_{1} x_{\gamma} x_{\eta}+\mathrm{b}_{2} x_{\beta} x_{\eta}^{2}\right) \\
=\mathrm{x}_{\alpha} \otimes x_{\delta}-\mathrm{b}_{1} \mathrm{x}_{\gamma} \otimes x_{\eta}-\mathrm{b}_{2} \mathrm{x}_{\beta} \otimes x_{\eta}^{2}-q_{\alpha \delta} \mathrm{x}_{\delta} \otimes x_{\alpha}
\end{gathered}
$$

Now assume that (10.1.47) holds for $n$. Using Remark 10.1.2, inductive hypothesis,

$$
\begin{aligned}
d\left(\mathrm{x}_{\alpha} \mathrm{x}_{\gamma}^{n+1} \mathrm{x}_{\delta} \otimes 1\right)=\mathrm{x}_{\alpha} \mathrm{x}_{\gamma}^{n+1} \otimes x_{\delta}-s\left(q_{\gamma \delta} \mathrm{x}_{\alpha} \mathrm{x}_{\gamma}^{n} \otimes x_{\delta} x_{\gamma}+\left(-q_{\alpha \gamma}\right)^{n+1} \mathrm{x}_{\gamma}^{n+1} \otimes x_{\alpha} x_{\delta}\right) \\
=\mathrm{x}_{\alpha} \mathrm{x}_{\gamma}^{n+1} \otimes x_{\delta}-q_{\gamma \delta} \mathrm{x}_{\alpha} \mathrm{x}_{\gamma}^{n} \mathrm{x}_{\delta} \otimes x_{\gamma}-s\left(\left(-q_{\alpha \gamma}\right)^{n+1} q_{\alpha \delta} \mathrm{x}_{\gamma}^{n+1} \otimes x_{\delta} x_{\alpha}\right. \\
\quad+\left(-q_{\alpha \gamma}\right)^{n+1}\left(1+\widetilde{q}_{\gamma \delta}(n+1)_{\widetilde{q}_{\delta \gamma}}\right) \mathrm{b}_{1} \mathrm{x}_{\gamma}^{n+1} \otimes x_{\gamma} x_{\eta}+\left(-q_{\alpha \gamma}\right)^{n+1} \mathrm{~b}_{2} \mathrm{x}_{\gamma}^{n+1} \otimes x_{\beta} x_{\eta}^{2} \\
\left.\quad+q_{\eta \gamma}^{2} q_{\gamma \delta}\left(-q_{\alpha \gamma}\right)^{n} \mathrm{~b}_{2} \mathrm{x}_{\gamma}^{n} \mathrm{x}_{\beta} \otimes x_{\gamma} x_{\eta}^{2}-\left(-q_{\alpha \gamma}\right)^{n+1} q_{\alpha \delta} q_{\gamma \delta} \mathrm{x}_{\gamma}^{n} \mathrm{x}_{\delta} \otimes x_{\gamma} x_{\alpha}\right) \\
=\mathrm{x}_{\alpha} \mathrm{x}_{\gamma}^{n+1} \otimes x_{\delta}-q_{\gamma \delta} \mathrm{x}_{\alpha} \mathrm{x}_{\gamma}^{n} \mathrm{x}_{\delta} \otimes x_{\gamma}-\left(-q_{\alpha \gamma}\right)^{n+1}(n+2)_{\widetilde{q}_{\delta \gamma}} \mathrm{b}_{1} \mathrm{x}_{\gamma}^{n+2} \otimes x_{\eta} \\
\quad-\left(-q_{\alpha \gamma}\right)^{n+1} q_{\alpha \delta} \mathrm{x}_{\gamma}^{n+1} \mathrm{x}_{\delta} \otimes x_{\alpha}-\left(-q_{\alpha \gamma}\right)^{n+1} \mathrm{~b}_{2} \mathrm{x}_{\gamma}^{n+1} \mathrm{x}_{\beta} \otimes x_{\eta}^{2} .
\end{aligned}
$$

Now we prove (10.1.44) by induction on $n$. When $n=0$,

$$
\begin{aligned}
& d\left(\mathrm{x}_{\alpha} \mathrm{x}_{\beta} \mathrm{x}_{\delta} \otimes 1\right)=\mathrm{x}_{\alpha} \mathrm{x}_{\beta} \otimes x_{\delta}-s\left(q_{\beta \delta} \mathrm{x}_{\alpha} \otimes x_{\delta} x_{\beta}-q_{\alpha \beta} \mathrm{x}_{\beta} \otimes x_{\alpha} x_{\delta}\right) \\
& =\mathrm{x}_{\alpha} \mathrm{x}_{\beta} \otimes x_{\delta}-q_{\beta \delta} \mathrm{x}_{\alpha} \mathrm{x}_{\delta} \otimes x_{\beta}-q_{\beta \delta} \mathrm{b}_{1} \mathrm{~b}_{3} \mathrm{x}_{\gamma}^{2} \otimes 1-q_{\eta \beta} q_{\beta \delta} \mathrm{b}_{1} \mathrm{x}_{\gamma} \mathrm{x}_{\beta} \otimes x_{\eta} \\
& \quad+q_{\alpha \beta}(2)_{\widetilde{q}_{\beta \delta}} \delta_{N_{\beta}, 2} \mathrm{~b}_{2} \mathrm{x}_{\beta}^{2} \otimes x_{\eta}^{2}+q_{\alpha \beta} q_{\alpha \delta} \mathrm{x}_{\beta} \mathrm{x}_{\delta} \otimes x_{\alpha},
\end{aligned}
$$


since $\mathrm{x}_{\beta} \otimes x_{\gamma} x_{\eta}=s\left(x_{\beta} x_{\gamma} x_{\eta}\right)$ and $s\left(\mathrm{x}_{\beta} \otimes x_{\beta} x_{\eta}^{2}\right)=0$ : either $\mathrm{x}_{\beta} \otimes x_{\beta} x_{\eta}^{2}=s\left(x_{\beta}^{2} x_{\eta}^{2}\right)$ if $N_{\beta} \neq 2$ or $q_{\beta \delta} q_{\eta \beta}^{2}=q_{\beta \delta} q_{\gamma \beta}^{2}=-q_{\alpha \beta} \widetilde{q}_{\beta \delta}$ if $N_{\beta}=2$. Now assume that (10.1.50) holds for $n$. Using Remark 10.1.2, (10.1.47) and inductive hypothesis,

$$
\begin{aligned}
& d\left(\mathrm{x}_{\alpha} \mathrm{x}_{\gamma}^{n+1} \mathrm{x}_{\beta} \mathrm{x}_{\delta} \otimes 1\right)=\mathrm{x}_{\alpha} \mathrm{x}_{\gamma}^{n+1} \mathrm{x}_{\beta} \otimes x_{\delta}-s\left(q_{\beta \delta} \mathrm{x}_{\alpha} \mathrm{x}_{\gamma}^{n+1} \otimes x_{\delta} x_{\beta}-q_{\gamma \beta} q_{\gamma \delta} \mathrm{x}_{\alpha} \mathrm{x}_{\gamma}^{n} \mathrm{x}_{\beta} \otimes x_{\delta} x_{\gamma}\right. \\
& \left.\quad-\left(-q_{\alpha \gamma}\right)^{n+1} q_{\alpha \beta} \mathrm{x}_{\gamma}^{n+1} \mathrm{x}_{\beta} \otimes\left(q_{\alpha \delta} x_{\delta} x_{\alpha}+\mathrm{b}_{1} x_{\gamma} x_{\eta}+\mathrm{b}_{2} x_{\beta} x_{\eta}^{2}\right)\right) \\
& =\mathrm{x}_{\alpha} \mathrm{x}_{\gamma}^{n+1} \mathrm{x}_{\beta} \otimes x_{\delta}-q_{\beta \delta} \mathrm{x}_{\alpha} \mathrm{x}_{\gamma}^{n+1} \mathrm{x}_{\delta} \otimes x_{\beta}+q_{\gamma \beta} q_{\gamma \delta} \mathrm{x}_{\alpha} \mathrm{x}_{\gamma}^{n} \mathrm{x}_{\beta} \mathrm{x}_{\delta} \otimes x_{\gamma} \\
& \quad-q_{\beta \delta}\left(-q_{\gamma \alpha}\right)^{-n+1} c_{\alpha \delta \gamma}^{(n+1)} \mathrm{b}_{1} \mathrm{~b}_{3} \mathrm{x}_{\gamma}^{n+3} \otimes 1-\left(-q_{\alpha \gamma}\right)^{n+1} q_{\eta \beta} q_{\beta \delta}(n+2)_{\widetilde{q}_{\delta \gamma}} \mathrm{b}_{1} \mathrm{x}_{\gamma}^{n+2} \mathrm{x}_{\beta} \otimes x_{\eta} \\
& \quad+q_{\alpha \beta}(n+2)_{\widetilde{q}_{\beta \delta}} \delta_{N_{\beta}, 2} \mathrm{~b}_{2} \mathrm{x}_{\gamma}^{n} \mathrm{x}_{\beta}^{2} \otimes x_{\eta}^{2}+\left(-q_{\alpha \gamma}\right)^{n+1} q_{\alpha \beta} q_{\alpha \delta} \mathrm{x}_{\gamma}^{n+1} \mathrm{x}_{\beta} \mathrm{x}_{\delta} \otimes x_{\alpha},
\end{aligned}
$$

which completes the inductive step.

Lemma 10.1.48. Let $\alpha<\beta<\gamma<\eta<\delta$ be positive roots such that $N_{\gamma}=2$ and the relations among the corresponding root vectors take the form

$$
x_{\beta} x_{\delta}=q_{\beta \delta} x_{\delta} x_{\beta}+\mathrm{b}_{1} x_{\eta} x_{\gamma}, \quad x_{\alpha} x_{\eta}=q_{\alpha \eta} x_{\eta} x_{\alpha}+\mathrm{b}_{2} x_{\gamma},
$$

for some scalars $\mathrm{b}_{1}, \mathrm{~b}_{2}$ and the other pairs of root vectors $q$-commute. Then, for all $n \geq 0$,

$$
\begin{gathered}
d\left(\mathrm{x}_{\alpha} \mathrm{x}_{\beta} \mathrm{x}_{\gamma}^{n} \mathrm{x}_{\delta} \otimes 1\right)=\mathrm{x}_{\alpha} \mathrm{x}_{\beta} \mathrm{x}_{\gamma}^{n} \otimes x_{\delta}-q_{\gamma \delta} \mathrm{x}_{\alpha} \mathrm{x}_{\beta} \mathrm{x}_{\gamma}^{n-1} \mathrm{x}_{\delta} \otimes x_{\gamma} \\
\quad-\left(-q_{\beta \gamma}\right)^{n} q_{\beta \delta} \mathrm{x}_{\alpha} \mathrm{x}_{\gamma}^{n} \mathrm{x}_{\delta} \otimes x_{\beta}+\left(-q_{\alpha \gamma}\right)^{n} q_{\alpha \beta} q_{\alpha \delta} \mathrm{x}_{\beta} \mathrm{x}_{\gamma}^{n} \mathrm{x}_{\delta} \otimes x_{\alpha} \\
\quad-\left(-q_{\beta \gamma}\right)^{n} \mathrm{~b}_{1} \mathrm{x}_{\alpha} \mathrm{x}_{\gamma}^{n} \mathrm{x}_{\eta} \otimes x_{\gamma}-q_{\gamma \delta}^{n} \mathrm{c}_{-\delta, \alpha, \gamma}^{(n)} \mathrm{b}_{1} \mathrm{~b}_{2} \mathrm{x}_{\gamma}^{n+2} \otimes 1 .
\end{gathered}
$$

Notice that the equalities in (10.1.49) force

$$
\gamma+\eta=\beta+\delta, \quad \eta+\alpha=\gamma .
$$

Hence the following equality also holds: $2 \gamma=\alpha+\beta+\delta$.

Proof. The following formula holds for all $n \geq 0$ :

$$
\begin{aligned}
& d\left(\mathrm{x}_{\beta} \mathrm{x}_{\gamma}^{n} \mathrm{x}_{\delta} \otimes 1\right)=\mathrm{x}_{\beta} \mathrm{x}_{\gamma}^{n} \otimes x_{\delta}-q_{\gamma \delta} \mathrm{x}_{\beta} \mathrm{x}_{\gamma}^{n-1} \mathrm{x}_{\delta} \otimes x_{\gamma}-\left(-q_{\beta \gamma}\right)^{n} q_{\beta \delta} \mathrm{x}_{\gamma}^{n} \mathrm{x}_{\delta} \otimes x_{\beta} \\
& -\left(-q_{\beta \gamma}\right)^{n} \mathrm{~b}_{1} \mathrm{x}_{\gamma}^{n} \mathrm{x}_{\eta} \otimes x_{\gamma} .
\end{aligned}
$$

The proof is analogous to (10.1.14), see also the proof of (10.1.30). Next, we apply Lemma 10.1.7 for $\alpha<\gamma<\eta$ (no other intermediate roots) to get

$$
\begin{aligned}
& d\left(\mathrm{x}_{\alpha} \mathrm{x}_{\gamma}^{n} \mathrm{x}_{\eta} \otimes 1\right)=\mathrm{x}_{\alpha} \mathrm{x}_{\gamma}^{n} \otimes x_{\eta}-q_{\gamma \eta} \mathrm{x}_{\alpha} \mathrm{x}_{\gamma}^{n-1} \mathrm{x}_{\eta} \otimes x_{\gamma}-q_{\alpha \eta}\left(-q_{\alpha \gamma}\right)^{n} \mathrm{x}_{\gamma}^{n} \mathrm{x}_{\eta} \otimes x_{\alpha} \\
& -\left(-q_{\alpha \gamma}\right)^{n}(n+1)_{\widetilde{q}_{\alpha \gamma}} \mathrm{b}_{2} \mathrm{x}_{\gamma}^{n+1} \otimes 1 .
\end{aligned}
$$

Now we prove (10.1.50) by induction on $n$. When $n=0$,

$$
\begin{aligned}
& d\left(\mathrm{x}_{\alpha} \mathrm{x}_{\beta} \mathrm{x}_{\delta} \otimes 1\right)=\mathrm{x}_{\alpha} \mathrm{x}_{\beta} \otimes x_{\delta}-s\left(q_{\beta \delta} \mathrm{x}_{\alpha} \otimes x_{\delta} x_{\beta}+\mathrm{b}_{1} \mathrm{x}_{\alpha} \otimes x_{\eta} x_{\gamma}-q_{\alpha \beta} q_{\alpha \delta} \mathrm{x}_{\beta} \otimes x_{\delta} x_{\alpha}\right) \\
& =\mathrm{x}_{\alpha} \mathrm{x}_{\beta} \otimes x_{\delta}-\mathrm{b}_{1} \mathrm{x}_{\alpha} \mathrm{x}_{\eta} \otimes x_{\gamma}-q_{\beta \delta} \mathrm{x}_{\alpha} \mathrm{x}_{\delta} \otimes x_{\beta}-s\left(q_{\alpha \beta} q_{\alpha \delta} q_{\beta \delta} \mathrm{x}_{\delta} \otimes x_{\beta} x_{\alpha}\right. \\
& \left.\quad+q_{\alpha \gamma} q_{\alpha \eta} \mathrm{b}_{1} \mathrm{x}_{\eta} \otimes x_{\gamma} x_{\alpha}+\mathrm{b}_{1} \mathrm{~b}_{2} \mathrm{x}_{\gamma} \otimes x_{\gamma}-q_{\alpha \beta} q_{\alpha \delta} \mathrm{x}_{\beta} \otimes x_{\delta} x_{\alpha}\right) \\
& =\mathrm{x}_{\alpha} \mathrm{x}_{\beta} \otimes x_{\delta}-\mathrm{b}_{1} \mathrm{x}_{\alpha} \mathrm{x}_{\eta} \otimes x_{\gamma}-q_{\beta \delta} \mathrm{x}_{\alpha} \mathrm{x}_{\delta} \otimes x_{\beta}+q_{\alpha \beta} q_{\alpha \delta} \mathrm{x}_{\beta} \mathrm{x}_{\delta} \otimes x_{\alpha}-\mathrm{b}_{1} \mathrm{~b}_{2} \mathrm{x}_{\gamma}^{2} \otimes 1 .
\end{aligned}
$$


Now assume that (10.1.50) holds for $n$. Using Remark 10.1.2, inductive hypothesis, the relation $x_{\gamma}^{2}=0$, (10.1.53), (10.1.52),

$$
\begin{aligned}
& d\left(\mathrm{x}_{\alpha} \mathrm{x}_{\beta} \mathrm{x}_{\gamma}^{n+1} \mathrm{x}_{\delta} \otimes 1\right)=\mathrm{x}_{\alpha} \mathrm{x}_{\beta} \mathrm{x}_{\gamma}^{n+1} \otimes x_{\delta}-s\left(q_{\gamma \delta} \mathrm{x}_{\alpha} \mathrm{x}_{\beta} \mathrm{x}_{\gamma}^{n} \otimes x_{\delta} x_{\gamma}+\left(-q_{\beta \gamma}\right)^{n+1} \mathrm{~b}_{1} \mathrm{x}_{\alpha} \mathrm{x}_{\gamma}^{n+1} \otimes x_{\eta} x_{\gamma}\right. \\
& \left.+\left(-q_{\beta \gamma}\right)^{n+1} q_{\beta \delta} \mathrm{x}_{\alpha} \mathrm{x}_{\gamma}^{n+1} \otimes x_{\delta} x_{\beta}-q_{\alpha \beta}\left(-q_{\alpha \gamma}\right)^{n+1} q_{\alpha \delta} \mathrm{x}_{\beta} \mathrm{x}_{\gamma}^{n+1} \otimes x_{\delta} x_{\alpha}\right) \\
& =\mathrm{x}_{\alpha} \mathrm{x}_{\beta} \mathrm{x}_{\gamma}^{n+1} \otimes x_{\delta}-q_{\gamma \delta} \mathrm{x}_{\alpha} \mathrm{x}_{\beta} \mathrm{x}_{\gamma}^{n} \mathrm{x}_{\delta} \otimes x_{\gamma}-\left(-q_{\beta \gamma}\right)^{n+1} \mathrm{~b}_{1} \mathrm{x}_{\alpha} \mathrm{x}_{\gamma}^{n+1} \mathrm{x}_{\eta} \otimes x_{\gamma} \\
& -\left(-q_{\beta \gamma}\right)^{n+1} q_{\beta \delta} \mathrm{x}_{\alpha} \mathrm{x}_{\gamma}^{n+1} \mathrm{x}_{\delta} \otimes x_{\beta}-s\left(-q_{\alpha \beta}\left(-q_{\alpha \gamma}\right)^{n+1} q_{\alpha \delta} \mathrm{x}_{\beta} \mathrm{x}_{\gamma}^{n+1} \otimes x_{\delta} x_{\alpha}\right. \\
& -q_{\alpha \gamma}\left(-q_{\alpha \gamma}\right)^{n} q_{\alpha \beta} q_{\alpha \delta} q_{\gamma \delta} \mathrm{x}_{\beta} \mathrm{x}_{\gamma}^{n} \mathrm{x}_{\delta} \otimes x_{\gamma} x_{\alpha} \\
& +\left(q_{\gamma \delta}^{n+1} \mathrm{c}_{-\delta, \alpha, \gamma}^{(n)}+q_{\beta \gamma}^{n+1} q_{\alpha \gamma}^{n+1}(n+2) \widetilde{q}_{\alpha \gamma}\right) \mathrm{b}_{1} \mathrm{~b}_{2} \mathrm{x}_{\gamma}^{n+2} \otimes x_{\gamma} \\
& \left.+q_{\beta \gamma}^{n+1} q_{\alpha \eta} q_{\alpha \gamma}^{n+2} \mathrm{~b}_{1} \mathrm{x}_{\gamma}^{n+1} \mathrm{x}_{\eta} \otimes x_{\gamma} x_{\alpha}-q_{\beta \gamma}^{n+1} q_{\beta \delta} q_{\alpha \beta} q_{\alpha \gamma}^{n+1} q_{\alpha \delta} \mathrm{x}_{\gamma}^{n+1} \mathrm{x}_{\delta} \otimes x_{\beta} x_{\alpha}\right) \\
& =\mathrm{x}_{\alpha} \mathrm{x}_{\beta} \mathrm{x}_{\gamma}^{n+1} \otimes x_{\delta}-q_{\gamma \delta} \mathrm{x}_{\alpha} \mathrm{x}_{\beta} \mathrm{x}_{\gamma}^{n} \mathrm{x}_{\delta} \otimes x_{\gamma}-\left(-q_{\beta \gamma}\right)^{n+1} \mathrm{~b}_{1} \mathrm{x}_{\alpha} \mathrm{x}_{\gamma}^{n+1} \mathrm{x}_{\eta} \otimes x_{\gamma} \\
& -\left(-q_{\beta \gamma}\right)^{n+1} q_{\beta \delta} \mathrm{x}_{\alpha} \mathrm{x}_{\gamma}^{n+1} \mathrm{x}_{\delta} \otimes x_{\beta}+q_{\alpha \beta}\left(-q_{\alpha \gamma}\right)^{n+1} q_{\alpha \delta} \mathrm{x}_{\beta} \mathrm{x}_{\gamma}^{n+1} \mathrm{x}_{\delta} \otimes x_{\alpha} \\
& -\left(q_{\gamma \delta}^{n+1} \mathrm{c}_{-\delta, \alpha, \gamma}^{(n)}+q_{\gamma \gamma}^{2(n+1)} q_{\delta \gamma}^{-n-1}(n+2) \widetilde{q}_{\alpha \gamma}\right) \mathrm{b}_{1} \mathrm{~b}_{2} \mathrm{x}_{\gamma}^{n+3} \otimes 1,
\end{aligned}
$$

and the inductive step follows.

Lemma 10.1.54. Let $\alpha<\tau<\beta<\gamma<\mu<\nu<\eta<\delta$ be positive roots such that $N_{\gamma}=2$ and the relations among the corresponding root vectors take the form

$$
\begin{array}{ll}
x_{\alpha} x_{\delta}=q_{\alpha \delta} x_{\delta} x_{\alpha}+\mathrm{b}_{1} x_{\eta} x_{\tau}, & x_{\beta} x_{\delta}=q_{\beta \delta} x_{\delta} x_{\beta}+\mathrm{b}_{2} x_{\nu} x_{\gamma}, \\
x_{\beta} x_{\eta}=q_{\beta \eta} x_{\eta} x_{\beta}+\mathrm{b}_{3} x_{\mu} x_{\gamma}, & x_{\alpha} x_{\nu}=q_{\alpha \nu} x_{\nu} x_{\alpha}+\mathrm{b}_{4} x_{\gamma},
\end{array}
$$

for some scalars $\mathrm{b}_{i}$ and the other pairs of root vectors $q$-commute. Then, for all $n \geq 0$,

$$
\begin{aligned}
& d\left(\mathrm{x}_{\alpha} \mathrm{x}_{\beta} \mathrm{x}_{\gamma}^{n} \mathrm{x}_{\delta} \otimes 1\right)=\mathrm{x}_{\alpha} \mathrm{x}_{\beta} \mathrm{x}_{\gamma}^{n} \otimes x_{\delta}-q_{\gamma \delta} \mathrm{x}_{\alpha} \mathrm{x}_{\beta} \mathrm{x}_{\gamma}^{n-1} \mathrm{x}_{\delta} \otimes x_{\gamma} \\
& \quad-\left(-q_{\beta \gamma}\right)^{n} q_{\beta \delta} \mathrm{x}_{\alpha} \mathrm{x}_{\gamma}^{n} \mathrm{x}_{\delta} \otimes x_{\beta}+\left(-q_{\alpha \gamma}\right)^{n} q_{\alpha \beta} q_{\alpha \delta} \mathrm{x}_{\beta} \mathrm{x}_{\gamma}^{n} \mathrm{x}_{\delta} \otimes x_{\alpha} \\
& \quad+q_{\alpha \beta}\left(-q_{\alpha \gamma}\right)^{n} \mathrm{~b}_{1} \mathrm{x}_{\beta} \mathrm{x}_{\gamma}^{n} \mathrm{x}_{\eta} \otimes x_{\tau}-\left(-q_{\beta \gamma}\right)^{n} \mathrm{~b}_{2} \mathrm{x}_{\alpha} \mathrm{x}_{\gamma}^{n} \mathrm{x}_{\nu} \otimes x_{\gamma} \\
& \quad-q_{\gamma \delta}^{n} \mathrm{c}_{-\delta, \alpha, \gamma}^{(n)} \mathrm{b}_{2} \mathrm{~b}_{4} \mathrm{x}_{\gamma}^{n+2} \otimes 1 .
\end{aligned}
$$

Notice that the equalities in (10.1.55) force

$$
\alpha+\delta=\eta+\tau, \quad \beta+\delta=\nu+\gamma, \quad \beta+\eta=\mu+\gamma, \quad \alpha+\nu=\gamma .
$$

Hence the following equality also holds: $2 \gamma=\alpha+\beta+\delta$.

Proof. We need some auxiliary computations. First we apply Lemma 10.1.7 to $\alpha<\gamma<\nu$ :

$$
\begin{aligned}
& d\left(\mathrm{x}_{\alpha} \mathrm{x}_{\gamma}^{n} \mathrm{x}_{\nu} \otimes 1\right)=\mathrm{x}_{\alpha} \mathrm{x}_{\gamma}^{n} \otimes x_{\nu}-q_{\gamma \nu} \mathrm{x}_{\alpha} \mathrm{x}_{\gamma}^{n-1} \mathrm{x}_{\nu} \otimes x_{\gamma}-q_{\alpha \nu}\left(-q_{\alpha \gamma}\right)^{n} \mathrm{x}_{\gamma}^{n} \mathrm{x}_{\nu} \otimes x_{\alpha} \\
& -\left(-q_{\alpha \gamma}\right)^{n}(n+1)_{\widetilde{q}_{\alpha \gamma}} \mathrm{b}_{4} \mathrm{x}_{\gamma}^{n+1} \otimes 1 .
\end{aligned}
$$

Next we claim that the following formulas hold for all $n \geq 0$ :

$$
\begin{aligned}
& d\left(\mathrm{x}_{\alpha} \mathrm{x}_{\gamma}^{n} \mathrm{x}_{\delta} \otimes 1\right)=\mathrm{x}_{\alpha} \mathrm{x}_{\gamma}^{n} \otimes x_{\delta}-q_{\gamma \delta} \mathrm{x}_{\alpha} \mathrm{x}_{\gamma}^{n-1} \mathrm{x}_{\delta} \otimes x_{\gamma}-\left(-q_{\alpha \gamma}\right)^{n} q_{\alpha \delta} \mathrm{x}_{\gamma}^{n} \mathrm{x}_{\delta} \otimes x_{\alpha} \\
& \quad-\left(-q_{\alpha \gamma}\right)^{n} \mathrm{~b}_{1} \mathrm{x}_{\gamma}^{n} \mathrm{x}_{\eta} \otimes x_{\tau}, \\
& d\left(\mathrm{x}_{\beta} \mathrm{x}_{\gamma}^{n} \mathrm{x}_{\delta} \otimes 1\right)=\mathrm{x}_{\beta} \mathrm{x}_{\gamma}^{n} \otimes x_{\delta}-q_{\gamma \delta} \mathrm{x}_{\beta} \mathrm{x}_{\gamma}^{n-1} \mathrm{x}_{\delta} \otimes x_{\gamma}-\left(-q_{\beta \gamma}\right)^{n} q_{\beta \delta} \mathrm{x}_{\gamma}^{n} \mathrm{x}_{\delta} \otimes x_{\beta} \\
& \quad-\left(-q_{\beta \gamma}\right)^{n} \mathrm{~b}_{2} \mathrm{x}_{\gamma}^{n} \mathrm{x}_{\nu} \otimes x_{\gamma},
\end{aligned}
$$




$$
\begin{aligned}
& d\left(\mathrm{x}_{\beta} \mathrm{x}_{\gamma}^{n} \mathrm{x}_{\eta} \otimes 1\right)=\mathrm{x}_{\beta} \mathrm{x}_{\gamma}^{n} \otimes x_{\eta}-q_{\gamma \eta} \mathrm{x}_{\beta} \mathrm{x}_{\gamma}^{n-1} \mathrm{x}_{\eta} \otimes x_{\gamma}-\left(-q_{\beta \gamma}\right)^{n} q_{\beta \eta} \mathrm{x}_{\gamma}^{n} \mathrm{x}_{\eta} \otimes x_{\beta} \\
& \quad-\left(-q_{\beta \gamma}\right)^{n} \mathrm{~b}_{3} \mathrm{x}_{\gamma}^{n} \mathrm{x}_{\mu} \otimes x_{\gamma} .
\end{aligned}
$$

The proof of each equality is analogous to (10.1.14).

Now we prove (10.1.56) by induction on $n$. When $n=0$,

$$
\begin{aligned}
d\left(\mathrm{x}_{\alpha} \mathrm{x}_{\beta} \mathrm{x}_{\delta} \otimes 1\right)=\mathrm{x}_{\alpha} \mathrm{x}_{\beta} \otimes x_{\delta}-s\left(q_{\beta \delta} \mathrm{x}_{\alpha} \otimes x_{\delta} x_{\beta}+\mathrm{b}_{2} \mathrm{x}_{\alpha} \otimes x_{\nu} x_{\gamma}-q_{\alpha \beta} \mathrm{x}_{\beta} \otimes x_{\alpha} x_{\delta}\right) \\
=\mathrm{x}_{\alpha} \mathrm{x}_{\beta} \otimes x_{\delta}-\mathrm{b}_{2} \mathrm{x}_{\alpha} \mathrm{x}_{\nu} \otimes x_{\gamma}-q_{\beta \delta} \mathrm{x}_{\alpha} \mathrm{x}_{\delta} \otimes x_{\beta}-s\left(-q_{\alpha \beta} q_{\alpha \delta} \mathrm{x}_{\beta} \otimes x_{\delta} x_{\alpha}-q_{\alpha \beta} \mathrm{b}_{1} \mathrm{x}_{\beta} \otimes x_{\eta} x_{\tau}\right. \\
\left.\quad+q_{\alpha \nu} \mathrm{b}_{2} \mathrm{x}_{\nu} \otimes x_{\alpha} x_{\gamma}+\mathrm{b}_{2} \mathrm{~b}_{4} \mathrm{x}_{\gamma} \otimes x_{\gamma}+q_{\alpha \beta} q_{\alpha \delta} q_{\beta \delta} \mathrm{x}_{\delta} \otimes x_{\beta} x_{\alpha}+q_{\tau \beta} q_{\beta \delta} \mathrm{b}_{1} \mathrm{x}_{\eta} \otimes x_{\beta} x_{\tau}\right) \\
=\mathrm{x}_{\alpha} \mathrm{x}_{\beta} \otimes x_{\delta}-\mathrm{b}_{2} \mathrm{x}_{\alpha} \mathrm{x}_{\nu} \otimes x_{\gamma}-q_{\beta \delta} \mathrm{x}_{\alpha} \mathrm{x}_{\delta} \otimes x_{\beta}+q_{\alpha \beta} \mathrm{b}_{1} \mathrm{x}_{\beta} \mathrm{x}_{\eta} \otimes x_{\tau}+q_{\alpha \beta} q_{\alpha \delta} \mathrm{x}_{\beta} \mathrm{x}_{\delta} \otimes x_{\alpha} \\
\quad-s\left(\mathrm{~b}_{2} \mathrm{~b}_{4} \mathrm{x}_{\gamma} \otimes x_{\gamma}-q_{\alpha \beta} \mathrm{b}_{1} \mathrm{~b}_{3} \mathrm{x}_{\mu} \otimes x_{\gamma} x_{\tau}\right),
\end{aligned}
$$

which is (10.1.56) for $n=0$ since

$$
s\left(\mathrm{x}_{\gamma} \otimes x_{\gamma}\right)=\mathrm{x}_{\gamma}^{2} \otimes 1, \quad s\left(\mathrm{x}_{\mu} \otimes x_{\gamma} x_{\tau}\right)=s \circ s\left(x_{\mu} x_{\gamma} x_{\tau}\right)=0 .
$$

Now assume that (10.1.50) holds for $n$. Using Remark 10.1.2, inductive hypothesis, the relation $x_{\gamma}^{2}=0$, (10.1.58), (10.1.59), (10.1.60), (10.1.61),

$$
\begin{aligned}
& d\left(\mathrm{x}_{\alpha} \mathrm{x}_{\beta} \mathrm{x}_{\gamma}^{n+1} \mathrm{x}_{\delta} \otimes 1\right)=\mathrm{x}_{\alpha} \mathrm{x}_{\beta} \mathrm{x}_{\gamma}^{n+1} \otimes x_{\delta}-s\left(q_{\gamma \delta} \mathrm{x}_{\alpha} \mathrm{x}_{\beta} \mathrm{x}_{\gamma}^{n} \otimes x_{\delta} x_{\gamma}+\left(-q_{\beta \gamma}\right)^{n+1} \mathrm{~b}_{2} \mathrm{x}_{\alpha} \mathrm{x}_{\gamma}^{n+1} \otimes x_{\nu} x_{\gamma}\right. \\
&+q_{\beta \delta}\left(-q_{\beta \gamma}\right)^{n+1} \mathrm{x}_{\alpha} \mathrm{x}_{\gamma}^{n+1} \otimes x_{\delta} x_{\beta}-q_{\alpha \beta}\left(-q_{\alpha \gamma}\right)^{n+1} q_{\alpha \delta} \mathrm{x}_{\beta} \mathrm{x}_{\gamma}^{n+1} \otimes x_{\delta} x_{\alpha} \\
&\left.-q_{\alpha \beta}\left(-q_{\alpha \gamma}\right)^{n+1} \mathrm{~b}_{1} \mathrm{x}_{\beta} \mathrm{x}_{\gamma}^{n+1} \otimes x_{\eta} x_{\tau}\right) \\
&= \mathrm{x}_{\alpha} \mathrm{x}_{\beta} \mathrm{x}_{\gamma}^{n+1} \otimes x_{\delta}-q_{\gamma \delta} \mathrm{x}_{\alpha} \mathrm{x}_{\beta} \mathrm{x}_{\gamma}^{n} \mathrm{x}_{\delta} \otimes x_{\gamma}-\left(-q_{\beta \gamma}\right)^{n+1} \mathrm{~b}_{2} \mathrm{x}_{\alpha} \mathrm{x}_{\gamma}^{n+1} \mathrm{x}_{\nu} \otimes x_{\gamma} \\
&-s\left(-q_{\gamma \delta}\left(-q_{\beta \gamma}\right)^{n+1} q_{\beta \delta} \mathrm{x}_{\alpha} \mathrm{x}_{\gamma}^{n} \mathrm{x}_{\delta} \otimes x_{\gamma} x_{\beta}+q_{\gamma \delta}\left(-q_{\alpha \gamma}\right)^{n+1} q_{\alpha \beta} q_{\alpha \delta} \mathrm{x}_{\beta} \mathrm{x}_{\gamma}^{n} \mathrm{x}_{\delta} \otimes x_{\gamma} x_{\alpha}\right. \\
&-q_{\gamma \delta} q_{\alpha \beta}\left(-q_{\alpha \gamma}\right)^{n} \mathrm{~b}_{1} \mathrm{x}_{\beta} \mathrm{x}_{\gamma}^{n} \mathrm{x}_{\eta} \otimes x_{\tau} x_{\gamma}+q_{\gamma \delta}\left(-q_{\beta \gamma}\right)^{n} \mathrm{~b}_{2} \mathrm{x}_{\alpha} \mathrm{x}_{\gamma}^{n} \mathrm{x}_{\nu} \otimes x_{\gamma}^{2} \\
&+\left(q_{\gamma \delta}^{n+1} c_{-\delta, \alpha, \gamma}^{(n)}+q_{\alpha \gamma}^{n+1} q_{\beta \gamma}^{n+1}(n+2)_{q_{\alpha \gamma}}\right) \mathrm{b}_{2} \mathrm{~b}_{4} \mathrm{x}_{\gamma}^{n+2} \otimes x_{\gamma} \\
&+q_{\beta \delta}\left(-q_{\beta \gamma}\right)^{n+1} \mathrm{x}_{\alpha} \mathrm{x}_{\gamma}^{n+1} \otimes x_{\delta} x_{\beta}-q_{\alpha \beta}\left(-q_{\alpha \gamma}\right)^{n+1} q_{\alpha \delta} \mathrm{x}_{\beta} \mathrm{x}_{\gamma}^{n+1} \otimes x_{\delta} x_{\alpha} \\
&\left.-q_{\alpha \beta}\left(-q_{\alpha \gamma}\right)^{n+1} \mathrm{~b}_{1} \mathrm{x}_{\beta} \mathrm{x}_{\gamma}^{n+1} \otimes x_{\eta} x_{\tau}+q_{\alpha \nu} q_{\alpha \gamma}^{n+2} q_{\beta \gamma}^{n+1} \mathrm{~b}_{2} \mathrm{x}_{\gamma}^{n+1} \mathrm{x}_{\nu} \otimes x_{\gamma} x_{\alpha}\right) \\
&= \mathrm{x}_{\alpha} \mathrm{x}_{\beta} \mathrm{x}_{\gamma}^{n+1} \otimes x_{\delta}-q_{\gamma \delta} \mathrm{x}_{\alpha} \mathrm{x}_{\beta} \mathrm{x}_{\gamma}^{n} \mathrm{x}_{\delta} \otimes x_{\gamma}-\left(-q_{\beta \gamma}\right)^{n+1} \mathrm{~b}_{2} \mathrm{x}_{\alpha} \mathrm{x}_{\gamma}^{n+1} \mathrm{x}_{\nu} \otimes x_{\gamma} \\
&-q_{\beta \delta}\left(-q_{\beta \gamma}\right)^{n+1} \mathrm{x}_{\alpha} \mathrm{x}_{\gamma}^{n+1} \mathrm{x}_{\delta} \otimes x_{\beta}+q_{\alpha \beta}\left(-q_{\alpha \gamma}\right)^{n+1} \mathrm{~b}_{1} \mathrm{x}_{\beta} \mathrm{x}_{\gamma}^{n+1} \mathrm{x}_{\eta} \otimes x_{\tau} \\
&+q_{\alpha \beta}\left(-q_{\alpha \gamma}\right)^{n+1} q_{\alpha \delta} \mathrm{x}_{\beta} \mathrm{x}_{\gamma}^{n+1} \mathrm{x}_{\delta} \otimes x_{\alpha}-s\left(-q_{\alpha \beta} q_{\alpha \gamma}^{n+1} q_{\beta \gamma}^{n+1} \mathrm{~b}_{1} \mathrm{~b}_{3} \mathrm{x}_{\gamma}^{n+1} \mathrm{x}_{\mu} \otimes x_{\gamma} x_{\tau}\right. \\
&\left.+q_{\gamma \delta}^{n+1}\left(c_{-\delta, \alpha, \gamma}^{(n)}+q_{\alpha \gamma}^{n+1} q_{\beta \gamma}^{n+1} q_{\gamma \delta}^{-n-1}(n+2)_{q_{\alpha \gamma}}\right) \mathrm{b}_{2} \mathrm{~b}_{4} \mathrm{x}_{\gamma}^{n+2} \otimes x_{\gamma}\right) .
\end{aligned}
$$

As $\alpha+\beta=2 \gamma-\delta$, we have $q_{\alpha \gamma}^{n+1} q_{\beta \gamma}^{n+1} q_{\gamma \delta}^{-n-1}=\widetilde{q}_{\gamma \delta}^{-n-1}$. Also, $s\left(\mathrm{x}_{\gamma}^{n+2} \otimes x_{\gamma}\right)=\mathrm{x}_{\gamma}^{n+3} \otimes 1$, so

$$
\begin{aligned}
& d\left(\mathrm{x}_{\alpha} \mathrm{x}_{\beta} \mathrm{x}_{\gamma}^{n+1} \mathrm{x}_{\delta} \otimes 1\right)=\mathrm{x}_{\alpha} \mathrm{x}_{\beta} \mathrm{x}_{\gamma}^{n+1} \otimes x_{\delta}-q_{\gamma \delta} \mathrm{x}_{\alpha} \mathrm{x}_{\beta} \mathrm{x}_{\gamma}^{n} \mathrm{x}_{\delta} \otimes x_{\gamma}-\left(-q_{\beta \gamma}\right)^{n+1} \mathrm{~b}_{2} \mathrm{x}_{\alpha} \mathrm{x}_{\gamma}^{n+1} \mathrm{x}_{\nu} \otimes x_{\gamma} \\
& \quad-q_{\beta \delta}\left(-q_{\beta \gamma}\right)^{n+1} \mathrm{x}_{\alpha} \mathrm{x}_{\gamma}^{n+1} \mathrm{x}_{\delta} \otimes x_{\beta}+q_{\alpha \beta}\left(-q_{\alpha \gamma}\right)^{n+1} \mathrm{~b}_{1} \mathrm{x}_{\beta} \mathrm{x}_{\gamma}^{n+1} \mathrm{x}_{\eta} \otimes x_{\tau} \\
& \quad+q_{\alpha \beta}\left(-q_{\alpha \gamma}\right)^{n+1} q_{\alpha \delta} \mathrm{x}_{\beta} \mathrm{x}_{\gamma}^{n+1} \mathrm{x}_{\delta} \otimes x_{\alpha}-q_{\gamma \delta}^{n+1} \mathrm{c}_{-\delta, \alpha, \gamma}^{(n+1)} \mathrm{b}_{2} \mathrm{~b}_{4} \mathrm{x}_{\gamma}^{n+3} \otimes 1 \\
& \quad+q_{\alpha \beta} q_{\alpha \gamma}^{n+1} q_{\beta \gamma}^{n+1} \mathrm{~b}_{1} \mathrm{~b}_{3} s\left(\mathrm{x}_{\gamma}^{n+1} \mathrm{x}_{\mu} \otimes x_{\gamma} x_{\tau}\right) .
\end{aligned}
$$


Next we claim that $\mathrm{x}_{\gamma}^{n+1} \mathrm{x}_{\mu} \otimes x_{\gamma} x_{\tau}=s\left(\mathrm{x}_{\gamma}^{n+1} \otimes x_{\mu} x_{\gamma} x_{\tau}\right)$. Indeed,

$$
d\left(\mathrm{x}_{\gamma}^{n+1} \otimes x_{\mu} x_{\gamma} x_{\tau}\right)=\mathrm{x}_{\gamma}^{n} \otimes x_{\gamma} x_{\mu} x_{\gamma} x_{\tau}=q_{\gamma \mu} \mathrm{x}_{\gamma}^{n} \otimes x_{\mu} x_{\gamma}^{2} x_{\tau}=0,
$$

so $\mathrm{x}_{\gamma}^{n+1} \otimes x_{\mu} x_{\gamma} x_{\tau} \in \operatorname{ker} d_{n}$, and we compute

$$
\begin{aligned}
s\left(\mathrm{x}_{\gamma}^{n+1}\right. & \left.\otimes x_{\mu} x_{\gamma} x_{\tau}\right)=\mathrm{x}_{\gamma}^{n+1} \mathrm{x}_{\mu} \otimes x_{\gamma} x_{\tau}+s\left(\mathrm{x}_{\gamma}^{n+1} \otimes x_{\mu} x_{\gamma} x_{\tau}-d\left(\mathrm{x}_{\gamma}^{n+1} \mathrm{x}_{\mu} \otimes x_{\gamma} x_{\tau}\right)\right) \\
& =\mathrm{x}_{\gamma}^{n+1} \mathrm{x}_{\mu} \otimes x_{\gamma} x_{\tau}+s\left(\mathrm{x}_{\gamma}^{n+1} \otimes x_{\mu} x_{\gamma} x_{\tau}-\left(\mathrm{x}_{\gamma}^{n+1} \otimes x_{\mu} x_{\gamma} x_{\tau}-q_{\gamma \mu} \mathrm{x}_{\gamma}^{n} \mathrm{x}_{\mu} \otimes x_{\gamma}^{2} x_{\tau}\right)\right) \\
& =\mathrm{x}_{\gamma}^{n+1} \mathrm{x}_{\mu} \otimes x_{\gamma} x_{\tau} .
\end{aligned}
$$

From this claim, $s\left(\mathrm{x}_{\gamma}^{n+1} \mathrm{x}_{\mu} \otimes x_{\gamma} x_{\tau}\right)=0$, and the inductive step follows.

Lemma 10.1.62. Let $\alpha<\beta<\nu<\gamma<\mu<\delta<\eta$ be positive roots such that $N_{\gamma}=2$ and the relations among the corresponding root vectors take the form

$$
\begin{aligned}
x_{\beta} x_{\delta} & =q_{\beta \delta} x_{\delta} x_{\beta}+\mathrm{b}_{1} x_{\gamma} x_{\nu}, \quad x_{\nu} x_{\eta}=q_{\nu \eta} x_{\eta} x_{\nu}+\mathrm{b}_{2} x_{\mu} x_{\gamma}, \\
x_{\alpha} x_{\mu} & =q_{\alpha \mu} x_{\mu} x_{\alpha}+\mathrm{b}_{3} x_{\gamma},
\end{aligned}
$$

for some scalars $\mathrm{b}_{i}, x_{\gamma} q$-commutes with the other root vectors and the following pairs of root vectors also q-commute: $\left(x_{\alpha}, x_{\beta}\right),\left(x_{\alpha}, x_{\nu}\right),\left(x_{\alpha}, x_{\delta}\right),\left(x_{\alpha}, x_{\eta}\right),\left(x_{\beta}, x_{\nu}\right),\left(x_{\beta}, x_{\eta}\right)$, $\left(x_{\nu}, x_{\delta}\right),\left(x_{\nu}, x_{\eta}\right),\left(x_{\mu}, x_{\delta}\right),\left(x_{\mu}, x_{\eta}\right)$. Then, for all $n \geq 0$,

$$
\begin{aligned}
& d\left(\mathrm{x}_{\alpha} \mathrm{x}_{\beta} \mathrm{x}_{\gamma}^{n} \mathrm{x}_{\delta} \mathrm{x}_{\eta} \otimes 1\right)=\mathrm{x}_{\alpha} \mathrm{x}_{\beta} \mathrm{x}_{\gamma}^{n} \mathrm{x}_{\delta} \otimes x_{\eta}+\left(-q_{\beta \gamma}\right)^{n}(n+1)_{\widetilde{q}_{\delta \gamma}} \mathrm{b}_{1} \mathrm{~b}_{2} \mathrm{x}_{\alpha} \mathrm{x}_{\gamma}^{n+1} \mathrm{x}_{\mu} \otimes x_{\gamma} \\
& \quad-q_{\delta \eta} \mathrm{x}_{\alpha} \mathrm{x}_{\beta} \mathrm{x}_{\gamma}^{n} \mathrm{x}_{\eta} \otimes x_{\delta}+q_{\nu \eta}\left(-q_{\beta \gamma}\right)^{n}(n+1)_{\widetilde{q}_{\delta \gamma}} \mathrm{b}_{1} \mathrm{x}_{\alpha} \mathrm{x}_{\gamma}^{n+1} \mathrm{x}_{\eta} \otimes x_{\nu} \\
& \quad+q_{\gamma \delta} q_{\gamma \eta} \mathrm{x}_{\alpha} \mathrm{x}_{\beta} \mathrm{x}_{\gamma}^{n-1} \mathrm{x}_{\delta} \mathrm{x}_{\eta} \otimes x_{\gamma}+\left(-q_{\beta \gamma}\right)^{n} q_{\beta \delta} q_{\beta \eta} \mathrm{x}_{\alpha} \mathrm{x}_{\gamma}^{n} \mathrm{x}_{\delta} \mathrm{x}_{\eta} \otimes x_{\beta} \\
& \quad-q_{\alpha \beta}\left(-q_{\alpha \gamma}\right)^{n} q_{\alpha \delta} q_{\alpha \eta} \mathrm{x}_{\beta} \mathrm{x}_{\gamma}^{n} \mathrm{x}_{\delta} \mathrm{x}_{\eta} \otimes x_{\alpha}-q_{\alpha \gamma} q_{\gamma \delta}^{n} q_{\gamma \eta}^{n} \mathrm{~d}_{\alpha+\beta, \delta, \alpha, \gamma}^{(n)} \mathrm{b}_{1} \mathrm{~b}_{2} \mathrm{~b}_{3} \mathrm{x}_{\gamma}^{n+3} \otimes 1 .
\end{aligned}
$$

Notice that the equalities in (10.1.63) force

$$
\beta+\delta=\gamma+\nu, \quad \nu+\eta=\mu+\gamma, \quad \alpha+\mu=\gamma .
$$

Hence the following equality also holds: $3 \gamma=\alpha+\beta+\delta+\eta$.

Proof. We need some auxiliary computations. By (10.1.14)

$$
\begin{aligned}
d\left(\mathrm{x}_{\beta} \mathrm{x}_{\gamma}^{n} \mathrm{x}_{\delta} \otimes 1\right) & =\mathrm{x}_{\beta} \mathrm{x}_{\gamma}^{n} \otimes x_{\delta}-q_{\gamma \delta} \mathrm{x}_{\beta} \mathrm{x}_{\gamma}^{n-1} \mathrm{x}_{\delta} \otimes x_{\gamma}-q_{\beta \delta}\left(-q_{\beta \gamma}\right)^{n} \mathrm{x}_{\gamma}^{n} \mathrm{x}_{\delta} \otimes x_{\beta} \\
& -\left(-q_{\beta \gamma}\right)^{n}(n+1)_{\widetilde{q}_{\delta \gamma}} \mathrm{b}_{1} \mathrm{x}_{\gamma}^{n+1} \otimes x_{\nu}
\end{aligned}
$$

Next we apply Lemma 10.1.7 to $\alpha<\gamma<\mu$ :

$$
\begin{aligned}
& d\left(\mathrm{x}_{\alpha} \mathrm{x}_{\gamma}^{n} \mathrm{x}_{\mu} \otimes 1\right)=\mathrm{x}_{\alpha} \mathrm{x}_{\gamma}^{n} \otimes x_{\mu}-q_{\gamma \mu} \mathrm{x}_{\alpha} \mathrm{x}_{\gamma}^{n-1} \mathrm{x}_{\mu} \otimes x_{\gamma}-q_{\alpha \mu}\left(-q_{\alpha \gamma}\right)^{n} \mathrm{x}_{\gamma}^{n} \mathrm{x}_{\mu} \otimes x_{\alpha} \\
& -\left(-q_{\alpha \gamma}\right)^{n}(n+1)_{\widetilde{q}_{\alpha \gamma}} \mathrm{b}_{3} \mathrm{x}_{\gamma}^{n+1} \otimes 1 .
\end{aligned}
$$

Now we prove the following equality by induction on $n$ :

$$
\begin{aligned}
& d\left(\mathrm{x}_{\alpha} \mathrm{x}_{\beta} \mathrm{x}_{\gamma}^{n} \mathrm{x}_{\delta} \otimes 1\right)=\mathrm{x}_{\alpha} \mathrm{x}_{\beta} \mathrm{x}_{\gamma}^{n} \otimes x_{\delta}-q_{\gamma \delta} \mathrm{x}_{\alpha} \mathrm{x}_{\beta} \mathrm{x}_{\gamma}^{n-1} \mathrm{x}_{\delta} \otimes x_{\gamma} \\
& \quad-\left(-q_{\beta \gamma}\right)^{n} q_{\beta \delta} \mathrm{x}_{\alpha} \mathrm{x}_{\gamma}^{n} \mathrm{x}_{\delta} \otimes x_{\beta}+q_{\alpha \beta}\left(-q_{\alpha \gamma}\right)^{n} q_{\alpha \delta} \mathrm{x}_{\beta} \mathrm{x}_{\gamma}^{n} \mathrm{x}_{\delta} \otimes x_{\alpha} \\
& \quad-\left(-q_{\beta \gamma}\right)^{n}(n+1) \widetilde{q}_{\delta \gamma} \mathrm{b}_{1} \mathrm{x}_{\alpha} \mathrm{x}_{\gamma}^{n+1} \otimes x_{\nu} .
\end{aligned}
$$




$$
\begin{aligned}
& d\left(\mathrm{x}_{\beta} \mathrm{x}_{\gamma}^{n} \mathrm{x}_{\delta} \mathrm{x}_{\eta} \otimes 1\right)=\mathrm{x}_{\beta} \mathrm{x}_{\gamma}^{n} \mathrm{x}_{\delta} \otimes x_{\eta}-q_{\delta \eta} \mathrm{x}_{\beta} \mathrm{x}_{\gamma}^{n} \mathrm{x}_{\eta} \otimes x_{\delta} \\
& \quad+q_{\gamma \delta} q_{\gamma \eta} \mathrm{x}_{\beta} \mathrm{x}_{\gamma}^{n-1} \mathrm{x}_{\delta} \mathrm{x}_{\eta} \otimes x_{\gamma}+\left(-q_{\beta \gamma}\right)^{n}(n+1)_{\widetilde{q}_{\delta \gamma}} \mathrm{b}_{1} \mathrm{~b}_{2} \mathrm{x}_{\gamma}^{n+1} \mathrm{x}_{\mu} \otimes x_{\gamma} \\
& \quad+\left(-q_{\beta \gamma}\right)^{n} q_{\beta \delta} q_{\beta \eta} \mathrm{x}_{\gamma}^{n} \mathrm{x}_{\delta} \mathrm{x}_{\eta} \otimes x_{\beta}+\left(-q_{\beta \gamma}\right)^{n} q_{\nu \eta}(n+1)_{\widetilde{q}_{\delta \gamma}} \mathrm{b}_{1} \mathrm{x}_{\gamma}^{n+1} \mathrm{x}_{\eta} \otimes x_{\nu}
\end{aligned}
$$

Indeed, for $n=0$ we have:

$$
\begin{gathered}
d\left(\mathrm{x}_{\alpha} \mathrm{x}_{\beta} \mathrm{x}_{\delta} \otimes 1\right)=\mathrm{x}_{\alpha} \mathrm{x}_{\beta} \otimes x_{\delta}-s\left(q_{\beta \delta} \mathrm{x}_{\alpha} \otimes x_{\delta} x_{\beta}+\mathrm{b}_{1} \mathrm{x}_{\alpha} \otimes x_{\gamma} x_{\nu}-q_{\alpha \beta} q_{\alpha \delta} \mathrm{x}_{\beta} \otimes x_{\delta} x_{\alpha}\right) \\
=\mathrm{x}_{\alpha} \mathrm{x}_{\beta} \otimes x_{\delta}-q_{\beta \delta} \mathrm{x}_{\alpha} \mathrm{x}_{\delta} \otimes x_{\beta}-\mathrm{b}_{1} \mathrm{x}_{\alpha} \mathrm{x}_{\gamma} \otimes x_{\nu}-s\left(-q_{\alpha \beta} q_{\alpha \delta} \mathrm{x}_{\beta} \otimes x_{\delta} x_{\alpha}\right. \\
\left.\quad+q_{\alpha \gamma} q_{\alpha \nu} \mathrm{b}_{1} \mathrm{x}_{\gamma} \otimes x_{\nu} x_{\alpha}+q_{\alpha \beta} q_{\beta \delta} q_{\alpha \delta} \mathrm{x}_{\delta} \otimes x_{\beta} x_{\alpha}\right) \\
=\mathrm{x}_{\alpha} \mathrm{x}_{\beta} \otimes x_{\delta}-q_{\beta \delta} \mathrm{x}_{\alpha} \mathrm{x}_{\delta} \otimes x_{\beta}-\mathrm{b}_{1} \mathrm{x}_{\alpha} \mathrm{x}_{\gamma} \otimes x_{\nu}+q_{\alpha \beta} q_{\alpha \delta} \mathrm{x}_{\beta} \mathrm{x}_{\delta} \otimes x_{\alpha} .
\end{gathered}
$$

And for the other equality,

$$
\begin{aligned}
d\left(\mathrm{x}_{\beta} \mathrm{x}_{\delta} \mathrm{x}_{\eta} \otimes 1\right)=\mathrm{x}_{\beta} \mathrm{x}_{\delta} \otimes x_{\eta}-s\left(q_{\delta \eta} \mathrm{x}_{\beta} \otimes x_{\eta} x_{\delta}-q_{\beta \delta} q_{\beta \eta} \mathrm{x}_{\delta} \otimes x_{\eta} x_{\beta}-\mathrm{b}_{1} \mathrm{x}_{\gamma} \otimes x_{\nu} x_{\eta}\right) \\
=\mathrm{x}_{\beta} \mathrm{x}_{\delta} \otimes x_{\eta}-q_{\delta \eta} \mathrm{x}_{\beta} \mathrm{x}_{\eta} \otimes x_{\delta}-s\left(-q_{\beta \delta} q_{\beta \eta} \mathrm{x}_{\delta} \otimes x_{\eta} x_{\beta}-q_{\nu \eta} \mathrm{b}_{1} \mathrm{x}_{\gamma} \otimes x_{\eta} x_{\nu}\right. \\
\left.\quad-\mathrm{b}_{1} \mathrm{~b}_{2} \mathrm{x}_{\gamma} \otimes x_{\mu} x_{\gamma}+q_{\beta \delta} q_{\beta \eta} q_{\delta \eta} \mathrm{x}_{\eta} \otimes x_{\delta} x_{\beta}+q_{\beta \eta} q_{\delta \eta} \mathrm{b}_{1} \mathrm{x}_{\eta} \otimes x_{\gamma} x_{\nu}\right) \\
=\mathrm{x}_{\beta} \mathrm{x}_{\delta} \otimes x_{\eta}-q_{\delta \eta} \mathrm{x}_{\beta} \mathrm{x}_{\eta} \otimes x_{\delta}+\mathrm{b}_{1} \mathrm{~b}_{2} \mathrm{x}_{\gamma} \mathrm{x}_{\mu} \otimes x_{\gamma}+q_{\nu \eta} \mathrm{b}_{1} \mathrm{x}_{\gamma} \mathrm{x}_{\eta} \otimes x_{\nu}+q_{\beta \delta} q_{\beta \eta} \mathrm{x}_{\delta} \mathrm{x}_{\eta} \otimes x_{\beta} .
\end{aligned}
$$

Now assume that (10.1.68) holds for $n$. Using Remark 10.1.2 repeatedly, inductive hypothesis, the relation $x_{\gamma}^{2}=0$ and (10.1.66),

$$
\begin{aligned}
& d\left(\mathrm{x}_{\alpha} \mathrm{x}_{\beta} \mathrm{x}_{\gamma}^{n+1} \mathrm{x}_{\delta} \otimes 1\right)=\mathrm{x}_{\alpha} \mathrm{x}_{\beta} \mathrm{x}_{\gamma}^{n+1} \otimes x_{\delta}-s\left(q_{\gamma \delta} \mathrm{x}_{\alpha} \mathrm{x}_{\beta} \mathrm{x}_{\gamma}^{n} \otimes x_{\delta} x_{\gamma}+\left(-q_{\beta \gamma}\right)^{n+1} \mathrm{~b}_{1} \mathrm{x}_{\alpha} \mathrm{x}_{\gamma}^{n+1} \otimes x_{\gamma} x_{\nu}\right. \\
&\left.\quad+\left(-q_{\beta \gamma}\right)^{n+1} q_{\beta \delta} \mathrm{x}_{\alpha} \mathrm{x}_{\gamma}^{n+1} \otimes x_{\delta} x_{\beta}-q_{\alpha \beta}\left(-q_{\alpha \gamma}\right)^{n+1} q_{\alpha \delta} \mathrm{x}_{\beta} \mathrm{x}_{\gamma}^{n+1} \otimes x_{\delta} x_{\alpha}\right) \\
&=\mathrm{x}_{\alpha} \mathrm{x}_{\beta} \mathrm{x}_{\gamma}^{n+1} \otimes x_{\delta}-q_{\gamma \delta} \mathrm{x}_{\alpha} \mathrm{x}_{\beta} \mathrm{x}_{\gamma}^{n} \mathrm{x}_{\delta} \otimes x_{\gamma}-s\left(\left(-q_{\beta \gamma}\right)^{n+1} q_{\beta \delta} \mathrm{x}_{\alpha} \mathrm{x}_{\gamma}^{n+1} \otimes x_{\delta} x_{\beta}\right. \\
&+\left(-q_{\beta \gamma}\right)^{n+1}\left(1+q_{\gamma \delta} q_{\nu \gamma}\left(-q_{\beta \gamma}\right)^{-1}\left(n+1 \widetilde{q}_{\delta \gamma}\right) \mathrm{b}_{1} \mathrm{x}_{\alpha} \mathrm{x}_{\gamma}^{n+1} \otimes x_{\gamma} x_{\nu}\right. \\
&-q_{\alpha \beta}\left(-q_{\alpha \gamma}\right)^{n+1} q_{\alpha \delta} \mathrm{x}_{\beta} \mathrm{x}_{\gamma}^{n+1} \otimes x_{\delta} x_{\alpha}-\left(-q_{\beta \gamma}\right)^{n+1} q_{\beta \delta} q_{\gamma \delta} \mathrm{x}_{\alpha} \mathrm{x}_{\gamma}^{n} \mathrm{x}_{\delta} \otimes x_{\gamma} x_{\beta} \\
&\left.+q_{\alpha \beta}\left(-q_{\alpha \gamma}\right)^{n+1} q_{\alpha \delta} q_{\gamma \delta} \mathrm{x}_{\beta} \mathrm{x}_{\gamma}^{n} \mathrm{x}_{\delta} \otimes x_{\gamma} x_{\alpha}\right) \\
&= \mathrm{x}_{\alpha} \mathrm{x}_{\beta} \mathrm{x}_{\gamma}^{n+1} \otimes x_{\delta}-q_{\gamma \delta} \mathrm{x}_{\alpha} \mathrm{x}_{\beta} \mathrm{x}_{\gamma}^{n} \mathrm{x}_{\delta} \otimes x_{\gamma}-\left(-q_{\beta \gamma}\right)^{n+1} q_{\beta \delta} \mathrm{x}_{\alpha} \mathrm{x}_{\gamma}^{n+1} \mathrm{x}_{\delta} \otimes x_{\beta} \\
&-\left(-q_{\beta \gamma}\right)^{n+1}(n+2)_{\widetilde{q}_{\delta \gamma}} \mathrm{b}_{1} \mathrm{x}_{\alpha} \mathrm{x}_{\gamma}^{n+2} \otimes x_{\nu}-s\left(-q_{\alpha \beta}\left(-q_{\alpha \gamma}\right)^{n+1} q_{\alpha \delta} \mathrm{x}_{\beta} \mathrm{x}_{\gamma}^{n+1} \otimes x_{\delta} x_{\alpha}\right. \\
&+q_{\alpha \beta}\left(-q_{\alpha \gamma}\right)^{n+1} q_{\alpha \delta} q_{\gamma \delta} \mathrm{x}_{\beta} \mathrm{x}_{\gamma}^{n} \mathrm{x}_{\delta} \otimes x_{\gamma} x_{\alpha}+q_{\alpha \beta} q_{\alpha \gamma}^{n+1} q_{\alpha \delta} q_{\beta \gamma}^{n+1} q_{\beta \delta} \mathrm{x}_{\gamma}^{n+1} \mathrm{x}_{\delta} \otimes x_{\beta} x_{\alpha} \\
&\left.+q_{\alpha \gamma}^{n+2} q_{\beta \gamma}^{n+1}(n+2)_{\widetilde{q}_{\delta \gamma}} \mathrm{b}_{1} \mathrm{x}_{\gamma}^{n+2} \otimes x_{\alpha} x_{\nu}\right) \\
&= \mathrm{x}_{\alpha} \mathrm{x}_{\beta} \mathrm{x}_{\gamma}^{n+1} \otimes x_{\delta}-q_{\gamma \delta} \mathrm{x}_{\alpha} \mathrm{x}_{\beta} \mathrm{x}_{\gamma}^{n} \mathrm{x}_{\delta} \otimes x_{\gamma}-\left(-q_{\beta \gamma}\right)^{n+1} q_{\beta \delta} \mathrm{x}_{\alpha} \mathrm{x}_{\gamma}^{n+1} \mathrm{x}_{\delta} \otimes x_{\beta} \\
&-\left(-q_{\beta \gamma}\right)^{n+1}(n+2)_{\widetilde{q}_{\delta \gamma}} \mathrm{b}_{1} \mathrm{x}_{\alpha} \mathrm{x}_{\gamma}^{n+2} \otimes x_{\nu}+q_{\alpha \beta}\left(-q_{\alpha \gamma}\right)^{n+1} q_{\alpha \delta} \mathrm{x}_{\beta} \mathrm{x}_{\gamma}^{n+1} \mathrm{x}_{\delta} \otimes x_{\alpha} .
\end{aligned}
$$

Next we assume that (10.1.69) holds for $n$. Using (10.1.66), inductive hypothesis, Remark 10.1.2, the relation $x_{\gamma}^{2}=0$,

$$
\begin{aligned}
& d\left(\mathrm{x}_{\beta} \mathrm{x}_{\gamma}^{n+1} \mathrm{x}_{\delta} \mathrm{x}_{\eta} \otimes 1\right)=\mathrm{x}_{\beta} \mathrm{x}_{\gamma}^{n+1} \mathrm{x}_{\delta} \otimes x_{\eta}-s\left(q_{\delta \eta} \mathrm{x}_{\beta} \mathrm{x}_{\gamma}^{n+1} \otimes x_{\eta} x_{\delta}-q_{\gamma \delta} q_{\gamma \eta} \mathrm{x}_{\beta} \mathrm{x}_{\gamma}^{n} \mathrm{x}_{\delta} \otimes x_{\eta} x_{\gamma}\right. \\
& \left.\quad-\left(-q_{\beta \gamma}\right)^{n+1} q_{\beta \delta} q_{\beta \eta} \mathrm{x}_{\gamma}^{n+1} \mathrm{x}_{\delta} \otimes x_{\eta} x_{\beta}-\left(-q_{\beta \gamma}\right)^{n+1}(n+2)_{\widetilde{q}_{\delta \gamma}} \mathrm{b}_{1} \mathrm{x}_{\gamma}^{n+2} \otimes x_{\nu} x_{\eta}\right) \\
& =\mathrm{x}_{\beta} \mathrm{x}_{\gamma}^{n+1} \mathrm{x}_{\delta} \otimes x_{\eta}-q_{\delta \eta} \mathrm{x}_{\beta} \mathrm{x}_{\gamma}^{n+1} \mathrm{x}_{\eta} \otimes x_{\delta}+q_{\gamma \delta} q_{\gamma \eta} \mathrm{x}_{\beta} \mathrm{x}_{\gamma}^{n} \mathrm{x}_{\delta} \mathrm{x}_{\eta} \otimes x_{\gamma}-s( \\
& \quad-\left(-q_{\beta \gamma}\right)^{n+1}(n+2)_{\widetilde{q}_{\delta \gamma}} \mathrm{b}_{1} \mathrm{~b}_{2} \mathrm{x}_{\gamma}^{n+2} \otimes x_{\mu} x_{\gamma}-\left(-q_{\beta \gamma}\right)^{n+1} q_{\nu \eta}(n+2)_{\widetilde{q}_{\delta \gamma}} \mathrm{b}_{1} \mathrm{x}_{\gamma}^{n+2} \otimes x_{\eta} x_{\nu}
\end{aligned}
$$




$$
\begin{aligned}
& -\left(-q_{\beta \gamma}\right)^{n+1} q_{\beta \delta} q_{\beta \eta} \mathrm{x}_{\gamma}^{n+1} \mathrm{x}_{\delta} \otimes x_{\eta} x_{\beta} \\
& +q_{\nu \eta} q_{\gamma \eta}\left(-q_{\beta \gamma}\right)^{n+1}\left(1+\widetilde{q}_{\gamma \delta}(n+1)_{\widetilde{q}_{\delta \gamma}}\right) \mathrm{b}_{1} \mathrm{x}_{\gamma}^{n+1} \mathrm{x}_{\eta} \otimes x_{\gamma} x_{\nu} \\
& \left.+q_{\delta \eta}\left(-q_{\beta \gamma}\right)^{n+1} q_{\beta \delta} q_{\beta \eta} \mathrm{x}_{\gamma}^{n+1} \mathbf{x}_{\eta} \otimes x_{\delta} x_{\beta}-q_{\gamma \delta} q_{\gamma \eta}\left(-q_{\beta \gamma}\right)^{n+1} q_{\beta \delta} q_{\beta \eta} \mathrm{x}_{\gamma}^{n} \mathrm{x}_{\delta} \mathrm{x}_{\eta} \otimes x_{\gamma} x_{\beta}\right) \\
= & \mathrm{x}_{\beta} \mathrm{x}_{\gamma}^{n+1} \mathrm{x}_{\delta} \otimes x_{\eta}-q_{\delta \eta} \mathrm{x}_{\beta} \mathrm{x}_{\gamma}^{n+1} \mathrm{x}_{\eta} \otimes x_{\delta}+q_{\gamma \delta} q_{\gamma \eta} \mathrm{x}_{\beta} \mathrm{x}_{\gamma}^{n} \mathrm{x}_{\delta} \mathrm{x}_{\eta} \otimes x_{\gamma} \\
& +\left(-q_{\beta \gamma}\right)^{n+1}(n+2)_{\widetilde{q}_{\delta \gamma}} \mathrm{b}_{1} \mathrm{~b}_{2} \mathrm{x}_{\gamma}^{n+2} \mathrm{x}_{\mu} \otimes x_{\gamma}+\left(-q_{\beta \gamma}\right)^{n+1} q_{\nu \eta}(n+2)_{\widetilde{q}_{\delta \gamma}} \mathrm{b}_{1} \mathrm{x}_{\gamma}^{n+2} \mathrm{x}_{\eta} \otimes x_{\nu} \\
& -s\left(-\left(-q_{\beta \gamma}\right)^{n+1} q_{\beta \delta} q_{\beta \eta} \mathrm{x}_{\gamma}^{n+1} \mathrm{x}_{\delta} \otimes x_{\eta} x_{\beta}+q_{\delta \eta}\left(-q_{\beta \gamma}\right)^{n+1} q_{\beta \delta} q_{\beta \eta} \mathrm{x}_{\gamma}^{n+1} \mathbf{x}_{\eta} \otimes x_{\delta} x_{\beta}\right. \\
& \left.-q_{\gamma \delta} q_{\gamma \eta}\left(-q_{\beta \gamma}\right)^{n+1} q_{\beta \delta} q_{\beta \eta} \mathrm{x}_{\gamma}^{n} \mathrm{x}_{\delta} \mathrm{x}_{\eta} \otimes x_{\gamma} x_{\beta}\right) \\
= & \mathrm{x}_{\beta} \mathrm{x}_{\gamma}^{n+1} \mathrm{x}_{\delta} \otimes x_{\eta}-q_{\delta \eta} \mathrm{x}_{\beta} \mathrm{x}_{\gamma}^{n+1} \mathrm{x}_{\eta} \otimes x_{\delta}+q_{\gamma \delta} q_{\gamma \eta} \mathrm{x}_{\beta} \mathrm{x}_{\gamma}^{n} \mathrm{x}_{\delta} \mathrm{x}_{\eta} \otimes x_{\gamma} \\
& +\left(-q_{\beta \gamma}\right)^{n+1}(n+2)_{\widetilde{q}_{\delta \gamma}} \mathrm{b}_{1} \mathrm{~b}_{2} \mathrm{x}_{\gamma}^{n+2} \mathrm{x}_{\mu} \otimes x_{\gamma}+\left(-q_{\beta \gamma}\right)^{n+1} q_{\nu \eta}(n+2)_{\widetilde{q}_{\delta \gamma}} \mathrm{b}_{1} \mathrm{x}_{\gamma}^{n+2} \mathrm{x}_{\eta} \otimes x_{\nu} \\
& +\left(-q_{\beta \gamma}\right)^{n+1} q_{\beta \delta} q_{\beta \eta} \mathrm{x}_{\gamma}^{n+1} \mathrm{x}_{\delta} \mathrm{x}_{\eta} \otimes x_{\beta} .
\end{aligned}
$$

Finally we prove (10.1.64) by induction on $n$. When $n=0$,

$$
\begin{aligned}
d\left(\mathrm{x}_{\alpha} \mathrm{x}_{\beta} \mathrm{x}_{\delta} \mathrm{x}_{\eta} \otimes 1\right)=\mathrm{x}_{\alpha} \mathrm{x}_{\beta} \mathrm{x}_{\delta} \otimes x_{\eta}-s\left(q_{\delta \eta} \mathrm{x}_{\alpha} \mathrm{x}_{\beta} \otimes x_{\eta} x_{\delta}-q_{\beta \eta} q_{\beta \delta} \mathrm{x}_{\alpha} \mathrm{x}_{\delta} \otimes x_{\eta} x_{\beta}\right. & \\
& \left.-q_{\nu \eta} \mathrm{b}_{1} \mathrm{x}_{\alpha} \mathrm{x}_{\gamma} \otimes x_{\eta} x_{\nu}-\mathrm{b}_{1} \mathrm{~b}_{2} \mathrm{x}_{\alpha} \mathrm{x}_{\gamma} \otimes x_{\mu} x_{\gamma}+q_{\alpha \beta} q_{\alpha \delta} q_{\alpha \eta} \mathrm{x}_{\beta} \mathrm{x}_{\delta} \otimes x_{\eta} x_{\alpha}\right) \\
= & \mathrm{x}_{\alpha} \mathrm{x}_{\beta} \mathrm{x}_{\delta} \otimes x_{\eta}-q_{\delta \eta} \mathrm{x}_{\alpha} \mathrm{x}_{\beta} \mathrm{x}_{\eta} \otimes x_{\delta}+q_{\beta \eta} q_{\beta \delta} \mathrm{x}_{\alpha} \mathrm{x}_{\delta} \mathrm{x}_{\eta} \otimes x_{\beta}-s\left(-q_{\nu \eta} \mathrm{b}_{1} \mathrm{x}_{\alpha} \mathrm{x}_{\gamma} \otimes x_{\eta} x_{\nu}\right. \\
& -\mathrm{b}_{1} \mathrm{~b}_{2} \mathrm{x}_{\alpha} \mathrm{x}_{\gamma} \otimes x_{\mu} x_{\gamma}+q_{\beta \eta} q_{\delta \eta} \mathrm{b}_{1} \mathrm{x}_{\alpha} \mathrm{x}_{\eta} \otimes x_{\gamma} x_{\nu}+q_{\alpha \beta} q_{\alpha \delta} q_{\alpha \eta} \mathrm{x}_{\beta} \mathrm{x}_{\delta} \otimes x_{\eta} x_{\alpha} \\
& \left.-q_{\alpha \beta} q_{\alpha \eta} q_{\alpha \delta} q_{\delta \eta} \mathrm{x}_{\beta} \mathrm{x}_{\eta} \otimes x_{\delta} x_{\alpha}+q_{\alpha \beta} q_{\alpha \delta} q_{\alpha \eta} q_{\beta \eta} q_{\beta \delta} \mathrm{x}_{\delta} \mathrm{x}_{\eta} \otimes x_{\beta} x_{\alpha}\right) \\
= & \mathrm{x}_{\alpha} \mathrm{x}_{\beta} \mathrm{x}_{\delta} \otimes x_{\eta}-q_{\delta \eta} \mathrm{x}_{\alpha} \mathrm{x}_{\beta} \mathrm{x}_{\eta} \otimes x_{\delta}+q_{\beta \eta} q_{\beta \delta} \mathrm{x}_{\alpha} \mathrm{x}_{\delta} \mathrm{x}_{\eta} \otimes x_{\beta}+q_{\nu \eta} \mathrm{b}_{1} \mathrm{x}_{\alpha} \mathrm{x}_{\gamma} \mathrm{x}_{\eta} \otimes x_{\nu} \\
& +\mathrm{b}_{1} \mathrm{~b}_{2} \mathrm{x}_{\alpha} \mathrm{x}_{\gamma} \mathrm{x}_{\mu} \otimes x_{\gamma}-s\left(q_{\alpha \beta} q_{\alpha \delta} q_{\alpha \eta} \mathrm{x}_{\beta} \mathrm{x}_{\delta} \otimes x_{\eta} x_{\alpha}+q_{\alpha \gamma}(2)_{\widetilde{q}_{\alpha \gamma}} \mathrm{b}_{1} \mathrm{~b}_{2} \mathrm{~b}_{3} \mathrm{x}_{\gamma}^{2} \otimes x_{\gamma}\right. \\
& -q_{\alpha \beta} q_{\alpha \eta} q_{\alpha \delta} q_{\delta \eta} \mathrm{x}_{\beta} \mathrm{x}_{\eta} \otimes x_{\delta} x_{\alpha}+q_{\alpha \beta} q_{\alpha \delta} q_{\alpha \eta} q_{\beta \eta} q_{\beta \delta} \mathrm{x}_{\delta} \mathrm{x}_{\eta} \otimes x_{\beta} x_{\alpha} \\
& \left.+q_{\alpha \gamma} q_{\alpha \eta} q_{\alpha \nu} q_{\nu \eta} \mathrm{b}_{1} \mathrm{x}_{\gamma} \mathrm{x}_{\eta} \otimes x_{\nu} x_{\alpha}+q_{\alpha \mu} q_{\alpha \gamma}^{2} \mathrm{~b}_{1} \mathrm{~b}_{2} \mathrm{x}_{\gamma} \mathrm{x}_{\mu} \otimes x_{\gamma} x_{\alpha}\right) \\
= & \mathrm{x}_{\alpha} \mathrm{x}_{\beta} \mathrm{x}_{\delta} \otimes x_{\eta}-q_{\delta \eta} \mathrm{x}_{\alpha} \mathrm{x}_{\beta} \mathrm{x}_{\eta} \otimes x_{\delta}+q_{\beta \eta} q_{\beta \delta} \mathrm{x}_{\alpha} \mathrm{x}_{\delta} \mathrm{x}_{\eta} \otimes x_{\beta}+q_{\nu \eta} \mathrm{b}_{1} \mathrm{x}_{\alpha} \mathrm{x}_{\gamma} \mathrm{x}_{\eta} \otimes x_{\nu} \\
& +\mathrm{b}_{1} \mathrm{~b}_{2} \mathrm{x}_{\alpha} \mathrm{x}_{\gamma} \mathrm{x}_{\mu} \otimes x_{\gamma}-q_{\alpha \beta} q_{\alpha \delta} q_{\alpha \eta} \mathrm{x}_{\beta} \mathrm{x}_{\delta} \mathrm{x}_{\eta} \otimes x_{\alpha}-q_{\alpha \gamma}(2)_{q_{\alpha \gamma}} \mathrm{b}_{1} \mathrm{~b}_{2} \mathrm{~b}_{3} \mathrm{x}_{\gamma}^{3} \otimes 1,
\end{aligned}
$$

which is (10.1.64) for $n=0$. Now assume that (10.1.64) holds for $n$. Using (10.1.68), Remark 10.1.2, inductive hypothesis, the relation $x_{\gamma}^{2}=0$, (10.1.67), (10.1.69),

$$
\begin{aligned}
d\left(\mathrm{x}_{\alpha} \mathrm{x}_{\beta} \mathrm{x}_{\gamma}^{n+1} \mathrm{x}_{\delta} \mathrm{x}_{\eta} \otimes 1\right)=\mathrm{x}_{\alpha} \mathrm{x}_{\beta} \mathrm{x}_{\gamma}^{n+1} \mathrm{x}_{\delta} \otimes x_{\eta}-s\left(q_{\delta \eta} \mathrm{x}_{\alpha} \mathrm{x}_{\beta} \mathrm{x}_{\gamma}^{n+1} \otimes x_{\eta} x_{\delta}\right. \\
\quad-\left(-q_{\beta \gamma}\right)^{n+1} q_{\beta \delta} q_{\beta \eta} \mathrm{x}_{\alpha} \mathrm{x}_{\gamma}^{n+1} \mathrm{x}_{\delta} \otimes x_{\eta} x_{\beta}+q_{\alpha \beta}\left(-q_{\alpha \gamma}\right)^{n+1} q_{\alpha \delta} q_{\alpha \eta} \mathrm{x}_{\beta} \mathrm{x}_{\gamma}^{n+1} \mathrm{x}_{\delta} \otimes x_{\eta} x_{\alpha} \\
\left.\quad-q_{\gamma \delta} q_{\gamma \eta} \mathrm{x}_{\alpha} \mathrm{x}_{\beta} \mathrm{x}_{\gamma}^{n} \mathrm{x}_{\delta} \otimes x_{\eta} x_{\gamma}-\left(-q_{\beta \gamma}\right)^{n+1}(n+2)_{\widetilde{q}_{\gamma}} \mathrm{b}_{1} \mathrm{x}_{\alpha} \mathrm{x}_{\gamma}^{n+2} \otimes\left(q_{\nu \eta} x_{\eta} x_{\nu}+\mathrm{b}_{2} x_{\mu} x_{\gamma}\right)\right) \\
=\mathrm{x}_{\alpha} \mathrm{x}_{\beta} \mathrm{x}_{\gamma}^{n+1} \mathrm{x}_{\delta} \otimes x_{\eta}-q_{\delta \eta} \mathrm{x}_{\alpha} \mathrm{x}_{\beta} \mathrm{x}_{\gamma}^{n+1} \mathrm{x}_{\eta} \otimes x_{\delta}-s\left(-\left(-q_{\beta \gamma}\right)^{n+1} q_{\beta \delta} q_{\beta \eta} \mathrm{x}_{\alpha} \mathrm{x}_{\gamma}^{n+1} \mathrm{x}_{\delta} \otimes x_{\eta} x_{\beta}\right. \\
\quad+q_{\alpha \beta}\left(-q_{\alpha \gamma}\right)^{n+1} q_{\alpha \delta} q_{\alpha \eta} \mathrm{x}_{\beta} \mathrm{x}_{\gamma}^{n+1} \mathrm{x}_{\delta} \otimes x_{\eta} x_{\alpha}-q_{\gamma \delta} q_{\gamma \eta} \mathrm{x}_{\alpha} \mathrm{x}_{\beta} \mathrm{x}_{\gamma}^{n} \mathrm{x}_{\delta} \otimes x_{\eta} x_{\gamma} \\
\quad-q_{\nu \eta}\left(-q_{\beta \gamma}\right)^{n+1}(n+2)_{\widetilde{q}_{\delta \gamma}} \mathrm{b}_{1} \mathrm{x}_{\alpha} \mathrm{x}_{\gamma}^{n+2} \otimes x_{\eta} x_{\nu}+q_{\gamma \eta} q_{\delta \eta} q_{\gamma \delta} \mathrm{x}_{\alpha} \mathrm{x}_{\beta} \mathrm{x}_{\gamma}^{n} \mathrm{x}_{\eta} \otimes x_{\delta} x_{\gamma} \\
\quad-\left(-q_{\beta \gamma}\right)^{n+1}(n+2)_{\widetilde{q}_{\delta \gamma}} \mathrm{b}_{1} \mathrm{~b}_{2} \mathrm{x}_{\alpha} \mathrm{x}_{\gamma}^{n+2} \otimes x_{\mu} x_{\gamma}-q_{\alpha \beta}\left(-q_{\alpha \gamma}\right)^{n+1} q_{\alpha \delta} q_{\alpha \eta} q_{\delta \eta} \mathrm{x}_{\beta} \mathrm{x}_{\gamma}^{n+1} \mathrm{x}_{\eta} \otimes x_{\delta} x_{\alpha} \\
\left.\quad+\left(-q_{\beta \gamma}\right)^{n+1} q_{\beta \eta} q_{\delta \eta} \mathrm{x}_{\alpha} \mathrm{x}_{\gamma}^{n+1} \mathrm{x}_{\eta} \otimes\left(q_{\beta \delta} x_{\delta} x_{\beta}+\mathrm{b}_{1} x_{\gamma} x_{\nu}\right)\right) \\
=\mathrm{x}_{\alpha} \mathrm{x}_{\beta} \mathrm{x}_{\gamma}^{n+1} \mathrm{x}_{\delta} \otimes x_{\eta}-q_{\delta \eta} \mathrm{x}_{\alpha} \mathrm{x}_{\beta} \mathrm{x}_{\gamma}^{n+1} \mathrm{x}_{\eta} \otimes x_{\delta}+q_{\gamma \delta} q_{\gamma \eta} \mathrm{x}_{\alpha} \mathrm{x}_{\beta} \mathrm{x}_{\gamma}^{n} \mathrm{x}_{\delta} \mathrm{x}_{\eta} \otimes x_{\gamma}
\end{aligned}
$$




$$
\begin{aligned}
& -s\left(q_{\alpha \beta}\left(-q_{\alpha \gamma}\right)^{n+1} q_{\alpha \delta} q_{\alpha \eta} \mathrm{x}_{\beta} \mathrm{x}_{\gamma}^{n+1} \mathrm{x}_{\delta} \otimes x_{\eta} x_{\alpha}-\left(-q_{\beta \gamma}\right)^{n+1}(n+2) \widetilde{q}_{\delta \gamma} \mathrm{b}_{1} \mathrm{~b}_{2} \mathrm{x}_{\alpha} \mathrm{x}_{\gamma}^{n+2} \otimes x_{\mu} x_{\gamma}\right. \\
& -\left(-q_{\beta \gamma}\right)^{n+1} q_{\beta \delta} q_{\beta \eta} \mathrm{x}_{\alpha} \mathrm{x}_{\gamma}^{n+1} \mathrm{x}_{\delta} \otimes x_{\eta} x_{\beta}-q_{\nu \eta}\left(-q_{\beta \gamma}\right)^{n+1}(n+2)_{\widetilde{q}_{\delta \gamma}} \mathrm{b}_{1} \mathrm{x}_{\alpha} \mathrm{x}_{\gamma}^{n+2} \otimes x_{\eta} x_{\nu} \\
& +\left(-q_{\beta \gamma}\right)^{n}\left(q_{\nu \eta}(n+1)_{\widetilde{q}_{\delta \gamma}} q_{\nu \gamma} q_{\gamma \delta} q_{\gamma \eta}-q_{\beta \gamma} q_{\beta \eta} q_{\delta \eta}\right) \mathrm{b}_{1} \mathrm{x}_{\alpha} \mathrm{x}_{\gamma}^{n+1} \mathrm{x}_{\eta} \otimes x_{\gamma} x_{\nu} \\
& -q_{\alpha \beta}\left(-q_{\alpha \gamma}\right)^{n+1} q_{\alpha \delta} q_{\alpha \eta} q_{\delta \eta} \mathrm{x}_{\beta} \mathrm{x}_{\gamma}^{n+1} \mathrm{x}_{\eta} \otimes x_{\delta} x_{\alpha}+\left(-q_{\beta \gamma}\right)^{n+1} q_{\beta \delta} q_{\beta \eta} q_{\delta \eta} \mathrm{x}_{\alpha} \mathrm{x}_{\gamma}^{n+1} \mathrm{x}_{\eta} \otimes x_{\delta} x_{\beta} \\
& -\left(-q_{\beta \gamma}\right)^{n+1} q_{\beta \delta} q_{\beta \eta} q_{\gamma \delta} q_{\gamma \eta} \mathrm{x}_{\alpha} \mathrm{x}_{\gamma}^{n} \mathrm{x}_{\delta} \mathrm{x}_{\eta} \otimes x_{\gamma} x_{\beta}-q_{\alpha \gamma} q_{\gamma \delta}^{n+1} q_{\gamma \eta}^{n+1} \mathrm{~d}_{\alpha+\beta, \delta, \alpha, \gamma}^{(n)} \mathrm{b}_{1} \mathrm{~b}_{2} \mathrm{~b}_{3} \mathrm{x}_{\gamma}^{n+3} \otimes x_{\gamma} \\
& \left.+q_{\alpha \beta}\left(-q_{\alpha \gamma}\right)^{n+1} q_{\alpha \delta} q_{\alpha \eta} q_{\gamma \delta} q_{\gamma \eta} \mathrm{x}_{\beta} \mathrm{x}_{\gamma}^{n} \mathrm{x}_{\delta} \mathrm{x}_{\eta} \otimes x_{\gamma} x_{\alpha}\right) \\
& =\mathrm{x}_{\alpha} \mathrm{x}_{\beta} \mathrm{x}_{\gamma}^{n+1} \mathrm{x}_{\delta} \otimes x_{\eta}-q_{\delta \eta} \mathrm{x}_{\alpha} \mathrm{x}_{\beta} \mathrm{x}_{\gamma}^{n+1} \mathrm{x}_{\eta} \otimes x_{\delta}+q_{\gamma \delta} q_{\gamma \eta} \mathrm{x}_{\alpha} \mathrm{x}_{\beta} \mathrm{x}_{\gamma}^{n} \mathrm{x}_{\delta} \mathrm{x}_{\eta} \otimes x_{\gamma} \\
& +\left(-q_{\beta \gamma}\right)^{n+1}(n+2)_{\widetilde{q}_{\delta \gamma}} \mathrm{b}_{1} \mathrm{~b}_{2} \mathrm{x}_{\alpha} \mathrm{x}_{\gamma}^{n+2} \mathrm{x}_{\mu} \otimes x_{\gamma}+q_{\nu \eta}\left(-q_{\beta \gamma}\right)^{n+1}(n+2)_{\widetilde{q}_{\delta \gamma}} \mathrm{b}_{1} \mathrm{x}_{\alpha} \mathrm{x}_{\gamma}^{n+2} \mathrm{x}_{\eta} \otimes x_{\nu} \\
& +\left(-q_{\beta \gamma}\right)^{n+1} q_{\beta \delta} q_{\beta \eta} \mathrm{x}_{\alpha} \mathrm{x}_{\gamma}^{n+1} \mathrm{x}_{\delta} \mathrm{x}_{\eta} \otimes x_{\beta}-s\left(-q_{\alpha \beta}\left(-q_{\alpha \gamma}\right)^{n+1} q_{\alpha \delta} q_{\alpha \eta} q_{\delta \eta} \mathrm{x}_{\beta} \mathrm{x}_{\gamma}^{n+1} \mathrm{x}_{\eta} \otimes x_{\delta} x_{\alpha}\right. \\
& +q_{\alpha \beta}\left(-q_{\alpha \gamma}\right)^{n+1} q_{\alpha \delta} q_{\alpha \eta} \mathbf{x}_{\beta} \mathbf{x}_{\gamma}^{n+1} \mathbf{x}_{\delta} \otimes x_{\eta} x_{\alpha}+q_{\alpha \beta} q_{\alpha \gamma}^{n+1} q_{\alpha \delta} q_{\alpha \eta} q_{\beta \gamma}^{n+1} q_{\beta \delta} q_{\beta \eta} \mathbf{x}_{\gamma}^{n+1} \mathbf{x}_{\delta} \mathbf{x}_{\eta} \otimes x_{\beta} x_{\alpha} \\
& +q_{\alpha \beta}\left(-q_{\alpha \gamma}\right)^{n+1} q_{\alpha \delta} q_{\alpha \eta} q_{\gamma \delta} q_{\gamma \eta} \mathrm{x}_{\beta} \mathrm{x}_{\gamma}^{n} \mathrm{x}_{\delta} \mathrm{x}_{\eta} \otimes x_{\gamma} x_{\alpha} \\
& -q_{\alpha \gamma} q_{\gamma \delta}^{n+1} q_{\gamma \eta}^{n+1}\left(\widetilde{q}_{\alpha \gamma}^{n+1} \widetilde{q}_{\beta \gamma}^{n+1}(n+2)_{\widetilde{q}_{\delta \gamma}}(n+3)_{\widetilde{q}_{\alpha \gamma}}+\mathrm{d}_{\alpha+\beta, \delta, \alpha, \gamma}^{(n)}\right) \mathrm{b}_{1} \mathrm{~b}_{2} \mathrm{~b}_{3} \mathrm{x}_{\gamma}^{n+3} \otimes x_{\gamma} \\
& +q_{\alpha \mu} q_{\alpha \gamma}^{n+3} q_{\beta \gamma}^{n+1}(n+2)_{\widetilde{q}_{\delta \gamma}} \mathrm{b}_{1} \mathrm{~b}_{2} \mathrm{x}_{\gamma}^{n+2} \mathrm{x}_{\mu} \otimes x_{\gamma} x_{\alpha} \\
& \left.+q_{\alpha \nu} q_{\alpha \gamma}^{n+2} q_{\alpha \eta} q_{\nu \eta} q_{\beta \gamma}^{n+1}(n+2)_{\widetilde{q}_{\delta \gamma}} \mathrm{b}_{1} \mathrm{x}_{\gamma}^{n+2} \mathrm{x}_{\eta} \otimes x_{\nu} x_{\alpha}\right) \\
& =\mathrm{x}_{\alpha} \mathrm{x}_{\beta} \mathrm{x}_{\gamma}^{n+1} \mathrm{x}_{\delta} \otimes x_{\eta}-q_{\delta \eta} \mathrm{x}_{\alpha} \mathrm{x}_{\beta} \mathrm{x}_{\gamma}^{n+1} \mathrm{x}_{\eta} \otimes x_{\delta}+q_{\gamma \delta} q_{\gamma \eta} \mathrm{x}_{\alpha} \mathrm{x}_{\beta} \mathrm{x}_{\gamma}^{n} \mathrm{x}_{\delta} \mathrm{x}_{\eta} \otimes x_{\gamma} \\
& +\left(-q_{\beta \gamma}\right)^{n+1}(n+2)_{\widetilde{q}_{\delta \gamma}} \mathrm{b}_{1} \mathrm{~b}_{2} \mathrm{x}_{\alpha} \mathrm{x}_{\gamma}^{n+2} \mathrm{x}_{\mu} \otimes x_{\gamma}+q_{\nu \eta}\left(-q_{\beta \gamma}\right)^{n+1}(n+2)_{\widetilde{q}_{\delta \gamma}} \mathrm{b}_{1} \mathrm{x}_{\alpha} \mathrm{x}_{\gamma}^{n+2} \mathrm{x}_{\eta} \otimes x_{\nu} \\
& +\left(-q_{\beta \gamma}\right)^{n+1} q_{\beta \delta} q_{\beta \eta} \mathrm{x}_{\alpha} \mathrm{x}_{\gamma}^{n+1} \mathrm{x}_{\delta} \mathrm{x}_{\eta} \otimes x_{\beta}-q_{\alpha \beta}\left(-q_{\alpha \gamma}\right)^{n+1} q_{\alpha \delta} q_{\alpha \eta} \mathrm{x}_{\beta} \mathrm{x}_{\gamma}^{n+1} \mathrm{x}_{\delta} \mathrm{x}_{\eta} \otimes x_{\alpha} \\
& -q_{\alpha \gamma} q_{\gamma \delta}^{n+1} q_{\gamma \eta}^{n+1} \mathrm{~d}_{\alpha+\beta, \delta, \alpha, \gamma}^{(n+1)} \mathrm{b}_{1} \mathrm{~b}_{2} \mathrm{~b}_{3} s\left(\mathrm{x}_{\gamma}^{n+3} \otimes x_{\gamma}\right),
\end{aligned}
$$

and the inductive step follows.

Lemma 10.1.70. Let $\alpha<\beta<\delta<\gamma<\mu<\nu<\eta$ be positive roots such that $N_{\gamma}=2$ and the relations among the corresponding root vectors take the form

$$
\begin{aligned}
& x_{\beta} x_{\eta}=q_{\beta \eta} x_{\eta} x_{\beta}+\mathrm{b}_{1} x_{\nu} x_{\gamma}, \\
& x_{\delta} x_{\nu}=q_{\delta \nu} x_{\nu} x_{\delta}+\mathrm{b}_{2} x_{\mu} x_{\gamma}, \quad x_{\alpha} x_{\mu}=q_{\alpha \mu} x_{\mu} x_{\alpha}+\mathrm{b}_{3} x_{\gamma},
\end{aligned}
$$

for some scalars $\mathrm{b}_{i}$ and all other pairs of root vectors q-commute except possibly $\left(x_{\beta}, x_{\mu}\right)$. Then, for all $n \geq 0$,

$$
\begin{aligned}
& d\left(\mathrm{x}_{\alpha} \mathrm{x}_{\beta} \mathrm{x}_{\delta} \mathrm{x}_{\gamma}^{n} \mathrm{x}_{\eta} \otimes 1\right)=\mathrm{x}_{\alpha} \mathrm{x}_{\beta} \mathrm{x}_{\delta} \mathrm{x}_{\gamma}^{n} \otimes x_{\eta}-q_{\gamma \eta} \mathrm{x}_{\alpha} \mathrm{x}_{\beta} \mathrm{x}_{\delta} \mathrm{x}_{\gamma}^{n-1} \mathrm{x}_{\eta} \otimes x_{\gamma} \\
& -\left(-q_{\delta \gamma}\right)^{n} q_{\delta \eta} \mathrm{x}_{\alpha} \mathrm{x}_{\beta} \mathrm{x}_{\gamma}^{n} \mathrm{x}_{\eta} \otimes x_{\delta}+q_{\beta \delta}\left(-q_{\beta \gamma}\right)^{n} q_{\beta \eta} \mathrm{x}_{\alpha} \mathrm{x}_{\delta} \mathrm{x}_{\gamma}^{n} \mathrm{x}_{\eta} \otimes x_{\beta} \\
& +q_{\beta \delta}\left(-q_{\beta \gamma}\right)^{n} \mathrm{~b}_{1} \mathrm{x}_{\alpha} \mathrm{x}_{\delta} \mathrm{x}_{\gamma}^{n} \mathrm{x}_{\nu} \otimes x_{\gamma}-q_{\alpha \beta} q_{\alpha \delta}\left(-q_{\alpha \gamma}\right)^{n} q_{\alpha \eta} \mathrm{x}_{\beta} \mathrm{x}_{\delta} \mathrm{x}_{\gamma}^{n} \mathrm{x}_{\eta} \otimes x_{\alpha} \\
& +q_{\beta \delta} q_{\gamma \eta}^{n} \mathrm{~d}_{\beta-\nu \alpha \gamma}^{(n)} \mathrm{b}_{1} \mathrm{~b}_{2} \mathrm{~b}_{3} \mathrm{x}_{\gamma}^{n+3} \otimes 1 \text {. }
\end{aligned}
$$

Notice that the equalities in (10.1.71) force

$$
\beta+\eta=\gamma+\nu, \quad \delta+\nu=\gamma+\mu, \quad \alpha+\mu=\gamma .
$$

Hence the following equality also holds: $3 \gamma=\alpha+\beta+\delta+\eta$. 
Proof. We need some auxiliary computations. By (10.1.60),

$$
\begin{aligned}
& d\left(\mathrm{x}_{\beta} \mathrm{x}_{\gamma}^{n} \mathrm{x}_{\eta} \otimes 1\right)=\mathrm{x}_{\beta} \mathrm{x}_{\gamma}^{n} \otimes x_{\eta}-q_{\gamma \eta} \mathrm{x}_{\beta} \mathrm{x}_{\gamma}^{n-1} \mathrm{x}_{\eta} \otimes x_{\gamma}-\left(-q_{\beta \gamma}\right)^{n} q_{\beta \eta} \mathrm{x}_{\gamma}^{n} \mathrm{x}_{\eta} \otimes x_{\beta} \\
& \quad-\left(-q_{\beta \gamma}\right)^{n} \mathrm{~b}_{1} \mathrm{x}_{\gamma}^{n} \mathrm{x}_{\nu} \otimes x_{\gamma}, \\
& d\left(\mathrm{x}_{\delta} \mathrm{x}_{\gamma}^{n} \mathrm{x}_{\nu} \otimes 1\right)=\mathrm{x}_{\delta} \mathrm{x}_{\gamma}^{n} \otimes x_{\nu}-q_{\gamma \nu} \mathrm{x}_{\delta} \mathrm{x}_{\gamma}^{n-1} \mathrm{x}_{\nu} \otimes x_{\gamma}-\left(-q_{\delta \gamma}\right)^{n} q_{\delta \nu} \mathrm{x}_{\gamma}^{n} \mathrm{x}_{\nu} \otimes x_{\delta} \\
& \quad-\left(-q_{\delta \gamma}\right)^{n} \mathrm{~b}_{2} \mathrm{x}_{\gamma}^{n} \mathrm{x}_{\mu} \otimes x_{\gamma} .
\end{aligned}
$$

Now we prove the following equality by induction on $n$ :

$$
\begin{aligned}
& d\left(\mathrm{x}_{\alpha} \mathrm{x}_{\beta} \mathrm{x}_{\gamma}^{n} \mathrm{x}_{\eta} \otimes 1\right)=\mathrm{x}_{\alpha} \mathrm{x}_{\beta} \mathrm{x}_{\gamma}^{n} \otimes x_{\eta}-q_{\gamma \eta} \mathrm{x}_{\alpha} \mathrm{x}_{\beta} \mathrm{x}_{\gamma}^{n-1} \mathrm{x}_{\eta} \otimes x_{\gamma}-\left(-q_{\beta \gamma}\right)^{n} \mathrm{~b}_{1} \mathrm{x}_{\alpha} \mathrm{x}_{\gamma}^{n} \mathrm{x}_{\nu} \otimes x_{\gamma} \\
& -\left(-q_{\beta \gamma}\right)^{n} q_{\beta \eta} \mathrm{x}_{\alpha} \mathrm{x}_{\gamma}^{n} \mathrm{x}_{\eta} \otimes x_{\beta}+q_{\alpha \beta}\left(-q_{\alpha \gamma}\right)^{n} q_{\alpha \eta} \mathrm{x}_{\beta} \mathrm{x}_{\gamma}^{n} \mathrm{x}_{\eta} \otimes x_{\alpha} .
\end{aligned}
$$

Indeed for $n=0$ we have:

$$
\begin{aligned}
& d\left(\mathrm{x}_{\alpha} \mathrm{x}_{\beta} \mathrm{x}_{\eta} \otimes 1\right)=\mathrm{x}_{\alpha} \mathrm{x}_{\beta} \otimes x_{\eta}-s\left(q_{\beta \eta} \mathrm{x}_{\alpha} \otimes x_{\eta} x_{\beta}+\mathrm{b}_{1} \mathrm{x}_{\alpha} \otimes x_{\nu} x_{\gamma}-q_{\alpha \beta} q_{\alpha \beta} \mathrm{x}_{\beta} \otimes x_{\eta} x_{\alpha}\right) \\
& \quad=\mathrm{x}_{\alpha} \mathrm{x}_{\beta} \otimes x_{\eta}-\mathrm{b}_{1} \mathrm{x}_{\alpha} \mathrm{x}_{\nu} \otimes x_{\gamma}-q_{\beta \eta} \mathrm{x}_{\alpha} \mathrm{x}_{\eta} \otimes x_{\beta}+q_{\alpha \beta} q_{\alpha \eta} \mathrm{x}_{\beta} \mathrm{x}_{\eta} \otimes x_{\alpha} .
\end{aligned}
$$

Now assume that (10.1.76) holds for $n$. By Remark 10.1.2, inductive hypothesis and (10.1.74),

$$
\begin{aligned}
& d\left(\mathrm{x}_{\alpha} \mathrm{x}_{\beta} \mathrm{x}_{\gamma}^{n+1} \mathrm{x}_{\eta} \otimes 1\right)=\mathrm{x}_{\alpha} \mathrm{x}_{\beta} \mathrm{x}_{\gamma}^{n+1} \otimes x_{\eta}-s\left(q_{\gamma \eta} \mathrm{x}_{\alpha} \mathrm{x}_{\beta} \mathrm{x}_{\gamma}^{n} \otimes x_{\eta} x_{\gamma}\right. \\
& \left.\quad+\left(-q_{\beta \gamma}\right)^{n+1} \mathrm{x}_{\alpha} \mathrm{x}_{\gamma}^{n+1} \otimes\left(q_{\beta \eta} x_{\eta} x_{\beta}+\mathrm{b}_{1} x_{\nu} x_{\gamma}\right)-q_{\alpha \beta}\left(-q_{\alpha \gamma}\right)^{n+1} q_{\alpha \eta} \mathrm{x}_{\beta} \mathrm{x}_{\gamma}^{n+1} \otimes x_{\eta} x_{\alpha}\right) \\
& =\mathrm{x}_{\alpha} \mathrm{x}_{\beta} \mathrm{x}_{\gamma}^{n+1} \otimes x_{\eta}-q_{\gamma \eta} \mathrm{x}_{\alpha} \mathrm{x}_{\beta} \mathrm{x}_{\gamma}^{n} \mathrm{x}_{\eta} \otimes x_{\gamma}-\left(-q_{\beta \gamma}\right)^{n+1} \mathrm{~b}_{1} \mathrm{x}_{\alpha} \mathrm{x}_{\gamma}^{n+1} \mathrm{x}_{\nu} \otimes x_{\gamma} \\
& \quad-\left(-q_{\beta \gamma}\right)^{n+1} q_{\beta \eta} \mathrm{x}_{\alpha} \mathrm{x}_{\gamma}^{n+1} \mathrm{x}_{\eta} \otimes x_{\beta}+q_{\alpha \beta}\left(-q_{\alpha \gamma}\right)^{n+1} q_{\alpha \eta} \mathrm{x}_{\beta} \mathrm{x}_{\gamma}^{n+1} \mathrm{x}_{\eta} \otimes x_{\alpha} .
\end{aligned}
$$

Next we apply Lemma 10.1 .48 to $\alpha<\delta<\gamma<\mu<\nu$ to get:

$$
\begin{gathered}
d\left(\mathrm{x}_{\alpha} \mathrm{x}_{\delta} \mathrm{x}_{\gamma}^{n} \mathrm{x}_{\nu} \otimes 1\right)=\mathrm{x}_{\alpha} \mathrm{x}_{\delta} \mathrm{x}_{\gamma}^{n} \otimes x_{\nu}-q_{\gamma \nu} \mathrm{x}_{\alpha} \mathrm{x}_{\delta} \mathrm{x}_{\gamma}^{n-1} \mathrm{x}_{\nu} \otimes x_{\gamma} \\
-\left(-q_{\delta \gamma}\right)^{n} q_{\delta \nu} \mathrm{x}_{\alpha} \mathrm{x}_{\gamma}^{n} \mathrm{x}_{\nu} \otimes x_{\delta}+\left(-q_{\alpha \gamma}\right)^{n} q_{\alpha \delta} q_{\alpha \nu} \mathrm{x}_{\delta} \mathrm{x}_{\gamma}^{n} \mathrm{x}_{\nu} \otimes x_{\alpha} \\
-\left(-q_{\delta \gamma}\right)^{n} \mathrm{~b}_{2} \mathrm{x}_{\alpha} \mathrm{x}_{\gamma}^{n} \mathrm{x}_{\mu} \otimes x_{\gamma}-q_{\gamma \nu}^{n} \mathrm{c}_{-\nu, \alpha, \gamma}^{(n)} \mathrm{b}_{2} \mathrm{~b}_{3} \mathrm{x}_{\gamma}^{n+2} \otimes 1 .
\end{gathered}
$$

Now we prove by induction on $n$ that

$$
\begin{aligned}
& d\left(\mathrm{x}_{\beta} \mathrm{x}_{\delta} \mathrm{x}_{\gamma}^{n} \mathrm{x}_{\eta} \otimes 1\right)=\mathrm{x}_{\beta} \mathrm{x}_{\delta} \mathrm{x}_{\gamma}^{n} \otimes x_{\eta}-q_{\gamma \eta} \mathrm{x}_{\beta} \mathrm{x}_{\delta} \mathrm{x}_{\gamma}^{n-1} \mathrm{x}_{\eta} \otimes x_{\gamma}-\left(-q_{\delta \gamma}\right)^{n} q_{\delta \eta} \mathrm{x}_{\beta} \mathrm{x}_{\gamma}^{n} \mathrm{x}_{\eta} \otimes x_{\delta} \\
& \quad+\left(-q_{\beta \gamma}\right)^{n} q_{\beta \delta} q_{\beta \eta} \mathrm{x}_{\delta} \mathrm{x}_{\gamma}^{n} \mathrm{x}_{\eta} \otimes x_{\beta}+q_{\beta \delta}\left(-q_{\beta \gamma}\right)^{n} \mathrm{~b}_{1} \mathrm{x}_{\delta} \mathrm{x}_{\gamma}^{n} \mathrm{x}_{\nu} \otimes x_{\gamma} .
\end{aligned}
$$

Indeed, for $n=0$ we have

$$
\begin{aligned}
& d\left(\mathrm{x}_{\beta} \mathrm{x}_{\delta} \mathrm{x}_{\eta} \otimes 1\right)=\mathrm{x}_{\beta} \mathrm{x}_{\delta} \otimes x_{\eta}-s\left(q_{\delta \eta} \mathrm{x}_{\beta} \otimes x_{\eta} x_{\delta}-q_{\beta \delta} q_{\beta \eta} \mathrm{x}_{\delta} \otimes x_{\eta} x_{\beta}-q_{\beta \delta} \mathrm{b}_{1} \mathrm{x}_{\delta} \otimes x_{\nu} x_{\gamma}\right) \\
& \quad=\mathrm{x}_{\beta} \mathrm{x}_{\delta} \otimes x_{\eta}-q_{\delta \eta} \mathrm{x}_{\beta} \mathrm{x}_{\eta} \otimes x_{\delta}+q_{\beta \delta} q_{\beta \eta} \mathrm{x}_{\delta} \mathrm{x}_{\eta} \otimes x_{\beta}+q_{\beta \delta} \mathrm{b}_{1} \mathrm{x}_{\delta} \mathrm{x}_{\nu} \otimes x_{\gamma} .
\end{aligned}
$$

Now we assume that (10.1.78) holds for $n$. Using Remark 10.1.2, inductive hypothesis, (10.1.74), (10.1.75),

$$
\begin{aligned}
& d\left(\mathrm{x}_{\beta} \mathrm{x}_{\delta} \mathrm{x}_{\gamma}^{n+1} \mathrm{x}_{\eta} \otimes 1\right)=\mathrm{x}_{\beta} \mathrm{x}_{\delta} \mathrm{x}_{\gamma}^{n+1} \otimes x_{\eta}-s\left(q_{\gamma \eta} \mathrm{x}_{\beta} \mathrm{x}_{\delta} \mathrm{x}_{\gamma}^{n} \otimes x_{\eta} x_{\gamma}+\left(-q_{\delta \gamma}\right)^{n+1} q_{\delta \eta} \mathrm{x}_{\beta} \mathrm{x}_{\gamma}^{n+1} \otimes x_{\eta} x_{\delta}\right. \\
& \left.-q_{\beta \delta}\left(-q_{\beta \gamma}\right)^{n+1} q_{\beta \eta} \mathrm{x}_{\delta} \mathrm{x}_{\gamma}^{n+1} \otimes x_{\eta} x_{\beta}-q_{\beta \delta}\left(-q_{\beta \gamma}\right)^{n+1} \mathrm{~b}_{1} \mathrm{x}_{\delta} \mathrm{x}_{\gamma}^{n+1} \otimes x_{\nu} x_{\gamma}\right) \\
& =\mathrm{x}_{\beta} \mathrm{x}_{\delta} \mathrm{x}_{\gamma}^{n+1} \otimes x_{\eta}-q_{\gamma \eta} \mathbf{x}_{\beta} \mathbf{x}_{\delta} \mathbf{x}_{\gamma}^{n} \mathbf{x}_{\eta} \otimes x_{\gamma}-\left(-q_{\delta \gamma}\right)^{n+1} q_{\delta \eta} \mathbf{x}_{\beta} \mathbf{x}_{\gamma}^{n+1} \mathbf{x}_{\eta} \otimes x_{\delta}
\end{aligned}
$$




$$
\begin{aligned}
& +q_{\beta \delta}\left(-q_{\beta \gamma}\right)^{n+1} \mathrm{~b}_{1} \mathrm{x}_{\delta} \mathrm{x}_{\gamma}^{n+1} \mathrm{x}_{\nu} \otimes x_{\gamma}+q_{\beta \delta}\left(-q_{\beta \gamma}\right)^{n+1} q_{\beta \eta} \mathrm{x}_{\delta} \mathrm{x}_{\gamma}^{n+1} \mathrm{x}_{\eta} \otimes x_{\beta} \\
& -q_{\beta \gamma}^{n+1} q_{\delta \gamma}^{n+1} q_{\delta \eta}\left(1-\widetilde{q}_{\beta \delta}\right) \mathrm{b}_{1} s\left(\mathrm{x}_{\gamma}^{n+1} \mathrm{x}_{\nu} \otimes x_{\gamma} x_{\delta}\right),
\end{aligned}
$$

and the inductive step follows since $\mathrm{x}_{\gamma}^{n+1} \mathbf{x}_{\nu} \otimes x_{\gamma} x_{\delta}=s\left(\mathrm{x}_{\gamma}^{n+1} \otimes x_{\nu} x_{\gamma} x_{\delta}\right)$.

Finally we prove (10.1.72) by induction on $n$. When $n=0$,

$$
\begin{aligned}
& d\left(\mathrm{x}_{\alpha} \mathrm{x}_{\beta} \mathrm{x}_{\delta} \mathrm{x}_{\eta} \otimes 1\right)=\mathrm{x}_{\alpha} \mathrm{x}_{\beta} \mathrm{x}_{\delta} \otimes x_{\eta}-s\left(q_{\delta \eta} \mathrm{x}_{\alpha} \mathrm{x}_{\beta} \otimes x_{\eta} x_{\delta}-q_{\beta \delta} q_{\beta \eta} \mathrm{x}_{\alpha} \mathrm{x}_{\delta} \otimes x_{\eta} x_{\beta}\right. \\
& \left.\quad-q_{\beta \delta} \mathrm{b}_{1} \mathrm{x}_{\alpha} \mathrm{x}_{\delta} \otimes x_{\nu} x_{\gamma}+q_{\alpha \beta} q_{\alpha \delta} q_{\alpha \eta} \mathrm{x}_{\beta} \mathrm{x}_{\delta} \otimes x_{\eta} x_{\alpha}\right) \\
& =\mathrm{x}_{\alpha} \mathrm{x}_{\beta} \mathrm{x}_{\delta} \otimes x_{\eta}-q_{\delta \eta} \mathrm{x}_{\alpha} \mathrm{x}_{\beta} \mathrm{x}_{\eta} \otimes x_{\delta}+q_{\beta \delta} q_{\beta \eta} \mathrm{x}_{\alpha} \mathrm{x}_{\delta} \mathrm{x}_{\eta} \otimes x_{\beta}+q_{\beta \delta} \mathrm{b}_{1} \mathrm{x}_{\alpha} \mathrm{x}_{\delta} \mathrm{x}_{\nu} \otimes x_{\gamma} \\
& \quad-q_{\alpha \beta} q_{\alpha \delta} q_{\alpha \eta} \mathrm{x}_{\beta} \mathrm{x}_{\delta} \mathrm{x}_{\eta} \otimes x_{\alpha}+q_{\beta \delta} \mathrm{b}_{1} \mathrm{~b}_{2} \mathrm{~b}_{3} \mathrm{x}_{\gamma}^{3} \otimes 1 .
\end{aligned}
$$

Now assume that (10.1.72) holds for $n$. Using Remark 10.1.2, inductive hypothesis, (10.1.76), (10.1.77) and (10.1.78)

$$
\begin{aligned}
& d\left(\mathrm{x}_{\alpha} \mathrm{x}_{\beta} \mathrm{x}_{\delta} \mathrm{x}_{\gamma}^{n+1} \mathrm{x}_{\eta} \otimes 1\right)=\mathrm{x}_{\alpha} \mathrm{x}_{\beta} \mathrm{x}_{\delta} \mathrm{x}_{\gamma}^{n+1} \otimes x_{\eta}-s\left(q_{\gamma \eta} \mathrm{x}_{\alpha} \mathrm{x}_{\beta} \mathrm{x}_{\delta} \mathrm{x}_{\gamma}^{n} \otimes x_{\eta} x_{\gamma}\right. \\
& +\left(-q_{\delta \gamma}\right)^{n+1} q_{\delta \eta} \mathrm{x}_{\alpha} \mathrm{x}_{\beta} \mathrm{x}_{\gamma}^{n+1} \otimes x_{\eta} x_{\delta}-q_{\beta \delta}\left(-q_{\beta \gamma}\right)^{n+1} q_{\beta \eta} \mathrm{x}_{\alpha} \mathrm{x}_{\delta} \mathrm{x}_{\gamma}^{n+1} \otimes x_{\eta} x_{\beta} \\
& \left.-q_{\beta \delta}\left(-q_{\beta \gamma}\right)^{n+1} \mathrm{~b}_{1} \mathrm{x}_{\alpha} \mathrm{x}_{\delta} \mathrm{x}_{\gamma}^{n+1} \otimes x_{\nu} x_{\gamma}+q_{\alpha \beta} q_{\alpha \delta}\left(-q_{\alpha \gamma}\right)^{n+1} q_{\alpha \eta} \mathrm{x}_{\beta} \mathrm{x}_{\delta} \mathrm{x}_{\gamma}^{n+1} \otimes x_{\eta} x_{\alpha}\right) \\
& =\mathrm{x}_{\alpha} \mathrm{x}_{\beta} \mathrm{x}_{\delta} \mathrm{x}_{\gamma}^{n+1} \otimes x_{\eta}-q_{\gamma \eta} \mathrm{x}_{\alpha} \mathrm{x}_{\beta} \mathrm{x}_{\delta} \mathrm{x}_{\gamma}^{n} \mathrm{x}_{\eta} \otimes x_{\gamma}-\left(-q_{\delta \gamma}\right)^{n+1} q_{\delta \eta} \mathrm{x}_{\alpha} \mathrm{x}_{\beta} \mathrm{x}_{\gamma}^{n+1} \mathrm{x}_{\eta} \otimes x_{\delta} \\
& +q_{\beta \delta}\left(-q_{\beta \gamma}\right)^{n+1} \mathbf{b}_{1} \mathbf{x}_{\alpha} \mathbf{x}_{\delta} \mathbf{x}_{\gamma}^{n+1} \mathbf{x}_{\nu} \otimes x_{\gamma}+q_{\beta \delta}\left(-q_{\beta \gamma}\right)^{n+1} q_{\beta \eta} \mathbf{x}_{\alpha} \mathbf{x}_{\delta} \mathbf{x}_{\gamma}^{n+1} \mathbf{x}_{\eta} \otimes x_{\beta} \\
& -s\left(q_{\alpha \beta} q_{\alpha \delta}\left(-q_{\alpha \gamma}\right)^{n+1} q_{\alpha \eta} \mathbf{x}_{\beta} \mathrm{x}_{\delta} \mathrm{x}_{\gamma}^{n+1} \otimes x_{\eta} x_{\alpha}-q_{\alpha \beta} q_{\alpha \delta} q_{\alpha \gamma}^{n+1} q_{\alpha \eta} q_{\delta \gamma}^{n+1} q_{\delta \eta} \mathrm{x}_{\beta} \mathrm{x}_{\gamma}^{n+1} \mathbf{x}_{\eta} \otimes x_{\delta} x_{\alpha}\right. \\
& -q_{\alpha \beta} q_{\alpha \delta}\left(-q_{\alpha \gamma}\right)^{n+1} q_{\alpha \eta} q_{\gamma \eta} \mathrm{x}_{\beta} \mathrm{x}_{\delta} \mathrm{x}_{\gamma}^{n} \mathrm{x}_{\eta} \otimes x_{\gamma} x_{\alpha}+q_{\beta \delta} q_{\beta \gamma}^{n+1} q_{\alpha \gamma}^{n+2} q_{\alpha \delta} q_{\alpha \nu} \mathrm{b}_{1} \mathrm{x}_{\delta} \mathrm{x}_{\gamma}^{n+1} \mathrm{x}_{\nu} \otimes x_{\gamma} x_{\alpha} \\
& -q_{\beta \delta}\left(q_{\gamma \eta}^{n+1} \mathrm{~d}_{\beta-\nu \alpha \gamma}^{(n)}+q_{\gamma \nu}^{n+1} \mathrm{c}_{-\nu, \alpha, \gamma}^{(n+1)}\left(-q_{\beta \gamma}\right)^{n+1}\right) \mathrm{b}_{1} \mathrm{~b}_{2} \mathrm{~b}_{3} \mathrm{x}_{\gamma}^{n+3} \otimes x_{\gamma} \\
& \left.+q_{\beta \delta} q_{\beta \gamma}^{n+1} q_{\beta \eta} q_{\alpha \beta} q_{\alpha \delta} q_{\alpha \gamma}^{n+1} q_{\alpha \eta} \mathrm{x}_{\delta} \mathrm{x}_{\gamma}^{n+1} \mathrm{x}_{\eta} \otimes x_{\beta} x_{\alpha}\right) \\
& =\mathrm{x}_{\alpha} \mathrm{x}_{\beta} \mathrm{x}_{\delta} \mathrm{x}_{\gamma}^{n+1} \otimes x_{\eta}-q_{\gamma \eta} \mathrm{x}_{\alpha} \mathrm{x}_{\beta} \mathrm{x}_{\delta} \mathrm{x}_{\gamma}^{n} \mathrm{x}_{\eta} \otimes x_{\gamma}-\left(-q_{\delta \gamma}\right)^{n+1} q_{\delta \eta} \mathrm{x}_{\alpha} \mathrm{x}_{\beta} \mathrm{x}_{\gamma}^{n+1} \mathrm{x}_{\eta} \otimes x_{\delta} \\
& +q_{\beta \delta}\left(-q_{\beta \gamma}\right)^{n+1} \mathrm{~b}_{1} \mathrm{x}_{\alpha} \mathrm{x}_{\delta} \mathrm{x}_{\gamma}^{n+1} \mathbf{x}_{\nu} \otimes x_{\gamma}+q_{\beta \delta}\left(-q_{\beta \gamma}\right)^{n+1} q_{\beta \eta} \mathrm{x}_{\alpha} \mathrm{x}_{\delta} \mathbf{x}_{\gamma}^{n+1} \mathbf{x}_{\eta} \otimes x_{\beta} \\
& -q_{\alpha \beta} q_{\alpha \delta}\left(-q_{\alpha \gamma}\right)^{n+1} q_{\alpha \eta} \mathrm{x}_{\beta} \mathrm{x}_{\delta} \mathrm{x}_{\gamma}^{n+1} \mathrm{x}_{\eta} \otimes x_{\alpha}+q_{\beta \delta} q_{\gamma \eta}^{n+1} \mathbf{d}_{\beta-\nu \alpha \gamma}^{(n+1)} \mathrm{b}_{1} \mathrm{~b}_{2} \mathrm{~b}_{3} \mathrm{x}_{\gamma}^{n+4} \otimes 1,
\end{aligned}
$$

and the inductive step follows.

Lemma 10.1.79. Let $\alpha<\beta<\tau<\delta<\mu<\nu<\gamma<\kappa<\iota<\eta$ be positive roots such that $N_{\gamma}=N_{\eta}=N_{\kappa}=2$ and the relations among the corresponding root vectors take the form

$$
\begin{array}{ll}
x_{\alpha} x_{\delta}=q_{\alpha \delta} x_{\delta} x_{\alpha}+\mathrm{b}_{1} x_{\tau}^{2} x_{\beta}, & x_{\delta} x_{\eta}=q_{\delta \eta} x_{\eta} x_{\delta}+\mathrm{b}_{2} x_{\kappa} x_{\gamma} x_{\mu}+\mathrm{b}_{3} x_{\gamma} x_{\nu}, \\
x_{\tau} x_{\eta}=q_{\tau \eta} x_{\eta} x_{\tau}+\mathrm{b}_{4} x_{\kappa} x_{\gamma}, & x_{\alpha} x_{\mu}=q_{\alpha \mu} x_{\mu} x_{\alpha}+\mathrm{b}_{5} x_{\tau} x_{\beta}, \\
x_{\mu} x_{\eta}=q_{\mu \eta} x_{\eta} x_{\mu}+\mathrm{b}_{6} x_{\iota} x_{\gamma}, & x_{\nu} x_{\eta}=q_{\nu \eta} x_{\eta} x_{\nu}+\mathrm{b}_{7} x_{\iota} x_{\kappa} x_{\gamma}, \\
x_{\beta} x_{\kappa}=q_{\beta \kappa} x_{\kappa} x_{\beta}+\mathrm{b}_{8} x_{\gamma}, & x_{\alpha} x_{\iota}=q_{\alpha \iota} x_{\iota} x_{\alpha}+\mathrm{b}_{9} x_{\gamma},
\end{array}
$$


for some scalars $\mathrm{b}_{j} \in \mathbb{k}$ and the other pairs of root vectors $q$-commute, except possibly $\left(x_{\tau}, x_{\mu}\right),\left(x_{\mu}, x_{\kappa}\right),\left(x_{\delta}, x_{\kappa}\right)$. Then

$$
\begin{aligned}
& d\left(\mathrm{x}_{\alpha} \mathrm{x}_{\beta} \mathrm{x}_{\delta} \mathrm{x}_{\gamma}^{2} \mathrm{x}_{\eta}^{2} \otimes 1\right)=\mathrm{x}_{\alpha} \mathrm{x}_{\beta} \mathrm{x}_{\delta} \mathrm{x}_{\gamma}^{2} \mathrm{x}_{\eta} \otimes x_{\eta}+q_{\gamma \eta}^{2} \mathrm{x}_{\alpha} \mathrm{x}_{\beta} \mathrm{x}_{\delta} \mathrm{x}_{\gamma} \mathrm{x}_{\eta}^{2} \otimes x_{\gamma} \\
& \quad+q_{\delta \gamma}^{2}(3)_{\widetilde{q}_{\gamma \eta}} \mathrm{b}_{3} \mathrm{~b}_{7} \mathrm{x}_{\alpha} \mathrm{x}_{\beta} \mathrm{x}_{\gamma}^{3} \mathrm{x}_{\iota} \otimes x_{\kappa} x_{\gamma}+q_{\delta \gamma}^{2} q_{\nu \eta}(3)_{\widetilde{q}_{\gamma \eta}} \mathrm{b}_{3} \mathrm{x}_{\alpha} \mathrm{x}_{\beta} \mathrm{x}_{\gamma}^{3} \mathrm{x}_{\eta} \otimes x_{\nu} \\
& \quad+q_{\delta \gamma}^{2} q_{\mu \eta} q_{\gamma \eta} \mathrm{b}_{2} \mathrm{x}_{\alpha} \mathrm{x}_{\beta} \mathrm{x}_{\gamma}^{2} \mathrm{x}_{\kappa} \mathrm{x}_{\eta} \otimes x_{\gamma} x_{\mu}+q_{\delta \gamma}^{2} q_{\delta \eta}^{2} \mathrm{x}_{\alpha} \mathrm{x}_{\beta} \mathrm{x}_{\gamma}^{2} \mathrm{x}_{\eta}^{2} \otimes x_{\delta} \\
& \quad+q_{\delta \gamma}^{2} q_{\gamma \kappa}^{2} \mathrm{c}_{\beta \eta \gamma}^{(3)} \mathrm{b}_{2} \mathrm{~b}_{6} \mathrm{~b}_{8} \mathrm{x}_{\alpha} \mathrm{x}_{\gamma}^{4} \mathrm{x}_{\iota} \otimes x_{\gamma}+q_{\delta \gamma}^{2} q_{\gamma \kappa}^{2} q_{\mu \eta} \mathrm{c}_{\beta \eta \gamma}^{(3)} \mathrm{b}_{2} \mathrm{~b}_{8} \mathrm{x}_{\alpha} \mathrm{x}_{\gamma}^{4} \mathrm{x}_{\eta} \otimes x_{\mu} \\
& \quad-q_{\beta \delta} q_{\beta \gamma}^{2} q_{\beta \eta}^{2} \mathrm{x}_{\alpha} \mathrm{x}_{\delta} \mathrm{x}_{\gamma}^{2} \mathrm{x}_{\eta}^{2} \otimes x_{\beta}+q_{\alpha \beta} q_{\beta \gamma}^{2} q_{\beta \eta}^{2} q_{\tau \gamma}^{2} q_{\gamma \eta} \mathrm{b}_{1} \mathrm{~b}_{4} \mathrm{x}_{\beta} \mathrm{x}_{\tau} \mathrm{x}_{\gamma}^{2} \mathrm{x}_{\kappa} \mathrm{x}_{\eta} \otimes x_{\gamma} x_{\beta} \\
& \quad-q_{\alpha \beta} q_{\beta \gamma}^{2} q_{\beta \eta}^{2} q_{\tau \gamma}^{2} q_{\tau \eta}^{2} \mathrm{~b}_{1} \mathrm{x}_{\beta} \mathrm{x}_{\tau} \mathrm{x}_{\gamma}^{2} \mathrm{x}_{\eta}^{2} \otimes x_{\tau} x_{\beta}+q_{\alpha \beta} q_{\alpha \delta} q_{\alpha \gamma}^{2} q_{\alpha \eta}^{2} \mathrm{x}_{\beta} \mathrm{x}_{\delta} \mathrm{x}_{\gamma}^{2} \mathrm{x}_{\eta}^{2} \otimes x_{\alpha} \\
& \quad-q_{\alpha \beta} q_{\beta \tau} q_{\beta \gamma}^{2} q_{\beta \eta}^{2} q_{\gamma \beta}^{-2} q_{\tau \gamma}^{2} c_{\beta \eta \gamma}^{(3)} \mathrm{b}_{1} \mathrm{~b}_{4} \mathrm{~b}_{8} \mathrm{x}_{\tau} \mathrm{x}_{\gamma}^{4} \mathrm{x}_{\eta} \otimes x_{\beta}-q_{\delta \gamma}^{2} q_{\gamma \kappa}^{2} \mathrm{~d}_{\alpha \beta \eta \gamma}^{(4)} \mathrm{b}_{2} \mathrm{~b}_{6} \mathrm{~b}_{8} \mathrm{~b}_{9} \mathrm{x}_{\gamma}^{6} \otimes 1
\end{aligned}
$$

Notice that the equalities in (10.1.16) forces

$$
\begin{aligned}
& \alpha+\delta=\beta+2 \tau, \quad \delta+\eta=\kappa+\gamma+\mu, \quad \delta+\eta=\gamma+\nu, \\
& \tau+\eta=\kappa+\gamma, \quad \alpha+\mu=\tau+\beta, \quad \mu+\eta=\iota+\gamma, \\
& \nu+\eta=\iota+\kappa+\gamma, \quad \beta+\kappa=\gamma, \quad \alpha+\iota=\gamma .
\end{aligned}
$$

Thus the following equality also holds: $4 \gamma=\alpha+\beta+\delta+2 \eta$.

Proof. First we note that Lemma 10.1.7 applies for $\alpha<\gamma<\iota$, and Lemma 10.1.48 applies for $\beta<\tau<\gamma<\kappa<\eta$. Hence the following formulas hold for all $n \geq 0$ :

$$
\begin{aligned}
& d\left(\mathrm{x}_{\alpha} \mathrm{x}_{\gamma}^{n} \mathrm{x}_{\iota} \otimes 1\right)=\mathrm{x}_{\alpha} \mathrm{x}_{\gamma}^{n} \otimes x_{\iota}-q_{\gamma \iota} \mathrm{x}_{\alpha} \mathrm{x}_{\gamma}^{n-1} \mathrm{x}_{\iota} \otimes x_{\gamma}-q_{\alpha \iota}\left(-q_{\alpha \gamma}\right)^{n} \mathrm{x}_{\gamma}^{n} \mathrm{x}_{\iota} \otimes x_{\alpha} \\
& \quad-\left(-q_{\alpha \gamma}\right)^{n}(n+1)_{\widetilde{q}_{\alpha \gamma}} \mathrm{b}_{9} \mathrm{x}_{\gamma}^{n+1} \otimes 1 \\
& d\left(\mathrm{x}_{\tau} \mathrm{x}_{\gamma}^{n} \mathrm{x}_{\eta} \otimes 1\right)=\mathrm{x}_{\tau} \mathrm{x}_{\gamma}^{n} \otimes x_{\eta}-q_{\gamma \eta} \mathrm{x}_{\tau} \mathrm{x}_{\gamma}^{n-1} \mathrm{x}_{\eta} \otimes x_{\gamma}-\left(-q_{\tau \gamma}\right)^{n} q_{\tau \eta} \mathrm{x}_{\gamma}^{n} \mathrm{x}_{\eta} \otimes x_{\tau} \\
& \quad-\left(-q_{\tau \gamma}\right)^{n} \mathrm{~b}_{4} \mathrm{x}_{\gamma}^{n} \mathrm{x}_{\kappa} \otimes x_{\gamma}, \\
& d\left(\mathrm{x}_{\beta} \mathrm{x}_{\gamma}^{n} \mathrm{x}_{\kappa} \otimes 1\right)=\mathrm{x}_{\beta} \mathrm{x}_{\gamma}^{n} \otimes x_{\kappa}-q_{\gamma \kappa} \mathrm{x}_{\beta} \mathrm{x}_{\gamma}^{n-1} \mathrm{x}_{\kappa} \otimes x_{\gamma}-q_{\beta \kappa}\left(-q_{\beta \gamma}\right)^{n} \mathrm{x}_{\gamma}^{n} \mathrm{x}_{\kappa} \otimes x_{\beta} \\
& \quad-\left(-q_{\beta \gamma}\right)^{n}(n+1)_{q_{\beta \gamma}} \mathrm{b}_{8} \mathrm{x}_{\gamma}^{n+1} \otimes 1, \\
& d\left(\mathrm{x}_{\beta} \mathrm{x}_{\tau} \mathrm{x}_{\gamma}^{n} \mathrm{x}_{\eta} \otimes 1\right)=\mathrm{x}_{\beta} \mathrm{x}_{\tau} \mathrm{x}_{\gamma}^{n} \otimes x_{\eta}-q_{\gamma \eta} \mathrm{x}_{\beta} \mathrm{x}_{\tau} \mathrm{x}_{\gamma}^{n-1} \mathrm{x}_{\eta} \otimes x_{\gamma} \\
& \quad-\left(-q_{\tau \gamma}\right)^{n} q_{\tau \eta} \mathrm{x}_{\beta} \mathrm{x}_{\gamma}^{n} \mathrm{x}_{\eta} \otimes x_{\tau}+\left(-q_{\beta \gamma}\right)^{n} q_{\beta \tau} q_{\beta \eta} \mathrm{x}_{\tau} \mathrm{x}_{\gamma}^{n} \mathrm{x}_{\eta} \otimes x_{\beta} \\
& \quad-\left(-q_{\tau \gamma}\right)^{n} \mathrm{~b}_{4} \mathrm{x}_{\beta} \mathrm{x}_{\gamma}^{n} \mathrm{x}_{\kappa} \otimes x_{\gamma}-q_{\gamma \eta}^{n} c_{-\eta, \beta, \gamma}^{(n)} \mathrm{b}_{4} \mathrm{~b}_{8} \mathrm{x}_{\gamma}^{n+2} \otimes 1 .
\end{aligned}
$$

We also need some auxiliar computations. These are straightforward and we omit the details:

$$
\begin{aligned}
& d\left(\mathrm{x}_{\alpha} \mathrm{x}_{\beta} \mathrm{x}_{\delta} \otimes 1\right)=\mathrm{x}_{\alpha} \mathrm{x}_{\beta} \otimes x_{\delta}-q_{\beta \delta} \mathrm{x}_{\alpha} \mathrm{x}_{\delta} \otimes x_{\beta}+q_{\alpha \beta} \mathrm{b}_{1} \mathrm{x}_{\beta} \mathrm{x}_{\tau} \otimes x_{\tau} x_{\beta} \\
& \quad+q_{\alpha \beta} q_{\alpha \delta} \mathrm{x}_{\beta} \mathrm{x}_{\delta} \otimes x_{\alpha} \\
& d\left(\mathrm{x}_{\alpha} \mathrm{x}_{\beta} \mathrm{x}_{\kappa} \otimes 1\right)=\mathrm{x}_{\alpha} \mathrm{x}_{\beta} \otimes x_{\kappa}-\mathrm{b}_{\delta} \mathrm{x}_{\alpha} \mathrm{x}_{\gamma} \otimes 1-q_{\beta \kappa} \mathrm{x}_{\alpha} \mathrm{x}_{\kappa} \otimes x_{\beta} \\
& \quad+q_{\alpha \beta} q_{\alpha \kappa} \mathrm{x}_{\beta} \mathrm{x}_{\kappa} \otimes x_{\alpha} \\
& \quad d\left(\mathrm{x}_{\alpha} \mathrm{x}_{\delta} \mathrm{x}_{\gamma} \otimes 1\right)=\mathrm{x}_{\alpha} \mathrm{x}_{\delta} \otimes x_{\gamma}-q_{\delta \gamma} \mathrm{x}_{\alpha} \mathrm{x}_{\gamma} \otimes x_{\delta}+q_{\alpha \delta} q_{\alpha \gamma} \mathrm{x}_{\delta} \mathrm{x}_{\gamma} \otimes x_{\alpha} \\
& \quad+q_{\tau \gamma} q_{\beta \gamma} \mathrm{b}_{1} \mathrm{x}_{\tau} \mathrm{x}_{\gamma} \otimes x_{\tau} x_{\beta}
\end{aligned}
$$




$$
\begin{aligned}
& d\left(\mathrm{x}_{\alpha} \mathrm{x}_{\delta} \mathrm{x}_{\eta} \otimes 1\right)=\mathrm{x}_{\alpha} \mathrm{x}_{\delta} \otimes x_{\eta}-q_{\delta \eta} \mathrm{x}_{\alpha} \mathrm{x}_{\eta} \otimes x_{\delta}-\mathrm{b}_{2} \mathrm{x}_{\alpha} \mathrm{x}_{\kappa} \otimes x_{\gamma} x_{\mu}-\mathrm{b}_{3} \mathrm{x}_{\alpha} \mathrm{x}_{\gamma} \otimes x_{\nu} \\
& +q_{\alpha \delta} q_{\alpha \eta} \mathrm{x}_{\delta} \mathrm{x}_{\eta} \otimes x_{\alpha}+q_{\beta \eta} \mathrm{b}_{1} \mathrm{~b}_{4} \mathrm{x}_{\tau} \mathrm{x}_{\kappa} \otimes x_{\gamma} x_{\beta}+q_{\beta \eta} q_{\tau \eta} \mathrm{b}_{1} \mathrm{x}_{\tau} \mathrm{x}_{\eta} \otimes x_{\tau} x_{\beta}, \\
& d\left(\mathrm{x}_{\beta} \mathrm{x}_{\tau} \mathrm{x}_{\kappa} \otimes 1\right)=\mathrm{x}_{\beta} \mathrm{x}_{\tau} \otimes x_{\kappa}-q_{\tau \kappa} \mathrm{x}_{\beta} \mathrm{x}_{\kappa} \otimes x_{\tau}+q_{\beta \tau} \mathrm{b}_{8} \mathrm{x}_{\tau} \mathrm{x}_{\gamma} \otimes 1 \\
& +q_{\beta \tau} q_{\beta \kappa} \mathrm{x}_{\tau} \mathrm{x}_{\kappa} \otimes x_{\beta}, \\
& d\left(\mathrm{x}_{\beta} \mathrm{x}_{\delta} \mathrm{x}_{\eta} \otimes 1\right)=\mathrm{x}_{\beta} \mathrm{x}_{\delta} \otimes x_{\eta}-q_{\delta \eta} \mathrm{x}_{\beta} \mathrm{x}_{\eta} \otimes x_{\delta}-\mathrm{b}_{2} \mathrm{x}_{\beta} \mathrm{x}_{\kappa} \otimes x_{\gamma} x_{\mu}-\mathrm{b}_{3} \mathrm{x}_{\beta} \mathrm{x}_{\gamma} \otimes x_{\nu} \\
& +q_{\beta \delta} q_{\beta \eta} \mathrm{x}_{\delta} \mathrm{x}_{\eta} \otimes x_{\beta}-\mathrm{b}_{2} \mathrm{~b}_{8} \mathrm{x}_{\gamma}^{2} \otimes x_{\mu}, \\
& d\left(\mathrm{x}_{\beta} \mathrm{x}_{\kappa}^{2} \otimes 1\right)=\mathrm{x}_{\beta} \mathrm{x}_{\kappa} \otimes x_{\kappa}+\mathrm{b}_{8} \mathrm{x}_{\gamma} \mathrm{x}_{\kappa} \otimes 1+q_{\beta \kappa}^{2} \mathrm{x}_{\kappa}^{2} \otimes x_{\beta}, \\
& d\left(\mathrm{x}_{\beta} \mathrm{x}_{\kappa} \mathrm{x}_{\eta} \otimes 1\right)=\mathrm{x}_{\beta} \mathbf{x}_{\kappa} \otimes x_{\eta}-q_{\kappa \eta} \mathbf{x}_{\beta} \mathbf{x}_{\eta} \otimes x_{\kappa}+\mathrm{b}_{8} \mathbf{x}_{\gamma} \mathbf{x}_{\eta} \otimes 1+q_{\beta \kappa} q_{\beta \eta} \mathbf{x}_{\kappa} \mathbf{x}_{\eta} \otimes x_{\beta}, \\
& d\left(\mathrm{x}_{\tau} \mathrm{x}_{\kappa} \mathrm{x}_{\eta} \otimes 1\right)=\mathrm{x}_{\tau} \mathrm{x}_{\kappa} \otimes x_{\eta}-q_{\kappa \eta} \mathrm{x}_{\tau} \mathrm{x}_{\eta} \otimes x_{\kappa}+q_{\tau \kappa} q_{\tau \eta} \mathrm{x}_{\kappa} \mathrm{x}_{\eta} \otimes x_{\tau} \\
& +q_{\kappa \tau}(2)_{\eta \kappa} \mathrm{b}_{4} \mathrm{x}_{\kappa}^{2} \otimes x_{\gamma}, \\
& d\left(\mathrm{x}_{\tau} \mathrm{x}_{\eta}^{2} \otimes 1\right)=\mathrm{x}_{\tau} \mathrm{x}_{\eta} \otimes x_{\eta}+q_{\gamma \eta} \mathrm{b}_{4} \mathrm{x}_{\kappa} \mathrm{x}_{\eta} \otimes x_{\gamma}+q_{\tau \eta}^{2} \mathrm{x}_{\eta}^{2} \otimes x_{\tau} \\
& d\left(\mathrm{x}_{\delta} \mathrm{x}_{\gamma} \mathrm{x}_{\eta} \otimes 1\right)=\mathrm{x}_{\delta} \mathrm{x}_{\gamma} \otimes x_{\eta}-q_{\gamma \eta} \mathrm{x}_{\delta} \mathrm{x}_{\eta} \otimes x_{\gamma}+q_{\delta \gamma} q_{\delta \eta} \mathrm{x}_{\gamma} \mathrm{x}_{\eta} \otimes x_{\delta} \\
& +q_{\delta \gamma} \mathrm{b}_{2} \mathrm{x}_{\gamma} \mathrm{x}_{\kappa} \otimes x_{\gamma} x_{\mu}+q_{\delta \gamma}(2)_{\widetilde{q}_{\gamma \eta}} \mathrm{b}_{3} \mathrm{x}_{\gamma}^{2} \otimes x_{\nu} . \\
& d\left(\mathrm{x}_{\delta} \mathrm{x}_{\eta}^{2} \otimes 1\right)=\mathrm{x}_{\delta} \mathrm{x}_{\eta} \otimes x_{\eta}+\mathrm{b}_{3} \mathrm{~b}_{7} \mathrm{x}_{\gamma} \mathrm{x}_{\iota} \otimes x_{\kappa} x_{\gamma}+q_{\nu \eta} \mathrm{b}_{3} \mathrm{x}_{\gamma} \mathrm{x}_{\eta} \otimes x_{\nu} \\
& +q_{\gamma \eta} q_{\mu \eta} \mathrm{b}_{2} \mathrm{x}_{\kappa} \mathrm{x}_{\eta} \otimes x_{\gamma} x_{\mu}+q_{\delta \eta}^{2} \mathrm{x}_{\eta}^{2} \otimes x_{\delta} \text {. }
\end{aligned}
$$

Next we compute differentials of some 4-chains, using the previous computations on 3-chains and Remark 10.1.2:

$$
\begin{aligned}
& d\left(\mathrm{x}_{\alpha} \mathrm{x}_{\beta} \mathrm{x}_{\delta} \mathrm{x}_{\gamma} \otimes 1\right)=\mathrm{x}_{\alpha} \mathrm{x}_{\beta} \mathrm{x}_{\delta} \otimes x_{\gamma}-q_{\delta \gamma} \mathrm{x}_{\alpha} \mathrm{x}_{\beta} \mathrm{x}_{\gamma} \otimes x_{\delta}+q_{\beta \delta} q_{\beta \gamma} \mathrm{x}_{\alpha} \mathrm{x}_{\delta} \mathrm{x}_{\gamma} \otimes x_{\beta} \\
& -q_{\alpha \beta} q_{\beta \gamma} q_{\tau \gamma} \mathrm{b}_{1} \mathrm{x}_{\beta} \mathrm{x}_{\tau} \mathrm{x}_{\gamma} \otimes x_{\tau} x_{\beta}-q_{\alpha \beta} q_{\alpha \delta} q_{\alpha \gamma} \mathrm{x}_{\beta} \mathrm{x}_{\delta} \mathrm{x}_{\gamma} \otimes x_{\alpha}, \\
& d\left(\mathrm{x}_{\alpha} \mathrm{x}_{\delta} \mathrm{x}_{\gamma}^{2} \otimes 1\right)=\mathrm{x}_{\alpha} \mathrm{x}_{\delta} \mathrm{x}_{\gamma} \otimes x_{\gamma}+q_{\delta \gamma}^{2} \mathrm{x}_{\alpha} \mathrm{x}_{\gamma}^{2} \otimes x_{\delta}-q_{\alpha \delta} q_{\alpha \gamma}^{2} \mathrm{x}_{\delta} \mathrm{x}_{\gamma}^{2} \otimes x_{\alpha} \\
& -q_{\tau \gamma}^{2} q_{\beta \gamma}^{2} \mathrm{~b}_{1} \mathrm{x}_{\tau} \mathrm{x}_{\gamma}^{2} \otimes x_{\tau} x_{\beta}, \\
& d\left(\mathrm{x}_{\alpha} \mathrm{x}_{\beta} \mathrm{x}_{\delta} \mathrm{x}_{\eta} \otimes 1\right)=\mathrm{x}_{\alpha} \mathrm{x}_{\beta} \mathrm{x}_{\delta} \otimes x_{\eta}-q_{\delta \eta} \mathrm{x}_{\alpha} \mathrm{x}_{\beta} \mathrm{x}_{\eta} \otimes x_{\delta}-\mathrm{b}_{3} \mathrm{x}_{\alpha} \mathrm{x}_{\beta} \mathrm{x}_{\gamma} \otimes x_{\nu} \\
& -\mathrm{b}_{2} \mathrm{x}_{\alpha} \mathrm{x}_{\beta} \mathrm{x}_{\kappa} \otimes x_{\gamma} x_{\mu}-\mathrm{b}_{2} \mathrm{~b}_{8} \mathrm{x}_{\alpha} \mathrm{x}_{\gamma}^{2} \otimes x_{\mu}+q_{\beta \delta} q_{\beta \eta} \mathrm{x}_{\alpha} \mathrm{x}_{\delta} \mathrm{x}_{\eta} \otimes x_{\beta} \\
& -q_{\alpha \beta} q_{\beta \eta} \mathrm{b}_{1} \mathrm{~b}_{4} \mathrm{x}_{\beta} \mathrm{x}_{\tau} \mathrm{x}_{\kappa} \otimes x_{\gamma} x_{\beta}-q_{\alpha \beta} q_{\beta \eta} q_{\tau \eta} \mathrm{b}_{1} \mathrm{x}_{\beta} \mathrm{x}_{\tau} \mathrm{x}_{\eta} \otimes x_{\tau} x_{\beta} \\
& -q_{\alpha \beta} q_{\alpha \delta} q_{\alpha \eta} \mathbf{x}_{\beta} \mathbf{x}_{\delta} \mathrm{x}_{\eta} \otimes x_{\alpha}+q_{\beta \tau} q_{\alpha \beta} q_{\beta \eta} \mathrm{b}_{1} \mathrm{~b}_{4} \mathrm{~b}_{8} \mathrm{x}_{\tau} \mathrm{x}_{\gamma}^{2} \otimes x_{\beta}, \\
& d\left(\mathrm{x}_{\alpha} \mathrm{x}_{\beta} \mathrm{x}_{\kappa} \mathrm{x}_{\eta} \otimes 1\right)=\mathrm{x}_{\alpha} \mathrm{x}_{\beta} \mathrm{x}_{\kappa} \otimes x_{\eta}-q_{\kappa \eta} \mathrm{x}_{\alpha} \mathrm{x}_{\beta} \mathrm{x}_{\eta} \otimes x_{\kappa}+q_{\beta \kappa} q_{\beta \eta} \mathrm{x}_{\alpha} \mathrm{x}_{\kappa} \mathrm{x}_{\eta} \otimes x_{\beta} \\
& +\mathrm{b}_{8} \mathrm{x}_{\alpha} \mathrm{x}_{\gamma} \mathrm{x}_{\eta} \otimes 1-q_{\alpha \beta} q_{\alpha \kappa} q_{\alpha \eta} \mathrm{x}_{\beta} \mathrm{x}_{\kappa} \mathrm{x}_{\eta} \otimes x_{\alpha} \\
& d\left(\mathrm{x}_{\alpha} \mathrm{x}_{\beta} \mathrm{x}_{\gamma} \mathrm{x}_{\kappa} \otimes 1\right)=\mathrm{x}_{\alpha} \mathrm{x}_{\beta} \mathrm{x}_{\gamma} \otimes x_{\kappa}-q_{\gamma \kappa} \mathrm{x}_{\alpha} \mathrm{x}_{\beta} \mathrm{x}_{\kappa} \otimes x_{\gamma}+q_{\beta \gamma} q_{\beta \kappa} \mathrm{x}_{\alpha} \mathrm{x}_{\gamma} \mathrm{x}_{\kappa} \otimes x_{\beta} \\
& +q_{\gamma \beta}^{-1}(2)_{\widetilde{q}_{\beta \gamma}} \mathrm{b}_{8} \mathrm{x}_{\alpha} \mathrm{x}_{\gamma}^{2} \otimes 1-q_{\alpha \beta} q_{\alpha \gamma} q_{\alpha \kappa} \mathrm{x}_{\beta} \mathrm{x}_{\gamma} \mathrm{x}_{\kappa} \otimes x_{\alpha}, \\
& d\left(\mathrm{x}_{\alpha} \mathrm{x}_{\delta} \mathrm{x}_{\gamma} \mathrm{x}_{\eta} \otimes 1\right)=\mathrm{x}_{\alpha} \mathrm{x}_{\delta} \mathrm{x}_{\gamma} \otimes x_{\eta}-q_{\gamma \eta} \mathrm{x}_{\alpha} \mathrm{x}_{\delta} \mathrm{x}_{\eta} \otimes x_{\gamma}+q_{\delta \gamma} q_{\delta \eta} \mathrm{x}_{\alpha} \mathrm{x}_{\gamma} \mathrm{x}_{\eta} \otimes x_{\delta} \\
& +q_{\delta \gamma} \mathrm{b}_{2} \mathrm{x}_{\alpha} \mathrm{x}_{\gamma} \mathrm{x}_{\kappa} \otimes x_{\gamma} x_{\mu}-q_{\alpha \delta} q_{\alpha \gamma} q_{\alpha \eta} \mathrm{x}_{\delta} \mathrm{x}_{\gamma} \mathrm{x}_{\eta} \otimes x_{\alpha} \\
& -q_{\tau \gamma} q_{\beta \gamma} q_{\beta \eta} \mathrm{b}_{1} \mathrm{~b}_{4} \mathrm{x}_{\tau} \mathrm{x}_{\gamma} \mathrm{x}_{\kappa} \otimes x_{\gamma} x_{\beta}+q_{\delta \gamma} \mathrm{b}_{3} \mathrm{x}_{\alpha} \mathrm{x}_{\gamma}^{2} \otimes x_{\nu} \\
& -q_{\beta \gamma} q_{\beta \eta} q_{\tau \gamma} q_{\tau \eta} \mathrm{b}_{1} \mathrm{x}_{\tau} \mathrm{x}_{\gamma} \mathrm{x}_{\eta} \otimes x_{\tau} x_{\beta}-q_{\alpha \gamma}^{2} q_{\alpha \kappa} q_{\delta \gamma} \mathrm{b}_{2} \mathrm{~b}_{5} \mathrm{x}_{\gamma}^{2} \mathrm{x}_{\kappa} \otimes x_{\tau} x_{\beta},
\end{aligned}
$$




$$
\begin{aligned}
& d\left(\mathrm{x}_{\alpha} \mathrm{x}_{\delta} \mathrm{x}_{\eta}^{2} \otimes 1\right)=\mathrm{x}_{\alpha} \mathrm{x}_{\delta} \mathrm{x}_{\eta} \otimes x_{\eta}+q_{\nu \eta} \mathrm{b}_{3} \mathrm{x}_{\alpha} \mathrm{x}_{\gamma} \mathrm{x}_{\eta} \otimes x_{\nu}+q_{\delta \eta}^{2} \mathrm{x}_{\alpha} \mathrm{x}_{\eta}^{2} \otimes x_{\delta} \\
& +q_{\mu \eta} q_{\gamma \eta} \mathrm{b}_{2} \mathrm{x}_{\alpha} \mathrm{x}_{\kappa} \mathrm{x}_{\eta} \otimes x_{\gamma} x_{\mu}-q_{\beta \eta}^{2} q_{\gamma \eta} \mathrm{b}_{1} \mathrm{~b}_{4} \mathrm{x}_{\tau} \mathrm{x}_{\kappa} \mathrm{x}_{\eta} \otimes x_{\gamma} x_{\beta} \\
& -q_{\beta \eta}^{2} q_{\tau \eta}^{2} \mathrm{~b}_{1} \mathrm{x}_{\tau} \mathrm{x}_{\eta}^{2} \otimes x_{\tau} x_{\beta}-q_{\alpha \delta} q_{\alpha \eta}^{2} \mathrm{x}_{\delta} \mathrm{x}_{\eta}^{2} \otimes x_{\alpha} \\
& d\left(\mathrm{x}_{\beta} \mathrm{x}_{\tau} \mathrm{x}_{\gamma} \mathrm{x}_{\kappa} \otimes 1\right)=\mathrm{x}_{\beta} \mathrm{x}_{\tau} \mathrm{x}_{\gamma} \otimes x_{\kappa}-q_{\gamma \kappa} \mathrm{x}_{\beta} \mathrm{x}_{\tau} \mathrm{x}_{\kappa} \otimes x_{\gamma}+q_{\tau \gamma} q_{\tau \kappa} \mathrm{x}_{\beta} \mathrm{x}_{\gamma} \mathrm{x}_{\kappa} \otimes x_{\tau} \\
& -q_{\beta \tau} q_{\gamma \beta}^{-1}(2)_{\widetilde{q}_{\beta \gamma}} \mathrm{b}_{8} \mathrm{x}_{\tau} \mathrm{x}_{\gamma}^{2} \otimes 1-q_{\beta \tau} q_{\beta \gamma} q_{\beta \kappa} \mathrm{x}_{\tau} \mathrm{x}_{\gamma} \mathrm{x}_{\kappa} \otimes x_{\beta}, \\
& d\left(\mathrm{x}_{\beta} \mathrm{x}_{\tau} \mathrm{x}_{\kappa} \mathrm{x}_{\eta} \otimes 1\right)=\mathrm{x}_{\beta} \mathrm{x}_{\tau} \mathrm{x}_{\kappa} \otimes x_{\eta}-q_{\kappa \eta} \mathrm{x}_{\beta} \mathrm{x}_{\tau} \mathrm{x}_{\eta} \otimes x_{\kappa}+q_{\tau \kappa} q_{\tau \eta} \mathrm{x}_{\beta} \mathrm{x}_{\kappa} \mathrm{x}_{\eta} \otimes x_{\tau} \\
& -q_{\beta \tau} \mathrm{b}_{8} \mathrm{x}_{\tau} \mathrm{x}_{\gamma} \mathrm{x}_{\eta} \otimes 1-q_{\gamma \kappa} q_{\eta \kappa}^{-1}(2)_{\widetilde{q}_{\kappa \eta}} \mathrm{b}_{4} \mathrm{x}_{\beta} \mathrm{x}_{\kappa}^{2} \otimes x_{\gamma} \\
& -q_{\beta \tau} q_{\beta \kappa} q_{\beta \eta} \mathrm{x}_{\tau} \mathrm{x}_{\kappa} \mathrm{x}_{\eta} \otimes x_{\beta}-q_{\kappa \eta} \mathrm{b}_{4} \mathrm{~b}_{8} \mathrm{x}_{\gamma}^{2} \mathrm{x}_{\kappa} \otimes 1 \\
& d\left(\mathrm{x}_{\beta} \mathrm{x}_{\tau} \mathrm{x}_{\eta}^{2} \otimes 1\right)=\mathrm{x}_{\beta} \mathrm{x}_{\tau} \mathrm{x}_{\eta} \otimes x_{\eta}+q_{\tau \eta}^{2} \mathrm{x}_{\beta} \mathrm{x}_{\eta}^{2} \otimes x_{\tau}-q_{\beta \tau} q_{\beta \eta}^{2} \mathrm{x}_{\tau} \mathrm{x}_{\eta}^{2} \otimes x_{\beta} \\
& +q_{\gamma \eta} \mathrm{b}_{4} \mathrm{x}_{\beta} \mathrm{x}_{\kappa} \mathrm{x}_{\eta} \otimes x_{\gamma}+\mathrm{b}_{4} \mathrm{~b}_{8} \mathrm{x}_{\gamma}^{2} \mathrm{x}_{\eta} \otimes 1 \\
& d\left(\mathrm{x}_{\beta} \mathrm{x}_{\delta} \mathrm{x}_{\gamma} \mathrm{x}_{\eta} \otimes 1\right)=\mathrm{x}_{\beta} \mathrm{x}_{\delta} \mathrm{x}_{\gamma} \otimes x_{\eta}-q_{\gamma \eta} \mathrm{x}_{\beta} \mathrm{x}_{\delta} \mathrm{x}_{\eta} \otimes x_{\gamma}+q_{\delta \gamma} q_{\delta \eta} \mathrm{x}_{\beta} \mathrm{x}_{\gamma} \mathrm{x}_{\eta} \otimes x_{\delta} \\
& +q_{\delta \gamma} \mathrm{b}_{2} \mathrm{x}_{\beta} \mathrm{x}_{\gamma} \mathrm{x}_{\kappa} \otimes x_{\gamma} x_{\mu}+q_{\delta \gamma}(2)_{\widetilde{q}_{\gamma \eta}} \mathrm{b}_{3} \mathrm{x}_{\beta} \mathrm{x}_{\gamma}^{2} \otimes x_{\nu} \\
& +q_{\beta \gamma} q_{\delta \gamma}(2) \tilde{q}_{\gamma \eta} \mathrm{b}_{2} \mathrm{~b}_{8} \mathrm{x}_{\gamma}^{3} \otimes x_{\mu}-q_{\beta \delta} q_{\beta \gamma} q_{\beta \eta} \mathrm{x}_{\delta} \mathrm{x}_{\gamma} \mathrm{x}_{\eta} \otimes x_{\beta} \text {, } \\
& d\left(\mathrm{x}_{\beta} \mathrm{x}_{\delta} \mathrm{x}_{\eta}^{2} \otimes 1\right)=\mathrm{x}_{\beta} \mathrm{x}_{\delta} \mathrm{x}_{\eta} \otimes x_{\eta}+\mathrm{b}_{3} \mathrm{~b}_{7} \mathrm{x}_{\beta} \mathrm{x}_{\gamma} \mathrm{x}_{\iota} \otimes x_{\kappa} x_{\gamma}+q_{\nu \eta} \mathrm{b}_{3} \mathrm{x}_{\beta} \mathrm{x}_{\gamma} \mathrm{x}_{\eta} \otimes x_{\nu} \\
& +q_{\mu \eta} q_{\gamma \eta} \mathrm{b}_{2} \mathrm{x}_{\beta} \mathrm{x}_{\kappa} \mathrm{x}_{\eta} \otimes x_{\gamma} x_{\mu}+q_{\delta \eta}^{2} \mathrm{x}_{\beta} \mathrm{x}_{\eta}^{2} \otimes x_{\delta}-q_{\beta \delta} q_{\beta \eta}^{2} \mathrm{x}_{\delta} \mathrm{x}_{\eta}^{2} \otimes x_{\beta} \\
& +q_{\mu \eta} \mathrm{b}_{2} \mathrm{~b}_{8} \mathrm{x}_{\gamma}^{2} \mathrm{x}_{\eta} \otimes x_{\mu}+\mathrm{b}_{2} \mathrm{~b}_{6} \mathrm{~b}_{8} \mathrm{x}_{\gamma}^{2} \mathrm{x}_{\iota} \otimes x_{\gamma} \text {, } \\
& d\left(\mathrm{x}_{\beta} \mathrm{x}_{\gamma} \mathrm{x}_{\kappa}^{2} \otimes 1\right)=\mathrm{x}_{\beta} \mathrm{x}_{\gamma} \mathrm{x}_{\kappa} \otimes x_{\kappa}+q_{\gamma \kappa}^{2} \mathrm{x}_{\beta} \mathrm{x}_{\kappa}^{2} \otimes x_{\gamma}-q_{\beta \gamma} \mathrm{b}_{8}(2)_{\widetilde{q}_{\gamma \kappa}} \mathrm{x}_{\gamma}^{2} \mathrm{x}_{\kappa} \otimes 1 \\
& -q_{\beta \gamma} q_{\beta \kappa}^{2} \mathrm{x}_{\gamma} \mathrm{x}_{\kappa}^{2} \otimes x_{\beta}, \\
& d\left(\mathrm{x}_{\beta} \mathrm{x}_{\gamma} \mathrm{x}_{\kappa} \mathrm{x}_{\eta} \otimes 1\right)=\mathrm{x}_{\beta} \mathrm{x}_{\gamma} \mathrm{x}_{\kappa} \otimes x_{\eta}-q_{\kappa \eta} \mathrm{x}_{\beta} \mathrm{x}_{\gamma} \mathrm{x}_{\eta} \otimes x_{\kappa}+q_{\gamma \kappa} q_{\gamma \eta} \mathrm{x}_{\beta} \mathrm{x}_{\kappa} \mathrm{x}_{\eta} \otimes x_{\gamma} \\
& -q_{\beta \gamma}(2)_{\widetilde{q}_{\gamma \kappa}} \mathrm{b}_{8} \mathrm{x}_{\gamma}^{2} \mathrm{x}_{\eta} \otimes 1-q_{\beta \gamma} q_{\beta \kappa} q_{\beta \eta} \mathrm{x}_{\gamma} \mathrm{x}_{\kappa} \mathrm{x}_{\eta} \otimes x_{\beta}, \\
& d\left(\mathrm{x}_{\tau} \mathrm{x}_{\gamma} \mathrm{x}_{\kappa} \mathrm{x}_{\eta} \otimes 1\right)=\mathrm{x}_{\tau} \mathrm{x}_{\gamma} \mathrm{x}_{\kappa} \otimes x_{\eta}-q_{\kappa \eta} \mathrm{x}_{\tau} \mathrm{x}_{\gamma} \mathrm{x}_{\eta} \otimes x_{\kappa}+q_{\gamma \kappa} q_{\gamma \eta} \mathrm{x}_{\tau} \mathrm{x}_{\kappa} \mathrm{x}_{\eta} \otimes x_{\gamma} \\
& -q_{\tau \gamma} q_{\tau \kappa}(2)_{\widetilde{q}_{\kappa \eta}} \mathrm{b}_{4} \mathrm{x}_{\gamma} \mathrm{x}_{\kappa}^{2} \otimes x_{\gamma}-q_{\tau \gamma} q_{\tau \kappa} q_{\tau \eta} \mathrm{x}_{\gamma} \mathrm{x}_{\kappa} \mathrm{x}_{\eta} \otimes x_{\tau} \text {, } \\
& d\left(\mathrm{x}_{\tau} \mathrm{x}_{\gamma} \mathrm{x}_{\eta}^{2} \otimes 1\right)=\mathrm{x}_{\tau} \mathrm{x}_{\gamma} \mathrm{x}_{\eta} \otimes x_{\eta}+q_{\gamma \eta}^{2} \mathrm{x}_{\tau} \mathrm{x}_{\eta}^{2} \otimes x_{\gamma}-q_{\tau \gamma} q_{\gamma \eta} \mathrm{b}_{4} \mathrm{x}_{\gamma} \mathrm{x}_{\kappa} \mathrm{x}_{\eta} \otimes x_{\gamma} \\
& -q_{\tau \gamma} q_{\tau \eta}^{2} \mathrm{x}_{\gamma} \mathrm{x}_{\eta}^{2} \otimes x_{\tau} \\
& d\left(\mathrm{x}_{\delta} \mathrm{x}_{\gamma}^{2} \mathrm{x}_{\eta} \otimes 1\right)=\mathrm{x}_{\delta} \mathrm{x}_{\gamma}^{2} \otimes x_{\eta}-q_{\gamma \eta} \mathrm{x}_{\delta} \mathrm{x}_{\gamma} \mathrm{x}_{\eta} \otimes x_{\gamma}-q_{\delta \gamma}^{2} \mathrm{~b}_{2} \mathrm{x}_{\gamma}^{2} \mathrm{x}_{\kappa} \otimes x_{\gamma} x_{\mu} \\
& -q_{\delta \gamma}^{2}(3)_{\widetilde{q}_{\gamma \eta}} \mathrm{b}_{3} \mathrm{x}_{\gamma}^{3} \otimes x_{\nu}-q_{\delta \gamma}^{2} q_{\delta \eta} \mathrm{x}_{\gamma}^{2} \mathrm{x}_{\eta} \otimes x_{\delta} \\
& d\left(\mathrm{x}_{\delta} \mathrm{x}_{\gamma} \mathrm{x}_{\eta}^{2} \otimes 1\right)=\mathrm{x}_{\delta} \mathrm{x}_{\gamma} \mathrm{x}_{\eta} \otimes x_{\eta}+q_{\gamma \eta}^{2} \mathrm{x}_{\delta} \mathrm{x}_{\eta}^{2} \otimes x_{\gamma}-q_{\delta \gamma}(2)_{\widetilde{q}_{\gamma \eta}} \mathrm{b}_{3} \mathrm{~b}_{7} \mathrm{x}_{\gamma}^{2} \mathrm{x}_{\iota} \otimes x_{\kappa} x_{\gamma} \\
& -q_{\delta \gamma} q_{\nu \eta}(2)_{\widetilde{q}_{\gamma \eta}} \mathrm{b}_{3} \mathrm{x}_{\gamma}^{2} \mathrm{x}_{\eta} \otimes x_{\nu}-q_{\delta \gamma} q_{\mu \eta} q_{\gamma \eta} \mathrm{b}_{2} \mathrm{x}_{\gamma} \mathrm{x}_{\kappa} \mathrm{x}_{\eta} \otimes x_{\gamma} x_{\mu}-q_{\delta \gamma} q_{\delta \eta}^{2} \mathrm{x}_{\gamma} \mathrm{x}_{\eta}^{2} \otimes x_{\delta} \text {. }
\end{aligned}
$$


Next we compute differentials of some 5-chains:

$$
\begin{gathered}
d\left(\mathrm{x}_{\alpha} \mathrm{x}_{\beta} \mathrm{x}_{\delta} \mathrm{x}_{\gamma}^{2} \otimes 1\right)=\mathrm{x}_{\alpha} \mathrm{x}_{\beta} \mathrm{x}_{\delta} \mathrm{x}_{\gamma} \otimes x_{\gamma}+q_{\delta \gamma}^{2} \mathrm{x}_{\alpha} \mathrm{x}_{\beta} \mathrm{x}_{\gamma}^{2} \otimes x_{\delta}-q_{\beta \delta} q_{\beta \gamma}^{2} \mathrm{x}_{\alpha} \mathrm{x}_{\delta} \mathrm{x}_{\gamma}^{2} \otimes x_{\beta} \\
+q_{\alpha \beta} q_{\beta \gamma}^{2} q_{\tau \gamma}^{2} \mathrm{~b}_{1} \mathrm{x}_{\beta} \mathrm{x}_{\tau} \mathrm{x}_{\gamma}^{2} \otimes x_{\tau} x_{\beta}+q_{\alpha \beta} q_{\alpha \delta} q_{\alpha \gamma}^{2} \mathrm{x}_{\beta} \mathrm{x}_{\delta} \mathrm{x}_{\gamma}^{2} \otimes x_{\alpha},
\end{gathered}
$$

$$
\begin{aligned}
& d\left(\mathrm{x}_{\alpha} \mathrm{x}_{\beta} \mathrm{x}_{\delta} \mathrm{x}_{\gamma} \mathrm{x}_{\eta} \otimes 1\right)=\mathrm{x}_{\alpha} \mathrm{x}_{\beta} \mathrm{x}_{\delta} \mathrm{x}_{\gamma} \otimes x_{\eta}+q_{\delta \gamma}(2)_{\widetilde{q}_{\gamma \eta}} \mathrm{b}_{3} \mathrm{x}_{\alpha} \mathrm{x}_{\beta} \mathrm{x}_{\gamma}^{2} \otimes x_{\nu} \\
& \quad-q_{\gamma \eta} \mathrm{x}_{\alpha} \mathrm{x}_{\beta} \mathrm{x}_{\delta} \mathrm{x}_{\eta} \otimes x_{\gamma}+q_{\delta \gamma} q_{\delta \eta} \mathrm{x}_{\alpha} \mathrm{x}_{\beta} \mathrm{x}_{\gamma} \mathrm{x}_{\eta} \otimes x_{\delta}+q_{\delta \gamma} \mathrm{b}_{2} \mathrm{x}_{\alpha} \mathrm{x}_{\beta} \mathrm{x}_{\gamma} \mathrm{x}_{\kappa} \otimes x_{\gamma} x_{\mu} \\
& \quad-q_{\beta \delta} q_{\beta \gamma} q_{\beta \eta} \mathrm{x}_{\alpha} \mathrm{x}_{\delta} \mathrm{x}_{\gamma} \mathrm{x}_{\eta} \otimes x_{\beta}+q_{\alpha \beta} q_{\beta \gamma} q_{\beta \eta} q_{\tau \gamma} q_{\tau \eta} \mathrm{b}_{1} \mathrm{x}_{\beta} \mathrm{x}_{\tau} \mathrm{x}_{\gamma} \mathrm{x}_{\eta} \otimes x_{\tau} x_{\beta} \\
& \quad-q_{\delta \gamma} q_{\gamma \beta}^{-1} c_{\beta \eta \gamma}^{(2)} \mathrm{b}_{2} \mathrm{~b}_{8} \mathbf{x}_{\alpha} \mathrm{x}_{\gamma}^{3} \otimes x_{\mu}+q_{\alpha \beta} q_{\beta \gamma} q_{\beta \eta} q_{\tau \gamma} \mathrm{b}_{1} \mathrm{~b}_{4} \mathrm{x}_{\beta} \mathrm{x}_{\tau} \mathrm{x}_{\gamma} \mathrm{x}_{\kappa} \otimes x_{\gamma} x_{\beta} \\
& \quad+q_{\alpha \beta} q_{\alpha \delta} q_{\alpha \gamma} q_{\alpha \eta} \mathrm{x}_{\beta} \mathrm{x}_{\delta} \mathrm{x}_{\gamma} \mathrm{x}_{\eta} \otimes x_{\alpha}+q_{\alpha \beta} q_{\beta \tau} q_{\beta \eta} q_{\eta \gamma}^{-1} q_{\gamma \beta}^{-1} c_{\beta \eta \gamma}^{(2)} \mathrm{b}_{1} \mathrm{~b}_{4} \mathrm{~b}_{8} \mathrm{x}_{\tau} \mathrm{x}_{\gamma}^{3} \otimes x_{\beta},
\end{aligned}
$$

$$
\begin{aligned}
& d\left(\mathrm{x}_{\alpha} \mathrm{x}_{\beta} \mathrm{x}_{\delta} \mathrm{x}_{\eta}^{2} \otimes 1\right)=\mathrm{x}_{\alpha} \mathrm{x}_{\beta} \mathrm{x}_{\delta} \mathrm{x}_{\eta} \otimes x_{\eta}+\mathrm{b}_{3} \mathrm{~b}_{7} \mathrm{x}_{\alpha} \mathrm{x}_{\beta} \mathrm{x}_{\gamma} \mathrm{x}_{\iota} \otimes x_{\kappa} x_{\gamma}+q_{\delta \eta}^{2} \mathrm{x}_{\alpha} \mathrm{x}_{\beta} \mathrm{x}_{\eta}^{2} \otimes x_{\delta} \\
& \quad+q_{\nu \eta} \mathrm{b}_{3} \mathrm{x}_{\alpha} \mathrm{x}_{\beta} \mathrm{x}_{\gamma} \mathrm{x}_{\eta} \otimes x_{\nu}+q_{\mu \gamma} q_{\mu \eta} q_{\gamma \eta} \mathrm{b}_{2} \mathrm{x}_{\alpha} \mathrm{x}_{\beta} \mathrm{x}_{\kappa} \mathrm{x}_{\eta} \otimes x_{\gamma} x_{\mu} \\
& \quad-q_{\beta \delta} q_{\beta \eta}^{2} \mathrm{x}_{\alpha} \mathrm{x}_{\delta} \mathrm{x}_{\eta}^{2} \otimes x_{\beta}+\mathrm{b}_{2} \mathrm{~b}_{6} \mathrm{~b}_{8} \mathrm{x}_{\alpha} \mathrm{x}_{\gamma}^{2} \mathrm{x}_{\iota} \otimes x_{\gamma}-(4)_{q_{\alpha \gamma}} \mathrm{b}_{2} \mathrm{~b}_{6} \mathrm{~b}_{8} \mathrm{~b}_{9} \mathrm{x}_{\gamma}^{4} \otimes 1 \\
& \quad+q_{\mu \eta} \mathrm{b}_{2} \mathrm{~b}_{8} \mathrm{x}_{\alpha} \mathrm{x}_{\gamma}^{2} \mathrm{x}_{\eta} \otimes x_{\mu}+q_{\alpha \beta} q_{\beta \eta}^{2} q_{\tau \eta}^{2} \mathrm{~b}_{1} \mathrm{x}_{\beta} \mathrm{x}_{\tau} \mathrm{x}_{\eta}^{2} \otimes x_{\tau} x_{\beta} \\
& \quad+q_{\alpha \beta} q_{\beta \gamma} q_{\beta \eta}^{2} q_{\gamma \eta} \mathrm{b}_{1} \mathrm{~b}_{4} \mathrm{x}_{\beta} \mathrm{x}_{\tau} \mathrm{x}_{\kappa} \mathrm{x}_{\eta} \otimes x_{\gamma} x_{\beta}+q_{\alpha \beta} q_{\alpha \delta} q_{\alpha \eta}^{2} \mathrm{x}_{\beta} \mathrm{x}_{\delta} \mathrm{x}_{\eta}^{2} \otimes x_{\alpha} \\
& \quad-q_{\beta \tau} q_{\alpha \beta} q_{\beta \eta}^{2} \mathrm{~b}_{1} \mathrm{~b}_{4} \mathrm{~b}_{8} \mathrm{x}_{\tau} \mathrm{x}_{\gamma}^{2} \mathrm{x}_{\eta} \otimes x_{\beta},
\end{aligned}
$$

$$
\begin{gathered}
d\left(\mathrm{x}_{\alpha} \mathrm{x}_{\beta} \mathrm{x}_{\gamma}^{2} \mathrm{x}_{\kappa} \otimes 1\right)=\mathrm{x}_{\alpha} \mathrm{x}_{\beta} \mathrm{x}_{\gamma}^{2} \otimes x_{\kappa}-q_{\gamma \kappa} \mathrm{x}_{\alpha} \mathrm{x}_{\beta} \mathrm{x}_{\gamma} \mathrm{x}_{\kappa} \otimes x_{\gamma}-q_{\beta \gamma}^{2} q_{\beta \kappa} \mathrm{x}_{\alpha} \mathrm{x}_{\gamma}^{2} \mathrm{x}_{\kappa} \otimes x_{\beta} \\
-q_{\gamma \beta}^{-2}(3)_{\widetilde{q}_{\beta \gamma}} \mathrm{b}_{8} \mathrm{x}_{\alpha} \mathrm{x}_{\gamma}^{3} \otimes 1+q_{\alpha \beta} q_{\alpha \gamma}^{2} q_{\alpha \kappa} \mathrm{x}_{\beta} \mathrm{x}_{\gamma}^{2} \mathrm{x}_{\kappa} \otimes x_{\alpha},
\end{gathered}
$$

$$
\begin{aligned}
& d\left(\mathrm{x}_{\alpha} \mathrm{x}_{\beta} \mathrm{x}_{\gamma} \mathrm{x}_{\kappa} \mathrm{x}_{\eta} \otimes 1\right)=\mathrm{x}_{\alpha} \mathrm{x}_{\beta} \mathrm{x}_{\gamma} \mathrm{x}_{\kappa} \otimes x_{\eta}-q_{\kappa \eta} \mathrm{x}_{\alpha} \mathrm{x}_{\beta} \mathrm{x}_{\gamma} \mathrm{x}_{\eta} \otimes x_{\kappa} \\
& \quad-q_{\gamma \beta}^{-1}(2)_{\widetilde{q}_{\beta \gamma}} \mathrm{b}_{8} \mathrm{x}_{\alpha} \mathrm{x}_{\gamma}^{2} \mathrm{x}_{\eta} \otimes 1+q_{\gamma \kappa} q_{\gamma \eta} \mathrm{x}_{\alpha} \mathrm{x}_{\beta} \mathrm{x}_{\kappa} \mathrm{x}_{\eta} \otimes x_{\gamma} \\
& \quad-q_{\beta \gamma} q_{\beta \kappa} q_{\beta \eta} \mathrm{x}_{\alpha} \mathrm{x}_{\gamma} \mathrm{x}_{\kappa} \mathrm{x}_{\eta} \otimes x_{\beta}+q_{\alpha \beta} q_{\alpha \gamma} q_{\alpha \kappa} q_{\alpha \eta} \mathrm{x}_{\beta} \mathrm{x}_{\gamma} \mathrm{x}_{\kappa} \mathrm{x}_{\eta} \otimes x_{\alpha},
\end{aligned}
$$

$$
\begin{gathered}
d\left(\mathrm{x}_{\alpha} \mathrm{x}_{\delta} \mathrm{x}_{\gamma}^{2} \mathrm{x}_{\eta} \otimes 1\right)=\mathrm{x}_{\alpha} \mathrm{x}_{\delta} \mathrm{x}_{\gamma}^{2} \otimes x_{\eta}-q_{\gamma \eta} \mathrm{x}_{\alpha} \mathrm{x}_{\delta} \mathrm{x}_{\gamma} \mathrm{x}_{\eta} \otimes x_{\gamma}-q_{\delta \gamma}^{2}(2)_{\widetilde{q}_{\gamma \eta}} \mathrm{b}_{3} \mathrm{x}_{\alpha} \mathrm{x}_{\gamma}^{3} \otimes x_{\nu} \\
\quad-q_{\delta \gamma}^{2} \mathrm{~b}_{2} \mathrm{x}_{\alpha} \mathrm{x}_{\gamma}^{2} \mathrm{x}_{\kappa} \otimes x_{\gamma} x_{\mu}-q_{\delta \gamma}^{2} q_{\delta \eta} \mathrm{x}_{\alpha} \mathrm{x}_{\gamma}^{2} \mathrm{x}_{\eta} \otimes x_{\delta}+q_{\alpha \delta} q_{\alpha \gamma}^{2} q_{\alpha \eta} \mathrm{x}_{\delta} \mathrm{x}_{\gamma}^{2} \mathrm{x}_{\eta} \otimes x_{\alpha} \\
+q_{\beta \eta} q_{\tau \gamma}^{2} q_{\beta \gamma}^{2} q_{\tau \eta} \mathrm{b}_{1} \mathrm{x}_{\tau} \mathrm{x}_{\gamma}^{2} \mathrm{x}_{\eta} \otimes x_{\tau} x_{\beta}+q_{\beta \eta} q_{\tau \gamma}^{2} q_{\beta \gamma}^{2} \mathrm{~b}_{1} \mathrm{~b}_{4} \mathrm{x}_{\tau} \mathrm{x}_{\gamma}^{2} \mathrm{x}_{\kappa} \otimes x_{\gamma} x_{\beta}
\end{gathered}
$$


$(10.1 .123)$

$$
\begin{aligned}
& d\left(\mathrm{x}_{\alpha} \mathrm{x}_{\delta} \mathrm{x}_{\gamma} \mathrm{x}_{\eta}^{2} \otimes 1\right)=\mathrm{x}_{\alpha} \mathrm{x}_{\delta} \mathrm{x}_{\gamma} \mathrm{x}_{\eta} \otimes x_{\eta}-q_{\delta \gamma} q_{\nu \eta}(2)_{\widetilde{q}_{\gamma \eta}} \mathrm{b}_{3} \mathrm{x}_{\alpha} \mathrm{x}_{\gamma}^{2} \mathrm{x}_{\eta} \otimes x_{\nu} \\
& \quad-q_{\delta \gamma}(2)_{\widetilde{q}_{\gamma \eta}} \mathrm{b}_{3} \mathrm{~b}_{7} \mathrm{x}_{\alpha} \mathrm{x}_{\gamma}^{2} \mathrm{x}_{\iota} \otimes x_{\kappa} x_{\gamma}+q_{\delta \gamma} \mathrm{c}_{\alpha \eta \gamma}^{(2)} \mathrm{b}_{3} \mathrm{~b}_{7} \mathrm{~b}_{9} \mathrm{x}_{\gamma}^{4} \otimes x_{\kappa} \\
& \quad-q_{\delta \gamma} q_{\mu \eta} q_{\gamma \eta} \mathrm{b}_{2} \mathrm{x}_{\alpha} \mathrm{x}_{\gamma} \mathrm{x}_{\kappa} \mathrm{x}_{\eta} \otimes x_{\gamma} x_{\mu}+q_{\gamma \eta}^{2} \mathrm{x}_{\alpha} \mathrm{x}_{\delta} \mathrm{x}_{\eta}^{2} \otimes x_{\gamma}-q_{\delta \gamma} q_{\delta \eta}^{2} \mathrm{x}_{\alpha} \mathrm{x}_{\gamma} \mathrm{x}_{\eta}^{2} \otimes x_{\delta} \\
& \quad+q_{\beta \gamma} q_{\beta \eta}^{2} q_{\tau \gamma} q_{\tau \eta}^{2} \mathrm{~b}_{1} \mathrm{x}_{\tau} \mathrm{x}_{\gamma} \mathrm{x}_{\eta}^{2} \otimes x_{\tau} x_{\beta}+q_{\tau \gamma} q_{\beta \gamma} q_{\beta \eta}^{2} q_{\gamma \eta} \mathrm{b}_{1} \mathrm{~b}_{4} \mathrm{x}_{\tau} \mathrm{x}_{\gamma} \mathrm{x}_{\kappa} \mathrm{x}_{\eta} \otimes x_{\gamma} x_{\beta} \\
& \quad+q_{\alpha \gamma}^{2} q_{\alpha \kappa} q_{\beta \eta} q_{\delta \gamma} \mathrm{b}_{2} \mathrm{~b}_{4} \mathrm{~b}_{5} \mathrm{x}_{\gamma}^{2} \mathrm{x}_{\kappa}^{2} \otimes x_{\gamma} x_{\beta}+q_{\alpha \delta} q_{\alpha \gamma} q_{\alpha \eta}^{2} \mathrm{x}_{\delta} \mathrm{x}_{\gamma} \mathrm{x}_{\eta}^{2} \otimes x_{\alpha} \\
& \quad+q_{\alpha \gamma}^{2} q_{\alpha \kappa} q_{\beta \eta} q_{\delta \gamma} q_{\tau \eta} \mathrm{b}_{2} \mathrm{~b}_{5} \mathrm{x}_{\gamma}^{2} \mathrm{x}_{\kappa} \mathrm{x}_{\eta} \otimes x_{\tau} x_{\beta},
\end{aligned}
$$

$(10.1 .124)$

$$
\begin{aligned}
& d\left(\mathrm{x}_{\beta} \mathrm{x}_{\tau} \mathrm{x}_{\gamma}^{2} \mathrm{x}_{\kappa} \otimes 1\right)=\mathrm{x}_{\beta} \mathrm{x}_{\tau} \mathrm{x}_{\gamma}^{2} \otimes x_{\kappa}-q_{\gamma \kappa} \mathrm{x}_{\beta} \mathrm{x}_{\tau} \mathrm{x}_{\gamma} \mathrm{x}_{\kappa} \otimes x_{\gamma}-q_{\tau \gamma}^{2} q_{\tau \kappa} \mathrm{x}_{\beta} \mathrm{x}_{\gamma}^{2} \mathrm{x}_{\kappa} \otimes x_{\tau} \\
& \quad+q_{\beta \tau} q_{\gamma \beta}^{-2}(3)_{\widetilde{q}_{\beta \gamma}} \mathrm{b}_{8} \mathrm{x}_{\tau} \mathrm{x}_{\gamma}^{3} \otimes 1+q_{\beta \tau} q_{\beta \gamma}^{2} q_{\beta \kappa} \mathrm{x}_{\tau} \mathrm{x}_{\gamma}^{2} \mathrm{x}_{\kappa} \otimes x_{\beta}
\end{aligned}
$$

$$
\begin{aligned}
& d\left(\mathrm{x}_{\beta} \mathrm{x}_{\tau} \mathrm{x}_{\gamma} \mathrm{x}_{\kappa} \mathrm{x}_{\eta} \otimes 1\right)=\mathrm{x}_{\beta} \mathrm{x}_{\tau} \mathrm{x}_{\gamma} \mathrm{x}_{\kappa} \otimes x_{\eta}-q_{\kappa \eta} \mathrm{x}_{\beta} \mathrm{x}_{\tau} \mathrm{x}_{\gamma} \mathrm{x}_{\eta} \otimes x_{\kappa} \\
& -q_{\tau \gamma} q_{\tau \kappa}(2)_{\widetilde{q}_{\kappa \eta}} \mathrm{b}_{4} \mathrm{x}_{\beta} \mathrm{x}_{\gamma} \mathrm{x}_{\kappa}^{2} \otimes x_{\gamma}+q_{\gamma \kappa} q_{\gamma \eta} \mathrm{x}_{\beta} \mathrm{x}_{\tau} \mathrm{x}_{\kappa} \mathrm{x}_{\eta} \otimes x_{\gamma} \\
& -q_{\tau \gamma} q_{\tau \kappa} q_{\tau \eta} \mathrm{x}_{\beta} \mathrm{x}_{\gamma} \mathrm{x}_{\kappa} \mathrm{x}_{\eta} \otimes x_{\tau}+q_{\beta \tau} q_{\gamma \beta}^{-1}(2)_{\widetilde{q}_{\beta \gamma}} \mathrm{b}_{8} \mathrm{x}_{\tau} \mathrm{x}_{\gamma}^{2} \mathrm{x}_{\eta} \otimes 1 \\
& +q_{\beta \tau} q_{\beta \gamma} q_{\beta \kappa} q_{\beta \eta} \mathrm{x}_{\tau} \mathrm{x}_{\gamma} \mathrm{x}_{\kappa} \mathrm{x}_{\eta} \otimes x_{\beta}-q_{\kappa \eta} q_{\eta \gamma}^{-1}(2)_{\widetilde{q}_{\gamma \eta}} \mathrm{b}_{4} \mathrm{~b}_{8} \mathrm{x}_{\gamma}^{3} \mathrm{x}_{\kappa} \otimes 1,
\end{aligned}
$$

$$
\begin{gathered}
d\left(\mathrm{x}_{\beta} \mathrm{x}_{\tau} \mathrm{x}_{\gamma} \mathrm{x}_{\eta}^{2} \otimes 1\right)=\mathrm{x}_{\beta} \mathrm{x}_{\tau} \mathrm{x}_{\gamma} \mathrm{x}_{\eta} \otimes x_{\eta}+q_{\gamma \eta}^{2} \mathrm{x}_{\beta} \mathrm{x}_{\tau} \mathrm{x}_{\eta}^{2} \otimes x_{\gamma}-q_{\tau \gamma} q_{\gamma \eta} \mathrm{b}_{4} \mathrm{x}_{\beta} \mathrm{x}_{\gamma} \mathrm{x}_{\kappa} \mathrm{x}_{\eta} \otimes x_{\gamma} \\
-q_{\tau \gamma} q_{\tau \eta}^{2} \mathrm{x}_{\beta} \mathrm{x}_{\gamma} \mathrm{x}_{\eta}^{2} \otimes x_{\tau}+q_{\beta \tau} q_{\beta \gamma} q_{\beta \eta}^{2} \mathrm{x}_{\tau} \mathrm{x}_{\gamma} \mathrm{x}_{\eta}^{2} \otimes x_{\beta}+q_{\gamma \eta}^{-1} c_{-\beta-\eta \gamma}^{(2)} \mathrm{b}_{4} \mathrm{~b}_{8} \mathrm{x}_{\gamma}^{3} \mathrm{x}_{\eta} \otimes 1
\end{gathered}
$$

$$
\begin{aligned}
& d\left(\mathrm{x}_{\beta} \mathrm{x}_{\delta} \mathrm{x}_{\gamma}^{2} \mathrm{x}_{\eta} \otimes 1\right)=\mathrm{x}_{\beta} \mathrm{x}_{\delta} \mathrm{x}_{\gamma}^{2} \otimes x_{\eta}-q_{\gamma \eta} \mathrm{x}_{\beta} \mathrm{x}_{\delta} \mathrm{x}_{\gamma} \mathrm{x}_{\eta} \otimes x_{\gamma}-q_{\delta \gamma}^{2} q_{\delta \eta} \mathrm{x}_{\beta} \mathrm{x}_{\gamma}^{2} \mathrm{x}_{\eta} \otimes x_{\delta} \\
& \quad-q_{\delta \gamma}^{2} \mathrm{~b}_{2} \mathrm{x}_{\beta} \mathrm{x}_{\gamma}^{2} \mathrm{x}_{\kappa} \otimes x_{\gamma} x_{\mu}-q_{\delta \gamma}^{2}(3)_{\widetilde{q}_{\gamma \eta}} \mathrm{b}_{3} \mathrm{x}_{\beta} \mathrm{x}_{\gamma}^{3} \otimes x_{\nu} \\
& \quad+q_{\beta \delta} q_{\beta \gamma}^{2} q_{\beta \eta} \mathrm{x}_{\delta} \mathrm{x}_{\gamma}^{2} \mathrm{x}_{\eta} \otimes x_{\beta}-q_{\beta \gamma} q_{\gamma \beta}^{-1} q_{\delta \gamma}^{2} \mathrm{c}_{\beta \eta}^{(3)} \mathrm{b}_{2} \mathrm{~b}_{8} \mathrm{x}_{\gamma}^{4} \otimes x_{\mu}
\end{aligned}
$$

$$
\begin{aligned}
& d\left(\mathrm{x}_{\beta} \mathrm{x}_{\delta} \mathrm{x}_{\gamma} \mathrm{x}_{\eta}^{2} \otimes 1\right)=\mathrm{x}_{\beta} \mathrm{x}_{\delta} \mathrm{x}_{\gamma} \mathrm{x}_{\eta} \otimes x_{\eta}-q_{\delta \gamma}(2)_{\widetilde{q}_{\gamma \eta}} \mathrm{b}_{3} \mathrm{~b}_{7} \mathrm{x}_{\beta} \mathrm{x}_{\gamma}^{2} \mathrm{x}_{\iota} \otimes x_{\kappa} x_{\gamma} \\
& -q_{\delta \gamma}(2)_{\widetilde{q}_{\gamma \eta}} q_{\nu \eta} \mathrm{b}_{3} \mathrm{x}_{\beta} \mathrm{x}_{\gamma}^{2} \mathrm{x}_{\eta} \otimes x_{\nu}-q_{\delta \gamma} q_{\mu \eta} q_{\gamma \eta} \mathrm{b}_{2} \mathrm{x}_{\beta} \mathrm{x}_{\gamma} \mathrm{x}_{\kappa} \mathrm{x}_{\eta} \otimes x_{\gamma} x_{\mu} \\
& +q_{\gamma \eta}^{2} \mathrm{x}_{\beta} \mathrm{x}_{\delta} \mathrm{x}_{\eta}^{2} \otimes x_{\gamma}-q_{\delta \gamma} q_{\delta \eta}^{2} \mathrm{x}_{\beta} \mathrm{x}_{\gamma} \mathbf{x}_{\eta}^{2} \otimes x_{\delta}+q_{\beta \delta} q_{\beta \gamma} q_{\beta \eta}^{2} \mathrm{x}_{\delta} \mathrm{x}_{\gamma} \mathbf{x}_{\eta}^{2} \otimes x_{\beta} \\
& -q_{\beta \gamma} q_{\delta \gamma}(2)_{\widetilde{q}_{\gamma \eta}} \mathrm{b}_{2} \mathrm{~b}_{6} \mathrm{~b}_{8} \mathrm{x}_{\gamma}^{3} \mathrm{x}_{\iota} \otimes x_{\gamma}-q_{\beta \gamma} q_{\delta \gamma} q_{\mu \eta}(2)_{\widetilde{q}_{\gamma \eta}} \mathrm{b}_{2} \mathrm{~b}_{8} \mathrm{x}_{\gamma}^{3} \mathrm{x}_{\eta} \otimes x_{\mu},
\end{aligned}
$$

$$
\begin{gathered}
d\left(\mathrm{x}_{\beta} \mathrm{x}_{\gamma}^{2} \mathrm{x}_{\kappa}^{2} \otimes 1\right)=\mathrm{x}_{\beta} \mathrm{x}_{\gamma}^{2} \mathrm{x}_{\kappa} \otimes x_{\kappa}+q_{\gamma \kappa}^{2} \mathrm{x}_{\beta} \mathrm{x}_{\gamma} \mathrm{x}_{\kappa}^{2} \otimes x_{\gamma} \\
+q_{\beta \gamma}^{2}(3)_{\widetilde{q}_{\beta \gamma}} \mathrm{b}_{8} \mathrm{x}_{\gamma}^{3} \mathrm{x}_{\kappa} \otimes 1+q_{\beta \kappa}^{2} q_{\beta \gamma}^{2} \mathrm{x}_{\gamma}^{2} \mathrm{x}_{\kappa}^{2} \otimes x_{\beta}
\end{gathered}
$$

$$
\begin{aligned}
& d\left(\mathrm{x}_{\beta} \mathrm{x}_{\gamma}^{2} \mathrm{x}_{\kappa} \mathrm{x}_{\eta} \otimes 1\right)=\mathrm{x}_{\beta} \mathrm{x}_{\gamma}^{2} \mathrm{x}_{\kappa} \otimes x_{\eta}-q_{\kappa \eta} \mathrm{x}_{\beta} \mathrm{x}_{\gamma}^{2} \mathrm{x}_{\eta} \otimes x_{\kappa}+q_{\gamma \kappa} q_{\gamma \eta} \mathrm{x}_{\beta} \mathrm{x}_{\gamma} \mathrm{x}_{\kappa} \mathrm{x}_{\eta} \otimes x_{\gamma} \\
& \quad+q_{\beta \kappa} q_{\beta \gamma}^{2} q_{\beta \eta} \mathrm{x}_{\gamma}^{2} \mathbf{x}_{\kappa} \mathrm{x}_{\eta} \otimes x_{\beta}+q_{\beta \gamma}^{2}(3)_{\widetilde{q}_{\beta \gamma}} \mathrm{b}_{8} \mathrm{x}_{\gamma}^{3} \mathrm{x}_{\eta} \otimes 1,
\end{aligned}
$$


$(10.1 .131)$

$$
\begin{aligned}
& d\left(\mathrm{x}_{\tau} \mathrm{x}_{\gamma}^{2} \mathrm{x}_{\kappa} \mathrm{x}_{\eta} \otimes 1\right)=\mathrm{x}_{\tau} \mathrm{x}_{\gamma}^{2} \mathrm{x}_{\kappa} \otimes x_{\eta}-q_{\kappa \eta} \mathrm{x}_{\tau} \mathrm{x}_{\gamma}^{2} \mathrm{x}_{\eta} \otimes x_{\kappa}+q_{\gamma \kappa} q_{\gamma \eta} \mathrm{x}_{\tau} \mathrm{x}_{\gamma} \mathrm{x}_{\kappa} \mathrm{x}_{\eta} \otimes x_{\gamma} \\
& +q_{\tau \gamma}^{2} q_{\tau \kappa}(2)_{\widetilde{q}_{\kappa \eta}} \mathrm{b}_{4} \mathrm{x}_{\gamma}^{2} \mathrm{x}_{\kappa}^{2} \otimes x_{\gamma}+q_{\tau \gamma}^{2} q_{\tau \kappa} q_{\tau \eta} \mathrm{x}_{\gamma}^{2} \mathrm{x}_{\kappa} \mathrm{x}_{\eta} \otimes x_{\tau}
\end{aligned}
$$

$$
\begin{array}{r}
d\left(\mathrm{x}_{\tau} \mathrm{x}_{\gamma}^{2} \mathrm{x}_{\eta}^{2} \otimes 1\right)=\mathrm{x}_{\tau} \mathrm{x}_{\gamma}^{2} \mathrm{x}_{\eta} \otimes x_{\eta}+q_{\gamma \eta}^{2} \mathrm{x}_{\tau} \mathrm{x}_{\gamma} \mathrm{x}_{\eta}^{2} \otimes x_{\gamma} \\
+q_{\tau \gamma}^{2} q_{\gamma \eta} \mathrm{b}_{4} \mathrm{x}_{\gamma}^{2} \mathrm{x}_{\kappa} \mathrm{x}_{\eta} \otimes x_{\gamma}+q_{\tau \gamma}^{2} q_{\tau \eta}^{2} \mathrm{x}_{\gamma}^{2} \mathrm{x}_{\eta}^{2} \otimes x_{\tau}
\end{array}
$$

$$
\begin{aligned}
& d\left(\mathrm{x}_{\delta} \mathrm{x}_{\gamma}^{2} \mathrm{x}_{\eta}^{2} \otimes 1\right)=\mathrm{x}_{\delta} \mathrm{x}_{\gamma}^{2} \mathrm{x}_{\eta} \otimes x_{\eta}+q_{\gamma \eta}^{2} \mathrm{x}_{\delta} \mathrm{x}_{\gamma} \mathrm{x}_{\eta}^{2} \otimes x_{\gamma} \\
& \quad+q_{\delta \gamma}^{2}(3)_{\widetilde{q}_{\gamma \eta}} \mathrm{b}_{3} \mathrm{~b}_{7} \mathrm{x}_{\gamma}^{3} \mathrm{x}_{\iota} \otimes x_{\kappa} x_{\gamma}+q_{\delta \gamma}^{2} q_{\nu \eta}(3)_{\widetilde{q}_{\gamma \eta}} \mathrm{b}_{3} \mathrm{x}_{\gamma}^{3} \mathrm{x}_{\eta} \otimes x_{\nu} \\
& \quad+q_{\delta \gamma}^{2} q_{\mu \eta} q_{\gamma \eta} \mathrm{b}_{2} \mathrm{x}_{\gamma}^{2} \mathrm{x}_{\kappa} \mathrm{x}_{\eta} \otimes x_{\gamma} x_{\mu}+q_{\delta \gamma}^{2} q_{\delta \eta}^{2} \mathrm{x}_{\gamma}^{2} \mathrm{x}_{\eta}^{2} \otimes x_{\delta}
\end{aligned}
$$

First we check (10.1.117). Using (10.1.99), Remark 10.1.2, (10.1.100),

$$
\begin{aligned}
d\left(\mathrm{x}_{\alpha} \mathrm{x}_{\beta} \mathrm{x}_{\delta} \mathrm{x}_{\gamma}^{2} \otimes 1\right)=\mathrm{x}_{\alpha} \mathrm{x}_{\beta} \mathrm{x}_{\delta} \mathrm{x}_{\gamma} \otimes x_{\gamma}-s\left(-q_{\delta \gamma}^{2} \mathrm{x}_{\alpha} \mathrm{x}_{\beta} \mathrm{x}_{\gamma} \otimes x_{\gamma} x_{\delta}+q_{\beta \delta} q_{\beta \gamma}^{2} \mathrm{x}_{\alpha} \mathrm{x}_{\delta} \mathrm{x}_{\gamma} \otimes x_{\gamma} x_{\beta}\right. \\
\left.\quad-q_{\alpha \beta} q_{\beta \gamma}^{2} q_{\tau \gamma}^{2} \mathrm{~b}_{1} \mathrm{x}_{\beta} \mathrm{x}_{\tau} \mathrm{x}_{\gamma} \otimes x_{\gamma} x_{\tau} x_{\beta}-q_{\alpha \beta} q_{\alpha \delta} q_{\alpha \gamma}^{2} \mathrm{x}_{\beta} \mathrm{x}_{\delta} \mathrm{x}_{\gamma} \otimes x_{\gamma} x_{\alpha}\right) \\
=\mathrm{x}_{\alpha} \mathrm{x}_{\beta} \mathrm{x}_{\delta} \mathrm{x}_{\gamma} \otimes x_{\gamma}+q_{\delta \gamma}^{2} \mathrm{x}_{\alpha} \mathrm{x}_{\beta} \mathrm{x}_{\gamma}^{2} \otimes x_{\delta}-q_{\beta \delta} q_{\beta \gamma}^{2} \mathrm{x}_{\alpha} \mathrm{x}_{\delta} \mathrm{x}_{\gamma}^{2} \otimes x_{\beta} \\
\quad-s\left(-q_{\alpha \beta} q_{\beta \gamma}^{2} q_{\tau \gamma}^{2} \mathrm{~b}_{1} \mathrm{x}_{\beta} \mathrm{x}_{\tau} \mathrm{x}_{\gamma} \otimes x_{\gamma} x_{\tau} x_{\beta}-q_{\alpha \beta} q_{\alpha \delta} q_{\alpha \gamma}^{2} \mathrm{x}_{\beta} \mathrm{x}_{\delta} \mathrm{x}_{\gamma} \otimes x_{\gamma} x_{\alpha}\right. \\
\quad-q_{\alpha \beta} q_{\alpha \gamma}^{2} q_{\alpha \delta} q_{\delta \gamma}^{2} \mathrm{x}_{\beta} \mathrm{x}_{\gamma}^{2} \otimes x_{\delta} x_{\alpha}-q_{\alpha \beta} q_{\alpha \gamma}^{2} q_{\delta \gamma}^{2} \mathrm{~b}_{1} \mathrm{x}_{\beta} \mathrm{x}_{\gamma}^{2} \otimes x_{\tau}^{2} x_{\beta} \\
\left.\quad+q_{\beta \delta} q_{\beta \gamma}^{4} q_{\tau \gamma}^{2} \mathrm{~b}_{1} \mathrm{x}_{\tau} \mathrm{x}_{\gamma}^{2} \otimes x_{\tau} x_{\beta}^{2}+q_{\alpha \beta} q_{\alpha \delta} q_{\alpha \gamma}^{2} q_{\beta \delta} q_{\beta \gamma}^{2} \mathrm{x}_{\delta} \mathrm{x}_{\gamma}^{2} \otimes x_{\beta} x_{\alpha}\right) \\
=\mathrm{x}_{\alpha} \mathrm{x}_{\beta} \mathrm{x}_{\delta} \mathrm{x}_{\gamma} \otimes x_{\gamma}+q_{\delta \gamma}^{2} \mathrm{x}_{\alpha} \mathrm{x}_{\beta} \mathrm{x}_{\gamma}^{2} \otimes x_{\delta}-q_{\beta \delta} q_{\beta \gamma}^{2} \mathrm{x}_{\alpha} \mathrm{x}_{\delta} \mathrm{x}_{\gamma}^{2} \otimes x_{\beta} \\
\quad+q_{\alpha \beta} q_{\beta \gamma}^{2} q_{\tau \gamma}^{2} \mathrm{~b}_{1} \mathrm{x}_{\beta} \mathrm{x}_{\tau} \mathrm{x}_{\gamma}^{2} \otimes x_{\tau} x_{\beta}+q_{\alpha \beta} q_{\alpha \delta} q_{\alpha \gamma}^{2} \mathrm{x}_{\beta} \mathrm{x}_{\delta} \mathrm{x}_{\gamma}^{2} \otimes x_{\alpha} .
\end{aligned}
$$

For (10.1.118), we use (10.1.99), (10.1.101), (10.1.104), (10.1.106), (10.1.86), Remark 10.1.2 (10.1.109), $\mathrm{x}_{\alpha} \mathrm{x}_{\gamma}^{2} \otimes x_{\nu} x_{\beta}=s\left(\mathrm{x}_{\alpha} \mathrm{x}_{\gamma} \otimes x_{\gamma} x_{\nu} x_{\beta}\right), \mathrm{x}_{\gamma}^{3} \otimes x_{\mu} x_{\alpha}=s\left(\mathrm{x}_{\gamma}^{2} \otimes x_{\gamma} x_{\mu} x_{\alpha}\right)$,

$$
\begin{aligned}
& d\left(\mathrm{x}_{\alpha} \mathrm{x}_{\beta} \mathrm{x}_{\delta} \mathrm{x}_{\gamma} \mathrm{x}_{\eta} \otimes 1\right)=\mathrm{x}_{\alpha} \mathrm{x}_{\beta} \mathrm{x}_{\delta} \mathrm{x}_{\gamma} \otimes x_{\eta}-s\left(q_{\gamma \eta} \mathrm{x}_{\alpha} \mathrm{x}_{\beta} \mathrm{x}_{\delta} \otimes x_{\eta} x_{\gamma}-q_{\delta \gamma} q_{\delta \eta} \mathrm{x}_{\alpha} \mathrm{x}_{\beta} \mathrm{x}_{\gamma} \otimes x_{\eta} x_{\delta}\right. \\
& +q_{\beta \delta} q_{\beta \gamma} q_{\beta \eta} \mathbf{x}_{\alpha} \mathbf{x}_{\delta} \mathbf{x}_{\gamma} \otimes x_{\eta} x_{\beta}-q_{\delta \gamma} \mathrm{b}_{2} \mathbf{x}_{\alpha} \mathbf{x}_{\beta} \mathbf{x}_{\gamma} \otimes x_{\kappa} x_{\gamma} x_{\mu}-q_{\delta \gamma} \mathrm{b}_{3} \mathbf{x}_{\alpha} \mathbf{x}_{\beta} \mathbf{x}_{\gamma} \otimes x_{\gamma} x_{\nu} \\
& -q_{\alpha \beta} q_{\beta \gamma} q_{\beta \eta} q_{\tau \gamma} q_{\tau \eta} \mathrm{b}_{1} \mathrm{x}_{\beta} \mathrm{x}_{\tau} \mathrm{x}_{\gamma} \otimes x_{\eta} x_{\tau} x_{\beta}-q_{\alpha \beta} q_{\beta \gamma} q_{\beta \eta} q_{\tau \gamma} \mathrm{b}_{1} \mathrm{~b}_{4} \mathrm{x}_{\beta} \mathrm{x}_{\tau} \mathrm{x}_{\gamma} \otimes x_{\kappa} x_{\gamma} x_{\beta} \\
& \left.-q_{\alpha \beta} q_{\alpha \delta} q_{\alpha \gamma} q_{\alpha \eta} \mathrm{x}_{\beta} \mathrm{x}_{\delta} \mathrm{x}_{\gamma} \otimes x_{\eta} x_{\alpha}\right) \\
& =\mathbf{x}_{\alpha} \mathbf{x}_{\beta} \mathbf{x}_{\delta} \mathbf{x}_{\gamma} \otimes x_{\eta}-q_{\gamma \eta} \mathbf{x}_{\alpha} \mathbf{x}_{\beta} \mathbf{x}_{\delta} \mathbf{x}_{\eta} \otimes x_{\gamma}+q_{\delta \gamma} q_{\delta \eta} \mathbf{x}_{\alpha} \mathbf{x}_{\beta} \mathbf{x}_{\gamma} \mathbf{x}_{\eta} \otimes x_{\delta}+q_{\delta \gamma} \mathrm{b}_{2} \mathbf{x}_{\alpha} \mathbf{x}_{\beta} \mathbf{x}_{\gamma} \mathbf{x}_{\kappa} \otimes x_{\gamma} x_{\mu} \\
& +q_{\delta \gamma}(2)_{\widetilde{q}_{\gamma \eta}} \mathrm{b}_{3} \mathrm{x}_{\alpha} \mathrm{x}_{\beta} \mathrm{x}_{\gamma}^{2} \otimes x_{\nu}-q_{\beta \delta} q_{\beta \gamma} q_{\beta \eta} \mathrm{x}_{\alpha} \mathrm{x}_{\delta} \mathrm{x}_{\gamma} \mathrm{x}_{\eta} \otimes x_{\beta}-q_{\delta \gamma} q_{\gamma \beta}^{-1} \mathrm{c}_{\beta \eta \gamma}^{(2)} \mathrm{b}_{2} \mathrm{~b}_{8} \mathrm{x}_{\alpha} \mathrm{x}_{\gamma}^{3} \otimes x_{\mu} \\
& +q_{\alpha \beta} q_{\beta \gamma} q_{\beta \eta} q_{\tau \gamma} q_{\tau \eta} \mathrm{b}_{1} \mathbf{x}_{\beta} \mathrm{x}_{\tau} \mathrm{x}_{\gamma} \mathrm{x}_{\eta} \otimes x_{\tau} x_{\beta}+q_{\alpha \beta} q_{\beta \gamma} q_{\beta \eta} q_{\tau \gamma} \mathrm{b}_{1} \mathrm{~b}_{4} \mathrm{x}_{\beta} \mathrm{x}_{\tau} \mathrm{x}_{\gamma} \mathrm{x}_{\kappa} \otimes x_{\gamma} x_{\beta} \\
& +q_{\alpha \beta} q_{\alpha \delta} q_{\alpha \gamma} q_{\alpha \eta} \mathrm{x}_{\beta} \mathrm{x}_{\delta} \mathrm{x}_{\gamma} \mathrm{x}_{\eta} \otimes x_{\alpha}+q_{\alpha \beta} q_{\beta \tau} q_{\beta \eta} q_{\eta \gamma}^{-1} q_{\gamma \beta}^{-1} \mathrm{c}_{\beta \eta \gamma}^{(2)} \mathrm{b}_{1} \mathrm{~b}_{4} \mathrm{~b}_{8} \mathrm{x}_{\tau} \mathrm{x}_{\gamma}^{3} \otimes x_{\beta} .
\end{aligned}
$$

For (10.1.119), we use (10.1.101), (10.1.102), (10.1.105), (10.1.83), (10.1.107), (10.1.108), Remark 10.1.2, (10.1.110) and (10.1.84):

$$
\begin{aligned}
& d\left(\mathrm{x}_{\alpha} \mathrm{x}_{\beta} \mathrm{x}_{\delta} \mathrm{x}_{\eta}^{2} \otimes 1\right)=\mathrm{x}_{\alpha} \mathrm{x}_{\beta} \mathrm{x}_{\delta} \mathrm{x}_{\eta} \otimes x_{\eta}-s\left(-q_{\nu \eta} \mathrm{b}_{3} \mathrm{x}_{\alpha} \mathrm{x}_{\beta} \mathrm{x}_{\gamma} \otimes x_{\eta} x_{\nu}-\mathrm{b}_{3} \mathrm{~b}_{7} \mathrm{x}_{\alpha} \mathrm{x}_{\beta} \mathrm{x}_{\gamma} \otimes x_{\iota} x_{\kappa} x_{\gamma}\right. \\
& -q_{\delta \eta}^{2} \mathrm{x}_{\alpha} \mathrm{x}_{\beta} \mathrm{x}_{\eta} \otimes x_{\eta} x_{\delta}-q_{\delta \eta} \mathrm{b}_{2} \mathrm{x}_{\alpha} \mathrm{x}_{\beta} \mathrm{x}_{\eta} \otimes x_{\kappa} x_{\gamma} x_{\mu}-q_{\delta \eta} \mathrm{b}_{3} \mathrm{x}_{\alpha} \mathrm{x}_{\beta} \mathrm{x}_{\eta} \otimes x_{\gamma} x_{\nu} \\
& -q_{\gamma \eta} q_{\mu \eta} \mathrm{b}_{2} \mathrm{x}_{\alpha} \mathrm{x}_{\beta} \mathrm{x}_{\kappa} \otimes x_{\eta} x_{\mu} x_{\gamma}-\mathrm{b}_{2} \mathrm{~b}_{8} \mathbf{x}_{\alpha} \mathrm{x}_{\gamma}^{2} \otimes\left(q_{\mu \eta} x_{\eta} x_{\mu}+\mathrm{b}_{6} x_{\iota} x_{\gamma}\right)+q_{\beta \delta} q_{\beta \eta}^{2} \mathrm{x}_{\alpha} \mathrm{x}_{\delta} \mathrm{x}_{\eta} \otimes x_{\eta} x_{\beta}
\end{aligned}
$$




$$
\begin{aligned}
& -q_{\alpha \beta} q_{\beta \eta}^{2} q_{\gamma \eta} \mathrm{b}_{1} \mathrm{~b}_{4} \mathrm{x}_{\beta} \mathrm{x}_{\tau} \mathrm{x}_{\kappa} \otimes x_{\eta} x_{\beta} x_{\gamma}-q_{\alpha \beta} q_{\beta \eta}^{2} q_{\tau \eta} \mathrm{b}_{1} \mathrm{x}_{\beta} \mathrm{x}_{\tau} \mathrm{x}_{\eta} \otimes\left(q_{\tau \eta} x_{\eta} x_{\tau}+\mathrm{b}_{4} x_{\kappa} x_{\gamma}\right) x_{\beta} \\
& \left.-q_{\alpha \beta} q_{\alpha \delta} q_{\alpha \eta}^{2} \mathrm{x}_{\beta} \mathrm{x}_{\delta} \mathrm{x}_{\eta} \otimes x_{\eta} x_{\alpha}+q_{\beta \tau} q_{\alpha \beta} q_{\beta \eta}^{2} \mathrm{~b}_{1} \mathrm{~b}_{4} \mathrm{~b}_{8} \mathrm{x}_{\tau} \mathrm{x}_{\gamma}^{2} \otimes x_{\eta} x_{\beta}\right) \\
= & \mathrm{x}_{\alpha} \mathrm{x}_{\beta} \mathrm{x}_{\delta} \mathrm{x}_{\eta} \otimes x_{\eta}+\mathrm{b}_{3} \mathrm{~b}_{7} \mathrm{x}_{\alpha} \mathrm{x}_{\beta} \mathrm{x}_{\gamma} \mathrm{x}_{\iota} \otimes x_{\kappa} x_{\gamma}+q_{\nu \eta} \mathrm{b}_{3} \mathrm{x}_{\alpha} \mathrm{x}_{\beta} \mathrm{x}_{\gamma} \mathrm{x}_{\eta} \otimes x_{\nu} \\
& +q_{\mu \gamma} q_{\mu \eta} q_{\gamma \eta} \mathrm{b}_{2} \mathrm{x}_{\alpha} \mathrm{x}_{\beta} \mathrm{x}_{\kappa} \mathrm{x}_{\eta} \otimes x_{\gamma} x_{\mu}+q_{\delta \eta}^{2} \mathrm{x}_{\alpha} \mathrm{x}_{\beta} \mathrm{x}_{\eta}^{2} \otimes x_{\delta}-q_{\beta \delta} q_{\beta \eta}^{2} \mathrm{x}_{\alpha} \mathrm{x}_{\delta} \mathrm{x}_{\eta}^{2} \otimes x_{\beta} \\
& +\mathrm{b}_{2} \mathrm{~b}_{6} \mathrm{~b}_{8} \mathrm{x}_{\alpha} \mathrm{x}_{\gamma}^{2} \mathrm{x}_{\iota} \otimes x_{\gamma}-(4)_{q_{\alpha \gamma}} \mathrm{b}_{2} \mathrm{~b}_{6} \mathrm{~b}_{8} \mathrm{~b}_{9} \mathrm{x}_{\gamma}^{4} \otimes 1+q_{\mu \eta} \mathrm{b}_{2} \mathrm{~b}_{8} \mathrm{x}_{\alpha} \mathrm{x}_{\gamma}^{2} \mathrm{x}_{\eta} \otimes x_{\mu} \\
& +q_{\alpha \beta} q_{\beta \eta}^{2} q_{\tau \eta}^{2} \mathrm{~b}_{1} \mathrm{x}_{\beta} \mathrm{x}_{\tau} \mathrm{x}_{\eta}^{2} \otimes x_{\tau} x_{\beta}+q_{\alpha \beta} q_{\beta \gamma} q_{\beta \eta}^{2} q_{\gamma \eta} \mathrm{b}_{1} \mathrm{~b}_{4} \mathrm{x}_{\beta} \mathrm{x}_{\tau} \mathrm{x}_{\kappa} \mathrm{x}_{\eta} \otimes x_{\gamma} x_{\beta} \\
& +q_{\alpha \beta} q_{\alpha \delta} q_{\alpha \eta}^{2} \mathrm{x}_{\beta} \mathrm{x}_{\delta} \mathrm{x}_{\eta}^{2} \otimes x_{\alpha}-q_{\beta \tau} q_{\alpha \beta} q_{\beta \eta}^{2} \mathrm{~b}_{1} \mathrm{~b}_{4} \mathrm{~b}_{8} \mathrm{x}_{\tau} \mathrm{x}_{\gamma}^{2} \mathrm{x}_{\eta} \otimes x_{\beta} .
\end{aligned}
$$

Next we check (10.1.120) using Remark 10.1.2, (10.1.103) and $\mathrm{x}_{\gamma}^{3} \otimes x_{\alpha}=s\left(\mathrm{x}_{\gamma}^{2} \otimes x_{\gamma} x_{\alpha}\right)$ :

$$
\begin{aligned}
& d\left(\mathrm{x}_{\alpha} \mathrm{x}_{\beta} \mathrm{x}_{\gamma}^{2} \mathrm{x}_{\kappa} \otimes 1\right)=\mathrm{x}_{\alpha} \mathrm{x}_{\beta} \mathrm{x}_{\gamma}^{2} \otimes x_{\kappa}-s\left(q_{\gamma \kappa} \mathrm{x}_{\alpha} \mathrm{x}_{\beta} \mathrm{x}_{\gamma} \otimes x_{\kappa} x_{\gamma}+q_{\beta \gamma}^{2} q_{\beta \kappa} \mathrm{x}_{\alpha} \mathrm{x}_{\gamma}^{2} \otimes x_{\kappa} x_{\beta}\right. \\
& \left.\quad+q_{\beta \gamma}^{2} \mathrm{~b}_{8} \mathrm{x}_{\alpha} \mathrm{x}_{\gamma}^{2} \otimes x_{\gamma}-q_{\alpha \beta} q_{\alpha \gamma}^{2} q_{\alpha \kappa} \mathrm{x}_{\beta} \mathrm{x}_{\gamma}^{2} \otimes x_{\kappa} x_{\alpha}\right) \\
& =\mathrm{x}_{\alpha} \mathrm{x}_{\beta} \mathrm{x}_{\gamma}^{2} \otimes x_{\kappa}-q_{\gamma \kappa} \mathrm{x}_{\alpha} \mathrm{x}_{\beta} \mathrm{x}_{\gamma} \mathrm{x}_{\kappa} \otimes x_{\gamma}-q_{\beta \gamma}^{2} q_{\beta \kappa} \mathrm{x}_{\alpha} \mathrm{x}_{\gamma}^{2} \mathrm{x}_{\kappa} \otimes x_{\beta}-q_{\gamma \beta}^{-2}(3)_{\widetilde{q}_{\beta \gamma}} \mathrm{b}_{8} \mathrm{x}_{\alpha} \mathrm{x}_{\gamma}^{3} \otimes 1 \\
& \quad+q_{\alpha \beta} q_{\alpha \gamma}^{2} q_{\alpha \kappa} \mathrm{x}_{\beta} \mathrm{x}_{\gamma}^{2} \mathrm{x}_{\kappa} \otimes x_{\alpha} .
\end{aligned}
$$

For (10.1.121) we use (10.1.103), Remark 10.1.2, (10.1.102) and (10.1.112):

$$
\begin{aligned}
& d\left(\mathrm{x}_{\alpha} \mathrm{x}_{\beta} \mathrm{x}_{\gamma} \mathrm{x}_{\kappa} \mathrm{x}_{\eta} \otimes 1\right)=\mathrm{x}_{\alpha} \mathrm{x}_{\beta} \mathrm{x}_{\gamma} \mathrm{x}_{\kappa} \otimes x_{\eta}-s\left(q_{\eta \kappa} \mathrm{x}_{\alpha} \mathrm{x}_{\beta} \mathrm{x}_{\gamma} \otimes x_{\eta} x_{\kappa}-q_{\gamma \kappa} q_{\gamma \eta} \mathrm{x}_{\alpha} \mathrm{x}_{\beta} \mathrm{x}_{\kappa} \otimes x_{\eta} x_{\gamma}\right. \\
& \left.+q_{\beta \gamma} q_{\beta \kappa} q_{\beta \eta} \mathrm{x}_{\alpha} \mathrm{x}_{\gamma} \mathrm{x}_{\kappa} \otimes x_{\eta} x_{\beta}+q_{\gamma \beta}^{-1}(2)_{\widetilde{q}_{\beta \gamma}} \mathrm{b}_{8} \mathrm{x}_{\alpha} \mathrm{x}_{\gamma}^{2} \otimes x_{\eta}-q_{\alpha \beta} q_{\alpha \gamma} q_{\alpha \kappa} \mathrm{x}_{\beta} \mathrm{x}_{\gamma} \mathrm{x}_{\kappa} \otimes x_{\alpha} x_{\eta}\right) \\
& =\mathrm{x}_{\alpha} \mathrm{x}_{\beta} \mathrm{x}_{\gamma} \mathrm{x}_{\kappa} \otimes x_{\eta}-q_{\kappa \eta} \mathrm{x}_{\alpha} \mathrm{x}_{\beta} \mathrm{x}_{\gamma} \mathrm{x}_{\eta} \otimes x_{\kappa}-q_{\gamma \beta}^{-1}(2)_{\widetilde{q}_{\beta \gamma}} \mathrm{b}_{8} \mathrm{x}_{\alpha} \mathrm{x}_{\gamma}^{2} \mathrm{x}_{\eta} \otimes 1 \\
& +q_{\gamma \kappa} q_{\gamma \eta} \mathrm{x}_{\alpha} \mathrm{x}_{\beta} \mathrm{x}_{\kappa} \mathrm{x}_{\eta} \otimes x_{\gamma}-q_{\beta \gamma} q_{\beta \kappa} q_{\beta \eta} \mathrm{x}_{\alpha} \mathrm{x}_{\gamma} \mathrm{x}_{\kappa} \mathrm{x}_{\eta} \otimes x_{\beta}+q_{\alpha \beta} q_{\alpha \gamma} q_{\alpha \kappa} q_{\alpha \eta} \mathrm{x}_{\beta} \mathrm{x}_{\gamma} \mathrm{x}_{\kappa} \mathrm{x}_{\eta} \otimes x_{\alpha} .
\end{aligned}
$$

To check (10.1.122) we use (10.1.100), (10.1.104), Remark10.1.2, (10.1.84) and (10.1.115):

$$
\begin{aligned}
d\left(\mathrm{x}_{\alpha} \mathrm{x}_{\delta} \mathrm{x}_{\gamma}^{2} \mathrm{x}_{\eta} \otimes 1\right)=\mathrm{x}_{\alpha} \mathrm{x}_{\delta} \mathrm{x}_{\gamma}^{2} \otimes x_{\eta}-s\left(q_{\gamma \eta} \mathrm{x}_{\alpha} \mathrm{x}_{\delta} \mathrm{x}_{\gamma} \otimes x_{\eta} x_{\gamma}+q_{\delta \gamma}^{2} q_{\delta \eta} \mathrm{x}_{\alpha} \mathrm{x}_{\gamma}^{2} \otimes x_{\eta} x_{\delta}\right. \\
\quad+q_{\delta \gamma}^{2} \mathrm{~b}_{2} \mathrm{x}_{\alpha} \mathrm{x}_{\gamma}^{2} \otimes x_{\kappa} x_{\gamma} x_{\mu}+q_{\delta \gamma}^{2} \mathrm{~b}_{3} \mathrm{x}_{\alpha} \mathrm{x}_{\gamma}^{2} \otimes x_{\gamma} x_{\nu}-q_{\beta \eta} q_{\tau \gamma}^{2} q_{\beta \gamma}^{2} q_{\tau \eta} \mathrm{b}_{1} \mathrm{x}_{\tau} \mathrm{x}_{\gamma}^{2} \otimes x_{\eta} x_{\tau} x_{\beta} \\
\left.\quad-q_{\beta \eta} q_{\tau \gamma}^{2} q_{\beta \gamma}^{2} \mathrm{~b}_{1} \mathrm{~b}_{4} \mathrm{x}_{\tau} \mathrm{x}_{\gamma}^{2} \otimes x_{\kappa} x_{\gamma} x_{\beta}-q_{\alpha \delta} q_{\alpha \gamma}^{2} q_{\alpha \eta} \mathrm{x}_{\delta} \mathrm{x}_{\gamma}^{2} \otimes x_{\eta} x_{\alpha}\right) \\
=\mathrm{x}_{\alpha} \mathrm{x}_{\delta} \mathrm{x}_{\gamma}^{2} \otimes x_{\eta}-q_{\gamma \eta} \mathrm{x}_{\alpha} \mathrm{x}_{\delta} \mathrm{x}_{\gamma} \mathrm{x}_{\eta} \otimes x_{\gamma}-q_{\delta \gamma}^{2}(2)_{\widetilde{q}_{\gamma \eta}} \mathrm{b}_{3} \mathrm{x}_{\alpha} \mathrm{x}_{\gamma}^{3} \otimes x_{\nu}-q_{\delta \gamma}^{2} \mathrm{~b}_{2} \mathrm{x}_{\alpha} \mathrm{x}_{\gamma}^{2} \mathrm{x}_{\kappa} \otimes x_{\gamma} x_{\mu} \\
\quad-q_{\delta \gamma}^{2} q_{\delta \eta} \mathrm{x}_{\alpha} \mathrm{x}_{\gamma}^{2} \mathrm{x}_{\eta} \otimes x_{\delta}+q_{\alpha \delta} q_{\alpha \gamma}^{2} q_{\alpha \eta} \mathrm{x}_{\delta} \mathrm{x}_{\gamma}^{2} \mathrm{x}_{\eta} \otimes x_{\alpha}+q_{\beta \eta} q_{\tau \gamma}^{2} q_{\beta \gamma}^{2} q_{\tau \eta} \mathrm{b}_{1} \mathrm{x}_{\tau} \mathrm{x}_{\gamma}^{2} \mathrm{x}_{\eta} \otimes x_{\tau} x_{\beta} \\
\quad+q_{\beta \eta} q_{\tau \gamma}^{2} q_{\beta \gamma}^{2} \mathrm{~b}_{1} \mathrm{~b}_{4} \mathrm{x}_{\tau} \mathrm{x}_{\gamma}^{2} \mathrm{x}_{\kappa} \otimes x_{\gamma} x_{\beta}+q_{\alpha \delta} q_{\alpha \gamma}^{2} q_{\alpha \eta} q_{\delta \gamma}^{2}(3)_{\widetilde{q}_{\gamma \eta}} \mathrm{b}_{3} s\left(\mathrm{x}_{\gamma}^{3} \otimes x_{\nu} x_{\alpha}\right),
\end{aligned}
$$

and we use that $\mathrm{x}_{\gamma}^{3} \otimes x_{\nu} x_{\alpha}=s\left(\mathrm{x}_{\gamma}^{2} \otimes x_{\gamma} x_{\nu} x_{\alpha}\right)$. To prove (10.1.123) we use (10.1.104), (10.1.105), (10.1.83), Remark 10.1.2, (10.1.113), (10.1.114) and (10.1.116):

$$
\begin{aligned}
d\left(\mathrm{x}_{\alpha} \mathrm{x}_{\delta} \mathrm{x}_{\gamma} \mathrm{x}_{\eta}^{2} \otimes 1\right)=\mathrm{x}_{\alpha} \mathrm{x}_{\delta} \mathrm{x}_{\gamma} \mathrm{x}_{\eta} \otimes x_{\eta}-s\left(-q_{\gamma \eta}^{2} \mathrm{x}_{\alpha} \mathrm{x}_{\delta} \mathrm{x}_{\eta} \otimes x_{\eta} x_{\gamma}+q_{\delta \gamma} q_{\delta \eta} \mathrm{x}_{\alpha} \mathrm{x}_{\gamma} \mathrm{x}_{\eta} \otimes x_{\delta} x_{\eta}\right. \\
\quad+q_{\delta \gamma} \mathrm{b}_{2} \mathrm{x}_{\alpha} \mathrm{x}_{\gamma} \mathrm{x}_{\kappa} \otimes x_{\gamma} x_{\mu} x_{\eta}-q_{\alpha \delta} q_{\alpha \gamma} q_{\alpha \eta} \mathrm{x}_{\delta} \mathrm{x}_{\gamma} \mathrm{x}_{\eta} \otimes x_{\alpha} x_{\eta} \\
\quad-q_{\tau \gamma} q_{\beta \gamma} q_{\beta \eta} \mathrm{b}_{1} \mathrm{~b}_{4} \mathrm{x}_{\tau} \mathrm{x}_{\gamma} \mathrm{x}_{\kappa} \otimes x_{\gamma} x_{\beta} x_{\eta}+q_{\delta \gamma} \mathrm{b}_{3} \mathrm{x}_{\alpha} \mathrm{x}_{\gamma}^{2} \otimes x_{\nu} x_{\eta} \\
\left.\quad-q_{\beta \gamma} q_{\beta \eta} q_{\tau \gamma} q_{\tau \eta} \mathrm{b}_{1} \mathrm{x}_{\tau} \mathrm{x}_{\gamma} \mathrm{x}_{\eta} \otimes x_{\tau} x_{\beta} x_{\eta}-q_{\alpha \gamma}^{2} q_{\alpha \kappa} q_{\delta \gamma} \mathrm{b}_{2} \mathrm{~b}_{5} \mathrm{x}_{\gamma}^{2} \mathrm{x}_{\kappa} \otimes x_{\tau} x_{\beta} x_{\eta}\right) \\
=\mathrm{x}_{\alpha} \mathrm{x}_{\delta} \mathrm{x}_{\gamma} \mathrm{x}_{\eta} \otimes x_{\eta}+q_{\gamma \eta}^{2} \mathrm{x}_{\alpha} \mathrm{x}_{\delta} \mathrm{x}_{\eta}^{2} \otimes x_{\gamma}-q_{\delta \gamma} q_{\nu \eta}(2)_{\widetilde{q}_{\gamma \eta}} \mathrm{b}_{3} \mathrm{x}_{\alpha} \mathrm{x}_{\gamma}^{2} \mathrm{x}_{\eta} \otimes x_{\nu} \\
\quad-q_{\delta \gamma}(2)_{\widetilde{q}_{\gamma \eta}} \mathrm{b}_{3} \mathrm{~b}_{7} \mathrm{x}_{\alpha} \mathrm{x}_{\gamma}^{2} \mathrm{x}_{\iota} \otimes x_{\kappa} x_{\gamma}+q_{\delta \gamma} c_{\alpha \eta \gamma}^{(2)} \mathrm{b}_{3} \mathrm{~b}_{7} \mathrm{~b}_{9} \mathrm{x}_{\gamma}^{4} \otimes x_{\kappa}-q_{\delta \gamma} q_{\delta \eta}^{2} \mathrm{x}_{\alpha} \mathrm{x}_{\gamma} \mathrm{x}_{\eta}^{2} \otimes x_{\delta}
\end{aligned}
$$




$$
\begin{aligned}
& -q_{\delta \gamma} q_{\mu \eta} q_{\gamma \eta} \mathrm{b}_{2} \mathrm{x}_{\alpha} \mathrm{x}_{\gamma} \mathrm{x}_{\kappa} \mathrm{x}_{\eta} \otimes x_{\gamma} x_{\mu}+q_{\tau \gamma} q_{\beta \gamma} q_{\beta \eta}^{2} q_{\gamma \eta} \mathrm{b}_{1} \mathrm{~b}_{4} \mathrm{x}_{\tau} \mathrm{x}_{\gamma} \mathrm{x}_{\kappa} \mathrm{x}_{\eta} \otimes x_{\gamma} x_{\beta} \\
& +q_{\beta \gamma} q_{\beta \eta}^{2} q_{\tau \gamma} q_{\tau \eta}^{2} \mathrm{~b}_{1} \mathrm{x}_{\tau} \mathrm{x}_{\gamma} \mathrm{x}_{\eta}^{2} \otimes x_{\tau} x_{\beta}+q_{\alpha \gamma}^{2} q_{\alpha \kappa} q_{\beta \eta} q_{\delta \gamma} \mathrm{b}_{2} \mathrm{~b}_{4} \mathrm{~b}_{5} \mathrm{x}_{\gamma}^{2} \mathrm{x}_{\kappa}^{2} \otimes x_{\gamma} x_{\beta} \\
& +q_{\alpha \delta} q_{\alpha \gamma} q_{\alpha \eta}^{2} \mathrm{x}_{\delta} \mathrm{x}_{\gamma} \mathrm{x}_{\eta}^{2} \otimes x_{\alpha}+q_{\alpha \gamma}^{2} q_{\alpha \kappa} q_{\beta \eta} q_{\delta \gamma} q_{\tau \eta} \mathrm{b}_{2} \mathrm{~b}_{5} \mathrm{x}_{\gamma}^{2} \mathrm{x}_{\kappa} \mathrm{x}_{\eta} \otimes x_{\tau} x_{\beta}
\end{aligned}
$$

We compute (10.1.125) using (10.1.106), (10.1.86), (10.1.107), (10.1.111), (10.1.112), (10.1.84), 10.1.113) and Remark 10.1.2.

$$
\begin{aligned}
& d\left(\mathrm{x}_{\beta} \mathrm{x}_{\tau} \mathrm{x}_{\gamma} \mathrm{x}_{\kappa} \mathrm{x}_{\eta} \otimes 1\right)=\mathrm{x}_{\beta} \mathrm{x}_{\tau} \mathrm{x}_{\gamma} \mathrm{x}_{\kappa} \otimes x_{\eta}-s\left(q_{\kappa \eta} \mathrm{x}_{\beta} \mathrm{x}_{\tau} \mathrm{x}_{\gamma} \otimes x_{\eta} x_{\kappa}-q_{\gamma \kappa} q_{\gamma \eta} \mathrm{x}_{\beta} \mathrm{x}_{\tau} \mathrm{x}_{\kappa} \otimes x_{\eta} x_{\gamma}\right. \\
& +q_{\tau \gamma} q_{\tau \kappa} q_{\tau \eta} \mathrm{x}_{\beta} \mathrm{x}_{\gamma} \mathrm{x}_{\kappa} \otimes x_{\eta} x_{\tau}+q_{\tau \gamma} q_{\tau \kappa} \mathrm{b}_{4} \mathrm{x}_{\beta} \mathrm{x}_{\gamma} \mathrm{x}_{\kappa} \otimes x_{\kappa} x_{\gamma} \\
& \left.-q_{\beta \tau} q_{\gamma \beta}^{-1}(2)_{\widetilde{q}_{\beta \gamma}} \mathrm{b}_{8} \mathrm{x}_{\tau} \mathrm{x}_{\gamma}^{2} \otimes x_{\eta}-q_{\beta \tau} q_{\beta \gamma} q_{\beta \kappa} q_{\beta \eta} \mathrm{x}_{\tau} \mathrm{x}_{\gamma} \mathrm{x}_{\kappa} \otimes x_{\eta} x_{\beta}\right) \\
& =\mathrm{x}_{\beta} \mathrm{x}_{\tau} \mathrm{x}_{\gamma} \mathrm{x}_{\kappa} \otimes x_{\eta}-q_{\kappa \eta} \mathrm{x}_{\beta} \mathrm{x}_{\tau} \mathrm{x}_{\gamma} \mathrm{x}_{\eta} \otimes x_{\kappa}-q_{\tau \gamma} q_{\tau \kappa}(2)_{\widetilde{q}_{\kappa \eta}} \mathrm{b}_{4} \mathrm{x}_{\beta} \mathrm{x}_{\gamma} \mathrm{x}_{\kappa}^{2} \otimes x_{\gamma} \\
& +q_{\gamma \kappa} q_{\gamma \eta} \mathrm{x}_{\beta} \mathrm{x}_{\tau} \mathrm{x}_{\kappa} \mathrm{x}_{\eta} \otimes x_{\gamma}-q_{\tau \gamma} q_{\tau \kappa} q_{\tau \eta} \mathrm{x}_{\beta} \mathrm{x}_{\gamma} \mathrm{x}_{\kappa} \mathrm{x}_{\eta} \otimes x_{\tau}+q_{\beta \tau} q_{\gamma \beta}^{-1}(2) \widetilde{q}_{\beta \gamma} \mathrm{b}_{8} \mathrm{x}_{\tau} \mathrm{x}_{\gamma}^{2} \mathrm{x}_{\eta} \otimes 1 \\
& +q_{\beta \tau} q_{\beta \gamma} q_{\beta \kappa} q_{\beta \eta} \mathrm{x}_{\tau} \mathrm{x}_{\gamma} \mathrm{x}_{\kappa} \mathrm{x}_{\eta} \otimes x_{\beta}-q_{\kappa \eta} q_{\eta \gamma}^{-1}(2)_{\widetilde{q}_{\gamma \eta}} \mathrm{b}_{4} \mathrm{~b}_{8} \mathrm{x}_{\gamma}^{3} \mathrm{x}_{\kappa} \otimes 1 \text {. }
\end{aligned}
$$

Next we check (10.1.124) using Remark 10.1.2, (10.1.106) and (10.1.85):

$$
\begin{aligned}
& d\left(\mathrm{x}_{\beta} \mathrm{x}_{\tau} \mathrm{x}_{\gamma}^{2} \mathrm{x}_{\kappa} \otimes 1\right)=\mathrm{x}_{\beta} \mathrm{x}_{\tau} \mathrm{x}_{\gamma}^{2} \otimes x_{\kappa}-s\left(q_{\gamma \kappa} \mathrm{x}_{\beta} \mathrm{x}_{\tau} \mathrm{x}_{\gamma} \otimes x_{\kappa} x_{\gamma}+q_{\tau \gamma}^{2} q_{\tau \kappa} \mathrm{x}_{\beta} \mathrm{x}_{\gamma}^{2} \otimes x_{\kappa} x_{\tau}\right. \\
& \left.\quad-q_{\beta \tau} q_{\beta \gamma}^{2} q_{\beta \kappa} \mathrm{x}_{\tau} \mathrm{x}_{\gamma}^{2} \otimes x_{\kappa} x_{\beta}-q_{\beta \tau} q_{\beta \gamma}^{2} \mathrm{~b}_{8} \mathrm{x}_{\tau} \mathrm{x}_{\gamma}^{2} \otimes x_{\gamma}\right) \\
& =\mathrm{x}_{\beta} \mathrm{x}_{\tau} \mathrm{x}_{\gamma}^{2} \otimes x_{\kappa}-q_{\gamma \kappa} \mathrm{x}_{\beta} \mathrm{x}_{\tau} \mathrm{x}_{\gamma} \mathrm{x}_{\kappa} \otimes x_{\gamma}-q_{\tau \gamma}^{2} q_{\tau \kappa} \mathrm{x}_{\beta} \mathrm{x}_{\gamma}^{2} \mathrm{x}_{\kappa} \otimes x_{\tau}+q_{\beta \tau} q_{\gamma \beta}^{-2}(3)_{\widetilde{q}_{\beta \gamma}} \mathrm{b}_{8} \mathrm{x}_{\tau} \mathrm{x}_{\gamma}^{3} \otimes 1 \\
& \quad+q_{\beta \tau} q_{\beta \gamma}^{2} q_{\beta \kappa} \mathrm{x}_{\tau} \mathrm{x}_{\gamma}^{2} \mathrm{x}_{\kappa} \otimes x_{\beta} .
\end{aligned}
$$

For (10.1.126) we use (10.1.86), (10.1.108), (10.1.112), Remark 10.1.2 and (10.1.114):

$$
\begin{aligned}
d\left(\mathrm{x}_{\beta} \mathrm{x}_{\tau} \mathrm{x}_{\gamma} \mathbf{x}_{\eta}^{2} \otimes 1\right)=\mathbf{x}_{\beta} \mathbf{x}_{\tau} \mathbf{x}_{\gamma} \mathbf{x}_{\eta} \otimes x_{\eta}-s\left(-q_{\gamma \eta}^{2} \mathbf{x}_{\beta} \mathbf{x}_{\tau} \mathbf{x}_{\eta} \otimes x_{\eta} x_{\gamma}+q_{\tau \gamma} q_{\tau \eta}^{2} \mathbf{x}_{\beta} \mathbf{x}_{\gamma} \mathbf{x}_{\eta} \otimes x_{\eta} x_{\tau}\right. \\
\quad+q_{\tau \gamma} q_{\tau \eta} \mathbf{b}_{4} \mathbf{x}_{\beta} \mathbf{x}_{\gamma} \mathbf{x}_{\eta} \otimes x_{\kappa} x_{\gamma}+q_{\tau \gamma} q_{\gamma \eta} \mathbf{b}_{4} \mathbf{x}_{\beta} \mathbf{x}_{\gamma} \mathbf{x}_{\kappa} \otimes x_{\eta} x_{\gamma} \\
\left.\quad-q_{\beta \tau} q_{\beta \gamma} q_{\beta \eta}^{2} \mathbf{x}_{\tau} \mathbf{x}_{\gamma} \mathbf{x}_{\eta} \otimes x_{\eta} x_{\beta}-q_{\eta \gamma}^{-1}(2)_{\widetilde{q}_{\gamma \eta}} \mathbf{b}_{4} \mathbf{b}_{8} \mathbf{x}_{\gamma}^{3} \otimes x_{\eta}\right) \\
=\mathbf{x}_{\beta} \mathbf{x}_{\tau} \mathbf{x}_{\gamma} \mathbf{x}_{\eta} \otimes x_{\eta}+q_{\gamma \eta}^{2} \mathbf{x}_{\beta} \mathbf{x}_{\tau} \mathbf{x}_{\eta}^{2} \otimes x_{\gamma}-q_{\tau \gamma} q_{\gamma \eta} \mathbf{b}_{4} \mathbf{x}_{\beta} \mathbf{x}_{\gamma} \mathbf{x}_{\kappa} \mathbf{x}_{\eta} \otimes x_{\gamma}-q_{\tau \gamma} q_{\tau \eta}^{2} \mathbf{x}_{\beta} \mathbf{x}_{\gamma} \mathbf{x}_{\eta}^{2} \otimes x_{\tau} \\
\quad+q_{\beta \tau} q_{\beta \gamma} q_{\beta \eta}^{2} \mathbf{x}_{\tau} \mathbf{x}_{\gamma} \mathbf{x}_{\eta}^{2} \otimes x_{\beta}+q_{\gamma \eta}^{-1} c_{-\beta-\eta \gamma}^{(2)} \mathbf{b}_{4} \mathbf{b}_{8} \mathbf{x}_{\gamma}^{3} \mathbf{x}_{\eta} \otimes 1 .
\end{aligned}
$$

The proof of (10.1.127) is similar, using Remark10.1.2, (10.1.109), (10.1.85) and (10.1.115):

$$
\begin{aligned}
& d\left(\mathrm{x}_{\beta} \mathrm{x}_{\delta} \mathrm{x}_{\gamma}^{2} \mathrm{x}_{\eta} \otimes 1\right)=\mathbf{x}_{\beta} \mathbf{x}_{\delta} \mathrm{x}_{\gamma}^{2} \otimes x_{\eta}-s\left(q_{\gamma \eta} \mathbf{x}_{\beta} \mathrm{x}_{\delta} \mathbf{x}_{\gamma} \otimes x_{\eta} x_{\gamma}+q_{\delta \gamma}^{2} q_{\delta \eta} \mathrm{x}_{\beta} \mathbf{x}_{\gamma}^{2} \otimes x_{\eta} x_{\delta}\right. \\
& \left.+q_{\delta \gamma}^{2} \mathrm{~b}_{2} \mathrm{x}_{\beta} \mathrm{x}_{\gamma}^{2} \otimes x_{\kappa} x_{\gamma} x_{\mu}+q_{\delta \gamma}^{2} \mathrm{~b}_{3} \mathrm{x}_{\beta} \mathrm{x}_{\gamma}^{2} \otimes x_{\gamma} x_{\nu}-q_{\beta \delta} q_{\beta \gamma}^{2} q_{\beta \eta} \mathrm{x}_{\delta} \mathrm{x}_{\gamma}^{2} \otimes x_{\eta} x_{\beta}\right) \\
& =\mathrm{x}_{\beta} \mathrm{x}_{\delta} \mathrm{x}_{\gamma}^{2} \otimes x_{\eta}-q_{\gamma \eta} \mathrm{x}_{\beta} \mathrm{x}_{\delta} \mathrm{x}_{\gamma} \mathrm{x}_{\eta} \otimes x_{\gamma}-q_{\delta \gamma}^{2} q_{\delta \eta} \mathrm{x}_{\beta} \mathrm{x}_{\gamma}^{2} \mathrm{x}_{\eta} \otimes x_{\delta}-q_{\delta \gamma}^{2} \mathrm{~b}_{2} \mathrm{x}_{\beta} \mathrm{x}_{\gamma}^{2} \mathrm{x}_{\kappa} \otimes x_{\gamma} x_{\mu} \\
& -q_{\delta \gamma}^{2}(3)_{\widetilde{q}_{\gamma \eta}} \mathrm{b}_{3} \mathrm{x}_{\beta} \mathrm{x}_{\gamma}^{3} \otimes x_{\nu}+q_{\beta \delta} q_{\beta \gamma}^{2} q_{\beta \eta} \mathrm{x}_{\delta} \mathrm{x}_{\gamma}^{2} \mathrm{x}_{\eta} \otimes x_{\beta}-q_{\beta \gamma} q_{\gamma \beta}^{-1} q_{\delta \gamma}^{2} \mathrm{c}_{\beta \eta}^{(3)} \mathrm{b}_{2} \mathrm{~b}_{8} \mathrm{x}_{\gamma}^{4} \otimes x_{\mu} .
\end{aligned}
$$

For (10.1.128) we use (10.1.109), (10.1.110), Remark 10.1.2, (10.1.112) and (10.1.116):

$$
\begin{aligned}
& d\left(\mathrm{x}_{\beta} \mathrm{x}_{\delta} \mathrm{x}_{\gamma} \mathrm{x}_{\eta}^{2} \otimes 1\right)=\mathrm{x}_{\beta} \mathrm{x}_{\delta} \mathrm{x}_{\gamma} \mathrm{x}_{\eta} \otimes x_{\eta}-s\left(-q_{\gamma \eta}^{2} \mathrm{x}_{\beta} \mathrm{x}_{\delta} \mathrm{x}_{\eta} \otimes x_{\eta} x_{\gamma}+q_{\delta \gamma} q_{\delta \eta}^{2} \mathrm{x}_{\beta} \mathrm{x}_{\gamma} \mathrm{x}_{\eta} \otimes x_{\eta} x_{\delta}\right. \\
& +q_{\delta \gamma} q_{\delta \eta} \mathrm{b}_{2} \mathrm{x}_{\beta} \mathrm{x}_{\gamma} \mathrm{x}_{\eta} \otimes x_{\kappa} x_{\gamma} x_{\mu}+q_{\delta \gamma} q_{\delta \eta} \mathrm{b}_{3} \mathrm{x}_{\beta} \mathrm{x}_{\gamma} \mathrm{x}_{\eta} \otimes x_{\gamma} x_{\nu} \\
& +q_{\delta \gamma} q_{\mu \eta} q_{\gamma \eta} \mathrm{b}_{2} \mathrm{x}_{\beta} \mathrm{x}_{\gamma} \mathrm{x}_{\kappa} \otimes x_{\eta} x_{\gamma} x_{\mu}+q_{\delta \gamma}(2)_{\widetilde{q}_{\gamma \eta}} q_{\nu \eta} \mathrm{b}_{3} \mathrm{x}_{\beta} \mathrm{x}_{\gamma}^{2} \otimes x_{\eta} x_{\nu} \\
& +q_{\delta \gamma}(2)_{\widetilde{q}_{\gamma \eta}} \mathrm{b}_{3} \mathrm{~b}_{7} \mathrm{x}_{\beta} \mathrm{x}_{\gamma}^{2} \otimes x_{\iota} x_{\kappa} x_{\gamma}+q_{\beta \gamma} q_{\delta \gamma} q_{\mu \eta}(2)_{\widetilde{q}_{\gamma \eta}} \mathrm{b}_{2} \mathrm{~b}_{8} \mathrm{x}_{\gamma}^{3} \otimes x_{\eta} x_{\mu}
\end{aligned}
$$




$$
\begin{aligned}
& \left.+q_{\beta \gamma} q_{\delta \gamma}(2)_{\widetilde{q}_{\gamma \eta}} \mathrm{b}_{2} \mathrm{~b}_{6} \mathrm{~b}_{8} \mathrm{x}_{\gamma}^{3} \otimes x_{\iota} x_{\gamma}-q_{\beta \delta} q_{\beta \gamma} q_{\beta \eta}^{2} \mathrm{x}_{\delta} \mathrm{x}_{\gamma} \mathrm{x}_{\eta} \otimes x_{\eta} x_{\beta}\right) \\
& =\mathrm{x}_{\beta} \mathrm{x}_{\delta} \mathrm{x}_{\gamma} \mathrm{x}_{\eta} \otimes x_{\eta}+q_{\gamma \eta}^{2} \mathrm{x}_{\beta} \mathbf{x}_{\delta} \mathrm{x}_{\eta}^{2} \otimes x_{\gamma}-q_{\delta \gamma}(2)_{\widetilde{q}_{\gamma \eta}} \mathrm{b}_{3} \mathrm{~b}_{7} \mathrm{x}_{\beta} \mathrm{x}_{\gamma}^{2} \mathrm{x}_{\iota} \otimes x_{\kappa} x_{\gamma} \\
& -q_{\delta \gamma}(2)_{\widetilde{q}_{\gamma \eta}} q_{\nu \eta} \mathrm{b}_{3} \mathrm{x}_{\beta} \mathrm{x}_{\gamma}^{2} \mathrm{x}_{\eta} \otimes x_{\nu}-q_{\delta \gamma} q_{\mu \eta} q_{\gamma \eta} \mathrm{b}_{2} \mathrm{x}_{\beta} \mathrm{x}_{\gamma} \mathrm{x}_{\kappa} \mathrm{x}_{\eta} \otimes x_{\gamma} x_{\mu} \\
& -q_{\delta \gamma} q_{\delta \eta}^{2} \mathrm{x}_{\beta} \mathrm{x}_{\gamma} \mathrm{x}_{\eta}^{2} \otimes x_{\delta}+q_{\beta \delta} q_{\beta \gamma} q_{\beta \eta}^{2} \mathrm{x}_{\delta} \mathrm{x}_{\gamma} \mathrm{x}_{\eta}^{2} \otimes x_{\beta} \\
& -q_{\beta \gamma} q_{\delta \gamma}(2)_{\widetilde{q}_{\gamma \eta}} \mathrm{b}_{2} \mathrm{~b}_{6} \mathrm{~b}_{8} \mathrm{x}_{\gamma}^{3} \mathrm{x}_{\iota} \otimes x_{\gamma}-q_{\beta \gamma} q_{\delta \gamma} q_{\mu \eta}(2)_{\widetilde{q}_{\gamma \eta}} \mathrm{b}_{2} \mathrm{~b}_{8} \mathrm{x}_{\gamma}^{3} \mathrm{x}_{\eta} \otimes x_{\mu} \text {. }
\end{aligned}
$$

We check (10.1.129) using (10.1.85), (10.1.111) and Remark 10.1.2:

$$
\begin{aligned}
& d\left(\mathrm{x}_{\beta} \mathrm{x}_{\gamma}^{2} \mathrm{x}_{\kappa}^{2} \otimes 1\right)=\mathrm{x}_{\beta} \mathrm{x}_{\gamma}^{2} \mathrm{x}_{\kappa} \otimes x_{\kappa}-s\left(-q_{\gamma \kappa}^{2} \mathrm{x}_{\beta} \mathrm{x}_{\gamma} \mathrm{x}_{\kappa} \otimes x_{\kappa} x_{\gamma}-q_{\beta \kappa} q_{\beta \gamma}^{2} \mathrm{~b}_{8} \mathrm{x}_{\gamma}^{2} \mathrm{x}_{\kappa} \otimes x_{\gamma}\right. \\
& \left.\quad-q_{\beta \kappa}^{2} q_{\beta \gamma}^{2} \mathrm{x}_{\gamma}^{2} \mathrm{x}_{\kappa} \otimes x_{\kappa} x_{\beta}-q_{\beta \gamma}^{2}(3)_{\widetilde{q}_{\beta \gamma}} \mathrm{b}_{8} \mathrm{x}_{\gamma}^{3} \otimes x_{\kappa}\right) \\
& =\mathrm{x}_{\beta} \mathrm{x}_{\gamma}^{2} \mathrm{x}_{\kappa} \otimes x_{\kappa}+q_{\gamma \kappa}^{2} \mathrm{x}_{\beta} \mathrm{x}_{\gamma} \mathrm{x}_{\kappa}^{2} \otimes x_{\gamma}+q_{\beta \gamma}^{2}(3)_{\widetilde{q}_{\beta \gamma}} \mathrm{b}_{8} \mathrm{x}_{\gamma}^{3} \mathrm{x}_{\kappa} \otimes 1+q_{\beta \kappa}^{2} q_{\beta \gamma}^{2} \mathrm{x}_{\gamma}^{2} \mathrm{x}_{\kappa}^{2} \otimes x_{\beta} .
\end{aligned}
$$

For (10.1.130) we use (10.1.85), Remark 10.1.2 and (10.1.112):

$$
\begin{aligned}
& d\left(\mathrm{x}_{\beta} \mathrm{x}_{\gamma}^{2} \mathrm{x}_{\kappa} \mathrm{x}_{\eta} \otimes 1\right)=\mathrm{x}_{\beta} \mathrm{x}_{\gamma}^{2} \mathrm{x}_{\kappa} \otimes x_{\eta}-s\left(q_{\kappa \eta} \mathrm{x}_{\beta} \mathrm{x}_{\gamma}^{2} \otimes x_{\eta} x_{\kappa}-q_{\gamma \kappa} q_{\gamma \eta} \mathrm{x}_{\beta} \mathrm{x}_{\gamma} \mathrm{x}_{\kappa} \otimes x_{\eta} x_{\gamma}\right. \\
& \left.\quad-q_{\beta \kappa} q_{\beta \gamma}^{2} q_{\beta \eta} \mathrm{x}_{\gamma}^{2} \mathrm{x}_{\kappa} \otimes x_{\eta} x_{\beta}-q_{\beta \gamma}^{2}(3)_{\widetilde{q}_{\beta \gamma}} \mathrm{b}_{8} \mathrm{x}_{\gamma}^{3} \otimes x_{\eta}\right) \\
& =\mathrm{x}_{\beta} \mathrm{x}_{\gamma}^{2} \mathrm{x}_{\kappa} \otimes x_{\eta}-q_{\kappa \eta} \mathrm{x}_{\beta} \mathrm{x}_{\gamma}^{2} \mathrm{x}_{\eta} \otimes x_{\kappa}+q_{\gamma \kappa} q_{\gamma \eta} \mathrm{x}_{\beta} \mathrm{x}_{\gamma} \mathrm{x}_{\kappa} \mathrm{x}_{\eta} \otimes x_{\gamma} \\
& \quad+q_{\beta \kappa} q_{\beta \gamma}^{2} q_{\beta \eta} \mathrm{x}_{\gamma}^{2} \mathrm{x}_{\kappa} \mathrm{x}_{\eta} \otimes x_{\beta}+q_{\beta \gamma}^{2}(3)_{\widetilde{q}_{\beta \gamma}} \mathrm{b}_{8} \mathrm{x}_{\gamma}^{3} \mathrm{x}_{\eta} \otimes 1 .
\end{aligned}
$$

Now we compute (10.1.131) using Remark 10.1.2, (10.1.84) and (10.1.113),

$$
\begin{aligned}
& d\left(\mathrm{x}_{\tau} \mathrm{x}_{\gamma}^{2} \mathrm{x}_{\kappa} \mathbf{x}_{\eta} \otimes 1\right)=\mathbf{x}_{\tau} \mathbf{x}_{\gamma}^{2} \mathbf{x}_{\kappa} \otimes x_{\eta}-s\left(q_{\kappa \eta} \mathbf{x}_{\tau} \mathbf{x}_{\gamma}^{2} \otimes x_{\eta} x_{\kappa}-q_{\gamma \eta}^{2} \mathbf{x}_{\tau} \mathbf{x}_{\gamma} \mathbf{x}_{\kappa} \otimes x_{\eta} x_{\gamma}\right. \\
& \left.\quad-q_{\tau \gamma}^{2} q_{\tau \kappa} q_{\tau \eta} \mathbf{x}_{\gamma}^{2} \mathbf{x}_{\kappa} \otimes x_{\eta} x_{\tau}-q_{\tau \gamma}^{2} q_{\tau \kappa} \mathrm{b}_{4} \mathrm{x}_{\gamma}^{2} \mathbf{x}_{\kappa} \otimes x_{\kappa} x_{\gamma}\right) \\
& =\mathbf{x}_{\tau} \mathbf{x}_{\gamma}^{2} \mathbf{x}_{\kappa} \otimes x_{\eta}-q_{\kappa \eta} \mathbf{x}_{\tau} \mathbf{x}_{\gamma}^{2} \mathbf{x}_{\eta} \otimes x_{\kappa}+q_{\gamma \kappa} q_{\gamma \eta} \mathbf{x}_{\tau} \mathbf{x}_{\gamma} \mathbf{x}_{\kappa} \mathbf{x}_{\eta} \otimes x_{\gamma}+q_{\tau \gamma}^{2} q_{\tau \kappa}(2)_{\widetilde{q}_{\kappa \eta}} \mathrm{b}_{4} \mathrm{x}_{\gamma}^{2} \mathbf{x}_{\kappa}^{2} \otimes x_{\gamma} \\
& \quad+q_{\tau \gamma}^{2} q_{\tau \kappa} q_{\tau \eta} \mathbf{x}_{\gamma}^{2} \mathbf{x}_{\kappa} \mathbf{x}_{\eta} \otimes x_{\tau} .
\end{aligned}
$$

For (10.1.132) we use (10.1.84), (10.1.114) and Remark 10.1.2,

$$
\begin{aligned}
d\left(\mathrm{x}_{\tau} \mathrm{x}_{\gamma}^{2} \mathrm{x}_{\eta}^{2} \otimes 1\right)=\mathrm{x}_{\tau} \mathrm{x}_{\gamma}^{2} \mathrm{x}_{\eta} \otimes x_{\eta}-s\left(-q_{\gamma \eta}^{2} \mathrm{x}_{\tau} \mathrm{x}_{\gamma} \mathrm{x}_{\eta} \otimes x_{\eta} x_{\gamma}-q_{\tau \gamma}^{2} q_{\tau \eta} \mathrm{b}_{4} \mathrm{x}_{\gamma}^{2} \mathrm{x}_{\eta} \otimes x_{\kappa} x_{\gamma}\right. \\
\left.\quad-q_{\tau \gamma}^{2} q_{\tau \eta}^{2} \mathrm{x}_{\gamma}^{2} \mathrm{x}_{\eta} \otimes x_{\eta} x_{\tau}-q_{\tau \gamma}^{2} q_{\gamma \eta} \mathrm{b}_{4} \mathrm{x}_{\gamma}^{2} \mathrm{x}_{\kappa} \otimes x_{\eta} x_{\gamma}\right) \\
=\mathrm{x}_{\tau} \mathrm{x}_{\gamma}^{2} \mathrm{x}_{\eta} \otimes x_{\eta}+q_{\gamma \eta}^{2} \mathrm{x}_{\tau} \mathrm{x}_{\gamma} \mathrm{x}_{\eta}^{2} \otimes x_{\gamma}+q_{\tau \gamma}^{2} q_{\gamma \eta} \mathrm{b}_{4} \mathrm{x}_{\gamma}^{2} \mathrm{x}_{\kappa} \mathrm{x}_{\eta} \otimes x_{\gamma}+q_{\tau \gamma}^{2} q_{\tau \eta}^{2} \mathrm{x}_{\gamma}^{2} \mathrm{x}_{\eta}^{2} \otimes x_{\tau}
\end{aligned}
$$

Finally we compute (10.1.133) using (10.1.115), (10.1.116) and Remark 10.1.2.

$$
\begin{aligned}
& d\left(\mathrm{x}_{\delta} \mathrm{x}_{\gamma}^{2} \mathrm{x}_{\eta}^{2} \otimes 1\right)=\mathrm{x}_{\delta} \mathrm{x}_{\gamma}^{2} \mathrm{x}_{\eta} \otimes x_{\eta}-s\left(-q_{\gamma \eta}^{2} \mathrm{x}_{\delta} \mathrm{x}_{\gamma} \mathrm{x}_{\eta} \otimes x_{\eta} x_{\gamma}-q_{\delta \gamma}^{2} q_{\mu \eta} q_{\gamma \eta} \mathrm{b}_{2} \mathrm{x}_{\gamma}^{2} \mathrm{x}_{\kappa} \otimes x_{\eta} x_{\gamma} x_{\mu}\right. \\
& -q_{\delta \gamma}^{2}(3)_{\widetilde{q}_{\gamma \eta}} \mathrm{b}_{3} \mathrm{~b}_{7} \mathrm{x}_{\gamma}^{3} \otimes x_{\iota} x_{\kappa} x_{\gamma}-q_{\delta \gamma}^{2} q_{\nu \eta}(3)_{\widetilde{q}_{\gamma \eta}} \mathrm{b}_{3} \mathrm{x}_{\gamma}^{3} \otimes x_{\eta} x_{\nu}-q_{\delta \gamma}^{2} q_{\delta \eta} \mathrm{b}_{3} \mathrm{x}_{\gamma}^{2} \mathrm{x}_{\eta} \otimes x_{\gamma} x_{\nu} \\
& \left.-q_{\delta \gamma}^{2} q_{\delta \eta} \mathrm{b}_{2} \mathrm{x}_{\gamma}^{2} \mathrm{x}_{\eta} \otimes x_{\kappa} x_{\gamma} x_{\mu}-q_{\delta \gamma}^{2} q_{\delta \eta}^{2} \mathrm{x}_{\gamma}^{2} \mathrm{x}_{\eta} \otimes x_{\eta} x_{\delta}\right) \\
& =\mathrm{x}_{\delta} \mathrm{x}_{\gamma}^{2} \mathrm{x}_{\eta} \otimes x_{\eta}+q_{\gamma \eta}^{2} \mathrm{x}_{\delta} \mathrm{x}_{\gamma} \mathrm{x}_{\eta}^{2} \otimes x_{\gamma}+q_{\delta \gamma}^{2}(3)_{\widetilde{q}_{\gamma \eta}} \mathrm{b}_{3} \mathrm{~b}_{7} \mathrm{x}_{\gamma}^{3} \mathrm{x}_{\iota} \otimes x_{\kappa} x_{\gamma} \\
& +q_{\delta \gamma}^{2} q_{\nu \eta}(3)_{\widetilde{q}_{\gamma \eta}} \mathrm{b}_{3} \mathrm{x}_{\gamma}^{3} \mathbf{x}_{\eta} \otimes x_{\nu}+q_{\delta \gamma}^{2} q_{\mu \eta} q_{\gamma \eta} \mathrm{b}_{2} \mathrm{x}_{\gamma}^{2} \mathbf{x}_{\kappa} \mathbf{x}_{\eta} \otimes x_{\gamma} x_{\mu}+q_{\delta \gamma}^{2} q_{\delta \eta}^{2} \mathrm{x}_{\gamma}^{2} \mathbf{x}_{\eta}^{2} \otimes x_{\delta} .
\end{aligned}
$$


Next we compute differentials of some 6-chains:

$$
\begin{aligned}
& d\left(\mathrm{x}_{\alpha} \mathrm{x}_{\beta} \mathrm{x}_{\delta} \mathrm{x}_{\gamma}^{2} \mathrm{x}_{\eta} \otimes 1\right)=\mathrm{x}_{\alpha} \mathrm{x}_{\beta} \mathrm{x}_{\delta} \mathrm{x}_{\gamma}^{2} \otimes x_{\eta}-q_{\gamma \eta} \mathrm{x}_{\alpha} \mathrm{x}_{\beta} \mathrm{x}_{\delta} \mathrm{x}_{\gamma} \mathrm{x}_{\eta} \otimes x_{\gamma}-q_{\delta \gamma}^{2}(3)_{\widetilde{q}_{\gamma \eta}} \mathrm{b}_{3} \mathrm{x}_{\alpha} \mathrm{x}_{\beta} \mathrm{x}_{\gamma}^{3} \otimes x_{\nu} \\
& -q_{\delta \gamma}^{2} \mathrm{~b}_{2} \mathbf{x}_{\alpha} \mathbf{x}_{\beta} \mathrm{x}_{\gamma}^{2} \mathbf{x}_{\kappa} \otimes x_{\gamma} x_{\mu}-q_{\delta \gamma}^{2} q_{\delta \eta} \mathbf{x}_{\alpha} \mathbf{x}_{\beta} \mathrm{x}_{\gamma}^{2} \mathbf{x}_{\eta} \otimes x_{\delta}+q_{\beta \delta} q_{\beta \gamma}^{2} q_{\beta \eta} \mathbf{x}_{\alpha} \mathbf{x}_{\delta} \mathrm{x}_{\gamma}^{2} \mathbf{x}_{\eta} \otimes x_{\beta} \\
& -q_{\delta \gamma}^{2} q_{\gamma \beta}^{-2} \mathrm{c}_{\beta \eta \gamma}^{(3)} \mathrm{b}_{2} \mathrm{~b}_{8} \mathrm{x}_{\alpha} \mathbf{x}_{\gamma}^{4} \otimes x_{\mu}-q_{\alpha \beta} q_{\beta \gamma}^{2} q_{\beta \eta} q_{\tau \gamma}^{2} \mathrm{~b}_{1} \mathrm{~b}_{4} \mathrm{x}_{\beta} \mathrm{x}_{\tau} \mathrm{x}_{\gamma}^{2} \mathrm{x}_{\kappa} \otimes x_{\gamma} x_{\beta} \\
& -q_{\alpha \beta} q_{\beta \gamma}^{2} q_{\beta \eta} q_{\tau \gamma}^{2} q_{\tau \eta} \mathrm{b}_{1} \mathrm{x}_{\beta} \mathrm{x}_{\tau} \mathrm{x}_{\gamma}^{2} \mathrm{x}_{\eta} \otimes x_{\tau} x_{\beta}-q_{\alpha \beta} q_{\alpha \delta} q_{\alpha \gamma}^{2} q_{\alpha \eta} \mathrm{x}_{\beta} \mathrm{x}_{\delta} \mathrm{x}_{\gamma}^{2} \mathrm{x}_{\eta} \otimes x_{\alpha} \\
& +q_{\alpha \beta} q_{\beta \gamma}^{2} q_{\beta \eta} q_{\beta \tau} q_{\gamma \beta}^{-2}(3)_{\widetilde{q}_{\beta \gamma}} q_{\tau \gamma}^{2} \mathrm{~b}_{1} \mathrm{~b}_{4} \mathrm{~b}_{8} \mathrm{x}_{\tau} \mathrm{x}_{\gamma}^{4} \otimes x_{\beta},
\end{aligned}
$$

$$
\begin{aligned}
& d\left(\mathrm{x}_{\alpha} \mathrm{x}_{\beta} \mathrm{x}_{\delta} \mathrm{x}_{\gamma} \mathrm{x}_{\eta}^{2} \otimes 1\right)=\mathrm{x}_{\alpha} \mathrm{x}_{\beta} \mathrm{x}_{\delta} \mathrm{x}_{\gamma} \mathrm{x}_{\eta} \otimes x_{\eta}+q_{\gamma \eta}^{2} \mathrm{x}_{\alpha} \mathrm{x}_{\beta} \mathrm{x}_{\delta} \mathrm{x}_{\eta}^{2} \otimes x_{\gamma}-q_{\delta \gamma}(2)_{\widetilde{q}_{\gamma \eta}} q_{\nu \eta} \mathrm{b}_{3} \mathrm{x}_{\alpha} \mathrm{x}_{\beta} \mathrm{x}_{\gamma}^{2} \mathrm{x}_{\eta} \otimes x_{\nu} \\
& -q_{\delta \gamma}(2)_{\widetilde{q}_{\gamma \eta}} \mathrm{b}_{3} \mathrm{~b}_{7} \mathrm{x}_{\alpha} \mathrm{x}_{\beta} \mathrm{x}_{\gamma}^{2} \mathrm{x}_{\iota} \otimes x_{\kappa} x_{\gamma}-q_{\delta \gamma} q_{\gamma \eta} q_{\mu \eta} \mathrm{b}_{2} \mathrm{x}_{\alpha} \mathrm{x}_{\beta} \mathrm{x}_{\gamma} \mathrm{x}_{\kappa} \mathrm{x}_{\eta} \otimes x_{\gamma} x_{\mu} \\
& -q_{\delta \gamma} q_{\delta \eta}^{2} \mathrm{x}_{\alpha} \mathrm{x}_{\beta} \mathrm{x}_{\gamma} \mathrm{x}_{\eta}^{2} \otimes x_{\delta}+q_{\alpha \beta} q_{\beta \gamma} q_{\beta \eta}^{2} q_{\tau \gamma} q_{\gamma \eta} \mathrm{b}_{1} \mathrm{~b}_{4} \mathrm{x}_{\beta} \mathrm{x}_{\tau} \mathrm{x}_{\gamma} \mathrm{x}_{\kappa} \mathrm{x}_{\eta} \otimes x_{\gamma} x_{\beta} \\
& +q_{\delta \gamma} q_{\gamma \beta}^{-1} \mathrm{c}_{\beta \eta \gamma}^{(2)} \mathrm{b}_{2} \mathrm{~b}_{6} \mathrm{~b}_{8} \mathrm{x}_{\alpha} \mathrm{x}_{\gamma}^{3} \mathrm{x}_{\iota} \otimes x_{\gamma}+q_{\delta \gamma} q_{\gamma \kappa} \mathrm{d}_{\alpha \beta \eta \gamma}^{(3)} \mathrm{b}_{2} \mathrm{~b}_{6} \mathrm{~b}_{8} \mathrm{~b}_{9} \mathrm{x}_{\gamma}^{5} \otimes 1 \\
& +q_{\delta \gamma} q_{\gamma \beta}^{-1} q_{\mu \eta} \mathrm{c}_{\beta \eta \gamma}^{(2)} \mathrm{b}_{2} \mathrm{~b}_{8} \mathrm{x}_{\alpha} \mathrm{x}_{\gamma}^{3} \mathrm{x}_{\eta} \otimes x_{\mu}+q_{\beta \delta} q_{\beta \gamma} q_{\beta \eta}^{2} \mathrm{x}_{\alpha} \mathrm{x}_{\delta} \mathrm{x}_{\gamma} \mathrm{x}_{\eta}^{2} \otimes x_{\beta} \\
& -q_{\alpha \beta} q_{\beta \gamma} q_{\beta \eta}^{2} q_{\tau \gamma} q_{\tau \eta}^{2} \mathrm{~b}_{1} \mathrm{x}_{\beta} \mathrm{x}_{\tau} \mathrm{x}_{\gamma} \mathrm{x}_{\eta}^{2} \otimes x_{\tau} x_{\beta}-q_{\alpha \beta} q_{\alpha \delta} q_{\alpha \gamma} q_{\alpha \eta}^{2} \mathrm{x}_{\beta} \mathrm{x}_{\delta} \mathrm{x}_{\gamma} \mathrm{x}_{\eta}^{2} \otimes x_{\alpha} \\
& -q_{\alpha \beta} q_{\beta \tau} q_{\beta \eta}^{2} q_{\eta \gamma}^{-1} q_{\gamma \beta}^{-1} \mathrm{c}_{\beta \eta \gamma}^{(2)} \mathrm{b}_{1} \mathrm{~b}_{4} \mathrm{~b}_{8} \mathrm{x}_{\tau} \mathrm{x}_{\gamma}^{3} \mathrm{x}_{\eta} \otimes x_{\beta},
\end{aligned}
$$

$$
\begin{aligned}
& d\left(\mathrm{x}_{\alpha} \mathrm{x}_{\beta} \mathrm{x}_{\gamma}^{2} \mathrm{x}_{\kappa} \mathrm{x}_{\eta} \otimes 1\right)=\mathrm{x}_{\alpha} \mathrm{x}_{\beta} \mathrm{x}_{\gamma}^{2} \mathrm{x}_{\kappa} \otimes x_{\eta}-q_{\kappa \eta} \mathrm{x}_{\alpha} \mathrm{x}_{\beta} \mathrm{x}_{\gamma}^{2} \mathrm{x}_{\eta} \otimes x_{\kappa}+q_{\gamma \kappa} q_{\gamma \eta} \mathrm{x}_{\alpha} \mathrm{x}_{\beta} \mathrm{x}_{\gamma} \mathrm{x}_{\kappa} \mathrm{x}_{\eta} \otimes x_{\gamma} \\
& \quad+q_{\gamma \beta}^{-2}(3)_{\widetilde{q}_{\beta \gamma}} \mathrm{b}_{8} \mathrm{x}_{\alpha} \mathrm{x}_{\gamma}^{3} \mathrm{x}_{\eta} \otimes 1+q_{\beta \gamma}^{2} q_{\beta \kappa} q_{\beta \eta} \mathrm{x}_{\alpha} \mathrm{x}_{\gamma}^{2} \mathrm{x}_{\kappa} \mathrm{x}_{\eta} \otimes x_{\beta}-q_{\alpha \beta} q_{\alpha \gamma}^{2} q_{\alpha \kappa} q_{\alpha \eta} \mathrm{x}_{\beta} \mathrm{x}_{\gamma}^{2} \mathrm{x}_{\kappa} \mathrm{x}_{\eta} \otimes x_{\alpha}
\end{aligned}
$$

$$
\begin{aligned}
& d\left(\mathrm{x}_{\alpha} \mathrm{x}_{\delta} \mathrm{x}_{\gamma}^{2} \mathrm{x}_{\eta}^{2} \otimes 1\right)=\mathbf{x}_{\alpha} \mathrm{x}_{\delta} \mathrm{x}_{\gamma}^{2} \mathrm{x}_{\eta} \otimes x_{\eta}+q_{\gamma \eta}^{2} \mathrm{x}_{\alpha} \mathrm{x}_{\delta} \mathrm{x}_{\gamma} \mathrm{x}_{\eta}^{2} \otimes x_{\gamma}+q_{\delta \gamma}^{2}(2)_{\widetilde{q}_{\gamma \eta}} \mathrm{b}_{3} \mathrm{~b}_{7} \mathrm{x}_{\alpha} \mathrm{x}_{\gamma}^{3} \mathrm{x}_{\iota} \otimes x_{\kappa} x_{\gamma} \\
& \quad-q_{\delta \gamma}^{2} \mathrm{c}_{\alpha \eta \gamma}^{(3)} \mathrm{b}_{3} \mathrm{~b}_{7} \mathrm{~b}_{9} \mathrm{x}_{\gamma}^{5} \otimes x_{\kappa}+q_{\delta \gamma}^{2} q_{\nu \eta}(2)_{\widetilde{q}_{\gamma \eta}} \mathrm{b}_{3} \mathrm{x}_{\alpha} \mathrm{x}_{\gamma}^{3} \mathrm{x}_{\eta} \otimes x_{\nu}+q_{\delta \gamma}^{2} q_{\delta \eta}^{2} \mathrm{x}_{\alpha} \mathrm{x}_{\gamma}^{2} \mathrm{x}_{\eta}^{2} \otimes x_{\delta} \\
& \quad+q_{\delta \gamma}^{2} q_{\mu \eta} q_{\gamma \eta} \mathrm{b}_{2} \mathrm{x}_{\alpha} \mathbf{x}_{\gamma}^{2} \mathrm{x}_{\kappa} \mathrm{x}_{\eta} \otimes x_{\gamma} x_{\mu}-q_{\beta \eta}^{2} q_{\tau \gamma}^{2} q_{\beta \gamma}^{2} q_{\gamma \eta} \mathrm{b}_{1} \mathrm{~b}_{4} \mathrm{x}_{\tau} \mathrm{x}_{\gamma}^{2} \mathrm{x}_{\kappa} \mathrm{x}_{\eta} \otimes x_{\gamma} x_{\beta} \\
& \quad-q_{\beta \eta}^{2} q_{\tau \gamma}^{2} q_{\beta \gamma}^{2} q_{\tau \eta}^{2} \mathrm{~b}_{1} \mathrm{x}_{\tau} \mathrm{x}_{\gamma}^{2} \mathrm{x}_{\eta}^{2} \otimes x_{\tau} x_{\beta}-q_{\alpha \delta} q_{\alpha \gamma}^{2} q_{\alpha \eta}^{2} \mathrm{x}_{\delta} \mathrm{x}_{\gamma}^{2} \mathrm{x}_{\eta}^{2} \otimes x_{\alpha},
\end{aligned}
$$

$$
\begin{aligned}
& d\left(\mathrm{x}_{\beta} \mathrm{x}_{\tau} \mathrm{x}_{\gamma}^{2} \mathrm{x}_{\kappa} \mathrm{x}_{\eta} \otimes 1\right)=\mathrm{x}_{\beta} \mathrm{x}_{\tau} \mathrm{x}_{\gamma}^{2} \mathrm{x}_{\kappa} \otimes x_{\eta}-q_{\kappa \eta} \mathrm{x}_{\beta} \mathrm{x}_{\tau} \mathrm{x}_{\gamma}^{2} \mathrm{x}_{\eta} \otimes x_{\kappa}+q_{\gamma \kappa} q_{\gamma \eta} \mathrm{x}_{\beta} \mathrm{x}_{\tau} \mathrm{x}_{\gamma} \mathrm{x}_{\kappa} \mathrm{x}_{\eta} \otimes x_{\gamma} \\
& \quad+q_{\tau \gamma}^{2} q_{\tau \kappa}(2)_{\widetilde{q}_{\kappa \eta}} \mathrm{b}_{4} \mathrm{x}_{\beta} \mathrm{x}_{\gamma}^{2} \mathrm{x}_{\kappa}^{2} \otimes x_{\gamma}+q_{\tau \gamma}^{2} q_{\tau \kappa} q_{\tau \eta} \mathrm{x}_{\beta} \mathrm{x}_{\gamma}^{2} \mathrm{x}_{\kappa} \mathrm{x}_{\eta} \otimes x_{\tau}-q_{\beta \tau} q_{\gamma \beta}^{-2}(3)_{\widetilde{q}_{\beta \gamma}} \mathrm{b}_{8} \mathrm{x}_{\tau} \mathrm{x}_{\gamma}^{3} \mathrm{x}_{\eta} \otimes 1 \\
& \quad-q_{\beta \tau} q_{\beta \gamma}^{2} q_{\beta \kappa} q_{\beta \eta} \mathrm{x}_{\tau} \mathrm{x}_{\gamma}^{2} \mathrm{x}_{\kappa} \mathrm{x}_{\eta} \otimes x_{\beta}-q_{\gamma \eta}^{2} q_{\kappa \eta} \mathrm{c}_{-\eta, \beta, \gamma}^{(2)} \mathrm{b}_{4} \mathrm{~b}_{8} \mathrm{x}_{\gamma}^{4} \mathrm{x}_{\kappa} \otimes 1
\end{aligned}
$$

$$
\begin{gathered}
d\left(\mathrm{x}_{\beta} \mathrm{x}_{\tau} \mathrm{x}_{\gamma}^{2} \mathrm{x}_{\eta}^{2} \otimes 1\right)=\mathrm{x}_{\beta} \mathrm{x}_{\tau} \mathrm{x}_{\gamma}^{2} \mathrm{x}_{\eta} \otimes x_{\eta}+q_{\gamma \eta}^{2} \mathrm{x}_{\beta} \mathrm{x}_{\tau} \mathrm{x}_{\gamma} \mathrm{x}_{\eta}^{2} \otimes x_{\gamma}+q_{\tau \gamma}^{2} q_{\gamma \eta} \mathrm{b}_{4} \mathrm{x}_{\beta} \mathrm{x}_{\gamma}^{2} \mathrm{x}_{\kappa} \mathrm{x}_{\eta} \otimes x_{\gamma} \\
+q_{\tau \gamma}^{2} q_{\tau \eta}^{2} \mathrm{x}_{\beta} \mathrm{x}_{\gamma}^{2} \mathrm{x}_{\eta}^{2} \otimes x_{\tau}-q_{\beta \tau} q_{\beta \gamma}^{2} q_{\beta \eta}^{2} \mathrm{x}_{\tau} \mathrm{x}_{\gamma}^{2} \mathrm{x}_{\eta}^{2} \otimes x_{\beta}+q_{\gamma \eta}^{2} c_{-\eta, \beta, \gamma}^{(2)} \mathrm{b}_{4} \mathrm{~b}_{8} \mathrm{x}_{\gamma}^{4} \otimes x_{\eta}
\end{gathered}
$$


$(10.1 .140)$

$$
\begin{aligned}
& d\left(\mathrm{x}_{\beta} \mathrm{x}_{\delta} \mathrm{x}_{\gamma}^{2} \mathrm{x}_{\eta}^{2} \otimes 1\right)=\mathrm{x}_{\beta} \mathrm{x}_{\delta} \mathrm{x}_{\gamma}^{2} \mathrm{x}_{\eta} \otimes x_{\eta}+q_{\gamma \eta}^{2} \mathrm{x}_{\beta} \mathrm{x}_{\delta} \mathrm{x}_{\gamma} \mathrm{x}_{\eta}^{2} \otimes x_{\gamma}+q_{\delta \gamma}^{2}(3)_{\widetilde{q}_{\gamma \eta}} \mathrm{b}_{3} \mathrm{~b}_{7} \mathrm{x}_{\beta} \mathrm{x}_{\gamma}^{3} \mathrm{x}_{\iota} \otimes x_{\kappa} x_{\gamma} \\
& \quad+q_{\delta \gamma}^{2} q_{\nu \eta}(3)_{\widetilde{q}_{\gamma \eta}} \mathrm{b}_{3} \mathrm{x}_{\beta} \mathrm{x}_{\gamma}^{3} \mathrm{x}_{\eta} \otimes x_{\nu}+q_{\delta \gamma}^{2} q_{\mu \eta} q_{\gamma \eta} \mathrm{b}_{2} \mathrm{x}_{\beta} \mathrm{x}_{\gamma}^{2} \mathrm{x}_{\kappa} \mathrm{x}_{\eta} \otimes x_{\gamma} x_{\mu} \\
& \quad+q_{\delta \gamma}^{2} q_{\delta \eta}^{2} \mathrm{x}_{\beta} \mathrm{x}_{\gamma}^{2} \mathrm{x}_{\eta}^{2} \otimes x_{\delta}-q_{\beta \delta} q_{\beta \gamma}^{2} q_{\beta \eta}^{2} \mathrm{x}_{\delta} \mathrm{x}_{\gamma}^{2} \mathrm{x}_{\eta}^{2} \otimes x_{\beta} \\
& \quad-q_{\beta \gamma} q_{\gamma \kappa} q_{\delta \gamma}^{2} c_{\beta \eta}^{(3)} \mathrm{b}_{2} \mathrm{~b}_{6} \mathrm{~b}_{8} \mathrm{x}_{\gamma}^{4} \mathrm{x}_{\iota} \otimes x_{\gamma}-q_{\beta \gamma} q_{\gamma \kappa} q_{\delta \gamma}^{2} c_{\beta \eta}^{(3)} q_{\mu \eta} \mathrm{b}_{2} \mathrm{~b}_{8} \mathrm{x}_{\gamma}^{4} \mathrm{x}_{\eta} \otimes x_{\mu} .
\end{aligned}
$$

First we deal with (10.1.134): using (10.1.117), Remark 10.1.2, (10.1.118), (10.1.120), (10.1.122), (10.1.124), (10.1.86) and (10.1.127):

$$
\begin{aligned}
& d\left(\mathrm{x}_{\alpha} \mathrm{x}_{\beta} \mathrm{x}_{\delta} \mathrm{x}_{\gamma}^{2} \mathrm{x}_{\eta} \otimes 1\right)=\mathrm{x}_{\alpha} \mathrm{x}_{\beta} \mathrm{x}_{\delta} \mathrm{x}_{\gamma}^{2} \otimes x_{\eta}-s\left(q_{\gamma \eta} \mathrm{x}_{\alpha} \mathrm{x}_{\beta} \mathrm{x}_{\delta} \mathrm{x}_{\gamma} \otimes x_{\eta} x_{\gamma}+q_{\delta \gamma}^{2} \mathrm{x}_{\alpha} \mathrm{x}_{\beta} \mathrm{x}_{\gamma}^{2} \otimes q_{\delta \eta} x_{\eta} x_{\delta}\right. \\
& +q_{\delta \gamma}^{2} \mathbf{x}_{\alpha} \mathbf{x}_{\beta} \mathbf{x}_{\gamma}^{2} \otimes \mathrm{b}_{2} x_{\kappa} x_{\gamma} x_{\mu}+q_{\delta \gamma}^{2} \mathbf{x}_{\alpha} \mathbf{x}_{\beta} \mathbf{x}_{\gamma}^{2} \otimes \mathrm{b}_{3} x_{\gamma} x_{\nu}-q_{\beta \delta} q_{\beta \gamma}^{2} q_{\beta \eta} \mathbf{x}_{\alpha} \mathbf{x}_{\delta} \mathbf{x}_{\gamma}^{2} \otimes x_{\eta} x_{\beta} \\
& \left.+q_{\alpha \beta} q_{\beta \gamma}^{2} q_{\beta \eta} q_{\tau \gamma}^{2} \mathrm{~b}_{1} \mathrm{x}_{\beta} \mathrm{x}_{\tau} \mathrm{x}_{\gamma}^{2} \otimes\left(q_{\tau \eta} x_{\eta} x_{\tau}+\mathrm{b}_{4} x_{\kappa} x_{\gamma}\right) x_{\beta}+q_{\alpha \beta} q_{\alpha \delta} q_{\alpha \gamma}^{2} q_{\alpha \eta} \mathrm{x}_{\beta} \mathrm{x}_{\delta} \mathrm{x}_{\gamma}^{2} \otimes x_{\eta} x_{\alpha}\right) \\
& =\mathrm{x}_{\alpha} \mathrm{x}_{\beta} \mathrm{x}_{\delta} \mathrm{x}_{\gamma}^{2} \otimes x_{\eta}-q_{\gamma \eta} \mathrm{x}_{\alpha} \mathrm{x}_{\beta} \mathrm{x}_{\delta} \mathrm{x}_{\gamma} \mathrm{x}_{\eta} \otimes x_{\gamma}-q_{\delta \gamma}^{2}(3)_{\widetilde{q}_{\gamma \eta}} \mathrm{b}_{3} \mathrm{x}_{\alpha} \mathrm{x}_{\beta} \mathrm{x}_{\gamma}^{3} \otimes x_{\nu} \\
& -q_{\delta \gamma}^{2} \mathrm{~b}_{2} \mathbf{x}_{\alpha} \mathbf{x}_{\beta} \mathbf{x}_{\gamma}^{2} \mathbf{x}_{\kappa} \otimes x_{\gamma} x_{\mu}-q_{\delta \gamma}^{2} q_{\delta \eta} \mathbf{x}_{\alpha} \mathbf{x}_{\beta} \mathbf{x}_{\gamma}^{2} \mathbf{x}_{\eta} \otimes x_{\delta}+q_{\beta \delta} q_{\beta \gamma}^{2} q_{\beta \eta} \mathbf{x}_{\alpha} \mathbf{x}_{\delta} \mathbf{x}_{\gamma}^{2} \mathbf{x}_{\eta} \otimes x_{\beta} \\
& -q_{\delta \gamma}^{2} q_{\gamma \beta}^{-2} \mathrm{c}_{\beta \eta \gamma}^{(3)} \mathrm{b}_{2} \mathrm{~b}_{8} \mathrm{x}_{\alpha} \mathrm{x}_{\gamma}^{4} \otimes x_{\mu}-q_{\alpha \beta} q_{\beta \gamma}^{2} q_{\beta \eta} q_{\tau \gamma}^{2} \mathrm{~b}_{1} \mathrm{~b}_{4} \mathrm{x}_{\beta} \mathrm{x}_{\tau} \mathrm{x}_{\gamma}^{2} \mathrm{x}_{\kappa} \otimes x_{\gamma} x_{\beta} \\
& -q_{\alpha \beta} q_{\beta \gamma}^{2} q_{\beta \eta} q_{\tau \gamma}^{2} q_{\tau \eta} \mathrm{b}_{1} \mathrm{x}_{\beta} \mathrm{x}_{\tau} \mathrm{x}_{\gamma}^{2} \mathrm{x}_{\eta} \otimes x_{\tau} x_{\beta}-q_{\alpha \beta} q_{\alpha \delta} q_{\alpha \gamma}^{2} q_{\alpha \eta} \mathrm{x}_{\beta} \mathrm{x}_{\delta} \mathrm{x}_{\gamma}^{2} \mathrm{x}_{\eta} \otimes x_{\alpha} \\
& +q_{\alpha \beta} q_{\beta \gamma}^{2} q_{\beta \eta} q_{\beta \tau} q_{\gamma \beta}^{-2}(3)_{\widetilde{q}_{\beta \gamma}} q_{\tau \gamma}^{2} \mathrm{~b}_{1} \mathrm{~b}_{4} \mathrm{~b}_{8} \mathrm{x}_{\tau} \mathrm{x}_{\gamma}^{4} \otimes x_{\beta} .
\end{aligned}
$$

Next we compute (10.1.135): using (10.1.118), (10.1.119), Remark 10.1.2, (10.1.121), (10.1.123), (10.1.83), (10.1.125), (10.1.126), (10.1.128) and (10.1.84):

$$
\begin{aligned}
& d\left(\mathrm{x}_{\alpha} \mathrm{x}_{\beta} \mathrm{x}_{\delta} \mathrm{x}_{\gamma} \mathrm{x}_{\eta}^{2} \otimes 1\right)=\mathrm{x}_{\alpha} \mathrm{x}_{\beta} \mathrm{x}_{\delta} \mathrm{x}_{\gamma} \mathrm{x}_{\eta} \otimes x_{\eta}-s\left(q_{\delta \gamma}(2)_{\widetilde{q}_{\gamma \eta}} \mathrm{b}_{3} \mathrm{x}_{\alpha} \mathrm{x}_{\beta} \mathrm{x}_{\gamma}^{2} \otimes\left(q_{\nu \eta} x_{\eta} x_{\nu}+\mathrm{b}_{7} x_{\iota} x_{\kappa} x_{\gamma}\right)\right. \\
& -q_{\gamma \eta}^{2} \mathrm{x}_{\alpha} \mathbf{x}_{\beta} \mathrm{x}_{\delta} \mathbf{x}_{\eta} \otimes x_{\eta} x_{\gamma}+q_{\delta \gamma} q_{\delta \eta} \mathrm{x}_{\alpha} \mathrm{x}_{\beta} \mathrm{x}_{\gamma} \mathrm{x}_{\eta} \otimes\left(q_{\delta \eta} x_{\eta} x_{\delta}+\mathrm{b}_{2} x_{\kappa} x_{\gamma} x_{\mu}+\mathrm{b}_{3} x_{\gamma} x_{\nu}\right) \\
& +q_{\delta \gamma} q_{\gamma \eta} q_{\mu \eta} \mathrm{b}_{2} \mathbf{x}_{\alpha} \mathbf{x}_{\beta} \mathbf{x}_{\gamma} \mathbf{x}_{\kappa} \otimes x_{\eta} x_{\gamma} x_{\mu}-q_{\beta \delta} q_{\beta \gamma} q_{\beta \eta}^{2} \mathbf{x}_{\alpha} \mathbf{x}_{\delta} \mathbf{x}_{\gamma} \mathbf{x}_{\eta} \otimes x_{\eta} x_{\beta} \\
& +q_{\alpha \beta} q_{\beta \gamma} q_{\beta \eta}^{2} q_{\tau \gamma} q_{\tau \eta} \mathrm{b}_{1} \mathrm{x}_{\beta} \mathrm{x}_{\tau} \mathrm{x}_{\gamma} \mathrm{x}_{\eta} \otimes\left(q_{\tau \eta} x_{\eta} x_{\tau}+\mathrm{b}_{4} x_{\kappa} x_{\gamma}\right) x_{\beta} \\
& -q_{\delta \gamma} q_{\gamma \beta}^{-1} \mathrm{c}_{\beta \eta \gamma}^{(2)} \mathrm{b}_{2} \mathrm{~b}_{8} \mathrm{x}_{\alpha} \mathrm{x}_{\gamma}^{3} \otimes x_{\mu} x_{\eta}+q_{\alpha \beta} q_{\beta \gamma} q_{\beta \eta}^{2} q_{\tau \gamma} q_{\gamma \eta} \mathrm{b}_{1} \mathrm{~b}_{4} \mathrm{x}_{\beta} \mathrm{x}_{\tau} \mathrm{x}_{\gamma} \mathrm{x}_{\kappa} \otimes x_{\eta} x_{\gamma} x_{\beta} \\
& \left.+q_{\alpha \beta} q_{\alpha \delta} q_{\alpha \gamma} q_{\alpha \eta}^{2} \mathrm{x}_{\beta} \mathrm{x}_{\delta} \mathrm{x}_{\gamma} \mathrm{x}_{\eta} \otimes x_{\eta} x_{\alpha}+q_{\alpha \beta} q_{\beta \tau} q_{\beta \eta}^{2} q_{\eta \gamma}^{-1} q_{\gamma \beta}^{-1} \mathrm{c}_{\beta \eta \gamma}^{(2)} \mathrm{b}_{1} \mathrm{~b}_{4} \mathrm{~b}_{8} \mathrm{x}_{\tau} \mathrm{x}_{\gamma}^{3} \otimes x_{\eta} x_{\beta}\right) \\
& =\mathrm{x}_{\alpha} \mathrm{x}_{\beta} \mathrm{x}_{\delta} \mathrm{x}_{\gamma} \mathrm{x}_{\eta} \otimes x_{\eta}+q_{\gamma \eta}^{2} \mathrm{x}_{\alpha} \mathrm{x}_{\beta} \mathrm{x}_{\delta} \mathrm{x}_{\eta}^{2} \otimes x_{\gamma}-q_{\delta \gamma}(2)_{\widetilde{q}_{\gamma \eta}} \mathrm{b}_{3} \mathrm{~b}_{7} \mathrm{x}_{\alpha} \mathrm{x}_{\beta} \mathrm{x}_{\gamma}^{2} \mathrm{x}_{\iota} \otimes x_{\kappa} x_{\gamma} \\
& -q_{\delta \gamma}(2)_{\widetilde{q}_{\gamma \eta}} q_{\nu \eta} \mathrm{b}_{3} \mathrm{x}_{\alpha} \mathrm{x}_{\beta} \mathrm{x}_{\gamma}^{2} \mathrm{x}_{\eta} \otimes x_{\nu}-q_{\delta \gamma} q_{\gamma \eta} q_{\mu \eta} \mathrm{b}_{2} \mathrm{x}_{\alpha} \mathrm{x}_{\beta} \mathrm{x}_{\gamma} \mathrm{x}_{\kappa} \mathrm{x}_{\eta} \otimes x_{\gamma} x_{\mu} \\
& -q_{\delta \gamma} q_{\delta \eta}^{2} \mathrm{x}_{\alpha} \mathrm{x}_{\beta} \mathrm{x}_{\gamma} \mathrm{x}_{\eta}^{2} \otimes x_{\delta}+q_{\alpha \beta} q_{\beta \gamma} q_{\beta \eta}^{2} q_{\tau \gamma} q_{\gamma \eta} \mathrm{b}_{1} \mathrm{~b}_{4} \mathrm{x}_{\beta} \mathrm{x}_{\tau} \mathrm{x}_{\gamma} \mathrm{x}_{\kappa} \mathrm{x}_{\eta} \otimes x_{\gamma} x_{\beta} \\
& +q_{\delta \gamma} q_{\gamma \beta}^{-1} \mathrm{c}_{\beta \eta \gamma}^{(2)} \mathrm{b}_{2} \mathrm{~b}_{6} \mathrm{~b}_{8} \mathrm{x}_{\alpha} \mathrm{x}_{\gamma}^{3} \mathrm{x}_{\iota} \otimes x_{\gamma}+q_{\delta \gamma} q_{\gamma \kappa} \mathrm{d}_{\alpha \beta \eta \gamma}^{(3)} \mathrm{b}_{2} \mathrm{~b}_{6} \mathrm{~b}_{8} \mathrm{~b}_{9} \mathrm{x}_{\gamma}^{5} \otimes 1+q_{\delta \gamma} q_{\gamma \beta}^{-1} q_{\mu \eta} \mathrm{c}_{\beta \eta \gamma}^{(2)} \mathrm{b}_{2} \mathrm{~b}_{8} \mathrm{x}_{\alpha} \mathrm{x}_{\gamma}^{3} \mathrm{x}_{\eta} \otimes x_{\mu} \\
& +q_{\beta \delta} q_{\beta \gamma} q_{\beta \eta}^{2} \mathrm{x}_{\alpha} \mathrm{x}_{\delta} \mathrm{x}_{\gamma} \mathrm{x}_{\eta}^{2} \otimes x_{\beta}-q_{\alpha \beta} q_{\beta \gamma} q_{\beta \eta}^{2} q_{\tau \gamma} q_{\tau \eta}^{2} \mathrm{~b}_{1} \mathrm{x}_{\beta} \mathrm{x}_{\tau} \mathrm{x}_{\gamma} \mathrm{x}_{\eta}^{2} \otimes x_{\tau} x_{\beta} \\
& -q_{\alpha \beta} q_{\alpha \delta} q_{\alpha \gamma} q_{\alpha \eta}^{2} \mathrm{x}_{\beta} \mathrm{x}_{\delta} \mathrm{x}_{\gamma} \mathrm{x}_{\eta}^{2} \otimes x_{\alpha}-q_{\alpha \beta} q_{\beta \tau} q_{\beta \eta}^{2} q_{\eta \gamma}^{-1} q_{\gamma \beta}^{-1} \mathrm{c}_{\beta \eta \gamma}^{(2)} \mathrm{b}_{1} \mathrm{~b}_{4} \mathrm{~b}_{8} \mathrm{x}_{\tau} \mathrm{x}_{\gamma}^{3} \mathrm{x}_{\eta} \otimes x_{\beta} .
\end{aligned}
$$

For (10.1.136) we use (10.1.120), Remark 10.1.2, (10.1.121) and (10.1.130):

$$
\begin{aligned}
& d\left(\mathrm{x}_{\alpha} \mathrm{x}_{\beta} \mathrm{x}_{\gamma}^{2} \mathrm{x}_{\kappa} \mathrm{x}_{\eta} \otimes 1\right)=\mathrm{x}_{\alpha} \mathrm{x}_{\beta} \mathrm{x}_{\gamma}^{2} \mathrm{x}_{\kappa} \otimes x_{\eta}-s\left(q_{\kappa \eta} \mathrm{x}_{\alpha} \mathrm{x}_{\beta} \mathrm{x}_{\gamma}^{2} \otimes x_{\eta} x_{\kappa}-q_{\gamma \kappa} q_{\gamma \eta} \mathrm{x}_{\alpha} \mathrm{x}_{\beta} \mathrm{x}_{\gamma} \mathrm{x}_{\kappa} \otimes x_{\eta} x_{\gamma}\right. \\
& \left.\quad-q_{\beta \gamma}^{2} q_{\beta \kappa} q_{\beta \eta} \mathrm{x}_{\alpha} \mathrm{x}_{\gamma}^{2} \mathrm{x}_{\kappa} \otimes x_{\eta} x_{\beta}-q_{\gamma \beta}^{-2}(3)_{\widetilde{q}_{\beta \gamma}} \mathrm{b}_{8} \mathrm{x}_{\alpha} \mathrm{x}_{\gamma}^{3} \otimes x_{\eta}+q_{\alpha \beta} q_{\alpha \gamma}^{2} q_{\alpha \kappa} q_{\alpha \eta} \mathrm{x}_{\beta} \mathrm{x}_{\gamma}^{2} \mathrm{x}_{\kappa} \otimes x_{\eta} x_{\alpha}\right)
\end{aligned}
$$




$$
\begin{aligned}
= & \mathrm{x}_{\alpha} \mathrm{x}_{\beta} \mathrm{x}_{\gamma}^{2} \mathrm{x}_{\kappa} \otimes x_{\eta}-q_{\kappa \eta} \mathrm{x}_{\alpha} \mathrm{x}_{\beta} \mathrm{x}_{\gamma}^{2} \mathrm{x}_{\eta} \otimes x_{\kappa}+q_{\gamma \kappa} q_{\gamma \eta} \mathrm{x}_{\alpha} \mathrm{x}_{\beta} \mathrm{x}_{\gamma} \mathrm{x}_{\kappa} \mathrm{x}_{\eta} \otimes x_{\gamma} \\
& +q_{\gamma \beta}^{-2}(3)_{\widetilde{q}_{\beta \gamma}} \mathrm{b}_{8} \mathrm{x}_{\alpha} \mathrm{x}_{\gamma}^{3} \mathrm{x}_{\eta} \otimes 1+q_{\beta \gamma}^{2} q_{\beta \kappa} q_{\beta \eta} \mathrm{x}_{\alpha} \mathrm{x}_{\gamma}^{2} \mathrm{x}_{\kappa} \mathrm{x}_{\eta} \otimes x_{\beta}-q_{\alpha \beta} q_{\alpha \gamma}^{2} q_{\alpha \kappa} q_{\alpha \eta} \mathrm{x}_{\beta} \mathrm{x}_{\gamma}^{2} \mathrm{x}_{\kappa} \mathrm{x}_{\eta} \otimes x_{\alpha} .
\end{aligned}
$$

For (10.1.137) we use (10.1.122), (10.1.123), (10.1.83), (10.1.131), (10.1.132) and (10.1.133):

$$
\begin{aligned}
& d\left(\mathrm{x}_{\alpha} \mathrm{x}_{\delta} \mathrm{x}_{\gamma}^{2} \mathrm{x}_{\eta}^{2} \otimes 1\right)=\mathrm{x}_{\alpha} \mathrm{x}_{\delta} \mathrm{x}_{\gamma}^{2} \mathrm{x}_{\eta} \otimes x_{\eta}-s\left(-q_{\gamma \eta}^{2} \mathrm{x}_{\alpha} \mathrm{x}_{\delta} \mathrm{x}_{\gamma} \mathrm{x}_{\eta} \otimes x_{\eta} x_{\gamma}-q_{\delta \gamma}^{2} q_{\nu \eta}(2)_{\widetilde{q}_{\gamma \eta}} \mathrm{b}_{3} \mathrm{x}_{\alpha} \mathrm{x}_{\gamma}^{3} \otimes x_{\eta} x_{\nu}\right. \\
& \quad-q_{\delta \gamma}^{2}(2)_{\widetilde{q}_{\gamma \eta}} \mathrm{b}_{3} \mathrm{~b}_{7} \mathrm{x}_{\alpha} \mathrm{x}_{\gamma}^{3} \otimes x_{\iota} x_{\kappa} x_{\gamma}-q_{\delta \gamma}^{2} q_{\mu \eta} q_{\gamma \eta} \mathrm{b}_{2} \mathrm{x}_{\alpha} \mathrm{x}_{\gamma}^{2} \mathrm{x}_{\kappa} \otimes x_{\eta} x_{\gamma} x_{\mu}-q_{\delta \gamma}^{2} q_{\delta \eta}^{2} \mathrm{x}_{\alpha} \mathrm{x}_{\gamma}^{2} \mathrm{x}_{\eta} \otimes x_{\eta} x_{\delta} \\
& \quad-q_{\delta \gamma}^{2} q_{\delta \eta} \mathrm{b}_{2} \mathrm{x}_{\alpha} \mathrm{x}_{\gamma}^{2} \mathrm{x}_{\eta} \otimes x_{\kappa} x_{\gamma} x_{\mu}-q_{\delta \gamma}^{2} q_{\delta \eta} \mathrm{b}_{3} \mathrm{x}_{\alpha} \mathrm{x}_{\gamma}^{2} \mathrm{x}_{\eta} \otimes x_{\gamma} x_{\nu}+q_{\alpha \delta} q_{\alpha \gamma}^{2} q_{\alpha \eta}^{2} \mathrm{x}_{\delta} \mathrm{x}_{\gamma}^{2} \mathrm{x}_{\eta} \otimes x_{\eta} x_{\alpha} \\
& \quad+q_{\beta \eta}^{2} q_{\tau \gamma}^{2} q_{\beta \gamma}^{2} q_{\tau \eta}^{2} \mathrm{~b}_{1} \mathrm{x}_{\tau} \mathrm{x}_{\gamma}^{2} \mathrm{x}_{\eta} \otimes x_{\eta} x_{\tau} x_{\beta}+q_{\beta \eta}^{2} q_{\tau \gamma}^{2} q_{\beta \gamma}^{2} q_{\tau \eta} \mathrm{b}_{1} \mathrm{~b}_{4} \mathrm{x}_{\tau} \mathrm{x}_{\gamma}^{2} \mathrm{x}_{\eta} \otimes x_{\kappa} x_{\gamma} x_{\beta} \\
& \left.\quad+q_{\beta \eta}^{2} q_{\tau \gamma}^{2} q_{\beta \gamma}^{2} q_{\gamma \eta} \mathrm{b}_{1} \mathrm{~b}_{4} \mathrm{x}_{\tau} \mathrm{x}_{\gamma}^{2} \mathrm{x}_{\kappa} \otimes x_{\eta} x_{\gamma} x_{\beta}\right) \\
& =\mathrm{x}_{\alpha} \mathrm{x}_{\delta} \mathrm{x}_{\gamma}^{2} \mathrm{x}_{\eta} \otimes x_{\eta}+q_{\gamma \eta}^{2} \mathrm{x}_{\alpha} \mathrm{x}_{\delta} \mathrm{x}_{\gamma} \mathrm{x}_{\eta}^{2} \otimes x_{\gamma}+q_{\delta \gamma}^{2}(2)_{\widetilde{q}_{\gamma \eta}} \mathrm{b}_{3} \mathrm{~b}_{7} \mathrm{x}_{\alpha} \mathrm{x}_{\gamma}^{3} \mathrm{x}_{\iota} \otimes x_{\kappa} x_{\gamma} \\
& \quad-q_{\delta \gamma}^{2} c_{\alpha \eta \gamma}^{(3)} \mathrm{b}_{3} \mathrm{~b}_{7} \mathrm{~b}_{9} \mathrm{x}_{\gamma}^{5} \otimes x_{\kappa}+q_{\delta \gamma}^{2} q_{\nu \eta}(2)_{\widetilde{q}_{\gamma \eta}} \mathrm{b}_{3} \mathrm{x}_{\alpha} \mathrm{x}_{\gamma}^{3} \mathrm{x}_{\eta} \otimes x_{\nu}+q_{\delta \gamma}^{2} q_{\mu \eta} q_{\gamma \eta} \mathrm{b}_{2} \mathrm{x}_{\alpha} \mathrm{x}_{\gamma}^{2} \mathrm{x}_{\kappa} \mathrm{x}_{\eta} \otimes x_{\gamma} x_{\mu} \\
& \quad+q_{\delta \gamma}^{2} q_{\delta \eta}^{2} \mathrm{x}_{\alpha} \mathrm{x}_{\gamma}^{2} \mathrm{x}_{\eta}^{2} \otimes x_{\delta}-q_{\beta \eta}^{2} q_{\tau \gamma}^{2} q_{\beta \gamma}^{2} q_{\gamma \eta} \mathrm{b}_{1} \mathrm{~b}_{4} \mathrm{x}_{\tau} \mathrm{x}_{\gamma}^{2} \mathrm{x}_{\kappa} \mathrm{x}_{\eta} \otimes x_{\gamma} x_{\beta} \\
& \quad-q_{\beta \eta}^{2} q_{\tau \gamma}^{2} q_{\beta \gamma}^{2} q_{\tau \eta}^{2} \mathrm{~b}_{1} \mathrm{x}_{\tau} \mathrm{x}_{\gamma}^{2} \mathrm{x}_{\eta}^{2} \otimes x_{\tau} x_{\beta}-q_{\alpha \delta} q_{\alpha \gamma}^{2} q_{\alpha \eta}^{2} \mathrm{x}_{\delta} \mathrm{x}_{\gamma}^{2} \mathrm{x}_{\eta}^{2} \otimes x_{\alpha} .
\end{aligned}
$$

Next we compute (10.1.138) using (10.1.124), (10.1.86), (10.1.125), (10.1.129), (10.1.130), (10.1.84) and (10.1.131):

$$
\begin{aligned}
& d\left(\mathrm{x}_{\beta} \mathrm{x}_{\tau} \mathrm{x}_{\gamma}^{2} \mathrm{x}_{\kappa} \mathrm{x}_{\eta} \otimes 1\right)=\mathrm{x}_{\beta} \mathrm{x}_{\tau} \mathrm{x}_{\gamma}^{2} \mathrm{x}_{\kappa} \otimes x_{\eta}-s\left(q_{\kappa \eta} \mathrm{x}_{\beta} \mathrm{x}_{\tau} \mathrm{x}_{\gamma}^{2} \otimes x_{\eta} x_{\kappa}-q_{\gamma \kappa} q_{\gamma \eta} \mathrm{x}_{\beta} \mathrm{x}_{\tau} \mathrm{x}_{\gamma} \mathrm{x}_{\kappa} \otimes x_{\eta} x_{\gamma}\right. \\
& -q_{\tau \gamma}^{2} q_{\tau \kappa} \mathrm{b}_{4} \mathrm{x}_{\beta} \mathrm{x}_{\gamma}^{2} \mathrm{x}_{\kappa} \otimes x_{\kappa} x_{\gamma}-q_{\tau \gamma}^{2} q_{\tau \kappa} q_{\tau \eta} \mathrm{x}_{\beta} \mathrm{x}_{\gamma}^{2} \mathrm{x}_{\kappa} \otimes x_{\eta} x_{\tau} \\
& \left.+q_{\beta \tau} q_{\gamma \beta}^{-2}(3)_{\widetilde{q}_{\beta \gamma}} \mathrm{b}_{8} \mathrm{x}_{\tau} \mathrm{x}_{\gamma}^{3} \otimes x_{\eta}+q_{\beta \tau} q_{\beta \gamma}^{2} q_{\beta \kappa} q_{\beta \eta} \mathrm{x}_{\tau} \mathrm{x}_{\gamma}^{2} \mathrm{x}_{\kappa} \otimes x_{\eta} x_{\beta}\right) \\
& =\mathrm{x}_{\beta} \mathrm{x}_{\tau} \mathrm{x}_{\gamma}^{2} \mathrm{x}_{\kappa} \otimes x_{\eta}-q_{\kappa \eta} \mathrm{x}_{\beta} \mathrm{x}_{\tau} \mathrm{x}_{\gamma}^{2} \mathrm{x}_{\eta} \otimes x_{\kappa}+q_{\gamma \kappa} q_{\gamma \eta} \mathrm{x}_{\beta} \mathrm{x}_{\tau} \mathrm{x}_{\gamma} \mathrm{x}_{\kappa} \mathrm{x}_{\eta} \otimes x_{\gamma} \\
& +q_{\tau \gamma}^{2} q_{\tau \kappa}(2)_{\widetilde{q}_{\kappa \eta}} \mathrm{b}_{4} \mathrm{x}_{\beta} \mathrm{x}_{\gamma}^{2} \mathrm{x}_{\kappa}^{2} \otimes x_{\gamma}+q_{\tau \gamma}^{2} q_{\tau \kappa} q_{\tau \eta} \mathrm{x}_{\beta} \mathrm{x}_{\gamma}^{2} \mathrm{x}_{\kappa} \mathrm{x}_{\eta} \otimes x_{\tau}-q_{\beta \tau} q_{\gamma \beta}^{-2}(3)_{\widetilde{q}_{\beta \gamma}} \mathrm{b}_{8} \mathrm{x}_{\tau} \mathrm{x}_{\gamma}^{3} \mathrm{x}_{\eta} \otimes 1 \\
& -q_{\beta \tau} q_{\beta \gamma}^{2} q_{\beta \kappa} q_{\beta \eta} \mathrm{x}_{\tau} \mathrm{x}_{\gamma}^{2} \mathrm{x}_{\kappa} \mathrm{x}_{\eta} \otimes x_{\beta}-q_{\gamma \eta}^{2} q_{\kappa \eta} \mathrm{c}_{-\eta, \beta, \gamma}^{(2)} \mathrm{b}_{4} \mathrm{~b}_{8} \mathrm{x}_{\gamma}^{4} \mathrm{x}_{\kappa} \otimes 1 .
\end{aligned}
$$

For (10.1.139) we use (10.1.86), (10.1.126), (10.1.130), Remark 10.1.2 and (10.1.132):

$$
\begin{aligned}
& d\left(\mathrm{x}_{\beta} \mathrm{x}_{\tau} \mathrm{x}_{\gamma}^{2} \mathrm{x}_{\eta}^{2} \otimes 1\right)=\mathrm{x}_{\beta} \mathrm{x}_{\tau} \mathrm{x}_{\gamma}^{2} \mathrm{x}_{\eta} \otimes x_{\eta}-s\left(-q_{\gamma \eta}^{2} \mathrm{x}_{\beta} \mathrm{x}_{\tau} \mathrm{x}_{\gamma} \mathrm{x}_{\eta} \otimes x_{\eta} x_{\gamma}-q_{\tau \gamma}^{2} q_{\tau \eta}^{2} \mathrm{x}_{\beta} \mathrm{x}_{\gamma}^{2} \mathrm{x}_{\eta} \otimes x_{\eta} x_{\tau}\right. \\
& -q_{\tau \gamma}^{2} q_{\gamma \eta} \mathrm{b}_{4} \mathrm{x}_{\beta} \mathrm{x}_{\gamma}^{2} \mathrm{x}_{\kappa} \otimes x_{\eta} x_{\gamma}-q_{\tau \gamma}^{2} q_{\tau \eta} \mathrm{b}_{4} \mathrm{x}_{\beta} \mathrm{x}_{\gamma}^{2} \mathrm{x}_{\eta} \otimes x_{\kappa} x_{\gamma} \\
& \left.+q_{\beta \tau} q_{\beta \gamma}^{2} q_{\beta \eta}^{2} \mathrm{x}_{\tau} \mathrm{x}_{\gamma}^{2} \mathrm{x}_{\eta} \otimes x_{\eta} x_{\beta}-q_{\gamma \eta}^{2} c_{-\eta, \beta, \gamma}^{(2)} \mathrm{b}_{4} \mathrm{~b}_{8} \mathrm{x}_{\gamma}^{4} \otimes x_{\eta}\right) \\
& =\mathrm{x}_{\beta} \mathrm{x}_{\tau} \mathrm{x}_{\gamma}^{2} \mathrm{x}_{\eta} \otimes x_{\eta}+q_{\gamma \eta}^{2} \mathrm{x}_{\beta} \mathrm{x}_{\tau} \mathrm{x}_{\gamma} \mathrm{x}_{\eta}^{2} \otimes x_{\gamma}+q_{\tau \gamma}^{2} q_{\gamma \eta} \mathrm{b}_{4} \mathrm{x}_{\beta} \mathrm{x}_{\gamma}^{2} \mathrm{x}_{\kappa} \mathrm{x}_{\eta} \otimes x_{\gamma}+q_{\tau \gamma}^{2} q_{\tau \eta}^{2} \mathrm{x}_{\beta} \mathrm{x}_{\gamma}^{2} \mathrm{x}_{\eta}^{2} \otimes x_{\tau} \\
& \quad-q_{\beta \tau} q_{\beta \gamma}^{2} q_{\beta \eta}^{2} \mathrm{x}_{\tau} \mathrm{x}_{\gamma}^{2} \mathrm{x}_{\eta}^{2} \otimes x_{\beta}+q_{\gamma \eta}^{2} c_{-\eta, \beta, \gamma}^{(2)} \mathrm{b}_{4} \mathrm{~b}_{8} \mathrm{x}_{\gamma}^{4} \otimes x_{\eta} .
\end{aligned}
$$

The proof of (10.1.140) is similar, using in this case (10.1.127), (10.1.128), Remark 10.1.2, (10.1.130) and (10.1.133):

$$
\begin{aligned}
& d\left(\mathrm{x}_{\beta} \mathrm{x}_{\delta} \mathrm{x}_{\gamma}^{2} \mathrm{x}_{\eta}^{2} \otimes 1\right)=\mathrm{x}_{\beta} \mathrm{x}_{\delta} \mathrm{x}_{\gamma}^{2} \mathrm{x}_{\eta} \otimes x_{\eta}-s\left(-q_{\gamma \eta}^{2} \mathrm{x}_{\beta} \mathrm{x}_{\delta} \mathrm{x}_{\gamma} \mathrm{x}_{\eta} \otimes x_{\eta} x_{\gamma}-q_{\delta \gamma}^{2} q_{\delta \eta} \mathrm{b}_{3} \mathrm{x}_{\beta} \mathrm{x}_{\gamma}^{2} \mathrm{x}_{\eta} \otimes x_{\gamma} x_{\nu}\right. \\
& \quad-q_{\delta \gamma}^{2} q_{\delta \eta} \mathrm{b}_{2} \mathrm{x}_{\beta} \mathrm{x}_{\gamma}^{2} \mathrm{x}_{\eta} \otimes x_{\kappa} x_{\gamma} x_{\mu}-q_{\delta \gamma}^{2} q_{\delta \eta}^{2} \mathrm{x}_{\beta} \mathrm{x}_{\gamma}^{2} \mathrm{x}_{\eta} \otimes x_{\eta} x_{\delta}-q_{\delta \gamma}^{2} q_{\mu \eta} q_{\gamma \eta} \mathrm{b}_{2} \mathrm{x}_{\beta} \mathrm{x}_{\gamma}^{2} \mathrm{x}_{\kappa} \otimes x_{\eta} x_{\gamma} x_{\mu} \\
& \quad-q_{\delta \gamma}^{2}(3)_{\widetilde{q}_{\gamma \eta}} \mathrm{b}_{3} \mathrm{~b}_{7} \mathrm{x}_{\beta} \mathrm{x}_{\gamma}^{3} \otimes x_{\iota} x_{\kappa} x_{\gamma}-q_{\delta \gamma}^{2} q_{\nu \eta}(3)_{\widetilde{q}_{\gamma \eta}} \mathrm{b}_{3} \mathrm{x}_{\beta} \mathrm{x}_{\gamma}^{3} \otimes x_{\eta} x_{\nu}+q_{\beta \delta} q_{\beta \gamma}^{2} q_{\beta \eta}^{2} \mathrm{x}_{\delta} \mathrm{x}_{\gamma}^{2} \mathbf{x}_{\eta} \otimes x_{\eta} x_{\beta} \\
& \left.\quad-q_{\beta \gamma} q_{\gamma \beta}^{-1} q_{\delta \gamma}^{2} c_{\beta \eta}^{(3)} \mathrm{b}_{2} \mathrm{~b}_{6} \mathrm{~b}_{8} \mathrm{x}_{\gamma}^{4} \otimes x_{\iota} x_{\gamma}-q_{\beta \gamma} q_{\gamma \beta}^{-1} q_{\delta \gamma}^{2} c_{\beta \eta}^{(3)} q_{\mu \eta} \mathrm{b}_{2} \mathrm{~b}_{8} \mathrm{x}_{\gamma}^{4} \otimes x_{\eta} x_{\mu}\right)
\end{aligned}
$$




$$
\begin{aligned}
= & \mathrm{x}_{\beta} \mathrm{x}_{\delta} \mathrm{x}_{\gamma}^{2} \mathrm{x}_{\eta} \otimes x_{\eta}+q_{\gamma \eta}^{2} \mathrm{x}_{\beta} \mathrm{x}_{\delta} \mathrm{x}_{\gamma} \mathrm{x}_{\eta}^{2} \otimes x_{\gamma}+q_{\delta \gamma}^{2}(3)_{\widetilde{q}_{\gamma \eta}} \mathrm{b}_{3} \mathrm{~b}_{7} \mathrm{x}_{\beta} \mathrm{x}_{\gamma}^{3} \mathrm{x}_{\iota} \otimes x_{\kappa} x_{\gamma} \\
& +q_{\delta \gamma}^{2} q_{\nu \eta}(3)_{\widetilde{q}_{\gamma \eta}} \mathrm{b}_{3} \mathrm{x}_{\beta} \mathrm{x}_{\gamma}^{3} \mathrm{x}_{\eta} \otimes x_{\nu}+q_{\delta \gamma}^{2} q_{\mu \eta} q_{\gamma \eta} \mathrm{b}_{2} \mathrm{x}_{\beta} \mathrm{x}_{\gamma}^{2} \mathrm{x}_{\kappa} \mathrm{x}_{\eta} \otimes x_{\gamma} x_{\mu} \\
& +q_{\delta \gamma}^{2} q_{\delta \eta}^{2} \mathrm{x}_{\beta} \mathbf{x}_{\gamma}^{2} \mathrm{x}_{\eta}^{2} \otimes x_{\delta}-q_{\beta \delta} q_{\beta \gamma}^{2} q_{\beta \eta}^{2} \mathrm{x}_{\delta} \mathrm{x}_{\gamma}^{2} \mathrm{x}_{\eta}^{2} \otimes x_{\beta} \\
& -q_{\beta \gamma} q_{\gamma \kappa} q_{\delta \gamma}^{2} \mathrm{c}_{\beta \eta}^{(3)} \mathrm{b}_{2} \mathrm{~b}_{6} \mathrm{~b}_{8} \mathrm{x}_{\gamma}^{4} \mathrm{x}_{\iota} \otimes x_{\gamma}-q_{\beta \gamma} q_{\gamma \kappa} q_{\delta \gamma}^{2} \mathrm{c}_{\beta \eta}^{(3)} q_{\mu \eta} \mathrm{b}_{2} \mathrm{~b}_{8} \mathrm{x}_{\gamma}^{4} \mathrm{x}_{\eta} \otimes x_{\mu} .
\end{aligned}
$$

Finally we compute (10.1.81). Using (10.1.134), (10.1.135), Remark 10.1.2, (10.1.136), (10.1.137), (10.1.138), (10.1.139), (10.1.140) and (10.1.84):

$$
\begin{aligned}
& d\left(\mathrm{x}_{\alpha} \mathrm{x}_{\beta} \mathrm{x}_{\delta} \mathrm{x}_{\gamma}^{2} \mathrm{x}_{\eta}^{2} \otimes 1\right)=\mathrm{x}_{\alpha} \mathrm{x}_{\beta} \mathrm{x}_{\delta} \mathrm{x}_{\gamma}^{2} \mathrm{x}_{\eta} \otimes x_{\eta}-s\left(-q_{\gamma \eta}^{2} \mathrm{x}_{\alpha} \mathrm{x}_{\beta} \mathrm{x}_{\delta} \mathrm{x}_{\gamma} \mathrm{x}_{\eta} \otimes x_{\eta} x_{\gamma}\right. \\
& -q_{\delta \gamma}^{2}(3)_{\widetilde{q}_{\gamma \eta}} \mathrm{b}_{3} \mathrm{x}_{\alpha} \mathrm{x}_{\beta} \mathrm{x}_{\gamma}^{3} \otimes x_{\nu} x_{\eta}-q_{\delta \gamma}^{2} \mathrm{~b}_{2} \mathrm{x}_{\alpha} \mathrm{x}_{\beta} \mathrm{x}_{\gamma}^{2} \mathrm{x}_{\kappa} \otimes x_{\gamma} x_{\mu} x_{\eta}-q_{\delta \gamma}^{2} q_{\delta \eta} \mathrm{x}_{\alpha} \mathrm{x}_{\beta} \mathrm{x}_{\gamma}^{2} \mathrm{x}_{\eta} \otimes x_{\delta} x_{\eta} \\
& +q_{\beta \delta} q_{\beta \gamma}^{2} q_{\beta \eta} \mathrm{x}_{\alpha} \mathrm{x}_{\delta} \mathrm{x}_{\gamma}^{2} \mathrm{x}_{\eta} \otimes x_{\beta} x_{\eta}-q_{\alpha \beta} q_{\beta \gamma}^{2} q_{\beta \eta} q_{\tau \gamma}^{2} \mathrm{~b}_{1} \mathrm{~b}_{4} \mathrm{x}_{\beta} \mathrm{x}_{\tau} \mathrm{x}_{\gamma}^{2} \mathrm{x}_{\kappa} \otimes x_{\gamma} x_{\beta} x_{\eta} \\
& -q_{\delta \gamma}^{2} q_{\gamma \beta}^{-2} \mathrm{c}_{\beta \eta \gamma}^{(3)} \mathrm{b}_{2} \mathrm{~b}_{8} \mathrm{x}_{\alpha} \mathrm{x}_{\gamma}^{4} \otimes x_{\mu} x_{\eta}-q_{\alpha \beta} q_{\beta \gamma}^{2} q_{\beta \eta} q_{\tau \gamma}^{2} q_{\tau \eta} \mathrm{b}_{1} \mathrm{x}_{\beta} \mathrm{x}_{\tau} \mathrm{x}_{\gamma}^{2} \mathrm{x}_{\eta} \otimes x_{\tau} x_{\beta} x_{\eta} \\
& \left.-q_{\alpha \beta} q_{\alpha \delta} q_{\alpha \gamma}^{2} q_{\alpha \eta} \mathrm{x}_{\beta} \mathrm{x}_{\delta} \mathrm{x}_{\gamma}^{2} \mathrm{x}_{\eta} \otimes x_{\alpha} x_{\eta}+q_{\alpha \beta} q_{\beta \gamma}^{2} q_{\beta \eta} q_{\beta \tau} q_{\gamma \beta}^{-2}(3)_{\widetilde{q}_{\beta \gamma}} q_{\tau \gamma}^{2} \mathrm{~b}_{1} \mathrm{~b}_{4} \mathrm{~b}_{8} \mathrm{x}_{\tau} \mathrm{x}_{\gamma}^{4} \otimes x_{\beta} x_{\eta}\right) \\
& =\mathrm{x}_{\alpha} \mathrm{x}_{\beta} \mathrm{x}_{\delta} \mathrm{x}_{\gamma}^{2} \mathrm{x}_{\eta} \otimes x_{\eta}+q_{\gamma \eta}^{2} \mathrm{x}_{\alpha} \mathrm{x}_{\beta} \mathrm{x}_{\delta} \mathrm{x}_{\gamma} \mathrm{x}_{\eta}^{2} \otimes x_{\gamma}+q_{\delta \gamma}^{2}(3)_{\widetilde{q}_{\gamma \eta}} \mathrm{b}_{3} \mathrm{~b}_{7} \mathrm{x}_{\alpha} \mathrm{x}_{\beta} \mathrm{x}_{\gamma}^{3} \mathrm{x}_{\iota} \otimes x_{\kappa} x_{\gamma} \\
& +q_{\delta \gamma}^{2} q_{\nu \eta}(3)_{\widetilde{q}_{\gamma \eta}} \mathrm{b}_{3} \mathrm{x}_{\alpha} \mathrm{x}_{\beta} \mathrm{x}_{\gamma}^{3} \mathrm{x}_{\eta} \otimes x_{\nu}+q_{\delta \gamma}^{2} q_{\mu \eta} q_{\gamma \eta} \mathrm{b}_{2} \mathrm{x}_{\alpha} \mathrm{x}_{\beta} \mathrm{x}_{\gamma}^{2} \mathrm{x}_{\kappa} \mathrm{x}_{\eta} \otimes x_{\gamma} x_{\mu} \\
& +q_{\delta \gamma}^{2} q_{\delta \eta}^{2} \mathrm{x}_{\alpha} \mathbf{x}_{\beta} \mathrm{x}_{\gamma}^{2} \mathbf{x}_{\eta}^{2} \otimes x_{\delta}-q_{\beta \delta} q_{\beta \gamma}^{2} q_{\beta \eta}^{2} \mathrm{x}_{\alpha} \mathrm{x}_{\delta} \mathrm{x}_{\gamma}^{2} \mathrm{x}_{\eta}^{2} \otimes x_{\beta} \\
& +q_{\delta \gamma}^{2} q_{\gamma \kappa}^{2} \mathrm{c}_{\beta \eta \gamma}^{(3)} \mathrm{b}_{2} \mathrm{~b}_{6} \mathrm{~b}_{8} \mathrm{x}_{\alpha} \mathrm{x}_{\gamma}^{4} \mathrm{x}_{\iota} \otimes x_{\gamma}+q_{\delta \gamma}^{2} q_{\gamma \kappa}^{2} q_{\mu \eta} \mathrm{c}_{\beta \eta \gamma}^{(3)} \mathrm{b}_{2} \mathrm{~b}_{8} \mathrm{x}_{\alpha} \mathrm{x}_{\gamma}^{4} \mathrm{x}_{\eta} \otimes x_{\mu} \\
& +q_{\alpha \beta} q_{\beta \gamma}^{2} q_{\beta \eta}^{2} q_{\tau \gamma}^{2} q_{\gamma \eta} \mathrm{b}_{1} \mathrm{~b}_{4} \mathrm{x}_{\beta} \mathrm{x}_{\tau} \mathrm{x}_{\gamma}^{2} \mathrm{x}_{\kappa} \mathrm{x}_{\eta} \otimes x_{\gamma} x_{\beta}-q_{\alpha \beta} q_{\beta \gamma}^{2} q_{\beta \eta}^{2} q_{\tau \gamma}^{2} q_{\tau \eta}^{2} \mathrm{~b}_{1} \mathrm{x}_{\beta} \mathrm{x}_{\tau} \mathrm{x}_{\gamma}^{2} \mathrm{x}_{\eta}^{2} \otimes x_{\tau} x_{\beta} \\
& +q_{\alpha \beta} q_{\alpha \delta} q_{\alpha \gamma}^{2} q_{\alpha \eta}^{2} \mathrm{x}_{\beta} \mathrm{x}_{\delta} \mathrm{x}_{\gamma}^{2} \mathrm{x}_{\eta}^{2} \otimes x_{\alpha}-q_{\alpha \beta} q_{\beta \tau} q_{\beta \gamma}^{2} q_{\beta \eta}^{2} q_{\gamma \beta}^{-2} q_{\tau \gamma}^{2} c_{\beta \eta \gamma}^{(3)} \mathrm{b}_{1} \mathrm{~b}_{4} \mathrm{~b}_{8} \mathrm{x}_{\tau} \mathrm{x}_{\gamma}^{4} \mathrm{x}_{\eta} \otimes x_{\beta} \\
& -q_{\delta \gamma}^{2} q_{\gamma \kappa}^{2} \mathrm{~d}_{\alpha \beta \eta \gamma}^{(4)} \mathrm{b}_{2} \mathrm{~b}_{6} \mathrm{~b}_{8} \mathrm{~b}_{9} \mathrm{x}_{\gamma}^{6} \otimes 1 .
\end{aligned}
$$

This completes the proof.

\section{REFERENCES}

[AJ] H. H. Andersen and J. C. Jantzen. Cohomology of induced representations for algebraic groups. Math. Ann. 269, pp. 487-525 (1984).

[A] N. Andruskiewitsch. An Introduction to Nichols Algebras: in "Quantization, Geometry and Noncommutative Structures in Mathematics and Physics". A. Cardona, P. Morales, H. Ocampo, S. Paycha, A. Reyes, eds., pp. 135-195, Springer (2017).

[AA] N. Andruskiewitsch and I. Angiono. On Finite dimensional Nichols algebras of diagonal type. Bull. Math. Sci. 7, pp. 353-573 (2017).

[AAG] N. Andruskiewitsch, I. Angiono, A. García Iglesias. Liftings of Nichols algebras of diagonal type I. Cartan type A. Int. Math. Res. Not. IMRN 2017 (9), pp. 2793-2884 (2017).

[AAH] N. Andruskiewitsch, I. Angiono and I. Heckenberger. On Nichols algebras of infinite rank with finite Gelfand-Kirillov dimension. Atti Accad. Naz. Lincei Rend. Lincei Mat. Appl., to appear.

[AAPPW] N. Andruskiewitsch, I. Angiono, Héctor Peña Pollastri, J. Pevtsova, and S. Witherspoon. Cohomology rings of finite-dimensional isolated Nichols algebras of diagonal type, in preparation.

[AC] N. Andruskiewitsch and J. Cuadra. On the structure of (co-Frobenius) Hopf algebras. J. Noncommut. Geom. 7, pp. 83-104 (2013).

[AG] N. Andruskiewitsch and G. A. García. Finite subgroups of a simple quantum group. Compositio Math. 145, pp. 476-500 (2009). 
[AGr] N. Andruskiewitsch and Graña, M. Braided Hopf algebras over non-abelian groups. Bol. Acad. Ciencias (Cordoba) 63, 45-78 (1999).

[ARS] N. Andruskiewitsch, D. E. Radford and H.-J. Schneider, Complete reducibility theorems for modules over pointed Hopf algebras. J. Algebra 324, 2932-2970 (2010).

[AS1] N. Andruskiewitsch and H.-J. Schneider. Lifting of quantum linear spaces and pointed Hopf algebras of order $p^{3}$. J. Algebra 209, pp.658-691 (1998).

[AS2] N. Andruskiewitsch and H.-J. Schneider. Pointed Hopf algebras; in "New directions in Hopf algebras". MSRI series Cambridge Univ. Press; pp. 1-68 (2002).

[AS3] N. Andruskiewitsch and H.-J. Schneider. On the classification of finite-dimensional pointed Hopf algebras. Ann. Math. 171, pp. 375-417 (2010).

[An1] I. Angiono. A presentation by generators and relations of Nichols algebras of diagonal type and convex orders on root systems. J. Eur. Math. Soc. 17, pp. 2643-2671 (2015).

[An2] I. Angiono. On Nichols algebras of diagonal type. J. Reine Angew. Math. 683, pp. 189-251 (2013).

[AnG] I. Angiono and A. García Iglesias. Liftings of Nichols algebras of diagonal type II. All liftings are cocycle deformations. Selecta Math. 25 (2019), to appear.

[Ani] D. J. Anick. On the homology of associative algebras. Trans. Amer. Math. Soc. 296, pp. 641-659 (1986).

[Be] M. Beattie. Duals of pointed Hopf algebras. J. Algebra 262, pp. 54-76 (2003).

$[\mathrm{BKN}]$ B. Boe, J. Kujawa and D. Nakano. Tensor triangular geometry for quantum groups. arXiv: 1702:01289v3 (2019).

[BNPP] C. Bendel, D. K. Nakano, B. J. Parshall and C. Pillen. Cohomology for quantum groups via the geometry of the nullcone. Mem. Amer. Math. Soc. 229 (2014), no. 1077.

[Ben] D. J. Benson. Representations and Cohomology I: Basic Representation Theory of Finite Groups and Associative Algebras. Cambridge University Press (1991).

[BO] P. A. Bergh and S. Oppermann. Cohomology of twisted tensor products. J. Algebra 320, pp. 33273338 (2008).

[CSV] A. Čap, H. Schichl, and J. Vanžura. On twisted tensor products of algebras. Comm. Algebra 23, pp. 4701-4735 (1995).

[CE] H. Cartan, S. Eilenberg. Homological Algebra. Princeton University Press, Princeton, NJ, (1956).

[CoU] S. Cojocaru and V. Ufnarovski. BERGMAN under MS-DOS and Anick's resolution. Discrete Math. and Theoretical Comp. Sci. 1, pp. 139-147 (1997).

[CuH] M. Cuntz and I. Heckenberger. Finite Weyl groupoids of rank three. Trans. Amer. Math. Soc. 364, pp. 1369-1393 (2012).

[DCK] C. De Concini and V. G. Kac. Representations of quantum groups at roots of 1. Progressin Math., 92, pp. 471-506; Basel: Birkhauser (1990).

[DoT] Y. Doi and M. Takeuchi. Multiplication alteration by two-cocycles. The quantum version. Commun. Algebra 22, pp. 5715-5732 (1994).

[Dr] V. G. Drinfeld. Quasi-Hopf algebras. Leningrad Math. J. 1, pp. 1419-1457 (1990).

[Dru1] C. Drupieski. Representations and cohomology for Frobenius-Lusztig kernels. J. Pure Appl. Algebra 215, pp. 1473-1491 (2011).

[Dru2] C. Drupieski. Cohomological finite-generation for finite supergroup schemes. Adv. Math. 288, pp. 1360-1432 (2016).

[ENO] P. Etingof, D. Nikshych and V. Ostrik. Weakly group-theoretical and solvable fusion categories. Adv. Math. 226, pp. 176-205 (2011).

[EO] P. Etingof and V. Ostrik. Finite tensor categories. Mosc. Math. J. 4, pp. 627-654, 782-783 (2004).

[EOW] K. Erdmann, O. Solberg and X. Wang. On the structure and cohomology ring of connected Hopf algebras. J. Algebra 527, pp. 366-398 (2019).

[Ev] L. Evens. The cohomology ring of a finite group. Trans. Amer. Math. Soc. 101, pp. $224-239$ (1961).

[Fa] D. Farkas. The Anick resolution. J. Pure Appl. Algebra 79, pp. 159-168 (1992).

[FN] E. Friedlander and C. Negron. Cohomology for Drinfeld doubles of some infinitesimal group schemes. Alg. Number Th. 12, pp. 1281-1309 (2018).

[FP] E. Friedlander and B. Parshall. On the cohomology of algebraic and related finite groups. Invent. Math. 74, pp. 85-117 (1983). 
[FS] E. Friedlander and A. Suslin. Cohomology of finite group schemes over a field. Invent. Math. 127, pp. 209-270 (1997).

[Ga] G. A. García. Quantum subgroups of $G L_{a, b}(n)$. J. Algebra 324, pp. 1392-1428 (2010).

[GaG] G. A. García and J. A. Gutiérrez. Quantum subgroups of simple twisted quantum groups at roots of one. Trans. Amer. Math. Soc. 370, pp. 3609-3637 (2018).

[GaJ] A. García Iglesias and J. M. Jury Giraldi. Liftings of Nichols algebras of diagonal type III. Cartan type $G_{2}$. J. Algebra 478, pp. 506-568 (2017).

[GK] V. Ginzburg and S. Kumar. Cohomology of quantum groups at roots of unity. Duke Math. J. 69, pp. 179-198 (1993).

[G] E. Golod. The cohomology ring of a finite p-group. Dokl. Akad. Nauk SSSR 235, pp. 703-706 (1959).

[Go] I. G. Gordon. Cohomology of quantized function algebras at roots of unity. Proc. London Math. Soc. (3) 80, pp. 337-359 (2000).

[Gr] M. Graña. A freeness theorem for Nichols algebras. J. Algebra 231, pp. 235-257 (2000).

[H1] I. Heckenberger. The Weyl groupoid of a Nichols algebra of diagonal type. Invent. Math. 164 175-188 (2006).

[H2] I. Heckenberger. Classification of arithmetic root systems. Adv. Math. 220, pp. 59-124 (2009).

[He] M. Helbig. On the lifting of Nichols algebras. Commun. Alg. 40, pp 3317-3351 (2012).

[Ja] J. C. Jantzen. Representations of Algebraic Groups. Mathematical Surveys and Monographs, Vol. 107, 2nd ed., Amer. Math. Soc. (2003).

[Kh] V. Kharchenko. Quantum Lie theory. Lect. Notes Math. 2150 (2015), Springer-Verlag.

[MPSW] M. Mastnak, J. Pevtsova, P. Schauenburg, and S. Witherspoon. Cohomology of finite-dimensional pointed Hopf algebras. Proc. London Math. Soc. (3) 100, pp. 377-404 (2010).

[May] P. May. The cohomology of restricted Lie algebras and of Hopf algebras. J. Algebra 3, pp. 123-146 (1966).

[Mo] S. Montgomery, Hopf Algebras and Their Actions on Rings. CBMS Lectures Vol. 82, Amer. Math. Soc. (1997).

[Mu] M. Müger. From subfactors to categories and topology I. Frobenius algebras in and Morita equivalence of tensor categories. J. Pure Appl. Algebra 180, pp. 81-157 (2003).

[N] C. Negron, Finite generation of cohomology for Drinfeld doubles of finite group schemes, arXiv:2005.14106.

[NPe] C. Negron, J. Pevtsova. Support for integrable Hopf algebras via noncommutative hypersurfaces, arXiv:2005.02965.

[NP] C. Negron, J. Y. Plavnik. Cohomology of finite tensor categories: duality and Drinfeld centers. arXiv: 1807.08854.

[NWW] Van C. Nguyen, X. Wang and S. Witherspoon. Finite generation of some cohomology rings via twisted tensor product and Anick resolutions. J. Pure Appl. Algebra 223, pp. 316-339 (2019).

[R] D. E. Radford. Hopf algebras. Series on Knots and Everything 49. Hackensack, NJ: World Scientific. xxii, 559 p. (2012).

[Re] N. Reshetikhin. Multiparameter quantum groups and twisted quasitriangular Hopf algebras. Lett. Math. Phys. 20, pp. 331-335 (1990).

[SW] A.V. Shepler and S. Witherspoon, Resolutions for twisted tensor products, Pacific J. Math. 298, pp. 445-469 (2019).

[Shr] P. Shroff, Finite generation of the cohomology of quotients of PBW algebras, J. Algebra 390, pp. 44-55 (2013).

[SV] D. Ştefan and C. Vay. The cohomology ring of the 12-dimensional Fomin-Kirillov algebra. Adv. Math. 291, pp. 584-620 (2016).

[V] B. B. Venkov. Cohomology algebras for some classifying spaces. Dokl. Akad. Nauk. SSSR 127, pp. 943-944 (1959).

[Wa] W. Waterhouse. Introduction to Affine Group Schemes, Springer Berlin, Heidelberg (1979).

[Wi] C. Wilkerson, The cohomology algebras of finite-dimensional Hopf algebras, Trans. Amer. Math. Soc. 264 no. 1, 137-150 (1981). 
Famaf-Ciem (COniCet), Universidad Nacional de Córdoba, Medina Allende s/n, Ciudad Universitaria, 5000 Córdoba, República Argentina.

Email address: nicolas.andruskiewitsch@unc.edu.ar

Email address: ivan.angiono@unc.edu.ar

Department of Mathematics University of Washington Seattle, WA 98195, USA

Email address: julia@math.washington.edu

Department of Mathematics Texas A\&M University College Station, TX 77843, USA

Email address: sjw@math.tamu.edu 